\title{
Increasing the potency of dendritic cell based vaccines for the treatment of cancer
}

\author{
Dianne Sika-Paotonu
}

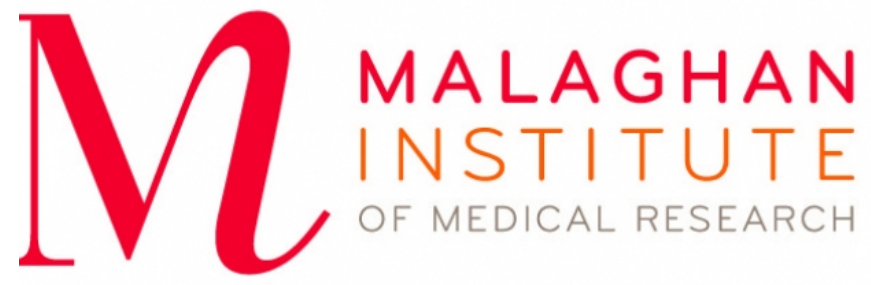

A thesis submitted for the degree of

Doctor of Philosophy at the University of Victoria, Wellington,

New Zealand 
'Oku ou fakamalo lahi ki he 'Otua 'i he 'Ene ngahi tokoni kotoape 'oku fai mai ma'aku.

Pea ki he 'eku tamai 'ofa David Sika, kae 'oua ke tau toe fema àta aki. 


\section{Abstract}

Tumours can be eradicated by $\mathrm{T}$ cells that recognise unique tumour-associated antigens. These $\mathrm{T}$ cells are initially stimulated by dendritic cells (DCs) that have acquired antigens from tumour tissue. Vaccination strategies that increase the frequencies of tumour-specific T cells by enhancing the activity of DCs are being evaluated in the clinic as novel cancer therapies. Our hypothesis is that existing DC-based vaccination strategies can be improved by stimulating toll-like receptor (TLR) signalling in the DCs, and also by encouraging interactions with iNKT cells, as these two activities are known to enhance DC function. It was also hypothesised that superior $\mathrm{T}$ cell responses could be induced by combining these two activities together.

We used the TLR 4 agonist monophosphoryl lipid A (MPL) alone and in combination with other TLR agonists to achieve effective activation of bone marrow-derived DCs (BM-DCs) cultured in-vitro, which was characterised by upregulated expression of maturation markers on the cell surface, and enhanced release of pro-inflammatory cytokines. Some TLR agonist combinations provided significantly enhanced activities in this regard, notably the combination of MPL with either the TLR 2 agonist Pam3Cys, or the TLR 7/8 agonist Resiquimod. Although in-vitro activated BM-DCs were unable to induce stronger antigen-specific $\mathrm{CD}^{+} \mathrm{T}$ cell responses after intravenous injection when compared to BMDCs without TLR stimulation, enhanced $\mathrm{CD}^{+} \mathrm{T}$ cell responses were achieved in-vivo with the co-administration of TLR ligands, implying that TLR stimulation needed to act on cells of the host. 
Further studies identified the langerin-expressing $\mathrm{CD} 8 \alpha^{+}$splenic DC subset in the spleen as recipients of antigen that was transferred from injected cells, and that these recipients were participants in the cross-presentation and $\mathrm{T}$ cell priming activities driving the $\mathrm{CD} 8^{+} \mathrm{T}$ cell response after vaccination.

Antigen-loaded BM-DCs carrying the NKT cell ligand $\alpha$-galactosylceramide ( $\alpha$-GalCer) were found to consistently increase antigen-specific $\mathrm{CD}^{+} \mathrm{T}$ cell responses in-vivo, and also cytotoxic responses as seen in cytotoxic killing assays. Again, langerin-expressing $\mathrm{CD} 8 \alpha^{+}$splenic DCs were shown to be involved in this response by acquiring antigen and $\alpha$-GalCer from the injected vaccine BM-DCs.

Finally, it was possible to achieve even greater $\mathrm{CD} 8^{+} \mathrm{T}$ cell responses in-vivo by injecting BM-DCs carrying antigen and $\alpha$-GalCer, together with timely co-administration of the TLR agonist. These results suggest a reassessment of the activities of DC-based vaccines to include the important role of "courier" to DCs already resident in the host that can be exploited to improve vaccination outcomes 


\section{Acknowledgements}

I would like to express sincere thanks to my primary supervisor Associate Professor Ian Hermans for his guidance during the course of this research project and for advice regarding the construction of this thesis. I also wish to recognise the support afforded me by Dr Troels Petersen particularly at the beginning of this research project. I would like to thank Associate Professor Franca Ronchese for her support and co-supervision of my work and extend my gratitude also to Professor Graham LeGros for the careful management of my $\mathrm{PhD}$ programme during my time at the Malaghan Institute of Medical Research - I wish you all the very best for the future.

I would like to thank my family Reno, Alisha, Mum and Dad (24 $4^{\text {th }}$ April 2006), Oli and Joey for your unconditional love, patience, unwavering support and encouragement, without which I would not have managed to achieve my goals. Please know that without any of you I could not fully appreciate any successes and I truly thank-you all again for always being there for me. Alisha you have grown up so quickly and I have so appreciated your patience with me while working on this project - I love you always and am so proud of the young woman you have become. I look forward to seeing what the future holds for you. To my dearest friend Api, words cannot express the gratitude I have for the care and support you have freely given to me over the years. You have been a light to many a $\mathrm{PhD}$ student and staff member at the Malaghan Institute over the years and it has been a privilege to call you friend. To Moana \& Ma'ave, Ofa, Alani, Jonathen and Neomai, Angie and Tuliasi I thank-you all for your support also and to Associate Professor Lumanavao Winnie Laban, I sincerely thank-you also for your friendship, invaluable support and encouragement. Ofa lahi atu.

To Peter Ferguson, Collin Brooks, Evelyn Bauer, Martin Hunn and the remainder of the IH group, I extend my gratitude to you for your support and friendship over the years and to my Malaghan office colleagues, I've certainly enjoyed your company and sincerely wish you all the best with your future endeavours - Joel Ma, Sabine Kuhn, Steffi Steiger, Misha Walton, Yen Yen Yee, Helen Martinen and Kylie Quinn to name a few. 
To Susie, I thank-you for your kindness and to Patrizia Stoitzner and Martin Hunn I thankyou also for your expert assistance with the RFA work. I also wish to acknowledge Kylie Price for her flow cytometry assistance and the BRU facility staff for their help and support with my various projects over the years. To the talented Mike Zablocki and Andrew Hamer-Jones, I sincerely thank each of you for your assistance with solving my computer complications and keeping my work safe. To all the Malaghan PhD students and staff past and present, I thank-you for your friendship and wish you all the best with future plans.

I wish to acknowledge and express my deepest appreciation to my colleagues at Victoria University of Welllington's Graduate School of Nursing, Midwifery \& Health based at Wellington Hospital. In particular I would like to thank Professor Jo Walton, Belinda Tuari and Billie-Jean, Professor Tim Maling, Dr Kathy Nelson, Dr Sara Quirke, Helen Rook, Caz Hales, Abbey McDonald, Annaliese Ellis, Dr Kay De Vries, Jan Westrate, Professor Mo Coombes, Dr Brian Robinson, Dr Annemarie Jutel, Richard Jenkins, Dr Robyn Maude, Dr Joan Skinner, Pam Green, Natalie Lindsay and Chris Fox. To my students past and present, I thank-you for your commitment to learning and wish you all the very best with your future studies.

To the Victoria University of Wellington staff I have known over the years particularly Patricia Stein, Professor Bill Jordan, Dr Paul Teesdale-Spittle, Mary Murray, Celia Simpson, Judith Bagley, Tara Fisher, Professor Phil Lester, the Pasifika team at VUW Rochelle, Ruth, Maggie, Gail, and the others who supported or assisted with my PhD programme at VUW, I thank-you for your help and guidance whilst at VUW. To my wider family, the Wellington SDA Pathfinders and Wellington City SDA Community Church, the ASANZ team, Dan Augsburger, Jake Ormsby, Hufanga Fui, David Bertelsen, Mel Trevena, I also thank-you and your families for your support and friendship over the years.

I kindly wish to acknowledge and extend my thanks to the Health Research Council of New Zealand and the Pacific Health Research committee, the Cancer Society and Wellington Medical Research Foundation for funding and giving me an opportunity to carry out my $\mathrm{PhD}$ research.

Ki he Eiki ‘Otua, Malo. 


\section{List of Publications}

Publications arising from this work and presented in this thesis are listed below:

1. Petersen, T. R., Sika-Paotonu, D., Knight, D. A., Dickgreber, N., Farrand, K. J., Ronchese, F., Hermans, I. F. (2010): Potent anti-tumour responses to immunization with dendritic cells loaded with tumour tissue and an NKT cell ligand. Immunology and Cell Biology. 88, 596-604, July 2010.

2. Petersen, T. R., Sika-Paotonu, D., Knight, D. A., Dickgreber, N., Simkins, H. M., Hermans, I. F. Exploiting the Role of Endogenous Lymphoid-Resident Dendritic Cells in the Priming of NKT Cells and $\mathrm{CD} 8^{+} \mathrm{T}$ Cells to Dendritic Cell-Based Vaccines. PLoS ONE. 6 March 2011, e17657.

Other publications contributed to during the course of my $\mathrm{PhD}$ :

1. Sika-Paotonu, D., Bethwaite, P. B., McCredie, M. R., Jordan, W. T., Delahunt, B. (2006): Nucleolar grade but not Fuhrman grade is applicable to renal cell carcinoma. American Journal of Surgical Pathology. 30 (9) 1091-1096.

2. Sika-Paotonu, D., Bethwaite, P. B., McCredie, M. R., Jordan, W. T., Delahunt, B. (2006): Nucleolar grade but not Fuhrman grade is applicable to renal cell carcinoma. American Journal of Surgical Pathology. 30 (9) 1091-1096.

Selected for republication in the Urological Survey section of Journal of Urology (2007). This highlights 5 to 10 influential publications per issue.

3. Delahunt, B., Sika-Paotonu, D., Bethwaite, P. B., McCredie, M. R., Martignoni, G., Eble, J. N., Jordan, W. T. (2007) Fuhrman grading is not appropriate for chromophobe renal cell carcinoma. American Journal of Surgical Pathology. 31 957-960.

4. Delahunt, B., Srigley, J. R., D., Bethwaite, P. B., Miller, R. J., Sika-Paotonu, D. (2010): Re Fuhrman grade provides higher prognostic accuracy than nucleolar grade for papillary renal cell carcinoma, Letter to the Editor. Journal of Urology 2010. 183: 2143-2147.

5. Delahunt, B., Sika-Paotonu, D., Bethwaite, Jordan, W. T., P. B., Magi-Galluzzi, C., Zhou, M., Samaratunga, H., Srigley, J. R. (2011) Grading of clear cell renal carcinoma should be based upon nucleolar prominence. American Journal of Surgical Pathology. 35(8): 1134-1139. 


\section{Permissions}

The following permissions have been sought and kindly granted for the use of selected images within this thesis document:

Page 5. Figure 1.1 Mummified head of Ramses V. Image (C) Mr Sandro Vannini, Italy and used with permission.

Page 8. Figure 1.2 Lady Mary Wortley Montague (1689-1762) wife of Lord Edward Wortley Montagu the British Ambassador to the Ottoman Empire. Portrait artist Christian Friedrich Zincke credit (C) National Portrait Gallery, London and used with permission.

Page 12. Figure 1.3 Dr Edward Jenner (1749-1823) considered the Father of Immunology for his research into vaccination to protect against disease. Portrait artist James Henry Lynch credit (C) National Portrait Gallery, London and used with permission.

Page 14. Figure 1.4 The hand of Sarah Nelmes the milkmaid showing her cowpox lesions. Picture Courtesy of the Historical Medical Library of The College of Physicians of Philadelphia and used with permission.

Page 19. Figure 1.5 Dr William B Coley $(1862$ - 1936) the "Father of Immunotherapy" and producer of Coley's toxins. Portrait courtesy of Cancer Research Institute (CRI) NY, USA and used with permission. 


\section{Table of Contents}

CHAPTER 1: GENERAL INTRODUCTION........................................................ 1

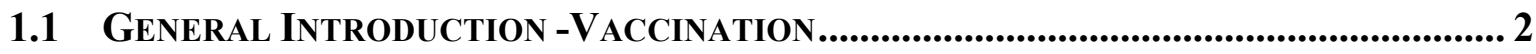

\subsection{History OF VACCINATION; FROM COWS AND MILKMAIDS TO A NEW}

TREATMENT MODALITY FOR CANCER.................................................................... 4

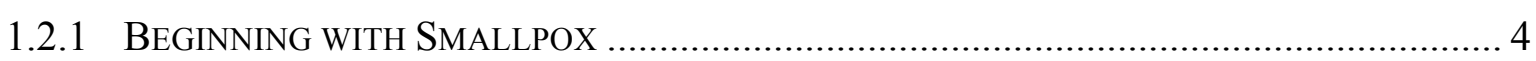

1.2.2 EARLY ATTEMPTS AT PROPHYLACTIC VACCINATION .............................................. 6

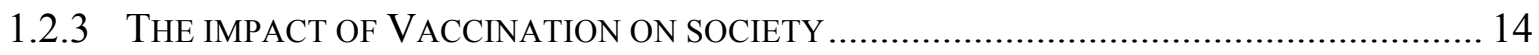

1.2.4 ORIGINS OF THERAPEUTIC VACCINATION TO TREAT CANCER ................................... 16

1.3 VACCINATION AND THE IMMUNE SYSTEM .............................................................. 21

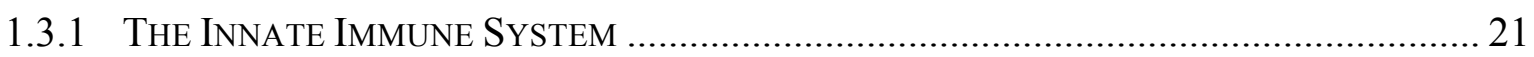

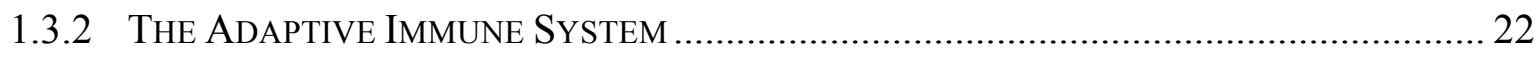

1.4 DCS AND THE ADAPTIVE IMMUNE RESPONSE ........................................................ 24

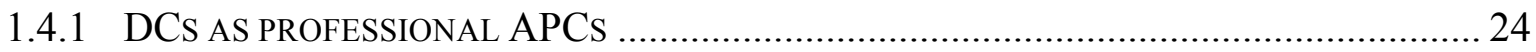

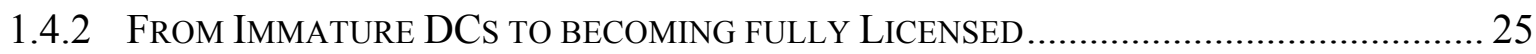

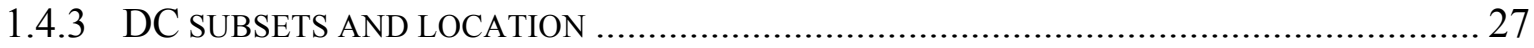

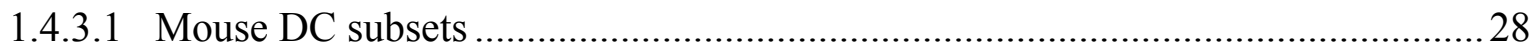

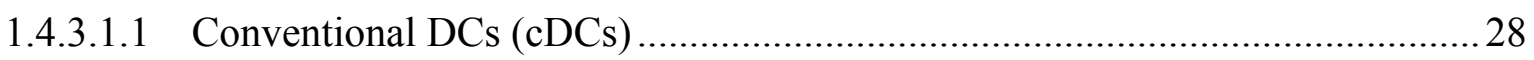

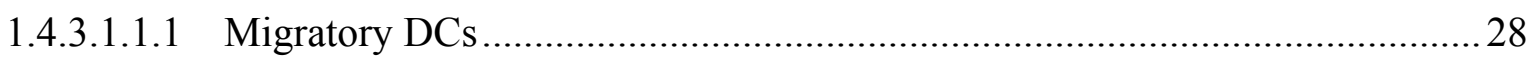

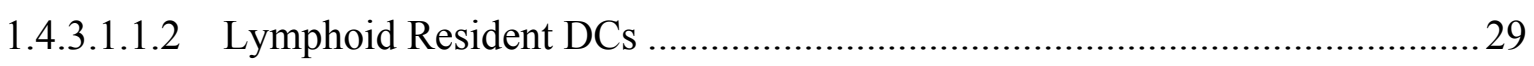

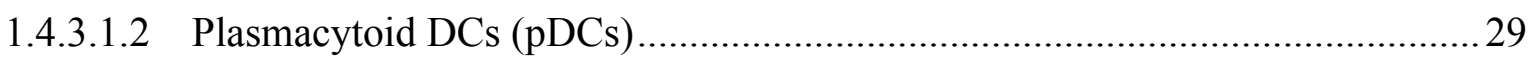

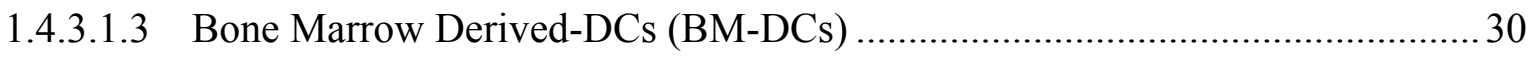

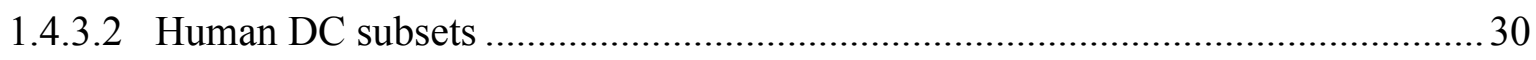

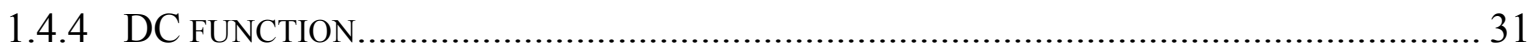

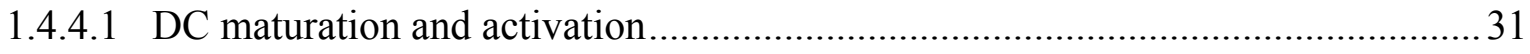

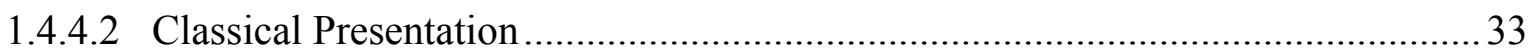

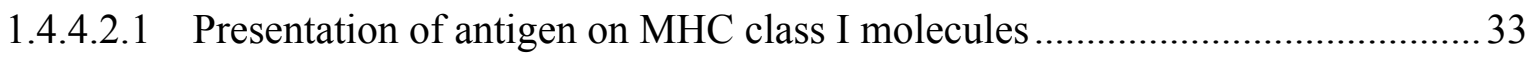

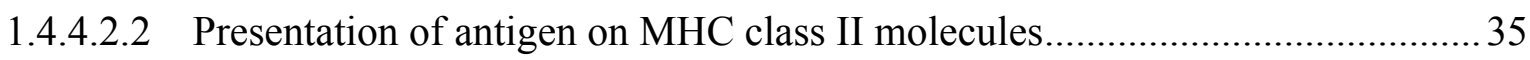

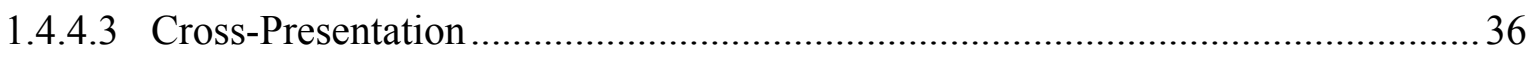

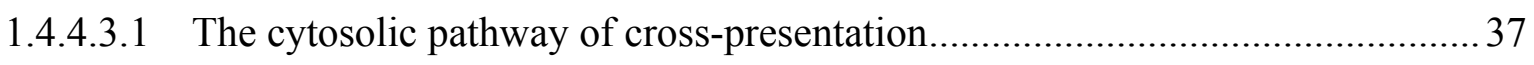

1.4.4.3.2 The ER-phagosome fusion pathway of cross-presentation ............................... 37 


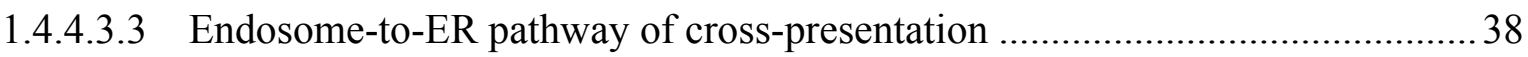

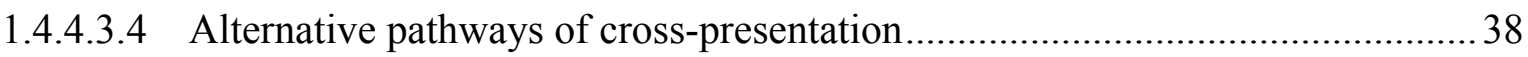

1.4.4.3.5 Preferential Cross presentation capability of DC subsets..................................39

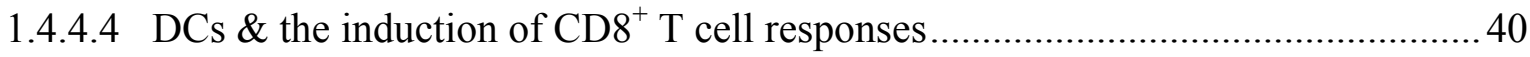

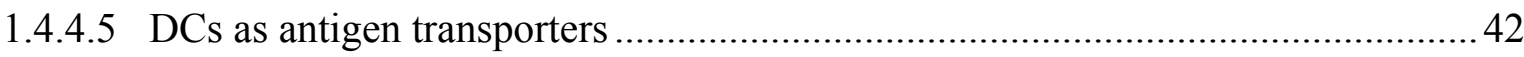

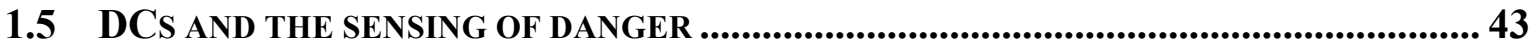

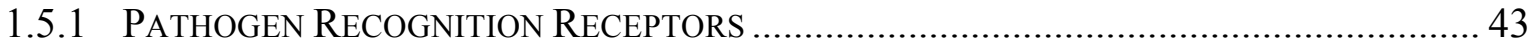

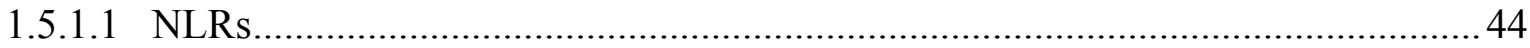

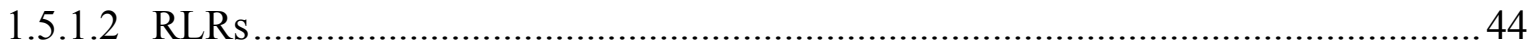

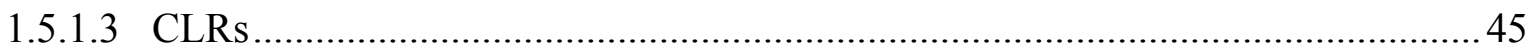

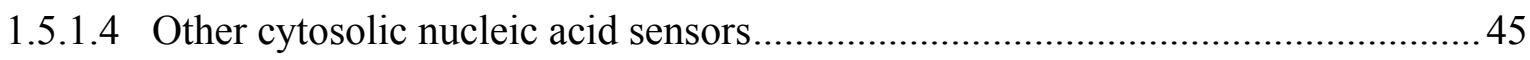

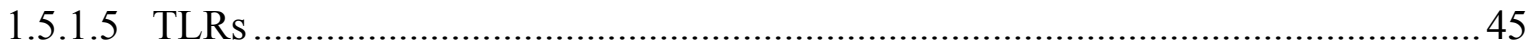

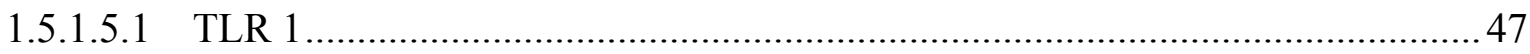

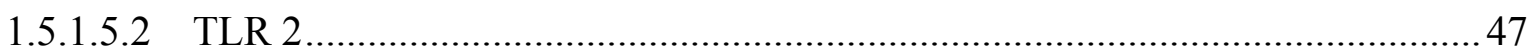

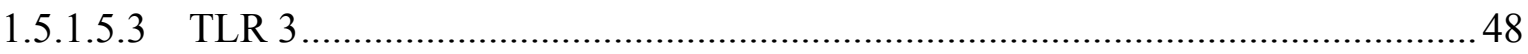

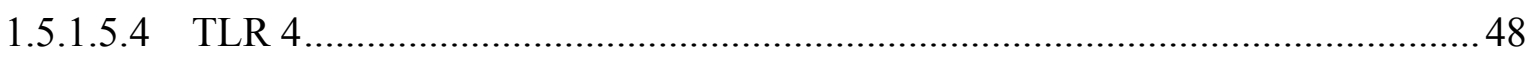

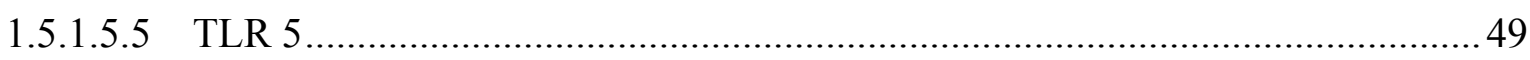

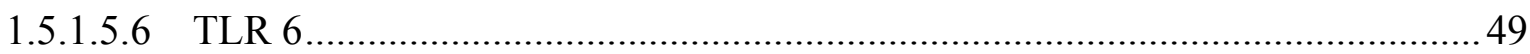

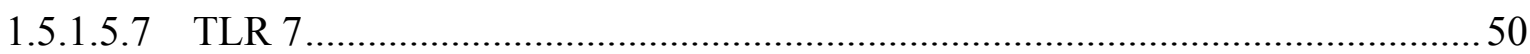

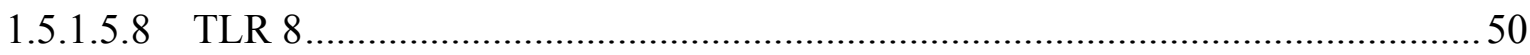

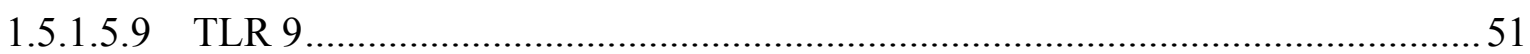

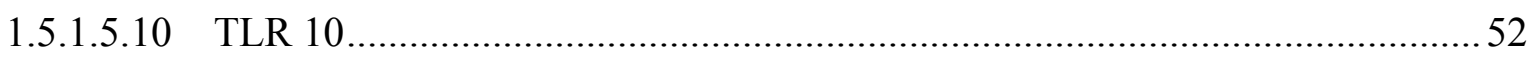

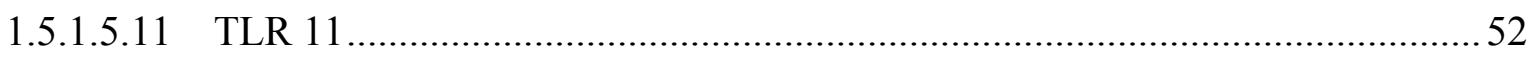

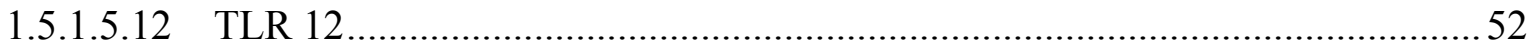

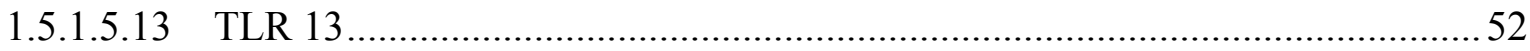

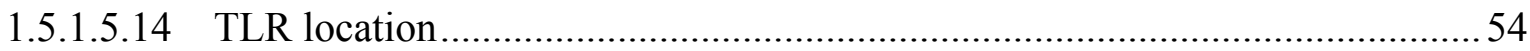

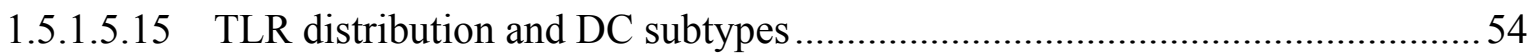

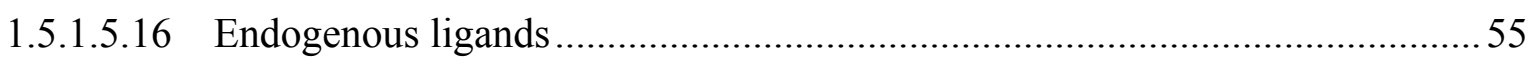

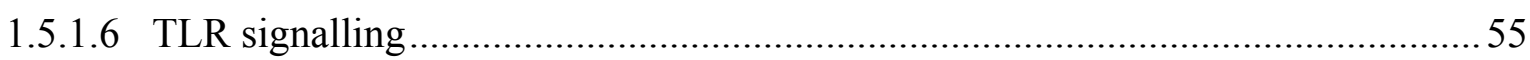

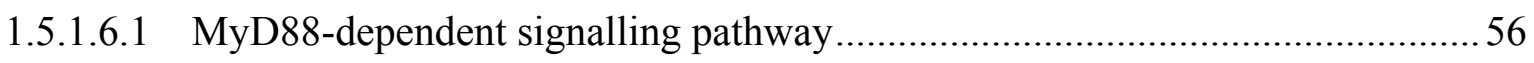

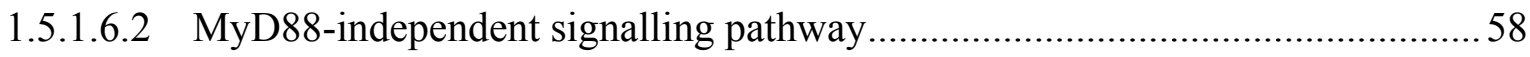

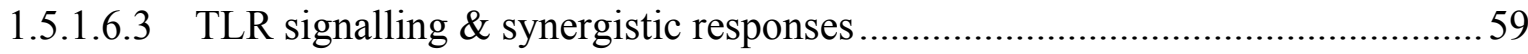




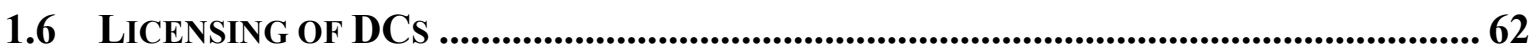

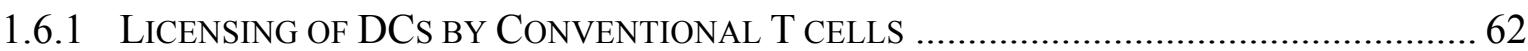

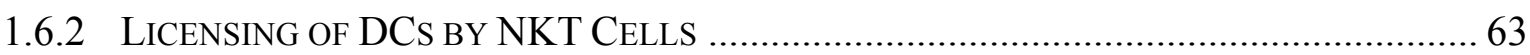

1.7 DENDRITIC CELL-BASED VACCINES \& CANCER ........................................................ 68

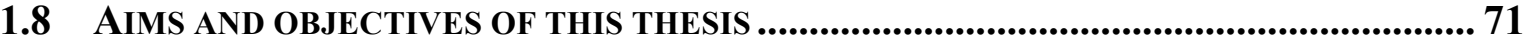

CHAPTER 2: MATERIALS \& METHODS ........................................................... 73

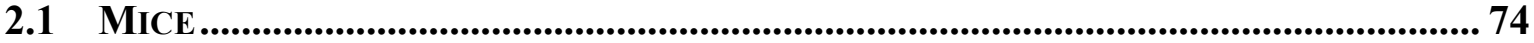

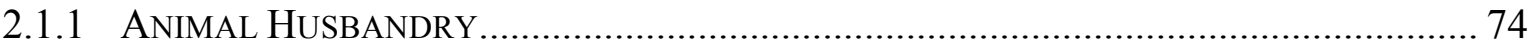

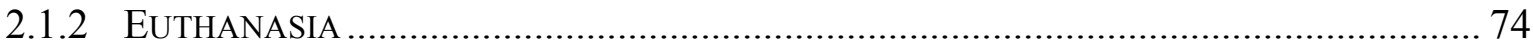

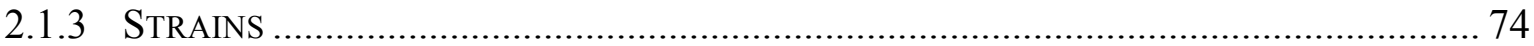

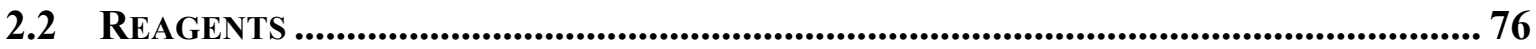

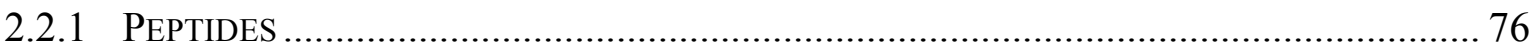

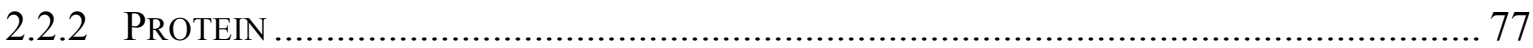

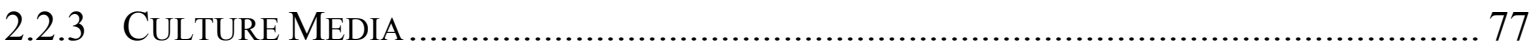

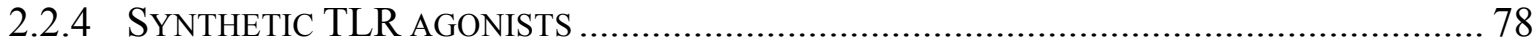

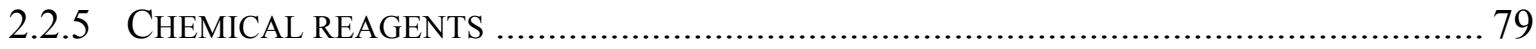

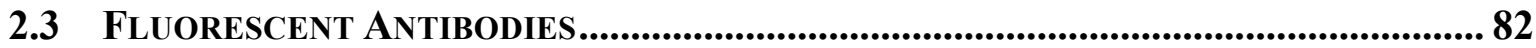

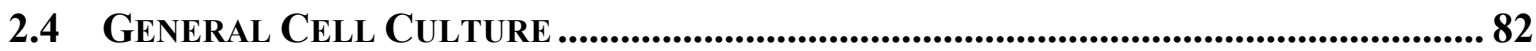

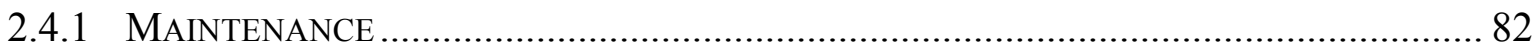

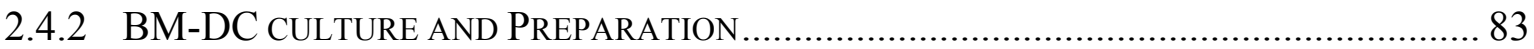

2.4.3 NIH3T3 CD40L EXPRESSING FIBROBLAST CELL LINE ........................................... 83

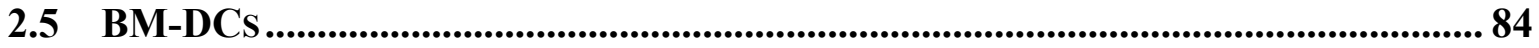

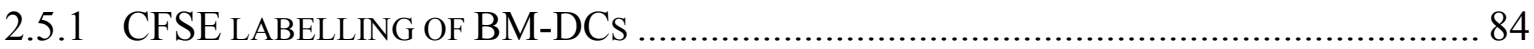

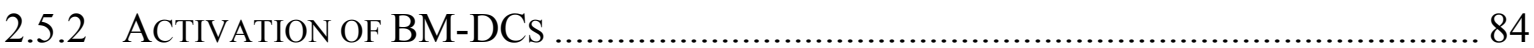

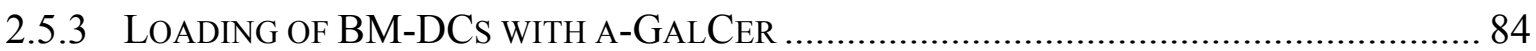

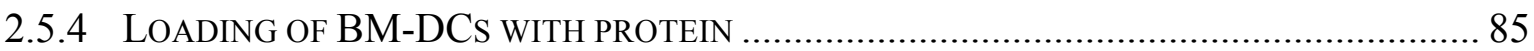

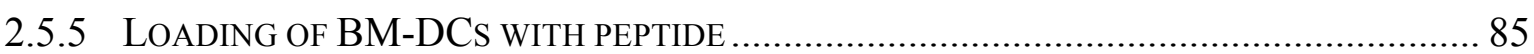

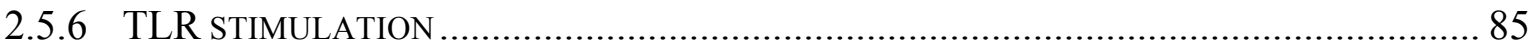

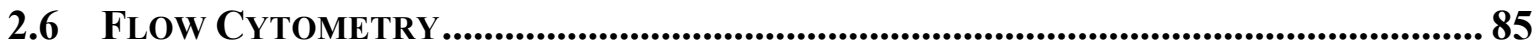

2.6.1 FLUORESCENCE ANTIBODY LABELLING OF CELLS ..................................................... 85 


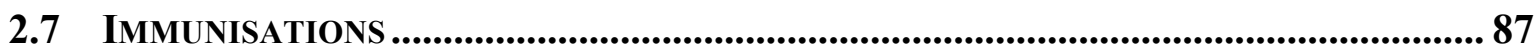

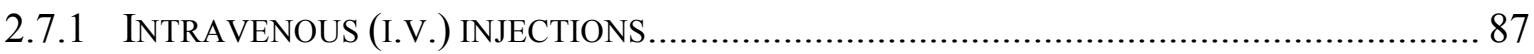

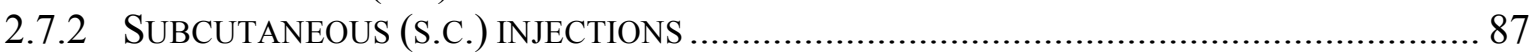

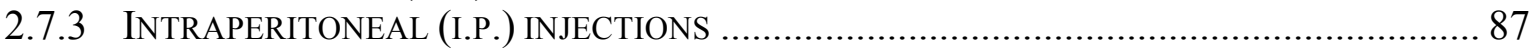

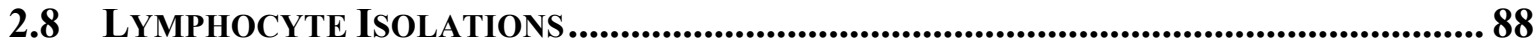

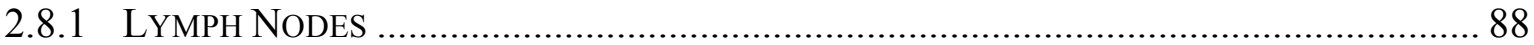

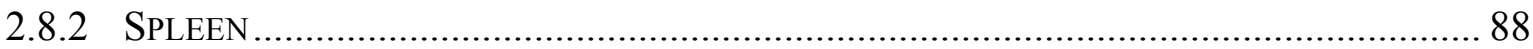

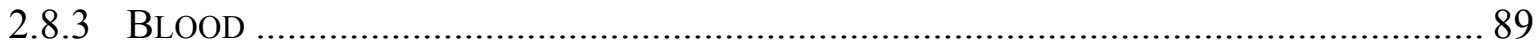

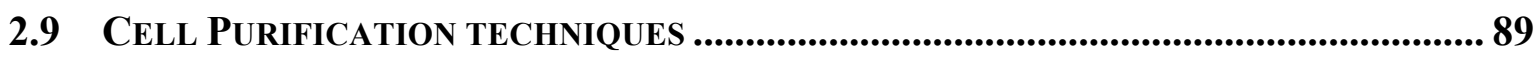

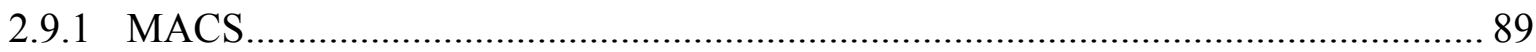

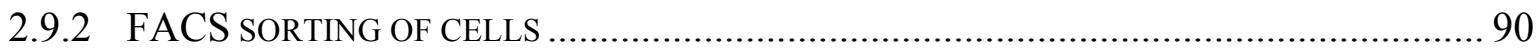

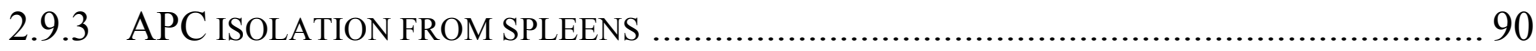

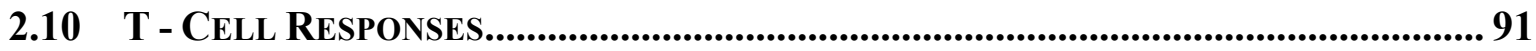

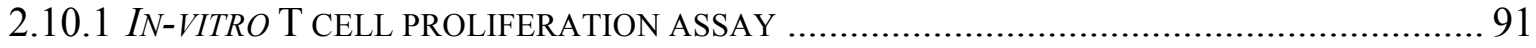

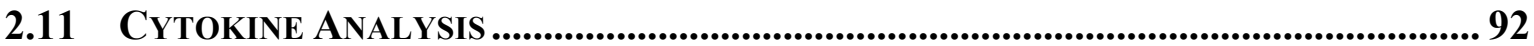

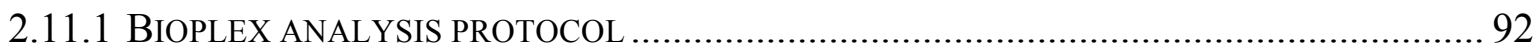

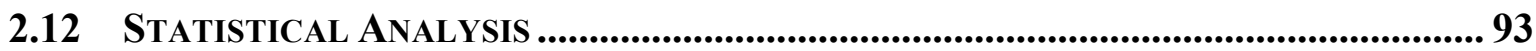

\section{CHAPTER 3: TLR STIMULATION ENHANCES ANTIGEN}

SPECIFIC RESPONSES TO DC-BASED VACCINES ..................... 94

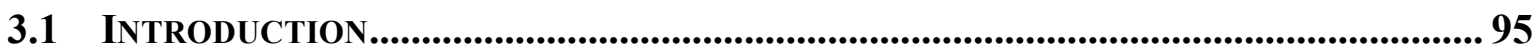

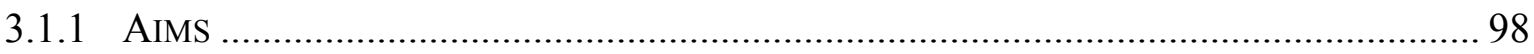

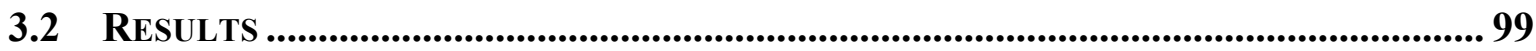

3.2.1 MPL STIMUlation IndUCES DC Maturation in Bone MarRow-Derived DENDRITIC CELL (BM-DC) CULTURES IN-VITRO ................................................... 99

3.2.2 THE UTILIZATION OF TLR AGONISTS FOR EFFECTIVE STIMULATION OF BM-

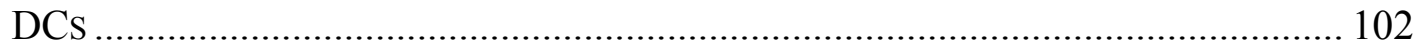

3.2.3 SPECIFIC MULTIPLE TLR AGONIST COMBINATIONS ENHANCE BM-DC

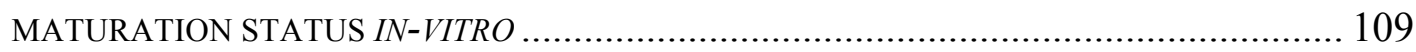

3.2.4 THE INFLUENCE OF CD40 STIMULATION ON BM-DC FUNCTION AFTER TLR

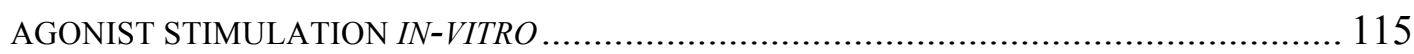

3.2.5 TLRS ALONE AND IN COMBINATION STIMULATE PEPTIDE LOADED BM-DCS TO INDUCE SUPERIOR T CELL RESPONSES IN-VITRO ............................................... 122

3.2.6 SPECIFIC TLR AGONISTS ALONE AND IN COMBINATION PROMOTE ANTIGEN PRESENTATION BY PROTEIN LOADED BM-DCS IN-VITRO....................................... 126

3.2.7 THE GENERATION OF IN-VIVO ANTIGEN SPECIFIC RESPONSES AGAINST TLR TREATED DC VACCINES 
3.2.8 TLR STIMULATED DC VACCINES DID NOT IMPROVE ANTIGEN SPECIFIC T CELL RESPONSES IN-VIVO

3.2.9 THE IN-VITRO MANIPULATION OF BM-DCS DOES NOT CORRELATE WITH MATURITY STATUS AFTER $I V$ ADMINISTRATION

3.2.10 SYSTEMIC ADMINISTRATION OF TLR AGONISTS ALONE AND IN COMBINATION IMPROVES MATURATION STATUS OF HOST DC POPULATIONS.

3.2.11 TLR STIMULATION TARGETING HOST DC POPULATIONS IMPROVES ANTIGEN SPECIFIC T CELL RESPONSES IN-VIVO.

3.2.12 THE TIMING OF SYSTEMIC TLR STIMULATION ….................................................. 142

3.2.13 SYSTEMIC TLR STIMULATION IMPROVES OVA-SPECIFIC T CELL RESPONSES........... 145

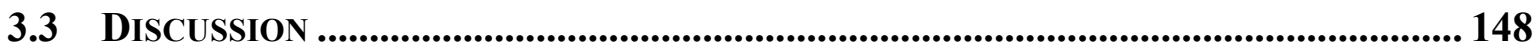

3.3.1 TLR STIMULATION IN-VITRO USING MPL AND OTHER LONE TLR AGONISTS ............ 148

3.3.2 TLR STIMULATION IN-VITRO USING MULTIPLE TLR AGONISTS ............................... 150

3.3.3 AMPLIFICATION OF CYTOKINE RESPONSES USING CD40L AFTER DUAL TLR

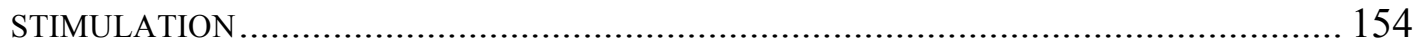

3.3.4 ANTIGEN SPECIFIC T CELL RESPONSES AFTER TLR TREATMENT OF BM-DCs ......... 157

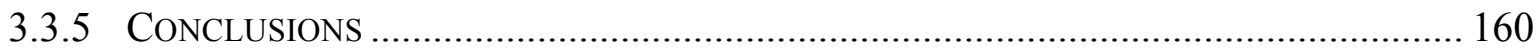

\section{CHAPTER 4: THE ROLE OF LANGERIN ${ }^{+}$CD8 $\alpha^{+}$DCS IN}

ANTIGEN PROCESSING AND T CELL PRIMING

ACTIVITIES

4.1 INTRODUCTION

4.1.1 AIMS

4.2 RESULTS

4.2.1 The EXISTENCE OF LANGERIN EXPRESSING CD $8 \alpha^{+}$DC SUB POPULATIONS IN THE MOUSE SPLEEN.

4.2.2 SELECTIVE DEPLETION OF LANGERIN ${ }^{+} \mathrm{CD} 8 \alpha^{+}$DCS IN LANGERIN-DTREGFP RECIPIENTS DOES NOT ADVERSELY AFFECT OTHER SPLENIC DC POPULATIONS

4.2.3 SYSTEMIC TLR STIMULATION TARGETS LANGERIN ${ }^{+} \mathrm{CD} 8 \alpha^{+} \mathrm{DC}$ POPULATIONS IN THE SPLEEN ....

4.2.4 ADJUVANT EFFECT OF SYSTEMIC MPL ADMINISTRATION IS NOT DUE TO TLR4 MEDIATED STIMULATION OF INJECTED VACCINE BM-DCS AFTER INVIVO TESTING ....

4.2.5 LANGERIN ${ }^{+}$CD $8 \alpha^{+}$DC POPULATIONS RESPOND TO $I V$ TLR STIMULATION ............... 178

4.2.6 LANGERIN $^{+} \mathrm{CD} 8 \alpha^{+}$DCS ARE RECIPIENTS OF PROTEIN ANTIGEN TRANSFERRED FROM PROTEIN LOADED VACCINE BM-DCS.

4.3 Discussion 183

4.3.1 CHARACTERIZATION OF THE MAJOR SPLENIC DC SUBSETS. 183 
4.3.2 THE UTILIZATION OF DT FOR CELLULAR DEPLETION WITHIN TRANSGENIC MODELS

4.3.3 THE ADJUVANT IMPACT OF SYSTEMIC MPL STIMULATION IS MEDIATED BY LANGERIN $^{+} \mathrm{CD} 8 \alpha^{+}$DCS IN-VIVO. 186

4.3.4 THE TRANSFER OF PROTEIN ANTIGEN TO LANGERIN ${ }^{+} \mathrm{CD} 8 \alpha^{+}$DCS SPLENIC DC POPULATIONS. 188

4.3.5 CONCLUSIONS 191

\section{CHAPTER 5: NKT STIMULATION ENHANCES ANTIGEN} SPECIFIC RESPONSES TO DC-BASED VACCINES

5.1 INTRODUCTION

5.1.1 AIMS 195

5.2 ReSUltS 196

5.2.1 NKT STIMULATION INCREASES ANTIGEN SPECIFIC T CELL RESPONSES IN-VIVO ........ 196

5.2.2 NKT STIMULATION IMPROVES ANTIGEN SPECIFIC T CELL RESPONSES TO PEPTIDE ANTIGEN LOADED BM-DC VACCINES.................................................... 200

5.2.3 SUBCUTANEOUS ADMINISTRATION OF $\alpha$-GALCER INDUCES INCREASED

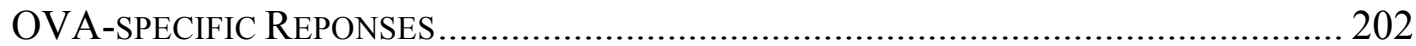

5.2.4 THE FATE OF $\alpha$-GALCER LOADED BM-DCS AFTER $I V$ ADMINISTRATION .................. 204

5.2.5 DIFFERENTIAL MATURATION OF HOST SPLENIC DC POPULATIONS IN

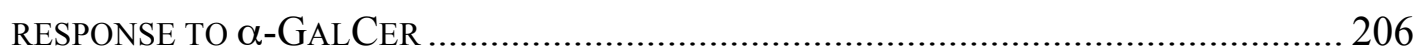

5.2.6 THE LOADING OF $\alpha$-GALCER IN-VITRO ONTO VACCINE BM-DCS COMPARED WITH SYSTEMIC ADMINISTRATION

5.2.7 LANGERIN ${ }^{+} \mathrm{CD} 8 \alpha^{+}$DCs MEDIATE THE INCREASED CD ${ }^{+}$T CELL RESPONSE TO $\alpha$-GALCER CONTAINING VACCINES (VIA ANTIGEN AND GLYCOLIPID TRANSFER).

5.2.8 ANTIGEN AND GLYCOLIPID TRANSFER FROM INJECTED VACCINE BM-DCS TO RESIDENT DC POPULATIONS

5.2.9 TLR STIMULATION DID NOT IMPROVE THE ACTION OF $\alpha$-GALCER LOADED

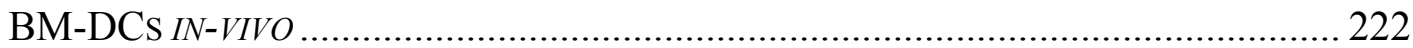

5.2.10 THE COMBINED SYSTEMIC EFFECTS OF NKT AND TLR STIMULATION ON SPLENIC DCS AND THE MAJOR SPLENIC DC SUBSETS ............................................. 228

5.2.11 SYSTEMIC $\alpha$-GALCER AND DC LOADED $\alpha$-GALCER + TLR STIMULATION .............. 232

5.2.12 TLR AND NKT CELL STIMULATION CO-OPERATE TO IMPROVE OVA SPECIFIC T CELL RESPONSES

5.3 DisCUSSION

5.3.1 SYSTEMIC $\alpha$-GALCER ADMINISTRATION VERSUS IN-VITRO LOADING ONTO BM-DCS.

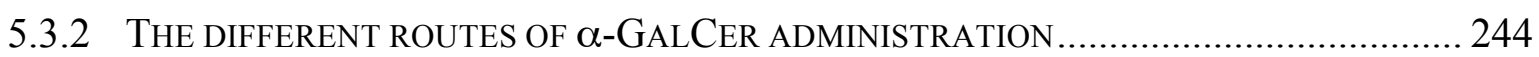

5.3.3 THE TRANSFER OF ANTIGEN AND PEPTIDE AFTER BM-DC VACCINATION ................. 245

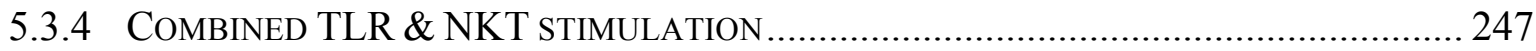


CHAPTER 6: GENERAL DISCUSSION ........................................................ 250

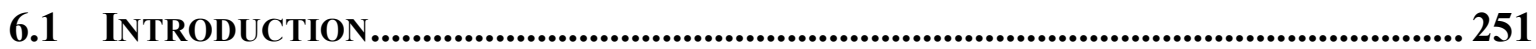

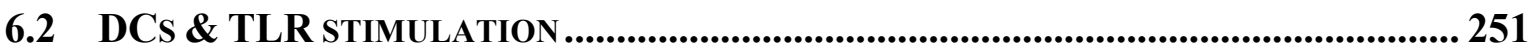

6.2.1 TLR STIMULATION INCREASES ANTIGEN SPECIFIC CD8 ${ }^{+}$T CELL RESPONSES

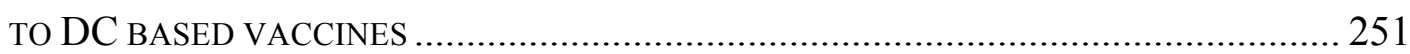

6.3 LANGERIN ${ }^{+}$CD8 $^{+} \alpha^{+}$DCS.................................................................................. 255

6.3.1 LANGERIN ${ }^{+} \mathrm{CD} 8 \alpha^{+}$DC' ARE INVOLVED IN PRIMING ANTIGEN SPECIFIC T CELL RESPONSES TO DC BASED VACCINES ….................................................. 255

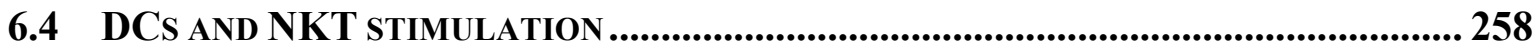

6.4.1 NKT STIMULATION INCREASES ANTIGEN SPECIFIC CD8 ${ }^{+}$T CELL RESPONSES

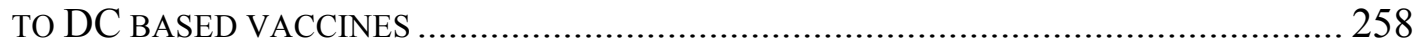

6.5 IMPLICATIONS FOR DC-BASED VACCINES........................................................ 259

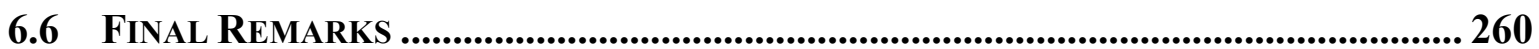

6.6.1 LIMITATIONS AND FUTURE WORK ................................................................... 260

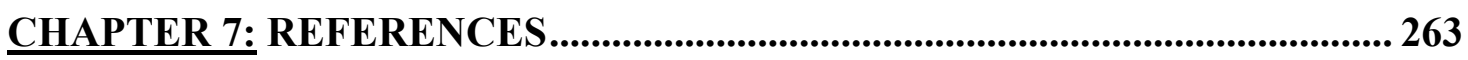

CHAPTER 8: APPENDIX TABLE 8.1 FLUORESCENT ANTIBODIES .......... 328

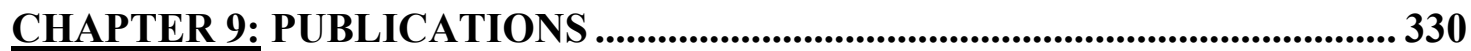




\section{List of Tables}

Table $1.1 \quad$ Vaccine Introduction Dates

Table 3.1 Levels of cytokine production by CD11 ${ }^{+}$BM-DCs after treatment 107 with TLR ligands

Table 3.2 Geometric MFI measures for activation marker expression

Table 3.3 Summary table highlighting in-vitro BM-DC assays

Table 3.4 Summary table highlighting in-vivo BM-DC assays

Table 8.1 Fluorescent Antibodies 


\section{List of Figures}

Figure 1.1 Mummified Head of Ramses V 5

$\begin{array}{llr}\text { Figure } 1.2 & \text { Lady Mary Wortley Montague } & 8\end{array}$

Figure 1.3 Dr Edward Jenner - Father of Immunotherapy 12

$\begin{array}{lll}\text { Figure 1.4 The hand of Sarah Nelmes } & 14\end{array}$

Figure 1.5 Dr William B Coley - Father of Immunotherapy 19

Figure 1.6 Schematic diagram highlighting the location of TLRs on DCs and 53 major signalling pathways and adaptor protein molecules

Figure 1.7 Schematic diagram highlighting molecular events involved in MyD88 57 signalling pathway

Figure 1.8 Schematic diagram comparing MyD88 dependent and MyD88 61 independent signalling pathways

Figure 1.9 Schematic diagram showing licensing of DCs by conventional T cells 63

Figure 1.10 The molecular structure of the potent iNKT cell activator $\alpha-65$ Galactosylceramide

Figure 1.11 Schematic diagram showing licensing of DCs by NKT cells and other 67 immune cell interactions

Figure 1.12 Designer vaccines: DC-based immunotherapy

Figure 3.1 The stimulation of BM-DCs with MPL induces maturation and 100 cytokine production in-vitro

Figure 3.2 Maturation status and cytokine production by MPL treated BM-DCs at 104 varied concentrations

Figure 3.3 Maturation status and cytokine production of BM-DCs after TLR 106 titration in-vitro 
Figure 3.4 TLR agonist combinations influence BM-DC maturation in-vitro

Figure 3.5 Selected TLR agonist combinations influence cytokine production by 114 BM-DCs in-vitro

Figure 3.6 CD40L increases in-vitro cytokine production of IL-12p70 by MPL 119 and Resiquimod treated BM-DCs over time

Figure 3.7 CD40L increases in-vitro cytokine production of IL-12p70 by MPL 120 and Pam3Cys treated BM-DCs

Figure 3.8 CD40L increases in-vitro cytokine production of IL-12p70 by CpG 121 and Resiquimod treated BM-DCs

Figure 3.9 TLR stimulation of BM-DCs in-vitro improves peptide antigen 123 presentation capability

Figure 3.10 Combined TLR agonists enhance peptide antigen presentation 125 capability of BM-DCs in-vitro

Figure 3.11 Selected TLR agonist combinations enhance both the antigen 127 processing and presentation capability of BM-DCs in-vitro

Figure 3.13 BM-DC vaccination strategy and manufacture protocol

Figure 3.14 TLR treatment of OVA loaded BM-DC vaccines did not influence 132 OVA specific $T$ cell responses in-vivo

Figure 3.15 The fate of CFSE labelled BM-DCs retrieved from spleen $24 \mathrm{~h}$ after $i v \quad 135$ administration

Figure 3.16 Systemic administration of TLR agonists alone and in combination 137 matures splenic DC populations

Figure 3.17 Systemic administration of TLR agonists influence antigen specific 140 responses to BM-DC vaccines

Figure 3.18 The kinetic influence of systemic Pam3Cys administration on protein 144 antigen uptake mechanisms by BM-DCs

Figure 3.19 Systemic administration of MPL enhances antigen-specific T cell 146 responses generated against BM-DCs

Figure 4.1 Characterisation of the major splenic DC subsets in langerin-EGFP 167 mice

Figure 4.2 Maturation marker expression of the major splenic DC subsets in 168 langerin-EGFP mice 
Figure 4.3 Diphtheria toxin treatment of langerin-DTREGFP recipients depletes 170 langerin ${ }^{+} \mathrm{CD} 8 \alpha^{+} \mathrm{DCs}$

Figure 4.4 The impact of Diphtheria toxin administration in langerin-DTREGFP 172 and $\mathrm{C} 57 \mathrm{BL} / 6$ recipients

Figure 4.5 Enhanced $\mathrm{CD}^{+} \mathrm{T}$ cell response to BM-DC based vaccine co- 175 administered with MPL is dependent on host langerin ${ }^{+}$DCs

Figure 4.6 Enhanced $\mathrm{CD}^{+} \mathrm{T}$ cell response to BM-DC based vaccines and 177 systemic MPL is not due to TLR 4 mediated stimulation of injected cells

Figure 4.7 The characterization of splenic DC subsets after systemic MPL 179 treatment for $5 \mathrm{~h}$ and $24 \mathrm{~h}$

Figure 4.8 Protein antigen transfer from vaccine BM-DCs to resident

langerin ${ }^{+} \mathrm{CD} 8 \alpha^{+} \mathrm{DC}$ populations is involved in cross-presentation

Figure 5.1 Protein loaded BM-DCs cultured with $\alpha$-GalCer increases the OVA- 197 specific T cell responses in-vivo

Figure 5.2 Enhanced OVA-specific T cell responses to $\alpha$-GalCer loaded BM- 199 DCs extends over time

Figure 5.3 Peptide loaded BM-DCs cultured with $\alpha$-GalCer and peptide antigen 201 increase OVA specific $\mathrm{T}$ cell responses in-vivo

Figure 5.4 Subcutaneous administration of $\alpha$-GalCer loaded BM-DCs induces 203 increased OVA specific $T$ cell responses

Figure 5.5 The fate of CFSE labelled BM-DCs retrieved from spleens $24 \mathrm{~h}$ after 205 systemic administration

Figure 5.6 Systemic administration of $\alpha-$ GalCer induces maturation of splenic 207 DCs and B cells

Figure 5.7 The characterization of splenic DC subsets after systemic $\alpha$-GalCer 210 treatment for 5 and $24 \mathrm{~h}$

Figure 5.8 The loading of BM-DCs with $\alpha$-GalCer in-vitro increases OVA 212 specific $\mathrm{T}$ cell responses

Figure 5.9 The depletion of langerin ${ }^{+} \mathrm{CD} 8 \alpha^{+}$DCs reduces the antigen specific T 214 cell response to $\alpha$-GalCer loaded BM-DC vaccines

Figure 5.10 Protein antigen transfer from vaccine BM-DCs to resident 216 langerin ${ }^{+} \mathrm{CD} 8 \alpha^{+} \mathrm{DC}$ populations is involved in cross presentation 
Figure 5.11 Peptide loaded BM-DCs treated with $\alpha$-GalCer in-vitro transfer 219 antigen in langerin-DTREGFP recipients

Figure 5.12 Peptide loaded BM-DCs treated with $\alpha$-GalCer in-vitro transfer 221 antigen in langerin-DTREGFP and CD1d-/- recipients

Figure 5.13 The maturation status of BM-DCs treated in-vitro with $\alpha$-GalCer and 223 TLR ligands

Figure 5.14 The in-vitro TLR treatment of $\alpha$-GalCer loaded BM-DC vaccines 225 does not enhance OVA-specific T cell responses generated in-vivo

Figure 5.15 TLR treatment of $\alpha$-GalCer loaded peptide vaccine BM-DCs in-vitro 227 does not enhance OVA-specific T cell responses generated in-vivo

Figure 5.16 The characterization of splenic DC subsets after systemic $\alpha$-GalCer 229 treatment for $16 \mathrm{~h}$

Figure 5.17 Characterization of the major splenic DC subsets after systemic 231 $\alpha-$ GalCer and MPL treatment for 5 and $24 \mathrm{~h}$

Figure 5.18 Systemic MPL improves antigen specific responses after vaccination 233 with soluble $\alpha$-GalCer and DC loaded $\alpha$-GalCer

Figure 5.19 Combined NKT and TLR stimulation increases the OVA-specific 236 responses in-vivo after vaccination 


\section{List of Abbreviations}

24G2

2ME

$\alpha$-GalCer

Ab

Ag

Alexa

APC

APC

B16

B16.Flt-3L

B16.OVA

B6

BCG

Bio

BM

BM-DC

BSA

C57

CCR7

cDC

CD

CD40L
Fc receptor blocker

2-Mercaptoethanol

alpha-Galactosylceramide

Antibody

Antigen

Alexa700

Antigen presenting cell

Allophycocyanin

Mouse melanoma cell line C57BL/6

FMS-like tyrosine kinase-3 ligand expressing mouse Melanoma cell line

Ova expressing B16 cell line

B6.SJL-Ptprc ${ }^{\mathrm{a}}$ Pepc $^{\mathrm{b}} /$ BoyJ mice

Bacille Calmette-Guerin

Biotinylated

Bone Marrow

Bone Marrow-derived Dendritic Cell

Bovine Serum Albumin

C57BL/6 mice

Chemokine (C-C motif) receptor 7

Conventional Dendritic Cell

Cluster of Differentiation

CD40 ligand 
CFSE

cIMDM

CLIP

$\mathrm{CO}_{2}$

CpG

cRPMI

CTL

CTLA4

CTO

CytC

DC

DLN

DMSO

DNA

DN

DT

dsRNA

EDTA

eGFP

ER

FACS

FBS

FITC

Flt-3
Carboxyfluorescein diacetate succinimidyl ester

Complete IMDM

Class II-associated invariant chain peptide

Carbon Dioxide

(Bacterial DNA repeats) binds to TLR 9

Complete RPMI Media 1640

Cytotoxic T lymphocyte

Cytotxic T lymphocyte associated antigen-4

Cell Tracker Orange

Cytochrome C

Dendritic Cell

Draining Lymph Node

Dimethylsulphoxide

Deoxyribonucleic Acid

Double Negative (langerin $\left.{ }^{-} \mathrm{CD} 8 \alpha^{-} \mathrm{DCs}\right)$

Diphtheria Toxin

Double Stranded RNA

Ethylenediaminetetraacetic Acid

Enhanced Green Fluorescent Protein

Endoplasmic Reticulum

Fluorescence Activated Cell Sorting

Foetal Bovine Serum

Fluorescein Isothiocyanate

FMS-like tyrosine kinase 3 
FSC

FSC-A

FSC-H

GFP

GM-CSF

${ }^{3} \mathrm{H}$ thymidine

HLA

IFN

IFN- $\alpha$

IFN- $\beta$

IFN- $\gamma$

Ig

IL

IMDM

ip

irr

iv

KO

$\mathbf{L}$

lang

LC

LN

LPS

mDC
Forward Scatter

Forward Scatter-Area

Forward Scatter-Height

Green Fluorescent Protein

Granulocyte-macrophage colony stimulating factor

Tritiated Thymidine

Human Leukocyte Antigen

Interferon

Interferon- $\alpha$

Interferon- $\beta$

Interferon- $\gamma$

Immunoglobulin

Interleukin

Iscove's Modified Dulbecco's Medium

Intraperitoneal, intraperitoneally

Irradiated

Intravenous, intravenously

Knock Out

Ligand

Langerin

Langerhans Cell

Lymph Node

Lipopolysaccharide binds to TLR 4

Myeloid-derived Dendritic Cell 
MACS

MBP

MDSCs

MFI

MHC

MPL

$\boldsymbol{n}$

NDLN

NK

NKT

OTI

OTII

OVA

Pam3Cys

PAMP

PBMC

PBS

pDC

PE

PECy7

PerCP

PI

Poly IC

PRR
Magnetic Activated Cell Sorting

Myelin Basic Protein

Myeloid-derived suppressor cells

Mean Fluorescence Intensity

Major Histocompatibility Complex

Monophosphoryl lipid A binds to TLR 4

Number of recipients per group

Non Draining Lymph Node

Natural Killer Cell

Natural Killer T Cell

Transgenic mice carrying OVA specific $\mathrm{CD8}^{+} \mathrm{T}$ cells

Transgenic mice carrying OVA specific $\mathrm{CD}^{+} \mathrm{T}$ cells

Ovalbumin

Pam-3 Cys-SKKKK binds to TLR 2

Pathogen Associated Molecular Pattern

Peripheral Blood Mononuclear Cells

Phosphate Buffered Saline

Plasmacytoid Dendritic Cell

Phycoerythrin

Phycoerythrin conjugated to cyanine dye 7

Peridinin Chlorophyll A Protein

Propidium Iodide

Multiple C repeats single stranded RNA binds to TLR3

Pattern Recognition Receptor 
$\mathbf{R}$

Res

RFA

RNA

rpm

RPMI

RT

SA

sc

SEM

SIINFEKL

SLE

SSC

SSC-A

SSC-H

SsRNA

TAP

TCR

TGF- $\beta$

TLR

TNF

TNF- $\alpha$

Treg

Tween-20
Receptor

Resiquimod binds to TLR 7/8

Radiofrequency Ablation

Ribonucleic Acid

Revolutions Per Minute

RPMI Media 1640

Room Temperature

Strepdavidin

Subcutaneous, subcutaneously

Standard error of the mean

OVA protein derived peptide presented on MHC class I

Systemic Lupus Erythematosus

Side Scatter

Side Scatter Area

Side Scatter Height

Single stranded RNA

Transporter associated with antigen processing

$T$ cell receptor

Transforming growth factor- $\beta$

Toll-like receptor

Tumour necrosis factor

Tumour necrosis factor- $\alpha$

Regulatory $\mathbf{T}$ cell

Polyoxyethylene-sorbitan monolaurate 
$\mathbf{V} \boldsymbol{\alpha}$

$\mathbf{V} \beta$

WT
Variable $\alpha$ region of $T$ cell receptor

Variable $\beta$ region of $T$ cell receptor

Wild type 
Chapter 1:

\section{General Introduction}




\subsection{General Introduction -Vaccination}

Vaccines can be utilized prophylactically to prevent diseases or therapeutically to treat or cure diseases. For either vaccine type, success is dependent upon mobilization of the immune system to generate appropriate and specific immune responses when required. In the prophylactic setting, an initial immune response is generated after vaccination that prepares the recipient for further encounters with the disease causing organism already contained within the vaccine in a weakened form. The secondary immune response generated after a subsequent encounter with the disease causing pathogen must be potent enough to eliminate the pathogen and prevent induction of disease. Therapeutic vaccines delivered to eliminate existing disease also rely upon stimulation of the immune system to resolve diseased states and prevent further occurrence.

The immune system is comprised of different cell types each contributing and supporting the generation of either innate or adaptive immunity. Features of the innate immune system include the induction of fierce and rapid immune and inflammatory responses designed to control and destroy invading pathogens quickly. The innate immune response has limited specificity, is quickly resolved and does not induce immunological memory of the pathogenic encounter. The adaptive immune response on the other hand is also capable of generating powerful immunity against invading pathogens. This response however is much slower, is pathogen specific and importantly induces immunological memory. The generation of immunological memory ensures rapid induction of the adaptive immune response upon subsequent encounter with the original pathogens. These powerful and efficient immune responses provide the basis for successful vaccination strategies capable of preventing disease.

This thesis will focus on adaptive immune responses to vaccination characterized by activation of $\mathrm{T}$ cells that recognize peptide fragments derived from foreign, mutated or dysregulated proteins (hereafter referred to as "antigens") displayed by specialised "presentation" molecules on the surface of infected or neoplastic cells. Important intermediaries in this process are dendritic cells (DCs), a class of "antigen-presenting cell" 
ultimately responsible for determining when and where $\mathrm{T}$ cell activation takes place. DCs form part of an innate network of cells capable of directly sensing infectious agents, or the tissue destruction associated with infection or neoplasia. These cells also sense the activity of the innate immune system components, and as such provide an important link between the innate and adaptive responses to disease.

The phagocytic and migratory function of DCs enables acquisition of antigens from injured tissues and the subsequent presentation of processed antigenic peptides to $\mathrm{T}$ cells located in the lymphoid tissues. T cells with antigen receptors specific for peptides carried on the DC surface are then provided with stimulatory signals to drive their clonal expansion. Large numbers of activated $\mathrm{T}$ cells then leave the lymphoid tissues and circulate through the body seeking the source and site of injury and possible infection in an effort to destroy invading pathogenic organisms and limit tissue destruction. An important feature of this adaptive immune response is that long-lived antigen-specific $\mathrm{T}$ cells are maintained in the host effectively providing immunological memory for a particular antigen and maintaining the capacity to respond quickly to the identical threat upon subsequent encounter. Other lymphocytes of the innate system such as NK cells and NKT cells also contribute to this response, although they are not believed to contribute to a pool of memory cells.

The overall theme of this thesis is that potent immune responses to specific antigens result from the context and environment in which the activities of cells of both the innate and adaptive immune systems are recruited. Vaccination strategies should therefore be designed with this concept in mind. This introductory chapter will begin by briefly covering the history and origins of prophylactic and therapeutic vaccination techniques, the function and characteristics of DCs and their interactions with other immune cell types. The importance of these interactions for cancer vaccine immune responses will also be highlighted. 


\subsection{History of Vaccination; from cows and milkmaids to a new treatment modality for cancer}

\subsubsection{Beginning with Smallpox}

Known as one of the scourges of mankind, smallpox was once a serious and dangerously infectious disease. Today considered a disease of antiquity, smallpox devastated human populations by killing and disfiguring millions of people around the world throughout the ages (WHO, 1980). After the World Health Organisation initiated a world-wide global eradication vaccination programme in 1967 (WHO, 1967) smallpox was declared eradicated in 1979 (Breman and Arita, 1980; WHO, 1980). Given the striking success of this vaccination programme it is now difficult to recall how devastating this disease once was.

One of the earliest cases of suspected smallpox was uncovered in the mummified remains of Pharaoh Ramses V (Figure 1.1). Discovered in 1898, his mummified head appears to display evidence of smallpox characterised by a rash-like collection of yellow blisters and pustules that resemble the pattern seen with smallpox infection with similar blisters found on his face neck shoulders arms, abdomen and scrotum (Lyons, 1987). A further two probable cases found in Egyptian mummies dating from the $20^{\text {th }}(1200-1100 \mathrm{BC})$ and $18^{\text {th }}(1580-1350 \mathrm{BC})$ dynasties are documented in the scientific literature (Ruffer and Moodie, 1921; Ruffer and Rietti, 1910).

The word variola was often used to describe smallpox, first coined by Bishop Marius of Avenches and derived from the Latin words varius-stained and varus-mark on the skin. The word small pockes (WHO, 1980) was used from the $15^{\text {th }}$ century onwards and was derived from pocke-sac, used to differentiate between large pockes caused by Syphilis (Riedel, 2005). Smallpox appeared in Europe sometime during the $5^{\text {th }}$ 


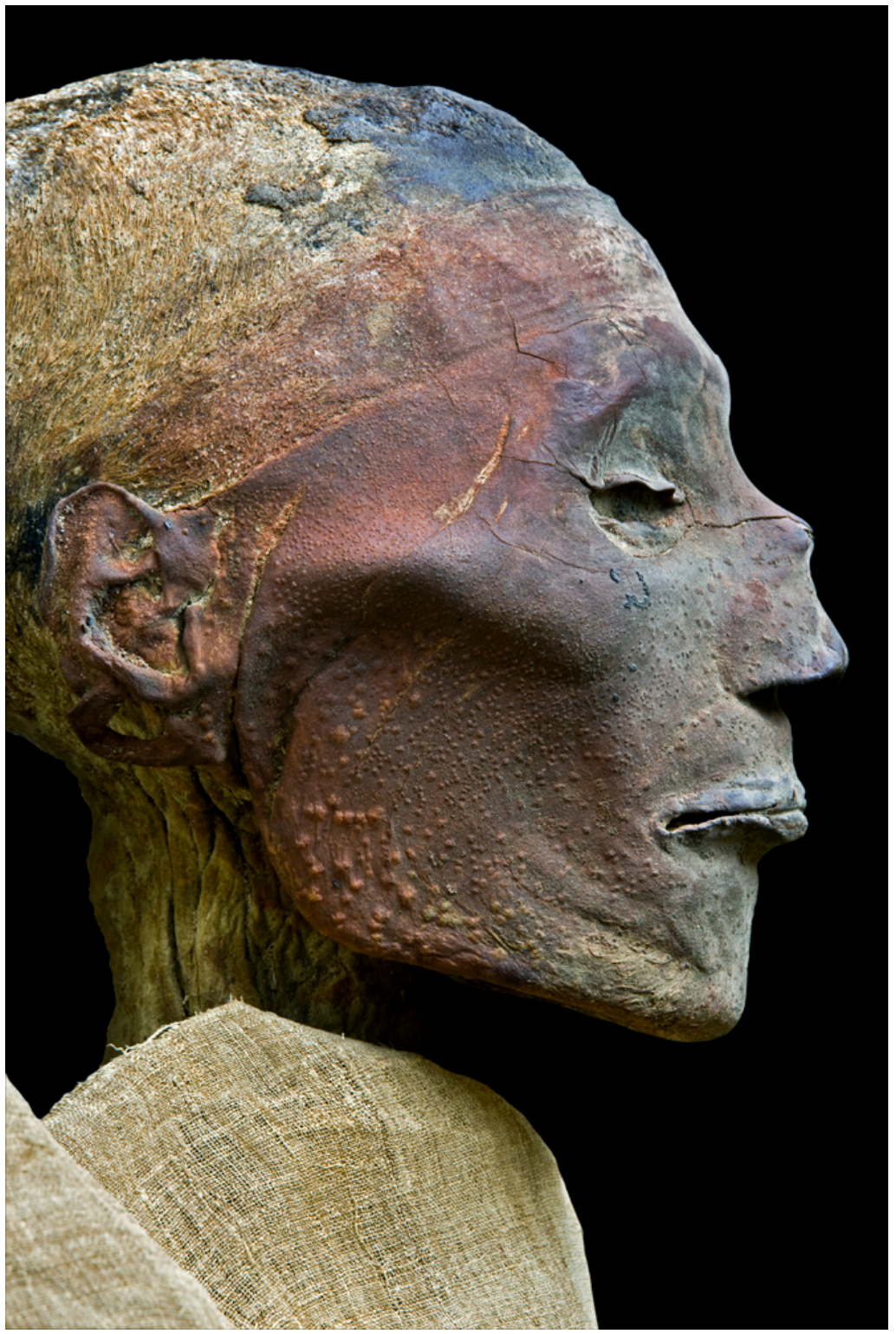

Figure 1.1 Mummified head of Pharaoh Ramses V. Copyright image courtesy of Sandro Vannini and used with permission. Ramses V died in 1157 BC and his mummified body discovered in 1898 and displayed in the Cairo Museum. It was reported that Ramses V had died suddenly at the age of 40 years after an acute illness. His mummy remains bear marks of the small-pox like rash comprised of yellow blisters or pustules seen on the lower part of his face. Evidence of this small-pox like rash was also found on his neck, shoulders, arms, lower portion of the scrotum and abdominal areas. In 1979 (Hopkins, 1983) Hopkins examined the mummy himself and made the following comments: "It is one of the best preserved royal mummies in the (Cairo) museum. Inspection of the mummy revealed a rash of elevated 'pustules", each about two to four millimetres in diameter, that was most distinct on the lower face, neck, and shoulders, but was also visible on the arms. Over the shoulders especially, these pustules were pale yellow against a dark brown-reddish background, the latter partly due to cosmetic compounds used in royal mummifications during that period. On his upper face, only smaller raised pimples (one to two millimetres) could be seen, which 
might have been shrunken more by tighter wrappings over the forehead. It was not possible to examine the palms or soles where the presence of pustules would be highly characteristic of smallpox, because his arms were folded across his chest with the palms down, and the shroud was stuck to his soles. No such rash could be seen on the chest or upper abdomen. Earlier photographs of this mummy, published by G. Elliot Smith (Smith, 1912) show that the rash is also prominent on the lower abdomen and scrotum.... Three folds in the skin over the left cheek suggest that his face may have been swollen when he died. The appearance of the larger pustules and the apparent distribution of the rash are similar to smallpox rashes I have seen in more recent victims." (Fenner, 1998, p. 211).

and $6^{\text {th }}$ centuries and during the Middle Ages caused frequent epidemics affecting millions of people. During the $18^{\text {th }}$ century it was estimated that smallpox was killing 400,000 persons annually while leaving one third of its survivors blind (Barquet and Domingo, 1997). During the French-Prussian war (1870 - 1871) 23,400 French soldiers succumbed to smallpox compared with 278 German soldiers. The reduced mortality amongst the German troops was attributed to the added protection against smallpox achieved by a process of "revaccination" which unfortunately had not been carried out for the French soldiers (Behbehani, 1983). The impact of this new "vaccination" therapy was profound and as discussed further has become a cornerstone of modern medicine.

\subsubsection{Early attempts at Prophylactic Vaccination}

Inoculation or variolation were the terms used to describe the practice of subcutaneous immunization of non-immune individuals with infectious material taken from smallpox lesions (Riedel, 2005). Variolation was known to have been practiced early in Africa, India and China before introduction into Europe in the $18^{\text {th }}$ century by travellers from Istanbul (Behbehani, 1983). Throughout the $17^{\text {th }}$ century, reports had been circulated by British, French and Italian physicians indicating that the practice of variolation served as a preventative measure against smallpox infection (Behbehani, 1983; Smith and McFadden, 2002). The introduction of variolation into Britain however was only achieved after the relentless effort of Lady Mary Wortley Montague whose face had been disfigured by the disease and had ordered the variolation of both her children one of which took place in the presence of royal court physicians (Figure 1.2). 
Variolation was not without risk to those treated since the mortality rate for persons dying of smallpox infection after variolation was $2-3 \%$ (Morgan and Parker, 2007). Others still contracted serious blood-borne disease such as syphilis and tuberculosis that added further complications to otherwise unwell persons. It was the death however of prominent members of royalty from smallpox that fuelled the drive towards variolation in Europe (Hopkins, 1983). After eventual acceptance in Britain, the practice of variolation became widespread throughout Western Europe (Behbehani, 1983; Riedel, 2005; Smith and McFadden, 2002). 


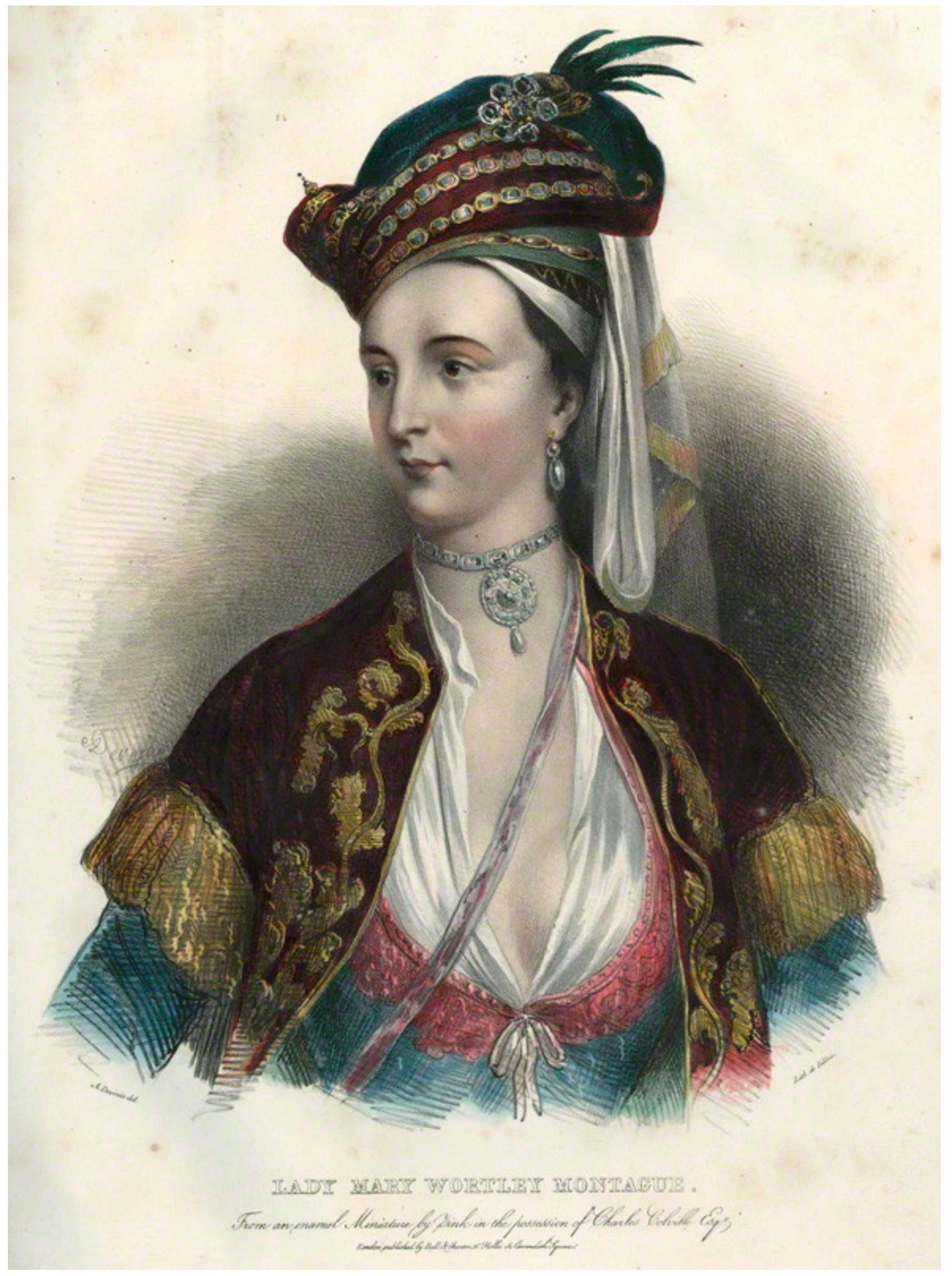


Figure 1.2 Lady Mary Wortley Montague (1689-1762) wife of Lord Edward Wortley Montagu the British Ambassador to the Ottoman Empire. Portrait artist Christian Friedrich Zincke credit (C) National Portrait Gallery, London and used with permission. In 1715, Lady Mary contracted smallpox at the age of 26 years. This left her permanently scarred with deep pockmarked skin on her face and no eyelashes. Her brother had died from smallpox and while living in Turkey, she had witnessed and became familiar with the technique of variolation to prevent smallpox. She was instrumental in raising the awareness and generating interest of the British general public and medical community in variolation at the time by utilizing her influence and successful immunization of her own two children Edward Jr aged 6 years, and Mary aged 3 years. In 1717 she described the variolation technique in a letter to her friend Sarah Chiswell in Nottingham, "The smallpox so fatal and so general among us, is here entirely harmless by the invention of ingrafting (i.e., inoculation) which is the term they gave it." (Behbehani, 1983, p. 461). Lady Mary was entirely convinced of the safety of the technique that she became determined to have her own children variolated and also to bring the practice into fashion in her homeland of Britain. The variolation of Lady Mary's daughter occurred in April 1721 after a fierce outbreak of smallpox in London and was carried out by Dr Charles Maitland (1668 - 1748) who had also successfully variolated Edward Jr. The Montagu variolation was the first ever in Britain and up until this time the Medical profession had largely regarded the practice as a "virtuoso amusement". Dr James Keith a member of the Royal College of Physicians was greatly encouraged by the results and having lost all but one of his children to smallpox. He had his only remaining 6 year old son variolated successfully shortly after on $11^{\text {th }}$ May 1721. A Portuguese Physician named Jacob de Castro Sarmento published the first English Treatise on Variolation in July 1721 while in the following month of August, an description of the Montagu variolation was appendixed to an address made by Dr Walter Harris Royal Physician to Queen Anne to the Royal College of Physicians on $17^{\text {th }}$ April 1721. Although Lady Mary's efforts to publicize the importance and acceptance of variolation as a preventative measure against smallpox was supported by other influential individuals such as the Princess of Wales and her husband, there remained some within her sphere of influence that did not approve of her efforts. Nevertheless, the gifted mother and widely admired aristocrat and famous letter writer, Lady Mary Montagu also became known for being a formidable and prominent advocate of variolation highlighting its importance in Britain at a time when the Medical establishment remained timid and reluctant to accept this new method for disease prevention.

In 1766, the American George Washington was defeated after many of his troops succumbed to an outbreak of smallpox against which the British troops he was engaged against were all variolated and therefore protected (Bardell, 1976; Behbehani, 1983). As a result American soldiers were variolated before commencement of new military operations (Stark, 1977; Thursfield, 1940). 
One of the key figures in the history of vaccination was Edward Jenner (Figure 1.3). Considered the "Father of Immunotherapy", Edward Jenner was born May 17 $7^{\text {th }}, 1749$ in Berkely, Gloucestershire, England. Having developed an early and keen interest in science and nature, Edward was destined for a career in medicine and by the age of 13 began working as an apprentice to a country surgeon and apothecary named Daniel Ludlow in Sodbury, near Bristol. According to (Parish, 1968) it was whilst working here that Jenner famously overheard a milkmaid exclaim: "I shall never have smallpox for I have had cowpox. I shall never have an ugly pockmarked face." (Barquet and Domingo, 1997). A commonly held belief at the time was that milkmaids were somehow protected from developing smallpox and it was this notion that later helped drive Jenner's future career to develop a successful vaccine against smallpox.

While working as a surgical and medical apprentice to George Harwicke in 1764, Jenner's interest focussed on the potential of cowpox to exert some degree of protective measure against smallpox (Willis, 1997). At this time in history it was estimated that approximately $10 \%$ of the population of Europe died each year from smallpox (Morgan and Parker, 2007). Interestingly, it would take Jenner another 32 years before he revisited this notion and began developing a vaccine against smallpox.

To address the idea that cowpox somehow conferred natural protection against smallpox and demonstrate that acquisition of immunity was possible, (Smith and McFadden, 2002) Jenner enlisted the help of a milkmaid Sarah Nelmes (Figure 1.4) who had recently developed cowpox from an infected cow and still had lesions present on her hands and arms (Andre, 2003; Behbehani, 1983). Jenner used fresh material extracted from one of these lesions to inoculate an 8-year old boy named James Phipps on $14^{\text {th }}$ May 1796. After being exposed to the cowpox matter, James developed a mild fever and was recorded as experiencing discomfort in the axillae. By day 10-post inoculation, James was feeling better and received a second inoculation using material extracted from a fresh smallpox lesion. This procedure failed to induce development of smallpox demonstrating that immune protection had indeed been generated (Behbehani, 1983; Morgan and Parker, 2007; Smith and McFadden, 2002; Willis, 1997). 
These experimental findings and observations from 13 other case histories were described by Jenner in a short communication manuscript submitted to the Royal Society on 10th July 1797 (Behbehani, 1983; Morgan and Parker, 2007; Riedel, 2005). After reviewed by the President of the Society Sir Joseph Banks and President of the Board of Agriculture Lord Somerville, the paper was rejected shortly thereafter due to what was described as insufficient data. Jenner remained unfazed and continued to collect evidence finally arranging for the private financing and publication of his work in a booklet dedicated to his dear friend Dr Caleb Hillier Parry in June 1798 and entitled "An Inquiry into the Causes and Effects of the Variolae Vaccinae, a disease discovered in some of the western countries of England, particularly Gloucestershire and Known by the Name CowPox." (Jenner, 1798). 


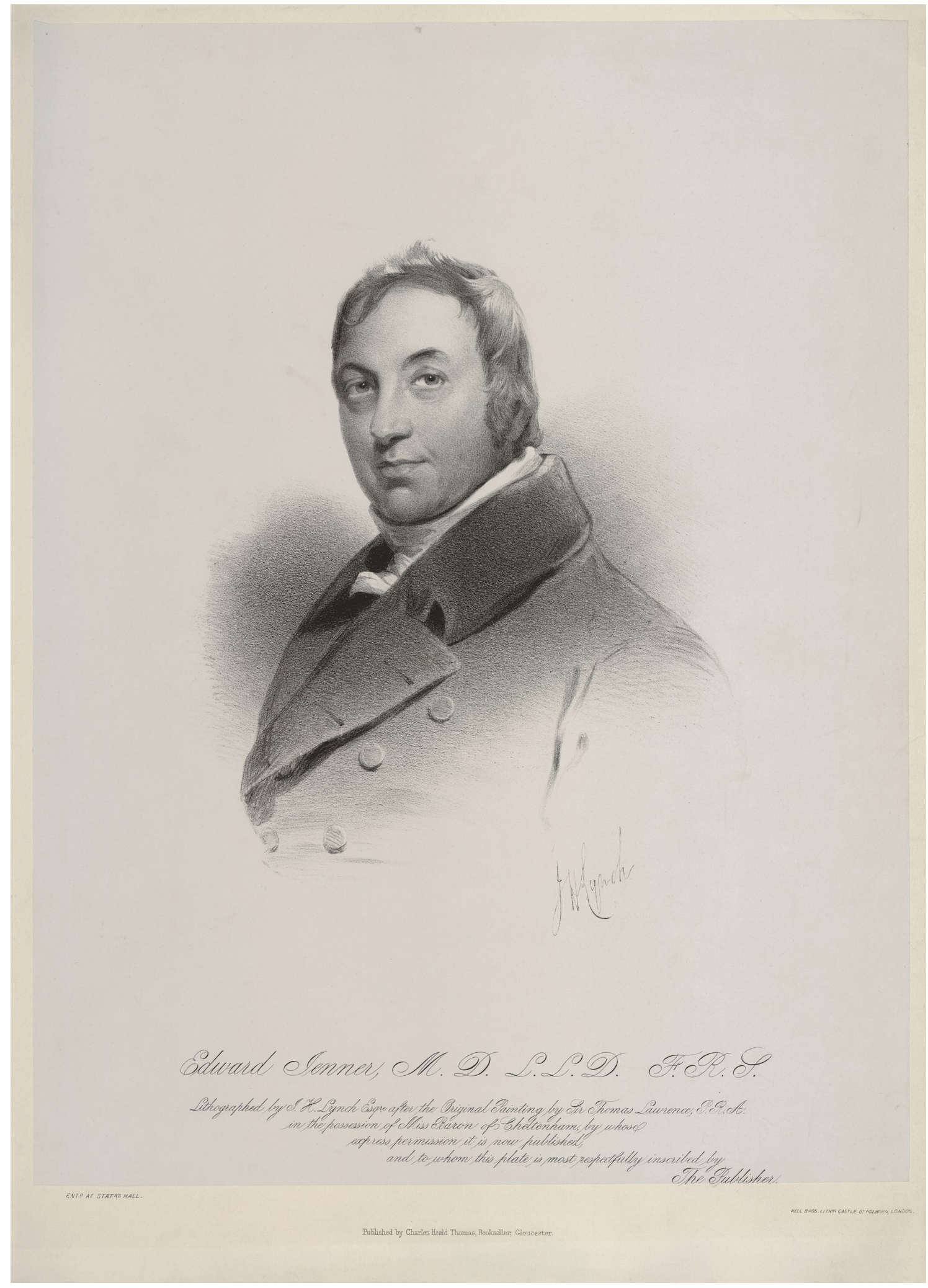


Figure 1.3 Dr Edward Jenner (1749-1823) considered the Father of Immunology for his research into vaccination to protect against disease. Portrait artist James Henry Lynch credit (C) National Portrait Gallery, London and used with permission. The sixth child and youngest of three sons he was orphaned at the age of 5 years and sent to live with his older brother Stephen who took over their Father's position of Vicar of Berkley. A keen interest in science and nature in his early years saw Edward Jenner grow up to become a surgical and medical student. At the age of 21, Jenner began his apprenticeship (1770 - 1773) working under the famous English clinical surgeon John Hunter at St George's hospital in London. Rumours concerning the protection of milkmaids from smallpox had already been in circulation for many years and after listening to these stories over time and making his own observations, Jenner attempted to revisit the idea that cowpox was capable of conferring natural protection against smallpox. His initial efforts to demonstrate the acquisition of immunity through vaccination were not accepted by some of the medical establishment. Although the medical community had reacted to the publication of his work with mixed responses, vaccination began to spread throughout England and by 1800, had reached Europe. Some who supported Jenner's work undertook to carry out vaccinations in their own patients including Dr John Hunter, who assisted to popularize vaccination by giving his own patients the inoculant supplied by Jenner. To garner further support for his work, Jenner conducted a nation-wide survey throughout England, which clearly proved his theory that vaccination with cowpox induced smallpox resistance. Although Jenner eventually received worldwide acclaim for his vaccination work, he did not seek to benefit financially from his discovery and efforts. In 1802 and 1807, Edward Jenner was publicly honoured by the British Parliament for his contributions to the vaccination programme and awarded him a total of $£ 30,000$.

Jenner was the first to coin the term variolae vaccinae to describe "cow smallpox" derived from the Latin words for cow-vacca and cowpox-vaccinia and from which the word vaccination originated. In 1800 Richard Dunning published a pamphlet entitled "Some Observations on Vaccination", however it was Louis Pasteur who proposed the use of the term vaccination after the development of his own anthrax vaccine to describe any protective inoculation in honour and recognition of Jenner and his work (Andre, 2003; Behbehani, 1983).

Although heavily criticized and publicly ridiculed by some of his peers, Jenner was to receive worldwide acclaim for his groundbreaking research and tireless effort to promote prophylactic vaccination as a means to protect against the development of infection. 


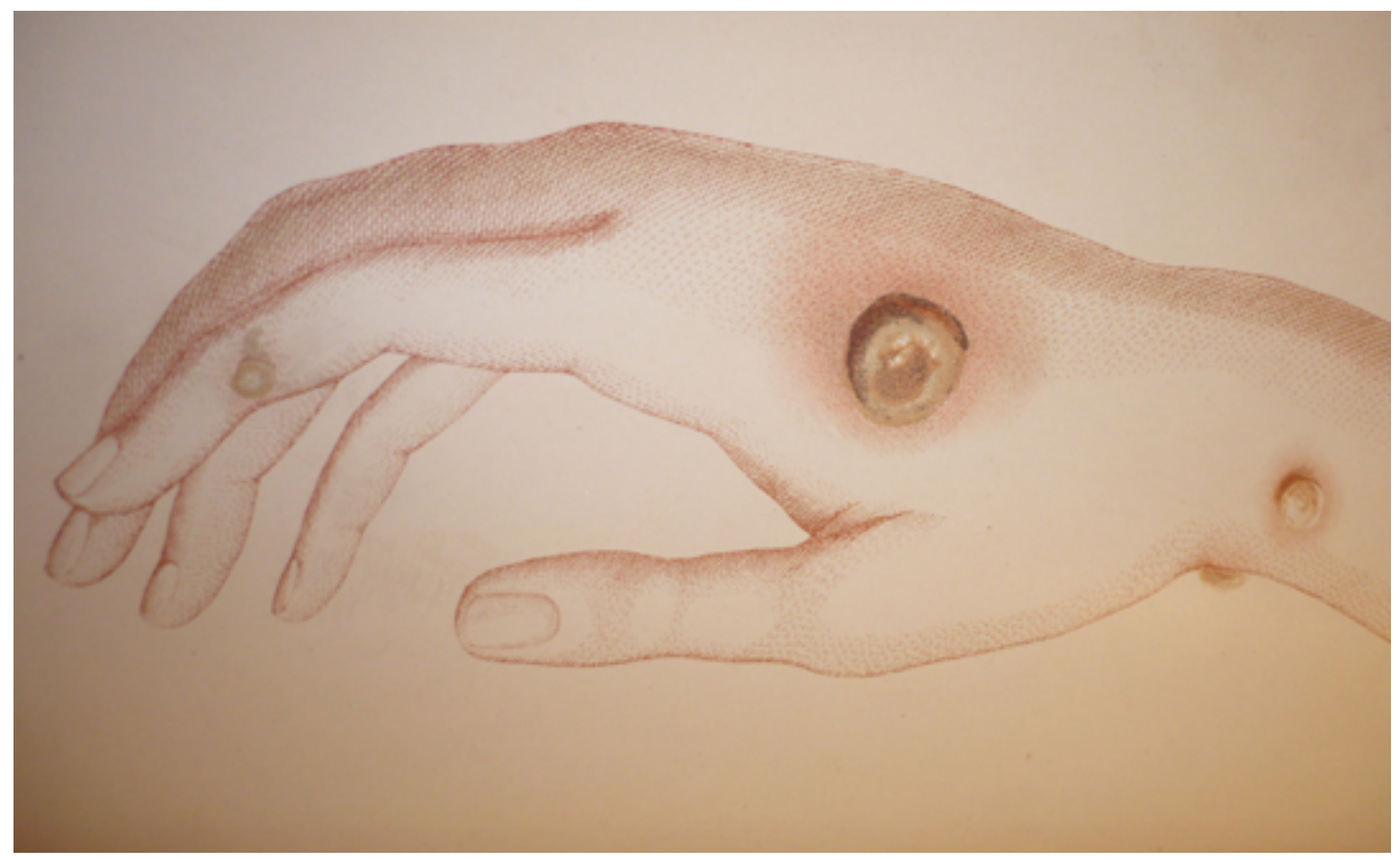

Figure 1.4 The hand of Sarah Nelmes the milkmaid showing her cowpox lesions. Picture Courtesy of the Historical Medical Library of The College of Physicians of Philadelphia and used with permission. James Phipps was inoculated in 1796 with fluid taken from a cowpox lesion found on Sarah Nelmes' arm and inserted beneath the skin of his arm via two superficial incisions. It was reported that one-week post vaccination James felt mildly unwell however recovered soon after. Six weeks post vaccination, he was inoculated this time with smallpox pustule material delivered into new incisions. No disease response was generated, even when inoculated again a few months later.

\subsubsection{The impact of Vaccination on society}

Vaccinology is the only branch of modern medicine that has achieved eradication of an infectious disease - smallpox. Other diseases such as poliomyelitis and measles are also targeted for control and elimination using vaccine technology. It is considered that the significant increase in life expectancy seen over the last two centuries has been largely attributed to the development and adoption of vaccination programmes which have totally eliminated smallpox, while in other cases reduced deadly infectious diseases such as diphtheria, tetanus, pertussis, measles, rubella, mumps, Hepatitis A and Hepatitis B, H, 
influenza b and paralytic poliomyelitis (Bonanni, 1999). Vaccines are available for twentysix diseases that are prevented after immunization (See Table 1.1).

Today the typical cost for the research, development, manufacture, launch and marketing of a new vaccine is estimated between US\$ 200 - \$400 million (Andre, 2003). The Global Alliance on Vaccines and Immunization (GAVI) was formed a few years ago for the purpose of attempting to bridge the equity gap between the poor and wealthy countries and ensure the provision of vaccines for poorer countries eligible for assistance to maintain vaccine uptake and usage for preventable diseases (Andre, 2003).

\begin{tabular}{|l|l|l|l|}
\hline Vaccine & Date & Vaccine & Date \\
\hline Smallpox & 1796 & Pneumococcus & 1976 \\
\hline Rabies & 1885 & Acellular P & 1981 \\
\hline Cholera & 1896 & Hepatitis B (HB) & 1981 \\
\hline Typhoid & 1896 & Varicella (V) & 1984 \\
\hline Plague & 1896 & rDNA HB & 1986 \\
\hline Diphtheria (D) & 1923 & H. influenzae b & 1988 \\
\hline Pertussis (Pw) & 1926 & Hepatitis A (HA) & 1991 \\
\hline Tetanus (T) & 1927 & DTPwIPVHib & 1993 \\
\hline Tuberculosis (BCG) & 1927 & DTPa & 1994 \\
\hline Yellow Fever & 1935 & DTPwHB & 1996 \\
\hline Influenza & 1936 & HBHA & 1996 \\
\hline Polio (IPV) & 1955 & DTPaHib & 1997 \\
\hline DTPw & 1957 & DTPaIPVHib & 1997 \\
\hline Polio (OPV) & 1958 & Lyme & 1998 \\
\hline DTIPV & 1961 & Rotavirus & 1998 \\
\hline Measles (M) & 1963 & Dtpa & 1999 \\
\hline DTPIPV & 1966 & HATy & 1999 \\
\hline Mumps (M) & 1967 & DTPaHBIPV & 2000 \\
\hline Rubella (R) & 1969 & DTPaHBIPVHib & 2000 \\
\hline MMR & 1971 & MCCV & 2000 \\
\hline Meningococcus & 1972 & PCV $^{\mathrm{b}}$ & 2000 \\
\hline
\end{tabular}

Table 1.1 Table shows introduction dates of commonly used vaccine and combinations for twenty-six vaccine preventable infectious diseases. ${ }^{a}$ Pneumococcus conjugate vaccine ${ }^{b}$ Meningococcus C conjugate vaccine. Adapted from (Bonanni, 1999). 
The use of prophylactic vaccines to achieve successful eradication of disease highlights the potential to develop therapeutic vaccines that also cure diseases by modification of the immune system.

\subsubsection{Origins of Therapeutic Vaccination to treat Cancer}

The cancer vaccination strategy being described in this thesis is not a referral to the mass application of a common preparation designed to prevent the development of cancer, but rather the administration of an individualised vaccine treatment for someone who has already developed cancer. This immunotherapeutic intervention is carried out in an effort to induce the patient's own immune system to generate an effective and efficient immune response capable of inducing tumour regression. The prevention of further tumours by the generation of a long-lived immune response capable of preventing the seeding of new tumours is also a desirable feature of this approach.

Some of the earliest recorded efforts to induce spontaneous tumour regression using immunotherapeutic approaches included the recommendation by the Egyptian physician Imhotep (2600 BC) that a poultice be applied to tumours followed by incision (Ebbell, 1937). This method of potentially inducing infection at the tumour (swelling) site was found in the Ebers Papyrus writings a 110 page scroll, 20 metres in length written in $\sim 1500$ BC containing medical knowledge copied from earlier texts. Another crude method of inducing tumour regression was reported in 1768 where a toad was applied to breast cancer lesions until its death, upon which a poultice would be utilized to keep the dead toad's body in contact with the lesion for a further several weeks (White, 1890). This method was utilized for several months to successfully treat a female suffering from metastatic breast cancer (Pennant, 1777).

Tanchou published a comprehensive cancer treatise in 1844 where he stated that: "One knows that often the affected lymph nodes and primary growths disappear during the course of concurrent illness, never to return. It is according to that idea...that a large 
number of observers have advised establishing issues (suppurating sores) on diverse portions of the body and even in the wounds remaining after operation." (as cited in Hoption Cann et al., 2003. p. 647). Further examples include the recommendation that infectious dressings should be applied to ulcerated tumours (Amoureaux, 1760; Tanchou, 1844), and infectious agents such as erysipelas (Busch, 1868; Fehleisen, 1886), gangrene (Cruveilhier, 1864; Dussaussoy, 1787; Robert, 1812; Tanchou, 1844) and even syphilis (Aliquie, 1851; Didot, 1852) were applied directly and deliberately to cause tumour infection in an effort to induce tumour regression.

Dr William B Coley (1862 - 1936) was an American surgeon and physician specialising in bone cancer working at New York Memorial Hospital in the late 1800's who through his pioneering work as a cancer researcher developed a cancer vaccine made from bacterial components (Figure 1.5) (Hoption Cann et al., 2003). Coley's interest in this area had been stimulated after the unexpected death of a young female patient following amputation of her right arm after initial presentation with persistent inflammation and pain.

Utterly devastated by the death of this patient, Coley sought out cases of spontaneous regression of established tumours in the hospital records for further investigation and noted one case in particular where a patient with a sarcoma on the cheek the size of an egg became infected with erysipelas (Streptococcus pyogenes) after surgery to remove the primary growth (Coley, 1893). The resultant wound did not heal and efforts to apply skin grafts were unsuccessful. Another round of surgery removed part of the tumour growth, but the wound subsequently became infected with erysipelas accompanied with a high fever, and the patient was not expected to recover. Astonishingly however, regression of the tumour and healing of the ulcer-like lesion was observed after each febrile attack, enough so that complete recovery was achieved after four and a half months after which the patient then discharged.

Coley proposed it was the infection that was responsible for this remarkable cure and set out to test this theory. He did this by deliberately infecting his cancer patients with erysipelas. However due to the unpredictability in outcome measures he decided to use 
killed bacteria components in an effort to control/prevent mortality from the disease. $\mathrm{He}$ attempted to simulate an infective immune response in a patient without actually causing infection and used killed Streptococcus pyogenes and Serratia marcescens to generate the first ever recorded cancer vaccine known as "Coley's toxins".

His first test case subject was a man who was bedridden with sarcoma affecting his abdominal wall, pelvis and bladder. Following treatment with Coley's toxins, complete tumour regression was achieved with the patient living for an additional 26 years (Nauts et al, 1953). Coley's toxins were used to treat other cancer types such as carcinomas, melanomas, myelomas and lymphomas and was shown time again that complete tumour regression and cures could be achieved even in the most seemingly hopeless of cases even when patients appeared to be in the final stages of their disease (Coley, 1914; Hoption Cann et al., 2003; Nauts, 1980; Nauts et al., 1953; Winberg, 1902).

Important features of the Coley's toxin treatment crucial to ensure patient survival included the imitation of acute infection and the absolute requirement for fever induction, the daily (or every $2^{\text {nd }}$ day) injections given to the patient for the first month or two followed by weekly injections for six months, and the gradual increase in the dose administered to avoid immune tolerance to the preparation and the intratumoural injection of the preparation into tumours when possible or into metastatic sites (Coley, 1906).

Coley had always maintained that the induction of fever was vital to successful treatment as it was the symptom most commonly associated with tumour regression. The subsequent use of antipyretics and antibiotics to suppress and prevent fever induction over the years was thought by some to be associated with reduced cases of spontaneous tumour regression. 


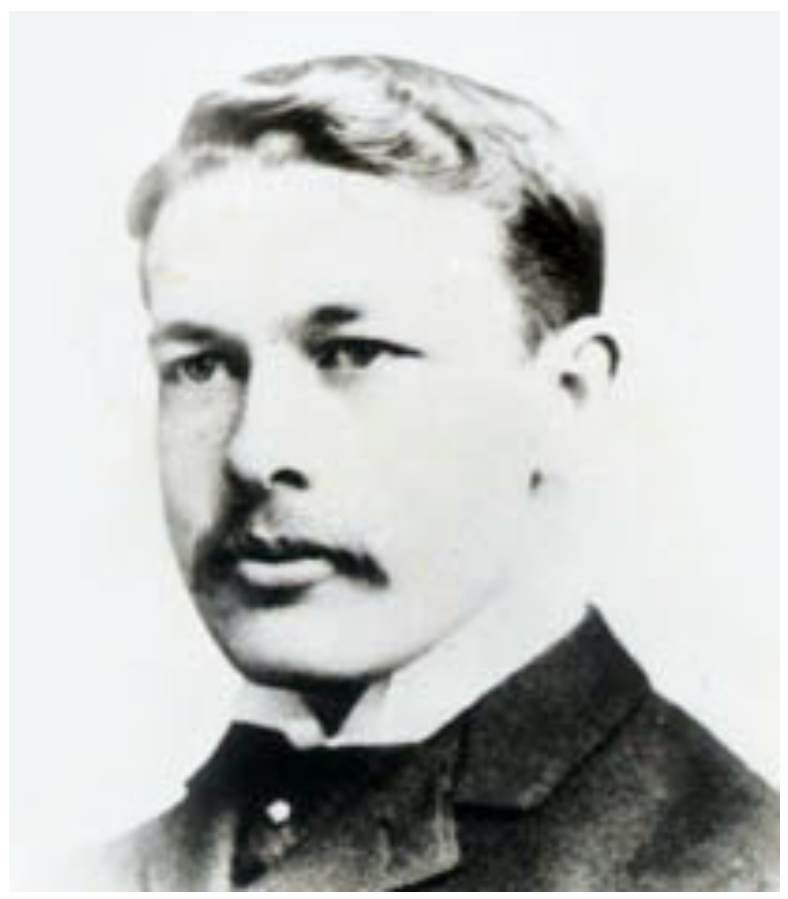

Figure 1.5 Dr William B Coley (1862 - 1936) the "Father of Immunotherapy" and producer of Coley's toxins. Portrait courtesy of Cancer Research Institute (CRI) NY, USA and used with permission. Dr William Coley was an American surgeon and physician specialising in bone cancer working at New York Memorial Hospital. After his first patient Elizabeth Dashiel died in 1891 after undergoing amputation of her right arm to treat persistent inflammation and pain, Dr William Coley sough to investigate the spontaneous regression of tumours. He came across a case where an immigrant gentleman had presented with a facial sarcoma and was treated with surgery three times and developed severe infections at the surgical site. His case was considered hopeless by the medical team treating him and he was not expected to survive after leaving hospital. Dr Coley met with this gentleman who had survived his ordeal and had been living in New York seven years after his discharge. Incredibly other than a scar on his cheek where the sarcoma had arisen, he was cancer free and claimed to be in good health. Dr Coley attempted to investigate the role of infection in spontaneous tumour regression as he attributed the cure and disappearance of the tumour in this case to the infection that occurred. He began testing his patients with what eventually developed into and became known as Coley's toxins - a solution mixture of bacterial strains that were used to inoculate tumour bearing patients in an effort to induce infection accompanied with high fever and eventual regression. $\mathrm{Dr}$ Coley experimented with application methods, concentrations, bacterial combinations and dosage regimes in an effort to develop and refine his methods and determine appropriate vaccination protocols. Although many patients were successfully treated, there were those who did not survive the infection. Nevertheless renewed interest in the role of infection and in particular fever and the impact on immune function within the tumour environment has occurred recently with clinical studies being conducted to examine the role of antipyretic drugs and fever on infection outcomes. 
Thanks to Lister's work on sterility (Lister, 1906), cancer surgery was fast becoming a sterile procedure and following the introduction of antibiotics, post surgical antibiotic use became standard after World War II; both these developments largely reduced the risk of developing fever associated infections and minimised the severity of those infections that did occur. The use of antipyretics came into use to further reduce the unpleasant impact of an active immune response which included eliminating high fevers. Reported spontaneous tumour regressions were markedly reduced in number, although when observed, this was nearly always accompanied by acute infection (Bowles and Perkins, 1999; Delmer et al., 1994; Fassas et al., 1991; Frick and Frick, 1993; Garcia-Rayo et al., 1996; Ifrah et al., 1985; Maekawa et al., 1989; Marcos Sanchez et al., 1991; Mitterbauer et al., 1996; Rebollo, 1990; Sureda et al., 1990; Tzankov et al., 2001).

The mechanism of tumour suppression was proposed by Coley as being due to the induction of a non-specific innate immune response given that the infection-induced regression was often initiated within hours of treatment and that primary adaptive immune responses required at least a few days to a week for initiation (Nauts et al., 1953). If tumour regression was partial, the tumour often regrew however regression would resume upon the reintroduction of infection accompanied by fever (Nauts, 1980). This was the reason Coley administered his toxin on a daily basis if tolerated by the patient - he had observed that withholding the treatment for even a few days resulted in tumour regrowth (Coley, 1906).

By the time of Coley's death in 1936, modern cancer therapies such as radiation and chemotherapeutic drugs were achieving popular acceptance. Compared with Coley's toxins these newer treatments could be more easily controlled and standardised. In a retrospective study carried out looking at the 10-year survival rate of patients treated with Coley's toxins versus those receiving modern costly treatments, patients treated with Coley's toxins more than 100 years ago did not fare any worse than those being treated with modern methods (Richardson et al., 1999). This finding was despite the billions of dollars being spent to develop modern cancer therapies over the years compared with the humble inexpensive preparation developed by Coley more than 100 years ago. 


\subsection{Vaccination and the Immune System}

As noted earlier, the innate immune system is responsible for a rapid immune response that is partially specific in nature, is quickly resolved and does not induce immunological memory. The adaptive immune response on the other hand is slower, is antigen-specific, and importantly induces immunological memory. While Coley and colleagues were convinced that innate immune responses accounted for tumour regression in their patients, it is likely that adaptive immune responses provided lasting regression. In fact, an element of both innate and adaptive immunity is likely to be involved in all successful vaccination strategies be they prophylactic or therapeutic. Co-ordinating these activities will be the key to providing effective vaccine-induced immunity. A review of the cell types of the innate and adaptive arms of the immune system that are of relevance to this thesis follows.

\subsubsection{The Innate Immune System}

Innate or "natural" immunity describes the immune response that is immediate, short-lived in duration, has no memory capacity and reproduces a similar response upon subsequent exposure to the same pathogen. When compared with adaptive immunity, the innate response has limited specificity and although some microbial recognition occurs it is in response to generalised structures that are common between microbes and viruses that are recognised.

Innate immune defence components are present in the body already and include physical and chemical barriers such as the skin and mucosal membranes. Cellular components include phagocytic cells such as neutrophils, macrophages, natural killer cells and DCs with inflammatory responses also considered part of the innate immune response operating to control infection within the local and systemic environment. The manifestation of fever occurs once localised mediators have entered the blood stream and communication has 
occurred with the thermoregulatory compartment of the hypothalamus. The uncontrolled systemic inflammatory response known as sepsis is a life-threatening condition that requires immediate specialised medical intervention.

The ability of innate immune cells to recognise conserved structures common to many pathogenic organisms involves receptors called "pattern recognition receptors" (PRRs). These receptors are found on the outer surface of innate immune cell types such as macrophages, DCs. Once binding with the appropriate "pathogen associated molecular pattern" (PAMP) occurs, these cells begin to secrete inflammatory cytokines, undergo increased expression of co-stimulatory molecules on their cell surface and become involved in amplification of inflammatory signals at the site of infection.

\subsubsection{The Adaptive Immune System}

Adaptive or "acquired" immunity describes the immune response that is delayed, longerlived in duration, has an effective memory capacity and usually generates a more powerful and amplified immune response upon subsequent exposure to the same pathogen. The adaptive response has a high degree of specificity when compared with innate immunity, since antigen is required for its generation. In addition, antigen acquisition, processing and presentation activities are carried out by the antigen presenting cell (APC) such as DCs, B cells and macrophages that allow the display of antigenic components on the outer surface of the APC on "major histocompatibility complex" (MHC) class I and II molecules.

The adaptive immune response is comprised of the cell-mediated (T lymphocytes) and humoral (B lymphocyte) response systems. Humoral immunity involves the elimination of pathogens after specific recognition of antigenic components by B cell receptors (BCRs) on the outer surface of B cells and their subsequent differentiation into antibody secreting plasma cells. Cell-mediated immunity involves the recognition of antigen fragments displayed by MHC molecules on the outer surface of APCs. These plasma membrane 
bound antigen MHC complexes are recognized by $\mathrm{T}$ cells that carry the corresponding $\mathrm{T}$ cell receptor (TCR) on its outer surface.

Antigen can be displayed on MHC class I or MHC class II molecules. MHC class I molecules present antigenic fragments to $\mathrm{CD}^{+} \mathrm{T}$ cell, while MHC class II molecules present antigen to $\mathrm{CD}^{+} \mathrm{T}$ cells. Under ideal conditions, $\mathrm{CD} 8^{+} \mathrm{T}$ cells differentiate into CTLs, while $\mathrm{CD}^{+} \mathrm{T}$ cells become $\mathrm{Th} 1$ or $\mathrm{Th} 2$ helper $\mathrm{T}$ cells that support cytotoxic $\mathrm{T}$ lymphocyte (CTL) or antibody responses. It is also possible for $\mathrm{CD}^{+} \mathrm{T}$ cells to differentiate into other T helper subtypes such as Th17 helper cells, Tfh follicular helper cells, T regulatory cells and Th9 helper cells.

Once binding occurs between a $\mathrm{CD}^{+} \mathrm{T}$ cell TCR and APC bound MHC I with antigen complex, the $\mathrm{T}$ cell undergoes cellular changes that causes differentiation and the proliferation of identical copies of itself capable of direct killing of specific pathogens. This is an example of clonal selection when a population of $\mathrm{T}$ cells (in this case) is generated that each bear an identical TCR corresponding to the antigen originally presented by the APC.

DCs and B cells are considered professional APCs as they are not only capable of presenting antigen, but also provide the necessary elements to create the ideal conditions for optimal $\mathrm{T}$ cell stimulation and antibody production. Professional antigen presentation will be discussed further. This ensures effective $\mathrm{B}$ and $\mathrm{T}$ cell responses are generated capable of destroying pathogens efficiently.

To destroy and eradicate pathogens successfully, the innate and adaptive immune responses are required to work in co-operation. DCs provide this important link between the innate and adaptive immune responses that enables appropriate and efficient immune responses to occur. 


\subsection{DCs and the Adaptive Immune Response}

DCs are professional antigen presenting cells vitally important for generating antigen specific T cell responses (Banchereau and Steinman, 1998). A promising approach for immunotherapeutic vaccination protocols is to use ex-vivo DCs loaded with tumour antigen in-vitro, prepared for re-administration into patients to allow for antitumour immunity to develop. Studies in mice have shown that peptide antigen loading of DCs in-vitro is indeed capable of inducing detectable antigen specific $\mathrm{T}$ cell responses and protecting against tumour challenge mediated via CTLs (Celluzzi, 1996). Although clinical responses have been reported in patients undergoing vaccine trials, the $\mathrm{T}$ cell response required for the destruction and elimination of tumour cells is generally low or even undetectable in some cases and has continued to correlate poorly with observed clinical outcomes (Yee et al., 2002).

\subsubsection{DCs as professional APCs}

While there are other APCs capable of presenting antigen, it is the biological features of DCs that make them superior immune system stimulators and regulators (Banchereau and Steinman, 1998; Steinman, 1991). These features include a high capacity for antigen uptake with a corresponding low rate of antigen degradation allowing prolonged antigen presentation (Trombetta and Mellman, 2005). DCs patrol the periphery and circulate through lymphoid organs searching for antigenic fragments for acquisition (Banchereau and Steinman, 1998). Once antigen has been captured, the DC undergoes maturation processes that involves the upregulation of surface maturation markers and the rapid migration of the antigen carrying DC to draining lymph nodes. Here these antigenic fragments are displayed on MHC class molecules on the surface of DCs and presented to naïve $\mathrm{T}$ cell populations; the clonal expansion of antigen specific $\mathrm{T}$ cells then takes place (Banchereau et al., 2000). 
The two criteria that have been proposed for defining professional or specialist APCs are that professional APCs must express MHC Class II - (in addition to MHC Class I) and therefore present antigen to $\mathrm{CD}^{+} \mathrm{T}$ cells, and that co-stimulatory molecules must exist and function in the generation of $\mathrm{T}$ cell responses (Trombetta and Mellman, 2005). When antigen is presented to $\mathrm{T}$ cells in the absence of sufficient co-stimulatory signals, antigenic tolerance occurs - this controls the production of autoreactive $\mathrm{T}$ cells capable of harm (Steinman et al., 2003; Steinman and Pope, 2002).

The suggestion has been made that DCs, B cells and macrophages are all professional APCs (de Jong et al., 2006). However although macrophages are capable of antigen uptake and presentation, they express low levels of MHC II and co-stimulatory molecules. Macrophages are inefficient $\mathrm{CD} 4^{+} \mathrm{T}$ cell stimulators while B cells are unable to stimulate $\mathrm{CD}^{+} \mathrm{T}$ cells unless memory $\mathrm{CD}^{+} \mathrm{T}$ cells, and while both cell types capable of presentation to $\mathrm{CD}^{+} \mathrm{T}$ cells, neither are as efficient as DCs (Ronchese and Hausmann, 1993). The existence of DC subpopulations and their ability to specialise in varied functional activities and being situated in favourable locations throughout the body combined with the aforementioned characteristics collectively support DCs as the most important professional APCs (Trombetta and Mellman, 2005).

\subsubsection{From Immature DCs to becoming fully Licensed}

Immature DCs can be found in the peripheral tissues and are characterised by low level surface expression of CD86, CD80 and CD40, MHC I and MHC II, high antigen capture and phagocytic capacity in addition to low antigen presentation capability. They are also found in lymphoid tissue. Mature DCs are characterised by the upregulation of CD86, CD80 and CD40, MHC I and MHC II molecules, the reduction in antigen capture and phagocytic abilities and enhanced antigen presentation capability (Banchereau and Steinman, 1998).

It is possible for immature DCs to undergo spontaneous maturation processes and migrate to the lymph nodes after the upregulation of surface maturation markers, MHC Class II 
molecules and LN homing receptors (Reis e Sousa, 2006). DCs that spontaneously mature in this manner and migrate to the LNs are termed "steady state" DCs and are responsible for the induction of tolerance to self antigens after their presentation to $\mathrm{T}$ cells in the $\mathrm{LN}$ (Hernandez, 2001; Liu et al., 2002). It has been reported that constitutive ablation of steady state DCs results in fatal autoimmunity highlighting the role of steady state DCs in T cell tolerance activities (Ohnmacht et al., 2009).

The conversion of immature to activated DCs occurs when inflammatory danger signals are encountered. This can be in response to viral and microbial products such as pathogen associated molecular patterns (PAMPs) that bind to pattern recognition receptors (PRRs) such as the toll-like receptors (TLRs) expressed by DCs (Akira, 2001; Kaisho and Akira, 2001b). The binding of PAMPs to their corresponding TLR causes conformational changes in the receptor ligand complex and the induction of specific intracellular signalling pathways that results in phenotypic and functional changes in DCs, in addition to the production of proinflammatory cytokines. The changes that occur after the activation of immature DCs include upregulation of CD86, CD80 and CD40, MHC I and MHC II molecules, the reduction in antigen capture and phagocytic abilities and enhanced antigen presentation capability and release of IL-6, TNF- $\alpha$ and IL1 $\beta$ (Banchereau and Steinman, 1998; Hart, 1997; Lipscomb and Masten, 2002). These changes promote the presentation of antigen to naïve $\mathrm{T}$ cells under conditions needed to generate successful antigen specific $\mathrm{T}$ cell responses.

The activation of peripheral DCs involves enhanced migration activity that allows these DCs to traffic to the draining lymph node and carry out antigen presentation activities with lymph node resident $\mathrm{T}$ cell populations. The migratory process allows trafficking through the extracellular matrix to reach the draining lymph node by the upregulation of matrix metalloproteases by the activated DCs (Ratzinger, 2002). DC expression of CCR7 is also upregulated and permits DC movement through draining lymph node to reach the naïve T cell rich paracortical area in response to chemokine gradients (Martin-Fontecha et al., 2003). 
It is possible for activated DCs to produce antigen specific T cell responses such as CD8 ${ }^{+}$ $\mathrm{T}$ cell populations. The cytotoxic $\mathrm{T}$ lymphocytes (CTLs) generated as a result however typically have reduced life spans, unfavourable cytotoxic activity and undergo TRAILmediated death (Janssen, 2003). Improved cytotoxic activity and the production of memory cells can be generated by the "licensing" of DCs (Bennett, 1998; Janssen, 2003; Ridge, 1998; Schoenberger et al, 1998; Smith, et al, 2004). This refers to the incorporation of CD40 signalling activities combined with DC activation to ensure successful antigen presentation with appropriate co-stimulatory activity occurs together with the cytokine production necessary for full effector and memory function to occur. The CD40 - CD40L interaction occurs between the CD40L bearing $\mathrm{CD}^{+} \mathrm{T}$ cell and CD40 found on the outer DC surface (Figure 1.9).

It is important to note that licensing of DCs can be induced by cells other than $\mathrm{CD}^{+} \mathrm{T}$ cells such as the natural killer (NK) and natural killer T cells (NKT) and will be discussed further as it is one of the major themes of this thesis (Mocikat et al., 2003; Semmling et al., 2010).

\subsubsection{DC subsets and location}

DCs have been found in many different locations and organs which include the skin, spleen, lymph nodes, lung, intestines, liver kidneys, pancreas and eyes (Forrester, 2010; Helft, 2010). Specific cell surface markers are used to identify different DC subtypes in tissue samples although there are some common features characteristic to all DC subsets and include MHC Class II and CD11c expression in addition to the DC morphological features and the ability to stimulate $\mathrm{T}$ cell responses.

Bone marrow DC precursors that migrate to specific tissue areas are responsible for the generation of the different DC subsets in these locations. The DCs that are generated from these bone marrow precursors work to maintain the DC supply by taking the place of tissue specific DCs that have undergone apoptosis or migrated to other tissues and exited their tissue of origin. The studies contained within this thesis focus on the murine BM-DCs and 
the resident splenic DC populations. In addition, the transfer of antigen that occurs between migratory DCs and resident DC populations is a feature of DCs that will also be explored further within this thesis.

\subsubsection{Mouse DC subsets}

In addition to their location and distribution, the functional properties and phenotypic characteristics of murine DCs can be used to define these DCs into specific categories (Anjuere, 1999; Vremec, 2000). Two main groups of DC subsets have been described in secondary murine lymphoid organs conventional (cDC) and plasmacytoid DCs (pDC) (Segura and Villadangos, 2009).

\subsection{Conventional DCs (cDCs)}

cDCs are characterized by CD11c expression and are further divided into migratory and resident DC subsets (Villadangos and Schnorrer, 2007).

\subsection{Migratory DCs}

Migratory DC subsets include the different subsets of epithelial DCs such as the pulmonary $\mathrm{CD} 103^{+} \mathrm{CD} 11 \mathrm{~b}^{-}$and $\mathrm{CD} 103^{-} \mathrm{CD} 11 \mathrm{~b}^{+}$DCs (Hintzen et al., 2006) and the dermal $\mathrm{CD} 103^{+} \mathrm{CD} 11 \mathrm{~b}{ }^{-}$langerin ${ }^{+}$and $\mathrm{CD} 103^{-} \mathrm{CD} 11 \mathrm{~b}^{+}$DCs (Bursch and Hogquist, 2007; Ginhoux, 2007; Poulin et al., 2007). The migratory Langerhans cells are characterized by the expression of $\mathrm{CD} 11 \mathrm{c}^{+}, \mathrm{CD} 4, \mathrm{CD} 8^{\text {low }}, \mathrm{DEC} 205^{\text {high }}, \mathrm{CD} 11 \mathrm{~b}^{+}$and when immature, are a typically found in the epidermis while mature LCs that have acquired antigen travel towards and are found in the draining lymph nodes (Anjuere, 1999; d'Ostiani, 2000; Henri, 2001; Langenkamp, 2000). Studies have shown that LCs are in fact capable of transferring acquired antigen to resident CD8 $\alpha^{+}$DCs (Allan et al., 2003; Allan et al., 2006; Belz et al., 2004).

Migratory DCs have also been shown to transfer antigen to resident DCs and this is a 
major feature that will be explored further within this thesis.

\subsection{Lymphoid Resident DCs}

Secondary lymphoid organ resident DC subsets can be defined by CD4 and CD8 $\alpha$ expression. The splenic CD $8 \alpha^{+}$DCs can be further categorized by langerin expression with a recently identified subset consisting of $\mathrm{CD} 8 \alpha^{+} \mathrm{CX}_{3} \mathrm{CR}^{+}$splenic DCs thought to be similar to plasmacytoid DCs (Bar-On et al., 2010). Work in our laboratory has also identified langerin ${ }^{-} \mathrm{CD} \alpha^{+} \mathrm{CX}_{3} \mathrm{CR}^{-}$cells within this splenic compartment (Troels Petersen, personal communication). These lymphoid resident DC subsets are generated from blood borne precursors (Liu et al., 2007; Naik, 2006) in contrast to migratory DCs which develop in the periphery and undergo constitutive migration to and from lymph nodes (Walton, 2006; Wilson et al., 2008). DC populations that are CD8 $\alpha$ - have been shown a greater propensity to present $\mathrm{Ag}$ to $\mathrm{CD} 4^{+} \mathrm{T}$ cells, while the $\mathrm{CD} 8^{+} \mathrm{DC}$ subset has been shown to possess superior antigen cross-presentation capabilities in comparison to other DC subpopulations (den Haan et al., 2000; Farrand et al., 2009; Pooley et al., 2001).

In the context of this thesis, the ability of splenic resident DC populations to cross-present antigen is thought to be required for tumour responses. The functional differences that exist among splenic resident DC populations will also be explored in subsequent chapters.

\subsection{Plasmacytoid DCs (pDCs)}

pDCs are important for antiviral responses and are the major producers of type 1 interferons (IFN's) (Dai et al., 2011; Lui et al., 2009). They can be found in the blood circulation and lymphoid tissues and convert to DC morphology after activation (Colonna et al., 2004; Liu, 2005). Although capable of inducing antigen specific T cell responses, pDCs are not as efficient as cDCs and it has been suggested that reduced levels of costimulatory molecules, MHC II expression and endocytic ability are responsible (Grouard et al., 1997). pDCs also respond to viral RNA and DNA by ligation of TLRs 7 and 9 and express B220 in addition to displaying intermediate levels of CD11c (Iwasaki and 
Medzhitov, 2004). It has been proposed they are further subdivided into pDCs that express RAG1 (Pelayo et al., 2005) or CCR9 (Hadeiba et al., 2008). Although pDCs are not examined in this thesis, they have been shown to play a role in supporting induced immune responses in mice (Lui et al., 2009) and in humans (Tel et al., 2012).

\subsection{Bone Marrow Derived-DCs (BM-DCs)}

Murine BM-DCs are a convenient source of DCs for in-vitro and in-vivo studies and are generated from bone marrow precursors and differentiated in the presence of the cytokines IL-4 and GM-CSF (See Chapter 2 for in-vitro culture details). The IL-4 cytokine prohibits the production of macrophages from BM precursors, while GM-CSF causes differentiation of myeloid cells (Jansen et al., 1989; Metcalf et al., 1986). BM-DCs cultured in the presence of IL4/GM-CSF produce CD11 $\mathrm{c}^{\text {hi }} \mathrm{CD} 11 \mathrm{~b}^{+}$DCs that are immature in function and phenotype. These DC populations are similar in phenotype to the "inflammatory DC" as able to generate measurable quantities of proinflammatory cytokines, include IL-12.

The low frequencies of DCs found in the body are a major barrier to DC based investigations. This BM-DC model of DC generation represents a simple, relatively inexpensive, rapid, easily reproducible method of producing large quantities of DC populations that can be manipulated in-vitro and reintroduced into the murine recipient readily for vaccine testing. Labelling of the injected DCs for ex-vivo studies can also be carried out in an effort to monitor vaccine responses.

The use of ex-vivo BM-DCs allows careful control and manipulation of culture conditions, antigenic products and concentrations in-vitro to generate the desired vaccine DCs.

\subsubsection{Human DC subsets}

Human DCs are characterized by their location such as the skin and blood DC subsets. The skin DC subsets include the Langerhans cells found in the epidermis, and the CD1 $\alpha^{+}$and 
CD14 ${ }^{+}$DC subsets found in the dermal layers of the skin. The human blood DC subsets include $\mathrm{CD}_{1}{ }^{+}$(BDCA-1) $\mathrm{Lin}^{\mathrm{neg}} \mathrm{HLA}^{-\mathrm{DR}}{ }^{+} \mathrm{CD} 11 \mathrm{c}^{+} \mathrm{DCs}, \mathrm{CD} 141^{+}$(thrombomodulin, BDCA-3) Lin $^{\text {neg }}{ }^{\text {HLA-DR }}{ }^{+}$CD $11 c^{+}$DCs and plasmacytoid DCs.

The second human blood DC subset characterized by $\mathrm{CD} 141^{+}$(thrombomodulin, BDCA-3) Lin ${ }^{\text {neg }} \mathrm{HLA}_{-\mathrm{DR}}^{+} \mathrm{CD} 11 \mathrm{c}^{+}$DCs or CD $141^{+}$DCs (Dzionek et al., 2000) have been found to express CLEC9A or (DNGR-1) a C-type lectin with ITAM-like motif (Huysamen et al., 2008) and has recently been reported to represent the human counterpart of the CD8 $\alpha^{+}$ mouse DC subset (Bachem et al., 2010; Crozat et al., 2010; Jongbloed et al., 2010; Poulin et al., 2010; Yamazaki et al., 2010). These DCs are also found in tonsils and spleen, and constitute less than 5\% of blood DC population (Bachem et al., 2010; Crozat et al., 2010; Jongbloed et al., 2010; Poulin et al., 2010; Yamazaki et al., 2010). Surface markers which are shared in common between mouse CD $8 \alpha^{+}$and CD141 ${ }^{+}$DC subset include NECL2 an adhesion molecule (Galibert et al., 2005; Poulin et al., 2010), CLEC9A (Caminschi et al., 2008; Jongbloed et al., 2010; Poulin et al., 2010; Sancho et al., 2008) and XCR1 the chemokine receptor (Bachem et al., 2010; Crozat et al., 2010; Jongbloed et al., 2010; Poulin et al., 2010; Yamazaki et al., 2010). CD8 $\alpha^{+}$DCs and CD141 ${ }^{+}$DCs also share the expression of TLR 3 and TLR 8 induce $\mathrm{CD}^{+} \mathrm{T}$ cell responses when stimulated via crosspresentation (Bachem et al., 2010; Crozat et al., 2010; Jongbloed et al., 2010; Poulin et al., 2010; Yamazaki et al., 2010).

\subsubsection{DC function}

\subsubsection{DC maturation and activation}

It is important to note the difference between DC maturation and activation activities. The process of DC maturation refers to the upregulation of specific markers and receptors on the DC surface, while DC activation refers to the release of cytokines and chemokines by matured DCs. Mature DCs are typically characterized by elevated surface expression of CD80 and CD86 (which both bind to CD28), and also by increased expression of CD40, 
CCR7 and MHC class II molecules. It has been reported however that in some circumstances, DCs that are phenotypically mature are not actually capable of generating $\mathrm{T}$ cell responses and that in some situations, may even contribute to the induction of tolerance (Albert et al., 2001; Menges et al., 2002).

The maturation of DCs is typically characterized by the upregulation of specific DC surface markers known as costimulatory molecules which interact with $\mathrm{T}$ cells and include CD40, CD80, CD86, CD70, 4-1BB-L, OX40L (Watts, 2005). DC activation involves the release of proinflammatory cytokines including IL-6, TNF- $\alpha$ and importantly the release of interleukin-12 (IL-12p70) important for driving Th1 T cell responses (Trinchieri et al., 1993). The upregulation of DC surface chemokine receptors associated with DC trafficking and release of specific chemokines involved in the attraction of other immune cell types also takes place (Allavena et al., 2000; Cyster, 1999; Dieu-Nosjean et al., 1999; Luster, 2002; Tang and Cyster, 1999). DC maturation and activation processes also impact on Class II MHC compartments driving the translocation of peptide-MHC (p-MHC) complexes to the cell surface allowing their prolonged persistence and priming of the $\mathrm{CD}^{+} \mathrm{T}$ cell response (Cella et al., 1997).

The term activation on the other hand, refers to DCs that have not only matured but are also capable of generating antigen specific effector $\mathrm{T}$ cells, the desired outcome for vaccination studies. The sequential events required for effective DC activation have been described as comprising signals 1-3 (Keppler et al., 2012; Pham et al., 2011). Signal 1 refers to the delivery of the $1^{\text {st }}$ activation signal received by the $T$ cell receptor on a naïve $T$ cell when presentation of pathogen derived peptides occurs by DCs on MHC class surface molecules. Signal 2 describes the $2^{\text {nd }}$ signal received by naïve $T$ cells which occurs when the pathogen derived peptide presenting DCs are matured and display upregulation of CD86 and CD80 (and other) co-stimulatory molecules capable of further stimulating the naïve $\mathrm{T}$ cell. Signal 3 refers to the final message required for effector $\mathrm{T}$ cell differentiation and occurs via mediators released by activated DCs - these are often cytokines such as IL12 which drives Th1 immunity. All 3 signals are delivered by activated DCs and are required for the development of effective effector antigen $\mathrm{T}$ cell responses. 
DC activation signals include the following: microbes which stimulate through C-type lectins (Cambi et al., 2005) and intracytoplasmic NOD-like receptors (NLRs) (Delbridge and O'Riordan, 2007; Mariathasan and Monack, 2007; Martinon and Tschopp, 2005; Ting and Davis, 2005); microbial and viral components which act on surface and intracellular TLRs, T cells, NKT and NK cells, CD40L; pro-inflammatory cytokines such as IL-1 $\beta$, TNF, IL-6 and prostaglandin E2 (PGE2). Apoptotic debris and dying cellular components (Gallucci et al., 1999) are capable of stimulating DCs in addition to endogenous molecules such as damage-associated molecular pattern molecules (DAMPS) which includes heat shock protein (HSP) (Srivastava and Heike, 1991), high mobility group box 1 protein (HMGB1) (Lotze and Tracey, 2005), $\beta$-defensin (Biragyn et al., 2002) and uric acid (Rock et al., 2005).

In summary, DC activation describes the conversion of naïve DCs into effector DCs that occurs after releasing tightly regulated cytokines such as the Th1 polarizing cytokine IL-12 (Lyakh et al., 2008; Sporri et al., 2006; Trinchieri, 2003). Proinflammatory cytokines such as IL-6 and TNF- $\alpha$ are also released by activated BM-DCs. Cytokines generated by matured and activated DCs are capable of influencing $\mathrm{T}$ cell responses which include $\mathrm{T}$ cell expansion, effector function and response duration (Curtsinger et al., 1999) maturation and activation processes are controlled by efficient regulatory signals that are present and experienced within the DC microenvironment.

\subsubsection{Classical Presentation}

The processing and presentation of antigen by DCs takes place via the two distinct pathways involving MHC class I and II molecules and are described here briefly.

\subsection{Presentation of antigen on MHC class I molecules}

MHC class I molecules are typically found on all nucleated cells and are capable of 
presenting peptides that are eight to ten amino acids in length. This peptide size restriction is due to a peptide-binding groove that is closed at both ends. The peptides presented by MHC I molecules are those which have originated from endogenous protein sources. After peptide degradation and processing via the proteasome found in the cytosol, peptides are transported into the Endoplasmic Reticulum (ER) via the heterodimeric "Transporters of Antigen-Processing" (TAP)-1 and 2 molecules (Uebel and Tampe, 1999). Peptide antigen is loaded onto MHC molecules I that are assembled in the ER with $\beta$-2-microglobulin $\left(\beta_{2} \mathrm{~m}\right)$ after which export takes place to the plasma membrane via Golgi cisternae (Moore et al., 1988; Uebel and Tampe, 1999; York and Rock, 1996). Components of the MHC class I peptide loading complex include tapasin which is a transmembrane protein that allows the connection between MHC class I molecules to TAP. Other members of this MHC class I peptide loading complex are the chaperone known as calreticulin and the oxidoreductase ERp57 (Jensen, 2007).

Proteasomal processing and degradation produces peptides which are typically between 2 to 25 amino acids in length and already contain the correct carboxy-terminal amino acid sequence required for MHC class I binding. Further trimming of the peptide amino terminus however is required at this stage and is carried out by a peptidase such as TPPII the cytosolic peptidase that trims peptide fragments greater than 15 amino acids in length (Reits et al., 2004; York et al., 2006). In addition, ERAAP (=ERAP1) the amino-peptidase found ubiquitously expressed in the ER also mediates the trimming of lengthy proteasomal peptide fragments (Saric et al., 2002; Serwold et al., 2002; York et al., 2002).

Sources of protein antigens for presentation on MHC Class I molecules include those found in the cytosol, translational and defective ribosomal products, proteins that have reentered the cytosol from the ER, proteins that have been internalized and are subsequently transferred into ER via the cytosol and internalized proteins that have been processed and entered the endocytic pathway to find MHC Class I molecules being recycled from the plasma membrane and thought to function independently of TAP (Heemels and Ploegh, 1995; Pamer and Cresswell, 1998; Shastri et al., 2002; York and Rock, 1996). 


\subsection{Presentation of antigen on MHC class II molecules}

The MHC Class II antigen-processing pathway is only found active in professional antigen presenting cells (APCs) and typically present peptide fragments which are derived from exogenous proteins that are not made by the APC. This pathway is found in cell types such as DCs, B cells, macrophages and thymic epithelial cells (Jensen, 2007). The MHC class II molecules are capable of presenting longer peptide chains as the groove into which they bind has open ends (Deng et al., 1993; Sette et al., 1989; Stern and Wiley, 1994). These MHC Class II molecules are assembled in the ER (Lamb and Cresswell, 1992) and require a specialized type II transmembrane chaperone protein called the invariant chain (Ii) for stabilization of the MHC II molecule and associated proteins. Ii contains an endosomal targeting signal which allows delivery of the MHCII-Ii complex into the endosomal compartment after which protease cleavage events take place to leave a peptide fragment know as CLIP (MHC class II-associated invariant-chain peptide) on its own protecting the binding groove (Jensen, 2007; Trombetta and Mellman, 2005). These proteolytic cleavage events are mediated by cathepsin $\mathrm{S}$ and beginning from the carboxyl terminus, release the peptide component responsible for endosomal retention (Jensen, 2007). These MHCII-Ii complexes are targeted to late endosomal 'MHC II compartments' which have been described to contain generous quantities of MHC II molecules in addition to numerous peptides, which can be loaded into the peptide-binding groove (Kleijmeer et al., 1997; Pierre et al., 1996). CLIP must become dissociated from the MHC Class II peptide-binding grove and this process is accelerated by the chaperone HLA-DM which allows peptide loading and binding prior to reaching the cell surface and subsequent presentation to $\mathrm{CD} 4^{+}$ T cells (Trombetta and Mellman, 2005).

What remains unknown is whether MHC II peptides are actually generated before or after binding to MHC class II molecules. The total degradation of larger MHC Class II antigenic peptides by lysosomal proteases is prevented by the binding of the peptide portions within the peptide binding groove leaving the exposed amino acid sequences available for destruction (Donermeyer and Allen, 1989). It has been reported also that some antigens require trimming prior to loading onto MHC Class II complexes (Trombetta and Mellman, 
2005).

\subsubsection{Cross-Presentation}

Bevan was first to describe a process whereby $\mathrm{CD}^{+} \mathrm{T}$ cells specific for an exogenous donor antigen were capable of being primed to differentiate into CTLs after being acquired by and presented by host APCs (Bevan, 1976). Because these CTL responses were generated from exogenous antigens, they were referred to as "cross-primed" and the processing pathway is referred to as cross-presentation. Initial attempts to propose a mechanism for cross-presentation described the processing of phagocytosed antigen into shorter peptides inside the phagosome prior to being loaded onto recycled MHC I molecules from the plasma membrane. This mechanism was known as the vacuolar route of cross presentation (Pfeifer et al., 1993).

Although a variety of cell types such as macrophages (Harding and Song, 1994; Norbury et al., 1995), B cells (Ke and Kapp, 1996) and neutrophils (Tvinnereim et al., 2004) have been shown capable of cross-presentation activities, it is the DCs which are most efficient at cross-presenting (Jung et al., 2002).

It has been suggested that the manner of antigen uptake by DCs is the determining factor regarding eventual cross-presentation of the antigen (Burgdorf et al., 2007), with DCs taking up antigen using phagocytic, macropinocytic and receptor mediated endocytic methods which is more likely to induce cross-presentation (Burgdorf et al., 2007; Inaba et al., 1993; Jiang et al., 1995; Sallusto et al., 1995). It has been shown that when antigen is acquired using endocytic processes through the Mannose-6-Phosphate receptor found on DCs or by scavenger receptors on macrophages, subsequent presentation occurs to $\mathrm{CD}^{+} \mathrm{T}$ cells caused by the transfer of internalized antigen to early endosomal compartments. In contrast, the uptake of antigen using pinocytic methods favoured presentation to $\mathrm{CD}^{+} \mathrm{T}$ cells after the internalized antigen has been transferred to lysosomes (Burgdorf et al., 2007). 
Different mechanisms describing intracellular processing pathways for cross-presentation have been described and are summarized in the next section, although the exact contribution and interplay between these mechanisms has yet to be fully defined, the process of cross presentation appears dependent upon antigen type, the specific uptake mechanism by APCs and nature of antigen processing.

\subsection{The cytosolic pathway of cross-presentation}

Otherwise known as the cytosolic route, this is a well defined cross presentation pathway that utilizes MHC class I components for the presentation of exogenous antigen. Antigen processing via this pathway requires proteasomal degradation within the cytosol and therefore the diversion of acquired antigen into the cytosolic area before transportation into the endoplasmic reticulum (ER) via TAP. The reduction of Golgi mediated exportation from the ER by Brefeldin A inhibits this pathway (Brossart and Bevan, 1997).

To enter the cytosol, antigen can move either by diffusion processes (Meier et al., 2002; Reis e Sousa and Germain, 1995) or the active transport utilizing specific transport mechanisms (Rodriguez et al., 1999) such as Sec61 already known to transport ER proteins into the cytosol for further processing and degradation (Ackerman et al., 2006; Imai et al., 2005; Wiertz et al., 1996).

\subsection{The ER-phagosome fusion pathway of cross-presentation}

This model was described later and involves fusion of the ER membrane to the phagosome (Hatsuzawa et al., 2006) during phagocytosis permitting the transfer of ER proteins such as MHC class I, TAP molecules and tapasin into the phagosome where MHC class I loading can take place after the diversion of antigen into the cytosol for processing and subsequent return into the phagosomal compartment (Ackerman et al., 2003; Guermonprez et al., 2003; Houde et al., 2003). The diversion of antigenic proteins into the cytosol within this framework is thought to be mediated by Sec61 the ER protein transporter, and the return into the phagosomal compartment after cytosolic processing and degradation into peptide 
fragments facilitated by TAP (Guermonprez, et al., 2003; Schmitz et al., 2000). The application of a TAP inhibitor that specifically targeted early endosomes was enough to lessen the cross presentation activity (Burgdorf and Kurts, 2008).

\subsection{Endosome-to-ER pathway of cross-presentation}

It has been proposed that antigen is also capable of direct movement across the ER after endocytosis (Ackerman et al., 2005) utilizing the same transport mechanisms associated with antigen transfer into the cytosol. When transport is inhibited by RNA interference of Sec61, cross presentation activity is markedly decreased (Imai, et al., 2005).

\subsection{Alternative pathways of cross-presentation}

The cross-presentation of antigen has been reported in the absence of traditional MHC Class I associated molecules and structures such as the proteasome and TAP (Bachmann et al., 1995; Kleijmeer et al., 2001; Pfeifer et al., 1993; Song and Harding, 1996; Wick and Pfeifer, 1996). The loading of antigen onto MHC Class I molecules for cross-presentation using this pathway was shown to take place after MHC class I export within Golgicisternae since inhibition was not achieved using Brefeldin A, a Golgi transport blocker (Song and Harding, 1996).

MHC Class I and II structure components are constantly being recycled and transported to the cell surface after uptake by endosomes (Dasgupta et al., 1988; Kleijmeer et al., 2001; Reid and Watts, 1990). It had already been shown that MHC Class I molecules being taken up from the cell surface contain peptide fragments (Abdel Motal et al., 1993) and it was suggested that a form of peptide exchange could be taking place within recycling endosomes given the absence of TAP within this cross-presentation pathway. Previous work had also indicated that empty MHC class I structures could be loaded with peptides within the endosomal compartment (Schirmbeck and Reimann, 1996). 


\subsection{Preferential Cross presentation capability of DC subsets}

DCs have been shown as the superior in-vivo cross presenters (Jung et al., 2002), largely due to their internalization of acquired antigen into phagosomal compartments with contents measuring a near neutral $\mathrm{pH}$ and containing oxidative activity that permits only low levels of proteolytic activity (Savina and Amigorena, 2007). This situation favours the preservation of antigenic fragments within DCs in contrast to the rapid degradation and complete destruction of antigenic components that occurs in other phagocytic APCs.

The superior cross presentation abilities of $\mathrm{CD} 8 \alpha^{+}$DCs highlights the existence of differential cross presentation function between DC subsets. The literature has suggested that differing antigen processing activities of the $\mathrm{CD} 8 \alpha^{+} \mathrm{DCs}$ rather than enhanced acquisition and uptake of antigen are responsible for this preferential capability for cross presentation (den Haan and Bevan, 2002; Pooley et al., 2001; Schnorrer et al., 2006; Villadangos and Schnorrer, 2007).

Other distinguishing features of the CD8 $\alpha^{+}$DCs include the elevated expression of MHC class I associated proteins and the efficient $\mathrm{pH}$ control causing elevated phagosomal $\mathrm{pH}$ levels necessary for preservation of antigen and subsequent cross presentation (Dudziak et al., 2007; Savina and Amigorena, 2007). Interestingly, CD4 ${ }^{+}$DCs were shown to express increased levels of MHC Class II associated proteins in contrast to CD8 ${ }^{+}$DCs (Dudziak, et al., 2007). Although cross presentation activity has been observed in CD8 $\alpha^{-}$DCs, additional activation such as the uptake of antigen though Fc receptors is essential (den Haan and Bevan, 2002).

Previous work has suggested that further heterogeneity exists within the $C D 8 \alpha^{+} \mathrm{DC}$ population with our own studies showing that the langerin ${ }^{+}$subset of $\mathrm{CD} 8 \alpha^{+} \mathrm{DCs}$ is intimately involved in the priming and differentiation of $\mathrm{T}$ cell responses to antigen which has been cross-presented (Farrand et al., 2009). Work by Lin et al, (2008) showed that murine recipients injected with the pro-apoptotic protein horse cytochrome c (cyt $c$ ) caused the selective death of cross presenting cells by the uptake of cyt $c$ into the cytosol with the 
exception of a subset of the CD8 $\alpha^{+}$DC population which surprisingly remained unaffected (Lin et al., 2008). It was therefore concluded that this subset of $\mathrm{CD} 8 \alpha^{+}$DCs was unaffected due to their inability to cross present antigen further emphasis of the heterogeneity that exists within the $\mathrm{CD} 8 \alpha^{+} \mathrm{DC}$ population. Work within our laboratory showed that the cytochrome c sensitive cells were langerin ${ }^{+}$(Farrand et al., 2009). Another study featured the $\mathrm{CD} 8 \alpha^{+} \mathrm{CD}_{103}{ }^{+}$langerin $(\mathrm{CD} 207)^{+}$splenic DC subset which showed these DCs responsible for the acquisition of apoptotic cells by phagocytic processes and to be the primary inducers of tolerance of cell-associated antigens via cross-presentation (Qiu et al., 2009). This was deemed possible by their strategic location within the marginal zone of the splenic compartment (Qiu et al., 2009).

The preferential specialization of cross presentation activity by mouse DC subsets suggests that perhaps similar heterogeneity exists within human DC subsets and that further exploration to identify efficient cross presenting human DC subsets could only improve immunotherapeutic vaccination target efforts.

\subsubsection{DCs \& the induction of $\mathrm{CDB}^{+} \mathrm{T}$ cell responses}

$\mathrm{CD}^{+} \mathrm{T}$ cells are derived from bone marrow precursors that differentiate in the thymus. After surviving positive and negative selection events that occur in the thymus, $\mathrm{CD} 8^{+} \mathrm{T}$ cells leave the thymus and traffic to the secondary lymphoid organs via the lymphatic system. Positive selection describes the process that causes deletion of T cells with TCRs that posses low affinity for self MHC I complexes. Negative selection on the other hand describes the deletion of $\mathrm{T}$ cells that are found to possess high affinity for self-antigens. These $\mathrm{T}$ cell developmental processes collectively ensure that $\mathrm{T}$ cells which leave the thymus are capable of recognising self MHC complexes, but not self reactive.

$\mathrm{T}$ cells are incapable of recognizing soluble antigens and must be presented with peptide antigens presented on MHC I or MHC II molecules. DCs present antigen to naïve T cells surface $\mathrm{T}$ cell receptors (TCRs) via MHC molecules on the DC cell surface that bear antigen and are recognised by TCRs. The immunologic synapse describes the interface 
between APC and naïve T cell. Once engagement of MHC antigen complex occurs with the appropriate TCR, stabilisation of this weak interaction occurs via CD4 or CD8 molecules. Initial DC-T cell interactions that occur over a couple of hours are described as transient and become stabilised over time. Stabilisation binding occurs between the nonpeptide binding portion of the MHC molecule and either the CD4 or CD8 molecule and allows intracellular signalling events to occur via the TCR-associated CD3 complex on the $\mathrm{T}$ cells. This is referred to as the $1^{\text {st }}$ signal required for effective $\mathrm{T}$ cell activation.

The $2^{\text {nd }}$ signal required for effective $\mathrm{T}$ cell activation is co-stimulation provided by the antigen presenting DC. The co-stimulatory CD86 and CD80 surface molecules found on DCs bind to CD28 on T cells and induce the progression of effector T cell development. T cells by this stage have upregulated CD69, CD44 and CD25 and begin to secrete IL-2 and IFN- $\gamma$. Clonal expansion occurs in response to intracellular signalling events that take place and cause the naïve $\mathrm{T}$ cell to differentiate into effector $\mathrm{T}$ cells such as CTLs.

The $3^{\text {rd }}$ signal necessary to induce full $\mathrm{T}$ cell activation of naïve $\mathrm{CD}^{+} \mathrm{T}$ cells is exposure to the IL-12 cytokine (Curtsinger et al., 2003a; Curtsinger et al., 2003b). It was shown that exposure to IL-12 in the presence of antigen presentation and co-stimulation for at least 40 hs was required to generate CTL effector function (Curtsinger et al., 2003a; Curtsinger et al., 2003b). Brief exposure to all 3 signals for 6 hs caused minimal effector function to develop even though clonal expansion did occur. The addition of signal 3 at a later stage was unable to rescue the abortive effector function generated when only signals 1 and 2 had occurred briefly, indicating the importance of all 3 signals and timing for avoidance of tolerogenic $\mathrm{T}$ cell responses.

Once naïve $\mathrm{CD}^{+} \mathrm{T}$ cells have differentiated into fully functional CTLs, cytokines such as IFN- $\gamma$, TNF- $\alpha$ and IL-2 are released. CTLs cause cellular death by the release of cytotoxic granules such as those utilized by the perforin/granzyme pathway, in addition to death receptor mediated apoptosis induced by Fas/FasL interactions. Once clonal expansion has occurred, the $\mathrm{T}$ cell dissociates from the presenting $\mathrm{DC}$ and leaves the lymph node. Functional memory cells are generated after the effective conversion of naïve $\mathrm{T}$ cells into 
CTLs that have the potential to mount a stronger and faster response to secondary exposure to the antigenic stimulus.

In the absence of or when inadequate levels of co-stimulatory molecules are expressed on the APC, naïve $\mathrm{T}$ cells can still undergo clonal expansion however express effector cytokines ineffectively. The inevitable outcome in this situation is that $\mathrm{T}$ cells either undergo apoptosis ( $\mathrm{T}$ cell deletional tolerance) or become anergic (selectively unresponsive). These particular tolerogenic and anergic events are important to ensure the prevention of autoimmunity.

The differentiation of naïve $\mathrm{T}$ cells has been described as occurring via 3 distinct phases: expansion, contraction and memory development (Ahmed and Gray, 1996; Williams and Bevan, 2007). Expansion covers the events that occur from creation of the immunological synapse at the time of antigen presentation by DCs to naïve T cells until approximately 7-8 days later when antigen specific T cells have clonally expanded. Contraction occurs after $\sim$ 8 days when the majority $90-95 \%$ of the antigen specific $\mathrm{T}$ cells die leaving behind a small population of survivors (Badovinac et al., 2002; Harty et al., 2000). These surviving antigen specific $\mathrm{T}$ cells enter the next phase of differentiation and become long-lived memory cells capable of eliciting stronger and faster immune responses upon re-encounter with the same antigenic stimulus.

\subsubsection{DCs as antigen transporters}

In addition to maturation, activation, licensing, presentation, cross-presentation and CTL induction activities, DCs have also been reported to function as antigen transporters. Antigen has been reportedly transferred from macrophages to DCs, (Backer et al., 2010) but more commonly described as taking place between DCs (Allan et al., 2006; Belz et al., 2004; Inaba et al., 1998; Kleindienst and Brocker, 2003; Qu et al., 2009).

The transfer of antigen between DCs has been shown to occur via secretion of exosomes (Luketic et al., 2007; Segura et al., 2005), acquisition of apoptotic DC material (Belz et al., 
2004; Inaba et al., 1998) and the transfer of plasma membrane components from DCs, through gap junctions (Neijssen et al., 2005) or nanotubules (Chinnery et al., 2008). It has also been shown that protein (Norbury et al., 2004; Shen and Rock, 2004), peptide (Neijssen, et al., 2005) and MHC complex-peptide complexes (Qu et al., 2009) have been transferred between DC populations. Why and how this occurs will become of direct relevance in the coming chapters and will be explored further.

\subsection{DCs and the sensing of danger}

As already noted, host innate and adaptive immune defense mechanisms are required for control and protection against invasive microbial pathogens. Pattern recognition receptors (PRRs) are capable of recognizing pathogen (or microbe) associated molecular patterns (PAMPs) (Akira and Takeda, 2004; Blander and Medzhitov, 2006; Granucci and Ricciardi-Castagnoli, 2003; Medzhitov and Janeway, 1998) and can be found on DCs neutrophils, macrophages and other immune cells including T cells and NK cells. PAMPs are conserved molecular patterns commonly found in pathogens.

\subsubsection{Pathogen Recognition Receptors}

It was Charles Janeway who first proposed the notion of PRRs that were capable of the detection of microbial pathogens or PAMPs (Janeway, 1989). In 1997 Medzhitov et al, published their discovery of what is now known as the Toll-like receptor 4 (TLR 4) (Medzhitov et al., 1997). Bruce Beutler and colleagues later showed the unresponsiveness of $\mathrm{C} 3 \mathrm{H} / \mathrm{HeJ}$ and $\mathrm{C} 57 \mathrm{BL} / 10 \mathrm{ScCr}$ mice to LPS was due to a mutation in the Tlr4 locus confirming TLR 4 as an LPS sensing receptor (Beutler, 2000; Poltorak et al, 1998). Subsequent TLRs were also identified along with other non-TLR PRRs such as NLRs, CLRs and RLRs which are briefly discussed in the following sections.

Once engagement has occurred between the PRR and its specific PAMP, signalling cascades are initiated within the cell that induces innate immune responses. PRRs can exist 
bound to the cell membrane surface as extracellular receptors for example TLRs, or as soluble intracellular cytoplasmic receptors such as NOD-like receptors. Transmembrane PRRs are membrane bound and recognize their specific PAMPs either in the extracellular domain or within phagosomal and endosomal compartments. Examples of transmembrane PRRs include the TLRs and C-type lectin Dectin-1. The TLRs represent an important subset of PRRs capable of inducing both innate and adaptive immune responses to pathogenic invasion and sensing of infection via specific PAMPs (Iwasaki and Medzhitov, 2004; Pasare and Medzhitov, 2005). Adaptive immune responses generated by TLR stimulation include $\operatorname{IgG}$ antibody production, $\mathrm{CD}^{+} \mathrm{T}$ cell induction and memory responses (Pasare and Medzhitov, 2004, 2005).

\subsubsection{NLRs}

NOD-like receptors (NLRs) are cytosolic receptors made up of more than 20 family members. The nucleotide-binding oligomerization domain protein 1 (NOD1) and nucleotide-binding oligomerization domain protein 2 (NOD2) were the first NLR members to be recognized. NOD1 and NOD2 are capable of recognizing cytosolic peptidoglycan bacterial cell wall components and inducing innate responses (Fritz and Girardin, 2005; Fritz et al., 2006; Girardin et al., 2003). Some NLRs such as NALP1 (NLRP1) and NALP3 (NLRP3) are important for inflammasome formation and the subsequent release of Il1B, while other NLRs family members are involved in Type 1 IFN production. NOD-2 mutations have been associated with Crohn's disease (Sancho et al., 2008) and a potential role in distinguishing pathogens from commensal organisms has been hypothesized for NODS. It has also been suggested that the combination of NOD1 and TLR stimulation is sufficient to induce adaptive immune responses.

\subsubsection{RLRs}

The RIG-1-like receptors are RNA helicases found in the cytosol and sense nucleic acids. They are involved in antiviral activities via production of Type 1 IFN. The three known 
RLRs are LGP2, melanoma differentiation-associated gene 5 (MDA5) and retinoic acidinducible gene (RIG-1). Creagh and O’Neill coined the name RIG-I-like receptor (RLR) for this family of receptors (Creagh and O'Neill, 2006).

\subsubsection{CLRs}

The C-type lectins are a large superfamily of proteins that contain one or more lectin-like domains and/or one or more ITAM's (immunoreceptor tyrosine-based activation motifs). The CLRs recognize fungal and bacterial PAMPS and respond specifically to carbohydrate ligands found on pathogens by generating inflammatory responses via SYK kinase enabling signalling via CARD9 (Caspase Recruitment Domain) that leads to NF- $\mathrm{BB}$ activation (Creagh and O'Neill, 2006; Drummond et al., 2011).

\subsubsection{Other cytosolic nucleic acid sensors}

DHX36 (DEAH/RHA protein RHAU) and the DEAD-box proteins DDX1 and DDX2 are helicases that sense double stranded RNA in the cytosol and induce IFN- $\beta$ transcription activity (Zhang et al., 2011). DAI (DNA-dependent activator or IFN-regulatory factors) is also known as DLM1 and ZBP1 is a type 1 IFN inducer and recognizes cytosolic DNA. Two PYHIN proteins IF116 (IFN- $\gamma$ inducible protein 16) and AIM2 have been proposed as members of a new family of ALRs AIM2-like receptors and recognise cytosolic DNA (Unterholzner et al., 2010).

\subsubsection{TLRs}

TLR agonists represent a major class of Pattern Recognition Receptors (PRRs) that recognise bacterial, viral and fungal components otherwise known as PAMPS (Pathogen Associated Molecular Patterns). Each TLR binds a specific PAMP and upon engagement with its respective ligand, induces the entry of the transcription factor NF-KB into the 
nucleus triggering the upregulation of co-stimulatory markers on DCs and the release of pro-inflammatory cytokines.

The Toll receptor found in Drosophila fruitflies was initially identified as being responsible for the dorso-ventral patterning in Drosophila embryos. It was later discovered that Toll was also involved in anti-fungal responses in insects and that in mammals, mediated immune responses against microorganisms (Imler and Hoffmann, 2003). Medzhitov and colleagues discovered hToll the mammalian homologue of the Drosophila toll receptor in 1997 - otherwise known as TLR 4 (Medzhitov et al., 1997).

In mammals, TLRs represent a large group of PRRs made up of members each classified with a number. In humans 10 functional TLRs have been identified while 12 functional TLRs have been found in mice (Kawai and Akira, 2011). The TLRs 1-9 are conserved in humans and mice, while TLR 10 is reportedly only functional in humans-even though it is still present in mice as a pseudogene (Takeda and Akira, 2005). Although the TLR 11 gene is present in mouse and humans, it is only translated in mice (Tauszig-Delamasure et al., 2002) while TLR 12 and 13 are found only in mice.

Specific microbial membrane and wall components are recognized by TLR 1, TLR 2, TLR 4, TLR 5 and TLR 6 which are localized to the cell surface, while TLR 3, TLR 7, TLR 8 and TLR 9 recognize nucleic acids and can be found within intracellular structures such as endosomes, lysosomes, endolysosomes and the endoplasmic reticulum (Blasius and Beutler, 2010; Kawai and Akira, 2011). TLRs can be found expressed on a variety of cell types in the spleen, lungs, blood, muscle and intestines (Abreu et al., 2005; Kabelitz and Medzhitov, 2007; Schuster and Nelson, 2000; Takeda et al., 2003; Zarember and Godowski, 2002) and their expression can be upregulated or downregulated in response to different stimuli (Hart et al., 2005; Karlsson et al., 2004; Salim et al., 2009). TLRs are designed to recognize invasive PAMPs, which include gram-negative and gram-positive bacterial fragments, viral DNA and RNA components in addition to fungi and protozoa (Liew et al., 2005). TLR engagement and recognition of specific PAMPs drives the induction of specific signalling events causing DC maturation and the induction of acute 
immune responses designed to destroy the invading pathogen (Akira and Takeda, 2004) with certain TLRs capable of recognizing and responding to a variety of host-derived stress signals released by different cell types.

Although the stimulation of immune cells via PAMP sensing by TLRs is typically regarded as a desirable process necessary for the elimination and destruction of invading pathogens, it is recognized that TLRs have been implicated as mediators in the pathogenesis of chronic inflammatory disorders, autoimmune and infectious diseases (Chaudhuri et al., 2005; Cook et al., 2005; Liew, et al., 2005). Examples of chronic inflammatory disorders that involve TLRs include asthma, rheumatoid arthritis and possibly even Alzheimer's disease (Chen et al., 2006; Cui et al., 2002) Autoimmune disorders also thought to involve TLRs include diabetes and experimental autoimmune encephalomyelitis (Liew et al., 2005). Dilated cardiomyopathy is a common cause of heart failure in young patients and has also been attributed to TLR stimulation (Eriksson et al., 2003).

\subsection{TLR 1}

TLR 1 is important for the recognition of lipopeptides and distinguishes between triacylated and diacylated forms (Takeuchi et al., 2002). TLR 1 and TLR 6 share a high degree of homology (Takeda et al., 2003) while heterodimers formed between TLR 1/TLR 2 recognize triacylated lipopeptides (Akira, 2006). This TLR 1/TLR 2 heterodimer is also required for the induction of responses against Neisseria meningitides (Wyllie et al., 2000).

\subsection{TLR 2}

TLR 2 recognizes a broad range of microorganisms that include both gram-positive and gram-negative bacterial components (Akira, 2006). TLR 2 associates functionally with either TLR 1 or TLR 6 forming heterodimers that allows discrimination between triacylated lipopeptides from gram-negative bacteria recognized by TLR 1/TLR 2, and diacylated lipopeptides from gram-positive bacteria recognized by TLR 2/TLR 6 (Akira, 
2006). Pam3Cys was the synthetic TLR 2 agonist utilized in the laboratory for experimental work highlighted in this thesis.

\subsection{TLR 3}

TLR 3 is a nucleic acid detector that specifically recognizes double stranded RNA (dsRNA) (Alexopoulou et al., 2001) generated by viruses during replication (Takeda, et al., 2003). dsRNA causes DC maturation and induces Type 1 IFN production (Takeda, et al., 2003) and activates NF-KB, IRF3 and proinflammatory cytokine production (Kawai and Akira, 2011). Although TLR 3 is found intracellulary mainly within endosomal structures (Funami et al., 2004; Matsumoto et al., 2003), it is also expressed within the endoplasmic reticulum, lysosomes and multivesicular bodies (Blasius and Beutler, 2010). TLR 3 does not utilize the MyD88 adaptor signalling molecule but relies on the alternative MyD88 independant signalling pathway utilizing the TRIF adaptor molecule for communication (Hoebe et al., 2003). The dsRNA analogue polyinosinic-polycytidylic acid (poly(I:C)) is also recognised by TLR 3 (Kawai and Akira, 2011).

\subsection{TLR 4}

TLR 4 responds to Lipopolysaccharide (LPS) an important component of gram-negative bacterial cell walls. The LPS structure is comprised of a short oligosaccharide, O-antigen polysaccharide and the bioactive component, lipid A (Leon et al., 2008). The TLR 4 undergoes homodimerization upon ligand engagement and downstream signalling events involving the adaptor molecules MyD88, TRIF, TRAM and TIRAP (Kawai and Akira, 2011; Leon et al., 2008). Important TLR 4 binding components include accessory molecules such as LPS-binding protein (LBP), the glycosylphosphatidylinositol (GPI)anchored molecule CD14 and MD-2 (Kawai and Akira, 2011; Leon et al., 2008). MD-2 is an essential constituent of the LPS receptor complex and associates with the extracellular TLR 4 complex while LBP is found in the serum and binds with the LPS molecule before connecting with the CD14 structure (Kawai and Akira, 2011). The LPS molecule is 
responsible for bringing the CD14 and TLR 4 complex close together (da Silva Correia et al., 2001; Jiang et al., 2000).

Other TLR 4 ligands include heat shock proteins (Ohashi et al., 2000; Vabulas et al., 2001), fibronectin EDA (Gondokaryono et al., 2007; Okamura et al., 2001) and the murine MMTV envelope viral glycoprotein (Jude et al., 2003). The activity of the Western Yew tree bark derived mitotic inhibitor drug Taxol/Paclitaxel is mediated by binding to the TLR 4 receptor even though its structure differs somewhat from that of LPS (Byrd-Leifer et al., 2001; Fitzpatrick and Wheeler, 2003). TLR 4 is also associated with the host response to the virulence factor pneumolysin produced by Streptococcus pneumoniae and to viral products generated by respiratory syncytial virus (RSV) infection (Kurt-Jones et al., 2000) (Malley et al., 2003). MPL is the synthetic TLR 4 agonist utilized in the lab for experimental work highlighted in this thesis. The potent TLR 4 agonist LPS was also utilized to generate positive control DC populations within this work.

\subsection{TLR 5}

Located on the cellular surface, TLR 5 is important for mucosal immunity (Hawn et al., 2003) and responds to flagellin the elemental component of bacterial flagella (Akira, 2006; Hayashi et al., 2001). Gram-negative bacteria use flagella attached to the outer surface membrane like propellers for transport using propulsive mechanisms through aqueous environments in addition to their utilization for attachment purposes during bacterial infection processes. TLR 5 signalling occurs via the MyD88 dependant pathway although it has been reported that TLR 5 expressing epithelia cells utilize TRIF (Choi et al., 2010).

\subsection{TLR 6}

TLR 6 is located on the cell surface and forms heterodimers with TLR 2 and recognizes diacylated lipopeptides from gram-positive bacteria (Akira, 2006). Signalling occurs via the MyD88 dependant pathway. 


\subsection{TLR 7}

TLR 7 recognizes single stranded RNA (ssRNA) is located intracellularly utilizing the MyD88 signalling pathway. In humans, the TLR 7 and TLR 8 genes show strong homology and are both located on the $\mathrm{X}$ chromosome. Guanosine or uridine-rich single stranded RNA (ssRNA) from different viruses have been shown to bind to TLR 7 and include human immunodeficiency virus (HIV), the influenza virus (Diebold et al., 2004; Heil et al., 2004; Lund et al., 2004). Although host ssRNA is widely available, it is not recognizable by TLR 7 under normal conditions due to the location of the receptor within the endosomal membrane preventing access by host ssRNA. Imidazoquinolines are a class of synthetic compounds that bind to TLR 7 and are capable of antiviral and antitumour properties by inducing the production of proinflammatory cytokines such as IFN- $\alpha$.

TLR 7 is also involved in the pathogenesis of immune disorders such as systemic lupus erythematosus (Link et al., 2006) and Sjogren's syndrome (Berland et al., 2006; Christensen et al., 2006; Marshak-Rothstein, 2006; Pisitkun et al., 2006). A possible cause includes the production of IFN- $\alpha$ as a result of contact and recognition of host ssRNA by TLR 7 after Fc receptor mediated endocytosis of the immune complexes formed between ssRNA with RNA-specific antibody in plasmacytoid DCs. Autoantibody production has also been reported after the recognition of ssRNA by the RNA-specific B cell receptor (BCR) found on autoreactive B cells (Barrat et al., 2005; Lau et al., 2005; Vollmer et al., 2005). Resiquimod was the synthetic TLR $7 / 8$ agonist we utilized in the laboratory for experimental work highlighted in this thesis.

\subsection{TLR 8}

Although expressed in both mice and humans, murine TLR 8 was initially believed nonfunctional (Forsbach et al., 2008; Jurk et al., 2002). TLR 8 is located within intracellular comportments with the human receptor recognizing ssRNA (Jurk, et al., 2002). Human TLR 8 causes TLR 7 and TLR 9 signalling downregulation while murine TLR 8 is a TLR 
7 inhibitor (Wang et al., 2006). TLR 8 has also been shown responsive to bacterial RNA (Davila et al., 2008; Gantier et al., 2010) while TLR 2 expression is upregulated in response to TLR 8 stimulation (Cervantes et al., 2011).

\subsection{TLR 9}

TLR 9 is located intracellularly and recognizes the unmethylated CpG motifs of bacterial and DNA viruses which include adenovirus (AdV), HSV, HCMV, MCMV, VZV, and baculovirus (Abe et al., 2005; Basner-Tschakarjan et al., 2006; Delale et al., 2005; Hochrein et al., 2004; Iacobelli-Martinez et al., 2007; Krug et al., 2004; Lund et al., 2003; Tabeta et al., 2004; Wang et al., 2005). The cysteine residues of vertebrate CpG motifs are highly methylated thereby preventing immunostimulatory activity. Vertebrate DNA can be recognized by TLR 9 if delivered to the endosome highlighting the importance of DNA location in recognition processes (Kawai and Akira, 2011). Synthetic oligodeoxynucleotides containing $\mathrm{CpG}$ motifs ( $\mathrm{CpG}$ ODN) mimic bacterial DNA recognizable by TLR 9 with CpG-A and CpG-B just two examples of synthetic CpGs that differ in structure yet still bind to TLR9 (Blasius and Beutler, 2010). The binding of CpGA with TLR 9 in plasmacytoid DCs induces production of Type 1 IFNs while CpG-B, the conventional type of $\mathrm{CpG}$ induces the proinflammatory cytokines (Blasius and Beutler, 2010).

TLR 9 is also involved in the autoimmune disorders systemic lupus erythematosus (SLE) and rheumatoid factor production. Rheumatoid factor is produced by autoreactive B cells after the binding of $\operatorname{IgG} 2 \alpha$-chromatin complex with the BCR, internalization of the complex that includes chromatin and hypomethylation CpG motifs with TLR 9 (Leadbetter et al., 2002). The pathogenesis of SLE is thought to involve the BCR or Fc receptor mediated internalization of immune complexes comprised of IgG and chromatin coming into contact with intracellular TLR 9 also causing IFN- $\alpha$ production by pDCs (Boule et al., 2004). 


\subsection{TLR 10}

TLR 10 is found only in humans and located on the cell surface (Blasius and Beutler, 2010).

\subsection{TLR 11}

TLR 11 does not produce a functional protein in humans (Yarovinsky et al., 2005; Zhang et al., 2004). In mice, TLR 11 is located on the cell surface and expressed in bladder epithelial cells offering protection from infection by uropathogenic bacteria (Zhang et al., 2004). The protozoan parasite Toxoplasma gondii was shown to stimulate DCs via TLR 11 using a profilin-like molecule resulting in potent IL-12 production (Chen et al., 2007).

\subsection{TLR 12}

TLR 12 is found in mice and located on the cell surface (Blasius and Beutler, 2010).

\subsection{TLR 13}

TLR 13 associates with the UNC93B1 protein found within intracellular endoplasmic reticular structures (Brinkmann et al., 2007). 


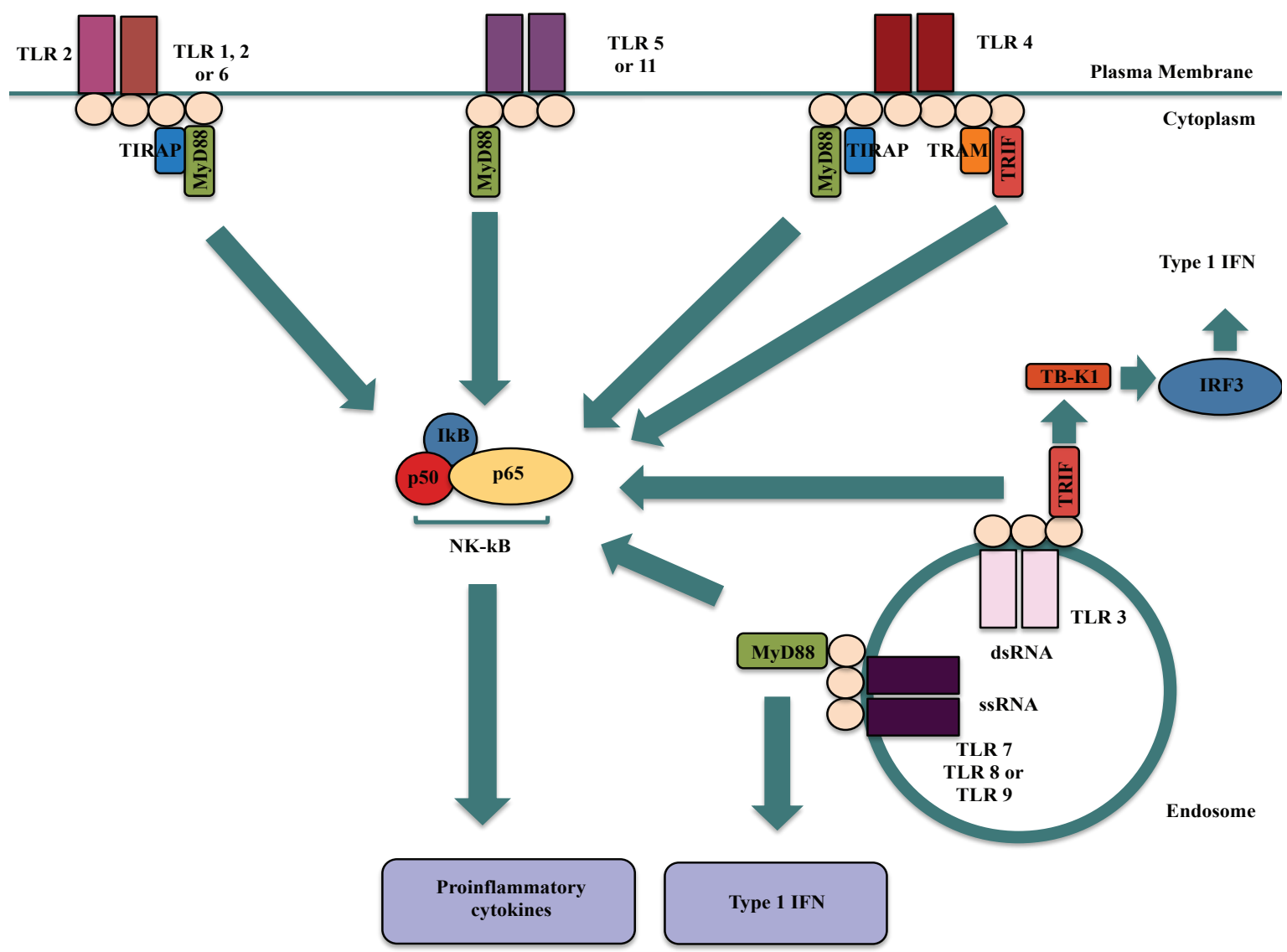

Figure 1.6 Schematic diagram showing location of TLRs on DCs and their connection to specific adaptor proteins and major signalling pathways. TLRs 1, 2, 4, 5, 6, 11 are found on the outer DC plasma membrane while TLRs 3, 7, 8 and 9 are found on the inner endosomal compartment. With the exception of the TLR 3 pathway, all TLRs signal via a signalling pathway that utilizes the MyD88 adaptor protein (myeloid differentiation primary response gene 88). The downstream outcome of the MyD88 signalling pathway is the production of proinflammatory cytokines via the NF-кB transcription factor. Signalling via this pathway causes the release of NF-KB from its inhibitor to allow movement into the nucleus and induction of gene expression. TLR 3 signals via a MyD88 independent pathway that utilizes an adaptor protein called TRIF (Toll/IL-1R(TIR)-domain containing adaptor protein inducing interferon- $\beta$ ) otherwise known as TICAM1). TLR 4 is capable of signalling via both the MyD88 dependent and MyD88 independent pathways. Adapted from (Trinchieri and Sher, 2007). 


\subsection{TLR location}

TLRs are found on the extracellular cell surface and intracellular compartments of both immune and non-immune cell types. DCs typically express a broad array of TLRs in both murine and human hosts where TLR 1, TLR 2, TLR 4, TLR 5, TLR 6 and TLR 11 are expressed and found on the extracellular cell surface while TLR 3, TLR 7, TLR 8 and TLR 9 are found within intracellular endosomal and endoplasmic reticular structures (Figure 1.6). UNC93B1 is a transmembrane protein found within the endoplasmic reticulum and is responsible for TLR transportation to intracellular expression sites (Brinkmann et al., 2007; Ewald et al., 2008; Kim et al., 2008; Takeda et al., 2003).

\subsection{TLR distribution and DC subtypes}

Conventional DCs (cDCs) in mice rats and humans express TLR 2, TLR 4 and TLR 6, which recognize peptidoglycans, gram-negative bacterial wall components such as LPS, in addition to lipopeptides.

The expression of TLR 3 mRNA appears to be restricted to the $\mathrm{CD} 8^{+} \mathrm{cDC}$ subset and generates cytokine and chemokine production. Plasmacytoid DCs (pDCs) in mice, rats and humans typically favour expression of the intracellular TLR types such as TLR 7, TLR 8 and TLR 9. TLR stimulation of pDCs results in the potent production of Type 1 IFNs (Guzylack-Piriou et al., 2004).

Although a variety of host derived (endogenous) and exogenous pathogen derived products have been identified as TLR ligands via investigations utilizing gene-knockout mice, there is however a relative dearth of pharmacological evidence showing direct ligand and TLR agonist interactions.

Immature DCs respond to microbial components (Hertz et al., 2001; Mellman and Steinman, 2001; Michelsen et al., 2001; Tsuji et al., 2000) and although immature DCs express TLR 1, TLR 2, TLR 4 and TLR 5, the DC maturation process reduces expression 
of these TLRs. Another example of the differential expression of TLRs based on maturity status is highlighted by the expression of TLR 3 on mature DCs only and not on immature DCs (Muzio et al., 2000).

\subsection{Endogenous ligands}

Danger signals released by stressed and damaged cells include HMGB1 and Heat shock proteins (Hsp) (HSP60 and HSP70). These molecules are typically generated by dying necrotic cells and when released, are also capable of inducing immune responses. HMGB1 and HSP60 exert immune stimulatory effects via recognition by TLR 4 (Asea et al., 2002; Ohashi et al., 2000; Vabulas et al., 2001). It has also been reported that Hsps such as Hsp60, Hsp70, Hsp90 (gp90) induce the maturation of DCs and induce the proinflammatory cytokine production by monocyte-macrophages. The cytokine-like effects of these endogenous molecules are mediated via TLR signal transduction pathways in a manner similar to lipopolysaccharide (via TLR 4) and bacterial lipoproteins (via TLR 2).

Controversy exists however concerning the exact nature of "host" derived TLR ligands and their interaction with TLRs. An example is the reported cytokine effects of Hsp being caused by contamination of LPS or LPS-associated molecules (Tsan and Gao, 2004).

\subsubsection{TLR signalling}

There are similarities and differences that exist between TLR signalling pathways in humans and Drosophila. Antifungal responses in Drosophila require the Toll pathway, while anti-gram-negative bacterial responses utilize the IMD pathway. The mammalian defense response consists of intracellular pathways that involve the TLR signalling pathway. This signalling pathway lies homologous to the IL-1R family with both pathways utilizing the (myeloid differentiation primary response protein 88) MyD88 adaptor protein. 
When activated, MyD88 recruits IRAK (IL-1R-associated kinase), a death domaincontaining serine/threonine kinase that undergoes phosphorylation to becomes activated and associate with TRAF6. The downstream impact of these early events is the activation of the JNK and NF-KB signalling pathways.

\subsection{MyD88-dependent signalling pathway}

The MyD88 adaptor protein is an essential component of the TLR signalling pathway and is required for the induction of proinflammatory cytokines. When treated with peptidoglycan, lipoproteins, CpG DNA, dsRNA, or imidazoquinolines, macrophages from MyD88 deficient mice were unable to generate inflammatory cytokines and were unable to activate NFKB or JNK (Alexopoulou, et al., 2001; Hemmi et al., 2002; Hacker et al., 2000; Schnare et al., 2000; Takeuchi et al., 2000; Takeuchi et al., 2000). Studies involving MyD88-deficient mice also showed that MyD88 was crucial for the response to IL-1 and LPS (Adachi et al., 1998; Kawai et al., 1999). In addition to being highly susceptible to Staphylococcus aureus infections (Takeuchi et al., 2000), MyD88-deficient mice were also unable to produce IL-6 in response to stimulation with flagellin (Hayashi et al., 2001).

The TRAF6 molecule is also a critical downstream component of the IL-1R and TLR signalling pathways given that TRAF6-deficient mice were incapable of responding to either IL-1 and LPS (Lomaga et al., 1999). IRAK-deficient mice have also highlighted the importance of the IRAK molecules in TLR signalling pathways (Kanakaraj et al., 1998; Suzuki et al., 2002; Swantek et al., 2000; Thomas et al., 1999) (See Figure 1.7). 


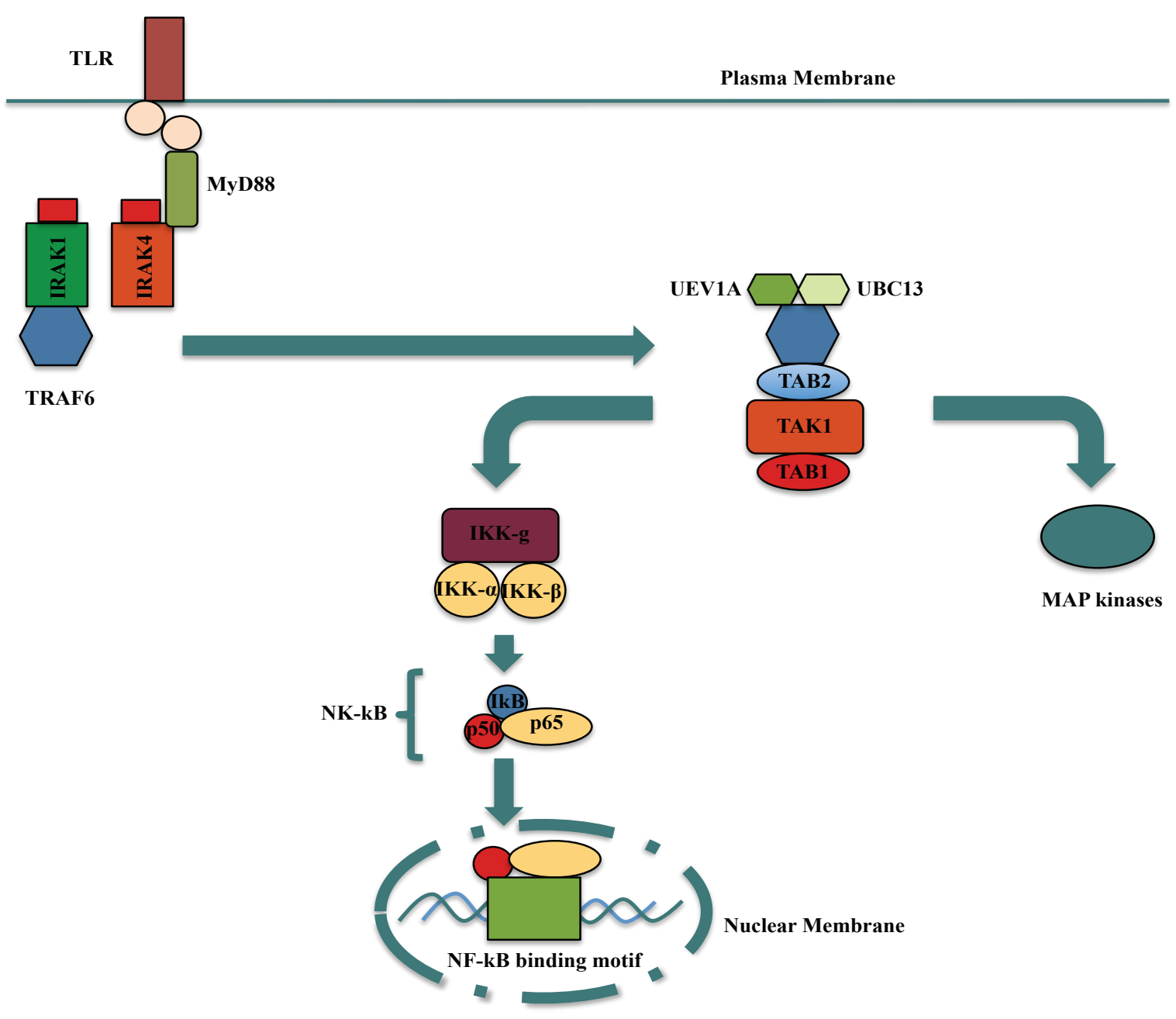

Figure 1.7 Schematic diagram showing major adaptor proteins and molecular signalling events of the TLR MyD88 dependent signalling pathway. Adapted from (Akira and Takeda, 2004). The MyD88-dependent signalling pathway utilizes adaptor proteins and signalling proteins that include MyD88, IRAK, and TRAF6 and leads to $\mathrm{NF}_{K} \mathrm{~B}$ activation. The TRAF6 molecule is also a critical downstream component of the IL$1 \mathrm{R}$ and TLR signalling pathways. The IKK components are also important for TLR signalling activities. Three molecules make up the IkB kinase (IKK) complex and include IKK- $\alpha$ and IKK- $\beta$ which are catalytic subunits joined to a scaffold protein IKK- $\gamma$. The IkB molecule sequesters NF-KB in the cytoplasm. When phosphorylation of the IkB serine residues occurs by the IkB kinase (IKK) complex, NF-KB becomes dissociated and translocates into the nucleus. The complex responsible for activating IKK is comprised of TAKI, Ubc13 and Uev1A. Together, Ubc13 and Uev1A make-up a ubiquitin conjugating enzyme complex that associates with TRAF6 to induce Lys 63 (K63)-linked polyubiquitination of TRAF6 (Wang et al., 2001). Once this occurs, TAKI becomes activated and is capable of mediating phosphorylation of the IKK complex (Kopp and Medzhitov, 1999). 
The IKK components are also important for TLR signalling activities. Three molecules make up the IkB kinase (IKK) complex and include IKK- $\alpha$ and IKK- $\beta$ which are catalytic subunits joined to a scaffold protein IKK- $\gamma$. The IkB molecule sequesters NF-KB in the cytoplasm (see Figure 1.7). When phosphorylation of the IkB serine residues occurs by the IkB kinase (IKK) complex, NF-KB becomes dissociated and translocates into the nucleus (Figure 1.7).

The complex responsible for activating IKK is comprised of TAKI, Ubc13 and Uev1A. Together, Ubc13 and Uev1A make-up a ubiquitin conjugating enzyme complex that associates with TRAF6 to induce Lys 63 (K63)-linked polyubiquitination of TRAF6 (Wang et al., 2001). Once this occurs, TAKI becomes activated and is capable of mediating phosphorylation of the IKK complex (Kopp and Medzhitov, 1999) (See Figure 1.7).

\subsection{MyD88-independent signalling pathway}

Although MyD88 is essential for the production of inflammatory cytokines in response to a range of microbial components, it has been shown that NF-KB and $\mathrm{NKK}$ can still be induced in MyD88-deficient macrophages in response to LPS but with delayed kinetics (Kawai et al., 1999). This provided evidence that MyD88-independent components existed within the TLR 4 MyD88 signalling pathway. It was also shown that DCs from MyD88 deficient mice were still capable of upregulation of co-stimulatory molecules in response to LPS while DCs from TLR 4-deficient mice were not, indicating an alternative to the MyD88 pathway must exist (Kaisho and Akira, 2001a; Kaisho et al., 2001).

The TIR domain containing adaptor protein TIRAP/Mal (MyD88 adaptor like) was identified as functioning within the MyD88-independent pathway in response to LPS (Fitzgerald et al., 2001; Horng et al., 2001). When wild-type and MyD88 deficient DCs were treated with a TIRAP peptide that blocked TIRAP signalling, the maturation response to LPS did not occur. 
The IRF3 transcription factor was also shown to be involved in the MyD88 independent signalling pathway in response to TLR 4 stimulation with LPS, but not TLR 2 stimulation (Kawai et al., 2001). This LPS induced stimulation of IRF3 also caused the expression of IFN-inducible genes (Fitzgerald et al., 2001). The MyD88-independent pathway was also activated by dsRNA and viral infection with IFN- $\alpha / \beta$ - and IFN-regulated genes being induced (Iwamura et al., 2001; Sato et al., 2000; Taniguchi and Takaoka, 2002).

The TRIF (TIR-domain-containing adaptor protein inducing IFN- $\beta$ ) adaptor molecule is also an important player that features in the MyD88-independent signalling pathway. The activation of MyD88/TRIF independent signalling cascade induces MAPK signalling pathways that cause NF- $\kappa$ B activation to induce IL-6, IL-12, TNF- $\alpha$ and (pro) IL-1 $\beta$ production. Induction of the MyD88/TRIF signalling cascade causes the activation of IRF3 (Interferon regulatory factor 3 ) that leads to Type 1 IFN production. TRIF promotes inflammasome activation that leads to the final conversion of (pro) IL-1 $\beta$ to IL-1 $\beta$.

\subsection{TLR signalling \& synergistic responses}

It has been suggested that synergistic responses generated by professional antigen presenting cells such as DCs are a regular feature of viral and microbial infection given that pathogens typically carry more than one PAMP (Napolitani et al., 2005). Bacterial degradation can expose additional TLR ligands, a specific example being infection by flagellated gram-negative bacteria from which multiple PAMPs such as LPS and flagellin can be derived simultaneously (Blander and Sander, 2012). The stimulation of multiple TLRs via multiple PAMPs from invading microorganisms ensures efficient and generous immune responses occur when invasion and infection have taken place (Napolitani et al., 2005). These synergistic responses are non-random and very specific in that only selected agonists are capable of inducing such events (Zhu et al., 2008).

The additive (combined) and synergistic (greater than additive) effects of using more than one TLR agonist to stimulate mouse and human DCs had been reported by a number of groups (Bagchi et al., 2007; Bohnenkamp et al., 2007; Gautier et al., 2005; Napolitani et 
al., 2005; Roelofs et al., 2005; Trinchieri and Sher, 2007; Underhill, 2007; Warger et al., 2006; Whitmore et al., 2004; Zhu et al., 2008). Selected TLR agonists combinations had been shown to induce DCs to mature and generate proinflammatory cytokines in-vitro at concentration doses which were suboptimal when compared to using the single TLR agonist dose for DC stimulation (Napolitani et al., 2005; Warger et al., 2006). Of particular interest was the increased production of IL-12p70 when using TLR agonists in combination that required lower doses to achieve the same level of production than when using either TLR agonist alone. Increased IL-12p70 was reportedly due to enhanced NK$\mathrm{kB}$ activity and nuclear translocation followed by the increased production of proinflammatory cytokines.

The ligation of TLR 4 by MPL or LPS can trigger signalling with both TRIF dependent and MyD88/TRIF independent signalling pathways (Figure 1.8), whereas Pam3Cys exerts its effects through the MyD88 dependent/TRIF independent pathway. Downstream effects of targeting the MyD88 signalling pathway include the production of proinflammatory cytokines such as IFN- $\beta$, IL-12, IL- 6 and TNF- $\alpha$ while targeting of the TRIF dependent signalling pathway induces the upregulation of CD86 and the production of IP-10 (Zhu et al., 2008). The successful use of MPL with Pam3Cys for inducing additive maturation and cytokine production effects is proposed to be resultant of the simultaneous targeting of these separate signalling pathways. The induction of the MyD88/TRIF dependent and TRIF independent signalling pathway by MPL is further amplified by the engagement of Pam3Cys that utilizes the MyD88 signalling pathway. It is important to note that the use of BCG (bacillus Calmettte-Guerin) to treat bladder cancer in humans is highly dependent on TLR 2 and TLR 4 mediated maturation of DCs (Tsuji et al., 2000; Uehori et al., 2005). 


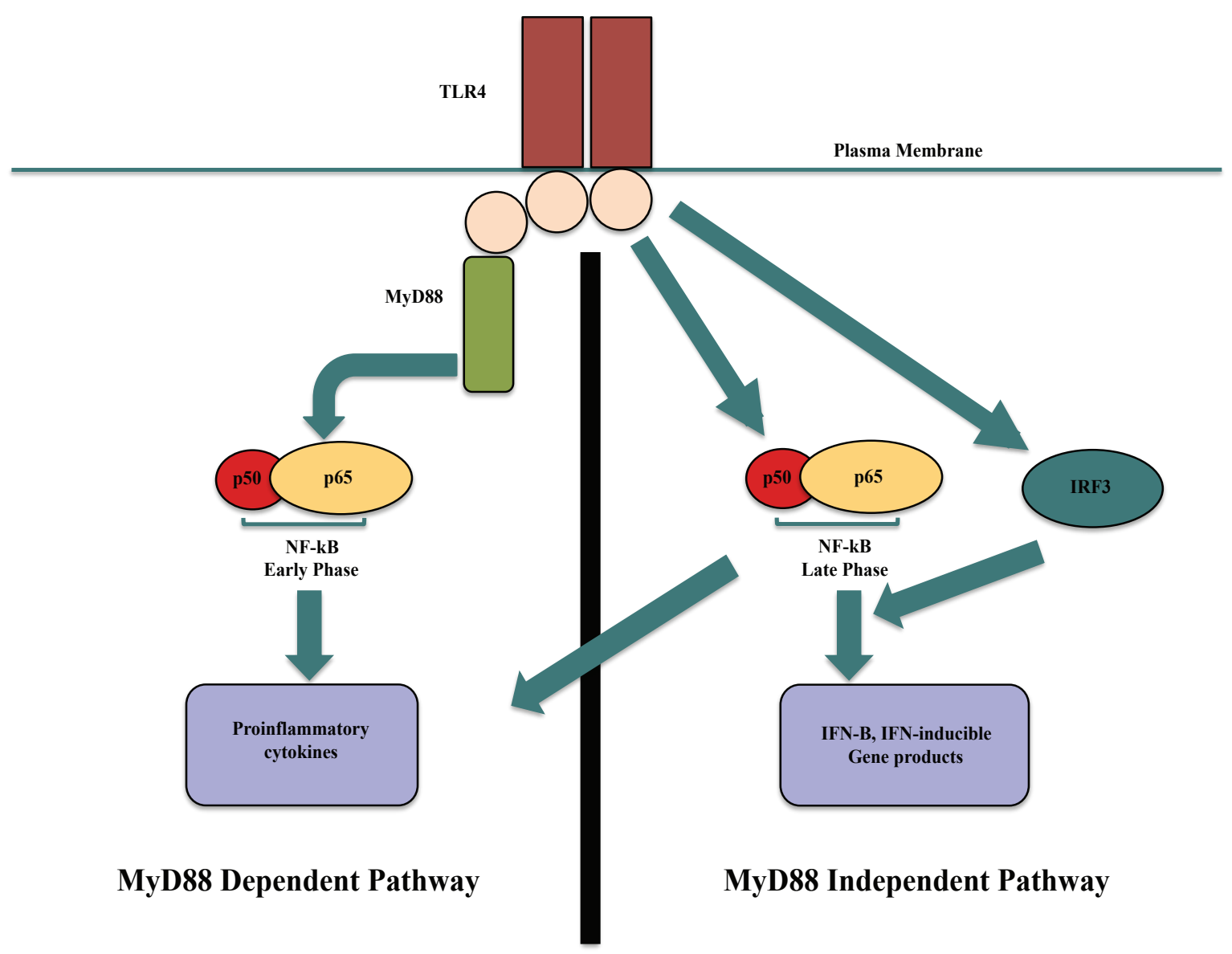

Figure 1.8 Schematic diagram showing the molecular events that occur on the MyD88 dependent signalling pathway compared with that of the MyD88 independent signalling pathway. Adapted from Akira \& Takeda, 2004. The MyD88 dependent and independent TLR signalling pathways feature mediators that are specific for different signalling pathways and include the MyD88 (myeloid differentiation primary response protein 88 ) and TRIF (TIR-domain-containing adaptor protein inducing IFN- $\beta$ adaptor molecules. The downstream signalling outcomes utilizing either the MyD88-dependent or MyD88-independent signalling pathways involve the induction of proinflammatory cytokines and generation of IFN- $\beta$ and the induction of IFN-inducible gene products. 


\subsection{Licensing of DCs}

Licensing" of DCs refers to the incorporation of CD40 signalling activities combined with DC activation. This ensures successful antigen presentation with appropriate costimulatory activity occurs together with the cytokine production necessary for full effector and memory function to occur. Please refer to (Section 1.4.2).

CD40 is a $45000-50000 \mathrm{MW}$ type 1 phosphoprotein expressed on a range of cell types such as DCs, B cells, non-hematopoietic cells and macrophages (Banchereau et al., 2000; Tan et al., 2002; van Kooten and Banchereau, 2000). The CD40 surface marker is a member of the tumour necrosis factor receptor superfamily (TNFR) that responds to CD40L expressed on T cells during DC antigen presentation events that causes IL-12 production by the DC and drives Th1 T cell responses (Cella et al., 1996; Koch et al., 1996; Moser and Murphy, 2000).

\subsubsection{Licensing of DCs by Conventional T cells}

The CD40 - CD40L interaction occurs between the CD40L bearing CD4 ${ }^{+} \mathrm{T}$ cell and CD40 found on the outer DC surface (Figure 1.9). It is important to note that licensing of DCs can be induced by cells other than $\mathrm{CD} 4^{+} \mathrm{T}$ cells such as the natural killer and natural killer T cells (NKT) and will be discussed further as it is one of the major themes of this thesis. 


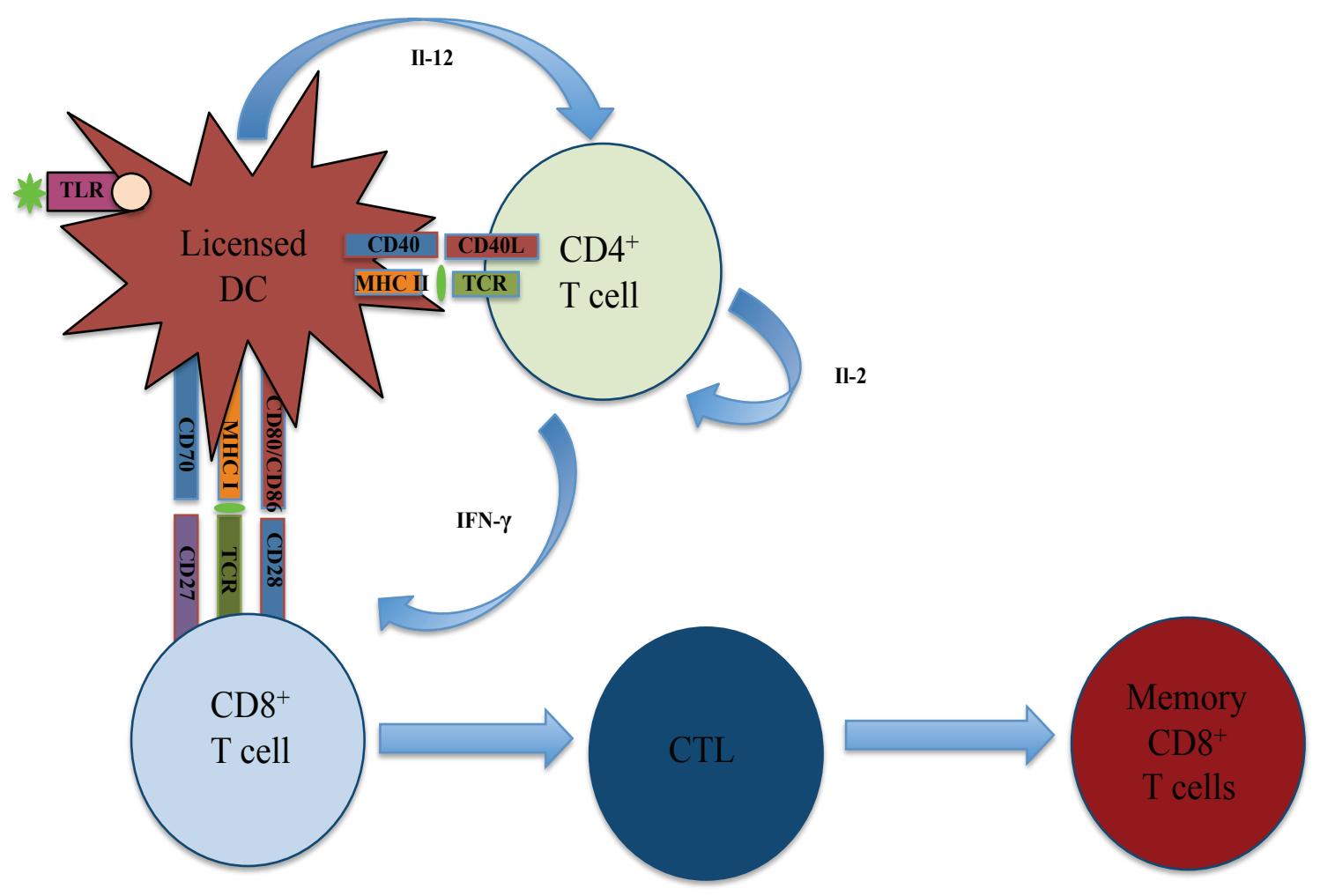

Figure 1.9 Schematic diagram showing molecular mechanisms involved in the licensing of DCs via CD4 ${ }^{+}$T cell "help". After antigen is acquired by DCs, it is presented to $\mathrm{CD} 4^{+} \mathrm{T}$ cells via MHC class II molecules. Antigenic fragments are also cross-presented to $\mathrm{MHC}$ class I molecules that cause the stimulation of $\mathrm{CD} 8^{+} \mathrm{T}$ cells. CD40 - CD40L interactions between the $\mathrm{DC}$ and $\mathrm{CD}^{+}{ }^{+} \mathrm{T}$ cells induce the upregulation of CD70, CD80 and CD86 on DCs and allows for the subsequent generation of CTLs. The downregulation of (programmed cell death ligand) PDL1 on DCs occurs. The survival of CTLs is caused by cessation of (TNF-related apoptosis-inducing ligand) TRAIL. TLR stimulation further activates DCs and increases cross-presentation activities.

\subsubsection{Licensing of DCs by NKT Cells}

NKT cells are unique lymphocytes that express cell surface markers typically associated with NK cells and TCR structures (Godfrey et al, 2004). NKT cells make up approximately $1-2 \%$ of the mouse splenic cells $(0.01-2 \%$ of human peripheral blood mononuclear cells) and are important for the regulation of $\mathrm{DCs}, \mathrm{CD} 8^{+} \mathrm{T}$ cells and $\mathrm{NK}$ 
cells, essentially connecting the innate and adaptive immune responses (Bendelac et al., 2007; Kronenberg et al, 2005; Taniguchi et al., 2003). NKT cells are a potent source of amplification signals and when fully activated can secrete copious amounts of cytokines such as IFN- $\gamma$, IL-4, IL-13, IL-17 and can induce DCs to produce the highly regulated IL12 cytokine (Matsuda et al., 2003; Rachitskaya et al., 2008; Stetson et al., 2003).

The NKT TCR is known to be semi-invariant and in mice consists of an invariant $\mathrm{V} \alpha 14 \mathrm{~J} \alpha 18 \alpha$-chain and restricted $\mathrm{V} \beta$ repertoire (V $\beta 2, \mathrm{~V} \beta 7$ and $\mathrm{V} \beta 8)$. In humans the NKT TCR is comprised of a V $\alpha 24 \mathrm{~J} \alpha 18$-chain paired with V $\beta 11$ (Lantz and Bendelac, 1994; Masuda et al., 1997; Prussin and Foster, 1997). Although many NKT cell express the NK cell marker NK1.1, this is not the case for all NKT cell subsets therefore the combination of using NK1.1 and TCR as markers is a more precise method of identifying NKT cell populations (Gumperz et al., 2002). NKT cells also express other NK markers, including NKG2A, NKG2D and Ly49 family members.

There are two major subsets of NKT cells, Type 1 and Type II NKT cells. Type 1 NKT cells are otherwise known as iNKT cells and recognize $\alpha$-GalCer presented on CD1d molecules (Izak et al., 2013). Type II iNKT cells express a more diverse TCR repertoire that can be activated by sulphatide but is non responsive to $\alpha$-GalCer (Jahng et al., 2004). The CD1d -/- mouse strain lacks Type I and Type II NKT cells while the J 1 18-/- lack only Type II NKT cells (Izak et al., 2013). These NKT cell-deficient mice have shown that Type I NKT cells are important for anti-tumour immunity while Type II NKT cells have been implicated in the suppression of tumour immunosurveillance (Crowe et al., 2002; Smyth et al., 2000; Terabe et al., 2005; Toura et al., 1999). The NKT cells referred to in the remainder of this thesis are the Type I iNKT cells.

NKT cells have been shown to be restricted to non-classical MHC class I-like CD1d molecules capable of presenting glycolipids as opposed to antigen peptides (Bendelac, 1995a, 1995b). In mice, fluorescently labeled CD1d tetramers loaded with $\alpha$ galactosylceramide ( $\alpha$-GalCer) a potent NKT cell ligand, have been utilized as an identification tool for NKT cells (Benlagha and Bendelac, 2000; Benlagha et al., 2000; 
Karadimitris et al., 2001; Matsuda et al., 2000). Most mammals have been shown to express CD1d and although found on most hematopoietic cells, the highest concentration is expressed on DCs, macrophages and B cells. It is the DCs however that are capable of inducing potent NKT responses.

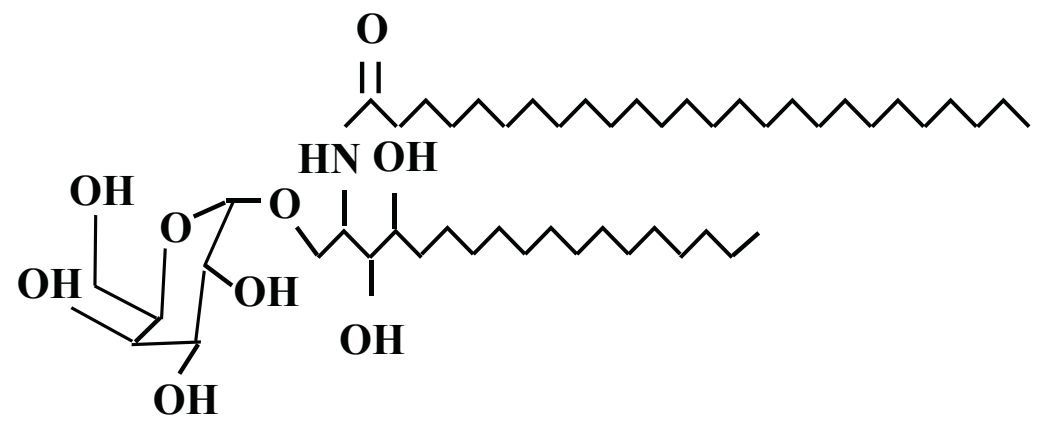

Figure 1.10 Molecular structure of the potent iNKT cell activator $\alpha$ Galactosylceramide ( $\alpha$-GalCer). Diagram shows synthetic ligand and marine sea sponge compound.

Once activated, NKT cells are able to produce a variety of cytokines and chemokines that include IL-2, IL-4, IL-5, IL-6, IL-10, IL-13, IL-17, IL-21, IFN- $\gamma$, TNF- $\alpha$, TGF- $\beta$, GMCSF (Coquet et al., 2008; Matsuda et al., 2008). It is the large amounts of IL-4, TNF- $\alpha$ and IFN- $\gamma$ that are produced rapidly that are useful for NKT-DC interactions. Transactivation of other immune cells such as DCs, NK cells, B cells, CD4 ${ }^{+} \mathrm{T}$ cells and $\mathrm{CD} 8^{+} \mathrm{T}$ cells can occur contributing to amplified immune responses (Figure 1.11).

The most widely studied Type I NKT cell ligand is the marine sponge derived glycolipid $\alpha$-GalCer (Kobayashi et al., 1995; Morita et al., 1995; Taniguchi et al., 2003). The $\alpha$ GalCer glycolipid comprised of a galactose head portion connected by an $\alpha$-linkage to a ceramide lipid tail component was originally discovered by Kirin Pharmaceuticals after screening marine compounds for antitumour activity (Figure 1.12). The powerful antitumour properties of $\alpha$-GalCer have been highlighted by tumour rejection reported within a number of murine models (Ambrosino et al., 2007; Chiodoni et al., 2001; Fuji et al., 2000; Hayakawa et al., 2001a, 2002, 2003; Kawano et al., 2008; Miyagi et al., 2003; 
Nakagawa et al., 2004; Osada et al., 2004). After being shown by Kawano et al in 1997 that $\alpha$-GalCer was the most potent activator of NKT cells, various synthetic ligands since then have been generated in an effort to reproduce, enhance and alter the activation and cytokine release profile generated after activation of NKT cells with $\alpha$-GalCer.

When stimulated by $\alpha$-GalCer, Type I NKT cells quickly release IFN- $\gamma$, IL-4 and IL-13 cytokines and facilitate antitumour immune responses. Anti-tumour protection generated in response to $\alpha$-GalCer stimulation has been shown reliant on IFN- $\gamma$ and IL-12 cytokines (Chidoni et al., 2001; Cui et al., 1997; Hayakawa et al., 2002; Smyth et al., 2002), the activation of $\mathrm{CD}^{+}$and $\mathrm{CD}^{+}$T cells (Hong et al., 2006; Nakagawa et al., 2004;) and the transactivation of NK cells by IFN- $\gamma$ (Hayakawa et al., 2001a; Smyth et al., 2001). The NKT - DC interaction occurs after presentation of $\alpha$-GalCer to the invariant TCR receptor on the NKT cells, by $\alpha$-GalCer bearing CD1d molecules found on the outer DC surface (Figure 1.11). This induces the maturation of the DC characterized by the upregulation of CD80, DC86 and the production of the tightly controlled IL-12 cytokine. IL-12 causes upregulation of the IL-12 receptor found on the NKT cells while creating amplification of the Th1 signals and responses being generated. IFN- $\gamma$ released by the NKT cells increases cytotoxicity activities in NK cells. iNKT cells are involved in the activation of B cells and are responsible for interactions that cause suppression of myeloid-derived suppressor cells (MDSCs) (Cerundolo et al., 2009).

The utilization of $\alpha$-GalCer within the vaccination strategies was to further explore the NKT mediated activity generated within the context of antigen loaded DC vaccines to improve the frequencies of antigen specific $\mathrm{T}$ cell responses. 


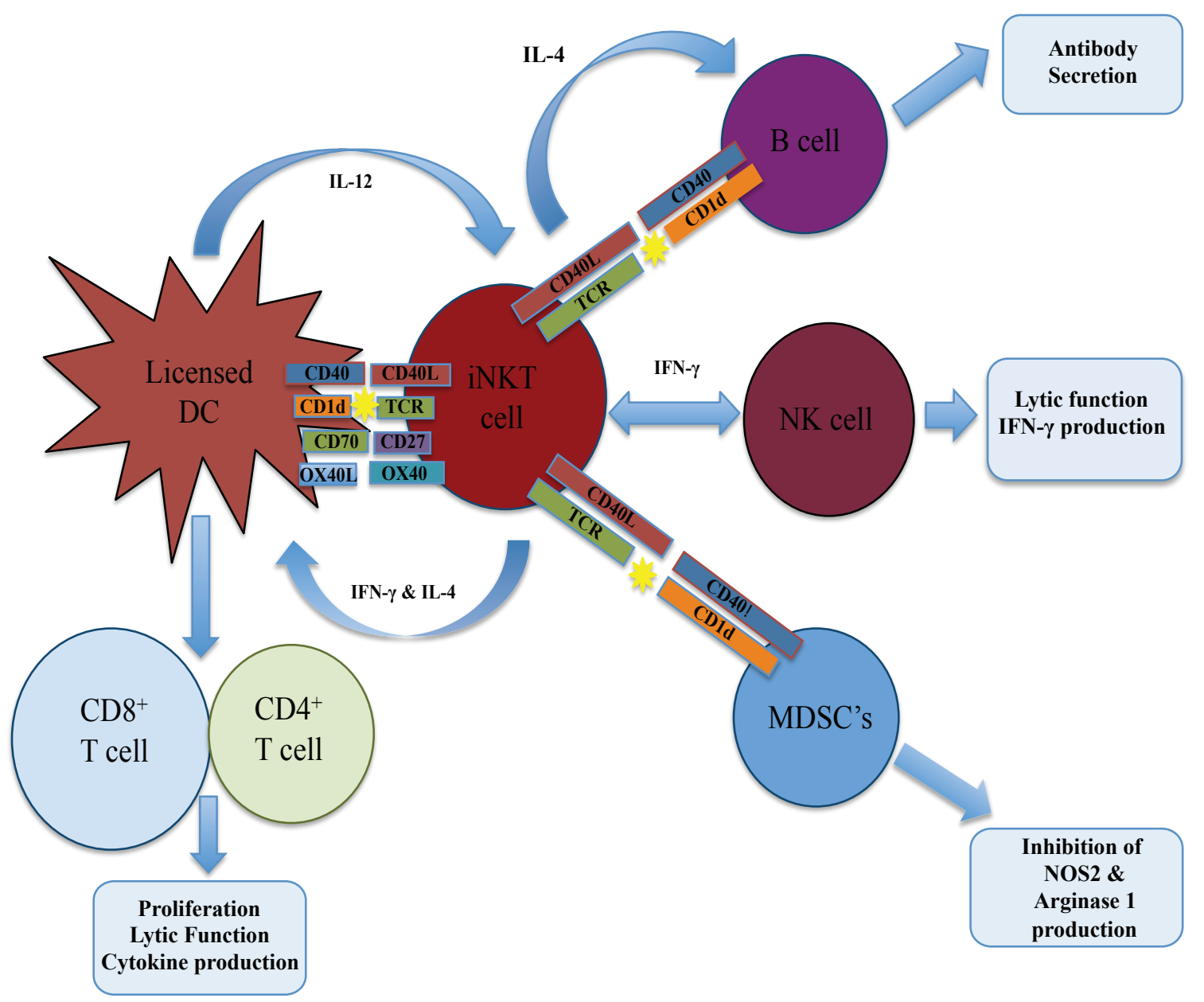

Figure 1.11 Schematic diagram showing iNKT cell interactions between DCs, that lead to licensing in addition to interactions with B cells, NK cell and MDSCs. The presentation of $\alpha$-GalCer by DCs on CD1d molecules and subsequent recognition by the invariant TCR receptor on the iNKT cell induces activation of the iNKT cells. Activated iNKT cells rapidly release large quantities of Th1 and Th2 cytokines that include IFN- $\gamma$ and IL-4. CD40L upregulation on the iNKT cells is also induced. In addition to providing help to B cells, iNKT cells are also capable of suppressing the function of myeloid-derived suppressor cells (MDSCs). Adapted from Cerundolo et al, 2009 (Cerundolo et al., 2009) . 


\subsection{Dendritic cell-based Vaccines \& Cancer}

Adoptive $\mathrm{T}$ cell therapy has been shown to cause tumour regression highlighting the important role of the immune system in anti-cancer responses (June, 2007a; June 2007b; Morgan et al., 2006; Yee, et al., 2002). DC-based vaccine immunotherapy (Figure 1.12) relies on the potent antigen-presenting properties of DCs and has the potential to cause the generation of tumour specific immune responses in addition to memory $\mathrm{T}$ cell immunity (Finn, 2008).

Early work to develop DC-based vaccines for the treatment of cancer started in 1994 when Romani et al published work featuring DC progenitors found in human blood that could be utilized for the ex-vivo generation of human DCs (Romani et al., 1994). Hsu et al soon followed publication of their work using autologous antigen-pulsed DCs to vaccinate patients with B-cell lymphoma (Hsu et al., 1996). At this stage immature DCs were being utilized in DC vaccination strategies with Nestle et al, publishing their vaccination work in melanoma patients comparing MHC I peptide pulsed DCs with tumour lysate pulsed DCs (Nestle et al., 1998). The utilization of matured ex-vivo DCs for subsequent DC vaccination work was carried out testing tumour lysate loaded DCs in renal cell carcinoma patients (Holtl et al., 1999; Holtl et al., 2002) and peptide loaded DCs in melanoma patients (Thurner et al., 1999). By 2001 it had been reported that FLT3L was being utilized to expand DC populations for vaccination purposes (Fong et al., 2001), while others were making use of $\mathrm{CD}_{3} 4^{+}$hematopoietic progenitor DC vaccines to treat melanoma patients (Banchereau et al., 2001; Mackensen et al., 2000).

Subsequent human studies were carried out to optimise DC-based vaccination protocols and included work to define and determine parameters such as the appropriate route of DC administration (Fong et al., 2001; Mullins et al., 2003), DC dose (Lau et al., 2001), the induction of tolerance when utilizing immature DCs (Dhodapkar and Steinman, 2002; Dhodapkar et al., 2001), the use of immature versus mature DC populations (de Vries et al., 2003; Jonuleit et al., 2001), MHC I peptides (Fong et al., 2001), the loading of antigen with MHC I and MHC II peptides (Schuler-Thurner et al., 2002), the use of RNA 
transfection of DCs (Heiser et al., 2002; Nair et al., 1999; Nair et al., 2002; Pecher et al., 2002; Su et al., 2003).

Further human work included testing allogeneic vaccine DCs (Holtl et al., 2005; Marten et al., 2003), the combination of DC vaccination with chemotherapy (Holtl et al., 2005) (Spisek et al., 2007), DC vaccination with T regulatory cell depletion (Dannull et al., 2005) IFN- $\gamma$ treatment (Hildenbrand et al., 2007) and the vaccination of patients with autologous DC derived exosomes (Escudier et al., 2005).

The clinical testing of DC-based vaccines typically results in the generation of 3 different groups of patients: the first of which is made up of patients who do not mount an immune response to the tumour antigen presented on the vaccine DCs. The second group is comprised of patients who show anti-tumour immunity after vaccination, but do not develop tumour regression-a durable objective response, whilst the third group of patients not only develop tumour specific immunity, but also exhibit clinical benefit such as tumour regression (Palucka et al., 2009).

The work presented in this thesis looks at the optimisation of a DC based vaccination protocol in murine subjects by testing the effect of TLR stimuli alone and in combination on ex-vivo $\mathrm{DC}$ populations and investigating the impact on antigen specific $\mathrm{T}$ cell responses. The resident DC subsets are also examined in response to DC vaccination and systemic delivery of TLR stimuli. The incorporation of NKT stimulation is also investigated in an effort to maximise antigen specific $\mathrm{T}$ cell responses quantities after DC vaccination with the developed protocol. 


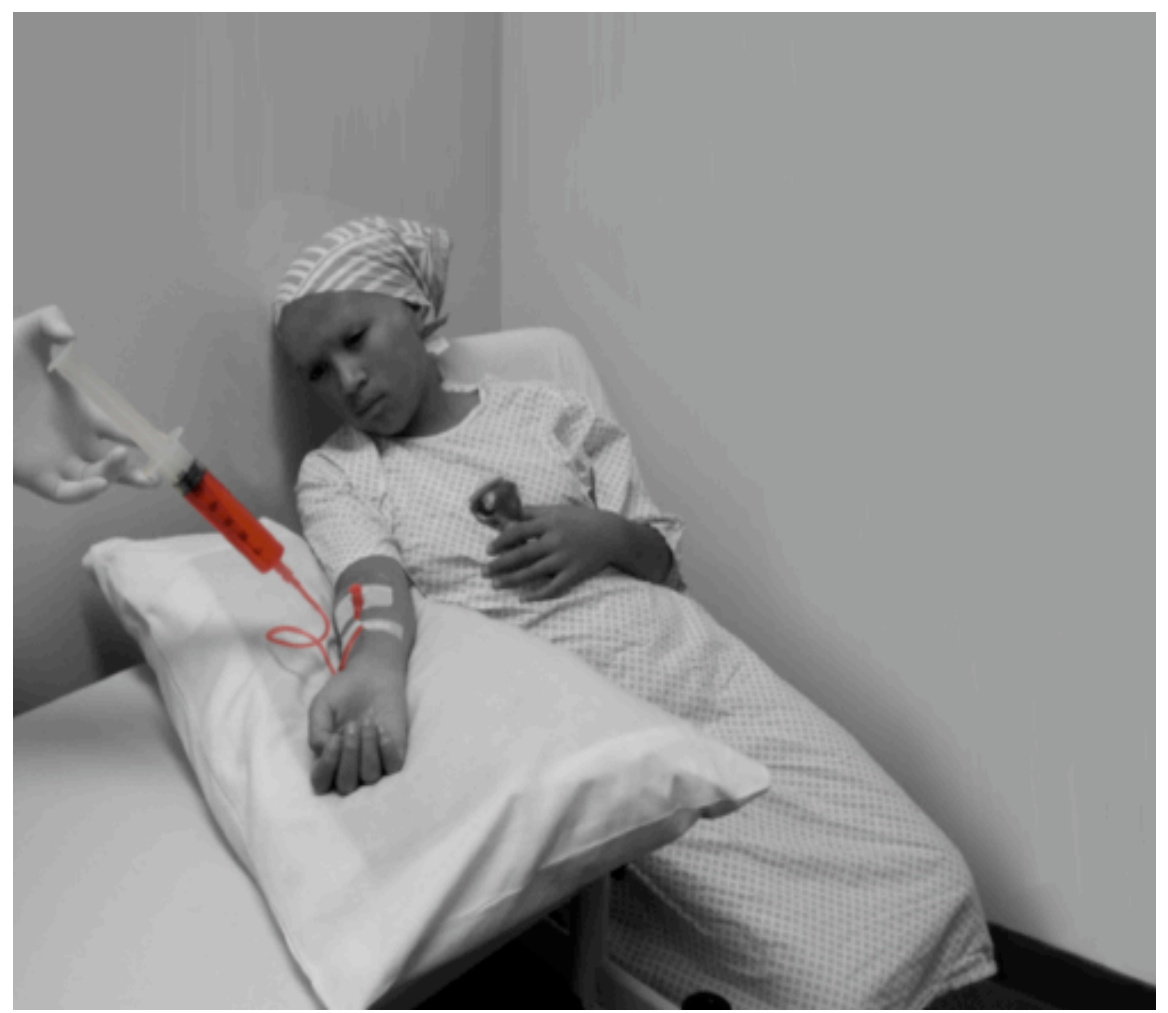

Figure 1.12 Designer vaccines: DC-based immunotherapy. Image (C) Dianne SikaPaotonu. DC-based immunotherapy treatment involves the generation of patient specific DC populations from ex-vivo progenitors from bone marrow precursors or the blood. These DCs can be co-cultured (loaded) with denatured tumour cells - from the same patient to allow the DCs to acquire tumour specific antigens. Once the DC vaccine preparation has undergone the appropriate culturing and stimulation processes in-vitro, the DC-based vaccine preparation is injected into the patient. The antigen laden DCs migrate and traffic to the nearest lymphoid organ where presentation by MHC I and MHC II molecules of acquired antigenic peptides occurs. The utilization of denatured tumour cells ensures the DCs are provided with a range of internalized antigens that can allow cross-priming of antigen specific $\mathrm{T}$ cells. This also avoids the use of selective known peptides that are haplotype specific. It means too that MHC II molecules also have a source of antigens available for presentation to antigen specific helper $\mathrm{T}$ cells. 


\subsection{Aims and objectives of this thesis}

Studies have demonstrated that the size and quality of the antigen specific $\mathrm{T}$ cell response is highly dependent on the DC phenotype at the time of antigen presentation to T cells. The first hypothesis therefore was that "BM-DC phenotype and function could be enhanced by the use of TLR stimulation generated using specific TLR agonists alone and in combination with each other".

The overall objective for the $1^{\text {st }}$ section of this thesis was:

- To examine the effect of lone and combined TLR stimulation on BM-DC function in-vitro with an emphasis on MPL, and analyse and optimise the in-vivo antigen specific response generated by TLR stimulated BM-DC based vaccines.

The next hypothesis addressed was that "NKT stimulation could increase antigen specific T cell responses being generated after vaccination”.

The overall objective for the following section of this thesis was:

- To explore the adjuvant effect, and its duration, when $\alpha$-GalCer was incorporated into antigen loaded BM-DC vaccines

The final hypothesis addressed was that "NKT and TLR stimulation could work cooperatively to further increase antigen specific $T$ cell responses being generated after vaccination".

The overall objective for the final section of this thesis was:

- To investigate the combined affects of NKT and TLR stimulation on antigen specific responses to BM-DC vaccines in-vivo 
Chapter 2:

\section{Materials \& Methods}




\subsection{Mice}

\subsubsection{Animal Husbandry}

All mice were bred and maintained at the Biomedical Research Unit of the Malaghan Institute of Medical Research, based at Victoria University of Wellington, New Zealand. Experimental protocols were approved by the Victoria University of Wellington Animal Ethics Committee, and carried out in accordance with the following ethical guidelines: 2006R14M, (Pilot study of assessment of interactions between radiofrequency ablation (RFA) and immunotherapy in the treatment of established murine tumours), 2007R17M (Improving Vaccines with adjuvants that stimulate NKT cells), 2009R8M (Use of mouse tissues for multiple research projects within the Malaghan Institute), 2009R13M (Mechanisms of induction of antitumour immune responses by dendritic cells).

\subsubsection{Euthanasia}

At the conclusion of experiments using the aforementioned protocol guidelines and prior to tissue harvesting procedures, mice were euthanised by $\mathrm{CO}_{2}$ asphyxiation followed by manual cervical dislocation. Alternatively mice were sacrificed using manual cervical dislocation alone.

\subsubsection{Strains}

\section{C57BL/6 (CD45.2 $\left.{ }^{+}\right)$}

Originally purchased from the Jackson Laboratories, Bar Harbour, ME, USA, and maintained in the BRU facility at the Malaghan Institute of Medical Research by brother sister matings. 


\section{B6.SJL-Ptprc ${ }^{\mathrm{a}} \operatorname{Pepc}^{\mathrm{b}} /{\text { BoyJ }\left(C D 45.1^{+}\right)}^{+}$}

Originally purchased from the Jackson Laboratories, Bar Harbour, ME, USA and were created by backcrossing the inbred SJL strain, expressing the $\operatorname{Ptprc}^{\mathrm{a}}$ gene (CD45.1), onto the (CD45.2) C57BL/6 background. This allowed the distinction between injected C57BL/6 cells isolated from OTI sources and recipient populations on the basis of CD45.2 and CD45.2 expression.

\section{OTI mice}

Obtained from Dr. Sarah Hook, School of Pharmacy, Dunedin, NZ, with permission from Prof. Frank Carbone, Melbourne University, Australia. These mice express the transgenic $\mathrm{V} \alpha 2$, V $\beta 5.1 / 5.2$ TCRs that are specific for the $\mathrm{H}-2 \mathrm{~K}^{\mathrm{b}}$-binding peptide of OVA (OVA 257 264) (Barnden et al., 1998; Hogquist et al., 1994). Fluorescence activated cell sorting was used to genotype these animals on a regular basis to ensure the expression of the transgenic TCR in peripheral blood lymphocyte populations.

\section{OTI x B6.SJL-Ptprc ${ }^{\mathrm{a}}$}

These OTI congenic mice were generated in-house at the Malaghan Institute of Medical Research, Wellington, New Zealand.

OTI congenic mice were maintained in-house by crossing OTI mice (CD45.2 ${ }^{+}$) with B6 congenic OTI populations, which could be differentiated from host populations on the basis of CD45.1 and CD45.2 expression.

\section{$\mathrm{CD}^{\top}{ }^{-}$}

These mice lack V $\alpha 14 i \mathrm{NKT}$ cells and were originally provided by C-R Wang from the Gwen Knapp centre of Lupus and Immunology Research, Department of Pathology at the University of Chicago, Illinois, USA (Chen et al., 1997). 
TLR $4^{\top}$

These knock-out mice lack the TLR 4 receptor were provided by Dr S. Akira, from Hyogo College of Medicine, Japan (Hoshino et al., 1999).

\section{Langerin-EGFP}

These Langerin knock-in mice express EGFP under control of the Langerin promoter and were provided by B. Malissen, Centre d'Immunologie Marseille-Luminy, France (Kissenpfennig et al., 2005). Fluorescence activated cell sorting can be used to identify langerin expressing cells within splenoctye preparations using the GFP fluorescence signal.

\section{Langerin-DTREGFP}

These Langerin knock-in mice express the human diphtheria toxin receptor (DTR) under control of the Langerin promoter and were provided by B. Malissen, Centre d'Immunologie Marseille-Luminy, France (Kissenpfennig et al., 2005). Fluorescence activated cell sorting can again be used to identify langerin expressing cells within splenoctye preparations using the GFP fluorescence signal.

\section{$2.2 \quad$ Reagents}

\subsubsection{Peptides}

SIINFEKL (OVA 257-264) peptide $\mathrm{CD}^{+} \mathrm{T}$ cell epitope was from Genscript Corp, Piscataway, NJ.

ISQAVHAAHAEINAGR (OVA $323-339$, ISQ in short) peptide $\mathrm{CD}^{+}$epitope was also from Genscript Corp, Piscataway, NJ. 


\subsubsection{Protein}

Endotoxin-free OVA protein was provided by Professor Thomas M. Moran, Mount Sinai School of Medicine, New York, USA.

\subsubsection{Culture Media}

\section{cIMDM}

Cell lines were maintained in complete medium Iscove's Modified Dulbecco's Medium supplemented with $2 \mathrm{mM}$ glutamine, $1 \%$ penicillin-streptomycin, $5 \times 10^{-5} \mathrm{M} 2$-mercatoethanol (all Invitrogen, Auckland, New Zealand) in addition to 5\% FCS (Sigma-Aldrich, Auckland, New Zealand). Solutions of cIMDM were stored for no more than 2 weeks at $4^{0}$ $\mathrm{C}$ until used to culture cells kept in an incubator at $37^{\circ} \mathrm{C}$ with $5 \% \mathrm{CO}_{2}$ and $95 \%$ humidity.

\section{IMDM}

Iscove's Modified Dulbecco's Medium supplemented with $2 \mathrm{mM}$ GlutaMAX ${ }^{\mathrm{TM}}, 25 \mathrm{mM}$ HEPES buffer and $3.024 \mathrm{mg} / \mathrm{L} \mathrm{NaHCO}_{3}$ was purchased from GIBCO (Invitrogen, Auckland, NZ) and stored at $4^{0} \mathrm{C}$ until used.

\section{cRPMI}

Some cell lines were maintained in complete RPMI medium 1640 containing Lglutamine but not phenol red (GIBCO, Invitrogen, Auckland, NZ), supplemented with $2 \mathrm{mM}$ glutamine, $1 \%$ penicillin-streptomycin, $5 \times 10^{-5} \mathrm{M}$ 2-mercato-ethanol (all Invitrogen, Auckland, New Zealand) in addition to 5\% FCS (Sigma-Aldrich, Auckland, New Zealand). Solutions of cRPMI were stored for no more than 2 weeks at $4^{0} \mathrm{C}$ until used to culture cells kept in an incubator at $37^{\circ} \mathrm{C}$ with $5 \% \mathrm{CO}_{2}$ and $95 \%$ humidity. 


\subsubsection{Synthetic TLR agonists}

Lipopolysaccharide (LPS)

LPS was purchased as lyophilised powder from Sigma Aldrich (St. Louis, Missouri, USA) and dissolved in sterile PBS at a stock concentration of $1 \mathrm{mg} / \mathrm{mL}$ and stored at $4{ }^{\circ} \mathrm{C}$. LPS sourced from Escherichia coli, serotype 0.111:B4.

\section{Monophosphoryl Lipid A (MPL)}

MPL was purchased as a lyophilised powder from Sigma Aldrich and was dissolved in sterile endotoxin-free physiological solution to give a final concentration of $1 \mathrm{mg} / \mathrm{mL}$ with working aliquots stored at $4{ }^{\circ} \mathrm{C}$.

\section{Oligodeoxynucleotide 1668 (CpG)}

CpG was purchased as lyophilised powder from GeneWorks (Thebatron, SA, Australia) and dissolved in sterile PBS with working aliquots stored at $4^{\circ} \mathrm{C}$ and remaining stock stored at $-20^{\circ} \mathrm{C}$.

\section{Pam3Cys}

Pam3Cys was purchased as a lyophilised powder (EMC microcollections, GmbH, Germany) and dissolved in sterile endotoxin-free physiological solution to give a final concentration of $1 \mathrm{mg} / \mathrm{mL}$ with working aliquots stored at $4^{\circ} \mathrm{C}$. (Pam3Cys-SKKKK x3 $\mathrm{HCl})$.

\section{Resiquimod}

Resiquimod was purchased as a lyophilised powder from PharmaTech Labs, Royston, GA, 
USA and dissolved in sterile endotoxin-free physiological solution to give a final concentration of $1 \mathrm{mg} / \mathrm{mL}$ with working aliquots stored at $4^{\circ} \mathrm{C}$ and remaining stock stored at $-20^{\circ} \mathrm{C}$.

\subsubsection{Chemical reagents}

\section{$\alpha$-Galactosylceramide ( $\alpha$-GalCer)}

The $\alpha$-GalCer glycolipid used for these studies was kindly provided by Dr. Gavin Painter from Industrial Research Limited (IRL) and manufactured according to protocols described in (Lee et al., 2006). The $\alpha$-GalCer was solubilised into a 10:10:3 methanol/chloroform/water solution and made up to a concentration of $5 \mathrm{mg} / \mathrm{ml}$. The solution was sonicated for 10 minutes and cooled at $4^{0} \mathrm{C}$ for between 2-3 days after which the solution was diluted to make a final concentration of $200 \mu \mathrm{g} / \mathrm{ml}$ in PBS (containing 0.5 $\%$ Tween 20).

\section{Carboxy Fluoroscein Succinimidyl Ester (CFSE) 5-(and-6)-carboxyfluorescein diacetate, succinimidyl ester}

Purchased from Molecular Probes, Invitrogen, CA, USA, and stored at $-20{ }^{\circ} \mathrm{C}$, as a $10 \mathrm{mM}$ solution in DMSO Sigma, St. Louis, MO, USA.

\section{Liberase}

Purchased from Roche, Mannheim, Germany.

\section{DNase I}

DNase I powder was sourced from Roche, Mannheim, Germany. This lyophilised powder was dissolved to a concentration of $10 \mathrm{mg} / \mathrm{mL}$ in IMDM and stored at $-20{ }^{\circ} \mathrm{C}$. 


\section{Fluorescence Activated Cell Sorting Buffer}

EDTA (Sigma, St. Louis, MO, USA), NaN3 (Sigma, St. Louis, MO, USA) and FBS were added to $1 \mathrm{~L}$ PBS to produce a final concentration of $10 \mathrm{mM}$ EDTA, $0.01 \% \mathrm{NaN}_{3}$ and $2 \%$ FBS in the PBS before storage at $4{ }^{\circ} \mathrm{C}$.

\section{FACS Sort Buffer}

$10 \mathrm{mM}$ EDTA and 2\% FBS were added to 0.5 L RPMI medium 1640, with L-glutamine but no phenol red (GIBCO, Invitrogen, Auckland, NZ) and stored at $4{ }^{\circ} \mathrm{C}$.

\section{Foetal Bovine Serum (FBS)}

FBS was purchased from GIBCO (Invitrogen, Auckland, NZ) and stored in $25 \mathrm{~mL}$ aliquots at $-20{ }^{\circ} \mathrm{C}$. After thawing, aliquots were stored at $4{ }^{\circ} \mathrm{C}$ for a maximum of 2 weeks. The endotoxin levels of the FBS was determined before purchase and found to be $27 \mathrm{EU} / \mathrm{mL}$. FBS was also screened for Mycoplasma and virus before purchase. Purchased from GIBCO Invitrogen, CA, USA, and stored at $-20{ }^{\circ} \mathrm{C}$.

\section{Geneticin(R) (G418)}

A selective neomyocin analogue that destroys tumour cells that do not express OVA Geneticin(R) (G418, GIBCO, Invitrogen, Auckland, NZ) was made into aliquots and stored at $-20{ }^{\circ} \mathrm{C}$. In use aliquots were stored at $4{ }^{\circ} \mathrm{C}$.

\section{Lipopolysaccharides (LPS)}

Lipopolysaccharides from Escherichia coli, serotype 0111:B4, were purchased from Sigma (St. Louis, MO, USA). IMDM was utilized to dissolve the lyophilised powder to a concentration of $1 \mathrm{mg} / \mathrm{mL}$ and stored at $-20{ }^{0} \mathrm{C}$. 


\section{Magnetic Cell Sorting Buffer (MACS Buffer)}

Final concentrations of $2 \mathrm{mM}$ EDTA and $0.5 \%$ BSA were achieved after addition to $1 \mathrm{~L}$ PBS. After filter-sterilisation the buffer solution was stored at $4{ }^{0} \mathrm{C}$.

\section{Mercaptoethanol (2 ME)}

2 ME was obtained from Sigma (St. Louis, MO, USA) as a $55 \mathrm{mM}(1000 \mathrm{x})$ solution in PBS and stored at $4{ }^{0} \mathrm{C}$.

\section{Penicillin-Streptomycin}

Penicillin-Streptomycin liquid was purchased from GIBCO (Invitrogen, Auckland, NZ) and stored as single use aliquots in $15 \mathrm{~mL}$ sterile conical tubes at $-20{ }^{\circ} \mathrm{C}$ until used.

\section{Phosphate Buffered Saline (PBS)}

$47.8 \mathrm{~g}$ of Dulbecco's PBS powder without $\mathrm{CaCl}_{2}$ and $\mathrm{MgCl}_{2}$ (Sigma Aldrich, Auckland, $\mathrm{NZ}$ ) was dissolved in $0.5 \mathrm{~L}$ distilled (MilliQ) $\mathrm{H}_{2} \mathrm{O}$ to make a 10 x solution (alternatively use $5 \mathrm{~L} \mathrm{dH}_{2} \mathrm{O}$ to make a $1 \mathrm{x}$ solution). After $\mathrm{pH}$ adjusted to 7.4 , the solution was filter sterilised and stored at $4{ }^{\circ} \mathrm{C}$.

\section{Sodium Azide $\left(\mathrm{NaN}_{3}\right)$}

Purchased from Sigma-Aldrich, MO, USA in powder form and dissolved in $\mathrm{dH}_{2} \mathrm{O}$ to give a stock concentration of $5 \%$. The solution was stored at room temperature.

\section{Tritiated Thymidine}

Stock solutions of $(5 \mathrm{mCi}) 6-\mathrm{Methyl}-3 \mathrm{H}$ thymidine with a specific activity of $5 \mathrm{Ci} / \mathrm{mmol}$ were purchased from Amersham Biosciences (Little Chalfont, UK) and when required, 
diluted to a working solution of $20 \mu \mathrm{Ci} / \mathrm{mL}$ in IMDM and stored at $4{ }^{\circ} \mathrm{C}$ until use.

\section{Granulocyte-macrophage colony stimulating factor (GM-CSF)}

X63 cell line cultures modified to secrete the full-length murine GM-CSF protein were used to produce recombinant murine GM-CSF. Kindly gifted by Dr Antonius Rolink, Basel Institute of Immunology, Basel, Switzerland.

\section{Interleukin 4 (IL-4)}

Stationary phase cultures of a Chinese Hamster Ovary cell line were modified to secrete the full-length murine IL-4 protein and utilized to generate recombinant murine IL-4. Kindly gifted by Dr Antonius Rolink (Basel Institute of Immunology, Basel, Switzerland.

\subsection{Fluorescent Antibodies}

See Appendix for list of fluorescent antibodies.

\subsection{General Cell Culture}

\subsubsection{Maintenance}

All cell cultures were maintained in cIMDM at $37{ }^{\circ} \mathrm{C}$ with $5 \% \mathrm{CO}_{2}$ and $95 \%$ humidity. Tumour cell cultures also contained $0.5 \mathrm{mg} / \mathrm{mL}$ G418 (GIBCO, Auckland, NZ). Adherent cells were incubated for 1 minute at $37^{\circ} \mathrm{C}$ in $3 \mathrm{~mL}$ Trypsin/EDTA to detach them from the flask. Proteolysis was stopped by the addition of an equal volume of FBS and cells were washed twice in IMDM before injecting into mice. 


\subsubsection{BM-DC culture and Preparation}

BM-DC cultures were generated from bone marrow precursors isolated from C57BL/6 female mice. Femur and tibial bones were removed and the marrow exposed by cutting bone ends with scissors at a $45^{\circ}$ angle inside the laboratory sterile hood. The marrow was flushed out using a 25-gauge needle attached to a $1 \mathrm{~mL}$ insulin syringe filled with IMDM and collected into a sterile 6-well plate with wells containing IMDM. Single cell suspensions were obtained by agitating the clumps using a pipette aid and passing the marrow pieces through a $70 \mathrm{uM}$ filter into a $50 \mathrm{~mL}$ falcon tube containing $10 \mathrm{~mL}$ cIMDM. After centrifugation at $1600 \mathrm{rpm}$ for 4-5 min, cells were counted and resuspended to allow for a final concentration of $2 \times 10^{6}$ DCs per well in $5 \mathrm{~mL}$ cIMDM supplemented with IL-4 and GM-CSF. IL-4 and GM-CSF cytokines were sourced from culture supernatants of Chinese hamster ovary (CHO) mIL-4 and X63/GM-CSF cells and titrations used to determine the optimal dose required to supplement BM-DC cultures. Cells were incubated at $37{ }^{\circ} \mathrm{C}$ and supplemented with fresh cytokine and cIMDM suspension on days 3 and 5 . This was done by replacing $2.5 \mathrm{~mL}$ of the BM-DC culture supernatant with $2.5 \mathrm{~mL}$ fresh cIMDM containing the appropriate proportions of IL-4 and GM-CSF.

\subsubsection{NIH3T3 CD40L expressing fibroblast cell line}

CD40L expressing NIH3T3 fibroblast cell lines were kindly provided by the NIH, USA and utilized for co-culture with BM-DCs. BM-DCs were generated as described in section 2.4.2 and plated at $2 \times 10^{6}$ cells/well into a 6-well plate with $5 \mathrm{ml}$ cIMDM5 and 100ul of IL-4 and GM-CSF. On day 3 of culture, NIH3T3/mock and NIH3T3/CD40L cells were thawed, washed and cultured into $75 \mathrm{~cm}^{3}$ flasks with $15 \mathrm{ml}$ fresh cIMDM5. On day 5 of culture, $2.5 \mathrm{ml} \mathrm{NIH} 3 \mathrm{~T} 3 /$ mock and NIH3T3/CD40L cell cultures were harvested and replated into flat-bottomed 96-well plates overnight at 25,000 cells/well. On day 6 BMDCs were harvested and combined with NIH3T3/mock and NIH3T3/CD40L cell cultures at 100,000 DCs/well (in 96-well plates). Appropriate TLR ligands at specific 
concentrations diluted with PBS were added to each well and cells harvested at $6 \mathrm{hrs}$ and $22 \mathrm{hrs}$ for flow cytometric analysis.

\subsection{BM-DCs}

\subsubsection{CFSE labelling of BM-DCs}

A single cell suspension of BM-DCs in PBS were labeled with $1.5 \mathrm{uM}$ Carboxy Fluoroscein Succinimidyl Ester (CFSE, Molecular Probes, Invitrogen) for $8 \mathrm{~min}$ at room temperature. After incubation, equal volume of FCS was added and cells were washed twice in complete medium and once in IMDM before injection into mice at $10 \times 10^{6} \mathrm{iv}$.

\subsubsection{Activation of BM-DCs}

During the final $\sim 16-24$ hs of the 7 day DC culture, DC were activated by the addition of $50 \mu \mathrm{L}$ of $10 \mu \mathrm{g} / \mathrm{mL}$ LPS to the cultures to give a final concentration of $100 \mathrm{ng} / \mathrm{mL}$ LPS. Adherent cells were harvested on day 7 by gently rinsing the plates with the supernatant 23 times before collecting the supernatant a final time.

\subsubsection{Loading of BM-DCs with $\alpha$-GalCer}

During the final $\sim 24$ hs of the 7 day DC culture, $\alpha$-GalCer was added to the culture at a final concentration of $100 \mathrm{ng} / \mathrm{mL}$. Adherent cells were harvested on day 7 by gently rinsing the plates with the supernatant 2-3 times before collecting the supernatant a final time. 


\subsubsection{Loading of BM-DCs with protein}

BM-DC cultures were treated with OVA protein added to DC cultures 24 hs before harvesting on day 7 at a concentration of $1 \mathrm{mg} / \mathrm{ml}$. After washing once with cIMDM followed by 2 more washes using IMDM, BM-DCs were resuspended in 200 ul IMDM per recipient for injection or co-culture with antigen specific $\mathrm{T}$ cells

\subsubsection{Loading of BM-DCs with peptide}

Day 7 LPS activated DC were harvested, washed and centrifuged at $320 \mathrm{~g}$ for $4 \mathrm{~min}$. The cells were counted and resuspended in fresh cIMDM and SIINFEKL peptide was added to a final concentration of $10 \mu \mathrm{M}$. The cells were then incubated at $37{ }^{\circ} \mathrm{C}$ for $2 \mathrm{hr}$, mixing frequently by inversion. Cells were then washed 3 times in IMDM, counted and resuspended in the appropriate volume of IMDM for i.v. injections.

\subsubsection{TLR stimulation}

BM-DC cultures were activated on day 6 by treatment with specific concentrations of TLR agonists for final $20-24 \mathrm{~h}$. Semi-adherent BM-DCs were harvested on day 7 by gentle agitation of culture supernatants using a pipette aid to gently wash over the surface of the plate 2 or 3 times. The maturation status of CD11 ${ }^{+}$BM-DC cultures was assessed by fluorescent antibody labelling for CD86 and CD40 and flow cytometric analysis.

\subsection{Flow Cytometry}

\subsubsection{Fluorescence antibody labelling of cells}

Flow cytometry was employed to determine the expression levels of surface markers after staining treatment with fluorescently labeled antibodies. FACS buffer was produced using 
PBS containing $1 \%$ FCS, $0.01 \%$ sodium azide and 2 mM EDTA. Single cell suspensions were counted, washed once in FACS buffer before transfer to 96 round bottom well plates such that each well contained up to $2 \times 10^{6}$ cells. Antibody labeling steps were carried out at $4{ }^{\circ} \mathrm{C}$. Plates were centrifuged for 2 minutes at $840 \mathrm{~g}$ and the supernatants removed by tipping the plate upside down and flicking once. Cellular pellets were resuspended in residual liquid or FACS buffer by gentle vortexing. Incubation for 10 minutes with the anti-CD16/32 antibody (2.4G2, made in house) was used to block non-specific Fc-receptor mediated antibody staining. After this incubation step, $200 \mu \mathrm{L}$ of FACS buffer was added to each well containing cells before centrifugation at $840 \mathrm{~g}$ for 2 minutes. Fluorescently labeled monoclonal antibodies for surface markers of interest were resuspended at the appropriate concentrations in FACS buffer and were added to each well to allow for a final volume of $50 \mu \mathrm{L}$ of antibody and FACS buffer solution. After incubation for a further 10 minutes on ice, cells were washed once with FACS buffer and then incubated with the appropriate streptavidin-conjugated fluorochrome for 10 minutes on ice, where required. Cells were then washed twice and resuspended in $200 \mu \mathrm{L}$ FACS buffer before transfer into titertubes (BioRad) for flow cytometric analysis.

For the exclusion of dead cells, $50 \mathrm{ng} / \mathrm{ml}$ propidium iodide (PI, BD PharMingen) or alternatively $0.1 \mu \mathrm{g} / \mathrm{ml} \mathrm{4',6-diamidino-2-phenylindole} \mathrm{(DAPI)} \mathrm{was} \mathrm{added} \mathrm{to} \mathrm{the} \mathrm{cells} \mathrm{prior}$ to acquisition. Flow cytometric data was generated using BD FACSort, BD FACSCalibur or BD LSRII SORP (all Becton Dickinson, San Jose, CA, USA) and analysed using the FlowJo software (Tree Star, San Carlos, CA, USA).

Flow cytometric analysis was carried out using FACSSort (BD). Unstained cell and tissue samples were used alongside those stained with single monoclonal antibody solutions to compensate for the natural overflow of specific fluorescence into other fluorescence channels by setting channel voltages to avoid this. Approximately 10,000 live cells were acquired per sample and were defined by their side scatter (SSC) and forward scatter (FSC) features. 


\subsection{Immunisations}

\subsubsection{Intravenous (i.v.) injections}

An insulin syringe tipped with a $26 \mathrm{G}$ needle was used to carry out intravenous injections using either of the lateral tail veins into vaccination recipients. BM-DC vaccine components were resuspended for intravenous administration using either IMDM or PBS and typically delivered using a $200 \mu \mathrm{L}$ volume.

\subsubsection{Subcutaneous (s.c.) injections}

An insulin syringe tipped with a $26 \mathrm{G}$ needle was used to carry out subcutaneous injections typically into the left flank of vaccination recipients. After applying a firm grip to injection recipients, a tuft of skin was lightly pinched on the left abdominal flank area to leave a raised area exposed for needle insertion just under the skin. The needle was inserted at an approximate angle of $35^{\circ}$. BM-DC vaccine components or tumour cells were resuspended for intravenous administration using either IMDM or PBS and typically delivered using a $50 \mu \mathrm{L}-100 \mathrm{uL}$ volume.

\subsubsection{Intraperitoneal (i.p.) injections}

An insulin syringe tipped with a $26 \mathrm{G}$ needle was used to carry out intraperitoneal injections typically using the central belly area of vaccination recipients. After firmly gripping vaccine recipient and tilting head down at a $30^{\circ}$ angle, the syringe needle tip is inserted into the central abdominal region at a $15^{\circ}$ angle relative to the abdomen in order to avoid the solid internal organs. BM-DC vaccine components were resuspended for 
intravenous administration using either IMDM or PBS and typically delivered using a 200 $\mu \mathrm{L}$ volume.

\subsection{Lymphocyte Isolations}

\subsubsection{Lymph Nodes}

Lymph nodes were isolated from euthanized participants following the exposure of surgical sites and sterilization using 70\% ethanol, then transferred into IMDM containing 6 well plates and placed on ice. To provide a single cell suspension, lymph nodes were perforated and tissue contents disrupted after being tightly sandwiched between 2 separate gauze pieces in Würzburger buffer. Red blood cells were removed from splenocyte preparations using $3 \mathrm{~mL}$ red blood cell lysing buffer (Qiagen, Maryland, USA) per LN sample. After incubation for 5 minutes, cells were washed twice with IMDM and then either washed a further 2 times before counting and resuspension, or for flow cytometric analysis. To allow for the intravenous administration of lymphocytes, 20,000 lymph node cells were prepared in a $200 \mathrm{uL}$ volume to allow delivery of 5,000 OTI cells. Alternatively the lymphocyte preparation was then analysed by flow cytometry. After spinning down $1500 \mathrm{rpm} 4$ minutes supernatants were removed and resuspended in $1 \mathrm{~mL}$ FACS buffer and $200 \mu \mathrm{L}$ used for fluorescence antibody staining.

\subsubsection{Spleen}

Spleens were isolated from euthanized participants after exposure of surgical site and sterilization using 70\% ethanol and transfer into IMDM containing 6 well plates and placed on ice. To provide a single cell suspension, spleens were perforated and tissue contents disrupted after being tightly sandwiched between 2 separate gauze pieces in Würzburger buffer. Red blood cells were removed from splenocyte preparations using 3 $\mathrm{mL}$ red blood cell lysing buffer (Qiagen, Maryland, USA) per LN sample. After incubation for 5 minutes, cells were washed twice with IMDM and then analysed by flow cytometry. 
After spinning down $1500 \mathrm{rpm} 4$ minutes supernatants were removed and resuspended in 1 mL FACS buffer and $200 \mu \mathrm{L}$ used for fluorescence antibody staining.

\subsubsection{Blood}

Blood was extracted from mice after accessing the lateral tail vein using a blade and collecting 6-10 drops in $1.7 \mathrm{~mL}$ microtubes containing $350 \mu \mathrm{L}$ of PBS with $10 \mathrm{mM}$ EDTA. After immediate collection of blood samples into EDTA containing PBS, contents were immediately shaken to prevent clotting. $1 \mathrm{~mL}$ red blood cell lysing buffer was added to each eppendorf tube to remove red blood cells before inverting and incubation for $30 \mathrm{~min}$ at $37{ }^{\circ} \mathrm{C}$. Once lysis of red blood cells had occurred and centrifugation at $664 \mathrm{~g}$ for $5 \mathrm{~min}$, at $4{ }^{\circ} \mathrm{C}$ was carried out, supernatants were removed from each eppendorf and vortexed before resuspending in $200 \mathrm{uL}$ FACS buffer and transferred into a round bottom 96-well plate. Antibody staining and flow cytometry was then utilized to analyse the samples.

\subsection{Cell Purification techniques}

\subsubsection{MACS}

An enriched DC fraction ( $>80 \%$ purity) was obtained by positive selection with antiCD11c coated magnetic beads (Miltenyi Biotec) that were used at $1 \mu \mathrm{L}$ beads and $4 \mu \mathrm{L}$ Würzburger buffer per $1 \times 10^{6}$ cells. After incubation on a rotating spinning wheel for 15 minutes to allow binding of the microbeads to the biotinylated antibodies, the preparation was washed in $10 \mathrm{~mL}$ Würzburger buffer before resuspension in $1 \mathrm{~mL}$ Automacs buffer and used to enrich for CD11c populations using the Automacs system (Miltenyi Biotec, Germany). Positive fractions collected were utilized for further studies after using flow cytometric analysis to check for purity. 


\subsubsection{FACS sorting of cells}

Purified splenic DC single cell suspensions were prepared and sorted using a FACSVantage SE DiVa (Becton Dickinson, CA, USA). Purified DCs were resuspended at $2 \times 10^{6}$ cells $/ \mathrm{mL}$ in Würzburger buffer and labeled with anti-CD8 and anti-CD11c fluorescent antibodies and kept on ice for 10 minutes. After staining, cells are spun in the centrifuge for 5 minutes at $1600 \mathrm{rpm} 4{ }^{\circ} \mathrm{C}$. Stained cells were then washed twice in sort buffer and then resuspended at $2 \times 10^{7}$ cells/ $\mathrm{mL}$ in sort buffer made up of RPMI without phenol red (Invitrogen) with $2 \%$ FCS and 5 mM EDTA. Unstained samples and samples stained with single antibodies were used to set fluorescence channel voltages and compensate for the overflow of specific fluorescence into neighboring fluorescent channels.

\subsubsection{APC isolation from spleens}

Harvested spleens were collected in 6 well plates containing IMDM and placed on ice. Spleens were then transferred into petri-dishes containing a solution of Würzburger buffer mixed with Liberase and DNase I (both Roche, Mannheim, Germany), and injected with 1 $\mathrm{mL}$ of the solution using an insulin syringe and 26 Gauge needle from either end of the actual spleen. A pair of scissors was used to cut up and expose splenic contents after which an 18 Gauge needle and $5 \mathrm{~mL}$ syringe was used to aspirate contents repeatedly to cause tissue disruption. After incubating for $20-30$ minutes at $37{ }^{\circ} \mathrm{C}, 0.1 \mathrm{M}$ of EDTA was added to the preparation for the final 5 minutes of incubation time to allow dissociation of DC and $\mathrm{T}$ cell interactions. A syringe was then used to collect digested splenic contents for filtration through a $70 \mu \mathrm{m}$ cell strainer to obtain a single cell suspension in Würzburger buffer before spinning down at $1600 \mathrm{rpm}$ for 5 minutes at $4{ }^{\circ} \mathrm{C}$.

After spinning and removal of supernatants, $10 \mathrm{~mL}$ of red blood cell (RBC) lysis buffer was added to each tube followed by incubation for 5 minutes at $37^{\circ} \mathrm{C}$. After washing with Würzburger buffer, cells were resuspended using $5 \mathrm{~mL}$ 14.1\% Nycodenz and gently 
overlaid with $2 \mathrm{~mL}$ Shortman buffer to generate a gradient designed to isolate splenic DC populations. After centrifugation at $1800 \mathrm{rpm}$ for 20 minutes $840 \mathrm{~g}$ at room temperature without breaking to allow separation of the different layers, the interphase layer was collected using a Pasteur pipette and transferred into a separate tube and washed using excess Würzburger buffer. An enriched DC fraction ( $>80 \%$ purity) was obtained by positive selection with anti-CD11c coated magnetic beads (Miltenyi Biotec) that were used at $1 \mu \mathrm{L}$ beads and $4 \mu \mathrm{L}$ Würzburger buffer per $1 \times 10^{6}$ cells. After incubation on a rotating spinning wheel for 15 minutes to allow binding of the microbeads to the biotinylated antibodies, the preparation was washed in $10 \mathrm{~mL}$ Würzburger buffer before resuspension in $1 \mathrm{~mL}$ Automacs buffer and used to enrich for CD11c populations using the Automacs system (Miltenyi Biotec, Germany). Positive fractions collected were utilized for further studies after using flow cytometric analysis to check for purity.

\subsection{T - Cell Responses}

\subsubsection{In-vitro $\mathrm{T}$ cell proliferation assay}

BM-DC cultures were generated from bone marrow precursors isolated from C57BL/6 female mice. Femur and tibial bones were removed and the marrow exposed by cutting bone ends with scissors at a $45^{\circ}$ angle inside the laboratory sterile hood. The marrow was flushed out using a 25 -gauge needle attached to a $1 \mathrm{~mL}$ insulin syringe filled with IMDM and collected into a sterile 6-well plate with wells containing IMDM. Single cell suspensions were obtained by agitating the clumps using a pipette aid and passing the marrow pieces through a $70 \mathrm{uM}$ filter into a $50 \mathrm{~mL}$ falcon tube containing $10 \mathrm{~mL}$ cIMDM. After centrifugation at $1600 \mathrm{rpm}$ for 4-5 min, cells were counted and resuspended to allow for a final concentration of $2 \times 10^{6}$ DCs per well in $5 \mathrm{~mL}$ cIMDM supplemented with IL-4 and GM-CSF. IL-4 and gm-CSF cytokines were sources from culture supernatants of Chinese hamster ovary mIL-4 and X63/GM-CSF cells and titrations used to determine the optimal dose required to supplement BM-DC cultures. Cells were incubated at $37^{\circ} \mathrm{C}$ and 
supplemented with fresh cytokine and cIMDM suspension on days 3 and 5. This was done by replacing $2.5 \mathrm{~mL}$ of the BM-DC culture supernatant with $2.5 \mathrm{~mL}$ fresh cIMDM containing the appropriate proportions of IL-4 and GM-CSF.

Purified LN cells are isolated from OT-I mice Lymph nodes were isolated from euthanized participants after exposure of surgical sites and sterilization using 70\% ethanol and transfer into IMDM containing 6 well plates and placed on ice. To provide a single cell suspension, lymph nodes were perforated and tissue contents disrupted after being tightly sandwiched between 2 separate gauze pieces in Würzburger buffer. Red blood cells were removed from splenocyte preparations using $3 \mathrm{~mL}$ red blood cell lysing buffer (Qiagen, Maryland, USA) per LN sample. After incubation for 5 minutes, cells were washed twice with IMDM and then either washed a further 2 times before counting and resuspension to allow for $1 \mathrm{x}$ $10^{6} \mathrm{LN}$ cells per well.

Proliferation assays were set up in 96-well plates at a range of DC concentrations per well between 20,000 - 250 DCs with 100,000 T cells per well using fold-dilution technique with the multi channel pipette. After $48 \mathrm{~h}$ incubation, $50 \mathrm{uL}$ of supernatant was removed and frozen down for cytokine analysis at a further date. Radioactive tritiated thymidine $3 \mathrm{H}-$ thymidine $(1 \mathrm{mCi} / \mathrm{ml}$; Amersham, Aylesbury, UK) was then added to each well in a volume of $50 \mathrm{uL}$ and cell cultures allowed to incubate for a further $16-18 \mathrm{hrs}$. After incubation cells were harvested using a Radioactive Cell Harvester and B-counter to determine tritiated thymidine uptake by $\mathrm{T}$ cells as a measure of $\mathrm{T}$ cell proliferation based on the efficiency of $\mathrm{T}$ cell proliferation.

\subsection{Cytokine Analysis}

\subsubsection{Bioplex analysis protocol}

Bioplex assay buffer was prepared using PBS containing 0.1\% BSA, $0.05 \%$ Tween20, $0.005 \%$ sodium azide and $2.5 \mathrm{mM}$ EDTA. Blood was taken from mice by nicking the 
lateral tail vein and 5-8 drops collected in $1.7 \mathrm{~mL}$ microtubes. The blood samples were incubated at room temperature for 4 to $6 \mathrm{~h}$ or overnight at $4^{\circ} \mathrm{C}$ to allow blood clotting. After centrifugation at $14,000 \mathrm{~g}$ for $5 \mathrm{~min}$, serum was transferred to a new microtube and stored at $-20{ }^{\circ} \mathrm{C}$ until analysis. Serum cytokine levels were analysed using the Bioplex system (BioRad, Auckland, New Zealand). Capture beads were transferred to 96 well filter plates (L-Plate, Abacus ALS, Auckland, New Zealand) with $0.65 \mu 1$ per cytokine and sample in $50 \mu$ assay buffer. Buffer was removed by vacuum and beads washed twice with assay buffer by adding $100 \mu \mathrm{l}$ buffer and removing by vacuum. Serum samples were diluted 1:2 in assay buffer before the plate mixed on a plate shaker for $30 \mathrm{~min}$ at room temperature and washed three times. Bioplex beads were incubated with assay buffer containing detection antibodies in 1:300 dilutions using a $25 \mu \mathrm{l}$ volume, followed by washing three times and incubation with SA-PE (50 $\mu$ volume, 1:1000 dilution) for $10 \mathrm{~min}$ at room temperature. Beads were washed three times before resuspension in $125 \mu$ assay buffer prior to analysis using the Bioplex system. Concentrations were determined using standards provided by BioRad.

\subsection{Statistical Analysis}

Statistical analysis comparing three or more groups together was carried out using one-way ANOVA with Tukey's post-test with means and standard errors of means shown in graphs. A two-way ANOVA with Tukey's post-test was utilized when analysing two factors in three groups or more to determine statistical significance. This post-test was carried out to correct for inference when comparing multiple groups. Statistical calculations were performed using Graphpad Prism ${ }^{\circledR}$ Version 4 statistical package (Graphpad Software Inc., San Diego, CA, USA). 


\section{Chapter 3:}

\section{TLR stimulation enhances antigen specific responses to DC-based vaccines}




\subsection{Introduction}

DCs that have been correctly stimulated are capable of delivering the appropriate instructive signals necessary for the generation of proper and effective $\mathrm{T}$ cell responses. Evidence points to the use of matured DC populations for vaccine studies which ensure the induction of antigen specific $\mathrm{T}$ cell responses that are superior to those generated if simply using immature DCs alone (Huck et al., 2008; Labeur et al., 1999; Martin-Fontecha et al., 2003). The DC maturation process can be initiated and influenced by cytokines, T cells and microbial product receptors found on the outer surface and internal components of DCs (Lanzavecchia and Sallusto, 2001a, 2001b; Reis e Sousa and Germain, 1999; Reis e Sousa et al., 1999).

DC activation describes the conversion of naïve DCs into effector DCs that occurs after releasing tightly regulated cytokines such as the Th1 polarizing cytokine IL-12 (Lyakh et al., 2008; Sporri et al., 2006; Trinchieri, 2003). Proinflammatory cytokines such as IL-6 and TNF- $\alpha$ are also released by activated BM-DCs. Cytokines generated by matured and activated DCs are capable of influencing $\mathrm{T}$ cell responses which include $\mathrm{T}$ cell expansion, effector function and response duration (Curtsinger et al., 1999; Haring et al., 2006). DC maturation and activation processes are controlled by efficient regulatory signals that are present and experienced within the DC microenvironment.

The stimulation of Toll-like Receptors (TLRs) expressed by DCs induces maturation and induces activation activity evidenced by the upregulation of DC surface maturation markers such as MHC II, CD86 and CD40 with production of proinflammatory cytokines such as IL-6 and TNF- $\alpha$ and IL-12. Examples of ligands that bind to DC TLRs include lipoproteins and lipopeptides (TLR2), double-stranded RNA (TLR 3), Lipopolysaccharide (TLR 4), flagellin (TLR 5), single-stranded RNA (TLR 7/8), CpG DNA (TLR 9) and profiling (TLR11). Examples of actual synthetic TLR agonists that bind to DC TLRs include Pam3Cys (TLR 2), Poly IC (TLR 3), LPS (TLR 4), MPL (TLR 4), flagellin (TLR 5), Resiquimod (TLR 7/8), CpG (TLR 9). 
For clinical applications, stimulation of in-vitro DCs with TLR ligands could be a useful system to enhance adaptive responses. For example, studies have shown lipopolysaccharide (LPS) to be an extremely potent adjuvant in-vitro and in-vivo, however the extreme toxicity typically associated with its use has remained a limitation for its clinical utilization (Ismaili et al., 2002). LPS is a gram-negative bacterial outer cell wall glycolipid composed of a hydrophobic lipid A portion and hydrophilic polysaccharide (Schletter et al., 1995; Schletter et al., 1995). The detoxified version of LPS otherwise known as monophosphoryl lipid A (MPL) has been designed and specifically developed to confer similar adjuvanticity effects but with reduced toxicity (Rietschel et al., 1994; Ulrich and Myers, 1995). The lipid A moiety of LPS is responsible for its adjuvant activity with manipulation of its structure achieved by the removal of fatty acid groups in addition to an acid labile phosphate group resulting in reduced pyrogenicity and toxicity (Ribi et al., 1984).

Published work has also shown that MPL is capable of activating APCs and inducing Th1 and Th2 immune responses in mice (De Becker et al., 2000; Moore et al., 1999). MPL has also been found capable of influencing both cellular and humoral immune responses with its safety being clearly demonstrated in human clinical trials (Thoelen et al., 1998; Ulrich and Myers, 1995). In addition, MPL has also been shown to induce Th1 type responses to co-administered antigen proteins in addition to influencing antibody responses (Hermans et al., 2007; Ulrich and Myers, 1995).

The stimulatory effect of TLR agonists in combination with MPL was examined in BMDC cultures and reported that combinations of multiple TLR agonists could induce synergistic maturation and activation responses (Napolitani et al., 2005; Warger et al., 2006). The combination of dual signalling pathways and associated mediators induced enhanced cytokine release, upregulation of maturation markers and improved $\mathrm{T}$ cell responses when compared with stimulation by the TLR agonists alone. A range of synthetic TLR agonists were selected for testing that targeted internal and externally situated TLRs in an effort to carefully mature vaccine BM-DCs prior to immunisation.

To achieve efficient production and release of polarizing cytokines by TLR stimulated DCs, a secondary feedback CD40L signal is required from associated T cells (Lee et al., 
2002). Work published by Napolitani et al (2005) showed that exposure to specific dual TLR agonist combinations increased DC functionality and was further enhanced after treatment with a CD40 antibody agonist. The generation of CTLs involves CD40 signalling and previous studies had reported that further amplification of BM-DC maturation and activation status could be provided by CD40L stimulation (Bennett, 1998; Schoenberger et al., 1998; van Mierlo et al., 2002).

The results presented in this chapter show the work carried out to determine the effect of in-vitro TLR stimulation of BM-DC cultures using MPL alone and then in combination with a second TLR agonist. Proliferation studies using protein and peptide antigen loaded BM-DCs treated with MPL alone and together with a second TLR agonist were conducted and used to measure antigen presentation and processing abilities of DCs for the various treatment groups. The in-vivo application of this work was tested by measuring antigen specific $\mathrm{T}$ cell responses after immunisation using this vaccination protocol. 


\subsubsection{Aims}

The size and quality of the antigen specific $\mathrm{T}$ cell response is highly dependent on the DC phenotype at the time of antigen presentation to $\mathrm{T}$ cells. The hypothesis therefore was that "BM-DC phenotype and function could be enhanced by the use of TLR stimulation generated using specific TLR agonists alone and in combination with each other". This was investigated by examining the surface marker phenotype, cytokine production, antigen processing and presentation activities of BM-DCs which had been cultured in-vitro with specific TLR agonists alone and in combination with each other at different concentrations. Information regarding the level of TLR stimulation achieved by in-vitro testing was then utilised in an attempt to enhance the antigen specific T cell responses mounted by BM-DC based vaccines in-vivo using the B16.OVA murine model of melanoma.

The specific objectives were:

- To examine the effect of TLR stimulation on BM-DC function in-vitro, with emphasis on MPL

- To evaluate combinations of TLR stimuli and their effect on BM-DC function in-vitro, with emphasis on combinations that include MPL

- To optimise and analyse the in-vivo antigen specific response generated by BM-DC based vaccines

- To explore the in-vivo antigen specific response generated by BM-DC based vaccines combined with TLR stimulation 


\subsection{Results}

\subsubsection{MPL stimulation induces DC maturation in Bone Marrow- Derived Dendritic Cell (BM-DC) cultures in-vitro}

DC maturation refers to the stimulation process that induces elevated expression of MHC and other co-stimulatory molecules such as CD86, CD80 that are necessary for effective $\mathrm{T}$ cell induction (Cella et al., 1997; Sporri et al., 2006). To provide an example of the capacity of BM-DCs to respond to TLR stimulation, a phenotypic examination of the DCs typically found in BM-DC in-vitro cultures was carried out. Testing was conducted to confirm maturation capability of MPL treated BM-DC in-vitro cultures relative to untreated groups. The cytokine release profile of non-MPL treated versus MPL treated BM-DCs was also examined by obtaining measures of IL-12p40, IL- 6 and TNF- $\alpha$ in invitro culture supernatants.

BM-DC cultures were generated using marrow isolated from $\mathrm{C} 57 \mathrm{BL} / 6$ female mice and supplemented with GM-CSF and IL-4 for 7 days. On day 6, selected groups were matured by the addition of $100 \mathrm{ng} / \mathrm{ml}$ (final concentration) of MPL for the final $22 \mathrm{hr}$ of culture. Fluorescent antibodies used to determine surface marker expression levels by flow cytometry analysis on day 7 were CD11c, CD86 and CD40. Propidium Iodide (PI) was utilized as a viability marker in order to eliminate dead or dying cellular populations from analysis. Dot plots illustrate the flow cytometry gating strategy employed to identify cell populations of interest (Figure 3.1 A). Viable BM-DCs were identified using CD11 $\mathrm{c}^{+} \mathrm{PI}-$ gate selections. Upregulation of the maturation CD86 and CD40 surface markers was observed in the MPL treated cultures (Figure 3.1 B). A proportion of 
A

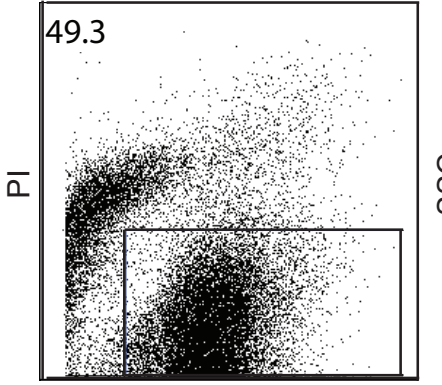

FSC

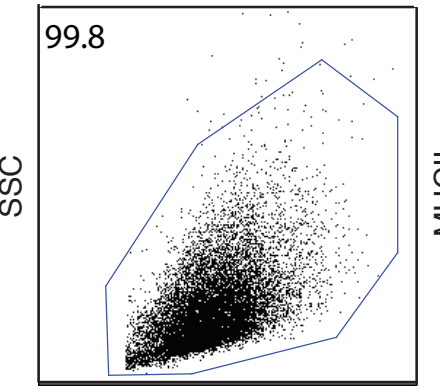

FSC
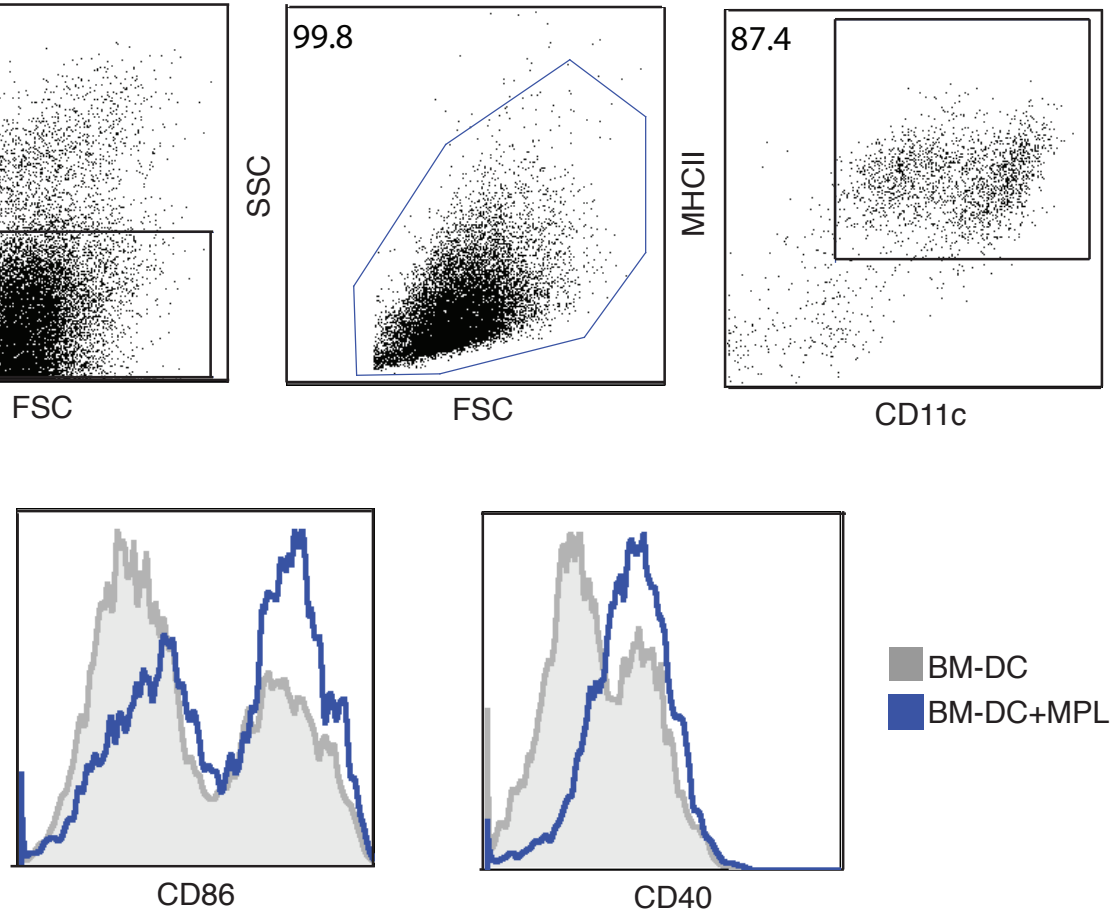

D

C
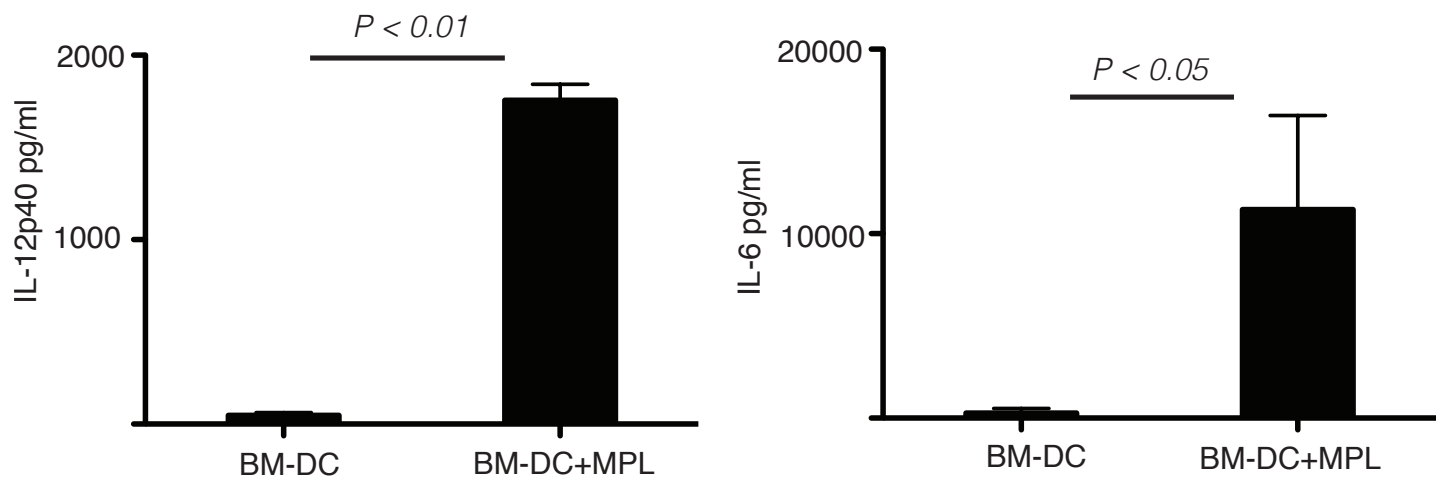

E

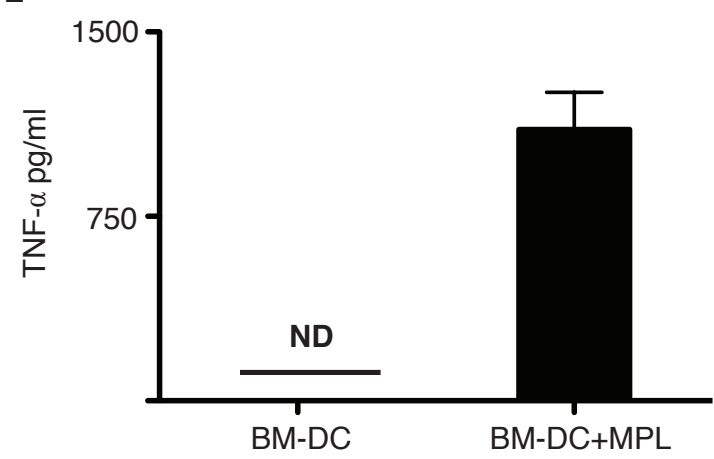

BM-DC

$B M-D C+M P L$ 
Figure 3.1 The stimulation of BM-DCs with MPL induces maturation and cytokine production in-vitro. BM-DCs were prepared from $\mathrm{C} 57 \mathrm{BL} / 6 \mathrm{~J}$ recipients and cultured invitro in the presence of GM-CSF and IL-4 for 7 days. To induce maturation some of the cultures were supplemented with $100 \mathrm{ng} / \mathrm{ml} \mathrm{MPL}$ and others with LPS $100 \mathrm{ng} / \mathrm{ml}$ as a positive control on day 6. After harvesting on day 7, cultured BM-DC cells were analysed using flow cytometric analysis to determine CD86 and CD40 expression levels. (A) Dot plots show the general gating strategy used to identify BM-DC populations from such invitro cultures as determined by antibody staining and flow cytometry on day 7 . (B) Histograms show naive untreated BM-DC samples depicted by light grey filled areas, with MPL treated samples indicated by coloured lines. Culture supernatants were removed on day 7 after $22 \mathrm{~h}$ exposure to MPL 100ng/ml. Cytokine analysis was conducted to measure levels for the following cytokines: (C) IL-12p40, (D) IL-6 and (E) TNF- $\alpha$. Each bar graph show measured cytokine levels for data combined from 2-3 separate experiments. Each test sample was carried out in triplicate with data pooled together from three individual samples for each experiment conducted. Statistical significance was determined using a 1way ANOVA test and Tukey's post-test where $* \mathrm{p}=0.05-0.01,{ }^{*} \mathrm{p}=0.01-0.001,{ }^{* * *} \mathrm{p}$ $<0.001, \mathrm{NS}=$ not significant; $\mathrm{ND}=$ levels not detected.

these BM-DCs exhibited a mature phenotype indicative of spontaneously maturing DC cells in-vitro. The final dot plot in Figure 3.1 A indicates that differing levels of CD11c expression exist within the BM-DC population. It has been shown that murine BM-DCs downregulate CD11c in response to TLR 4 stimulation (Singh-Jasuja et al., 2013). The concentration of MPL used was determined based on separate titration studies (Figure 3.2).

To obtain indication of BM-DC activation, production and release of IL- 6 and TNF- $\alpha$ and IL-12p40 was measured in in-vitro culture supernatants. Day 6 BM-DCs cultures were harvested and replated in triplicate into 96-well plates in the presence or absence of MPL at a $100 \mathrm{ng} / \mathrm{ml}$ final concentration per well. On day 7 after $22 \mathrm{~h}$, culture supernatants were collected and tested for IL-12p40 IL-6 and TNF- $\alpha$ levels (Figure 3.1). Cytokine levels were barely detectable in the immature DC only culture supernatants while results from mature MPL treated cultures showed that MPL was capable of inducing the production of IL-12-p40 (Figure 3.1 C), IL-6 (Figure 3.1 D) and TNF- $\alpha$ (Figure 3.1 E) from BM-DCs invitro.

In summary, the in-vitro stimulation of BM-DCs using the TLR 4 agonist MPL consistently induced maturation of BM-DCs, evidenced by the upregulation of their CD86 and CD40 surface expression. Cytokine production levels of IL-6, IL-12p40 and TNF- $\alpha$ were also enhanced after treatment with MPL 


\subsubsection{The utilization of TLR agonists for effective stimulation of BM- DCs}

Although MPL was the featured TLR agonist of interest, it was also important to identify additional TLR agonists that could be used in combination studies to further enhance BMDC stimulation after dual TLR stimulation. It was also important to ensure that appropriate concentrations of these additional TLR agonists were identified so that overstimulation of the BM-DCs did not occur after combining its action with MPL. Titration studies were therefore conducted to ensure effective maturation and activation of MPL treated BMDCs. Maturation marker levels for CD86 and CD40 were measured in addition to cytokine release profiles for IL-12p40, IL-6 and TNF- $\alpha$ after treatment with various MPL concentrations.

Similar studies were conducted with selected TLR agonists such as Resiquimod (TLR7/8 agonist), Pam3Cys (TLR2 agonist), CpG (TLR9 agonist) and LPS (TLR4 agonist) was used to generate a positive control sample. BM-DC cultures were again generated using marrow isolated from $\mathrm{C} 57 \mathrm{BL} / 6$ female mice. On day 6 , selected groups were replated into 96-well plates and matured by the addition of various concentrations for the selected TLR agonists for the final $22 \mathrm{hr}$ of culture. Samples were arranged in triplicate and maturation surface marker expression of CD86 and CD40 levels for $\mathrm{CD} 11 \mathrm{c}^{+} \mathrm{DC}$ populations were determined by flow cytometry analysis on day 7. BM-DC culture supernatants were also removed on day 7 after exposure to different TLR agonists at specific doses and analysed for cytokine production.

TLR agonist concentrations selected for further in-vitro studies were based on the resultant titration curves for the surface marker expression of CD86 and CD40 shown in Figures 3.2 and 3.3. Cytokine responses detected for IL-12p40, IL-6 and TNF- $\alpha$ in pg/ml are also shown in Figures 3.2 and 3.3. Multiple TLR agonist concentrations within the linear region of their respective titration curves were selected for use in order to avoid saturation effects preventing the distinction between beneficial and redundant TLR agonist combinations. At least two suboptimal concentrations were selected for each TLR agonist to be used in subsequent experiments. These titration curves are the combination of more than 3 separate 
experiments with standardised geometric mean fluorescence intensity (MFI) values expressed as percentage of max values (Figure 3.2 and Figure 3.3). Based on these results, the MPL doses selected for use were $10 \mathrm{ng} / \mathrm{ml}$ and $100 \mathrm{ng} / \mathrm{ml}$ (Figure $3.2 \mathrm{~B}$ ), Resiquimod doses were $10 \mathrm{ng} / \mathrm{ml}, 100 \mathrm{ng} / \mathrm{ml}$ and $1 \mathrm{ug} / \mathrm{ml}$ (Figure 3.3 B), Pam3Cys were $10 \mathrm{ng} / \mathrm{ml}$ and $100 \mathrm{ng} / \mathrm{ml}$ (Figure $3.3 \mathrm{~A}$ ), and $\mathrm{CpG}$ were $10 \mathrm{nM}$ and $100 \mathrm{nM}$ (Figure $3.3 \mathrm{C}$ ).

After establishing a suitable maturation response range to each TLR agonist tested, the cytokine release profile was examined within these limits. Maximal IL-6 production by the BM-DCs occurred towards the higher end of the concentration selections for all TLR agonists, while TNF- $\alpha$ was released at more modest levels in comparison. IL-12p40 was produced across MPL, Resiquimod, Pam3Cys and CpG treated BM-DC cultures while TNF- $\alpha$ production was evident only when high doses of MPL, Resiquimod and Pam3Cys were used. No TNF- $\alpha$ was produced when $\mathrm{CpG}$ was used to stimulate BM-DC cultures and although IL-6 levels peaked rapidly, this was followed by a rapid decline in production after $100 \mathrm{ng} / \mathrm{ml}$ for each TLR agonist except for Resiquimod. 
A

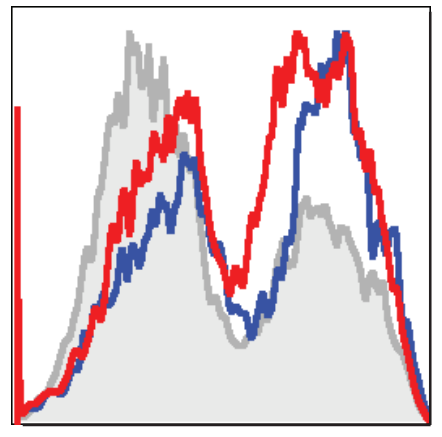

CD86

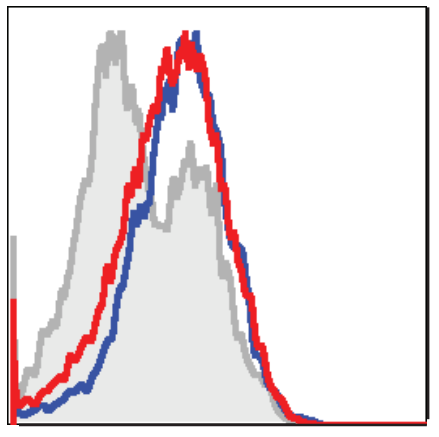

CD40

BM-DC

BM-DC+MPL 10ng/ml

BM-DC+MPL 100ng/ml

B

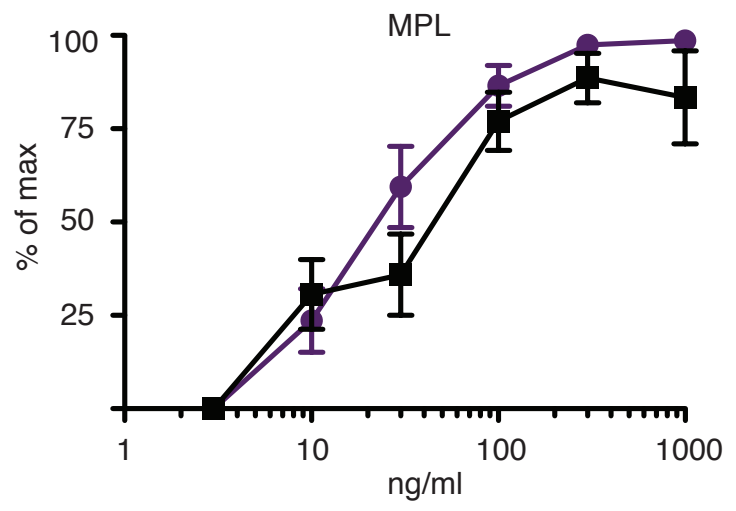

C

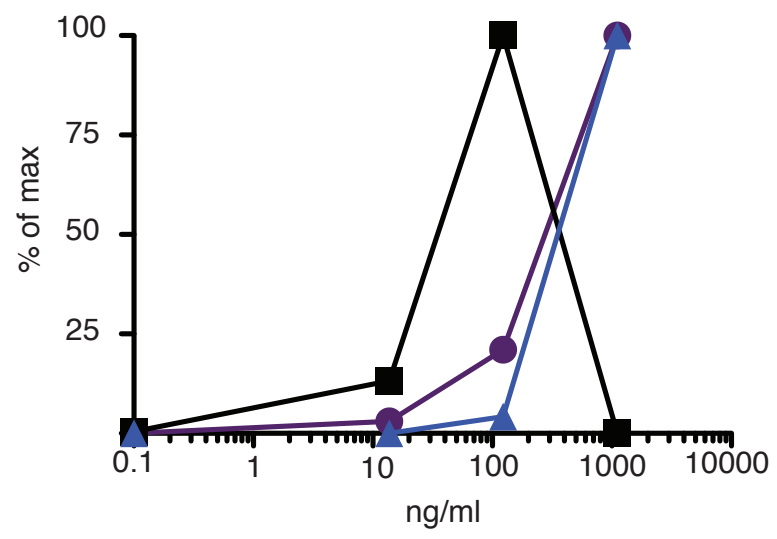

$\mathrm{IL}-12 \mathrm{p} 40$

IL-6

TNF-a

Figure 3.2 Maturation status and cytokine production by MPL treated BM-DCs at varied concentrations. BM-DC cultures were generated as described previously (in section 3.2.1 and Figure 3.1). (A) Histograms illustrate the impact of different concentrations of MPL $100 \mathrm{ng} / \mathrm{ml}$ and $10 \mathrm{ng} / \mathrm{ml}$ tested to determine their effect on CD86 and CD40 maturation marker status. BM-DC cultures were generated as described previously (in section 3.2.1 and Figure 3.1). MPL was used at various concentrations to 
stimulate BM-DC cultures on day 6. Flow cytometric analysis was used to determine the CD86 and CD40 expression levels of the BM-DC cultures after $22 \mathrm{~h}$ exposure to MPL. Graph (B) shows results from three separate experiments with individual samples tested in triplicate and adjusted for inter-experimental variation by using $\%$ of max values (actual value/maximal value) and are expressed as mean $\pm \mathrm{SD}$ values. (C) Culture supernatants were also collected after $22 \mathrm{~h}$ following MPL stimulation at different concentrations and used for detection of the following cytokines IL-12p40, IL-6 and TNF- $\alpha$. Each test sample was carried out in triplicate with data pooled together from three individual samples for each experiment conducted. Cytokine levels were initially measured in $\mathrm{pg} / \mathrm{ml}$, then standardised by adjusting for inter-experimental variation by using $\%$ of max values (actual value/maximal value) and are displayed on the same graph for comparison. 


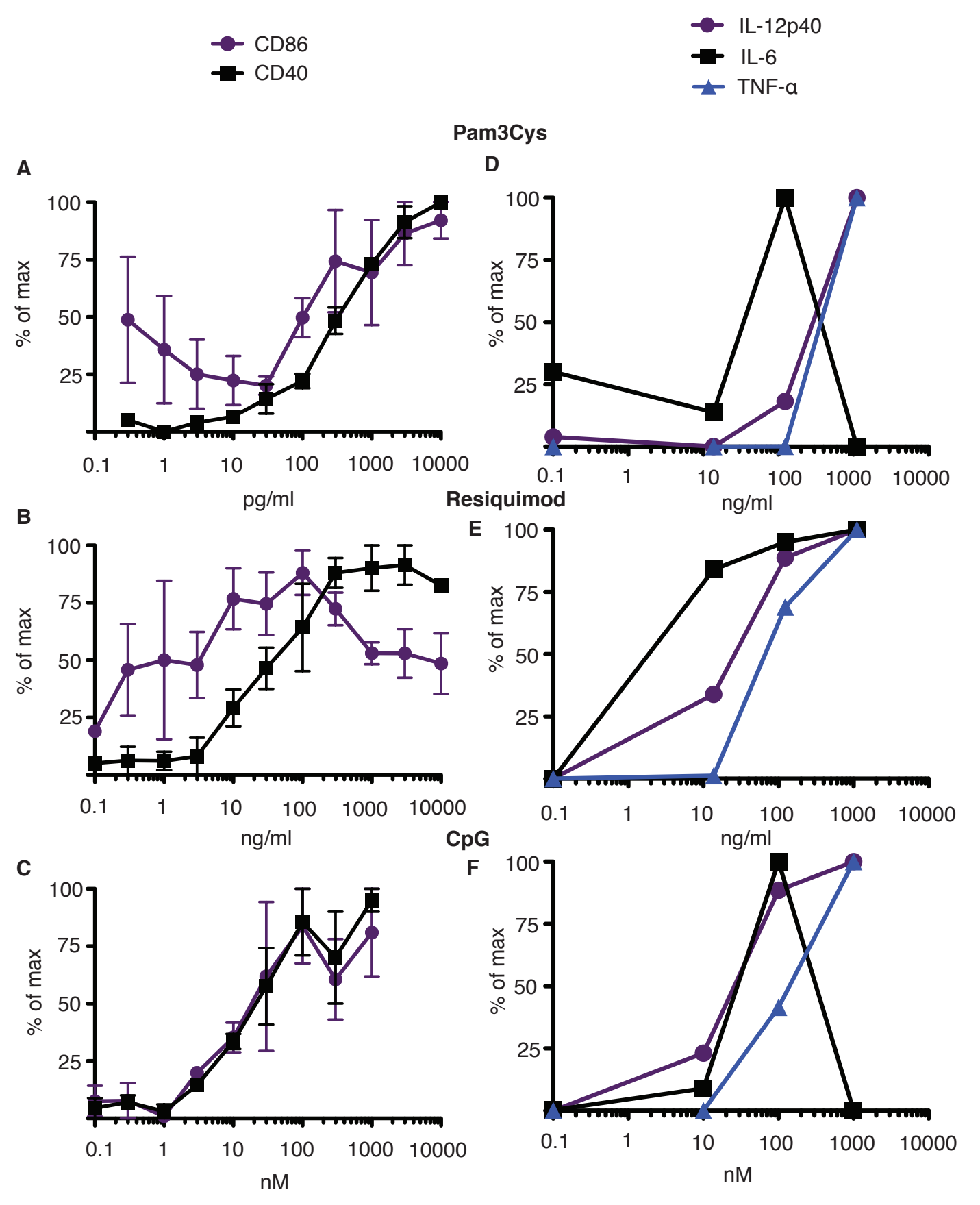

Figure 3.3 Maturation status and cytokine production of BM-DCs after TLR titration in-vitro. Selected TLR agonists were used at various concentrations to stimulate BM-DC cultures for the final $22 \mathrm{~h}$ of incubation on day 6. BM-DC cultures were generated as described previously (in section 3.2.1 and Figure 3.1). Flow cytometric analysis was used to determine CD86 and CD40 expression levels of BM-DCs after $22 \mathrm{~h}$ culture with individual synthetic TLR agonists tested: Pam3Cys (A), Resiquimod (B) and CpG (C). Results are displayed after adjusting for inter-experimental variation by using \% of max values (actual value/maximal value) and are expressed as mean $\pm \mathrm{SD}$ values. Graphs seen in (A), (B) and (C) show data from 2-3 separate experiments with individual samples tested in triplicate. Culture supernatants were also collected $22 \mathrm{hrs}$ after TLR agonist stimulation using different TLR concentrations and used for detection of the following 
cytokines: IL-12p40, IL-6 and TNF- $\alpha$. Titration results with Pam3Cys are shown in (D), Resiquimod (E) and $\mathrm{CpG}(\mathrm{F})$. Each sample was tested in triplicate with data pooled together from three individual samples for each cytokine tested. Cytokine levels were initially measured in $\mathrm{pg} / \mathrm{ml}$, then standardised by adjusting for inter-experimental variation by using \% of max values (actual value/maximal value) and are displayed on the same graph for comparison.

A

\begin{tabular}{|l|c|c|c|}
\hline \multicolumn{5}{|c|}{ MPL } \\
\hline Concentration & IL-12p40 & IL-6 & TNF- $\alpha$ \\
\hline $\mathbf{0 . 1} \mathbf{~ n g / m l ~}$ & 87.96 & 23.38 & 0 \\
\hline $\mathbf{1 3 . 7} \mathbf{~ n g} / \mathbf{m l}$ & 223.5 & 673.63 & 0 \\
\hline $\mathbf{1 2 3} \mathbf{~ n g} / \mathbf{m l}$ & 1011.29 & 5112.64 & 25.18 \\
\hline $\mathbf{1 1 1 1} \mathbf{~ n g} / \mathrm{ml}$ & 4483.73 & 0 & 628.59 \\
\hline
\end{tabular}

B

\begin{tabular}{|l|c|c|c|}
\hline \multicolumn{5}{|c|}{ Resiquimod } \\
\hline Concentration & IL-12p40 & IL-6 & TNF- $\alpha$ \\
\hline $\mathbf{0 . 1} \mathbf{~ n g / m l ~}$ & 73.04 & 30.72 & 0 \\
\hline $\mathbf{1 3 . 7} \mathbf{~ n g} / \mathbf{m l}$ & 1418.08 & 17299.31 & 19.86 \\
\hline $\mathbf{1 2 3} \mathbf{~ n g / m l}$ & 5490.45 & 0 & 812.28 \\
\hline $\mathbf{1 1 1 1} \mathbf{~ n g / m l}$ & 7649.96 & 0 & 1151.4 \\
\hline
\end{tabular}

C

\begin{tabular}{|l|c|c|c|}
\hline \multicolumn{5}{|c|}{ Pam3Cys } \\
\hline Concentration & IL-12p40 & IL-6 & TNF- $\alpha$ \\
\hline $\mathbf{0 . 1} \mathbf{~ n g / m l ~}$ & 166.81 & 804.27 & 0 \\
\hline $\mathbf{1 3 . 7} \mathbf{~ n g / m l}$ & 119.04 & 369.21 & 0 \\
\hline $\mathbf{1 2 3} \mathbf{~ g / m l}$ & 343.36 & 2678.59 & 0 \\
\hline $\mathbf{1 1 1 1} \mathbf{~ n g / m l}$ & 1350.79 & 0 & 214.51 \\
\hline
\end{tabular}

D

\begin{tabular}{|l|c|c|c|}
\hline \multicolumn{4}{|c|}{ CpG } \\
\hline Concentration & IL-12p40 & IL-6 & TNF- $\alpha$ \\
\hline $\mathbf{0 . 1} \mathbf{~ M}$ & 107.2 & 49.5 & 0 \\
\hline $\mathbf{1 3 . 7} \mathbf{~ n M}$ & 1077.97 & 1450.79 & 0 \\
\hline $\mathbf{1 2 3} \mathbf{~ M}$ & 3830.15 & 16250.23 & 28.47 \\
\hline $\mathbf{1 1 1 1} \mathbf{n M}$ & 4312.29 & 0 & 68.48 \\
\hline
\end{tabular}

Table 3.1 Levels of Cytokine production produced by CD11c $\mathrm{c}^{+}$BM-DCs treated with the respective TLR agonists at varying concentrations displayed in Figure 3.2 and Figure 3.3. 
Chapter 3: TLR stimulation enhances antigen specific responses to DC based vaccines 108

A

\begin{tabular}{|c|c|c|c|}
\hline \multicolumn{4}{|c|}{ MPL } \\
\hline \begin{tabular}{|l} 
Concentration \\
\end{tabular} & CD86 & CD40 & MHC II \\
\hline $0.0 \mathrm{ng} / \mathrm{ml}$ & 22.1 & 13.6 & 87.9 \\
\hline $3 \mathrm{ng} / \mathrm{ml}$ & 28.4 & 14 & 93.5 \\
\hline $10 \mathrm{ng} / \mathrm{ml}$ & 31.1 & 16 & 98.1 \\
\hline $30 \mathrm{ng} / \mathrm{ml}$ & 47.9 & 20.3 & 133 \\
\hline $100 \mathrm{ng} / \mathrm{ml}$ & 65.6 & 28.4 & 171 \\
\hline $300 \mathrm{ng} / \mathrm{ml}$ & 62.5 & 30.9 & 165 \\
\hline $1000 \mathrm{ng} / \mathrm{ml}$ & 64.3 & 26.3 & 153 \\
\hline
\end{tabular}

B

\begin{tabular}{|c|c|c|c|}
\hline \multicolumn{4}{|c|}{ Resiquimod } \\
\hline Concentration & CD86 & CD40 & MHC II \\
\hline $0.0 \mathrm{ng} / \mathrm{ml}$ & 19 & 13.4 & 77.3 \\
\hline $0.1 \mathrm{ng} / \mathrm{ml}$ & 24.4 & 12.9 & 82 \\
\hline $1 \mathrm{ng} / \mathrm{ml}$ & 26.6 & 13.7 & 86.2 \\
\hline $3 \mathrm{ng} / \mathrm{ml}$ & 31.6 & 16.6 & 103 \\
\hline $10 \mathrm{ng} / \mathrm{ml}$ & 48.4 & 22.9 & 132 \\
\hline $30 \mathrm{ng} / \mathrm{ml}$ & 48.3 & 26.2 & 129 \\
\hline $100 \mathrm{ng} / \mathrm{ml}$ & 75 & 35.4 & 199 \\
\hline $300 \mathrm{ng} / \mathrm{ml}$ & 57.6 & 32.6 & 154 \\
\hline $1000 \mathrm{ng} / \mathrm{ml}$ & 45.3 & 31.2 & 128 \\
\hline $3000 \mathrm{ng} / \mathrm{ml}$ & 42 & 35.7 & 130 \\
\hline $1000 \mathrm{ng} / \mathrm{ml}$ & 37.9 & 32.3 & 133 \\
\hline
\end{tabular}

C

\begin{tabular}{|c|c|c|c|}
\hline \multicolumn{4}{|c|}{ Pam3Cys } \\
\hline Concentration & CD86 & CD40 & MHC II \\
\hline $0.0 \mathrm{ng} / \mathrm{ml}$ & 23.4 & 16.1 & 92.3 \\
\hline $0.1 \mathrm{ng} / \mathrm{ml}$ & 28.2 & 14.8 & 90 \\
\hline $1 \mathrm{ng} / \mathrm{ml}$ & 24.8 & 14.2 & 85.5 \\
\hline $3 \mathrm{ng} / \mathrm{ml}$ & 23.9 & 14.5 & 80.7 \\
\hline $10 \mathrm{ng} / \mathrm{ml}$ & 24.5 & 15.1 & 87.3 \\
\hline $30 \mathrm{ng} / \mathrm{ml}$ & 26.2 & 16.1 & 93.4 \\
\hline $100 \mathrm{ng} / \mathrm{ml}$ & 35.7 & 20.3 & 115 \\
\hline $300 \mathrm{ng} / \mathrm{ml}$ & 39.9 & 24.5 & 122 \\
\hline $1000 \mathrm{ng} / \mathrm{ml}$ & 55.1 & 31.2 & 157 \\
\hline $3000 \mathrm{ng} / \mathrm{ml}$ & 58 & 38 & 166 \\
\hline $10000 \mathrm{ng} / \mathrm{ml}$ & 52 & 38.4 & 160 \\
\hline
\end{tabular}

D

\begin{tabular}{|c|c|c|c|}
\hline \multicolumn{4}{|c|}{ CpG } \\
\hline Concentration & CD86 & CD40 & MHC II \\
\hline $0.0 \mathrm{nM}$ & 26.2 & 15.3 & 95.2 \\
\hline $0.1 \mathrm{nM}$ & 26.2 & 15.9 & 96.9 \\
\hline $0.3 \mathrm{nM}$ & 26.5 & 16.1 & 98 \\
\hline $1 \mathrm{nM}$ & 22.7 & 14.2 & 85.6 \\
\hline $3 \mathrm{nM}$ & 27.2 & 17.3 & 87.2 \\
\hline $10 \mathrm{nM}$ & 33 & 21.2 & 99.7 \\
\hline $30 \mathrm{nM}$ & 46 & 28.3 & 129 \\
\hline $100 \mathrm{nM}$ & 47.4 & 33.2 & 145 \\
\hline $300 \mathrm{nM}$ & 42 & 31.3 & 137 \\
\hline $1000 \mathrm{nM}$ & 38 & 31.3 & 133 \\
\hline
\end{tabular}

Table 3.2 Geometric MFI measures of activation marker expression found on CD11 ${ }^{+}$ BM-DCs treated with the respective TLR agonists at varying concentrations displayed in Figure 3.2 and Figure 3.3. 


\subsubsection{Specific multiple TLR agonist combinations enhance BM-DC maturation status in-vitro}

The titration studies presented in the previous section provided useful guideline measures to assist with the appropriate concentration selections for utilization in subsequent investigations. Studies have also shown that selected dual TLR agonist combinations are capable of stimulating DCs to induce synergistic production of cytokines and enhanced $\mathrm{T}$ cell responses (Bagchi et al., 2007; Bohnenkamp et al., 2007; Napolitani et al., 2005; Pufnock et al., 2011; Warger et al., 2006; Whitmore et al., 2004; Zheng et al., 2008; Zhu et al., 2008). To assess the utility of using dual combinations of TLR agonists to enhance the maturation and activation status of BM-DCs, combinations of the selected TLR agonists MPL with Resiquimod, MPL with Pam3Cys, Resiquimod and CpG, Pam3Cys and CpG Resiquimod and Pam3Cys at the predetermined concentrations were used to stimulate cultures in-vitro, using the same experimental approach as in Figure 3.2 and 3.3 (see section 3.2.2).

The TLR agonist combination of MPL $100 \mathrm{ng} / \mathrm{ml}$ and Resiquimod 0.1ug/ml was shown to be fully capable of enhancing CD86 surface marker expression of treated BM-DCs beyond that achieved using these TLR agonists alone (Figure 3.4). When using MPL $100 \mathrm{ng} / \mathrm{ml}$ and Pam3Cys $100 \mathrm{ng} / \mathrm{ml}$ together in this particular experiment, a trend towards additive CD86 upregulation was seen at these TLR agonist concentrations, with repeated experiments statistically significant levels of CD86 levels were achieved (data not shown). The combinations of Pam3Cys $100 \mathrm{ng} / \mathrm{ml}$ with Resiquimod $0.1 \mathrm{ug} / \mathrm{ml}$, and CpG $100 \mathrm{nM}$ with Pam3Cys 100 ng/ml, Resiquimod 0.1 ug/ml with Pam3Cys 100 ng/ml were unable to significantly enhance the CD40 and CD86 expression levels above those observed when a single TLR agonist was used to stimulate BM-DC cultures (Figure 3.4). Although CD40 surface marker expression was upregulated after treatment of BM-DC cultures with the specified TLR agonists tested when compared with untreated BM-DC cultures, there was no statistically significant advantage to using TLR agonists alone or in combination (Figure 3.4). 
The TLR combinations of MPL $100 \mathrm{ng} / \mathrm{ml}$ with Resiquimod $0.1 \mathrm{ug} / \mathrm{ml}$, MPL $100 \mathrm{ng} / \mathrm{ml}$ with Pam3Cys 100 ng/ml, also induced IL-12p40 cytokine production by BM-DC cultures that was statistically higher when compared to utilising either TLR agonist alone 
A

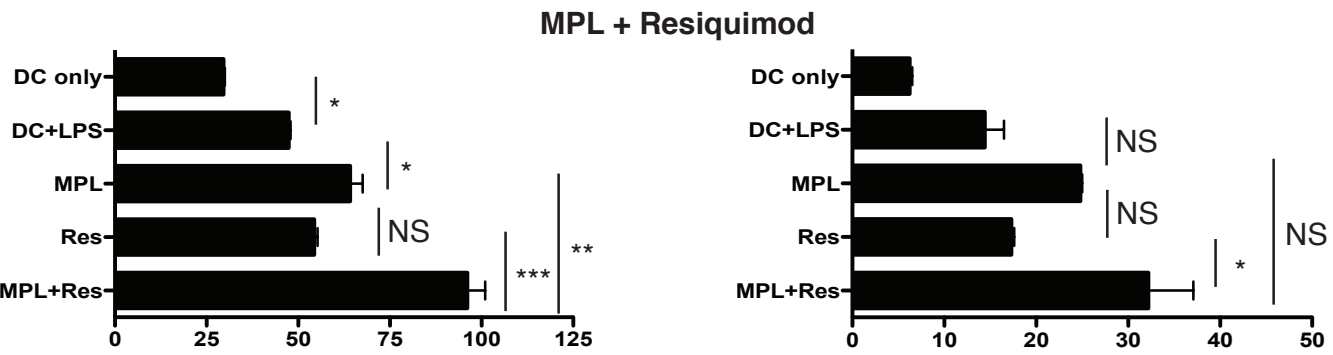

B

MPL + Pam3Cys
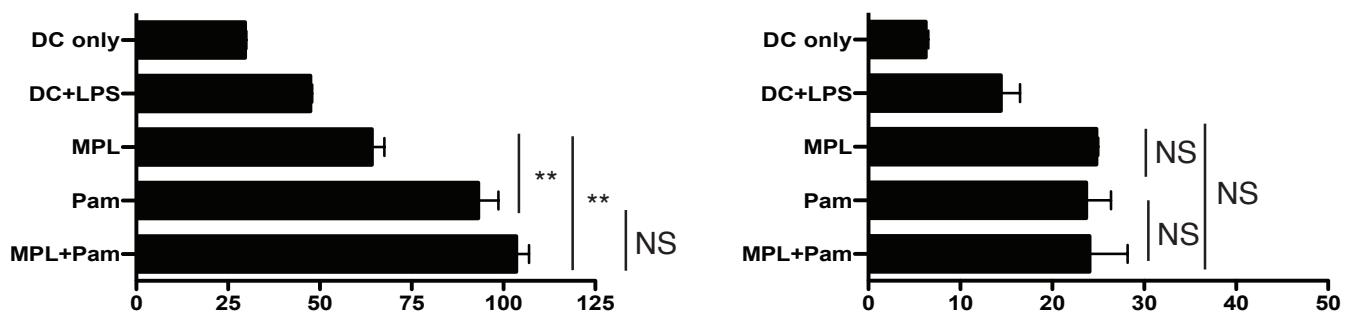

C

Resiquimod + Pam3Cys
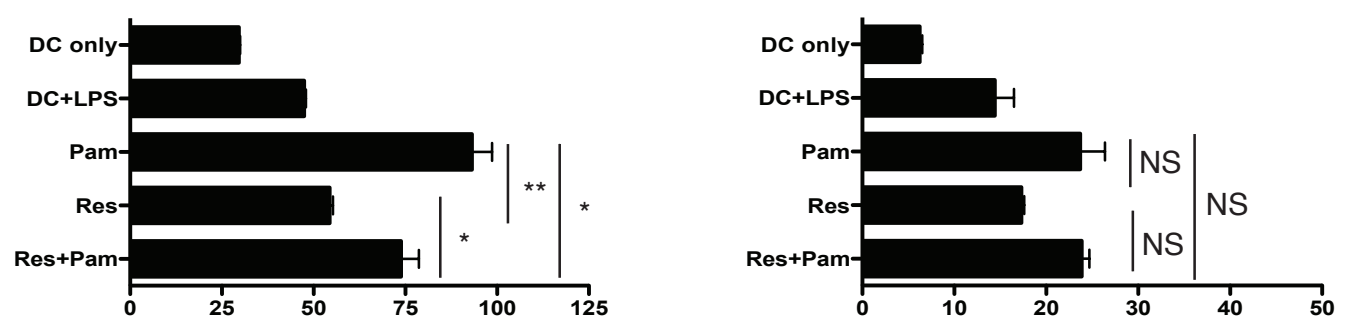

D

CpG + Pam3Cys
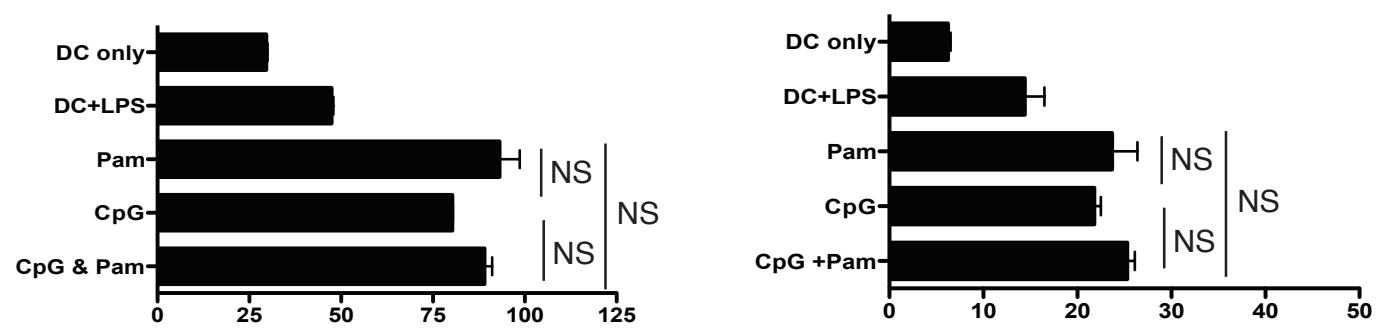

E

CpG + Resiquimod

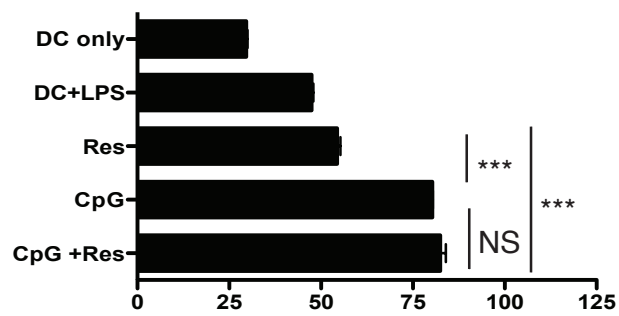

Geometric MFI CD86

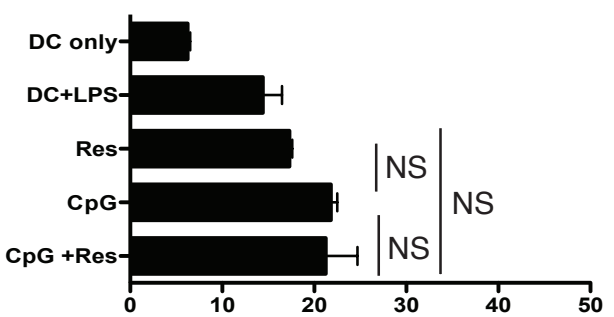

Geometric MFI CD40 
Figure 3.4 TLR agonist combinations influence BM-DC maturation in-vitro. Multiple TLR agonists were used alone and in combination with each other at various doses to stimulate BM-DC cultures on day 6. BM-DC cultures were generated as described previously (in section 3.2.1 and Figure 3.1). After $22 \mathrm{~h} \mathrm{BM-DCs}$ were harvested and flow cytometry used to determine CD86 and CD40 expression on day 7. TLR agonist combinations and concentrations shown are: (A) MPL $100 \mathrm{ng} / \mathrm{ml}$ and Resiquimod 0.1 $\mathrm{ug} / \mathrm{ml}$, (B) MPL $100 \mathrm{ng} / \mathrm{ml}$ and Pam3Cys 100ng/ml, (C) Resiquimod $0.1 \mathrm{ug} / \mathrm{ml}$ and Pam3Cys 100 ng/ml, (D) CpG $100 \mathrm{nM}$ and Pam3Cys $100 \mathrm{ng} / \mathrm{ml}$, (E) CpG $100 \mathrm{nM}$ Resiquimod 0.1ug/ml, (F) CpG $100 \mathrm{nM}$ and Resiquimod 0.1ug/ml. Bar graphs show mean values of the geometric MFI measures for CD86 and CD40 expression levels \pm SEM for each TLR combination group tested. Results shown are from one representative of 2-3 separate experiments with testing of individual samples carried out in triplicate. Statistical significance was determined using a 1-way ANOVA test and Tukey's post-test where * $\mathrm{p}=$ $0.05-0.01, * * \mathrm{p}=0.01-0.001, * * * \mathrm{p}<0.001, \mathrm{NS}=$ non significant.

(Figure 3.5). Additive IL-12p40 measures were not statistically significant when combinations of Pam3Cys $100 \mathrm{ng} / \mathrm{ml}$ with Resiquimod $0.1 \mathrm{ug} / \mathrm{ml}$, CpG $100 \mathrm{nM}$ and Pam3Cys 100 ng/ml, CpG $100 \mathrm{nM}$ and Resiquimod $0.1 \mathrm{ug} / \mathrm{ml}$ were utilised (Figure 3.5). At lower MPL concentrations, an additive effect for IL-6 production was observed after combining MPL 10 ng/ml with Resiquimod 0.1 ug/ml, MPL 10 ng/ml with Pam3Cys 100 $\mathrm{ng} / \mathrm{ml}$ (results not shown). A similar trend was seen in Figure 3.5 although statistical significance was not demonstrated. When MPL was combined with Resiquimod or with Pam3Cys, additive levels of TNF- $\alpha$ were generated which exceeded those produced when these TLR agonists were used alone (Figure 3.5).

It was the same TLR agonist combinations seen in Figure 3.4 that induced additive expression of CD86 and CD40 that also demonstrated additive induction of IL-12p40, IL-6 (at lower concentrations) and TNF- $\alpha$ cytokine production when used to treat BM-DCs invitro. These combinations were MPL with Resiquimod and MPL with Pam3Cys.

These results show that selected TLR agonist combinations are capable of inducing potent maturation and activation of BM-DCs in-vitro cultures. This occurred only for specific TLR agonist combinations and not for others, and at certain concentrations. MPL combined with Resiquimod and Pam3Cys consistently provided BM-DCs with potent TLR stimulation capable of enhancing maturation marker expression and cytokine release. 
Other TLR agonist combinations that were unsuccessful in this regard were CpG and Resiquimod, CpG and Pam3Cys.

These results provide some evidence to support the use of TLR agonist combinations for stimulating BM-DCs in-vitro. The consistent success achieved in BM-DC maturation after using MPL \& Pam3Cys and MPL with Resiquimod gave strong indication that these specific TLR agonist combinations would be worthwhile exploring further. Interestingly other TLR ligands did not work in combinations highlighting the differences in modes of signalling from the different receptors. These observations are consistent with work showing that a TLR 4 agonist combines well when used with a TLR 7/8 agonist (Napolitani et al., 2005; Warger et al., 2006). 


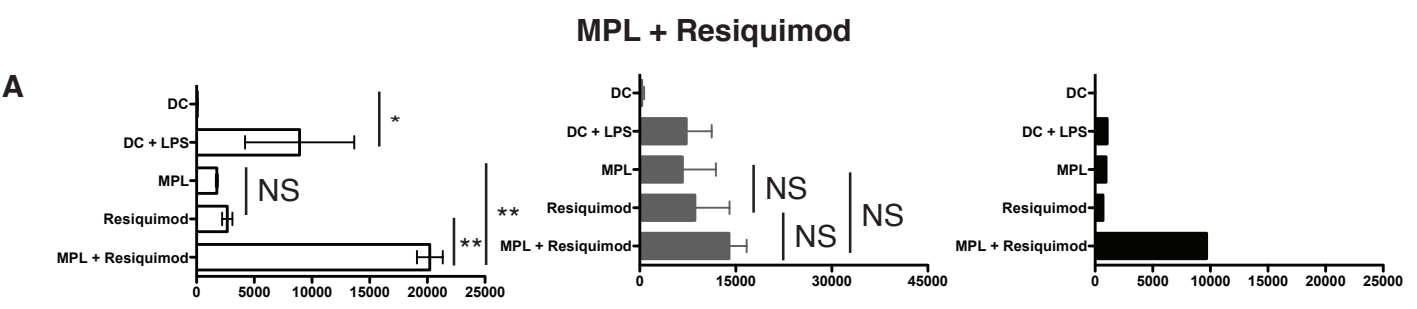

$$
M P L+\text { Pam3Cys }
$$

B
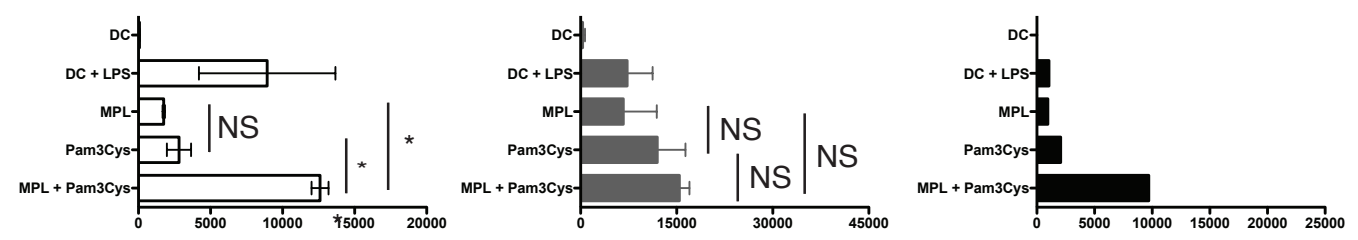

C

Pam3Cys + Resiquimod

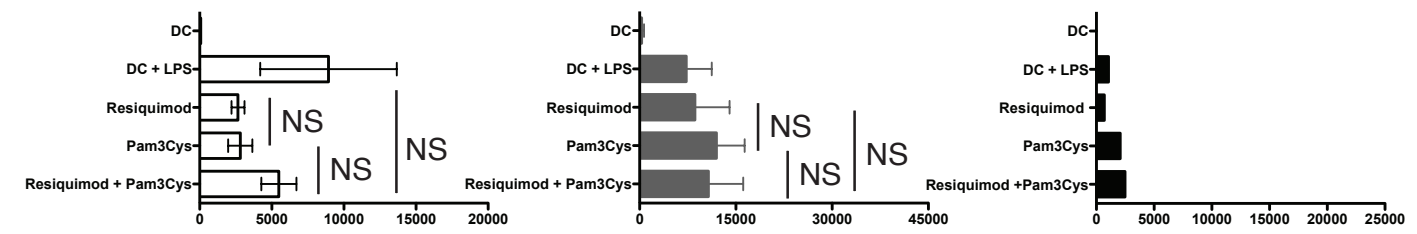

D

CpG + Pam3Cys
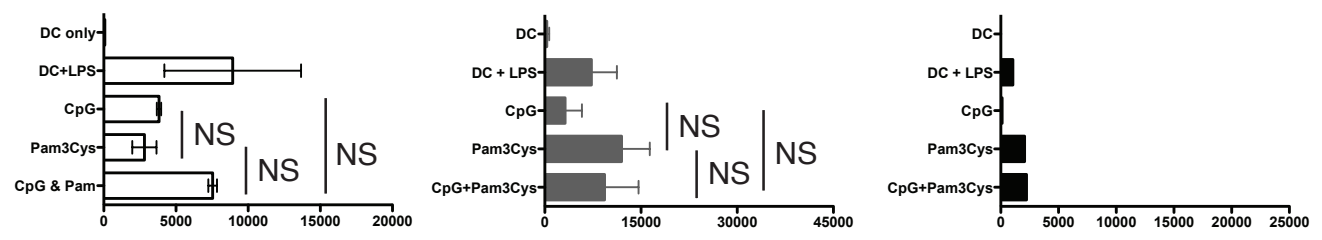

E

$$
\mathrm{CpG}+\text { Resiquimod }
$$
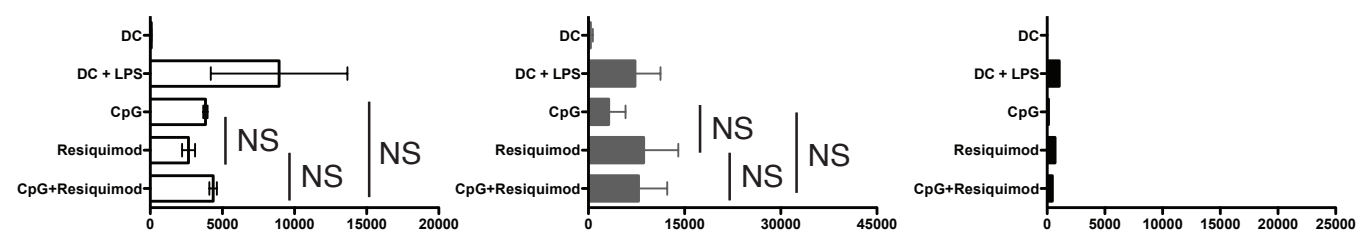

IL-12p40 pg/ml

IL-6 pg/ml

TNF-a pg/ml

Figure 3.5 Selected TLR agonist combinations influence cytokine production by BMDCs in-vitro. Multiple TLR agonists were used alone and in combination with each other at various doses to stimulate BM-DC cultures on day 6. BM-DC cultures were generated as described previously (in section 3.2.1 and Figure 3.1). After $22 \mathrm{~h}$ BM-DCs were harvested and culture supernatants collected on day 7 after stimulation with various TLR agonists alone and in combination with each other at different concentrations to quantify production of the following cytokines: IL-12p40, IL-6 and TNF- $\alpha$. Each sample was tested in 
triplicate with data pooled together from three individual samples for each cytokine tested. Cytokine levels were initially measured in $\mathrm{pg} / \mathrm{ml}$. Bar graphs show the specified cytokine levels after testing with the following TLR agonists alone and in combination with each other: (A) MPL $100 \mathrm{ng} / \mathrm{ml}$ and Resiquimod 0.1ug/ml, (B) MPL 100ng/ml and Pam3ys 100 ng/ml, (C) Pam3Cys 100ng/ml and Resiquimod 0.1ug/ml, (D) CpG $100 \mathrm{nM}$ and Pam3Cys $100 \mathrm{ng} / \mathrm{ml}$, (E) CpG $100 \mathrm{nM}$ and Resiquimod $0.1 \mathrm{ug} / \mathrm{ml}$. Results shown for IL-12p40 (1 ${ }^{\text {st }}$ column) and IL-6 ( $2^{\text {nd }}$ column) are from 2 separate experiments with testing of individual samples carried out in triplicate, while results for TNF- $\alpha$ ( $3^{\text {rd }}$ column) was generated by three individual pooled samples from 1 experiment. Statistical significance was determined using a 1-way ANOVA test and Tukey's post-test where *p $=0.05-0.01,{ }^{* *} \mathrm{p}=0.01-$ $0.001, * * * \mathrm{p}<0.001, \mathrm{NS}=$ non significant.

\subsubsection{The influence of CD40 stimulation on BM-DC function after TLR agonist stimulation in-vitro}

The generation of effective CTL responses involves CD40 signalling. Work published by Napolitani et al (2005) showed that exposure to specific dual TLR agonist combinations increased DC functionality and could be further enhanced after treatment with a CD40 antibody agonist. Other studies reported that further amplification of BM-DC maturation and activation status could also be provided by CD40L stimulation (Bennett, 1998; Ridge, 1998; Schoenberger et al., 1998; van Mierlo et al., 2002). To test the capacity of BM-DCs to respond to CD40 ligation after single and dual TLR agonist stimulation, CD40L expressing fibroblast cell lines were co-cultured with BM-DCs and cytokine production measured in culture supernatants.

BM-DCs were prepared as outlined in 3.2.2. TLR agonists alone and in combination with each other were added to BM-DC cultures already containing CD40L expressing NIH3T3 fibroblast cell lines. Culture supernatants were removed after 6 and $22 \mathrm{~h}$ time points. These were examined for IL-12p40, IL-12p70 and IL-6 production and compared between single and combination TLR agonist treated BM-DCs.

In the absence of CD40L stimulation, a trend indicating increased levels of the cytokines IL-12p70, IL-12p40 and IL-6 were detected at $22 \mathrm{~h}$ compared with $6 \mathrm{~h}$ after treatment with the selected TLR agonists alone and in combination (Figure 3.6, Figure 3.7 and Figure 3.8). At $22 \mathrm{~h}$ for all TLRs tested alone and in combination, elevated levels of IL-12p70 
were detected in the presence of CD40L stimulation when compared to levels seen at $6 \mathrm{~h}$ and was a statistically significant observation (Figure 3.6, Figure 3.7 and Figure 3.8). CD40L stimulation appeared to induce the early production of IL-12p70 for all single and TLR combination treated BM-DCs at $6 \mathrm{~h}$, however this trend did not achieve statistical significance for MPL with Resiquimod combination, but did so for the MPL with Pam3Cys and CpG with Resiquimod combinations (Figure 3.6, Figure 3.7 and Figure 3.8).

By $22 \mathrm{~h}, \mathrm{IL}-12 \mathrm{p} 70$ and IL-12p40 production by non-CD40 L treated cultures had exceeded cytokine levels seen with CD40L stimulation at $6 \mathrm{~h}$, with maximal IL-12p70 production was detected at $22 \mathrm{~h}$ in the presence of CD40L stimulation. The non-TLR treated CD40L stimulated BM-DCs were induced to produce IL-12p40 levels similar to TLR treated BMDCs (Figure 3.6, Figure 3.7 and Figure 3.8).

When BM-DCs were treated with MPL and Resiquimod, MPL and Pam3Cys combinations, the presence of CD40L stimulation caused the early and potent release of bioactive IL-12p70 at $6 \mathrm{~h}$ (Figure 3.6 and Figure 3.7). This additive advantage however is lost by $22 \mathrm{~h}$ when single TLR agonist treated BM-DCs were being released in comparable quantities (Figure 3.6 Figure 3.7). Usage of the MPL and Resiquimod combination to treat BM-DCs induced rapid and potent IL-12p70 production at $6 \mathrm{~h}$, which exceeded IL-12p70 production by MPL and Pam3Cys, $\mathrm{CpG}$ and Resiquimod combinations (Figure 3.6, Figure 3.7 and Figure 3.8).

In summary, more IL-12p70 was produced over time for all CD40L treated groups (Figure 3.6, Figure 3.7, Figure 3.8). An early trend towards increased IL-12p70 levels was seen between non-treated and all other CD40L treated groups at $6 \mathrm{~h}$, although statistical significance was achieved for MPL with Resiquimod and $\mathrm{CpG}$ with Resiquimod combinations (Figure 3.6, Figure 3.7, Figure 3.8). By 22 h, all TLR treated CD40L stimulated groups had produced statistically significant higher levels of IL-12p70 than the non-CD40L treated TLR stimulated groups (Figure 3.6, Figure 3.7, Figure 3.8). There appeared to be no added advantage regarding cytokine production of IL-12p70 when utilizing a dual TLR combination to treat BM-DCs in the presence of CD40L stimulation for the MPL with Resiquimod and MPL with Pam3Cys combinations (Figure 3.6, Figure 3.7). There was however a statistically relevant outcome evident for the $\mathrm{CpG}$ with 
Resiquimod combination indicating that added advantage could be achieved using a dual TLR combination to stimulated BM-DCs in the presence of CD40L stimulation (Figure $3.8)$.

CD40L also appears to induce early IL-6 and IL-12p40 production at $6 \mathrm{~h}$ for all combinations tested, although this trend was not statistically significant at $6 \mathrm{~h}$, or at $22 \mathrm{~h}$ within this assay with cytokine levels varying for IL-12p40 and IL-6 measures particularly at the $22 \mathrm{~h}$ timepoint (Figure 3.6, Figure 3.7, Figure 3.8).

In summary, CD40L stimulation induces IL-12p70 production with TLR stimulation sustaining production. The impact of combining MPL and Resiquimod is the induction of early IL-12p70 manufacture. CD40L stimulation also showed a tendency to enhance IL-6, particularly in combination with MPL and Resiquimod but this production was not sustained relative to DCs treated with the TLR agonists alone. When using MPL together with Pam3Cys, CD40L stimulation induced early and sustained IL-12p70 production again with TLR stimulation contributing to this response, although the combination of MPL and Pam3Cys was not superior to each TLR agonists alone in this regard. As seen previously with MPL and Resiquimod, the IL-6 production generated after lengthy CD40L stimulation was not sustained when compared to early IL-6 production and that compared with cultures without CD40L stimulation.

The CpG and Resiquimod TLR agonists were a combination that had been identified in previous studies as being ineffective of improving BM-DC activation above that achieved when using either TLR agonist alone. The results shown in Figure 3.8 however show that this inefficiency can be overcome by the incorporation of CD40 stimulation into the culture preparation, which was seen to boost IL-12p70 and IL-12p40 production levels at the $6 \mathrm{~h}$ and $22 \mathrm{~h}$ time points.

CD40L stimulation successfully induced early IL-12p70 and IL-6 cytokine release and sustained IL-12p70 production. There was a trend towards the early release of IL-12p40 and IL- 6 being enhanced by the TLR combinations selected as effective on the previous section. The TLR combinations selected as being ineffective by previous results did still 
effectively combine with CD40L stimulation, but the early release of IL-12p40 and IL-6 was not as evident.

These results show that the provision of CD40L stimulation to TLR treated BM-DCs potently increases cytokine production as measured in-vitro. This increase was such that previously inactive TLR agonist combinations became more efficient at stimulating BMDC function. Thus the incorporation of both CD40 signalling in combination with TLR stimulation provides a means of improving BM-DC function in-vitro and an opportunity to investigate this dual action in-vivo. 
A

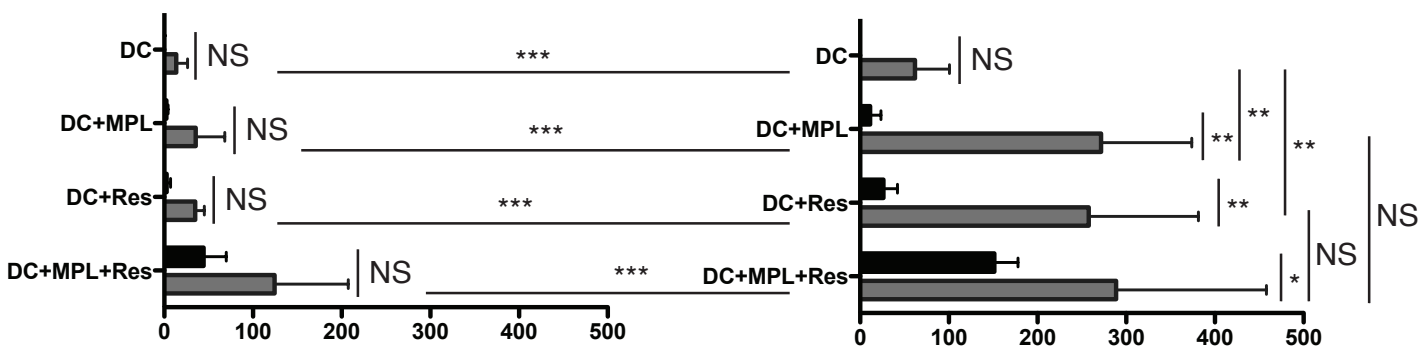

IL-12p40

B
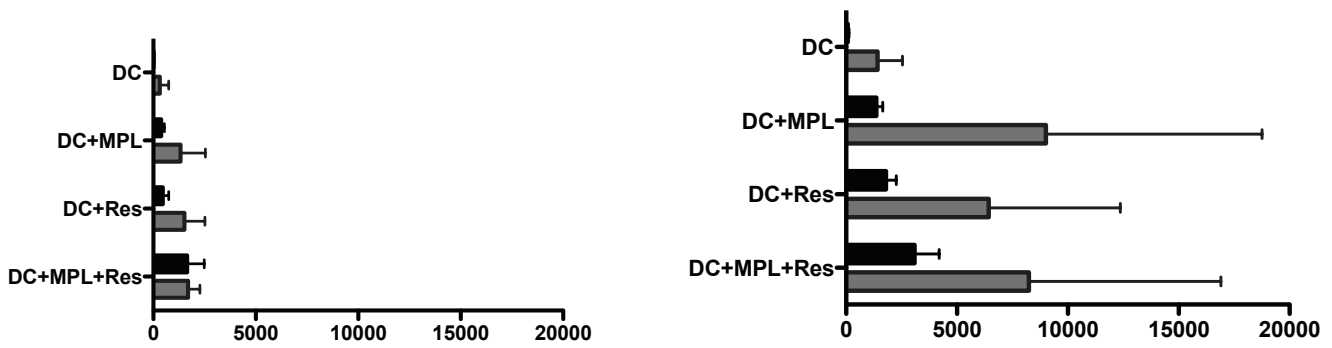

IL-6

C
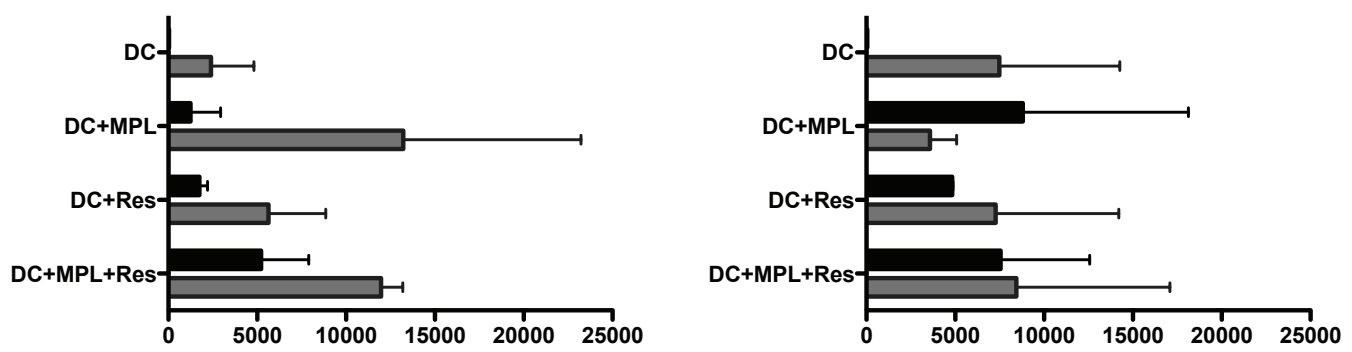

$\mathrm{pg} / \mathrm{ml}$

BM-DC

$\mathrm{pg} / \mathrm{ml}$

BM-DC + CD40L

Figure 3.6 CD40L increases in-vitro cytokine production of IL-12p70 by MPL and Resiquimod treated BM-DCs over time. BM-DC cells were co-cultured with CD40L expressing NIH3T3 fibroblastic cell lines and treated with MPL alone and in combination with Resiquimod. BM-DC cultures were generated as described previously (in section 3.2.1 and Figure 3.1). BM-DC culture supernatants were harvested at $6 \mathrm{~h}$ and $22 \mathrm{~h}$ time points to determine (A) IL-12p70, (B) IL-12p40 and (C) IL-6 levels in the absence or presence of CD40L support. Cytokine levels were measured in $\mathrm{pg} / \mathrm{ml}$. Bar graphs show pooled data from triplicate samples combined from 2 separate experiments. The specified cytokine levels were measured after treatment with MPL $100 \mathrm{ng} / \mathrm{ml}$ and Resiquimod 0.1 $\mathrm{ug} / \mathrm{ml}$ alone and in combination with each other. Statistical significance was determined using a 2-way ANOVA and Bonferroni's post-test where $* p=0.05-0.01,{ }^{*} p=0.01-$ $0.001, * * * p<0.001, \mathrm{NS}=$ non significant. 


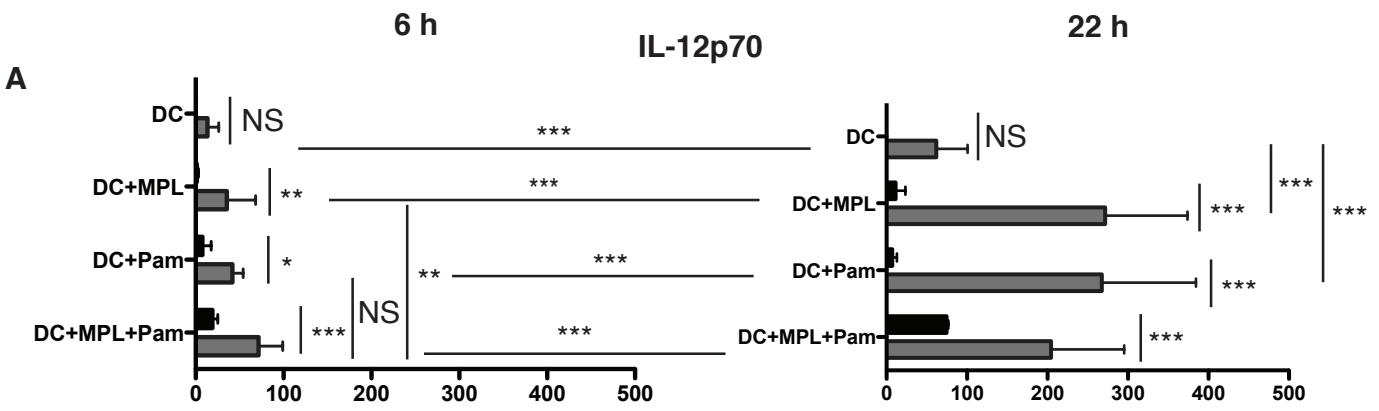

IL-12p40

B
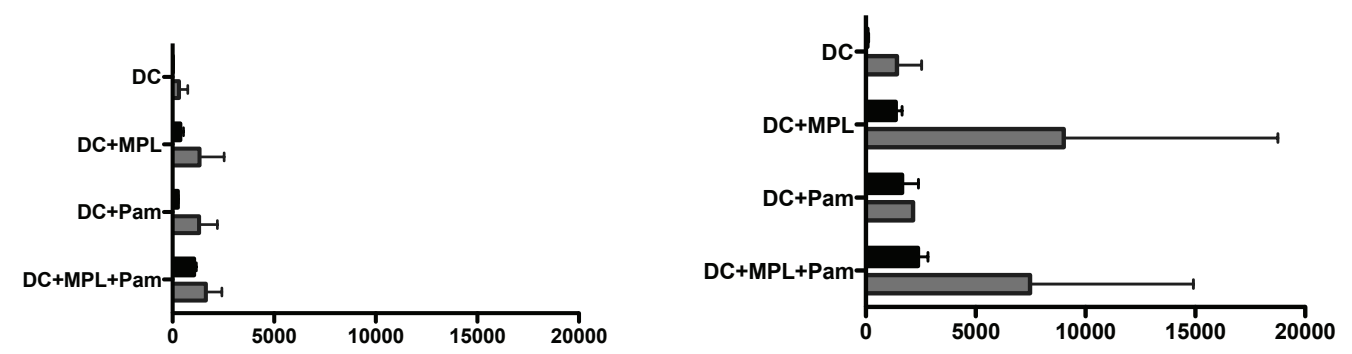

IL-6

C
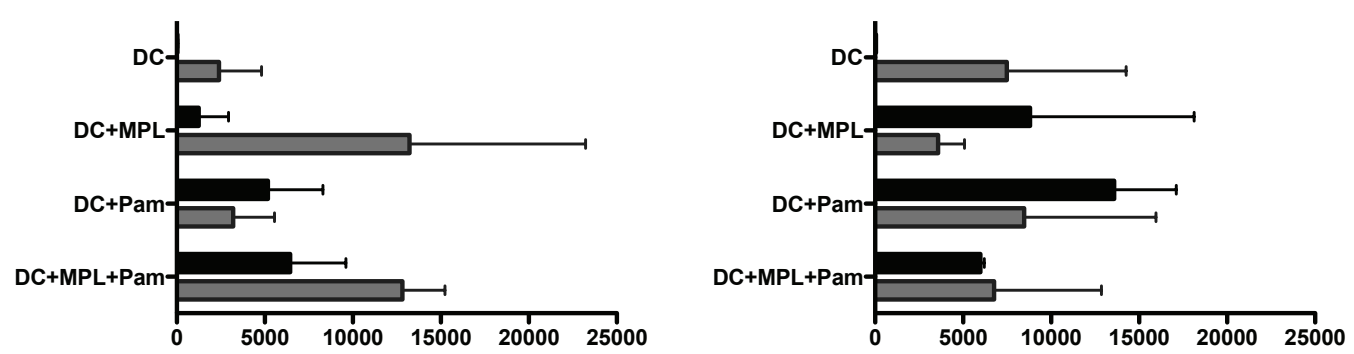

$\mathrm{pg} / \mathrm{ml}$

$\mathrm{pg} / \mathrm{ml}$

BM-DC

$\mathrm{BM}-\mathrm{DC}+\mathrm{CD} 40 \mathrm{~L}$

Figure 3.7 CD40L increases in-vitro cytokine production of IL-12p70 by MPL and Pam3Cys treated BM-DCs. BM-DC cells were co-cultured with CD40L expressing NIH3T3 fibroblastic cell lines and treated with MPL and Pam3Cys alone and in combination with each other. BM-DC cultures were generated as described previously (in section 3.2.1 and Figure 3.1). BM-DC culture supernatants were harvested at $6 \mathrm{~h}$ and $22 \mathrm{~h}$ time points to determine (A) IL-12p70, (B) IL-12p40 and (C) IL-6 levels in the absence or presence of CD40L support. Cytokine levels were measured in $\mathrm{pg} / \mathrm{ml}$. Bar graphs show pooled data from triplicate samples combined from 2 separate experiments. The specified cytokine levels were measured after treatment with MPL $100 \mathrm{ng} / \mathrm{ml}$ and Pam3Cys $100 \mathrm{ng} / \mathrm{ml}$ alone and in combination with each other. Statistical significance was determined using a 2-way ANOVA and Bonferroni's post-test where $* p=0.05-0.01, * * p$ $=0.01-0.001, * * * p<0.001, \mathrm{NS}=$ non significant. 


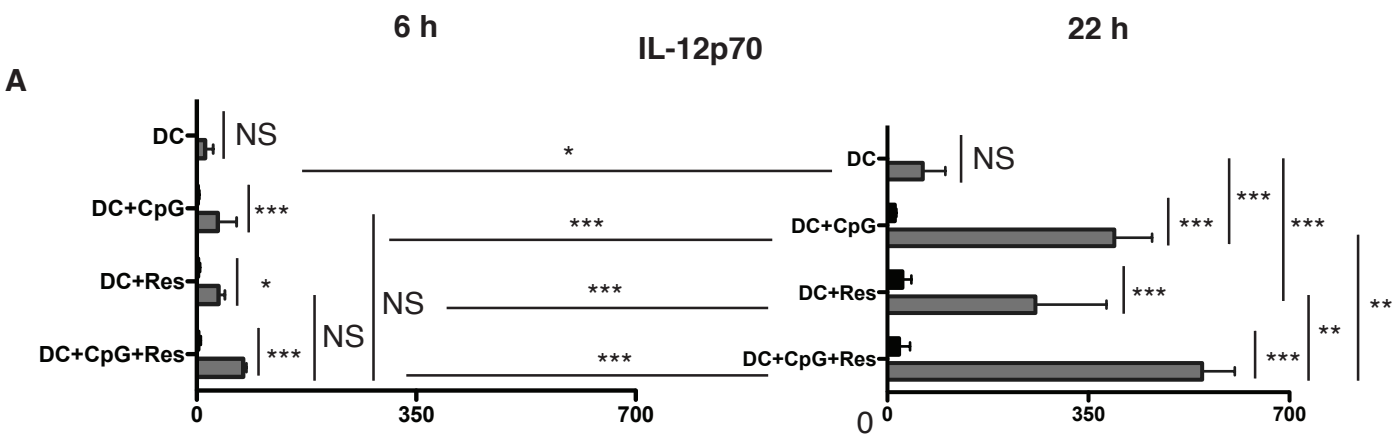

IL-12p40

B
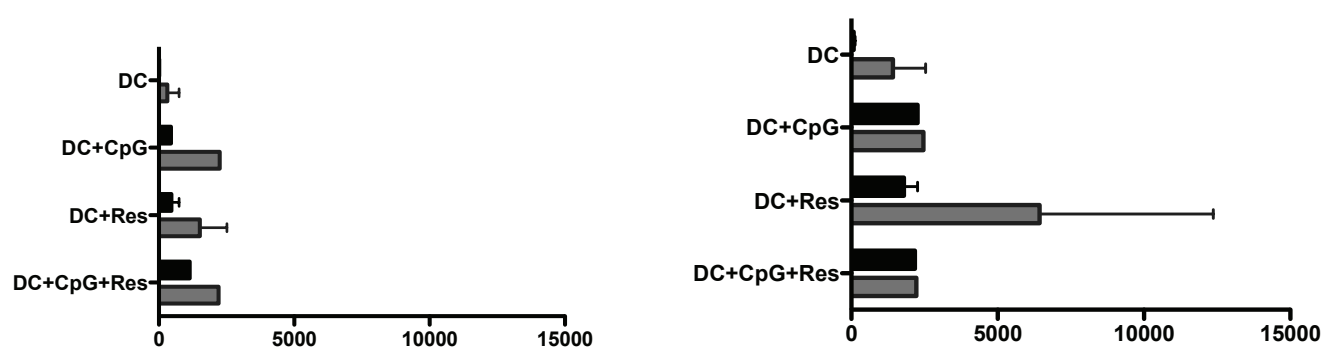

IL-6

C
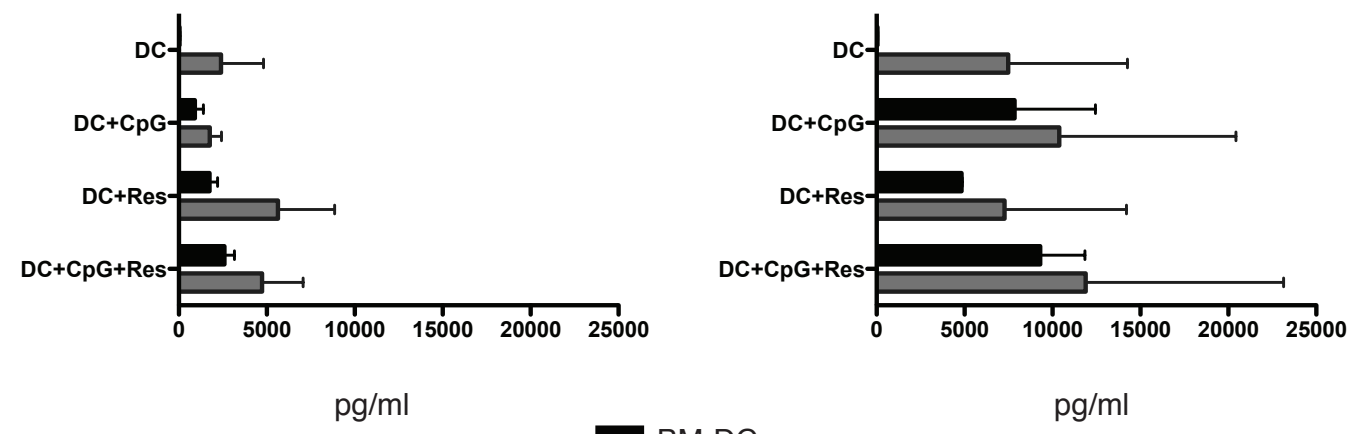

$B M-D C$

$\mathrm{pg} / \mathrm{ml}$

BM-DC + CD40L

Figure 3.8 CD40L increases in-vitro cytokine production of IL-12p70 by CpG and Resiquimod treated BM-DCs. BM-DC cells were co-cultured with CD40L expressing NIH3T3 fibroblastic cell lines and treated with $\mathrm{CpG}$ alone and in combination with Resiquimod. BM-DC cultures were generated as described previously (in section 3.2.1 and Figure 3.1). BM-DC culture supernatants were harvested at $6 \mathrm{~h}$ and $22 \mathrm{~h}$ time points to determine (A) IL-12p70, (B) IL-12p40 and (C) IL-6 levels in the absence or presence of CD40L support. Cytokine levels were measured in $\mathrm{pg} / \mathrm{ml}$. Bar graphs show pooled data from triplicate samples combined from 2 separate experiments. The specified cytokine levels were measured after treatment with $\mathrm{CpG} 100 \mathrm{nM}$ and Resiquimod $0.1 \mathrm{ug} / \mathrm{ml}$ alone and in combination with each other. Statistical significance was determined using a 2-way ANOVA and Bonferroni's post-test where $* \mathrm{p}=0.05-0.01,{ }^{*} \mathrm{p}=0.01-0.001,{ }^{* * *} \mathrm{p}<$ $0.001, \mathrm{NS}=$ non significant. 


\subsubsection{TLRs alone and in combination stimulate peptide loaded BM- DCs to induce superior $T$ cell responses in-vitro}

Previous studies had shown that stimulation of DCs with selected TLR agonist alone and in combination were capable of inducing DCs to generate superior $\mathrm{CD} 4^{+}$and $\mathrm{CD} 8^{+} \mathrm{T}$ cell responses in mice and humans (Lore et al., 2003; Pufnock et al., 2011; Warger et al., 2006). The TLR agonist combinations of MPL with Resiquimod and MPL and Pam3Cys were utilized in further investigations as they were deemed effective in-vitro BM-DC stimulators. The ability of TLR treated BM-DCs to influence T cell function was examined in response to using TLR agonists alone and in combination with each other.

The T cell stimulation capacity of TLR treated BM-DCs was assayed by examining ${ }^{3} \mathrm{H}-$ thymidine incorporation by proliferating OVA specific T cells in-vitro. Based on previous work identifying the successful BM-DC maturing TLR agonist combinations, MPL, Resiquimod and Pam3Cys were tested individually and in combination together, with stimulation with LPS-used as a positive control. Both protein and peptide antigens were used in separate experiments, the latter to eliminate any confounding factors associated with protein antigen processing.

BM-DC cultures were generated as described in Section 3.2.2 and exposed to TLR agonists alone and in combination with each other for $22 \mathrm{~h}$ of culture. BM-DCs were loaded with SIINFEKL peptide for the last $2 \mathrm{~h}$ of culture with TLR agonists. LN cells containing OVA-specific $\mathrm{CD} 8^{+} \mathrm{T}$ cells were then isolated from OTI donors and co cultured with the TLR treated BM-DCs for $48 \mathrm{~h}$ after which ${ }^{3} \mathrm{H}$-thymidine was added to wells for a final $16 \mathrm{~h}$. Incorporation of ${ }^{3} \mathrm{H}$-thymidine was used as a measure of $\mathrm{T}$ cell proliferation and direct correlate of the peptide antigen presentation capacity of the TLR treated BM-DCs. 

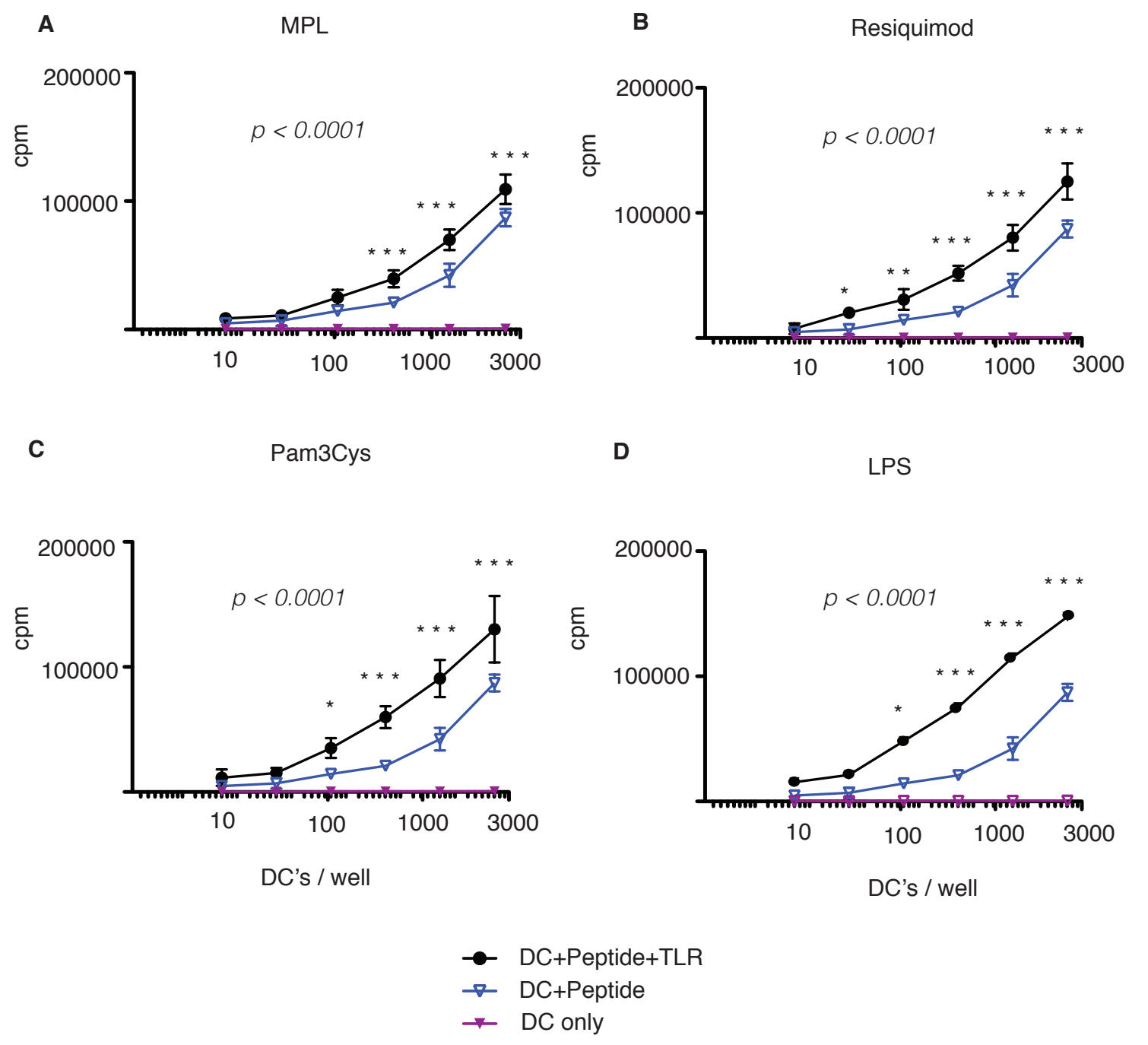

Figure 3.9 TLR stimulation of BM-DCs in-vitro improves peptide antigen presentation capability. BM-DC cultures were generated as described previously (in section 3.2.1 and Figure 3.1). Specific doses for each TLR agonist were used to stimulate BM-DC cultures on day 6 for $22 \mathrm{~h}$. Following TLR stimulation for $22 \mathrm{~h}$, BM-DCs were loaded with SIINFEKL peptide antigen on Day 7. After washing to remove excess antigen, BM-DCs were then co-cultured with OVA specific $\mathrm{CD}^{+} \mathrm{T}$ cell populations for a further $48 \mathrm{~h} .{ }^{3} \mathrm{H}$-thymidine was then added to wells for further $18 \mathrm{~h}$ and its incorporation into $\mathrm{CD} 8^{+} \mathrm{T}$ cells utilised to determine a measure of $\mathrm{T}$ cell proliferation. Graphs show measures of ${ }^{3} \mathrm{H}$-thymidine uptake by TLR simulated BM-DCs loaded with peptide antigen and cultured with titrated BM-DC numbers per well. The TLR agonists were used at previously titrated doses and were tested at the following concentrations (A) MPL $100 \mathrm{ng} / \mathrm{ml}$, (B) Resiquimod $0.1 \mathrm{ug} / \mathrm{ml}$ (C) Pam3Cys $100 \mathrm{ng} / \mathrm{ml}$ and (D) LPS $100 \mathrm{ng} / \mathrm{ml}$. Results for LPS $100 \mathrm{ng} / \mathrm{ml}$ treated BM-DCs, BM-DC only and BM-DC loaded with peptide only control samples are shown in (D). Results shown are from one representative of 2 separate experiments with testing of individual samples carried out in triplicate. Statistical significance was determined using a 2-way ANOVA and Bonferroni's post-test where ${ }^{*} \mathrm{p}=$ $0.05-0.01, * * \mathrm{p}=0.01-0.001, * * * \mathrm{p}<0.001, \mathrm{NS}=$ non significant. 
MPL, Pam3Cys and Resiquimod treated BM-DCs induced enhanced T cell proliferation of OVA specific $\mathrm{T}$ cell populations and modest peptide antigen presentation capacity for the specified TLR agonist concentrations tested (Figure 3.9). A separate study addressed the effect on BM-DC antigen presentation ability when TLR agonists were used in combination with each other (Figure 3.10). The MPL and Resiquimod combination induced more efficient stimulation of OVA specific $\mathrm{T}$ cells populations relative to the individual TLRs (Table 3.3). The stimulatory capacity of BM-DCs treated with the MPL and Pam3Cys, or Resiquimod was therefore improved by the combined stimuli.

In summary, MPL with Resiquimod, MPL with Pam3Cys and Resiquimod with Pam3Cys TLR agonist combinations increased the peptide antigen presentation capability of treated BM-DCs compared with lone TLR agonist treatment when $\mathrm{T}$ cell proliferation was measured in-vitro. 


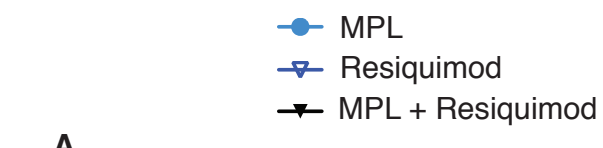

\section{A}

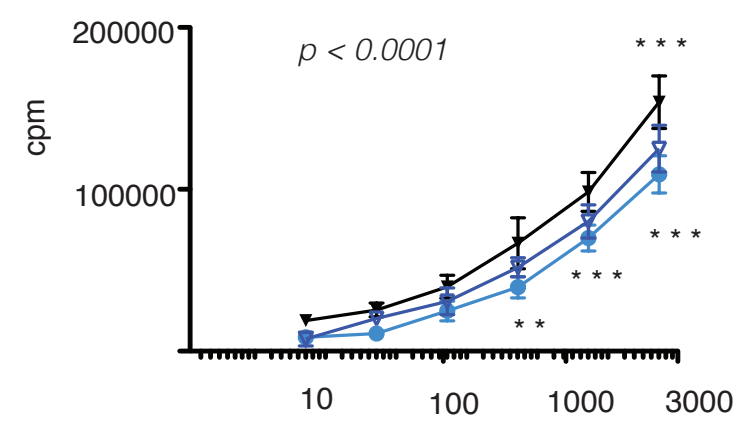

C

$$
\begin{aligned}
& \rightarrow \text { Resiquimod } \\
& \rightarrow \text { Pam3Cys } \\
& \rightarrow \text { Resiquimod + Pam3Cys }
\end{aligned}
$$

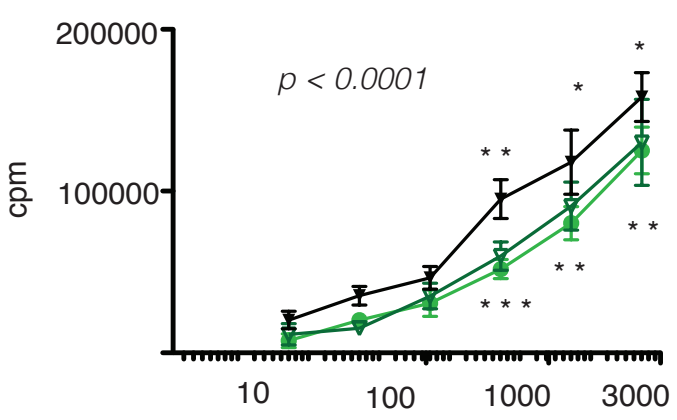

DC's / well

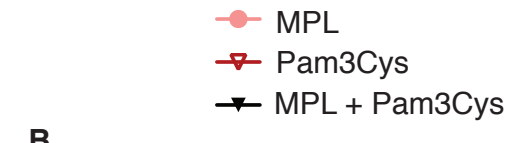

B

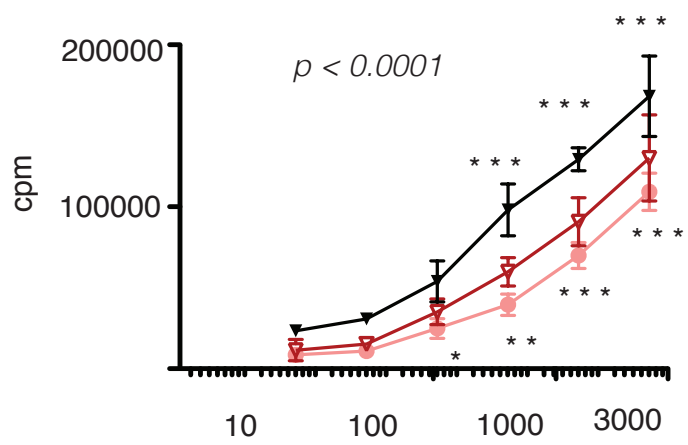

D
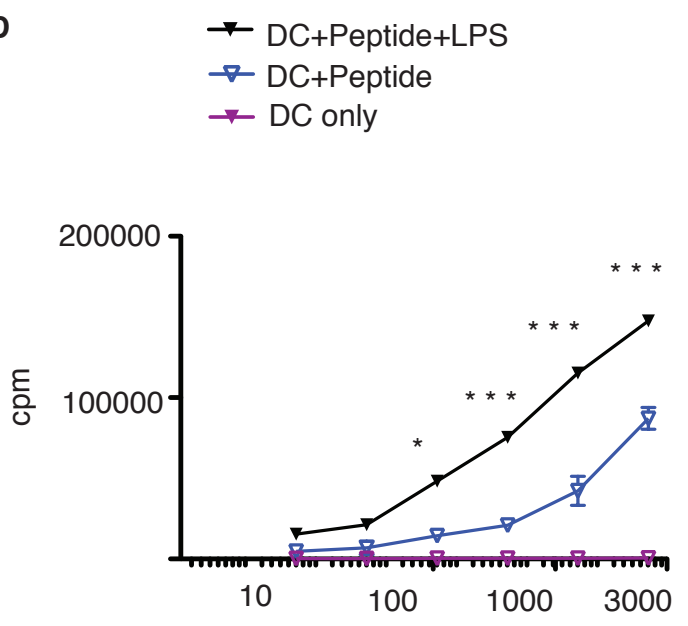

DC's / well

Figure 3.10 Combined TLR agonists enhance peptide antigen presentation capability by BM-DCs in-vitro. Selected TLR agonist combinations were used at specific doses to stimulate day 6 BM-DC cultures for $22 \mathrm{~h}$. BM-DC cultures were generated as described previously (in section 3.2.1 and Figure 3.1). Following TLR stimulation for $22 \mathrm{~h}$, BM-DCs were loaded with SIINFEKL peptide antigen on Day 7. After washing to remove excess antigen, BM-DCs were then co-cultured with OVA specific $\mathrm{CD} 8^{+} \mathrm{T}$ cell populations for a further $48 \mathrm{~h} .{ }^{3} \mathrm{H}$-thymidine was then added to wells for further $18 \mathrm{~h}$ and its incorporation into $\mathrm{CD} 8^{+} \mathrm{T}$ cells utilised to determine a measure of $\mathrm{T}$ cell proliferation. Graphs show measures of ${ }^{3} \mathrm{H}$-thymidine uptake by TLR simulated BM-DCs loaded with peptide antigen and cultured with titrated BM-DC numbers per well. Tested TLR agonist combinations were (A) MPL $100 \mathrm{ng} / \mathrm{ml}$ and Resiquimod $0.1 \mathrm{ug} / \mathrm{ml}$, (B) MPL $100 \mathrm{ng} / \mathrm{ml}$ and Pam3Cys 100ng/ml, (C) Pam3Cys $100 \mathrm{ng} / \mathrm{ml}$ and Resiquimod $0.1 \mathrm{ug} / \mathrm{ml}$. Results for LPS $100 \mathrm{ng} / \mathrm{ml}$ treated BM-DCs, BM-DC only and BM-DC loaded with peptide only control samples are shown in (D). Results shown are from one representative of 2 separate experiments with testing of individual samples carried out in triplicate. Statistical significance was determined using a 2-way ANOVA and Bonferroni's post-test where $* p=0.05-0.01, * * p$ $=0.01-0.001, * * * \mathrm{p}<0.001, \mathrm{NS}=$ not significant. 


\subsubsection{Specific TLR agonists alone and in combination promote antigen presentation by protein loaded BM-DCs in-vitro.}

As TLR stimulation has been shown to affect many facets of DC function including antigen acquisition and processing, in-vitro $\mathrm{T}$ cell proliferation studies (as described in 3.2.4) were employed to determine the stimulatory capacity of OVA loaded BM-DCs treated with either single or multiple TLR agonist combinations.

BM-DC cultures were generated as described in 3.2.2 and co-cultured with OVA protein and exposed to TLR agonists alone and in combination with each other for $22 \mathrm{~h}$ of culture. OVA-specific $\mathrm{CD} 8^{+} \mathrm{T}$ cells were then isolated from OTI donors as previously described and co cultured with the TLR treated BM-DCs for $48 \mathrm{~h}$ after which ${ }^{3} \mathrm{H}$-thymidine was added to wells for final $16 \mathrm{~h}$ culture.

Protein processing and presentation was improved when BM-DCs were treated with some individual TLR agonists at the specified concentrations (Figure 3.11). Although this effect was not as marked for the MPL treated BM-DC cultures, when compared with the MPL and Resiquimod combination treated cultures (Figure 3.11), the combined TLR agonists were still capable of enhancing proliferation of OVA specific $\mathrm{CD}^{+} \mathrm{T}$ cells in the assay. The MPL and Pam3Cys combination was also able to improve the OVA specific CD $8^{+} \mathrm{T}$ cell proliferation and therefore protein antigen processing and presentation over the BMDCs treated with MPL and Pam3Cys alone. The Pam3Cys with Resiquimod TLR agonist combination also showed antigen processing and presentation capacity of both peptide and protein antigen. This was in contrast to the failure of this TLR agonist combination to induce significant maturation marker upregulation of CD86 and CD40 in BM-DC cultures as seen in Figure 3.4, or to induce enhanced IL-12p40, IL-6 or TNF-a production as seen in Figure 3.5 .

MPL with Resiquimod, MPL with Pam3Cys combinations were able to improve the antigen processing and presentation capacity of both peptide and protein antigen loaded BM-DCs to OVA specific $\mathrm{CD}^{+} \mathrm{T}$ cells in-vitro. 

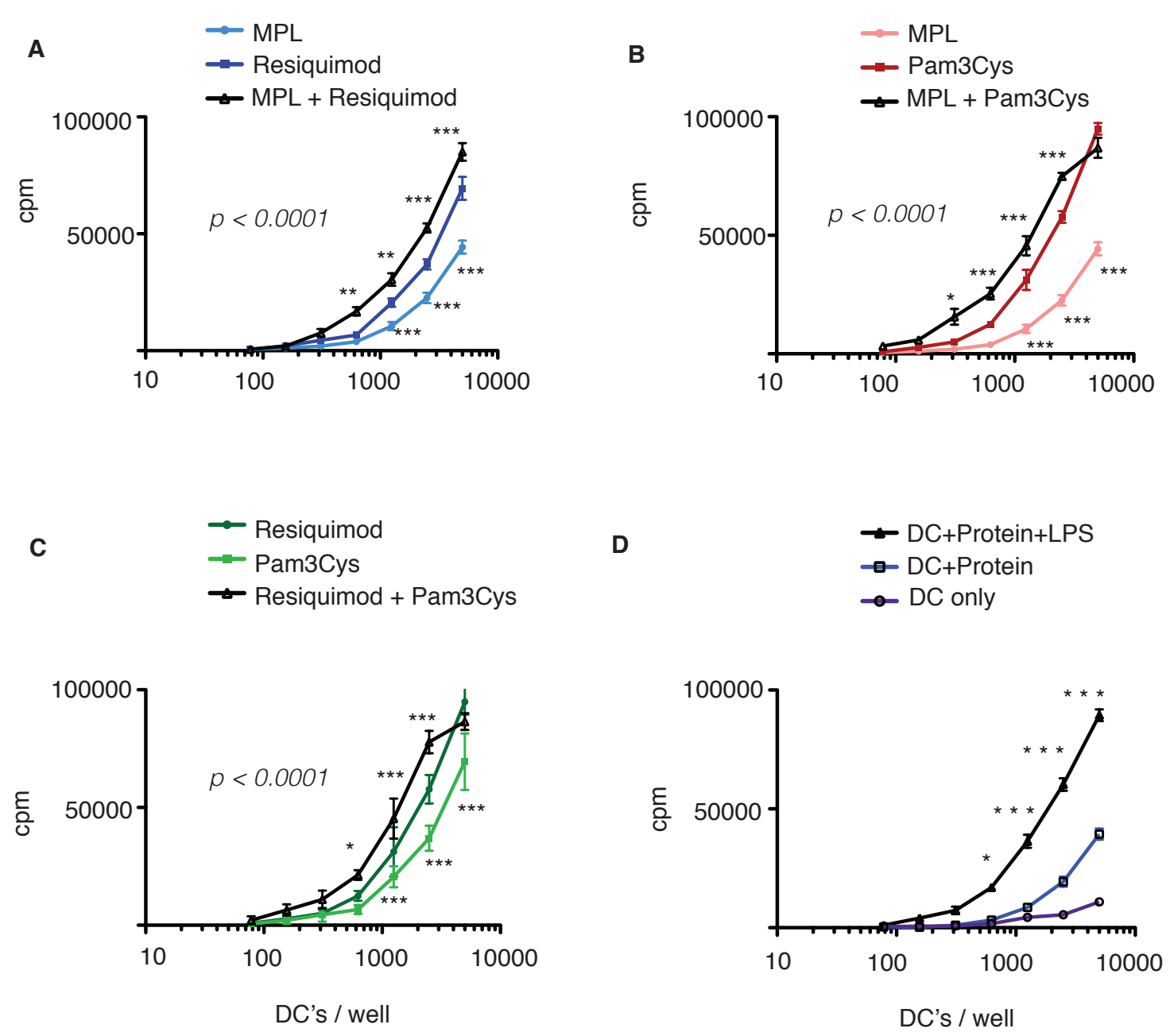

Figure 3.11 Selected TLR agonist combinations enhance both the antigen processing and presentation capabilities of BM-DCs in-vitro. Selected TLR agonist combinations were used at specific doses to stimulate day 6 BM-DC cultures for $22 \mathrm{~h}$. BM-DC cultures were generated as described previously (in section 3.2.1 and Figure 3.1). BM-DC cultures were also co-cultured with OVA protein antigen for $22 \mathrm{~h}$ on Day 6. After washing to remove excess antigen on Day 7, BM-DCs were then co-cultured with OVA specific CD8 ${ }^{+}$ $\mathrm{T}$ cell populations for a further $48 \mathrm{~h} .{ }^{3} \mathrm{H}$-thymidine was then added to wells for further $18 \mathrm{~h}$ and its incorporation into $\mathrm{CD}^{+} \mathrm{T}$ cells utilised to determine a measure of $\mathrm{T}$ cell proliferation. Graphs show measures of ${ }^{3} \mathrm{H}$-thymidine uptake by TLR simulated BM-DCs loaded with peptide antigen and cultured with titrated BM-DC numbers per well. Tested TLR agonist combinations were (A) MPL $100 \mathrm{ng} / \mathrm{ml}$ and Resiquimod $0.1 \mathrm{ug} / \mathrm{ml}$, (B) MPL $100 \mathrm{ng} / \mathrm{ml}$ and Pam3Cys 100ng/ml, (C) Pam3Cys $100 \mathrm{ng} / \mathrm{ml}$ and Resiquimod $0.1 \mathrm{ug} / \mathrm{ml}$. Results for LPS $100 \mathrm{ng} / \mathrm{ml}$ treated BM-DCs, BM-DC only and BM-DC loaded with peptide only control samples are shown in (D). Results shown are from one representative of 2 separate experiments with testing of individual samples carried out in triplicate. Statistical significance was determined using a 2-way ANOVA and Bonferroni's post-test where $* \mathrm{p}=0.05-0.01, * * \mathrm{p}=0.01-0.001, * * * \mathrm{p}<0.001, \mathrm{NS}=$ non significant. 


\begin{tabular}{|c|c|c|c|c|c|c|c|c|c|}
\hline \multicolumn{7}{|c|}{ Summary of in-vitro EXPERIMENTS utilizing TLR combinations } \\
\hline ASSAY & MPL & Res & Pam & CpG & $\begin{array}{c}\text { MPL } \\
+ \\
\text { Res }\end{array}$ & $\begin{array}{c}\text { MPL } \\
+\end{array}$ & $\begin{array}{c}\text { Res } \\
+ \\
\text { Pam }\end{array}$ & $\begin{array}{c}\text { CpG } \\
+ \\
\text { Pam }\end{array}$ & $\begin{array}{c}\text { CpG } \\
+ \\
\text { Res }\end{array}$ \\
\hline $\begin{array}{c}\text { DC culture } \\
\text { maturation } \\
\text { CD86 }\end{array}$ & Yes & Yes & Yes & Yes & Yes & Yes & No & No & No \\
\hline $\begin{array}{c}\text { DC culture } \\
\text { maturation } \\
\text { CD40 }\end{array}$ & Yes & Yes & Yes & Yes & No & No & No & No & No \\
\hline $\begin{array}{c}\text { DC culture } \\
\text { cytokines } \\
\text { IL-12p40 }\end{array}$ & Yes & Yes & Yes & Yes & Yes & Yes & No & No & No \\
\hline $\begin{array}{c}\text { DC culture } \\
\text { cytokines } \\
\text { IL-6 }\end{array}$ & No & No & No & No & No & No & No & No & No \\
\hline $\begin{array}{c}\text { DC culture } \\
\text { cytokines } \\
\text { TNF- } \alpha\end{array}$ & No & No & No & No & (Yes) & (Yes) & No & No & No \\
\hline $\begin{array}{c}\text { DC culture } \\
\& \\
\text { CD40L } \\
\text { IL-12p70 }\end{array}$ & Yes & Yes & Yes & Yes & No & No & - & - & Yes \\
\hline $\begin{array}{c}\text { DC culture } \\
\& \\
\text { CD40L } \\
\text { IL-12p40 }\end{array}$ & No & No & No & No & No & No & - & - & No \\
\hline $\begin{array}{c}\text { DC culture } \\
\& \\
\text { cD40L } \\
\text { IL-6 }\end{array}$ & No & No & No & No & No & No & - & - & No \\
\hline $\begin{array}{c}\text { T cell } \\
\text { Proliferation } \\
\text { Assay } \\
\text { PEPTIDE }\end{array}$ & Yes & Yes & Yes & - & Yes & Yes & Yes & - & - \\
\hline $\begin{array}{c}\text { T cell } \\
\text { Proliferation } \\
\text { Assay } \\
\text { PROTEIN }\end{array}$ & Yes & Yes & Yes & - & Yes & Yes & Yes & - & - \\
\hline
\end{tabular}

Table 3.3 Summary table of in-vitro assays highlighting successful TLR agonists and combinations. "No" "Yes" responses are indicative of positive assay response trends seen across experiments for specific parameters being measured. (For example: With reference to assay utilized to determine the presence or absence of DC maturation after exposure of in-vitro BM-DC cultures to TLR agonists alone "Yes" is indicative of CD86 upregulation on BM-DC cells as determined by flow cytometric analysis, while "No" indicates that CD86 was not upregulated above control levels. When TLR agonists were tested in combination, Yes indicated an additive responses such that the CD86 upregulation measured on BM-DCs was above that seen when either TLR agonist was utilized alone). 


\subsubsection{The generation of in-vivo antigen specific responses against TLR treated DC vaccines}

Having established that MPL alone or in combination with Pam3Cys or Resiquimod can improve aspects of DC function, antigen specific $\mathrm{T}$ cell responses were measured in-vivo using the OVA protein loaded BM-DCs. The OVA antigen specific $\mathrm{CD} 8^{+} \mathrm{T}$ cell response generated against in-vitro TLR treated BM-DC based vaccines was therefore compared to vaccines that were not TLR treated.

BM-DC cultures were generated as described in Figure 3.1 with $1 \mathrm{mg} / \mathrm{ml}$ OVA protein being loaded onto day 6 TLR treated cultures. The TLR treated OVA loaded BM-DC vaccines were injected $i v$ on day 7, after $22 \mathrm{~h}$ culture, and the OVA specific T cell response was measured in blood 7, 14 and 21 days post vaccination. A cohort of OVA specific $\mathrm{CD}^{+} \mathrm{T}$ cells were injected $i v$ into vaccine recipients 1 day prior to $\mathrm{DC}$ vaccine delivery to improve detection of the induced response. To allow the distinction between host versus injected $\mathrm{T}$ cell population responses, B6.SJL-Ptprc ${ }^{\mathrm{a}} \mathrm{Pepc} \mathrm{c}^{\mathrm{b}} / \mathrm{BoyJ}\left(\mathrm{CD} 45.1^{+}\right)$congenic mice were utilized for further studies. These mice are generated by backcrossing the inbred SJL strain, expressing the Ptprc ${ }^{\mathrm{a}}$ gene (CD45.1), onto the (CD45.2) C57BL/6 background which then permitted the distinction between injected (C57BL/6 background) OTI cells and recipient populations on the basis of CD45.1 and CD45.2 expression.

To determine the number of $\mathrm{CD}^{+}$OVA specific $\mathrm{T}$ cells required for subsequent vaccine experiments, LN cells were administered at various doses and the respective OVA specific $\mathrm{T}$ cell response measured by assessing accumulation in the blood by flow cytometry 7 days later (Figure 3.13). The transgenic TCR antibody markers used were V $\alpha-2$, CD45.2, CD8 while PI was used as a viability marker. A proportion of each LN preparation was comprised of $\mathrm{CD}^{+}$OVA specific $\mathrm{T}$ cells - previous work had confirmed a $40 \%$ composition for these LN samples (results not shown). The administration of 50,000 LN OTI cells therefore provided 20,000 CD $8^{+}$OVA specific T cells. This was the intermediate $\mathrm{LN}$ dose selected for future experiments to ensure provision of $20,000 \mathrm{CD} 8^{+} \mathrm{OVA}$ specific T cells. 


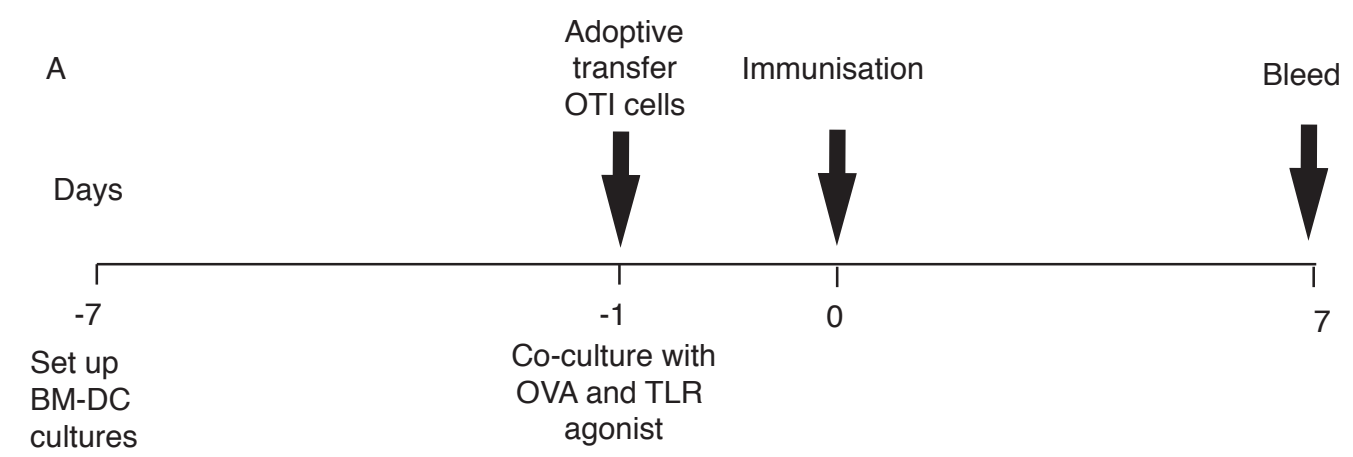

B

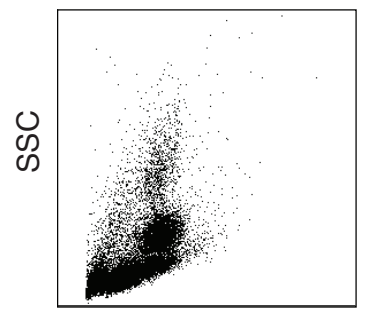

FSC

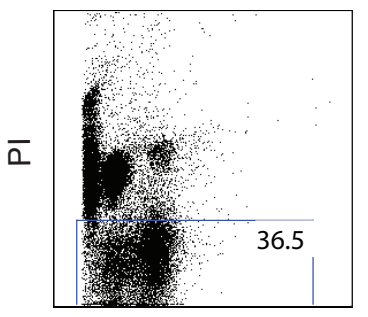

FSC

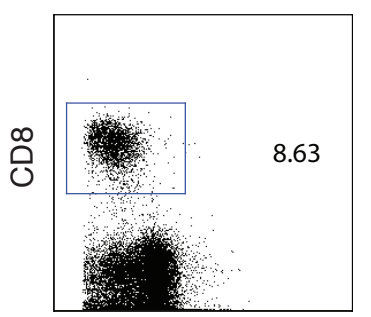

FSC

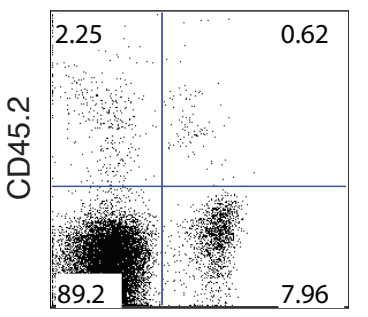

$\mathrm{Va}-2$

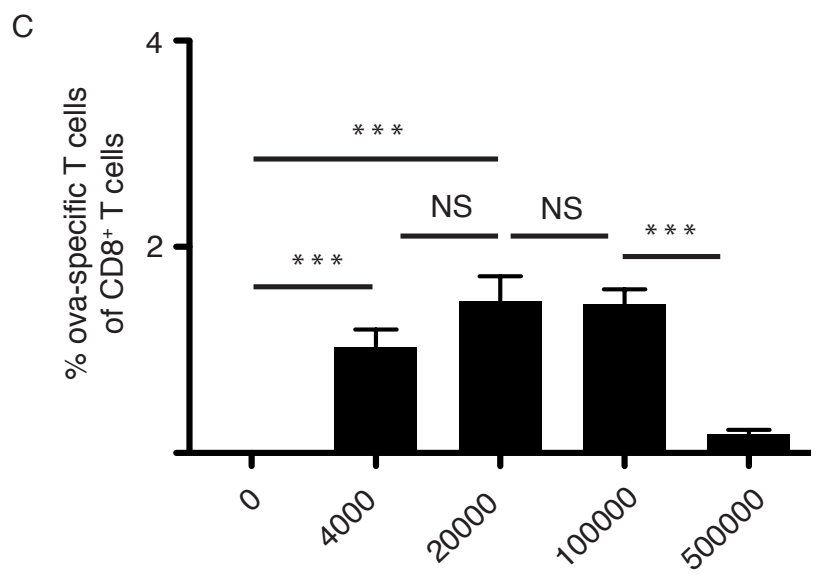

Figure 3.13 BM-DC vaccination strategy and manufacture protocol. (A) Timeline showing BM-DC manufacture and vaccination protocol for in-vivo vaccine testing model. (B) Dot plots show representative gating strategy showing isolation method of injected OVA-specific $T$ cells 7 days post vaccination for flow cytometric analysis. (C) OVA specific $\mathrm{T}$ cell responses generated against titrated numbers of OTI cells and measured using TCR marker antibodies to identify OVA specific T cells. "OVA specific $\mathrm{CD}^{+} \mathrm{T}$ cells" refers to $\mathrm{CD} 8^{+} \mathrm{CD} 45^{+} \mathrm{V} \alpha 2^{+} \mathrm{T}$ cells. Bar graph shows the mean percentages of the OVA specific $\mathrm{T}$ cell response expressed as a percentage of $\mathrm{CD} 8^{+} \mathrm{T}$ cells $( \pm \mathrm{SEM})$ as seen in the blood 7 days post BM-DC vaccination. Statistical significance was determined using a 1-way ANOVA test and Tukey's post-test where $* p=0.05-0.01, * * p=0.01-0.001$, $* * * p<0.001, \mathrm{NS}=$ not significant. Results shown are from one experiment. 


\subsubsection{TLR stimulated DC vaccines did not improve antigen specific $T$ cell responses in-vivo}

Published work had shown that $\mathrm{CD}^{+}$and $\mathrm{CD}^{+} \mathrm{T}$ cell responses could be generated in human subjects after the administration of DCs matured ex-vivo and loaded with antigen (Dhodapkar et al., 2000; Dhodapkar et al., 1999; Thurner et al., 1999). The magnitude and efficacy of the in-vitro generated TLR treated OVA protein loaded DC vaccine was assessed in this optimised in-vivo model system. OVA specific $\mathrm{T}$ cell responses were observed against vaccination with OVA loaded BM-DCs treated with single TLR agonists and multiple TLR agonist combinations.

A timeline outlining the BM-DC manufacture and vaccination protocol is shown in Figure 3.14 A. Antibodies were used to identify adoptively transferred $\mathrm{CD}^{+}$OVA specific T cell populations and distinguish these from host generated $\mathrm{CD} 8^{+} \mathrm{OVA}$ specific $\mathrm{T}$ cell responses 7 days after DC vaccine administration.

The OVA specific T cell response seen when BM-DCs alone were administered did not differ significantly from that seen when the BM-DCs were loaded with OVA protein in this particular experiment. Subsequent repeated experiments showed that BM-DCs loaded with OVA protein were capable of significantly enhancing OVA-specific T cell responses when compared to that seen after immunisation with BM-DCs alone. Surprisingly, no significant differences in the OVA specific $\mathrm{T}$ cell responses were evident between TLR treated vaccine groups and non-TLR treated vaccine groups Figure 3.14 B. In addition, TLR agonist combinations found to improve BM-DC maturation marker status and cytokine production in previous in-vitro studies also failed to enhance the in-vivo OVA specific $\mathrm{T}$ cell response seen 7 days post vaccination. TLR stimulation did not alter the kinetics of the response as repeated analysis on days 14 and 21 showed the same trend. 

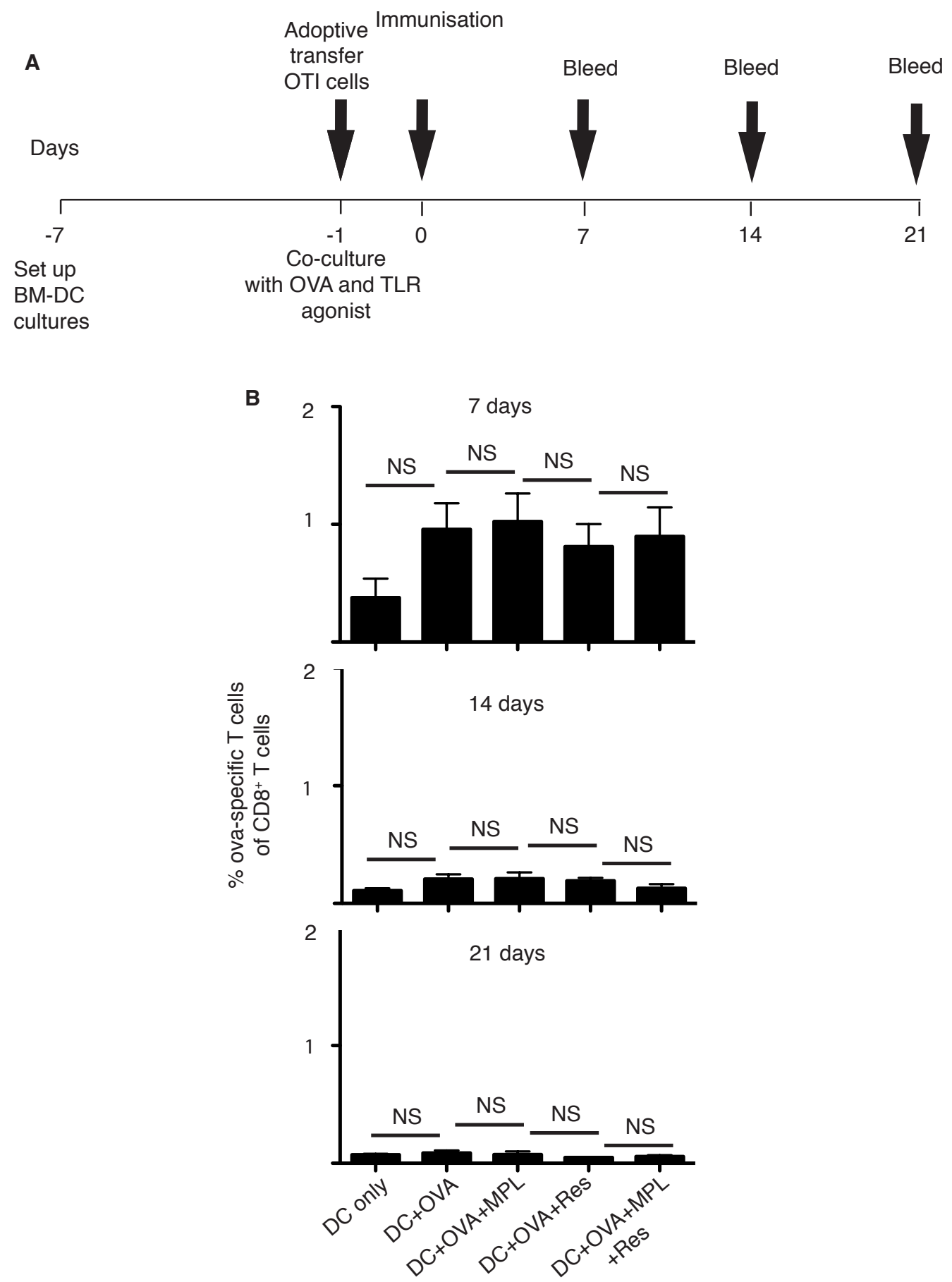

Figure 3.14 TLR treatment of OVA loaded BM-DCs vaccines did not influence OVA specific $\mathbf{T}$ cell response in-vivo. (A) Timeline depicting BM-DC vaccination protocol. (B) Bar graphs show the mean percentage of CD45.1 ${ }^{+}$TCRV $\alpha-2^{+}$(OVA-specific) T cells as a percentage of $\mathrm{CD}^{+} \mathrm{T}$ cells in the blood at day 7, 14 and 21 days post vaccination. Statistical significance was determined using a 1-way ANOVA test and Tukey's post-test where ${ }^{*} p=0.05-0.01,{ }^{* *} p=0.01-0.001, * * * p<0.001$, NS $=$ not significant. Results shown are from one experiment with 2 repeats conducted yielding similar results. 
Unfortunately these results highlight the inability of in-vitro TLR treatment on BM-DC based vaccines in this model system to induce any improvement in the OVA specific T cell response as measured in the blood 7 days post DC vaccination, and warranted further exploration.

\subsubsection{The in-vitro manipulation of BM-DCs does not correlate with maturity status after $i v$ administration}

An attempt was made to find an explanation for the failure of TLR treated BM-DCs to enhance OVA specific T cell responses in this in-vivo model. Specifically, the phenotype of in-vitro TLR treated BM-DCs was examined after $i v$ administration and retrieval from the spleen to determine the maturation status post in-vivo vaccination.

BM-DC cultures were generated as previously described in Figure 5.1 and treated with MPL on day 6 for $22 \mathrm{~h}$. On day 7, BM-DCs were harvested and labelled with CFSE before injecting into recipients. Spleens were retrieved $24 \mathrm{~h}$ later and enriched for CD11c DCs using the anti-CD11c coated magnetic MACS sorting beads. FACS analysis was utilized to identify the injected CFSE labelled BM-DC populations and the status of the maturation marker CD86 determined for each treatment group.

The in-vitro maturation status of the treated BM-DC groups was checked before iv injection and found to exhibit differences in maturation marker expression according to TLR treatment protocols described earlier (data not shown). The gating strategy used for this experiment is displayed in Figure 3.15 A. Distinctive CFSE high populations representative of the injected BM-DCs were evident for each recipient group. A second CFSE low positive CD11c population was also identified as a host DC population likely to have acquired injected CFSE labelled BM-DCs. All CFSE high BM-DC populations retrieved from spleens displayed similar CD86 expression across all treatment groups regardless of whether they were stimulated with TLR ligands before injection or not. Even when MPL was delivered at the same time as BM-DCs intravenously, the CFSE ${ }^{\text {high }}$ DCs recovered had similar expression levels of CD86. Endogenous CD11c ${ }^{+}$DC populations 
were shown to upregulate CD86 expression in response to BM-DCs co-administered with systemic MPL and deemed more responsive to systemic TLR stimulation than endogenous bystander $\mathrm{CD} 11 \mathrm{c}^{+}$populations displaying $\mathrm{CFSE}^{\text {low }}$ expression after possibly taking up BMDCs treated with MPL in-vitro.

These results show that although vaccine DCs were immature prior to administration, once these vaccine BM-DCs had reached the spleen, they had matured regardless of the in-vitro manipulation protocol used Figure 3.15 C. These results also highlight the importance of giving consideration to the role of endogenous DC populations in the context of BM-DC vaccination strategies when aiming to achieve enhanced cell responses utilizing TLR stimulation. 
A

CFSE label Retrieve treated DC's CFSE labelled then inject DC's from spleens

Days
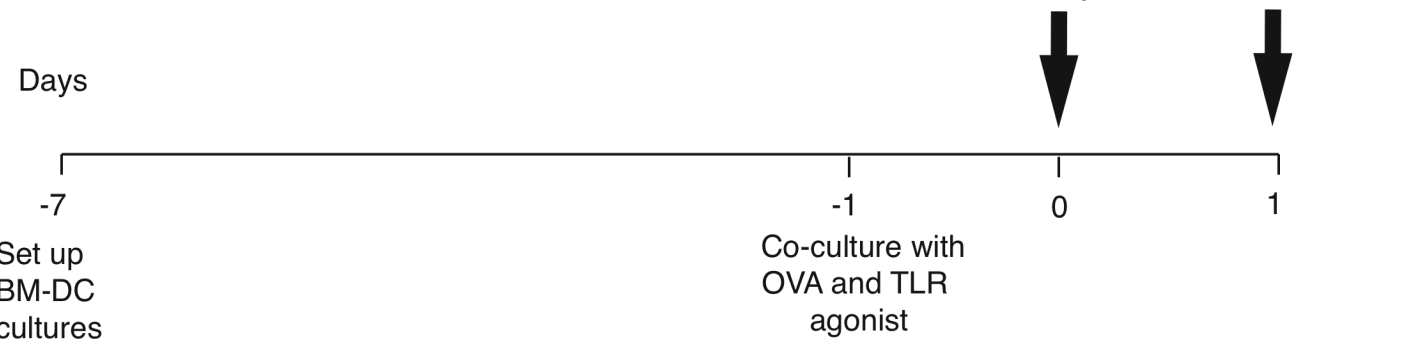

B

No BM-DC's

CFSE BM-DC

CFSE BM-DC + MPL

CFSE BM-DC+MPL iv

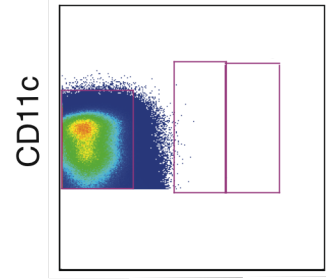

CFSE

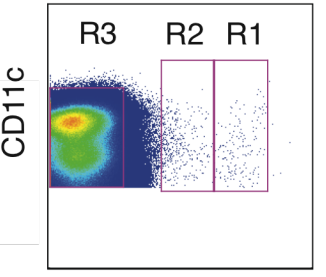

CFSE

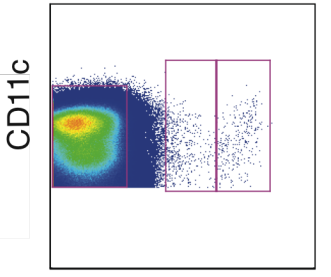

CFSE

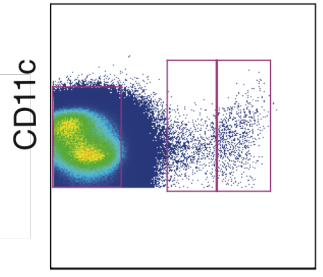

CFSE
C
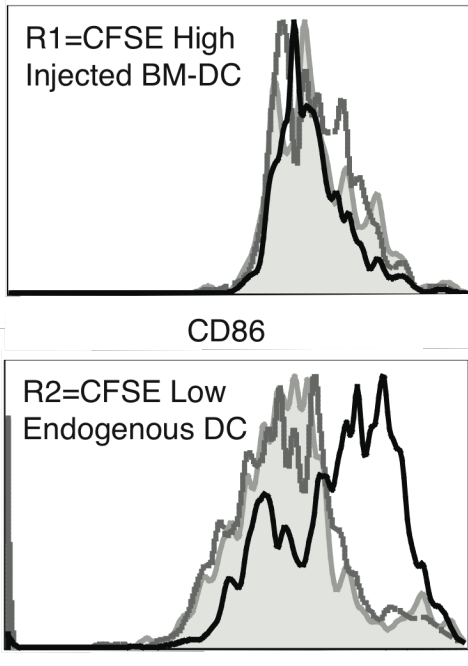

CD86

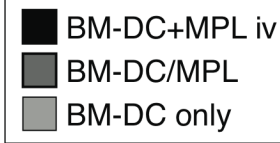

R3=CFSE negative

D

Endogenous DC

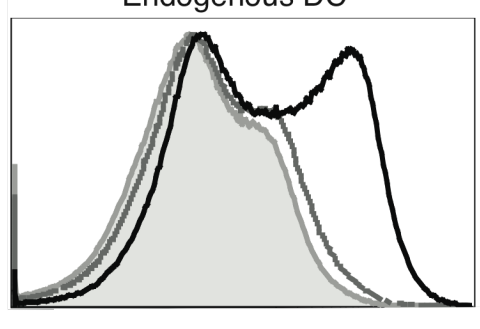

CD86

Figure 3.15 The fate of CFSE labelled BM-DCs retrieved from spleen 24 hrs after iv administration. (A) Timeline showing BM-DC vaccine manufacture and CFSE labelling prior to $i v$ administration. B Dots plots show CFSE labelled CD $11 \mathrm{c}^{+} \mathrm{BM}-\mathrm{DCs}$ retrieved from splenic DC populations $24 \mathrm{~h}$ post vaccination. Endogenous $\mathrm{CD} 11 \mathrm{c}^{+}$populations also evident in dot plots alongside populations of DCs exhibiting CFSE low expression. (C) Histograms showing maturation status of retrieved CFSE labelled BM-DC populations. (D) Histograms showing maturation status of endogenous CD11 $\mathrm{c}^{+}$DC populations $24 \mathrm{~h}$ after treatment with CFSE labelled BM-DC vaccines. Results shown are from one experiment. 


\subsubsection{Systemic administration of TLR agonists alone and in combination improves maturation status of host DC populations}

Although our previous work had demonstrated the capability of TLR agonists to stimulate BM-DCs in-vitro, this strategy failed to improve $\mathrm{CD} 8^{+} \mathrm{T}$ cell responses to vaccination in our conditions. It was therefore considered whether targeting of resident DC populations could be achieved by the systemic administration of TLR agonists alone or in combination. Preclinical and clinical studies utilising intravenously administered TLR agonists for therapeutic purposes have been published and describe the use of TLR 3, TLR 4, TLR 7 and TLR 9 in humans (Dudek et al., 2007; Dummer et al., 2008; Engelhardt et al., 1991; Link, et al., 2006; Otto et al., 1996; Spaner et al., 2010; Strayer et al., 1994; Thompson et al., 1996; Vosika et al., 1984a; Vosika et al., 1984b).

The TLR agonists MPL, Pam3Cys and Resiquimod were administered systemically into C57BL/6 recipients alone and in combination with each other. TLR agonist concentrations were selected from separate titration studies and were consistent with those found in the literature. After $16 \mathrm{~h}$, spleens were collected and expression of CD86 by CD11 ${ }^{+}$cells determined by flow cytometry.

As can be seen from Figure 3.16 B, B220 was used to distinguish between the cDCs of interest and $\mathrm{B} 220^{+} \mathrm{CD} 11 \mathrm{c}^{+} \mathrm{pDC}$ populations that were not investigated any further. Systemically administered MPL, Resiquimod and Pam3Cys were each able to significantly increase CD86 marker expression on resident CD11 ${ }^{+}$cells (Figure 3.16). As seen with BM-DC TLR agonist combinations using MPL and Resiquimod, MPL and Pam3Cys were able to further significantly enhance CD86 expression (Figure 3.16).

Thus, resident splenic DC populations were able to respond to TLR stimulation applied systemically when used alone and in combination with each other. 
A

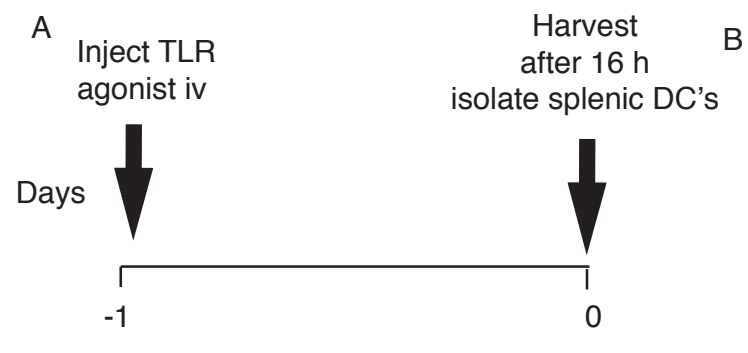

C
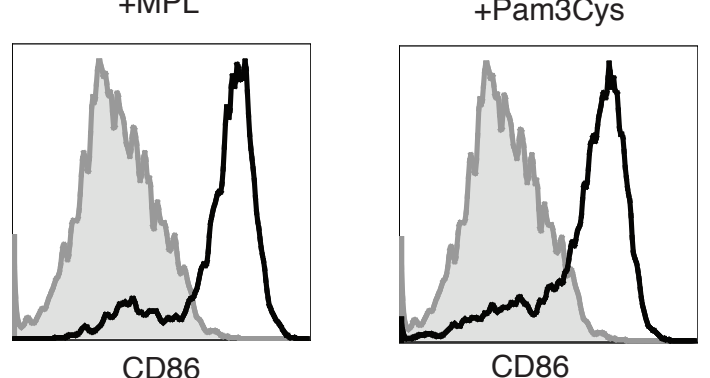

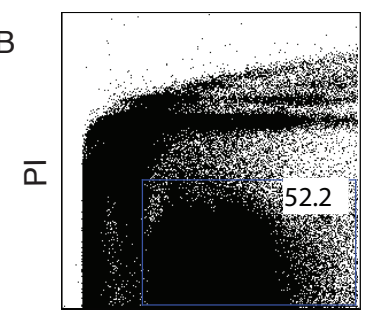

FSC

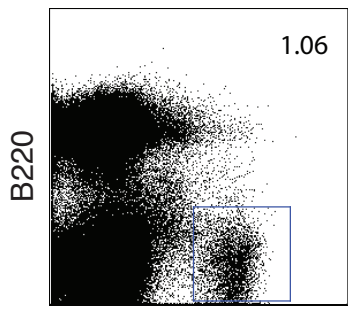

CD11C

$\mathrm{MPL}+$ Pam3Cys

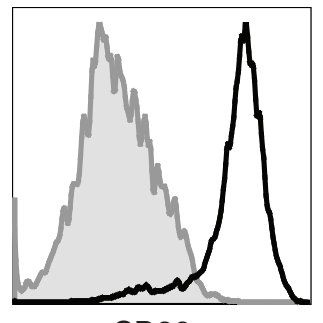

CD86

D

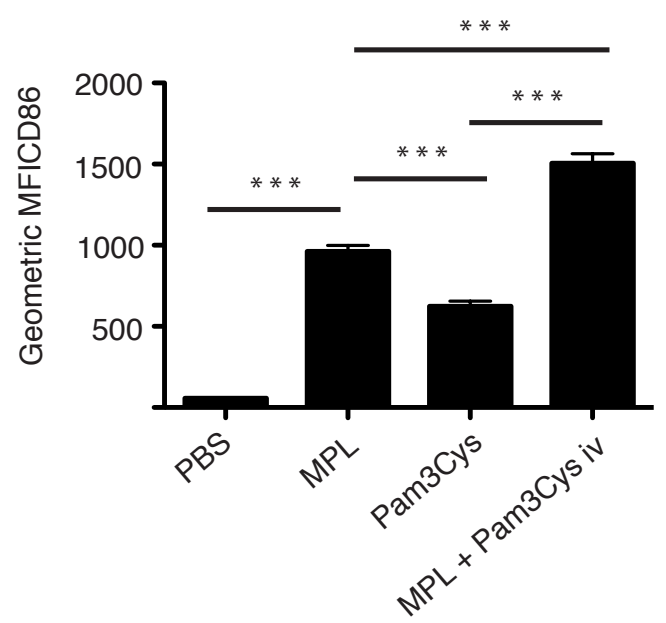

E

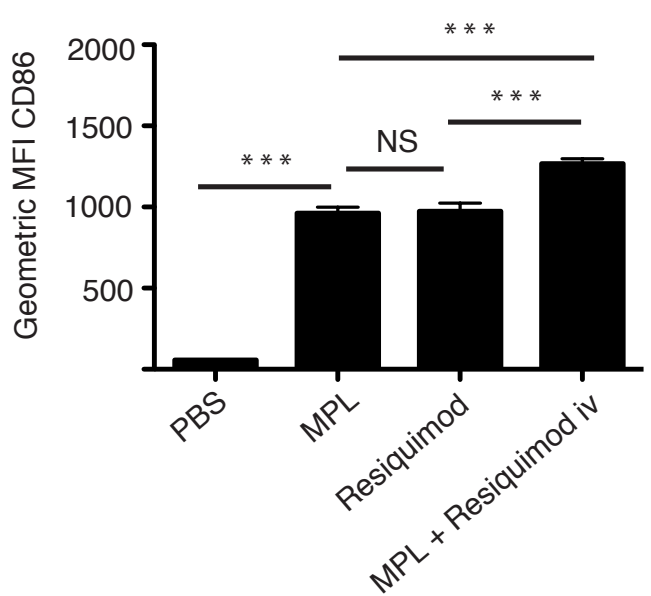

Figure 3.16 Systemic administration of TLR agonists alone and in combination matures splenic DC populations. (A) Timeline indicating timing of systemic TLR agonist administration. (B) Representative flow cytometry dot plots show general gating strategy showing splenic CD11 $\mathrm{c}^{+}$DC population. (C) Histograms show relative geometric MFI CD86 expression levels on CD11 ${ }^{+}$splenic DC $16 \mathrm{~h}$ after recipients injected with PBS, MPL $100 \mathrm{ng} / \mathrm{ml}$, Pam3Cys $100 \mathrm{ng} / \mathrm{ml}$ alone and combined, as determined by antibody staining and flow cytometry. Histograms show naive BM-DC only samples depicted by light grey filled areas, with treated samples indicated by darkened lines. (D) Bar graph shows geometric MFI levels for CD86 expression on CD11 ${ }^{+}$splenic DC after recipients injected with MPL $100 \mathrm{ng} / \mathrm{ml}$, and Pam3Cys $100 \mathrm{ng} / \mathrm{ml}$ alone and in combination $16 \mathrm{~h}$ earlier ( $n=2-3$ per group). (E) Bar graph shows geometric MFI levels for CD86 expression on CD11 $\mathrm{c}^{+}$splenic DC $16 \mathrm{~h}$ after recipients injected with MPL 100 $\mathrm{ng} / \mathrm{ml}$ and Resiquimod $0.1 \mathrm{ug} / \mathrm{ml}$. Statistical significance was determined using a 1-way ANOVA test and Tukey's post-test where $* \mathrm{p}=0.05-0.01, * * \mathrm{p}=0.01-0.001, * * * \mathrm{p}<$ $0.001, \mathrm{NS}=$ not significant. Results shown are representative of 2 similar experiments. 


\subsubsection{TLR stimulation targeting host DC populations improves antigen specific $T$ cell responses in-vivo}

Given that results in section 3.2.9 suggested cellular material from injected CFSE high BM-DCs was being transferred to resident $\mathrm{CD} 11 \mathrm{c}^{+}$cells, attention was turned to strategies that might exploit this phenomenon. In this context it might be possible that resident cells captured the antigens that had been loaded onto the injected DCs and would then be capable of processing and presenting this antigen to $\mathrm{CD}^{+} \mathrm{T}$ cells. Results in section 3.2.10 show that these resident antigen presenting cells are likely to be in an immature state, and would be unlikely to promote $\mathrm{T}$ cell stimulation unless provided with an external stimulus. In order to achieve this, it may be possible to co-administer TLR ligands with the injected BM-DC-based vaccines. Furthermore, it may be possible to exploit multiple TLR agonists to maximise this response, if the resident cells, like in-vitro stimulated BM-DCs respond additively or synergistically to TLR agonists in combination.

An attempt was made to improve the vaccine efficacy by targeting TLR stimulation to host DC populations by intravenous administration. Thus, the in-vivo application of using TLR stimulation to target resident DC populations was tested by using systemic MPL administration in co-operation with DC vaccination, using BM-DCs that had been loaded with protein antigen in-vitro. The in-vivo $\mathrm{CD}^{+} \mathrm{T}$ cell response to co-administration of MPL and Pam3Cys alone and together, combined with protein loaded vaccines DCs, would be compared with that seen after in-vitro TLR stimulation of the protein loaded vaccine BM-DCs.

In-vitro BM-DC cultures were from C57BL/6 mice and treated with TLR agonists alone and in combination on day 6 , and loaded with OVA protein at the same time. TLR treated and OVA loaded BM-DCs were cultured for a further $22 \mathrm{~h}$ before harvesting, washing and injection at 300,000 DCs per recipient. Adoptive transfer of OTI x B6 congenic donor T cells was administered to recipients also carried out on day 6 . This enabled discrimination of the transferred $\mathrm{T}$ cell population on the basis of CD45.1 expression. Expansion of this injected $\mathrm{CD}^{+} \mathrm{T}$ cell population was monitored for each recipient by testing blood samples 
taken 7 days post vaccination and used as a measure of DC vaccine efficacy. Systemic administration of MPL at the time of DC vaccination with OVA loaded DCs was carried out by co-injecting MPL into the contra-lateral tail vein immediately after DC vaccine administration.

A timeline for the experimental procedure followed is shown in Figure 3.17 A. Antibodies were used to identify adoptively transferred $\mathrm{CD} 8^{+} \mathrm{OVA}$ specific $\mathrm{T}$ cell populations and distinguish these from host generated $\mathrm{CD}^{+}$OVA specific $\mathrm{T}$ cell responses 7 days after DC vaccine administration. The transgenic TCR antibody markers used were v $\alpha-2$, CD45.2, CD8 while PI was used as a viability marker. The antigen specific $\mathrm{CD} 8^{+} \mathrm{T}$ cell response was measured in the blood 7 days post vaccination. The co-administration of MPL was capable of significantly boosting the OVA specific $\mathrm{CD}^{+} \mathrm{T}$ cell response seen in blood 7 days post vaccination, whereas the in-vitro application of MPL to BM-DC cultures for subsequent use in DC vaccines was unable to improve the OVA specific $\mathrm{CD}^{+} \mathrm{T}$ cell response over untreated vaccines.

The inclusion of systemically administered Pam3Cys into the vaccination protocol did not improve observed OVA specific T cell responses, and in addition appeared to negate and impede the action of systemic MPL when co-administered together. Thus these results suggest that targeting of MPL stimulation to resident DC populations significantly enhances the antigen specific $\mathrm{T}$ cell response generated against $i v$ administered $\mathrm{DC}$ vaccines, however despite Pam3Cys being capable of driving the maturation of resident APCs this TLR agonist failed to enhance vaccine-induced responses generated by the transfer of antigen to resident APCs. 


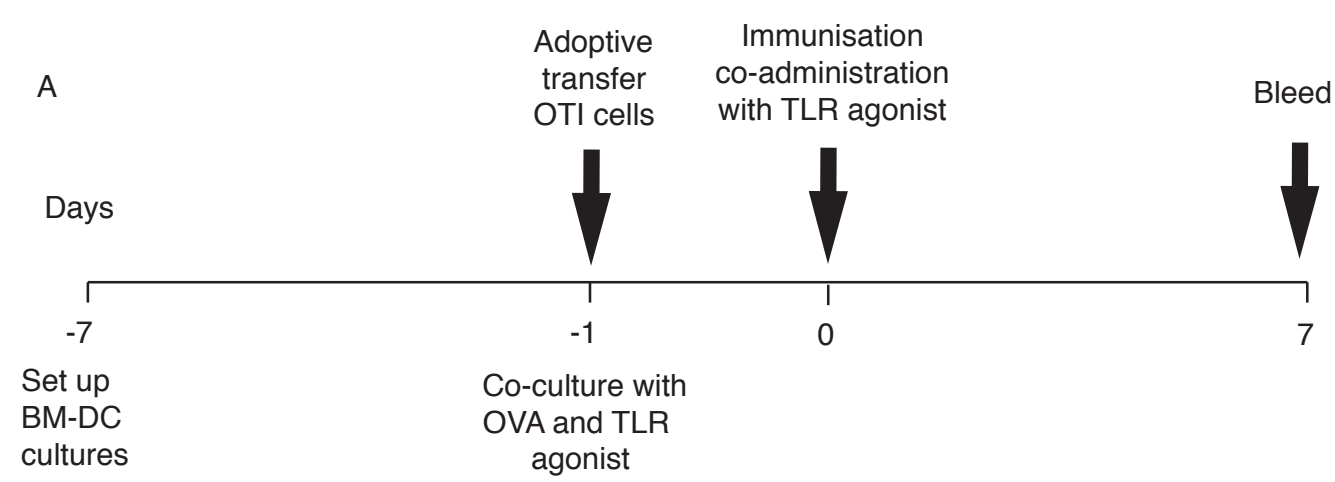

B

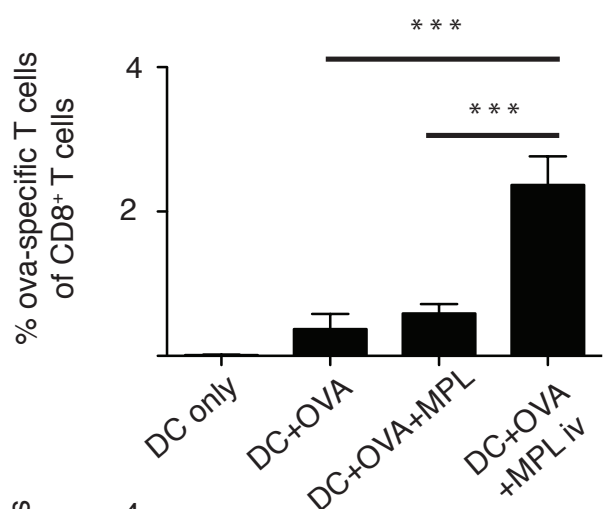

C
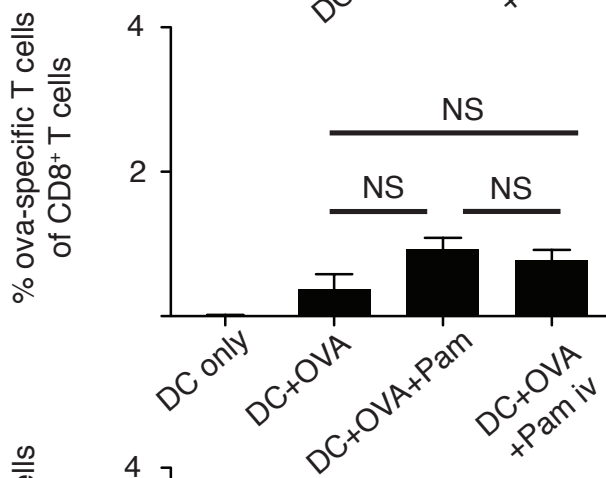

$\mathrm{D}$

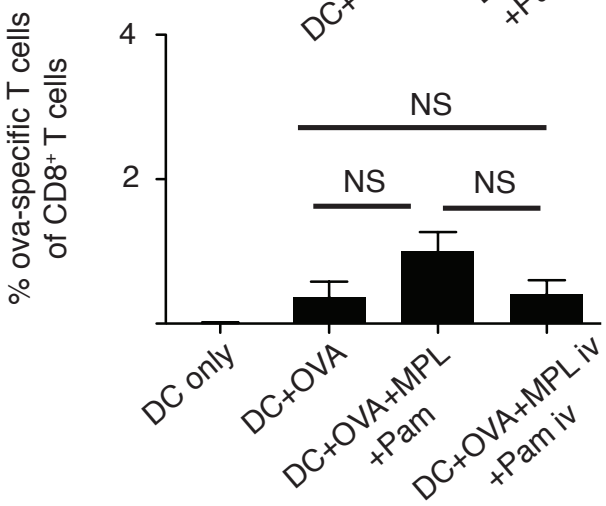

Figure 3.17 Systemic administration of TLR agonists influence antigen specific responses to BM-DC vaccines. (A) Timeline showing BM-DC vaccine manufacture protocol highlighting the co-administration of TLR agonists alongside OVA-loaded BMDC vaccines. (B), (C), (D) Bar graphs show the mean percentage of OVA-specific T cells as a percentage of $\mathrm{CD}^{+} \mathrm{T}$ cells in the blood at day 7 post immunisation with BM-DC vaccines tested with vaccine inclusive versus systemic administration of (B) MPL 100 ng/ml, (C) Pam3Cys C 100 ng/ml, and MPL combined with Pam3Cys. Bar graph shows the mean percentage OVA specific $\mathrm{T}$ cell response expressed as a percent of $\mathrm{CD}^{+} \mathrm{T}$ cells 
$( \pm$ SEM) seen in the blood 7 days post vaccination. Results shown are from one experiment with 2 repeats conducted yielding similar results. Statistical significance was determined using a 1-way ANOVA test and Tukey's post-test where $* \mathrm{p}=0.05-0.01, * * \mathrm{p}$ $=0.01-0.001, * * * \mathrm{p}<0.001, \mathrm{NS}=$ not significant. 


\subsubsection{The timing of systemic TLR stimulation}

After showing that systemic co-delivery of MPL was able to improve the OVA specific T cell response generated in-vivo, the failure of Pam3Cys to induce a similar result was investigated. An attempt was made to examine the kinetic effect of using Pam3Cys on antigen processing and $\mathrm{T}$ cell presentation activities after OVA protein loaded BM-DC vaccine administration. This was to address the question of whether Pam3Cys used in combination was interfering with the antigen uptake and processing of protein antigen OVA.

BM-DCs were prepared as previously described in section 3.2.1 and after loading with OVA on day 6 were administered on day 7. Recipients received iv Pam3Cys in the presence or absence of before, during and after BM-DC vaccine administration (Figure $3.18 \mathrm{~A}$ ). The antigen specific $\mathrm{T}$ cell response was measured in treatment groups one week after vaccination. The results in Figure 3.18 A show that Pam3Cys does appear to influence the OVA specific T cell response being generated if given either before or at the time of vaccine delivery. If however systemic Pam3Cys is administered one $\mathrm{h}$ after vaccine injection then the OVA specific T cell response is significantly improved. After 4 and $9 \mathrm{~h}$ post BM-DC vaccination the OVA specific $\mathrm{T}$ cell response measured has fallen back to baseline levels that are comparable to that achieved if vaccinating alone with OVA loaded BM-DCs administered with Pam3Cys $1 \mathrm{~h}$ before and during vaccine delivery. In this experiment, intravenous Pam3Cys co-administered at the time of vaccine delivery did show a trend towards an enhanced response, although this was not statistically significant. However, if Pam3Cys was delayed for $1 \mathrm{~h}$, the improvement in $\mathrm{CD} 8^{+} \mathrm{T}$ cell responses over non-TLR treated vaccines became significant.

These results show that intravenous Pam3Cys is able to improve response to OVA loaded BM-DCs if given after a short delay of $1 \mathrm{hr}$. Therefore if to be used for further testing, the kinetic influence of its action would need to be taken into account. These results suggest that Pam3Cys may be interfering with the protein processing activities of the resident DC populations. 
When Pam3Cys is co-delivered with the antigen carrying vaccine BM-DCs, the increased antigen specific $\mathrm{T}$ cell response typically seen with co-delivery of MPL and vaccine is absent. However when Pam3Cys is administered $1 \mathrm{~h}$ post vaccination, this elevated antigen specific $\mathrm{T}$ cell response is restored. It may be that signalling via the MyD88 dependent pathway when triggered by TLR 2 stimulation is occurring faster with Pam3Cys than MPL, therefore BM-DC maturation is more rapid and antigen acquisition capability is shutting down faster. There is also a possibility that TLR 2 expression is higher on the cross presenting resident DC, other splenic DC subsets and additional immune cell populations that could respond to Pam3Cys causing increased levels of cytokines being generated and available for maturing cross presenting DCs, again leading to a more rapid loss of antigen acquisition capability and possibly antigen processing capability of the cross presenting DCs. The rapid shut down of antigen processing has been reported previously with systemic TLR stimulation (Wilson et al., 2006). 


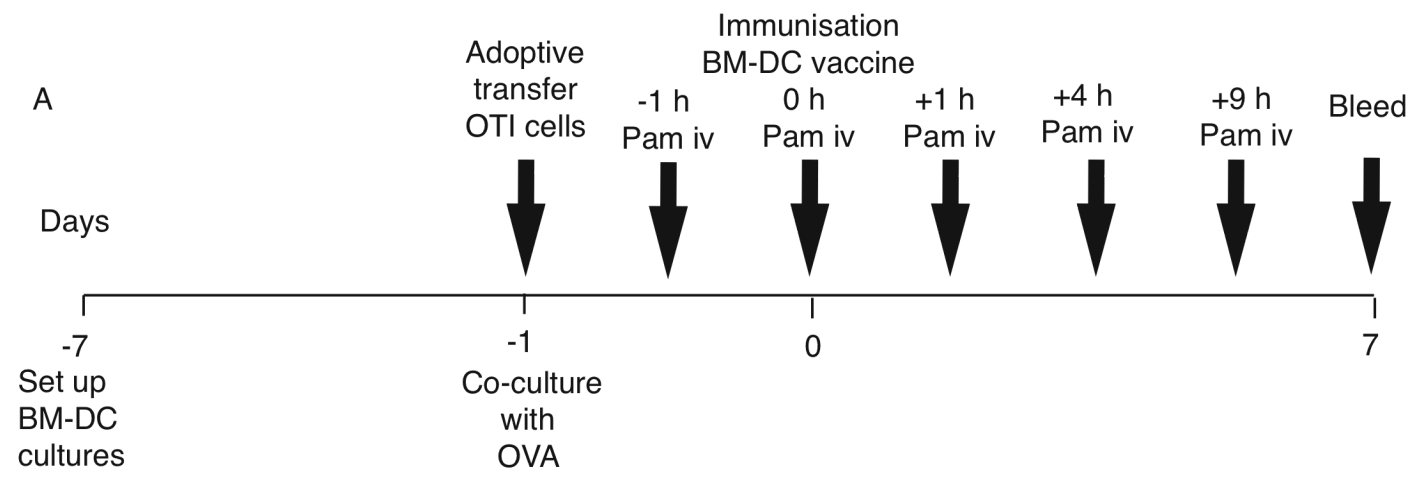

B

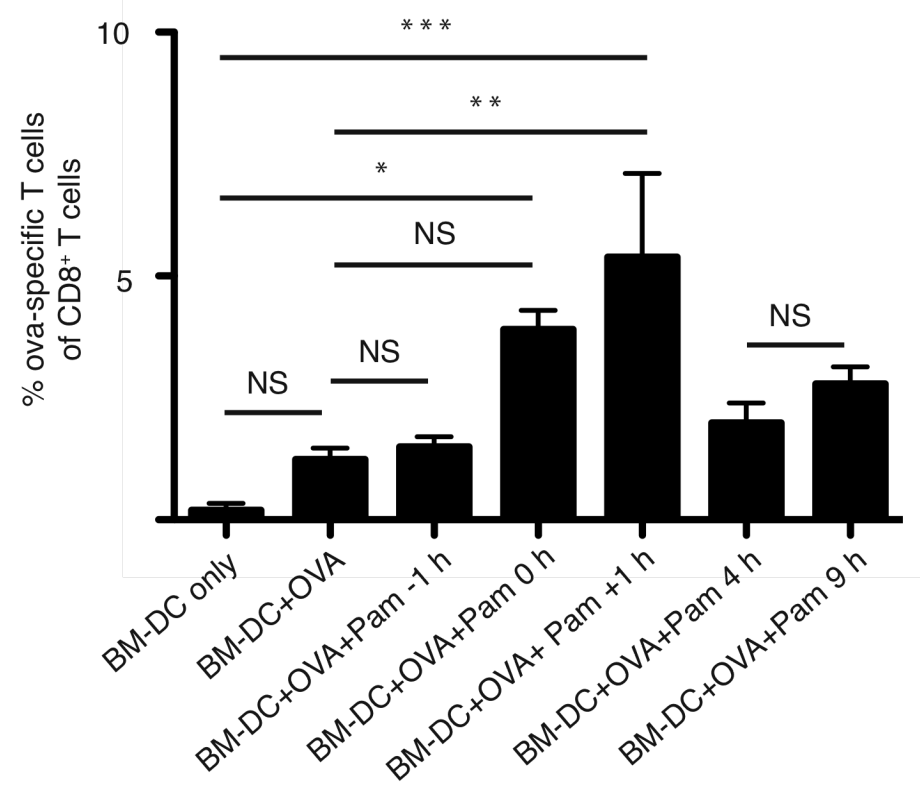

Figure 3.18 The kinetic influence of systemic Pam3Cys administration on protein antigen uptake mechanisms by BM-DCs. (A) Timeline depicting BM-DC vaccine manufacture protocol and the various time points at which Pam3Cys was administered to C57BL/6 recipients in relation to OVA loaded BM-DCs. (B) Bar graph shows the mean percentages of the OVA specific $\mathrm{T}$ cell response expressed as a percentage of $\mathrm{CD}^{+} \mathrm{T}$ cells $( \pm$ SEM) as seen in the blood 7 days post BM-DC vaccination. Results shown are from one experiment. Statistical significance was determined using a 1-way ANOVA test and Tukey's post-test where ${ }^{*} \mathrm{p}=0.05-0.01, * * \mathrm{p}=0.01-0.001, * * * \mathrm{p}<0.001$, NS $=$ not significant. 


\subsubsection{Systemic TLR stimulation improves OVA-specific $T$ cell responses}

At this stage, in-vivo studies were developed by the availability of the generation of congenic OVA specific OTI cells generated by crossing CD45 congenic B6 mice with OTI mice. Adoptively transferring these congenic OTI cells into C57BL/6 mice as recipients allowed for their identification using flow cytometric analysis after antibody staining for the relevant surface markers. This new system was therefore validated using BM-DCs and TLR stimulation.

BM-DCs were prepared as previously described in section 3.2.1 and loaded with OVA protein on day 6 followed by vaccination on day 7 (Figure 3.19 A). MPL was co-delivered with the vaccine and the OVA specific $\mathrm{T}$ cell response measured one week post vaccination. Dot plots in Figure 3.19 B show the gating strategy and flow cytometry profiles used to identify the adoptively transferred congenic OTI cells. The bar graph in Figure 3.19 C shows the significantly increased OVA specific $\mathrm{T}$ cell response generated when using MPL co-delivered systemically with the OVA loaded vaccine.

To look at the duration of action induced by the current BM-DC vaccine protocol using systemic MPL co-delivery, OVA specific $\mathrm{T}$ cell responses for all the treatment groups were again tested 2 weeks post vaccination (Figure $3.19 \mathrm{D}$ ). The bar graph presented in Figure 3.19 D shows that the same trends seen after one week are still evident for the OVA specific $\mathrm{T}$ cell responses measured after two, although they are markedly lessened across all groups.

These results provided validation of the in-vivo model and showed that it was possible to improve the antigen specific $\mathrm{T}$ cell response to OVA loaded BM-DCs using systemic TLR stimulation in the form of MPL co-delivered at the time of vaccine administration. 


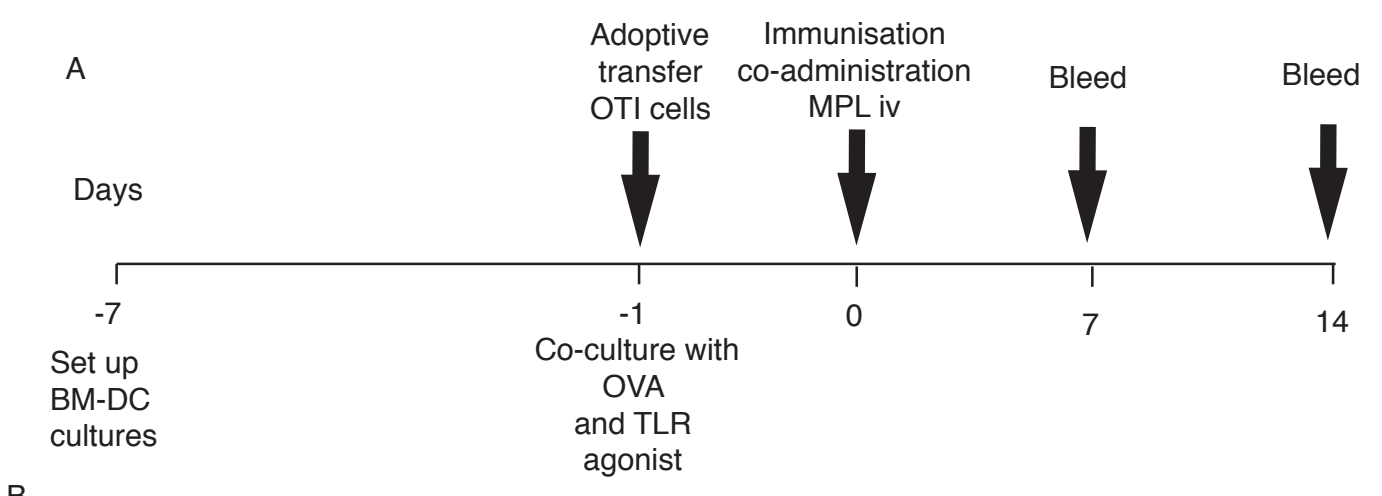

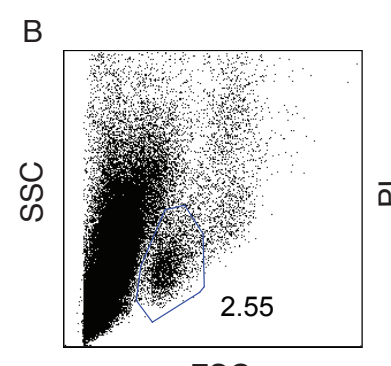

FSC

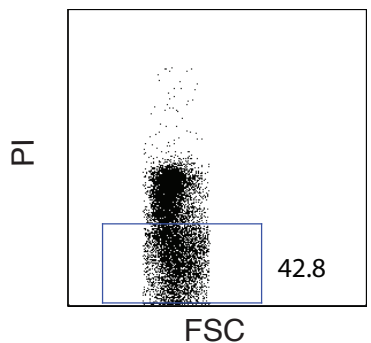

Day 7

$$
\begin{gathered}
C \\
0 \\
0 \\
0 \\
0 \\
10 \\
0 \\
: 0 \\
0 \\
0 \\
0 \\
0 \\
0 \\
0 \\
0 \\
0 \\
0
\end{gathered}
$$

C
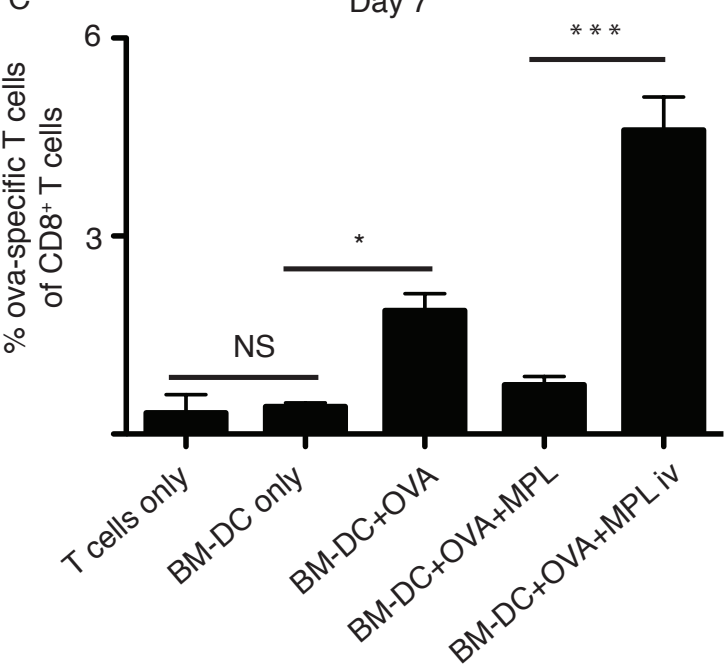

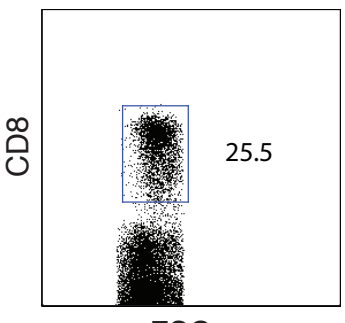

FSC
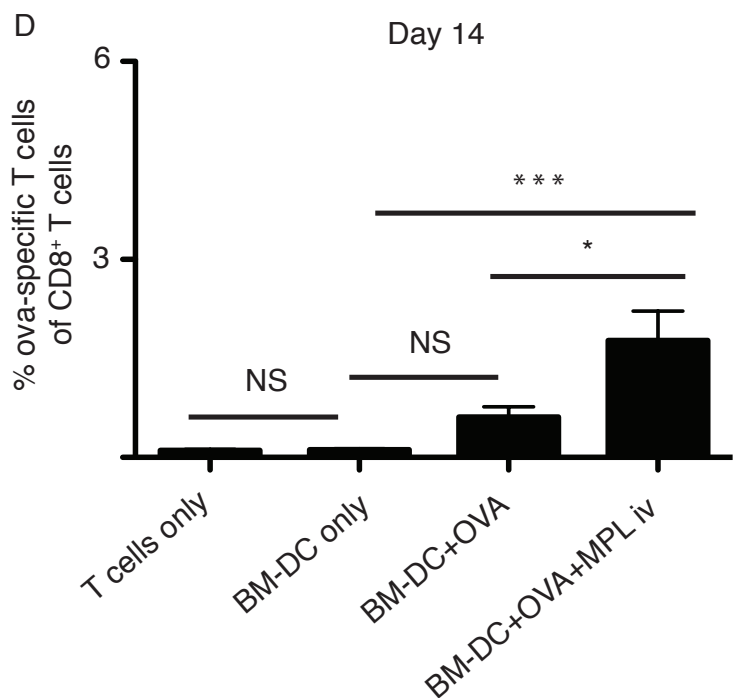

Figure 3.19 Systemic administration of MPL stimulation enhances antigen-specific $T$ cell responses generated against BM-DCs. (A) Timeline depicts BM-DC vaccine manufacture protocol and indicates systemic administration of MPL co-delivered on day 0 . (B) Representative dot plots are shown to highlight the general gating strategy used to identify a cohort of injected OTI x B6 cells into C57BL/6 recipients for flow cytometry analysis. (C) Bar graph shows the mean percentages of the OVA specific $\mathrm{T}$ cell response expressed as a percentage of $\mathrm{CD}^{+} \mathrm{T}$ cells $( \pm \mathrm{SEM})$ as seen in the blood 7 days post BMDC vaccination. (D) Bar graph shows the mean percentages of the OVA specific $T$ cell response expressed as a percentage of $\mathrm{CD}^{+} \mathrm{T}$ cells $( \pm$ SEM) as seen in the blood 14 days post BM-DC vaccination. Statistical significance was determined using a 1-way ANOVA test and Tukey's post-test where ${ }^{*} p=0.05-0.01,{ }^{* *} p=0.01-0.001,{ }^{* * *} p<0.001, \mathrm{NS}=$ not significant. 


\begin{tabular}{|c|c|c|c|c|c|}
\hline \multicolumn{6}{|c|}{ Summary of in-vivo EXPERIMENTS utilizing TLR combinations } \\
\hline ASSAY & MPL & Res & Pam & MPL+Res & MPL+Pam \\
\hline $\begin{array}{c}\text { DC vaccine } \\
\text { treated with } \\
\text { TLR's prior to } \\
\text { administration }\end{array}$ & No & No & No & No & No \\
\hline $\begin{array}{c}\text { Systemic } \\
\text { TLR's } \\
\text { delivered in } \\
\text { absence of } \\
\text { vaccine }\end{array}$ & Yes & Yes & Yes & Yes & Yes \\
\hline $\begin{array}{c}\text { DC vaccine co- } \\
\text { administered } \\
\text { with systemic } \\
\text { TLR's }\end{array}$ & Yes & No & No & No & No \\
\hline
\end{tabular}

Table 3. 4 Summary table highlighting in-vivo assays with successful TLR agonists and combinations. "No" "Yes" responses are indicative of assay outcomes for specific parameters being measured. When testing lone TLR agonists, Yes refers to in-vivo responses that were measured above that seen with control groups, while No refers to responses that were not greater than controls. When testing TLR agonists in combination, Yes refers to in-vivo responses to vaccines that were additive and greater than that seen with either TLR agonist alone. 


\subsection{Discussion}

This chapter explored the use of TLR stimulation to enhance antigen specific $\mathrm{T}$ cell responses generated after vaccination with BM-DC based vaccines. It was shown that the use of MPL alone and in combination with either Resiquimod or Pam3Cys was capable of inducing increased maturation marker expression and cytokine production by BM-DCs cultured in-vitro. Vaccination protocols that utilized in-vitro TLR treated BM-DCs failed to generate antigen specific $\mathrm{T}$ cell responses that were superior to those seen with non-TLR treatment groups. Further study and investigation however showed that the administration of selected TLR ligands given systemically at the time of BM-DC vaccination (or shortly after) was capable of significantly enhancing the antigen specific $\mathrm{T}$ cell response above that seen in the absence of TLR stimulation. In addition, it was shown that the actual timing of subsequent TLR stimulation administration within the vaccination protocol tested was of relevance and suggested an alternative avenue with potential of influencing antigen specific $\mathrm{T}$ cell responses.

\subsubsection{TLR stimulation in-vitro using MPL and other lone TLR agonists}

Although a selection of synthetic TLR agonists were used for in-vitro testing on BM-DC cultures, emphasis was placed on the use of MPL in particular. MPL is one of only three TLR agonists that the FDA has approved for use in humans-in addition to bacillus Calmette Guerin (BCG) which targets TLR 2/4, and imiquimod that targets TLR 7 (Vacchelli et al., 2012). Recent approval has been afforded for the use MPL as an adjuvant for cancer vaccines (Casella and Mitchell, 2008; Cluff, 2009; Mata-Haro et al., 2007), with OK-432 a lyophilized bacterial preparation that stimulates TLR 4 being utilized for the treatment of cervical, gastric, and oral squamous cell carcinoma in humans (Hironaka et al., 2006; Kikkawa et al., 1993; Maehara et al., 1994; Okamoto et al., 1967; Okamoto et al., 2006). In addition, work already published highlighted TLR agonist combinations that contained a TLR 4 agonist were capable of inducing synergistic maturation, cytokine and T cell responses (Napolitani et al., 2005; Pufnock et al., 2011). 
Specific maturation and activation responses were evaluated for MPL and each additional agonist used with a view to select the agonist(s) exhibiting the most desirable outcomes for further investigative efforts. Since DCs have been reported to express the broadest range of TLRs, a variety of synthetic TLR agonists were selected for testing which included TLRs localized to the plasma membrane (TLR 2, TLR 4) and those localized to the endosomal membrane (TLR 3, TLR 7, TLR 9) (Blander and Sander, 2012; Napolitani et al., 2005). MPL, Pam3Cys, CpG and Resiquimod were used to stimulate BM-DC culture in-vitro and the maturation marker status, and cytokine production profile examined after 24-48 $\mathrm{h}$. The incorporation of LPS was for the use of a positive control sample. The upregulation of CD86 and CD40 surface marker expression was usually observed for all single TLR agonists tested.

Overall increased production of the pro-inflammatory cytokines IL-6, TNF- $\alpha$ and IL12 p40 was observed for all TLR agonists selected for testing, although some quantitative variation was seen based on the individual agonists used. Cytokine production initially remained the more consistent measure for BM-DC culture activation in these studies. The IL-12 production capacity of the TLR treated BM-DCs needed for strong Th1 responses, was measured given that DCs are considered a potent source of IL-12 production (Trinchieri, 2003). Construction of cytokine release profiles for BM-DC cultures treated with TLR agonists initially included the IL-12p40 subunit, however once further activation signals were provided by the incorporation of CD40 stimulation into experimental procedures, bioactive levels of the IL-12p70 subunit were then monitored.

Titration studies were carried out to determine the appropriate TLR agonist concentrations required for further experimentation. This was to ensure that TLR agonist doses could be selected that would not be confounded by saturation effects for maturation and cytokine production. This would also permit the identification of synergistic effects after in-vitro BM-DC cultures were treated using MPL combined with a second TLR agonist. These studies examined CD40 and CD86 surface maturation marker expression in addition to cytokine production for IL-12p40, IL-6 and TNF- $\alpha$ after in-vitro culture.

It was observed that MPL and the single TLR agonists tested (Pam3Cys, Resiquimod and $\mathrm{CpG}$ ) induced similar maturation and cytokine release responses - for CD40 and CD86 
expression and IL-12p40, IL-6 and TNF- $\alpha$ production. Predetermined MPL and lone TLR agonist concentration ranges were then utilized for subsequent experiments investigating the impact of dual TLR agonist stimulation upon in-vitro BM-DC cultures.

\subsubsection{TLR stimulation in-vitro using multiple TLR agonists}

It has been suggested that synergistic responses generated by professional antigen presenting cells such as DCs are a regular feature of viral and microbial infection given that pathogens typically carry more than one PAMP (Napolitani et al., 2005). Bacterial degradation can expose additional TLR ligands, a specific example being infection by flagellated gram-negative bacteria from which multiple PAMPs such as LPS and flagellin can be derived simultaneously (Blander and Sander, 2012). The stimulation of multiple TLRs via multiple PAMPs from invading microorganisms ensures efficient and generous immune responses occur when invasion and infection have taken place (Napolitani et al., 2005). These synergistic responses are non-random and very specific in that only selected agonists are capable of inducing such events (Zhu et al., 2008).

The additive (combined) and synergistic (greater than additive) effects of using more than one TLR agonist to stimulate mouse and human DCs had been reported by a number of groups (Bagchi et al., 2007; Bohnenkamp et al., 2007; Gautier et al., 2005; Napolitani et al., 2005; Roelofs et al., 2005; Trinchieri and Sher, 2007; Underhill, 2007; Warger et al., 2006; Whitmore et al., 2004; Zhu et al., 2008). Selected TLR agonists combinations had been shown to induce DCs to mature and generate proinflammatory cytokines in-vitro at concentration doses which were suboptimal when compared to using the single TLR agonist dose for DC stimulation (Napolitani et al., 2005; Warger et al., 2006). Of particular interest was the increased production of IL-12p70 when using TLR agonists in combination that required lower doses to achieve the same level of production than if using either TLR agonist alone. Increased IL-12p70 was reportedly due to enhanced NF-KB activity and nuclear translocation followed by the increased production of proinflammatory cytokines. 
According to the aforementioned studies, TLR agonist combinations that worked in synergy were those directed at TLR4 and TLR7/8 (Napolitani et al., 2005; Warger et al., 2006); TLR 4 and TLR 9, while those that did not synergise were TLR 3 and TLR 4; TLR 7/8 and TLR 9 (Napolitani et al., 2005). The combination of TLR 4 with TLR 7/8 agonists was also reported to increase the Th1 polarising capacity of DCs measured by the increased IFN- $\gamma$ production observed after in-vitro culture, while in the presence of additional IFN- $\gamma$ and CD40, measured IL-12p70 responses were further amplified (Napolitani et al., 2005).

An attempt was made to expose BM-DCs to the PAMPSs that may be present within the phagocytic environment during infection by the utilization of dual synthetic TLR agonist combinations to stimulate in-vitro BM-DC cultures. Results presented in this chapter showed that BM-DC cultures treated with either MPL (TLR 4) combined with either Pam3Cys (TLR 2) or with Resiquimod (TLR 7/8), were capable of generating potent maturation and cytokine IL-12p40 and TNF- $\alpha$ productive responses in-vitro. The increased cytokine and maturation results seen after combining TLR 4 with a TLR 7/8 agonist, was consistent with published studies (Napolitani et al., 2005; Warger et al., 2006) although the TLR4 agonist of choice for these prior investigations was LPS. Improved vaccine responses were also seen after combining TLR 4 and TLR 7 utilization (Kasturi et al., 2011). These findings provided some support for Napolitani's theory that superior DC responses could be achieved by targeting both an external (plasma membrane localized) and internal (endosomal membrane localized) TLR. It is important to note however that our work found efficient BM-DC stimulation was also achieved by targeting 2 separate TLR agonists found on the outer plasma membrane surface of the BM-DC by combining MPL with Pam3Cys.

Intracellular TLR signalling pathways employ the use of characteristic molecules and mediators that are specific for different signalling pathways. Two such key players are the MyD88 (myeloid differentiation primary response protein 88) and TRIF (TIR-domaincontaining adaptor protein inducing IFN- $\beta$ ) adaptor molecules which feature consistently in specific TLR signalling cascades. The activation of MyD88/TRIF independent signalling cascade induces MAPK signalling pathways that cause NF- $\kappa \mathrm{B}$ activation to 
induce IL-6, IL-12, TNF- $\alpha$ and (pro) IL-1 $\beta$ production. Induction of the MyD88/TRIF signalling cascade causes the activation of IRF3 (Interferon regulatory factor 3 ) that leads to Type 1 IFN production. TRIF promotes inflammasome activation that leads to the final conversion of (pro) IL-1 $\beta$ to IL-1 $\beta$.

The ligation of TLR 4 by MPL or LPS can trigger signalling with both TRIF dependent and MyD88/TRIF independent signalling pathways, whereas Pam3Cys exerts its effects through the MyD88 dependent/TRIF independent pathway. Downstream effects of targeting the MyD88 signalling pathway include the production of proinflammatory cytokines such as IFN- $\beta$, IL-12, IL-6 and TNF- $\alpha$ while targeting of the TRIF dependent signalling pathway induces the upregulation of CD86 and the production of IP-10 (Zhu et al., 2008). IP-10 (CXCL10) is a chemokine produced by DCs important for protective $\mathrm{CD}^{+} \mathrm{T}$ cell responses and is important for antimumour, antiviral and antifungal activities (Enderlin et al., 2009; Pertl et al., 2001; Uicker et al., 2005; Weinzierl et al., 2008) and can be triggered by TLR stimulation (Majumder et al., 2012; Zhu et al., 2008). IP-10 binds to the seven transmembrane $\mathrm{G}$ protein-coupled receptor CXCR3 which is expressed on $\mathrm{T}$ cells and is important for chemotaxis (Dar and Knechtle, 2007; Taub et al., 1996).

The successful use of MPL with Pam3Cys for inducing additive maturation and cytokine production effects is proposed to be resultant of the simultaneous targeting of these separate signalling pathways. The induction of the MyD88/TRIF dependent and TRIF independent signalling pathway by MPL is further amplified by the engagement of Pam3Cys that utilizes the MyD88 signalling pathway. It is important to note that the use of BCG (bacillus Calmettte-Guerin) to treat bladder cancer in humans is highly dependent on TLR 2 and TLR 4 mediated maturation of DCs (Tsuji et al., 2000; Uehori et al., 2005).

Warger et al (2006) proposed that the combination of TLR agonists that use the MyD88 adaptor molecule-signalling pathway with an agonist that uses a MyD88 independent pathway would induce synergistic stimulatory responses. The MyD88 signalling pathway had been shown by Yamamoto et al in 2002 to act independently from the TRIF pathway and that TLR3 acts independently of the MyD88 pathway. Warger et al showed that MyD88 vs TRIF dependent and independent signalling pathways could synergize to increase maturation marker expression for CD86, CD80 and CD40, and the increased 
cytokine production for IL-6 and IL-12p70. The combinations of TLR7/8 with TLR4 and TLR7/8 with TLR4 were shown to increase the DC production of IL-6 and RANTES. DC maturation was induced more rapidly when using these specific TLR agonist combinations. A key feature of this work was to demonstrate that DCs treated with TLR agonist combination in-vitro were able to overcome the immunosuppressive effects of $\mathrm{T}$ regulatory cells (Warger et al., 2006). A separate study showed that TLR 3 and TLR 7 increased production of IL12p70 and Type 1 IFNs (Gautier et al., 2005).

Other examples of TLR agonist combinations tested that included plasma membrane localized versus endosomal localized TLRs were Pam3Cys (TLR 2) and Resiquimod (TLR 7/8), CpG (TLR 9) and Pam3Cys (TLR 2) (see Figure 3.5). Although combining Pam3Cys and Resiquimod achieved an additive cytokine response for IL-12p40 and TNF- $\alpha$ production, the measure of this cytokine response was inferior to that seen for the MPL and Pam3Cys, and MPL and Resiquimod combinations. In contrast, CpG and Pam3Cys together did not produce additive cytokine production for IL-12p40, IL-6 and TNF- $\alpha$ (Figure 3.5). The most effective maturation inducing and cytokine producing combinations and those containing MPL were selected for further investigation.

The results in initial portion of this chapter showed overall that some TLR agonist combinations were able to induce enhanced DC stimulation of BM-DC cultures, while others were not, indicative of definitive non-random synergistic responses consistent with the literature at the time (Napolitani et al., 2005; Warger et al., 2006; Zhu et al., 2008). The combination of MPL with other TLR agonists always appeared to enhance BM-DC stimulation and it was suggested this may be due to signalling via the both the MyD88 adaptor pathway working in co-operation with the indirectly connected TRIF signalling pathway. It was suggested that IL-12p40 levels were controlled primarily by the triggering of TLR signalling pathways that utilized MyD88, while IL-12p35 levels were controlled by the TRIF pathways TLR signalling pathways; therefore synergistic production of IL12 p70 in mouse and humans requires co-operation between both these pathways.

In summary, the combination use of synthetic agonists for TLR 4 together with TLR 7/8 also induced maturation and increased cytokine production, and so did TLR 4 and TLR 2/6. The integration of signalling pathways triggered by multiple PAMPs (mimicked by the 
utilization of dual synthetic TLR agonist combinations) which amplifies the impact of the original signals, ensures robust immune responses are generated that enable destruction and eradication of the invading microorganism. It is important to note however that our work also demonstrated that using three synthetic TLR agonists together did not enhance BM-DC maturation or activation. The combination of three TLR agonists failed to produce synergistic stimulation and if anything, an exhaustive DC profile was generated where the resultant maturation status and cytokine release profile was no greater than that seen after using a single or dual TLR agonist combination (data not shown). The term exhaustive is used here to describe DCs that have become refractory to further TLR simulation however it has been shown that exhausted DCs are capable of responding to $\mathrm{T}$ cell products and signals such as CD40L, IFN- $\gamma$ (Abdi et al., 2012).

\subsubsection{Amplification of cytokine responses using CD40L after dual TLR stimulation}

The CD40 receptors are members of the TNF molecular superfamily, a large family of receptors located on a variety of cell types that include immune cells such as DCs. The triggering of these receptors by their respective ligands can cause cell death, inflammation or even promote cell survival. It has been reported that activated T cells increase IL-12p70 production by DC via CD40L and CD40 receptor interactions (Schulz et al., 2000). Ligation of the CD40 receptors on the actual DC cell surface leads to trimerization of the receptor followed by the recruitment of multiple intracellular TRAFs (TNF-receptor associated factors) necessary for receptor coupling to downstream signalling mediators (Summers deLuca and Gommerman, 2012). Once the CD40 receptor engages with a CD40L, signalling occurs via TRAF1, TRAF2, TRAF3, TRAF5 and TRAF6 molecules with downstream effects causing activation of $\mathrm{p} 38$, AKT, PKC (protein kinase C), JNK (JUN N-terminal kinase), classical and alternative nuclear factor pathways (NF-KB) (Summers deLuca and Gommerman, 2012). Upon TLR stimulation of DCs, expression of CD40 is upregulated and CD40 signalling is induced upon ligation with a CD40L such as a $\mathrm{CD}^{+} \mathrm{T}$ cell. CD40 signalling induces IL-12 production, CD70, CD86, 4-1BBL, OX40L and GITRL upregulation (Summers deLuca and Gommerman, 2012). To employ the use of CD40 signalling events to enhance BM-DC function, CD40L expressing fibroblast cell 
lines were utilized for in-vitro co-culture preparations and functioned as external sources of CD40L. Levels of IL-12p40, IL-12p70 and TNF- $\alpha$ cytokine production by the treated BM-DCs were measured in response.

The heterodimeric IL-12p70 cytokine is made up of the IL-12p40 and IL-p35 chain subunits, which combine together to make the bioactive cytokine (Gautier et al., 2005). The IL-12p40 subunit is also capable of combining with the IL-12p19 to produce the IL-23 cytokine. Human and mouse studies had previously shown that efficient IL-12p70 production required a secondary signal that was additional to that provided by TLR stimulation (Schulz et al., 2000; Snijders et al., 1998). Early studies presented in this chapter measured the production of IL-12p40 rather than Il-12p70 production largely due to the absence of CD40 ligation that would provide the optimal induction for the treated DCs. The incorporation of CD40L stimulation into culture protocols was conducted in an effort to determine whether IL-12p70 cytokine production could be further enhanced following dual TLR stimulation of in-vitro BM-DC co-cultures using CD40L expressing fibroblastic cell lines as described in section 5.2.4.

What was observed in these studies was that early production of IL-12p70 in the presence of CD40 ligation was greater than that seen in the absence of CD40 stimulation using single TLR stimulated BM-DCs. IL-12p70 production was further enhanced in CD40L treated BM-DC co-cultures if either MPL and Resiquimod, or MPL and Pam3Cys were used in combination. However by $22 \mathrm{~h}$, the advantage of early IL-12p70 production after CD40L stimulation for dual treated TLRs was lost since BM-DC co-cultures treated with a lone TLR agonist in the presence of CD40 stimulation had produced levels of IL-12p70 that were similar. Improved production of IL- 6 was also seen at the early timepoint $(6 \mathrm{~h})$ after treatment with single and dual TLR agonists in the presence of CD40 stimulation, however by $22 \mathrm{~h}$ this advantage was lost as this IL-6 production was not sustained and that being produced by cultures without CD40L was greater in all cases. The production of IL12 p40 was also improved by CD40 stimulation at earlier timepoints, however was rapid and no additional advantage was seen between using single versus dual TLR agonists.

These results showing improved early IL-12p70 production after treatment with single and dual TLR stimulation appear consistent with those obtained by Napolitani et al (2005) after 
lone and dual TLR agonist treatment of MoDCs in the presence of CD40L. However Napolitani's measures of amplification were undertaken after $48 \mathrm{~h}$ while the latest timepoint used for our experiments was $22 \mathrm{~h}$. In addition, IL-6 and IL-12p40 measures were not undertaken in their study, however the addition of exogenous IFN- $\gamma$ and was carried out in separate treatment protocols and used for comparative purposes. As mentioned previously, the advantage of using dual TLR agonist stimulation in addition to CD40 ligation was lost given that IL-12p70 production levels by single TLR treated BMDC and CD40L co-cultures was similar to that seen with the dual TLR treated groups. Further measures of Il-12p70 cytokine production taken at $48 \mathrm{~h}$ in our experiments may have allowed enough time for additional amplification events to have taken place to generate $\mathrm{pg} / \mathrm{ml}$ values consistent with those seen in the Napolitani study.

Type 1 IFNs are important for IL-12p70 production while partially inhibiting IL-12p40 production (Trinchieri and Sher, 2007). Therefore TLR agonist combinations that favour production of Type 1 IFNs via the MyD88 independent/TRIF dependent signalling pathways may limit IL-12p40 production yet promote IL-12p70 generation.

Reported TLR agonist combinations capable of inducing synergistic production levels of IL-12p70 included combining TLR 3 with either TLR 7/8, TLR 9 or TLR 11, in addition to combining TLR 4 with TLR 7/8, TLR 9 and TLR 11 (Bekeredjian-Ding et al., 2006) (Gautier et al., 2005; Napolitani et al., 2005; Roelofs et al., 2005). What was interesting to note was the moderate impact on IL-12p40, IL-6, TNF- $\alpha$ and IL-12p70 production when combining a TLR 2 agonist together with a TLR 3, TLR 4 or TLR 9 agonist (Bafica et al., 2006; Bafica et al., 2005; Gautier et al., 2005) which differed with our findings. It has been proposed that the co-adaptor molecules TIRAP (otherwise known as MAL, TIR-domaincontaining adaptor protein) and TRAM (TRIF-related adaptor molecule) may allow further distinction between finer details of the intracellular signalling pathways (Trinchieri and Sher, 2007). Additionally, it was noted that IRF3 utilized by the TRIF associated pathway can suppress IL-12p35 mRNA production (Dahlberg et al., 2006). The location of specific TLRs may possibly impact on their synergistic ability given the vast array of accessory molecules that require contact with specific components of the signalling pathways (Honda et al., 2005) in addition to the impact of simultaneous ligation of the different TLRs and 
potential effects of negative regulatory mechanisms that govern TLR responses (Liew et al., 2005).

\subsubsection{Antigen specific $T$ cell responses after TLR treatment of BM- DCs}

The utilization of selected dual TLR agonist combinations for BM-DC stimulation to generate functional $\mathrm{T}$ cell populations was tested in-vitro by the use of $\mathrm{T}$ cell proliferation assays. This was in an effort to explore $\mathrm{T}$ cell stimulation capability using both protein and peptide antigens in separate experiments, the latter to eliminate any confounding factors associated with protein antigen processing by the TLR stimulated DC.

Our work showed that both peptide and protein carrying BM-DCs treated with a single TLR agonist appeared capable of stimulating $\mathrm{CD} 8^{+}$antigen specific $\mathrm{T}$ cell proliferative responses in-vitro that were increased when compared to controls. The $\mathrm{T}$ cell proliferative response seen with BM-DC cultures treated with dual TLR agonist combinations also appeared to improve the $\mathrm{T}$ cell presentation capability even further when tested in-vitro for the selected TLR agonist combinations chosen for investigation-MPL \& Resiquimod and MPL with Pam3Cys.

These results were partially consistent with the published literature, which showed that dual TLR treated BM-DCs co-cultured with $\mathrm{CD}^{+}$and $\mathrm{CD} 8^{+}$antigen specific $\mathrm{T}$ cells were able to induce superior $\mathrm{T}$ cell responses, induce enhanced cytokine production and confer greater resistance to the Treg mediated suppression (Warger et al., 2006). The TLR agonist combinations tested by Warger et al in this particular study were PolyIC and Resiquimod both alone and in combination together. Warger et al also demonstrated enhanced antigen specific $\mathrm{T}$ cell responses, cytotoxic killing and IFN- $\gamma$ production responses after the in-vivo stimulation using BM-DCs pretreated with the TLR agonist combination PolyIC and Resiquimod. Although the PolyIC with Resiquimod combination was not tested in our studies, the TLR agonist combinations we did select based on the in-vitro maturation and cytokine production data, yielded results consistent with their selection as effective BMDC stimulators and $\mathrm{T}$ cell inducers. It should be noted that not all TLR agonist 
combinations were effective $\mathrm{T}$ cell inducers in this context and that only specific combinations of TLR agonists were able to also improve the presentation and processing capacity of in-vitro TLR BM-DCs (data not shown).

Antigen specific $\mathrm{T}$ cell responses were next explored in-vivo after the optimisation of a vaccination protocol designed to test the utility of this work in the context of immunotherapeutic vaccination strategies. It was endeavoured to use the information generated by initial in-vitro studies testing TLR treatment of BM-DC cultures to generate improved vaccination outcomes with regards to generating antigen specific $\mathrm{T}$ cell responses.

The failure of TLR treated vaccine BM-DCs to generate antigen specific $\mathrm{T}$ cell responses that were improved above that seen in the absence of TLR treatment was inconsistent with published work. A study by Huck et al et al (2008) had showed that increased antigen specific T cell response were observed after using LPS treated BM-DCs for vaccination. A possible reason for this failure may have been the exhaustion of the TLR treated BM-DC cultures after a lengthy in-vitro incubation period of $22 \mathrm{~h}$. To address this possibility a shorter TLR treatment time of $16 \mathrm{~h}$ was adopted however similar results were seen even after this change (data not shown). The route of administration was also tested in an attempt to improve antigen specific responses after vaccination with TLR treated BM-DCs however no improved responses were seen even after intravenous or subcutaneous administration (data not shown).

This inability of TLR pretreated vaccine BM-DCs to generate increased antigen specific T cell responses in-vivo was unexpected. When LPS is employed to deliver stimulation to vaccine BM-DCs in-vitro, strong in-vivo antigen specific $\mathrm{T}$ cell responses have been seen (Huck et al., 2008). With MPL developed as the non-toxic form of LPS, it was expected that if using MPL as an adjuvant the in-vivo antigen specific $\mathrm{T}$ cell responses would be observed however be decreased in magnitude. It was surprising to find that in-vitro MPL treated BM-DC vaccines were unable to improve in-vivo responses even though TLR stimulation of our BM-DC cultures was sufficient to induce phenotypic and functional properties in-vitro. 
It was possible that the combination of in-vitro TLR stimulation with $i v$ administration of recipients was leading to over-saturation effects resulting in the inability of the administered vaccine BM-DCs to present antigen to antigen specific $\mathrm{T}$ cell populations within secondary lymphoid organs. In addition, it may have been possible that the TLR stimulation was shutting down the antigen processing and presentation capacity of the treated BM-DCs in-vivo due to over-saturation effects. Although this may seem to contrast with our T cell proliferation studies that showed TLR treated BM-DCs were capable of antigen presentation, the added trauma of being administered $i v$ may have caused overstimulation and interfered with antigen processing and presentation within the vaccine recipients.

After examining the fate of BM-DCs after intravenous administration, it was observed that BM-DC retrieved from the spleens of recipients for different TLR treatment groups exhibited a similar maturation status regardless of the in-vitro manipulation protocol followed. This indicated that BM-DCs were maturing after $i v$ injection on route to the spleen. It was also observed that MPL administered systemically was able to induce maturation of endogenous DC populations and that the use of MPL to treat BM-DCs in vitro was ineffective at inducing maturation of endogenous DC populations in the spleen. The focus of this study became the use of systemic TLR delivery in an effort to target resident DC populations for the effective induction of relevant antigen specific $\mathrm{T}$ cell responses.

The impact of systemic Pam3Cys co-administration on antigen specific $\mathrm{T}$ cell responses after vaccination with protein treated BM-DCs was also interesting. It would appear that kinetic influences may interfere with the protein antigen processing capability of the vaccine BM-DCs. The delayed administration of Pam3Cys can occur up to $1 \mathrm{~h}$ post vaccination before negative effects on antigen specific $\mathrm{T}$ cell responses are evident. The antigen specific $\mathrm{T}$ cell response seen when Pam3Cys is given $1 \mathrm{~h}$ post vaccination is superior to that when co-delivered with the vaccine at time 0. It is possible that Pam3Cys works potently as a TLR stimulator that shuts down the antigen processing and presentation capacity of the administered vaccine BM-DCs immediately, and possibly does the same to endogenous DC populations. 
MPL remained the TLR agonist of choice due to its current clinical utility in addition to its ability to target both arms of the TLR signalling adaptor pathways-MyD88 and TRIF signalling pathways. The systemic delivery of MPL was tested in-vivo by coadministration with protein loaded BM-DCs at the time of vaccination. Our results clearly showed that systemic delivery of MPL was able to significantly improve the antigen specific $\mathrm{CD}^{+} \mathrm{T}$ cell response seen in the blood one week post vaccination.

\subsubsection{Conclusions}

- MPL worked well in combination with Resiquimod and Pam3Cys to mature and activate BM-DCs in-vitro

- Antigen loaded BM-DCs treated with TLR stimulation in-vitro alone or in combination did not increase antigen specific $\mathrm{T}$ cell responses after vaccination.

- BM-DCs treated with TLRs in-vitro and administered intravenously all displayed a similar maturation status after reaching the spleen regardless of the in-vitro manipulation protocol used.

- Systemic TLR stimulation co-delivered with the BM-DC vaccine was able to increase the antigen specific $\mathrm{CD}^{+} \mathrm{T}$ cell response in the blood one-week post vaccination. 
Chapter 3: TLR stimulation enhances antigen specific responses to DC-based vaccines 161 


\section{Chapter 4:}

\section{The role of Langerin ${ }^{+} \mathrm{CD8} \alpha^{+}$DCs in antigen processing and $T$ cell priming activities}

In part published in:

Petersen, T. R., Sika-Paotonu, D., Knight, D. A., Dickgreber, N., Simkins, H. M., Hermans, I. F. Exploiting the Role of Endogenous Lymphoid-Resident Dendritic Cells in the Priming of NKT Cells and CD ${ }^{+}$T Cells to Dendritic Cell-Based Vaccines. PLoS ONE 6 March 2011, e17657. 


\subsection{Introduction}

Results shown in the previous chapter suggest that antigen is being transferred from injected antigen loaded vaccine BM-DCs to resident DC populations. Previous work had provided evidence for transfer of antigen (Inaba et al., 1990a; Inaba et al., 1990b; Ma et al., 2012; Suleman et al., 2011; Yewdall et al., 2010) and results from our laboratory at the time this work was done using allogeneic BM-DCs for vaccination protocols also provided support for this concept (Petersen TR personal communication) What remained unclear however was which host cell type was the recipient of the transferred antigen and became the subsequent antigen specific $\mathrm{T}$ cell response primer.

Within the spleen, $\mathrm{CD} 8 \alpha^{+} \mathrm{DCs}$ have been shown to conduct superior cross-presentation activities when compared with other DC subsets and they were most likely to be a strong candidate in this case. Other results from our laboratory also suggested that within the $\mathrm{CD} 8 \alpha^{+} \mathrm{DC}$ population it is the langerin ${ }^{+} \mathrm{DC}$ subset that cross-present soluble antigens most efficiently (Farrand et al., 2009). It was therefore feasible to suggest that this splenic DC subset may be the actual recipients of transferred antigen and the subsequent producer of the antigen specific $\mathrm{T}$ cell response after $\mathrm{T}$ cell priming.

This chapter addresses the possibility that langerin ${ }^{+}$splenic DCs are involved in the generation of antigen specific $\mathrm{T}$ cell responses to BM-DC-based vaccines with specific emphasis on vaccines co-delivered with TLR stimulation. Two transgenic mouse strains were employed to uncover the nature and determine the role of the major splenic DC subsets after BM-DC vaccination: the langerin-EGFP mice were utilized to examine the role of the major DC subsets found in the spleen in generating the OVA-specific T cell response seen after BM-DC vaccination, with particular attention being focused on the langerin ${ }^{+} \mathrm{CD} 8 \alpha^{+} \mathrm{DC}$ subset. These mice contain DNA inserts which encode the EGFP protein within the langerin gene locus allowing the visualization of langerin ${ }^{+}$cells within the spleen using flow cytometric analysis to track the EGFP signal found in langerin containing cells. 
To examine the role of the langerin ${ }^{+} \mathrm{CD} 8 \alpha^{+} \mathrm{DC}$ subset on the induction of $\mathrm{CD} 8^{+} \mathrm{T}$ cell response, langerin-DTREGFP knock-in mice were employed for further studies. LangerinDTREGFP mice carry the DNA sequence for the human diphtheria toxin (DT) receptor under control of the langerin promoter. This allows the selective depletion of langerin expressing cells upon the administration of DT providing a means for closer inspection of the function of the langerin ${ }^{+} \mathrm{CD} 8 \alpha^{+} \mathrm{DC}$ subset in cross presentation and $\mathrm{T}$ cell priming activities. These mice also contain DNA inserts which encode the EGFP protein within the langerin gene locus allowing the visualization of langerin ${ }^{+}$cells within the spleen using flow cytometric analysis to track the EGFP signal found in langerin containing cells.

Langerin-DTREGFP knock-in mice were also utilized for experimental work presented in this chapter. These mice carry either an IRES-EGFP or an IRES-DTR-EGFP cassette knocked-in to the sixth exon, in the 30-untranslated region of langerin (Kissenpfennig and Malissen, 2006). DT is a heterodimeric protein where binding of the $\beta$ subunit to the heparin-binding EGF-like growth factor (H $\beta$-EGF) precursor found on the surface of mammalian cells, facilitates entry of DTA into the cell. Once entry into the cell has taken place, DTA works to inhibit protein biosynthesis and induces a rapid cell death. The rodent homologue of HB-EGF precursor does not bind to the $\beta$ subunit of DT, which prevents internalization of DTA and renders mice resistant to DT (Saito et al., 2001). This is why the human DT receptor components are utilized in these transgenic mice. The selective depletion of the langerin ${ }^{+} \mathrm{CD} 8 \alpha^{+}$DCs after ip administration of DT permitted closer examination of their role in antigen acquisition and $\mathrm{T}$ cell priming activities (Kissenpfennig and Malissen, 2006).

The results presented in this chapter show the work carried out to define the role of the langerin ${ }^{+} \mathrm{CD} 8 \alpha^{+}$splenic DC subset for in-vivo antigen processing and $\mathrm{T}$ cell priming activities following BM-DC vaccination. The adjuvant activity of systemic TLR stimulation for improving antigen specific $\mathrm{T}$ cell responses after vaccination was also investigated. 


\subsubsection{Aims}

A series of studies was carried out to determine the role (if any) the host splenic langerin ${ }^{+} \mathrm{CD} 8 \alpha^{+} \mathrm{DC}$ subset played in the cross presentation and $\mathrm{T}$ cell priming activities in response in recipients vaccinated with our in-vitro manufactured BM-DC vaccine. Our hypothesis was that the langerin ${ }^{+} \mathrm{CD} 8 \alpha^{+}$host splenic DC subset was responsible for driving the antigen specific $\mathrm{T}$ cell response measured in the blood and that this process could be made more effective by the use of adjuvant activity targeted at this DC subset.

The specific objectives were:

- To examine the role of langerin ${ }^{+} \mathrm{CD} 8 \alpha^{+} \mathrm{DCs}$ in antigen processing and presentation activities after vaccination with antigen loaded BM-DCs

- To explore the role of langerin ${ }^{+} \mathrm{CD} 8 \alpha^{+} \mathrm{DCs}$ in immunization strategies utilizing TLR stimulation 


\subsection{Results}

\subsubsection{The existence of langerin expressing $C D 8 \alpha^{+} \mathrm{DC}$ sub populations in the mouse spleen}

Langerin-EGFP mice were used to examine the phenotype of DC subsets found in the spleen, with particular attention being focused on the langerin ${ }^{+} \mathrm{CD} 8 \alpha^{+} \mathrm{DC}$ subset. The expression of the EGFP protein from the langerin gene locus allowed the visualization of langerin ${ }^{+}$cells within the spleen using flow cytometric analysis.

The gating strategy used to identify the live major splenic DC populations is shown in Figure 4.1 A while histograms in Fig 4.1 B reveal the baseline expression levels of CD103, CD24 and CD205 on the major splenic DC subsets isolated using this protocol from langerin-EGFP mice. It is clear that langerin ${ }^{+} \mathrm{CD} 8 \alpha^{+} \mathrm{DC}$ subsets express $\mathrm{CD} 24$ and CD205. This experiment was conducted by laboratory colleague Dr Troels Petersen.

Maturation marker expression of the major splenic DC populations was also explored in langerin-EGFP mice (Figure 4.2). The bar graphs in Figure 4.2 C illustrate the differences in CD86 and CD40 expression between each of these splenic DC subsets with the baseline expression of CD40 low in all subsets with a higher baseline expression of CD86 on the langerin ${ }^{+} \mathrm{CD} 8 \alpha^{+} \mathrm{DC}$ subset.

A comparison between the langerin-EGFP and langerin-DTREGFP mouse strains was also undertaken which showed that in the absence of stimulation higher baseline levels of CD86 were found on langerin ${ }^{+} \mathrm{CD} 8 \alpha^{+} \mathrm{DCs}$ when compared to other DC subsets and was evident for each of the transgenic mice strains. CD40 expression did not differ significantly across all splenic DC subsets found in langerin-EGFP and langerinDTREGFP mice however CD86 expression was found to be higher in the langerin ${ }^{-} \mathrm{CD} 8 \alpha^{+}$ DC subset of the langerin-DTREGFP mouse strains (data not shown). 


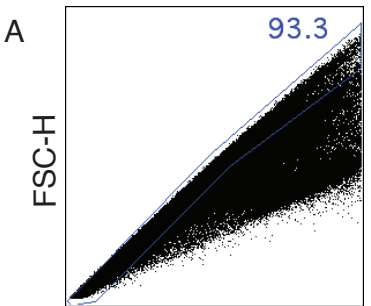

FSC-A

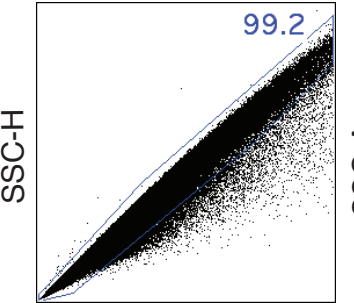

SSC-A

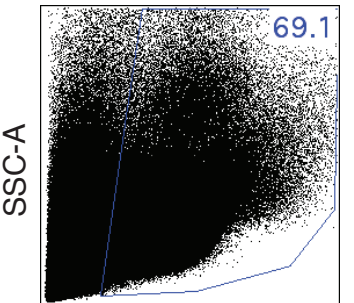

FCS-A

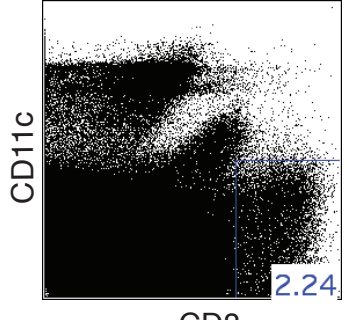

CD8

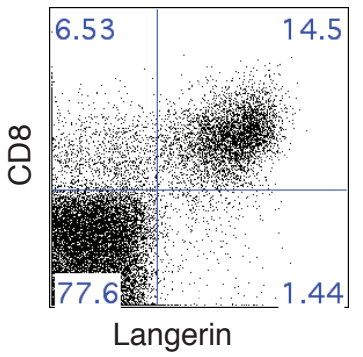

B
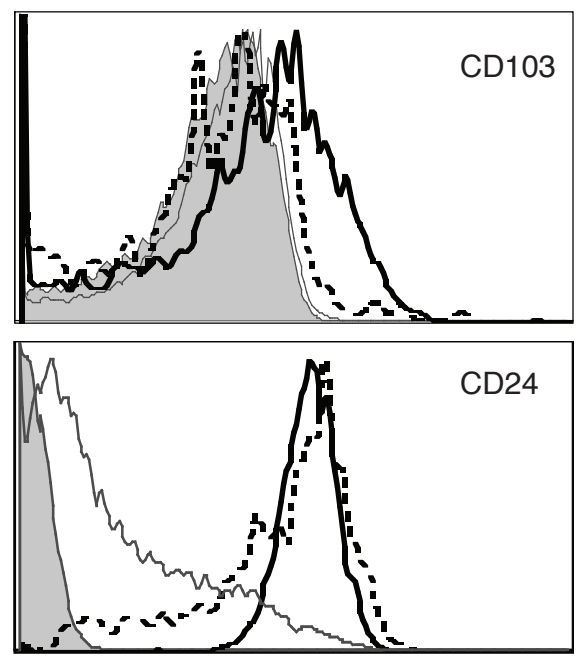

$$
\begin{array}{r}
\text { unstained (fmo) } \\
\text { langerin+ CD8 } \alpha+D C= \\
\text { langerin- CD8 } \alpha+D C= \\
\text { CD8 } 8 \text { - DC = }
\end{array}
$$

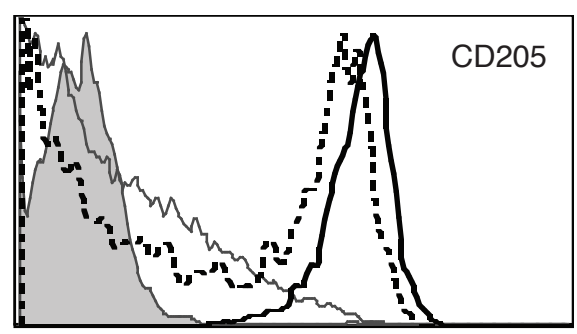

Figure 4.1 Characterisation of the major splenic DC subsets in langerin-EGFP mice. (A) Dot plots show the gating strategy used to identify major splenic DC populations in langerin-EGFP recipients. (B) Histograms show expression of CD103, CD24 and CD205 in $\mathrm{CD} 11 \mathrm{c}$ high splenic DC populations in langerin-EGFP mice expressed as Mean Fluorescence Intensity (MFI). This experiment was performed by Dr Troels Petersen. Results shown are from one experiment. 


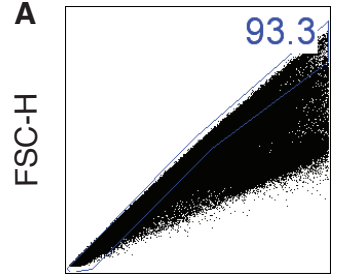

FSC-A

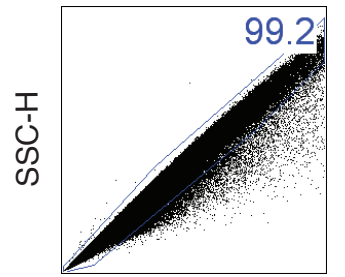

SSC-H

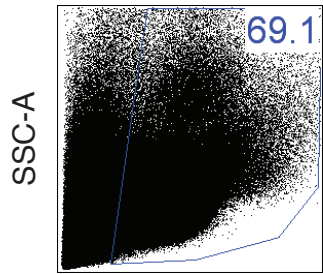

FSC-A

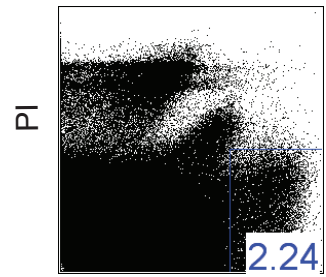

CD11c

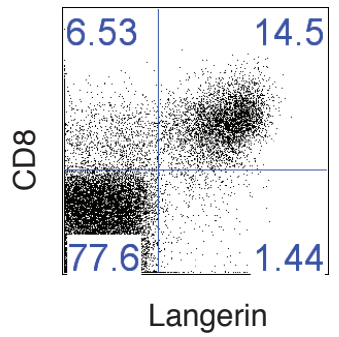

B

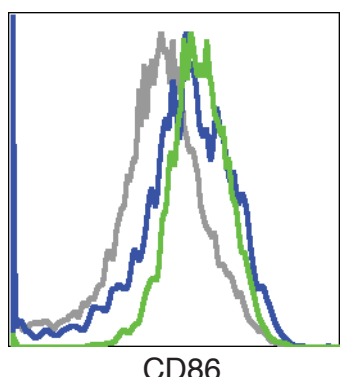

CD86
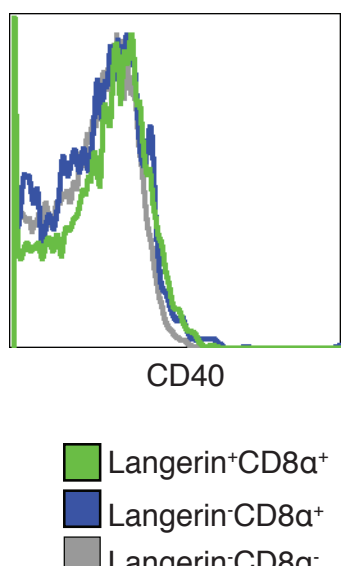

C
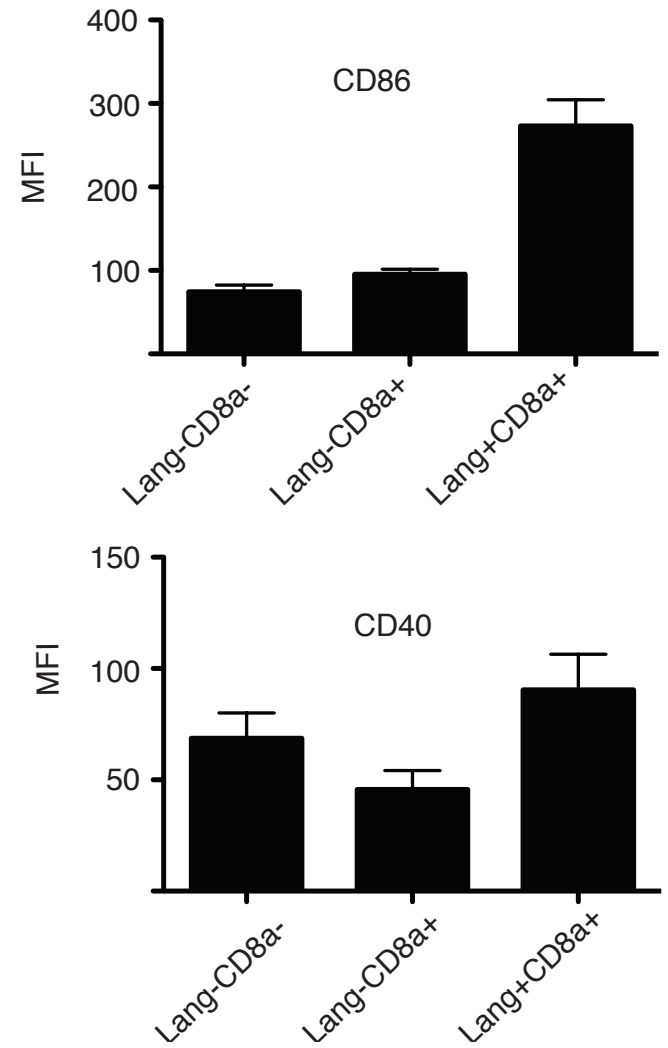

Figure 4.2 Maturation marker expression of the major splenic DC subsets in langerin-EGFP mice. (A) The typical gating strategy utilised to identify major splenic DC populations in langerin-EGFP recipients. (B) Histograms showing expression of CD86 and $\mathrm{CD} 40$ in CD11c high splenic DC populations in langerin-EGFP mice. (C) Bar graphs illustrate differences expressed as Mean Fluorescence Intensity (MFI) for CD86 and CD40 expression between the major splenic DC subsets identified in langerin-EGFP mice. Results shown are from one experiment $(n=3)$. 


\subsubsection{Selective depletion of langerin $^{+} \mathrm{CD}^{+} \alpha^{+} \mathrm{DCs}$ in langerin-DTREGFP recipients does not adversely affect other splenic DC populations}

To examine the role of the langerin ${ }^{+} \mathrm{CD} 8 \alpha^{+} \mathrm{DC}$ subset on the induction of $\mathrm{CD} 8^{+} \mathrm{T}$ cell response, langerin-DTREGFP knock-in mice were employed for further studies. LangerinDTREGFP mice carry the DNA sequence for the human diphtheria toxin (DT) receptor under control of the langerin promoter. This allows the selective depletion of langerin expressing cells upon the administration of DT providing a means for closer inspection of the function of the langerin ${ }^{+} \mathrm{CD} 8 \alpha^{+} \mathrm{DC}$ subset in cross presentation and $\mathrm{T}$ cell priming activities. These mice also contain DNA inserts, which encode the EGFP protein within the langerin gene locus allowing the visualization of langerin ${ }^{+}$cells within the spleen using flow cytometric analysis to track the EGFP signal found in langerin containing cells.

To examine the impact of langerin depletion on splenic DC populations, langerinDTREGFP recipients were treated with 2 separate intraperitoneal injections of $350 \mathrm{ng}$ of DT given $24 \mathrm{~h}$ apart on day -2 and day -1 prior to vaccination, with the control groups also treated at the same time points with PBS. Spleens were harvested at different time points following the DT or PBS treatment regime in order to track the specific depletion seen in the langerin ${ }^{+} \mathrm{CD} 8 \alpha^{+} \mathrm{DC}$ population. The subsequent replenishment of this population over time was also tracked by flow cytometry using antibodies to identify CD $11 \mathrm{c}^{+}$splenic DC populations and subsets. Flow cytometric analysis was used to gate on $\mathrm{CD} 11 \mathrm{c}^{+}$populations and $\mathrm{CD} 8 \alpha^{+}$langerin expression examined.

Figure 4.3 A shows the dot plots generated after using the general gating strategy to identify the live major splenic DC populations in langerin-DTREGFP recipients. These dot plots track the specific depletion levels of langerin ${ }^{+}$cells in the major splenic DC subsets after treatment with DT toxin using the method specified in this protocol. 

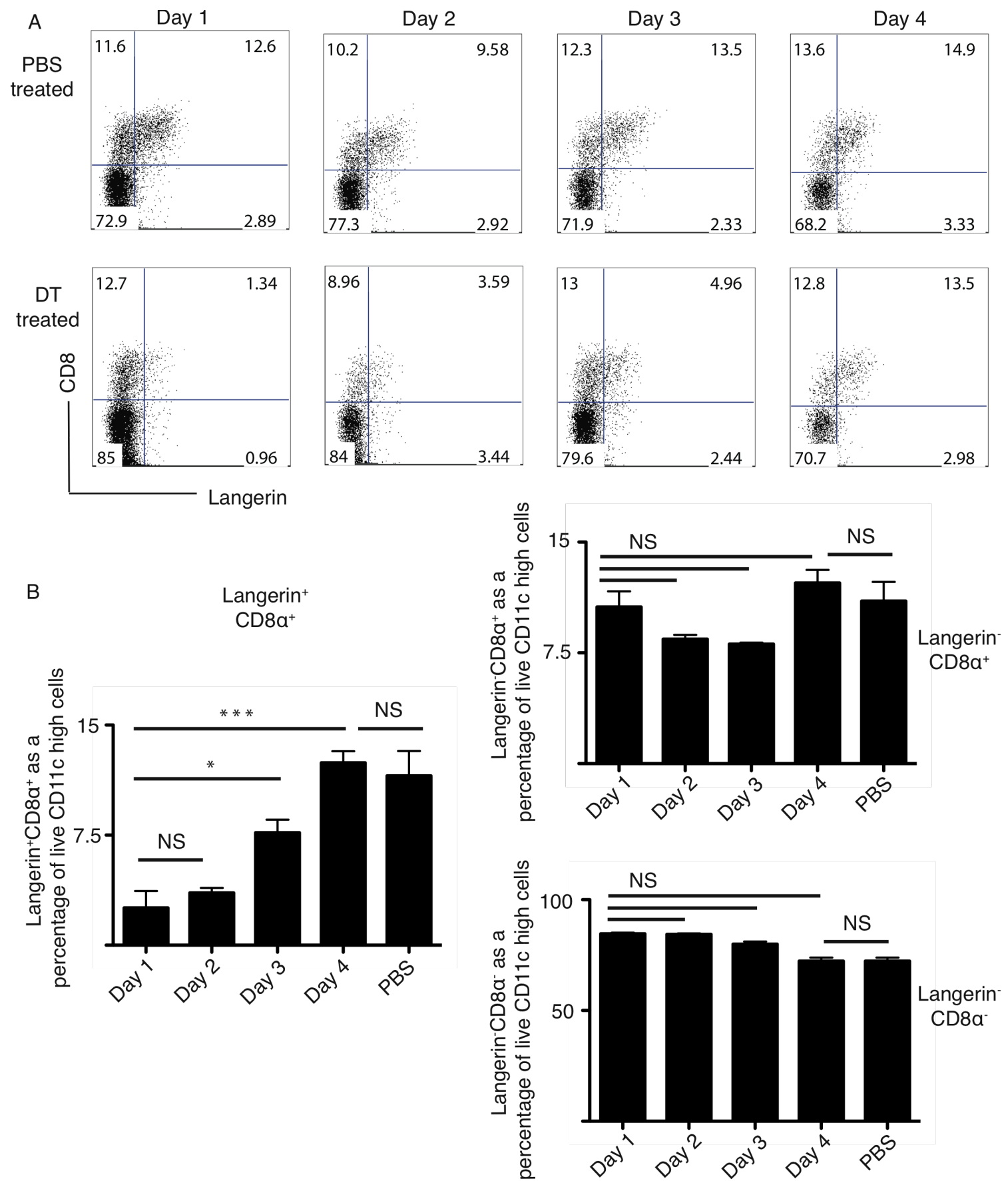

Figure 4.3 Diphtheria toxin treatment of langerin-DTREGFP recipients depletes langerin ${ }^{+} \mathrm{CD} \alpha^{+}$DCs. (A) Dot plots show major CD11c ${ }^{\text {high }}$ splenic DC populations in DT treated langerin-DTREGFP mice on day 1, 2, 3 and 4 after single doses administered on days -2 and -1 with 350ng DT injected ip. Representative plots for each treatment group are shown plus PBS treated controls. (B) Bar graphs show quantification of major CD11c high splenic DC populations after treatment with diphtheria toxin (DT) 1, 2, 3 and 4 days post DT administration in langerin-DTREGFP recipients $(n=3)$. Statistical significance was determined using a 1-way ANOVA test and Tukey's post-test where ${ }^{*} p=0.01-0.05$, $* * \mathrm{p}=0.001-0.01, * * * \mathrm{p}<0.001, \mathrm{NS}=$ not significant. Results shown are from one experiment. 
Bar graphs in Figure 4.3 B show levels of specific splenic DC subsets expressed as a percentage of live CD11c hi splenocytes. Dot plots from flow cytometric analysis results showed that 1-2 days after administration of the $2^{\text {nd }}$ dose of DT, langerin-DTREGFP recipients were depleted of langerin ${ }^{+} \mathrm{CD} 8 \alpha^{+}$DCs. By day 3 post treatment, the langerin ${ }^{+}$ $\mathrm{CD} 8 \alpha^{+}$DC population had commenced restoration and by day 4 , levels were completely replenished and comparable to PBS only treated group controls. Analysis of CD86 expression showed that DT treatment did not enhance maturation of DCs (figure not shown).

Thus DT administration into langerin-DTREGFP recipients allows the selective depletion of langerin ${ }^{+} \mathrm{CD} 8 \alpha^{+}$DCs without significantly affecting other splenic DC populations. This period of depletion lasts for 2 days.

Although it is presumed that only langerin expressing cells in langerin-DTREGFP transgenic recipients were capable of responding to DT because they carry the human DT receptor, it was nevertheless deemed necessary to ensure that DT did not have a nonspecific response on DC unrelated to expression of the human DT receptor. Thus, DT alone was given to $\mathrm{C} 57 \mathrm{BL} / 6$ control recipients and the CD11c DC populations quantified between DT toxin treated and PBS control groups (Figure 4.4). Although langerin ${ }^{+}$cells are present in these mice, no ablation of the langerin ${ }^{+}$DCs should occur in response to DT treatment due to the absence of the human DT receptor. Overall no impact of DT treatment was observed on DC populations in these animals. Again DT specifically depleted langerin ${ }^{+}$DCs in langerin-DTREGFP recipients for this experiment. 
A

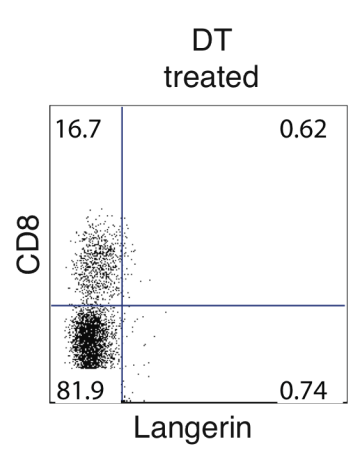

C57BL/6 recipients

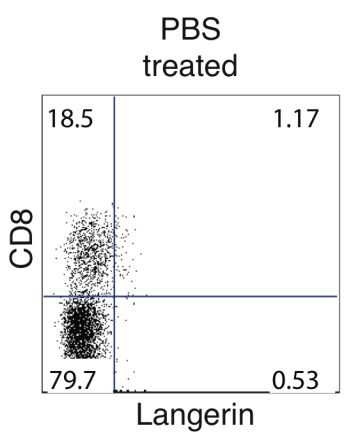

C
B Langerin-DTREGFP recipients
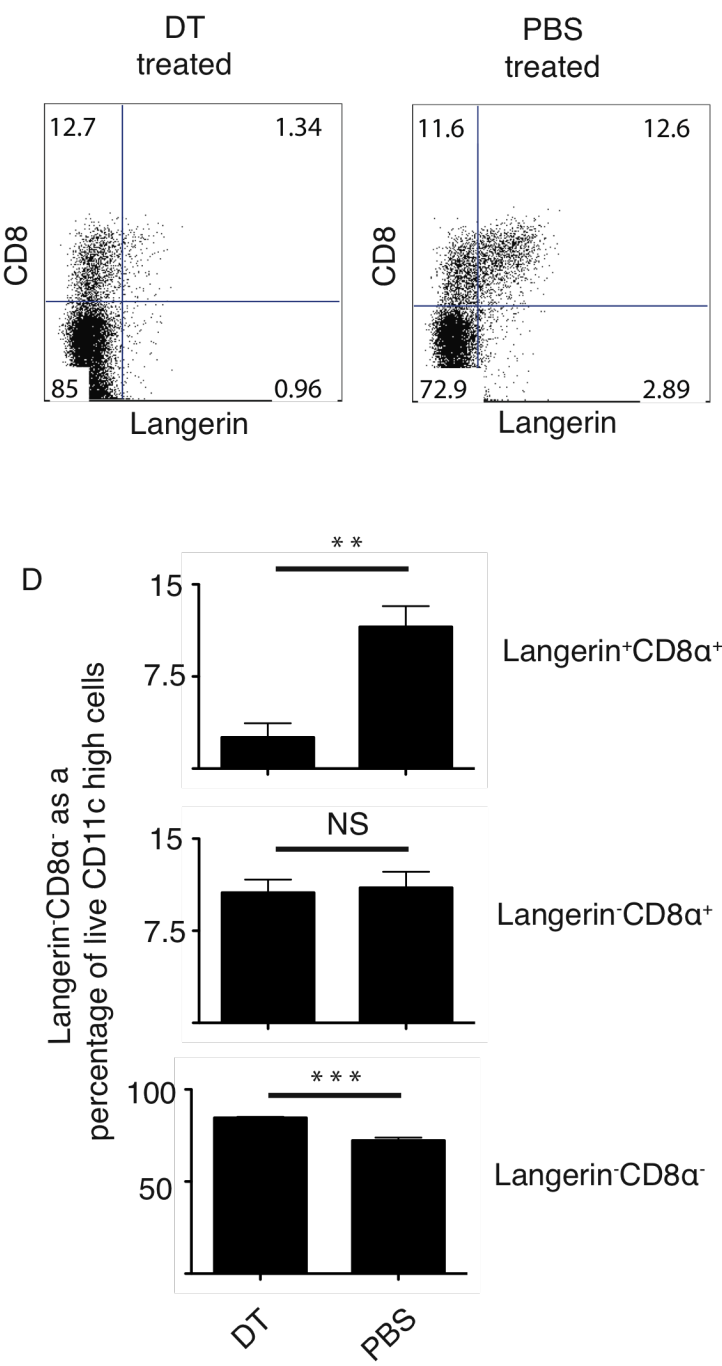

Figure 4.4 The impact of Diphtheria toxin administration in langerin-DTREGFP and C57BL/6 recipients. (A) Representative flow cytometry dot plots show langerin ${ }^{-} \mathrm{CD} 8 \alpha^{+}$ and langerin ${ }^{-} \mathrm{CD} 8 \alpha^{-}$splenic DC populations after treatment with DT or PBS in C57BL/6 recipients and (B) langerin-DTREGFP recipients. (C) Bar graphs show quantification of major CD11c hi splenic DC populations after treatment with diphtheria toxin (DT) or PBS 1, 2, 3 and 4 days post DT or PBS administration into C57BL/6 recipients (D) and langerin-DTREGFP recipients $(n=3)$. Statistical significance was determined using a 1way ANOVA test and Tukey's post-test where $* p=0.01-0.05,{ }^{* *} p=0.001-0.01$, $* * * \mathrm{p}<0.001, \mathrm{NS}=$ not significant. Results shown are from one representative experiment of two repeats that showed similar results. 
Collectively these results confirm the selective action of DT on the langerin ${ }^{+}$DC subsets found in langerin-DTREGFP recipients after intraperitoneal administration using the described protocol, and suggest the impact of DT toxin on non-langerin expressing CD11 $\mathrm{c}^{\text {high }}$ DC populations in both langerin-DTREGFP and C57BL/6 recipients is minimal. This method is therefore acceptable to examine the role of langerin ${ }^{+} \mathrm{CD} 8 \alpha^{+} \mathrm{DCs}$ in response to vaccination with antigen-loaded BM-DCs.

\subsubsection{Systemic TLR stimulation targets langerin $^{+} \mathrm{CD8} \alpha^{+} \mathrm{DC}$ populations in the spleen}

To examine the action of systemic TLR stimulation when used in combination with BMDC based vaccines, langerin-DTREGFP mice were used as recipients so that the impact of selective depletion of the langerin ${ }^{+} \mathrm{CD} 8 \alpha^{+}$DCs could be examined on the induction of $\mathrm{CD}^{+} \mathrm{T}$ cell responses. Specifically, it was aimed to distinguish whether the stimulatory effect of systemic TLR stimulation is a consequence of improving function of host langerin ${ }^{+} \mathrm{CD} 8 \alpha^{+} \mathrm{DC}$ populations that had received antigen from the injected BM-DC vaccines exclusively or other splenic DC subsets.

For these experiments BM-DC-based vaccines were generated from C57BL/6 mice and injected into langerin-DTREGFP recipients. BM-DCs were not prepared from langerinDTREGFP mice to avoid the possibility that DT targets the injected cells. In-vitro BM-DC cultures were loaded with OVA protein on day 6 as described in section 3.2.1. Before DCs were administered LN cells isolated from OTI x B6 congenic donors and administered to langerin-DTREGFP recipients was also carried out on day 6 to provide a cohort of approximately 20,000 OVA specific $\mathrm{CD}^{+} \mathrm{T}$ cells per recipient. The expansion of this injected $\mathrm{CD}^{+} \mathrm{T}$ cell population was monitored for each recipient by testing blood samples taken 7 days post vaccination. Systemic administration of MPL at the time of DC vaccination with OVA protein loaded DCs was carried out by co-injecting MPL into the contra-lateral tail vein immediately after DC vaccine administration (see Figure 4.5 A). 
DT administration at $350 \mathrm{ng}$ per mouse was carried out on days -2 and -1 so as to ensure maximal depletion of the langerin ${ }^{+} \mathrm{CD} 8 \alpha^{+}$splenic DC populations upon DC vaccination with systemic MPL administration on day 0. A subsequent repeat of this experiment clearly showed that systemic MPL administration significantly improved the OVA-specific $\mathrm{T}$ cell response generated when co-administered together with vaccine BM-DCs, however this enhanced response is eliminated in the group where DT is given to deplete the langerin ${ }^{+} \mathrm{CD} 8 \alpha^{+} \mathrm{DC}$ population. Indeed, depletion of the langerin ${ }^{+} \mathrm{CD} 8 \alpha^{+}$population provides an OVA-specific $\mathrm{T}$ cell response level which is no different to that generated to BM-DCs loaded with OVA protein alone.

Control groups were used to reveal if any detrimental effects of DT toxin administration could be shown on the induced $\mathrm{T}$ cell response using $\mathrm{C} 57 \mathrm{BL} / 6$ groups that received vaccination treatments in combination with DT toxin injections. No significant differences in the OVA-specific T cell responses were evident between the vaccine and MPL treated group, and the vaccine and MPL treated groups that received DT in these C57BL6 recipients which suggests, DT does not in itself impede T cell responses. 
A

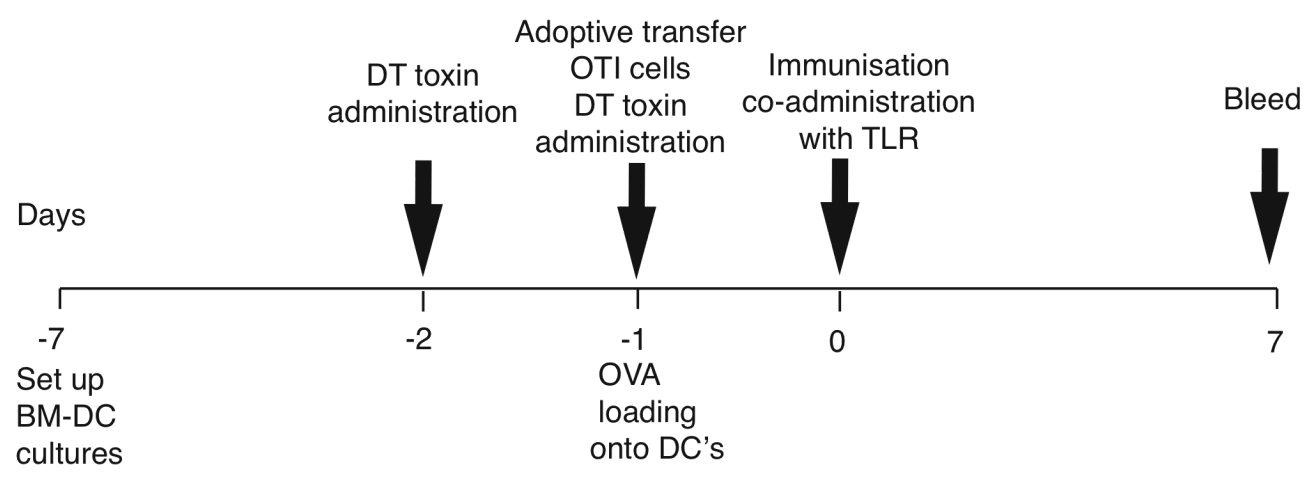

B

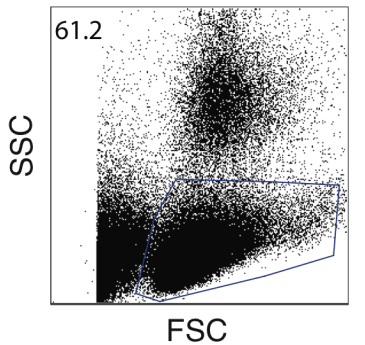

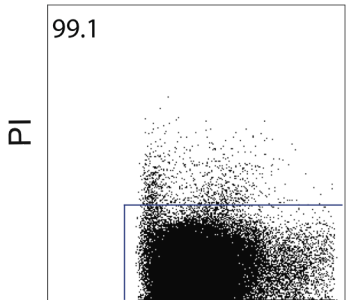

FSC

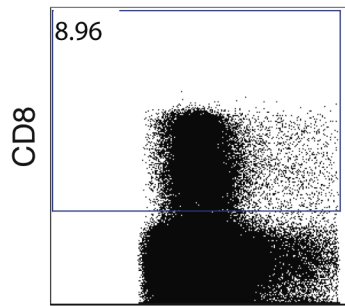

FSC

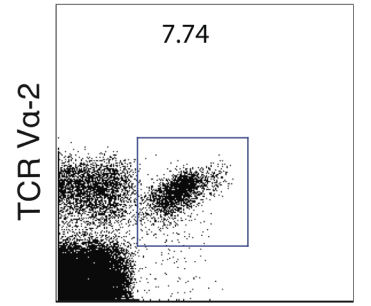

CD45.1
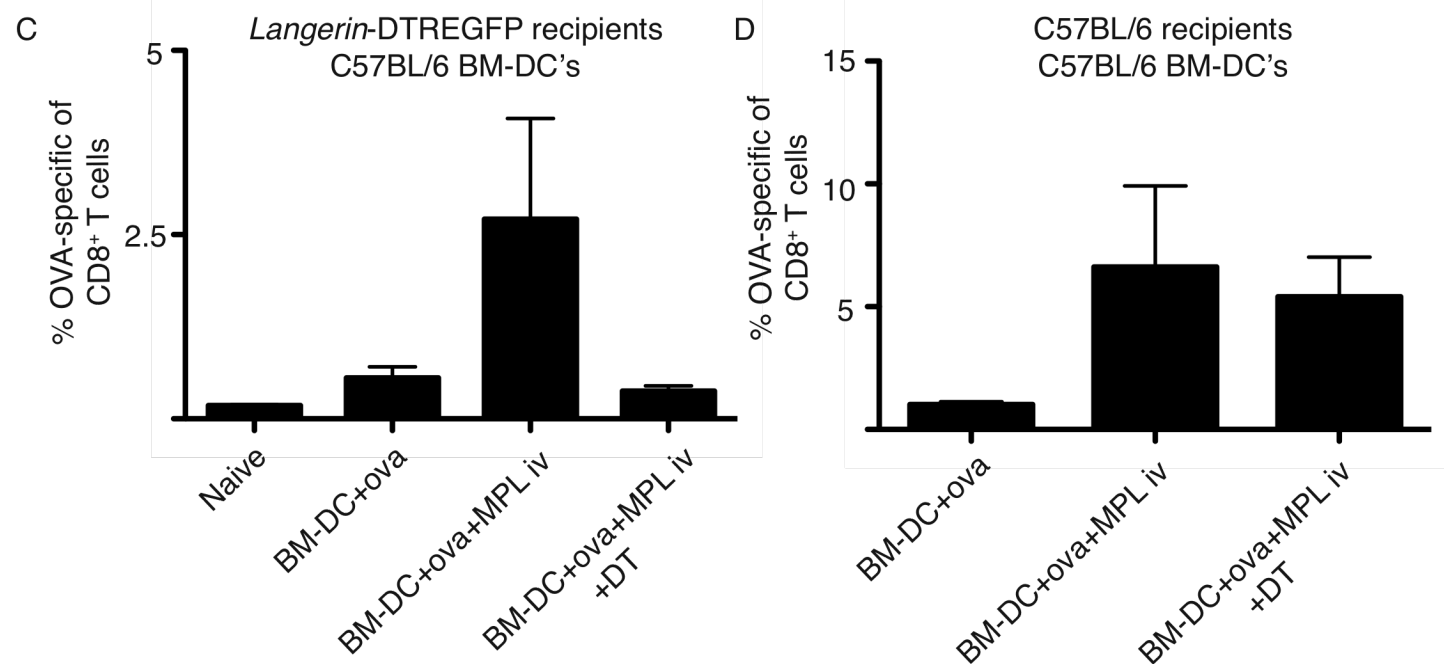

Figure 4.5 Enhanced $\mathrm{CD8}^{+} \mathrm{T}$ cell response to $\mathrm{BM}-\mathrm{DC}$ based vaccine co-administered with MPL is dependent on host langerin ${ }^{+}$DCs. (A) Timeline showing BM-DC vaccine manufacture protocol and diphtheria toxin (DT) administration strategy. (B) Gating strategy used to identify adoptively transferred OTI cell populations 7 days post vaccination into langerin-DTREGFP recipients. (C) Bar graph shows (ova-specific) $\mathrm{CD} 45.1^{+} \mathrm{TCR} \mathrm{V \alpha -2^{+ }}$ expansion of $\mathrm{CD} 8 \alpha^{+} \mathrm{T}$ cells 7 days post vaccination with $\mathrm{C} 57 \mathrm{BL} / 6$ BM-DCs co-administered with MPL iv injected into the contralateral tail vein of langerinDTREGFP recipients ( $n=4$ - 5 recipients per group). Diphtheria toxin was injected on days -2 and -1 to deplete langerin ${ }^{+} \mathrm{CD} 8 \alpha^{+}$splenic DC populations. Bar graph shows the OVA specific $T$ cell response expressed as a percentage of $\mathrm{CD} 8^{+} \mathrm{T}$ cells $( \pm \mathrm{SEM})$ as seen in the blood 7 days post BM-DC vaccination. (D) As in (C) but with C57BL/6 recipients with DT toxin administered at day -2 and -1 to determine adverse effects of the diphtheria toxin itself ( $n=4$ - 5 recipients per group). Statistical significance was determined using 
a 1-way ANOVA test and Tukey's post-test where $* p=0.01-0.05, * * p=0.001-0.01$, $* * * \mathrm{p}<0.001, \mathrm{NS}=$ not significant. Results shown are from one representative experiment of two repeats that yielded similar results.

\subsubsection{Adjuvant effect of systemic MPL administration is not due to TLR4 mediated stimulation of injected vaccine BM-DCs after in-vivo testing}

A plausible explanation for the enhanced $\mathrm{CD}^{+} \mathrm{T}$ cell response in Figure 3.2.1 with intravenous TLR stimulation was that protein antigen was being transferred from the injected BM-DCs to the host langerin ${ }^{+} \mathrm{CD} 8 \alpha^{+} \mathrm{DC}$ population, and that this population was indirectly priming the $\mathrm{CD}^{+} \mathrm{T}$ cell response and responding to the systemic TLR stimulation. Another possibility was that in addition to the indirect priming activity of the host langerin ${ }^{+} \mathrm{CD} 8 \alpha^{+}$DC population, the systemic TLR stimulation provided was acting on surrounding or neighbouring cell types-including the injected vaccine BM-DCs, and that these cells were releasing cytokines that would assist the indirect priming activity and ultimately produce the enhanced $\mathrm{CD}^{+} \mathrm{T}$ cell response observed.

To test the possibility that the injected cells were responding, TLR $4 \%$ mice were used to generate the BM-DCs used as part of the general vaccination protocol. Results in Figure 4.6 shows that the adjuvant effect of systemic TLR stimulation with MPL is not dependent on the injected cells being capable of responding to the TLR ligand. It is therefore concluded that the enhanced OVA-specific T cell effect seen with TLR stimulation is due to TLR action on the resident DC populations. 
A

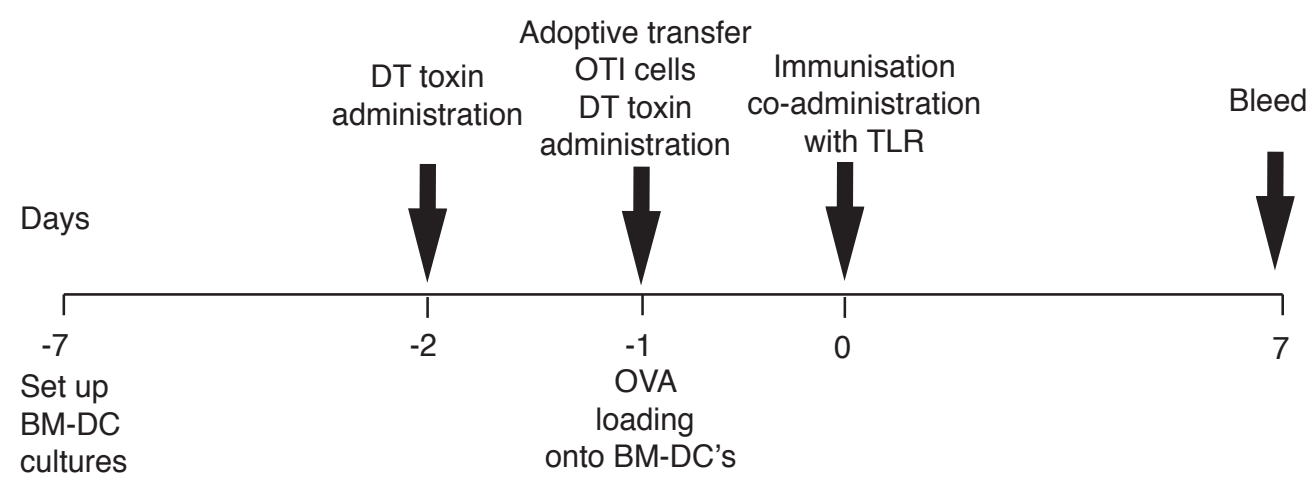

B

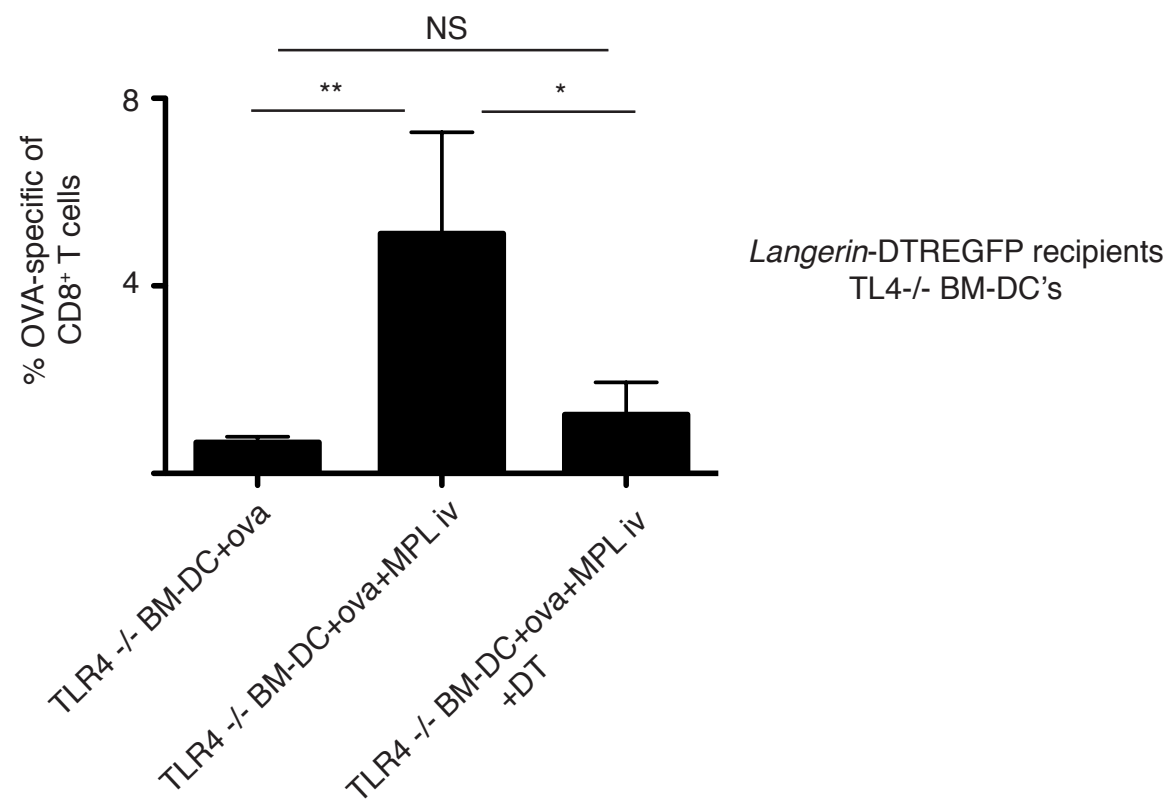

Figure 4.6 Enhanced $\mathrm{CD8}^{+} \mathrm{T}$ cell response to $\mathrm{BM}-\mathrm{DC}$ based vaccines and systemic MPL is not due to TLR 4 mediated stimulation of injected cells. (A) Timeline showing BM-DC vaccine manufacture protocol and diphtheria toxin (DT) administration strategy. (B) Bar graph shows mean percentage of CD45. $1^{+} \mathrm{TCRV} \alpha-2^{+} \mathrm{CD} 8^{+} \mathrm{T}$ cells (OVAspecific) as a proportion of $\mathrm{CD}^{+} \mathrm{T}$ cells in the blood at day 7 post vaccination with TLR4/- vaccine BM-DCs co-administered with MPL $i v$. Diphtheria toxin was injected on days -2 and -1 to deplete langerin ${ }^{+} \mathrm{CD} 8 \alpha^{+}$splenic DC populations. Results shown are from one representative experiment of two repeats that yielded similar results $(n=3-5)$. Statistical significance was determined using a 2-way ANOVA test and Bonferroni's post-test where $* \mathrm{p}=0.01-0.05, * * \mathrm{p}=0.001-0.01, * * * \mathrm{p}<0.001, \mathrm{NS}=$ not significant. 


\subsubsection{Langerin $^{+} \mathrm{CD8} \alpha^{+}$DC populations respond to iv TLR stimulation}

Having shown systemic TLR stimulation capable of improving OVA-specific $\mathrm{T}$ cell responses after DC vaccination and that this appears to involve langerin ${ }^{+} \mathrm{CD} 8 \alpha^{+} \mathrm{DCs}$, the response of this subset to MPL was studied and compared to other major DC subsets of the spleen. Thus, MPL was administered iv to langerin-EGFP recipients and the phenotypic changes produced were observed using flow cytometric analysis to asses maturation marker status of the major splenic DC populations.

Spleens were harvested $5 \mathrm{~h}$ and $24 \mathrm{~h}$ after MPL was administered and expression of CD40 and CD86 examined on splenic DC subsets by flow cytometry. The histograms in Fig 4.7 $\mathrm{B}$ and $\mathrm{D}$ show the maturation status of these splenic DC populations for CD86 and CD40 expression.

Bar graphs in Figure 4.7 A and C show CD86 and CD40 expression differs across all 3 major subsets and basal CD86 expression is higher on the langerin ${ }^{+} \mathrm{CD} 8 \alpha^{+} \mathrm{DC}$ subset. It is also clear that CD86 expression is retained at higher levels $24 \mathrm{~h}$ after MPL albeit there being lower numbers of cells. Results for Figure 4.7 A and Figure 4.7 C were measured seperately at different times with the $24 \mathrm{~h}$ analysis of primary interest. Elevated levels of CD86 overall at $5 \mathrm{~h}$ reflect interexperimental variability. It is also important to note that CD40 expression increases from basal levels earlier in the langerin ${ }^{+} \mathrm{CD} \alpha^{+} \mathrm{DCs}$ in response to MPL and that this expression is retained at higher levels again, albeit on a lower overall numbers of cells.

The faster kinetics of upregulation of maturation markers on the langerin ${ }^{+} \mathrm{CD} 8 \alpha^{+}$splenic DC subset suggests these DCs are better designed for $\mathrm{T}$ cell priming activities than other splenic DC counterparts. This may partially explain the critical role of these langerin ${ }^{+}$ $\mathrm{CD} 8 \alpha^{+} \mathrm{DCs}$ for antigen cross presentation to $\mathrm{CD} 8^{+} \mathrm{T}$ cell populations as it appears they are better adapted to undertake these responsibilities more rapidly than the other splenic DC subtypes. 
A
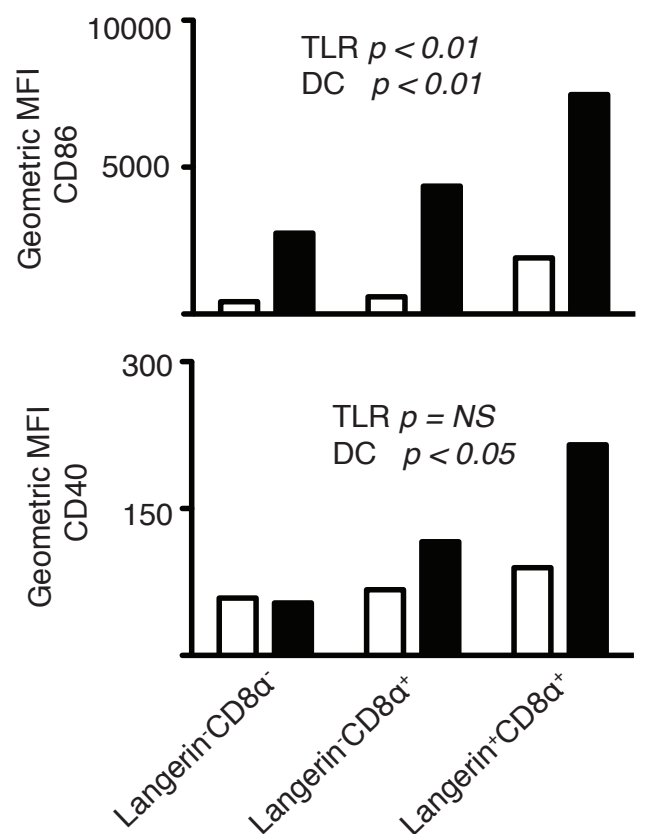

B
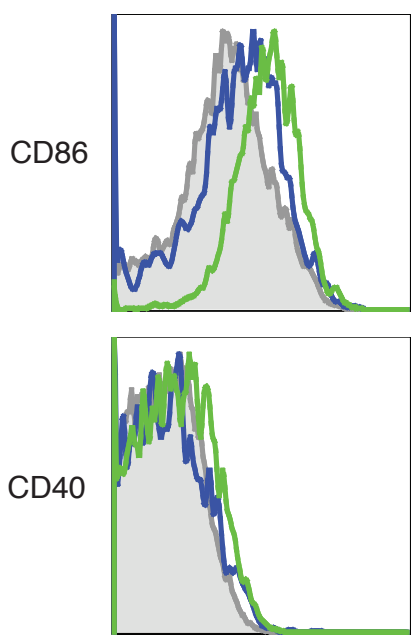

Naive
$5 \mathrm{~h}$
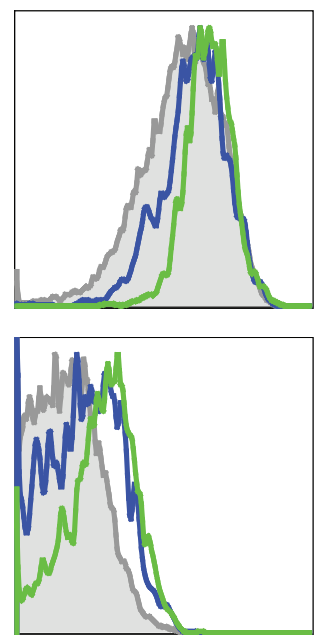

+ MPL
C

$24 \mathrm{~h}$
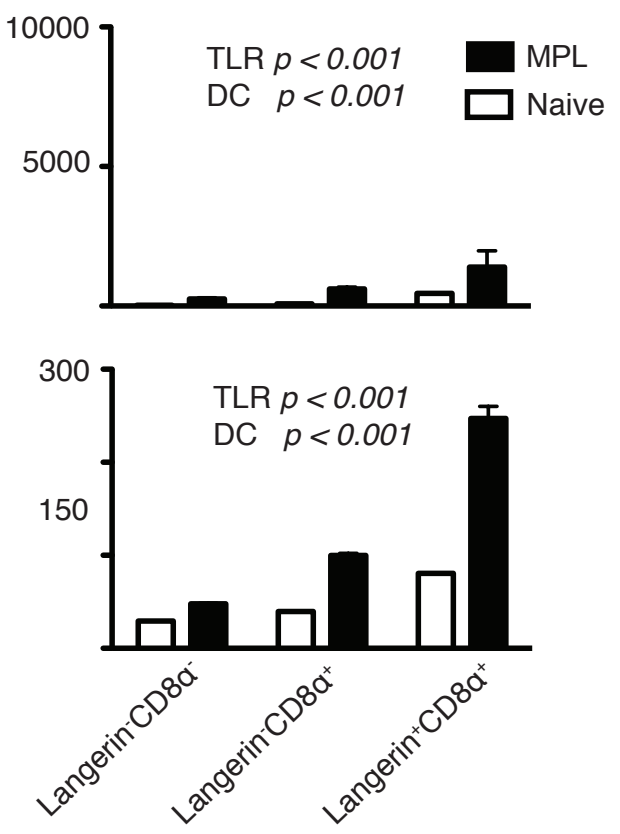

D

$24 \mathrm{~h}$
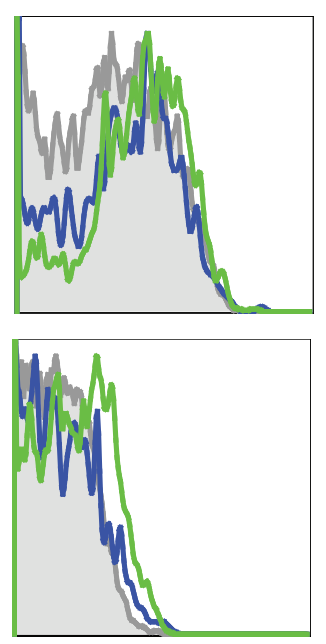

Naive
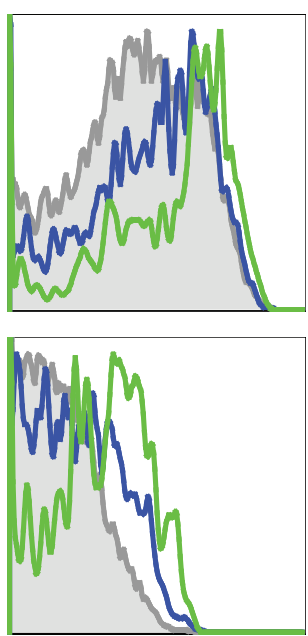

$+\mathrm{MPL}$

$\square$ Langerin ${ }^{+} \mathrm{CD} 8 \mathrm{a}^{+}$

$\square$ Langerin $\mathrm{CD}^{-} \mathrm{a}^{+}$

Langerin ${ }^{C D} 8 a^{-}$ 
Figure 4.7 The characterization of splenic DC subsets after systemic MPL treatment for $5 \mathbf{h}$ and $24 \mathbf{h}$. (A) Bar graphs show levels of CD86 (top graph) and CD40 (bottom graph) expression on major splenic DC populations in naive and MPL treated langerinEGFP recipients $5 \mathrm{~h}$ and $24 \mathrm{~h}(\mathrm{C})$ after $i v$ administration. (B) Histograms show CD86 and CD40 expression across all major splenic DC subsets in naive and systemic MPL treated langerin-EGFP subjects after $5 \mathrm{~h}$ (B) and after $24 \mathrm{~h}$ (D). Results shown are from one experiment. Statistical significance was determined using a 1-way ANOVA test and Tukey's post-test where $* \mathrm{p}=0.01-0.05,{ }^{*} \mathrm{p}=0.001-0.01,{ }^{* * *} \mathrm{p}<0.001$, NS $=$ not significant.

\subsubsection{Langerin ${ }^{+} \mathrm{CD8} \alpha^{+} \mathrm{DCs}$ are recipients of protein antigen transferred from protein loaded vaccine $B M-D C s$}

After noting the possible major involvement of the resident DC populations in $\mathrm{T}$ cell priming of the OVA-specific T cell responses after BM-DC vaccination, the fate of protein antigen initially loaded onto vaccine BM-DCs was investigated further. Data from within our laboratory suggested that allogeneic DCs could also stimulate OTI-T cell responses (Petersen TR personal communication). As these allogeneic DCs do not express the H-2 $\mathrm{K}^{\mathrm{b}}$ restriction element required to stimulate OT-1 cell, a resultant antigen specific $\mathrm{T}$ cell response would indicate antigen transfer had occurred to resident $\mathrm{H}-2 \mathrm{~K}^{\mathrm{b}+}$ cell populations. The possibility that antigen was transferred from allogeneic DCs to resident langerin ${ }^{+} \mathrm{DCs}$ was therefore investigated.

B10Q mice were used to generate allogeneic BM-DCs that were loaded with OVA protein and used to vaccinate C57BL/6 and langerin-DTREGFP recipients. Syngeneic BM-DCs were also prepared using $\mathrm{C} 57 \mathrm{BL} / 6$ mice, and the OVA-specific immune responses compared with those seen after using the B10Q BM-DCs. B10Q mice (B10.Q; H-2 ${ }^{\mathrm{q}}$ ) differ in MHC haplotype from C57BL/6 mice (C57BL/6; H-2 ${ }^{b}$ ), and therefore BM-DCs generated from B10Q mice cannot directly present processed antigen to OTI cells and prime the OVA-specific T cell response.

As shown in Figure 4.12 B, a significant OVA-specific $\mathrm{T}$ cell response was observed following injection of the allogeneic vaccine BM-DCs in the presence of systemic TLR stimulation, suggesting antigen had been transferred to resident cells. This response was 
A

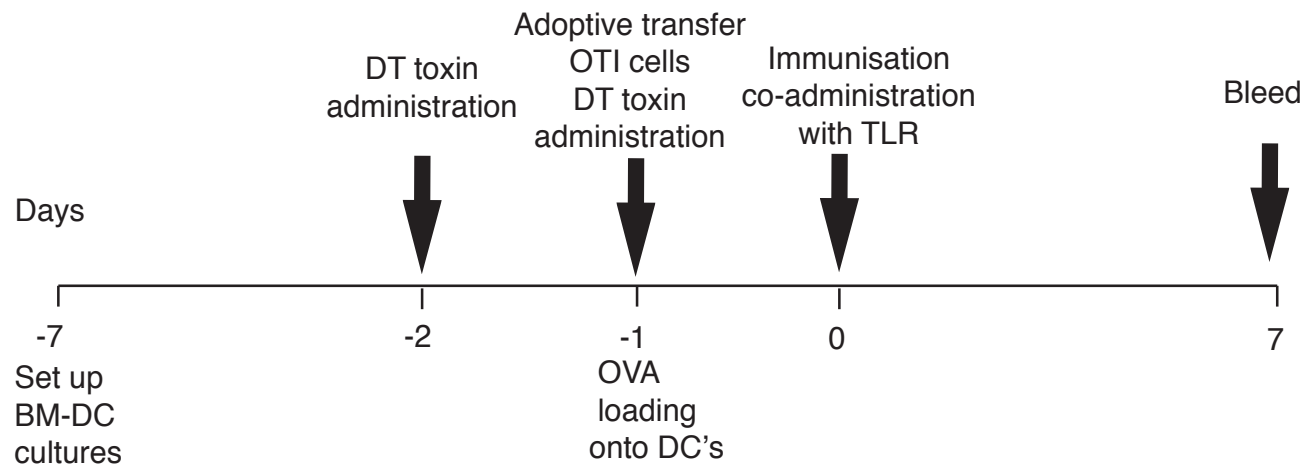

B

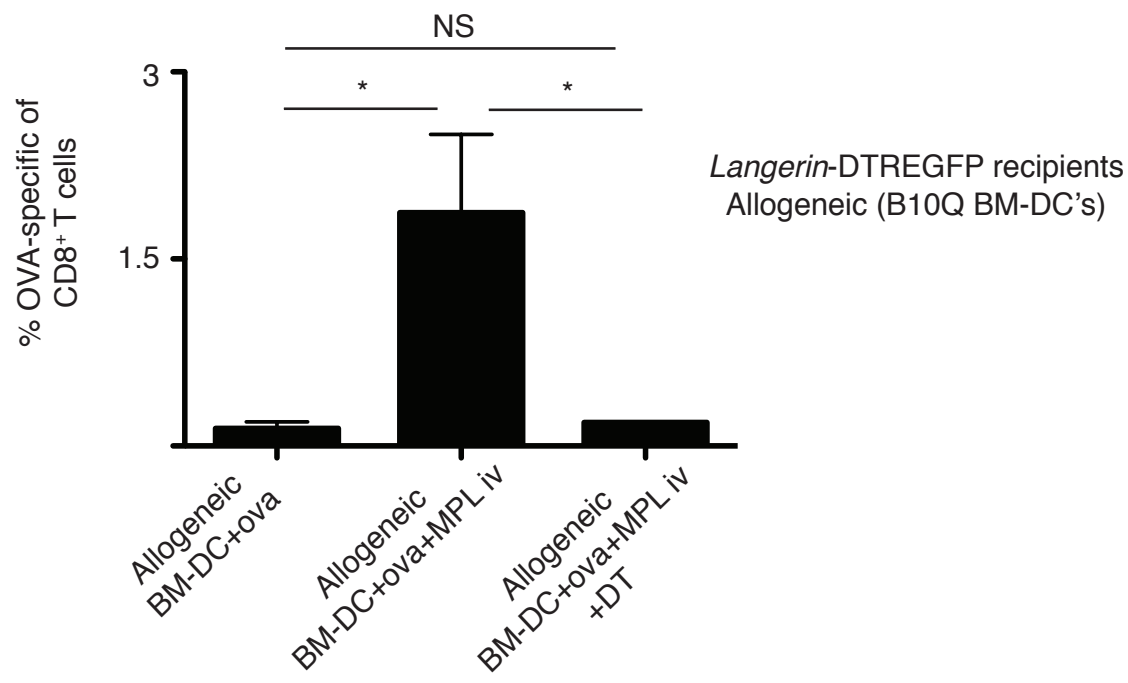

Figure 4.8 Protein antigen transfer from vaccine BM-DCs to resident langerin ${ }^{+} \mathrm{CD} \alpha^{+}$DC populations is involved in cross-presentation. (A) Timeline showing allogeneic BM-DC vaccine manufacture protocol and diphtheria toxin (DT) administration strategy. (B) Bar graph shows mean percentage of CD45.1 ${ }^{+}$TCRV $\alpha-2^{+}$ $\mathrm{CD}^{+} \mathrm{T}$ cells (OVA-specific) as a proportion of $\mathrm{CD}^{+} \mathrm{T}$ cells in the blood at day 7 post vaccination with MPL administered systemically. Diphtheria toxin was injected on days -2 and -1 to deplete langerin ${ }^{+} \mathrm{CD} 8 \alpha^{+}$splenic DC populations. Results shown are from one representative experiment of two repeats that yielded similar results $(n=4-5$ recipients per group). Statistical significance was determined using a 1-way ANOVA test and Tukey's post-test where ${ }^{*} \mathrm{p}=0.01-0.05,{ }^{* *} \mathrm{p}=0.001-0.01,{ }^{* * *} \mathrm{p}<0.001$, NS $=$ not significant. 
eliminated in langerin mice where DT was also administered to deplete the langerin ${ }^{+}$ $\mathrm{CD} 8 \alpha^{+} \mathrm{DC}$ population suggesting transfer of antigen to this resident DC population is critical to inducing the cross-presented OVA-specific T cell response.

Collectively these experiments have provided strong evidence to support a previously described but less mainstream notion that vaccine DCs can act as antigen carriers, and that under appropriate conditions the antigen-specific $\mathrm{T}$ cell response can be primed by resident DC populations. 


\subsection{Discussion}

This chapter presents studies which feature the utilization of two transgenic mouse strains used to investigate the role of langerin ${ }^{+} \mathrm{CD} 8 \alpha^{+} \mathrm{DCs}$ in BM-DC vaccine responses. Langerin-EGFP recipients were used to identify langerin ${ }^{+} \mathrm{CD} 8 \alpha^{+} \mathrm{DC}$ subsets in the spleen and examine the response to systemic TLR stimulation. The administration of DT was shown to achieve selective depletion of the langerin ${ }^{+} \mathrm{CD} 8 \alpha^{+} \mathrm{DC}$ subset in the langerinDTREGFP strain and was used to explore facets of the antigen specific $\mathrm{T}$ cell response generated after vaccination with antigen loaded BM-DC vaccines.

It was shown that the langerin ${ }^{+} \mathrm{CD} 8 \alpha^{+} \mathrm{DC}$ subset was very important for the adjuvant response seen after co-administration of systemic MPL with BM-DC vaccines. The contribution of langerin ${ }^{+} \mathrm{CD} 8 \alpha^{+} \mathrm{DCs}$ as recipients of transferred antigenic components from injected vaccine BM-DCs was also confirmed after showing a significant reduction in the antigen specific $\mathrm{T}$ cell response following depletion of this subset in vaccine recipients using DT. Further vaccine development could involve the selective targeting of BM-DC vaccine components and TLR stimulation to the endogenous langerin ${ }^{+} \mathrm{CD} 8 \alpha^{+} \mathrm{DCs}$ in an effort to achieve optimal antigen specific $\mathrm{T}$ cell responses.

\subsubsection{Characterization of the major splenic DC subsets}

Three major DC populations that reside within the mouse spleen include the plasmacytoid DCs, conventional $\mathrm{CD} 8^{+} \mathrm{DCs}\left(\mathrm{CD} 8^{+} \mathrm{CD} 24^{+}\right)$and conventional $\mathrm{CD} 8^{-} \mathrm{DCs}\left(\mathrm{CD} 8^{-} \mathrm{CD} 24^{-}\right)$ DCs (Bedoui et al., 2009). It has been shown that the $\mathrm{CD} 8^{+} \mathrm{DC}$ subset were cross presentation specialist DCs and were largely responsible for antiviral cytotoxic $\mathrm{T}$ lymphocyte responses in-vivo (Bedoui et al., 2009; den Haan et al., 2000; Shortman and Heath, 2010). CD8 $\alpha^{+}$DCs are located in the paracortical regions of the secondary lymphoid organs with the langerin ${ }^{+} \mathrm{CD} 8 \alpha^{+}$DCs residing in the splenic marginal zone (Kissenpfennig et al., 2005; Kissenpfennig and Malissen, 2006; Qiu et al., 2009). It is now clear that CD8 $\alpha$ DCs can be further subdivided into subsets based on langerin and very 
recently $\mathrm{CX}_{3} \mathrm{CR} 1$ (Bar-On et al., 2010). This thesis examined the role of langerin ${ }^{+} \mathrm{DCs}$ since $\mathrm{CX}_{3} \mathrm{CR} 1 \mathrm{DCs}$ appear to differ from the langerin ${ }^{+} \mathrm{DC}$ subset given their lack of cross presentation ability, and inability to generate the IL-12 cytokine (Bar-On et al., 2010).

This chapter presented studies that were conducted using the langerin-EGFP and langerinDTREGF transgenic mouse strains. An effort to characterize the major splenic DC subsets within langerin-EGFP mice was undertaken with a major focus being on the langerin ${ }^{+}$ (CD207) expressing DC subsets (Farrand et al., 2009; Qiu et al., 2009). Expression of the C-type lectins CD205 (DEC205) and CD207 was examined on langerin ${ }^{+}$CD8 $\alpha^{+}$, langerin ${ }^{-}$ $\mathrm{CD} \alpha^{+}$and langerin ${ }^{-} \mathrm{CD} 8 \alpha^{-} \mathrm{DCs}$ and results showed that CD205 (DEC205) expression was found to be higher on the langerin ${ }^{+} \mathrm{CD} 8 \alpha^{+}$DCs when compared with the remaining two subsets. CD205 (DEC205) functions as an endocytic receptor found on the DC outer surface involved in the delivery of antigen to degradation sites within the DC interior such as late endosomes or lysosomal compartments (Platt et al., 2010). These results were consistent with previous reports that had also shown enhanced expression of CD205 on DCs and indicated enhanced cross-presentation function (Dudziak et al., 2007; Schnorrer et al., 2006).

The expression of CD24 $4^{+}$on DCs is indicative of CD $8 \alpha^{+}$DC precursor identity in addition to highlighting function as cross-presenting DCs (Bedoui et al., 2009; Bedoui et al., 2009). $\mathrm{CD} 24^{+}$expression was evident on the CD $8 \alpha^{+}$DC subsets and not on the CD8 $\alpha^{-}$DCs. The expression of the CD103 marker important for cross presentation activities involving foreign and self antigens was examined and indeed found to be higher on langerin ${ }^{+} \mathrm{CD} 8 \alpha^{+}$ DCs, although present on langerin ${ }^{-} \mathrm{CD} 8 \alpha^{+}$and langerin ${ }^{-} \mathrm{CD} 8 \alpha^{-} \mathrm{DCs}$ in agreement with previous studies (Bedoui et al., 2009; Bedoui et al., 2009; del Rio et al., 2008; del Rio et al., 2007; Jaensson et al., 2008; Jakubzick et al., 2008; Qiu et al., 2009). It has also been reported that the $\mathrm{CD} 103^{+}$marker is found on effector memory $\mathrm{CD} 8^{+} \mathrm{T}$ cells, is also found on DCs involved in adhesion interactions, can mediate the motility and cellular shapes, and have also been implicated in the formation of $\mathrm{CD}^{+} \mathrm{CD} 25^{+} \mathrm{FoxP} 3^{+} \mathrm{T}$ regulatory cells (Coombes et al., 2007; Feng et al., 2002; Jaensson et al., 2008; Johansson-Lindbom et al., 2005; Lehmann et al., 2002; Uss et al., 2006; Zhao et al., 2008). It is also estimated that 
between $50 \%$ and $70 \%$ of splenic DCs also co-express the CD103 marker (del Rio et al., 2008; del Rio et al., 2007; Pribila et al., 2004; Qiu et al., 2009).

Maturation marker expression patterns were also examined on the splenic DCs langerin ${ }^{+} \mathrm{CD} 8 \alpha^{+}$, langerin ${ }^{-} \mathrm{CD} 8 \alpha^{+}$and langerin ${ }^{-} \mathrm{CD} 8 \alpha^{-}$DCs. CD86 and CD40 expression was preferentially higher on the langerin ${ }^{+} \mathrm{CD} 8 \alpha^{+} \mathrm{DCs}$ perhaps indicating more efficient $\mathrm{T}$ cell stimulation, although these markers were also present on the remaining DC subsets langerin ${ }^{-} \mathrm{CD} 8 \alpha^{+}$and langerin ${ }^{-} \mathrm{CD} 8 \alpha^{-} \mathrm{DCs}$.

These results show that distinctive expression patterns exist between the major splenic DC subsets for selected DC markers involved in cross-presentation activities, endocytosis and maturation. The preferential elevation of the markers on the resident langerin ${ }^{+} \mathrm{CD} 8 \alpha^{+} \mathrm{DC}$ subset suggested their superior functionality as mature cross-presenting DCs which served as a desirable target for systemic TLR stimulation utilizing the DC vaccination strategy developed within this $\mathrm{PhD}$ project. Work in Chapter 3 had clearly shown that systemic TLR stimulation significantly increased antigen specific $\mathrm{T}$ cell responses post vaccination with DC based antigen carrying DC vaccines. The mechanism responsible for TLR enhanced antigen specific T cell responses to DC vaccination, and the direct targeting TLR stimulation to the resident langerin ${ }^{+} \mathrm{CD} 8 \alpha^{+} \mathrm{DC}$ subset were both explored further and results presented in this chapter.

\subsubsection{The utilization of DT for cellular depletion within transgenic models}

Initial experiments were conducted to establish the duration of langerin ${ }^{+} \mathrm{CD} 8 \alpha^{+} \mathrm{DC}$ depletion after DT administration. Results showed that 1-2 days after administration of the $2^{\text {nd }}$ dose of DT, langerin-DTREGFP recipients were depleted of langerin ${ }^{+} \mathrm{CD} 8 \alpha^{+}$DCs in their spleens. By day 3 post treatment, the langerin ${ }^{+} \mathrm{CD} 8 \alpha^{+} \mathrm{DC}$ population had commenced restoration and by day 4 , levels were completely replenished and comparable to PBS only treated group controls. The duration and magnitude of langerin ${ }^{+} \mathrm{CD} 8 \alpha^{+}$ 
depletion was consistent with previous work published by our laboratory (Farrand et al., 2009 ) and enabled the exploration of langerin ${ }^{+} \mathrm{CD} 8 \alpha^{+} \mathrm{DC}$ functionality.

\subsubsection{The adjuvant impact of systemic MPL stimulation is mediated by langerin ${ }^{+} \mathrm{CD8} \alpha^{+} \mathrm{DCs}$ in-vivo}

Having confirmed the utility of the langerin-EGFP and langerin-DTREGFP transgenic mouse models, an investigation into the action of systemic TLR stimulation coadministered with protein antigen loaded BM-DC vaccines was undertaken to uncover the nature of the involvement of the splenic langerin ${ }^{+} \mathrm{CD} 8 \alpha^{+} \mathrm{DCs}$ on the induction of $\mathrm{CD} 8^{+} \mathrm{T}$ cell responses.

An attempt was made to determine whether the adjuvant effect of systemic MPL coadministration was a consequence of TLR agonist action on the injected protein antigen carrying vaccine BM-DCs. The alternative scenario being explored was that TLR stimulation was acting primarily on resident APCs that had acquired antigenic fragments from injected BM-DC vaccine populations and were in the process of priming the observed antigen specific $\mathrm{T}$ cell responses. An alternative to this situation was the possibility that TLR stimulation was targeting bystander cells responsive by the production of proinflammatory cytokines that improved the function of host langerin ${ }^{+} \mathrm{CD} 8 \alpha^{+} \mathrm{DC}$ populations that had received antigen from the injected BM-DC vaccines and were priming antigen specific $\mathrm{T}$ cell responses.

Work by Liu et al (2002) showed that DCs found in the spleen express TLR 2, TLR 4, TLR 5, TLR 6 and TLR 9 while langerin expressing Langerhans DCs found in skin differed in TLR expression pattern by expressing only TLR 2, TLR 4 and TLR 9 (Liu et al., 2002; Mitsui et al., 2004).

Although systemic MPL administration improved the antigen specific $\mathrm{T}$ cell response generated when co-administered together with vaccine BM-DCs, pre-treatment with DT to eliminate langerin ${ }^{+} \mathrm{CD} 8 \alpha^{+} \mathrm{DC}$ populations ablated this $\mathrm{T}$ cell response. What was unclear 
was whether MPL action was targeting antigen carrying injected BM-DCs initially assumed to be driving the $\mathrm{T}$ cell response. These results suggested that $\mathrm{T}$ cell priming could actually be carried out indirectly by resident langerin ${ }^{+} \mathrm{CD} 8 \alpha^{+}$DCs which had received antigenic components from the injected vaccine BM-DCs. What was also unclear was whether the adjuvant action of the co-administered MPL was targeting the langerin ${ }^{+}$ $\mathrm{CD} 8 \alpha^{+} \mathrm{DCs}$ indirectly priming the $\mathrm{T}$ cells response, or targeting bystander DCs to release the appropriate cytokine milieu needed to support the efficient induction of $\mathrm{T}$ cell responses.

To further examine the $\mathrm{T}$ cell priming activity of injected vaccine BM-DCs used in vaccination protocols, vaccine BM-DCs were generated using TLR4 $\%$ mice. This would effectively eliminate the direct contribution of injected vaccine BM-DC populations in any enhanced OVA-specific T cell response caused by systemic TLR stimulation and its effect on $\mathrm{T}$ cell priming activity. Increased antigen specific $\mathrm{T}$ cell responses could only be produced by MPL action on either resident langerin ${ }^{+} \mathrm{CD} 8 \alpha^{+} \mathrm{DCs}$ priming $\mathrm{T}$ cell responses or by ligation of bystander cells producing cytokines supporting the $\mathrm{T}$ cell priming actions of host langerin ${ }^{+} \mathrm{CD} 8 \alpha^{+} \mathrm{DC}$ populations.

The adjuvant effect of systemic MPL stimulation was not shown to be dependent on the injected vaccine BM-DC cells, but rather these results provide further evidence that any TLR adjuvant activity used to improve this BM-DC vaccination protocol should be targeted to the resident DC populations.

B10Q allogeneic BM-DCs were used to generate vaccine DCs that were co-administered to langerin-DTREGFP recipients with systemic MPL. These allogeneic BM-DCs are incapable of priming the OVA specific $\mathrm{T}$ cell response and were utilized to confirm the transfer of antigen from injected BM-DCs to resident DC populations. Results showed the generation of an antigen specific $\mathrm{T}$ cell response after immunisation using allogeneic BMDCs in the presence of systemic MPL stimulation indeed confirming the transfer of antigen from injected BM-DC populations to resident langerin ${ }^{+} \mathrm{CD} 8 \alpha^{+}$DCs after vaccination with protein loaded BM-DCs. The antigen specific $\mathrm{T}$ cell response was ablated after DT administration caused depletion of the langerin ${ }^{+} \mathrm{CD} 8 \alpha^{+} \mathrm{DC}$ subset suggesting transfer of 
antigen to this resident DC population is critical to inducing the cross-presented OVAspecific $\mathrm{T}$ cell response.

Collectively these experiments have provided strong evidence to support a previously described but less mainstream notion that vaccine DCs can act as antigen carriers, and that under appropriate conditions the antigen-specific $\mathrm{T}$ cell response can be primed by resident DC populations.

\subsubsection{The transfer of protein antigen to langerin ${ }^{+} \mathrm{CD} 8 \alpha^{+} \mathrm{DCs}$ splenic DC populations}

Work presented in this chapter showed that langerin ${ }^{+} \mathrm{CD} 8 \alpha^{+} \mathrm{DCs}$ are recipients of protein antigen transferred from vaccine BM-DCs. This was demonstrated after allogeneic vaccine BM-DCs loaded with OVA protein and co-administered with MPL $i v$, were utilized to vaccinate langerin-DTREGFP recipients. The method by which OVA protein was being transferred from vaccine BM-DCs to endogenous langerin ${ }^{+} \mathrm{CD} 8 \alpha^{+}$DCs was not confirmed, however it was suggested that specific processes may have been taking place.

Previous work had also provided evidence for the transfer of antigen from injected DC populations to host DCs (Kleindienst and Brocker, 2003; Kuipers et al., 2009; Luketic et al., 2007; Yewdall et al., 2010). The splenic marginal zone is the prime target site for trapping antigen (Kraal, 2002) with the splenic CD8 $\alpha^{+}$DCs being identified as the DC subset responsible for removal of apoptotic bodies from the peripheral blood (Iyoda et al., 2002). It is also possible that OVA protein fragments may have been released after the original vaccine BM-DCs became apoptotic enroute to the spleen, although it has been shown that the $\mathrm{CD} 8 \alpha^{+} \mathrm{CD} 103^{+} \mathrm{CD} 207^{+}$splenic DC subset acquires apoptotic material and is responsible for the induction of tolerance which was not seen here (Qiu et al., 2009). Gene expression studies and functional assays have also shown that the CD $8 \alpha^{+} \mathrm{CD} 207^{+}$ $\mathrm{CD}_{103^{+}} \mathrm{DCs}$ are apoptotic cell uptake specialists that migrate into the $\mathrm{T}$ cell zone area to cross-present acquired antigen to $\mathrm{CD}^{+} \mathrm{T}$ cells (Lin et al., 2008; Qiu et al., 2009). This 
uptake mechanism if involved here induces immunity and therefore must involve stimulatory signals from the vaccines BM-DCs to avoid tolerance.

It is also feasible that direct transfer of membranous fragments is taking place between protein loaded vaccine BM-DCs and the splenic langerin ${ }^{+} \mathrm{CD} 8 \alpha^{+}$DCs that have come into direct contact with each other. Previous work has shown transfer of membrane components between live cells can occur when in direct contact with each other (Harshyne et al., 2001). Results from our own laboratory also showed that langerin ${ }^{+} \mathrm{CD} 8 \alpha^{+} \mathrm{DCs}$ acquired more fluorescent material from CFSE labelled BM-DCs after $i v$ administration when compared with langerin ${ }^{-C D} 8 \alpha^{-}$DCs (Petersen et al., 2011).

It has also been shown that it is possible for protein antigens that have been internally loaded and processed by DCs to be transferred to host cells for MHC I presentation after the phagocytosis of apoptotic (Albert et al., 1998a; Albert et al., 1998b) and necrotic OVA bearing DCs (Sauter et al., 2000). Dolan et al (2006) also showed that antigen could be transferred to endogenous APC populations by peptide shedding from injected DC populations (Dolan et al., 2006). It was also shown by these same groups that intact MHC/peptide complexes could also be transferred intact. Yewdall et al (2010) showed that externally loaded peptide antigen loaded to the outer surface of MHC molecules was more readily transferred to host populations during $\mathrm{T}$ cell priming activities when compared to internally loaded peptide/MHC complexes (Yewdall et al., 2010). Antigen transfer between DC subsets via direct contact was described and visualized histological analysis carried out by (Kleindienst and Brocker, 2003). Immunotransplantation studies also showed that the immunosuppressive impact of DCs injected $i v$ were mediated by endogenous DC populations (Divito et al., 2010).

It is also possible that small membrane bound vesicles called exosomes secreted by DCs and other cell types, are responsible for antigen supply and delivery to splenic langerin ${ }^{+} \mathrm{CD} 8 \alpha^{+}$DCs involved in cross presentation and $\mathrm{T}$ cell priming activities after vaccination with protein loaded BM-DCs (Luketic et al., 2007; Thery et al., 2002a; Thery et al., 2002b). Direct transfer of peptide loaded MHC molecule complexes has reportedly occurred via exosomes (Andre et al., 2004a; Andre et al., 2004b; Luketic et al., 2007). 
Montecalvo et al (2012) described a mechanism for the transfer of mRNA between murine DCs via exosomal function (Montecalvo et al., 2012).

It had been reported that the intravenous administration of LPS and MPL causes the phenotypical and functional maturation of splenic DCs (De Becker et al., 2000; De Smedt et al., 1996). In particular it was also shown that following MPL stimulation, DCs found in the marginal zone migrated and were found in the $\mathrm{T}$ cell areas of the spleen (De Becker et al., 2000). Qiu et al 2009 reported that the langerin ${ }^{+}\left(103^{+}, \mathrm{CD} 207^{+}\right) \mathrm{CD} \alpha^{+} \mathrm{DC}$ subset found in the spleen was also largely found in the marginal zone areas (Banchereau et al., 2000; Dudziak et al., 2007; Qiu et al., 2009) until after uptake of intravenously administered apoptotic material when migration took place to the $\mathrm{T}$ cell zone areas (Iyoda et al., 2002; Schnorrer et al., 2006; Schulz and Reis e Sousa, 2002). The Qiu studies also reported that depletion of this langerin ${ }^{+}\left(103^{+}, \mathrm{CD} 207^{+}\right) \mathrm{CD} 8 \alpha^{+} \mathrm{DC}$ subset using of cytochrome c, caused the loss of tolerance mechanisms against self-antigens.

Interestingly they also showed that the systemic TLR stimulation induced by the intravenous administration of either LPS or PolyIC caused the disappearance of the langerin $^{+}\left(103^{+}, \mathrm{CD} 207^{+}\right) \mathrm{CD} \alpha^{+} \mathrm{DC}$ subset $24 \mathrm{~h}$ after administration (Qiu et al., 2009). This finding was consistent to observations made during our investigations where numbers of splenic langerin ${ }^{+} \mathrm{CD} 8 \alpha^{+}$DCs were markedly reduced $24 \mathrm{~h}$ post intravenous administration into langerin-EGFP recipients. We reported that those langerin ${ }^{+} \mathrm{CD} 8 \alpha^{+} \mathrm{DCs}$ that did remain were found to express high levels of CD86. The disappearance of the langerin ${ }^{+} \mathrm{CD} 8 \alpha^{+}$DC subset was also noted after the administration of $\alpha$-GalCer and when $\alpha$-GalCer was administered together with MPL (data not shown).

The disappearance of the langerin ${ }^{+} \mathrm{CD} 8 \alpha^{+} \mathrm{DC}$ subset after TLR stimulation appears to defeat the purpose of inducing enhanced stimulation of the appropriate splenic DC subset designed for optimal $\mathrm{T}$ cell stimulation activity. One possible explanation is that the langerin ${ }^{+} \mathrm{CD} 8 \alpha^{+}$DCs die after migration from the marginal zone having picked up antigen and received TLR stimulation signals, followed by $\mathrm{T}$ cell presentation activity in the $\mathrm{T}$ cell area of the spleen. 


\subsubsection{Conclusions}

- Langerin $^{+} \mathrm{CD} 8 \alpha^{+}$DCs are a distinct subset of the CD8 $\alpha^{+}$splenic DCs and can be selectively depleted in langerin-DTREGFP recipients after DT administration

- $\quad$ Langerin ${ }^{+} \mathrm{CD} 8 \alpha^{+}$splenic DC subsets are the recipients of antigens derived from injected antigen loaded vaccine BM-DCs and are capable of contributing to the stimulation of $\mathrm{CD} 8^{+} \mathrm{T}$ cell response of appropriately vaccinated recipients

- The adjuvant effect of systemic TLR stimulation is mediated by langerin ${ }^{+} \mathrm{CD} 8 \alpha^{+}$ splenic DC subset in-vivo 


\section{Chapter 5:}

\section{NKT stimulation enhances antigen specific responses to $\mathrm{DC}$-based vaccines}

In part published in:

Petersen TR, Sika-Paotonu D, Knight DA, Simkins HM, Anderson RJ, Painter GF, Hermans IF. Exploiting the Role of Endogenous Lymphoid-Resident Dendritic Cells in the Priming of NKT Cells and $\mathrm{CD}^{+} \mathrm{T}$ Cells to Dendritic Cell-Based Vaccines. PLoS ONE 6 March 2011. 


\subsection{Introduction}

Original studies with $\alpha$-GalCer demonstrated potent anti-tumour properties after showing that $\alpha$-GalCer loaded BM-DCs not only caused the inhibition of tumour metastasis in a B16 melanoma model, but that complete regression could be induced in established metastatic tumours of murine recipients (Toura et al., 1999). The discovery of V $\alpha-24 J \alpha 18$ NKT cells - the human counterpart of the murine V $\alpha 14 \mathrm{~J} \alpha 18$ NKT cells which could also bind to DCs presenting $\alpha$-GalCer, led to the subsequent intense worldwide interest in its development as a potential cancer-fighting agent (Kawano et al., 1997; Kawano et al., 1999).

The range of anti-tumour activities induced by $\alpha$-GalCer vary from generating DCs with an improved maturation and activation status able to contribute to long lasting adaptive immune responses, to the iNKT and NK-mediated perforin-dependent cytolytic destruction of tumour cells and anti-angiogenic function of IFN- $\gamma$ (Colombo and Trinchieri, 2002; Kawano et al., 1998; Zamai et al., 2007). Previous studies have also shown that $\alpha$-GalCer loaded BM-DCs were indeed capable of enhancing T cell responses (Fujii et al., 2004; Fujii et al., 2003; Hermans et al., 2007; Hermans et al., 2003; Silk et al., 2004; Stober et al., 2003). Vaccination strategies that exploit DC and NKT cell activity by the use of $\alpha$ GalCer may serve to increase frequencies of tumour-specific $\mathrm{T}$ cell responses and thereby generate more effective antitumour immunity.

This chapter presents work that investigated whether numbers of $\mathrm{CD} 8^{+} \mathrm{T}$ cells generated after BM-DC vaccination could be increased by inducing in-vivo NKT stimulation utilizing the synthetic glycolipid $\alpha$-GalCer within this vaccination protocol. It has been reported that the co-administration of $\alpha$-GalCer with antigen increases the $\operatorname{CD} 8^{+} \mathrm{T}$ cell responses generated $2-6$ weeks post vaccination (Gonzalez-Aseguinolaza et al., 2002). Studies using $\alpha$-GalCer co-administered with soluble antigen into murine recipients showed that antigen specific $\mathrm{T}$ cell responses could also be enhanced after $i v$ administration (Fujii et al., 2003; Hermans et al., 2003). This effect was shown to peak at day 7 post vaccination and that protein antigen needed to be co-delivered with $\alpha$-GalCer at the same time. The co- 
delivery of antigen and $\alpha$-GalCer was shown by other work to confer partial prophylactic protection of murine recipients from tumour challenge after $i v$ vaccination (Hermans et al., 2003). It was also shown that the combination of NKT and TLR stimulation was capable of enhancing $\mathrm{CD}^{+}$antigen specific T cell responses and that treatment of DCs with both $\alpha$-GalCer and TLR ligand stimulation was able to induce increased IFN- $\gamma$ production by antigen specific T cells after in-vitro culture (Hermans et al., 2007).

As discussed in the previous chapter, an alternative method of antigen acquisition by host DCs involves antigen transfer between migratory DCs and lymphoid-resident DCs. Superior cross-presentation abilities have been demonstrated by the CD $8 \alpha^{+}$DCs (den Haan et al., 2000; Iyoda et al., 2002; Pooley et al., 2001; Schnorrer et al., 2006) with the langerin ${ }^{+} \mathrm{CD} 8 \alpha^{+} \mathrm{DC}$ subset being shown to be the major cross-presenting DC subset capable of generating IL-12 post injection of adjuvant co-administered with soluble antigen (Farrand et al., 2009). The previous chapter also highlighted work that indicated protein and peptide antigen was being transferred from injected vaccine BM-DCs by an unknown mechanism to host populations that were involved in the generation of $\mathrm{CD}^{+} \mathrm{T}$ cell responses. It was deemed a logical move to investigate the role of a-GalCer in the generation of $\mathrm{CD}^{+} \mathrm{T}$ cell responses and to determine whether glycolipid antigen was being transferred to host populations also.

The results presented in this chapter initially show the work carried out to establish the adjuvant activity of $\alpha$-GalCer for improving antigen specific T cell responses in this BMDC vaccine model system. Since work presented in the previous chapter showed that DC vaccines could transfer antigen to resident $\mathrm{DCs}$ with langerin ${ }^{+} \mathrm{CD} 8 \alpha^{+}$DCs being implicated in this process, the in-vivo action of $\alpha$-GalCer loaded BM-DCs was explored using the langerin-DTREGFP and langerin-EGFP transgenic mouse strains. Finally synthetic TLR agonists were also incorporated into the vaccines to provide TLR stimulation designed to improve the antigen specific $\mathrm{T}$ cell response in co-operation with NKT stimulation. 


\subsubsection{Aims}

NKT stimulation was tested for antigen specific $T$ cell response enhancing capability invivo by conducting studies that involved the in-vitro loading of $\alpha$-GalCer onto vaccine BM-DCs prior to $i v$ or $s c$ administration. The first hypothesis tested was that NKT stimulation could improve antigen specific T cell responses to $\alpha$-GalCer loaded BM-DC vaccines. The second hypothesis addressed was that both NKT and TLR stimulation could work co-operatively to further increase antigen specific $\mathrm{T}$ cell responses being generated after vaccination with antigen loaded BM-DC vaccines. Further studies were carried out to determine the specific action of $\alpha$-GalCer in-vivo with specific emphasis on the involvement of endogenous langerin ${ }^{+} \mathrm{CD} 8 \alpha^{+} \mathrm{DCs}$ in the induced $\mathrm{CD} 8^{+} \mathrm{T}$ cell response.

Specific Objectives were:

- To explore the adjuvant effect, and its duration, when $\alpha$-GalCer was incorporated into antigen loaded BM-DC vaccines

- To examine the role for antigen transfer between an injected vaccine consisting of antigen and $\alpha$-GalCer loaded BM-DCs, and host DC populations

- To investigate the combined effects of NKT and TLR stimulation on antigen specific responses to BM-DC vaccines 


\subsection{Results}

\subsubsection{NKT stimulation increases antigen specific $T$ cell responses in- vivo}

An attempt was made to further improve the $\mathrm{CD} 8^{+} \mathrm{T}$ cell response being generated in-vivo using the current BM-DC vaccine model by the use of NKT stimulation. It was anticipated that molecular interactions such as CD40 signalling and pro-inflammatory cytokines released by iNKT cells engaged with antigen presenting cells bearing CD1d and bound with $\alpha$-GalCer would further mature antigen presenting DCs and hence increase the antigen specific $\mathrm{T}$ cell response being generated against the vaccine.

BM-DC cultures were prepared using C57BL/6 mice as described in section 3.2.1 and loaded with $\alpha$-GalCer and OVA protein in-vitro on day 6 for $24 \mathrm{~h}$. Blood samples were tested for OVA specific $\mathrm{CD}^{+} \mathrm{T}$ cell responses measured 1, 2, 3 and 4 weeks after the $i v$ vaccination of B6 congenic recipients and differences in quantity compared between treatment groups. Figure 5.1 A shows the experimental schedule followed.

Dot plots presented in Figure 5.1 B depict the expansion of adoptively transferred OVA specific $\mathrm{T}$ cell populations one week after immunisation with this vaccine preparation. The bar graph in Figure 5.1 C shows that the inclusion of $\alpha$-GalCer into the BM-DC vaccination protocol markedly improved the OVA specific $\mathrm{CD}^{+} \mathrm{T}$ cell response generated when compared with non $\alpha$-GalCer-containing BM-DC vaccine groups. The loading of $\alpha-$ GalCer alone onto BM-DCs in the absence of protein antigen did not improve the OVA specific $\mathrm{T}$ cell response after vaccination.

To examine the duration of the $T$ cell response elicited by $\alpha$-GalCer containing BM-DC vaccine preparations, the OVA specific $T$ cell response was also measured again at 1 , then 2, 3 and 4 weeks post vaccination. Figure 5.2 A shows the timeline followed for this testing protocol. Bar graphs in Figure 5.2 B show that BM-DC vaccines containing 

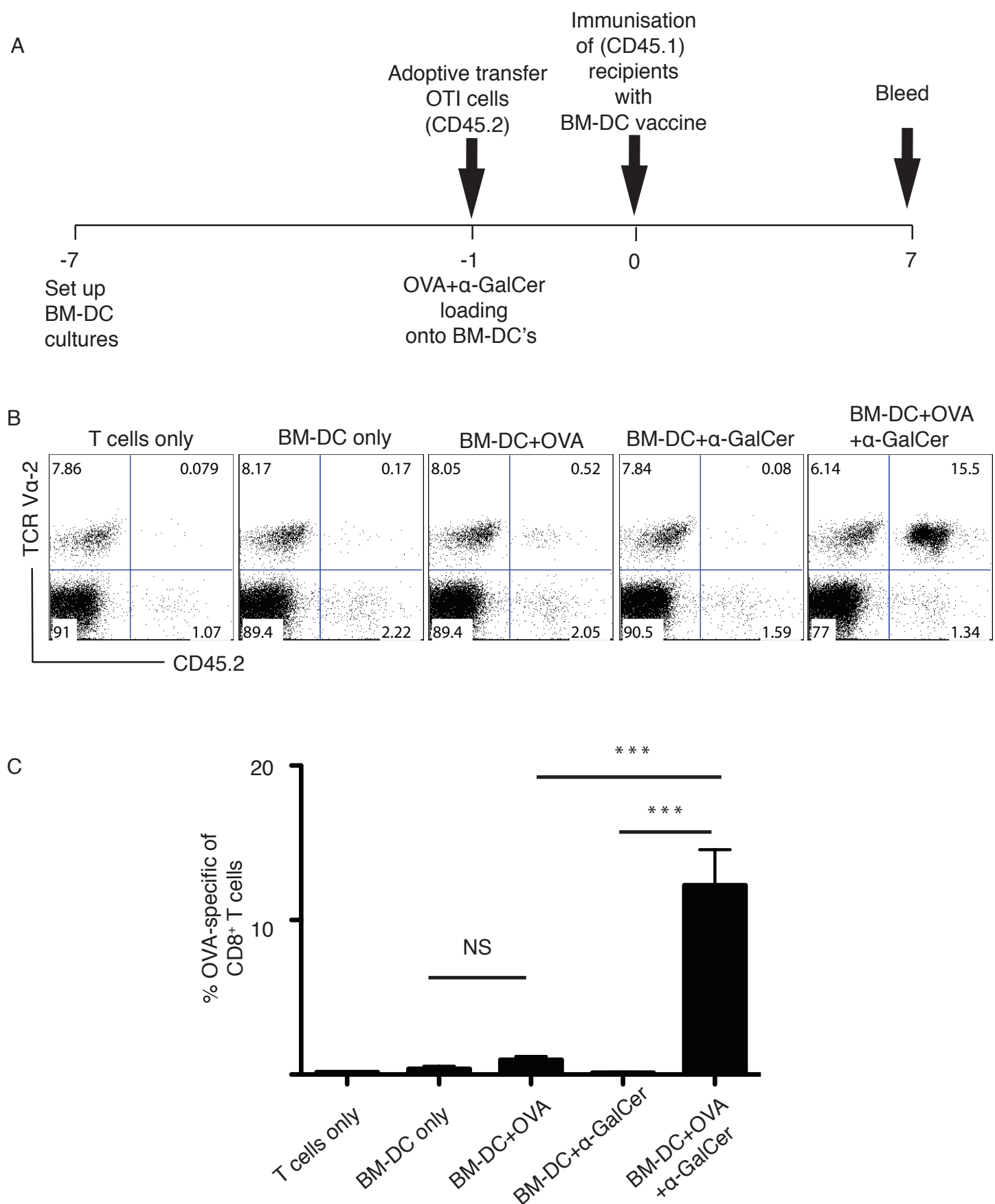

Figure 5.1 Protein loaded BM-DCs cultured with $\alpha$-GalCer increases the OVAspecific T cell responses in-vivo. (A) Timeline depicts the experimental schedule. (B) Dot plots illustrate the OVA-specific $\mathrm{T}$ cell response generated with the adoptively transferred OVA specific $\mathrm{T}$ cells represented as TCRV $\alpha 2^{+} \mathrm{CD} 45.2^{+}$cells. (C) Bar graph shows the mean percentages of the OVA specific $\mathrm{T}$ cell response expressed as a percentage of CD8 ${ }^{+}$ $\mathrm{T}$ cells $( \pm \mathrm{SEM})$ in the blood 7 days post BM-DC vaccination. One representative experiment of 3 is shown ( $n=4-5$ recipients per group) with \pm SEM. Statistical significance was determined using a 1-way ANOVA test and Tukey's post-test where ${ }^{*} \mathrm{p}=$ $0.05-0.01,{ }^{* *} \mathrm{p}=0.01-0.001,{ }^{* * *} \mathrm{p}<0.001$, NS $=$ not significant. 
Chapter 5: NKT stimulation enhances antigen specific responses to DC-based vaccines 198

$\alpha$-GalCer were able to exert an increased OVA specific $T$ cell response that persisted for more than 2 weeks post vaccination. 

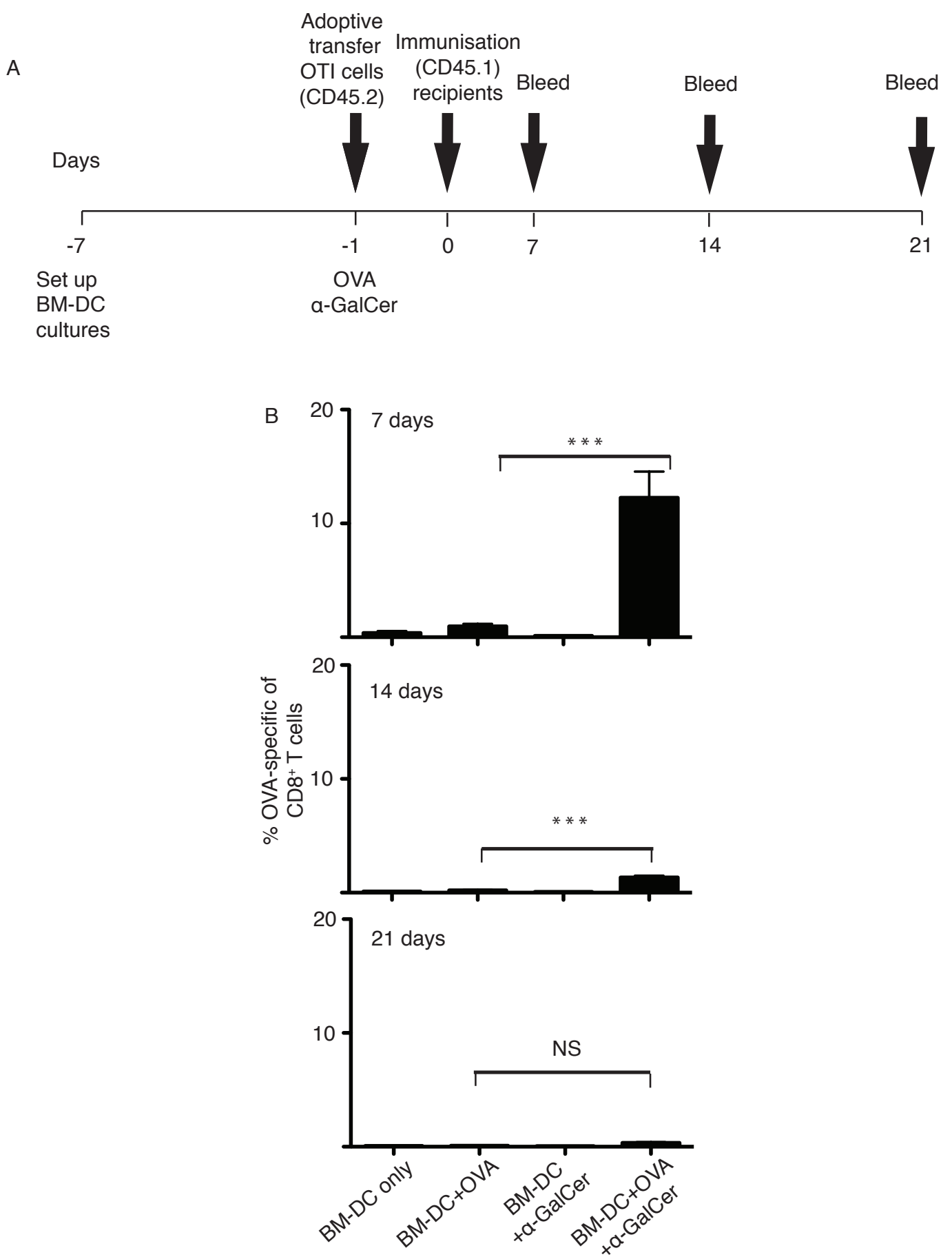

Figure 5.2 Enhanced OVA-specific $T$ cell responses to $\alpha$-GalCer loaded BM-DCs extends over time. (A) Timeline depicting the experimental set-up. (B) Bar graphs show the mean percentage of OVA-specific CD8 $\mathrm{T}$ cells as a percentage of $\mathrm{CD} 8^{+} \mathrm{T}$ cells $( \pm$ SEM) in the blood at day 7, 14, and 21 post vaccination. Results are shown from one experiment ( $n=4$ - 5 recipients per group). Statistical significance was determined using a 1-way ANOVA test and Tukey's post-test where $* \mathrm{p}=0.01-0.05, * * \mathrm{p}=0.001-0.01$, $* * * \mathrm{p}<0.001, \mathrm{NS}=$ not significant. 


\subsubsection{NKT stimulation improves antigen specific $T$ cell responses to peptide antigen loaded BM-DC vaccines}

To explore the use of peptide antigen and $\alpha$-GalCer loaded vaccine BM-DCs and determine the impact on the antigen specific responses. BM-DC cultures were treated with SIINFEKL peptide in-vitro to substitute the OVA protein previously tested for the BM-DC vaccination protocol. This permits the direct loading of peptide antigen onto BM-DC surface MHC complexes and eliminates the internal processing requirement necessary for protein antigen by the BM-DCs. The subsequent impact on antigen specific $\mathrm{T}$ cell responses was then measured in-vivo.

SIINFEKL peptide antigen was loaded onto $\alpha$-GalCer treated BM-DCs that were prepared as described in section 3.2.1. As seen previously when vaccinating recipients using protein loaded BM-DC vaccines treated with $\alpha$-GalCer (Figure 5.1 C), the bar graph in Figure 5.3 $\mathrm{C}$ also shows the same response where an increased OVA-specific $\mathrm{T}$ cell response being generated in recipients vaccinated with $\alpha$-GalCer even when BM-DC vaccines are loaded with peptide.

These results indicate the increased antigen specific $\mathrm{T}$ cell response seen after combining with $\alpha$-GalCer occurs independently of internal antigen processing steps. 
A
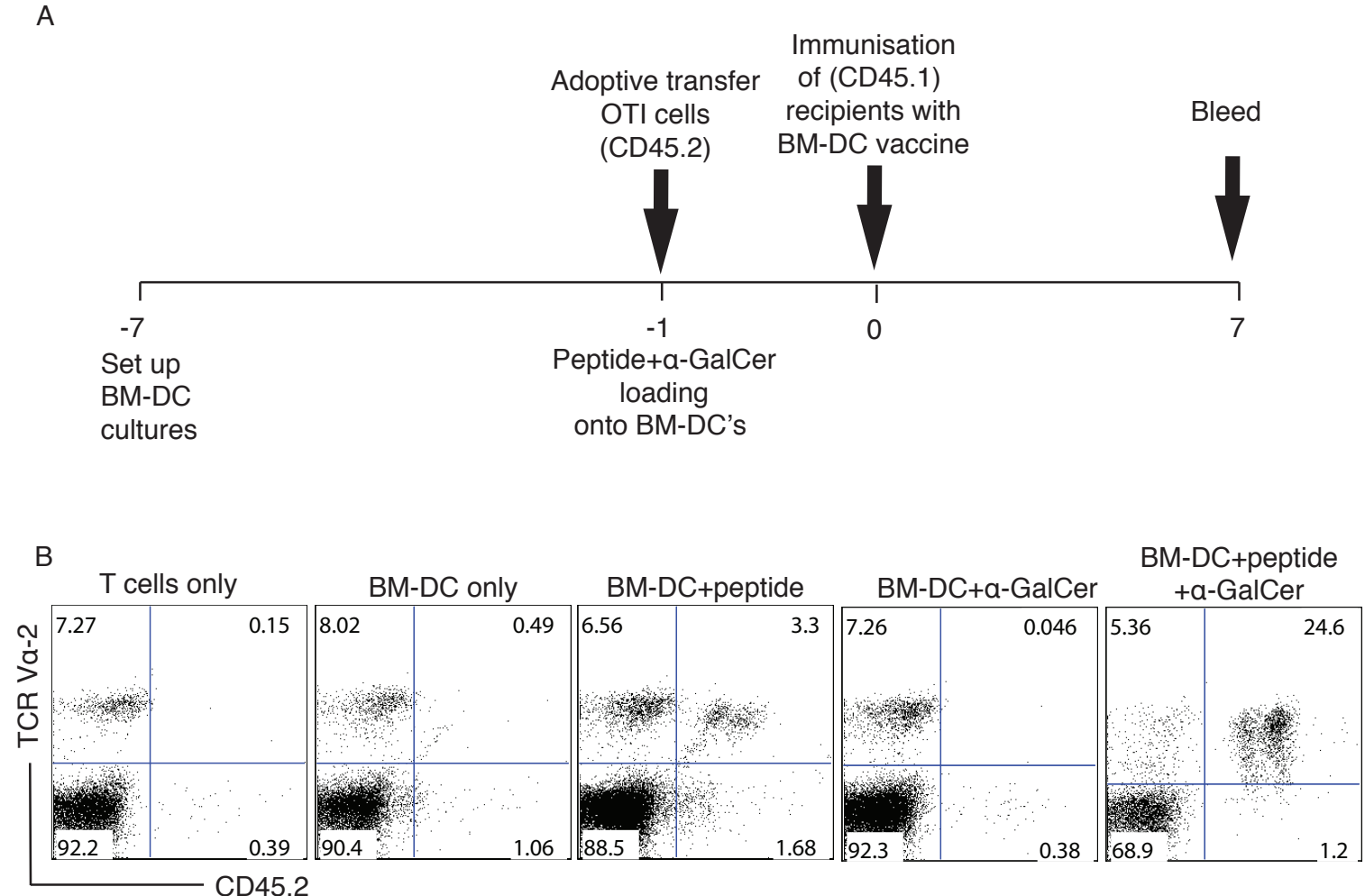

C

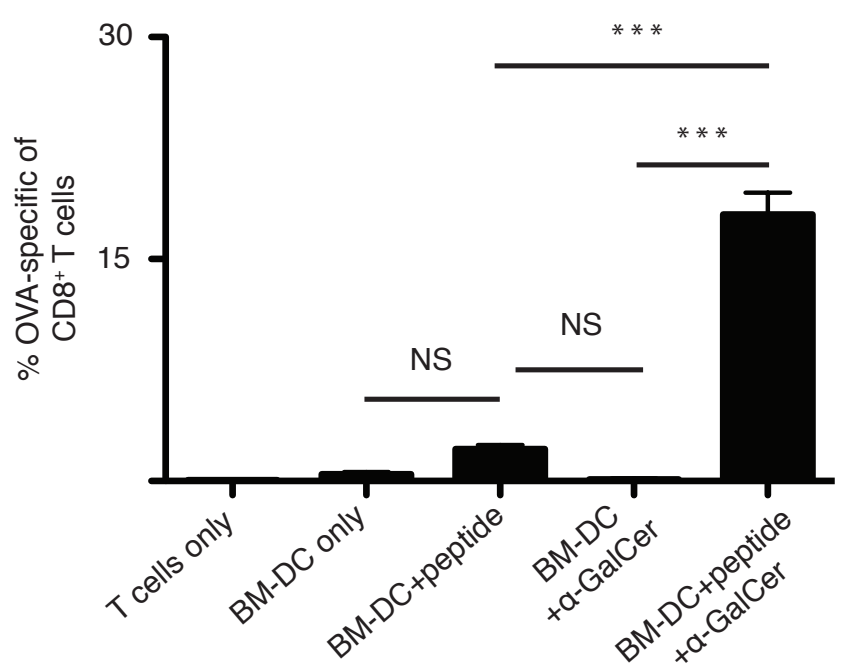

Figure 5.3 Peptide loaded BM-DCs cultured with $\alpha$-GalCer and peptide increase OVA specific $\mathbf{T}$ cell responses in-vivo. (A) Timeline depicts the experimental set-up followed. (B) Dot plots illustrate the OVA-specific $\mathrm{T}$ cell response generated with the adoptively transferred OVA specific T cells represented as TCRV $\alpha 2^{+} \mathrm{CD} 45.2^{+}$cells. (C) Bar graph shows the mean percentages of the OVA specific $\mathrm{T}$ cell response expressed as a percentage of $\mathrm{CD}^{+} \mathrm{T}$ cells $( \pm \mathrm{SEM})$ as seen in the blood 7 days post BM-DC vaccination. One representative experiment of 3 is shown ( $n=4-5$ recipients per group). Statistical significance was determined using a 1-way ANOVA test and Tukey's post-test where * $\mathrm{p}=$ $0.05-0.01,{ }^{*} \mathrm{p}=0.01-0.001,{ }^{* * *} \mathrm{p}<0.001, \mathrm{NS}=$ not significant. 


\subsubsection{Subcutaneous administration of $\alpha$-GalCer induces increased OVA-specific Reponses}

Although DC based vaccines used in human clinical trials typically use the intradermal/subcutaneous route, the intravenous administration of antigen loaded $\alpha$-GalCer treated BM-DC vaccines was initially the route of choice for our studies due to the prevalence of iNKT cells found in the mouse spleen. The influence of vaccination route on induction of antigen specific immune responses after immunisation with $\alpha$-GalCer loaded BM-DC vaccines in this model system had not been tested. A study was therefore conducted to measure the antigen specific $\mathrm{T}$ cell response generated after vaccination using the subcutaneous route of application.

The BM-DCs were prepared as previously described in section 3.2.1 and loaded with $\alpha$ GalCer and protein antigen OVA on day 6 (Figure $5.4 \mathrm{~A}$ ). The bar graph shown in Figure 5.4 $\mathrm{C}$ shows that $\alpha$-GalCer containing BM-DC vaccines administered subcutaneously were able to incorporate the use of NKT cell stimulation to increase the OVA specific T cell response generated. Although the OVA specific T cell response was not as large as that seen after $i v$ administration (Figure $5.1 \mathrm{C}$ ), the degree of expansion was nevertheless significantly improved over recipient groups that received the OVA loaded BM-DC vaccines in the absence of $\alpha$-GalCer.

These results indicate that $\alpha$-GalCer loaded BM-DCs administered subcutaneously are indeed capable of inducing increased antigen specific $\mathrm{T}$ cell responses via iNKT cell mediation. The implication of this experiment is that $\alpha$-GalCer loaded onto BM-DCs is either being directly presented to iNKT cells on CD1d molecules found on the injected vaccine BM-DCs, or that $\alpha$-GalCer may even be transferred to another antigen presenting 


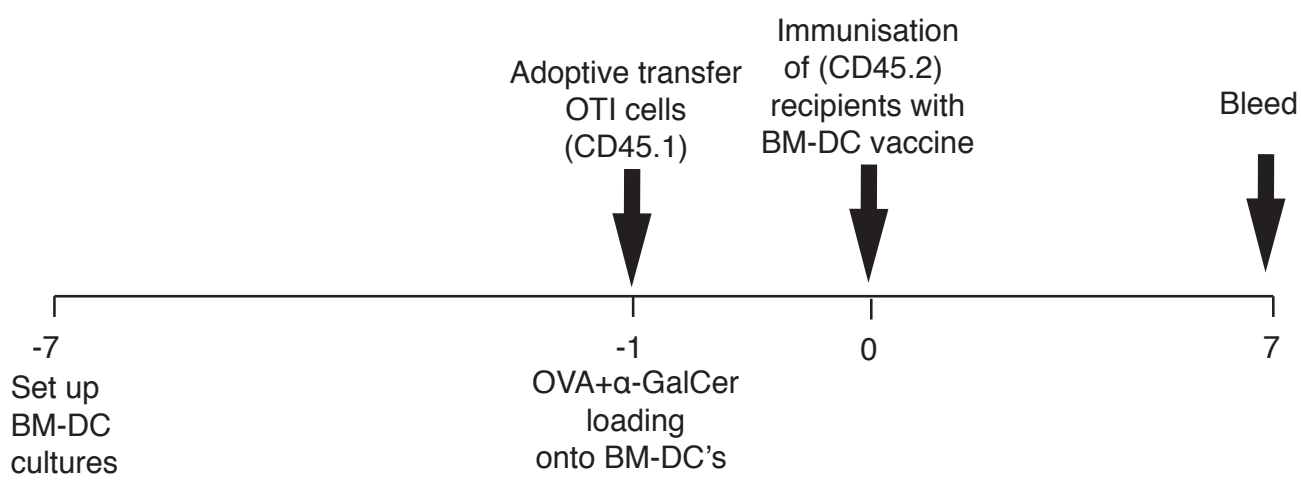

B

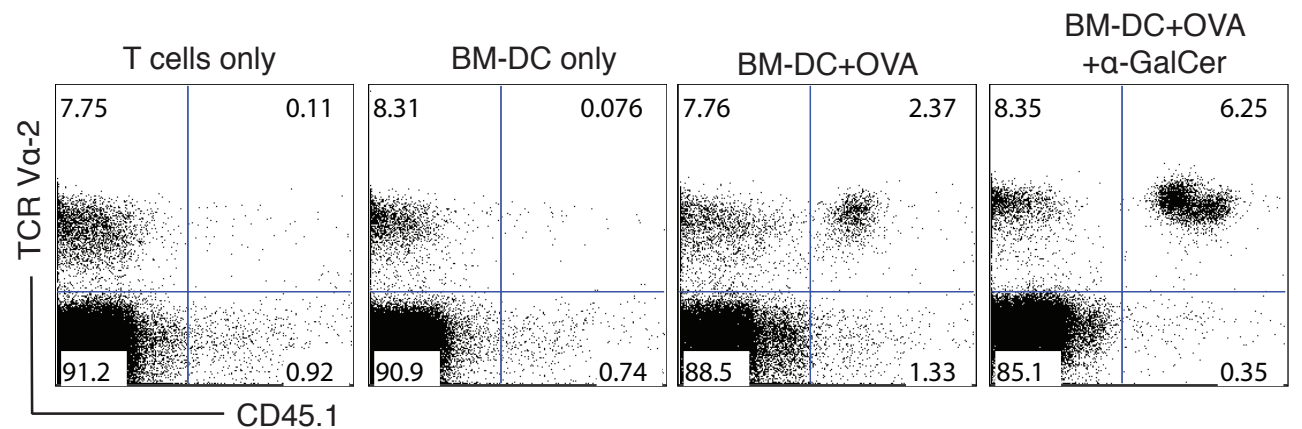

C

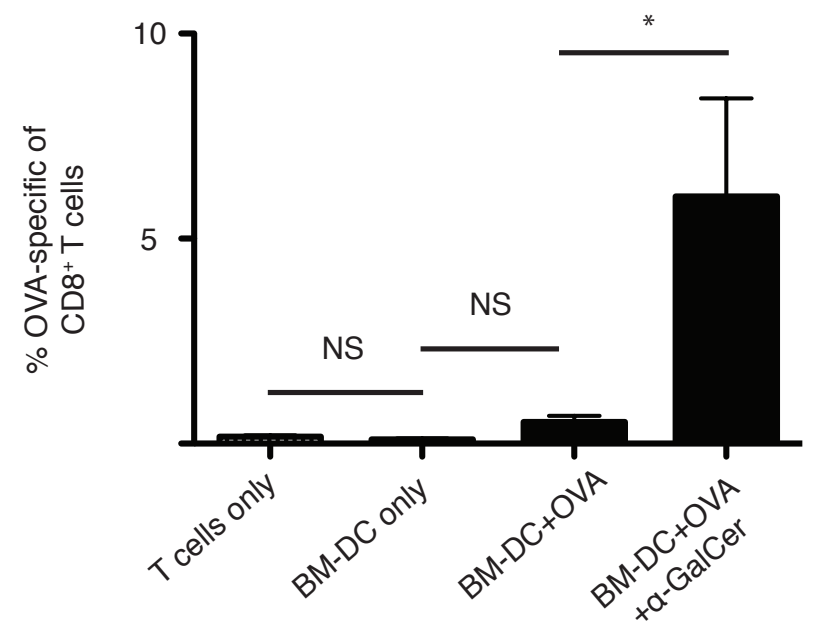

Figure 5.4 Subcutaneous administration of $\alpha$-GalCer loaded BM-DCs induces increased OVA specific $T$ cell responses. (A) Timeline depicting the BM-DC, OVA and $\alpha-$ GalCer loading vaccination protocol strategy. (B) Representative flow cytometry dot plots illustrate the OVA-specific T cell response generated with the adoptively transferred OVA specific T cells represented as TCRV $22^{+} \mathrm{CD} 45.1^{+}$cells into $\mathrm{C} 57 \mathrm{BL} / 6$ recipients $(n=$ 4-5). One representative experiment of 3 is shown. (C) Bar graph shows the mean percentages of the OVA specific $\mathrm{T}$ cell response expressed as a percentage of $\mathrm{CD} 8^{+} \mathrm{T}$ cells $( \pm$ SEM) as seen in the blood 7 days post BM-DC vaccination. Statistical significance was determined using a 1-way ANOVA test and Tukey's post-test where $* p=0.05-0.01,{ }^{*} p$ $=0.01-0.001, * * * \mathrm{p}<0.001, \mathrm{NS}=$ not significant. 
cell type that is able to then present the $\alpha$-GalCer to iNKT cells and induce enhanced OVA specific $\mathrm{T}$ cell responses in an indirect manner.

\subsubsection{The fate of $\alpha$-GalCer loaded BM-DCs after $i v$ administration}

The increased antigen specific $T$ cell response generated in response to using $\alpha$-GalCer loaded BM-DCs given $i v$ or $s c$, was seen consistently in vaccine recipients. iNKT mediated stimulation was accepted as a contributing factor responsible for boosting the OVA specific $\mathrm{T}$ cell responses throughout these studies. Efforts were now directed to determine the effect of $\alpha$-GalCer loading on both injected BM-DC and host splenic DC populations in-vivo and determine the source of $\alpha$-GalCer presentation to the iNKT cells.

This was done by retrieving CFSE labelled $\alpha$-GalCer treated BM-DCs in the spleen $24 \mathrm{~h}$ after $i v$ administration and checking their maturation status. Injected $\alpha$-GalCer loaded BMDCs were expected to reach the spleen by $24 \mathrm{~h}$ after $i v$ injection, therefore it was decided to harvest splenocytes and isolate $\mathrm{CFSE}^{\text {high }}$ and $\mathrm{CFSE}^{\text {low }}$ populations that were present at the $24 \mathrm{~h}$ time point to assess maturation of the injected cells (CFSE ${ }^{\text {high }}$ ) versus endogenous DCs that had acquired cellular material from the vaccine (CFSE $\left.{ }^{\text {low }}\right)$.

CFSE was used to label $\alpha$-GalCer loaded BM-DCs prior to $i v$ injection and after $24 \mathrm{~h}$, splenic DC populations were isolated and the phenotype of injected and host DC populations examined using flow cytometric analysis (Figure 5.5 A). Dot plots presented

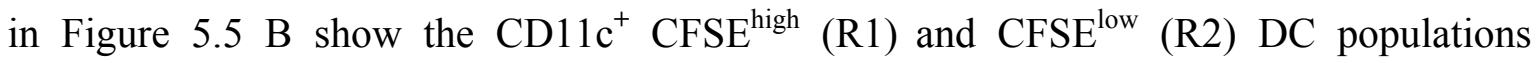
retrieved. Endogenous splenic DC populations that had not acquired material are also seen in R3. Histograms in Figure 5.5 C show that the maturation status of injected BM-DCs is similar once they have reached the spleen whether or not they have been loaded with $\alpha$ GalCer (R1). In contrast, the endogenous DCs were matured in response to administration of $\alpha$-GalCer loaded DCs, regardless of whether they had acquired fluorescent material from the vaccine or not. 


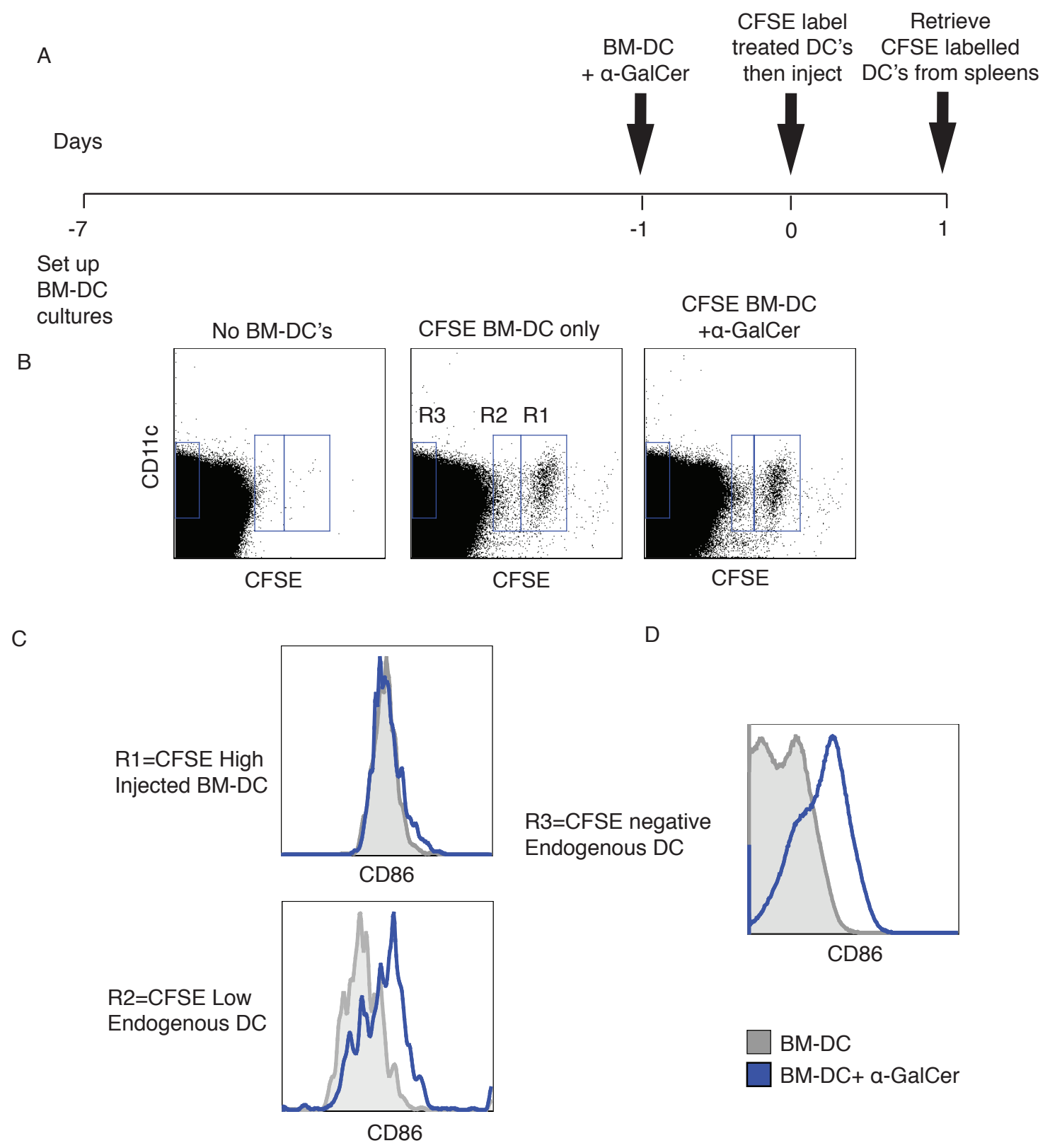

Figure 5.5 The fate of CFSE labelled BM-DCs retrieved from spleens $24 \mathrm{~h}$ after systemic administration. (A) Timeline depicting BM-DC vaccine manufacture and CFSE labelling prior to $i v$ administration. (B) Flow cytometry dots plots show CFSE labelled CD11 $\mathrm{c}^{+}$BM-DCs retrieved from splenic DC populations $24 \mathrm{~h}$ post vaccination. Endogenous $\mathrm{CD} 11 \mathrm{c}^{+}$populations also evident in dot plots alongside populations of DCs exhibiting CFSE low expression. (C) Histograms show maturation status of retrieved CFSE labelled BM-DC populations. (D) Histogram showing maturation status of endogenous CD11 $\mathrm{c}^{+}$DC populations $24 \mathrm{~h}$ after treatment with CFSE labelled BM-DC vaccines. Results from one experiment are shown ( $n=3$ recipients per group). 
This finding shows that $\alpha$-GalCer treated BM-DCs are capable of influencing the maturation status of host DC populations whether this was through cytokine effects, or the actual acquisition of $\alpha$-GalCer was unknown at this stage.

These data are similar to groups treated with TLR ligands (see Figure 3.16) again emphasising that regardless of the in-vitro manipulation protocol adopted, by the time $i v$ administered BM-DC populations reach the spleen their maturation status is remarkably similar. It would appear that loading $\alpha$-GalCer onto BM-DC populations allows not only the injected DCs but also host DC populations to be influenced by NKT cell stimulatory effects.

\subsubsection{Differential maturation of host splenic DC populations in response to $\alpha$-GalCer}

Experiments thus far have consisted of the in-vitro loading of BM-DC populations using $\alpha$-GalCer for vaccination studies. Because data presented in the previous chapter has indicated a key role for transfer of antigen from injected BM-DCs to resident langerin ${ }^{+} \mathrm{CD} 8 \alpha^{+} \mathrm{DCs}$ in the induction of $\mathrm{CD}^{+} \mathrm{T}$ cell responses to $\mathrm{DC}$ vaccination, it was likely that NKT-cell-mediated improvements to vaccination were through the effects on these endogenous DCs. To examine the effect of direct NKT stimulation on host splenic DC populations in-vivo, $\alpha$-GalCer was administered systemically into C57BL/6 recipients and the maturation effect on host DC populations and other cell types determined using flow cytometric analysis as seen in Figure 5.6 A. These experiments were also conducted in langerin-EGFP recipients so as to assess the impact of NKT cell stimulation on langerin ${ }^{+} \mathrm{CD} 8 \alpha^{+} \mathrm{DCs}$.

The bar graph in Figure 5.6 C shows the increased CD86 expression on splenic CD11c ${ }^{+}$ DCs isolated $16 \mathrm{~h}$ after systemic $\alpha$-GalCer administration of C57BL/6 recipient groups. The histogram in Figure 5.6 B shows the geometric MFI values measured between control 
A

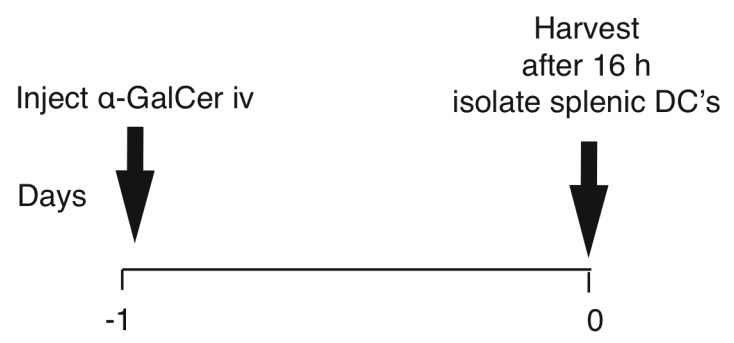

B

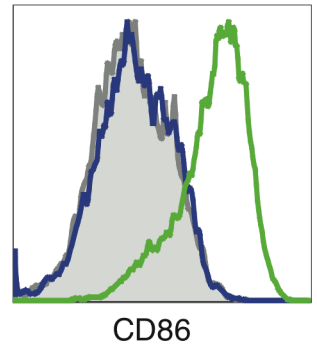

a-GalCer

PBS

Naive

D

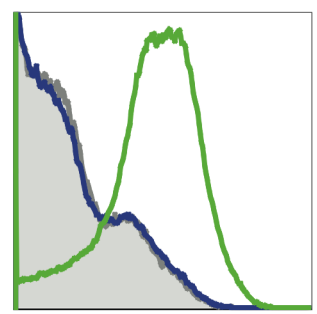

CD86

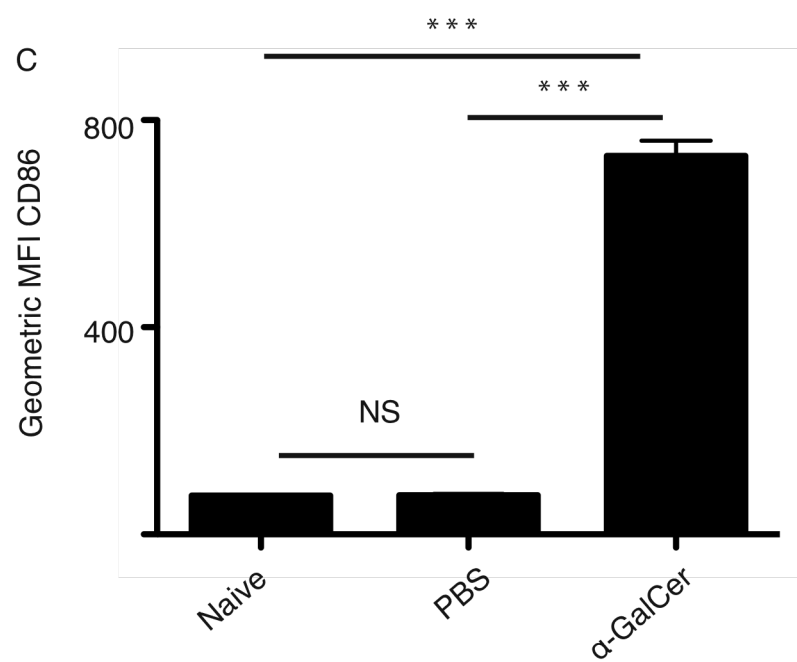

$E$

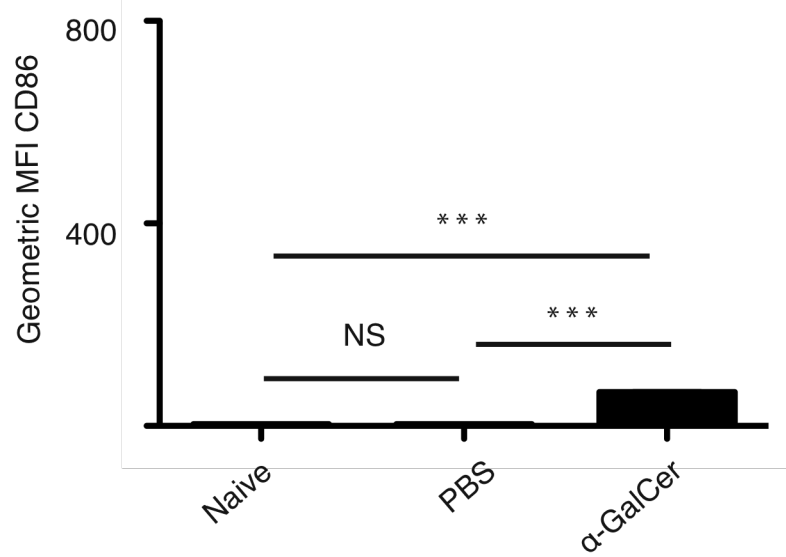

Figure 5.6 Systemic administration of $\alpha$-GalCer induces maturation of splenic DCs and $B$ cells. A. Timeline indicating experimental procedure. B. Histogram shows relative geometric MFI CD86 expression on splenic CD11 ${ }^{+}$DCs. C. Bar graphs show geometric MFI values for CD86 expression on splenic DCs $16 \mathrm{~h}$ after systemic administration of PBS or $\alpha$-GalCer $(n=3$ recipients). D. Histogram shows relative geometric MFI CD86 expression on splenic B cells. E. Bar graph shows geometric MFI values for CD86 expression on splenic B cells $16 \mathrm{~h}$ after systemic administration of PBS or $\alpha$-GalCer. One representative experiment of 3 is shown ( $n=3$ C57BL6 recipients per group). Statistical significance was determined using 1-way ANOVA test and Tukey's post-test where $* \mathrm{p}=$ $0.05-0.01,{ }^{* *} \mathrm{p}=0.01-0.001,{ }^{* * *} \mathrm{p}<0.001, \mathrm{NS}=$ not significant. 
Chapter 5: NKT stimulation enhances antigen specific responses to DC-based vaccines 208

and $\alpha$-GalCer treated groups. A similar maturation trend was seen for B cells in Figure 5.6 D and Figure 5.6 E, although lower levels of CD86 expression were seen after stimulation with $\alpha$-GalCer when compared with splenic DC measures in Figure 5.6 B and C. This may be reflective of differences in CD1d expression between B cells and DCs.

To explore the effect of $\alpha$-GalCer in more detail, $\alpha$-GalCer was given systemically to langerin-EGFP recipients and the major splenic DC subsets recognized using flow cytometric analysis. Langerin ${ }^{+} \mathrm{CD} 8 \alpha^{+}$langerin ${ }^{-} \mathrm{CD} 8 \alpha^{+}$and langerin ${ }^{-} \mathrm{CD} 8 \alpha^{-} \mathrm{DC}$ populations were identified from splenocyte populations isolated from langerin-EGFP recipients treated with or without $\alpha$-GalCer, and the maturation status determined using CD86 and CD40 surface marker expression.

Figure 5.7 presents a panel of histograms (A) and (C) and bar graphs (B) and (D) that show the CD86 (top row) and CD40 (bottom row) geometric MFI levels as seen in $\alpha$-GalCer treated and non $\alpha$-GalCer treated groups after $5 \mathrm{~h}$ (first column) and $24 \mathrm{~h}$ (second column) for each of the major splenic DC subsets isolated from these recipients. What is immediately evident is that baseline expression of CD40 and CD86 expression appears higher on the langerin ${ }^{+} \mathrm{CD} 8 \alpha^{+} \mathrm{DC}$ subsets and that treatment with $\alpha$-GalCer after $5 \mathrm{~h}$ further enhances this increased expression. Systemic administration of $\alpha$-GalCer was also able to increase the CD86 expression on the remaining splenic DC subsets: langerin ${ }^{-}$ $\mathrm{CD} 8 \alpha^{+}$, langerin ${ }^{-} \mathrm{CD} 8 \alpha^{-}$after 5 h. systemic administration. Although the magnitude of CD86 expression at $24 \mathrm{~h}$ (Figure $5.7 \mathrm{D}$ ) appeared less than that seen at $5 \mathrm{~h}$ (Figure $5.7 \mathrm{~B}$ ), this was caused by inter-experimental variation since the $5 \mathrm{~h}$ timepoint was examined initially with the impact of $\alpha$-GalCer treatment on splenic DC subsets overall after $24 \mathrm{~h}$ being the primary concern. The elevation in CD86 expression from baseline levels for the splenic DC subsets at both timepoints was observed.

Although treatment with $\alpha$-GalCer increased the expression of CD40 and CD86 when compared with controls, by $24 \mathrm{~h}$ this trend still remained although the extent of the CD86 expression in particular was markedly less overall when compared to results at $5 \mathrm{~h}$. 
Chapter 5: NKT stimulation enhances antigen specific responses to DC-based vaccines209

When $\alpha$-GalCer was administered systemically, the percentage of langerin ${ }^{+} \mathrm{CD} 8 \alpha^{+}$splenic DCs was significantly reduced by $24 \mathrm{~h}$. What was unclear was whether this was due to down-regulation of the langerin marker in response to the $\alpha$-GalCer, or that whether cell death was occurring (results not shown) The langerin ${ }^{+} \mathrm{CD} 8 \alpha^{+}$DCs that remained were assessed for their CD86 and CD40 expression and incorporated into these results.

These results indicate that systemic administration of $\alpha$-GalCer is able to increase the maturation status of all splenic DC subsets-but in particular the langerin ${ }^{+} \mathrm{CD} 8 \alpha^{+} \mathrm{DC}$ subset. It was likely that $\alpha$-GalCer was binding to CD1d molecules on splenic DC subsets 
A
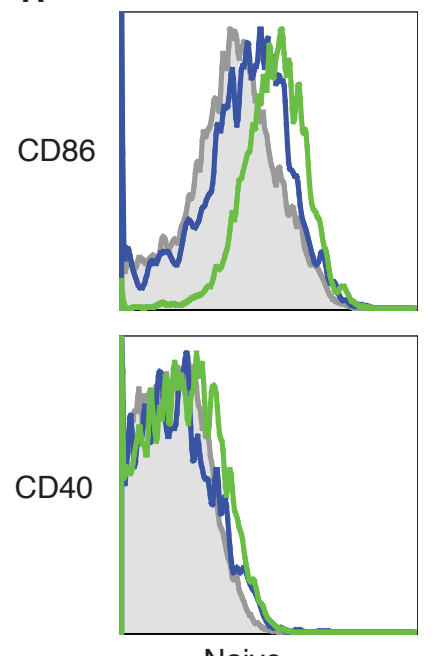

Naive
$5 \mathrm{~h}$
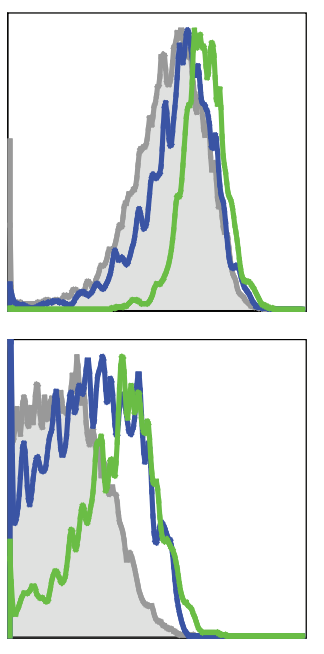

$+\mathrm{a}-\mathrm{GalCer}$
C
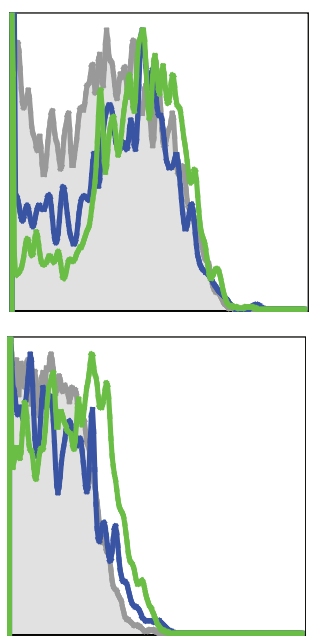

Naive
$24 \mathrm{~h}$
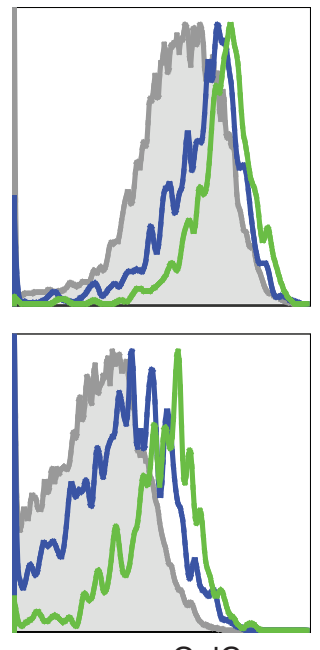

+ a-GalCer
B

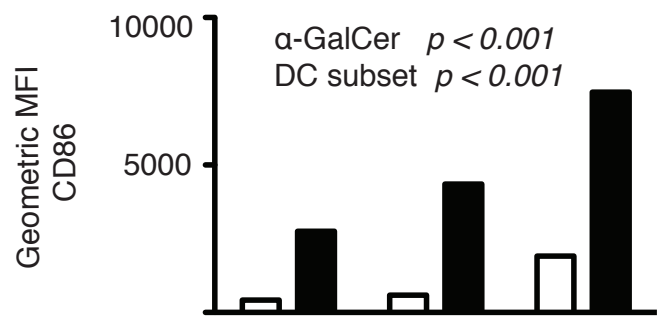

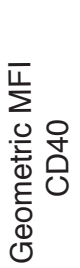

300

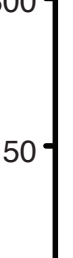

a-GalCer $\quad p<0.001$

DC subsets $p<0.001$

50
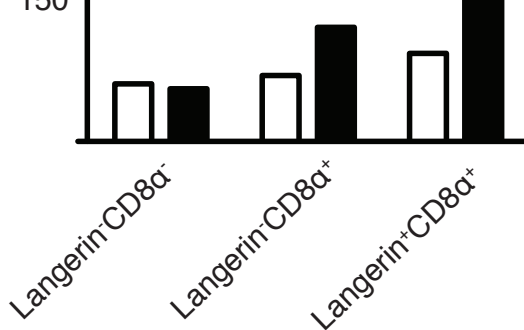

Langerin ${ }^{+} \mathrm{CD} 8 \mathrm{a}^{+}$

Langerin-CD8 ${ }^{+}$

Langerin ${ }^{-C D 8} a^{-}$

D

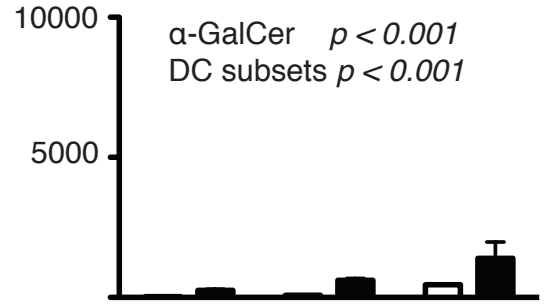

$24 \mathrm{~h}$

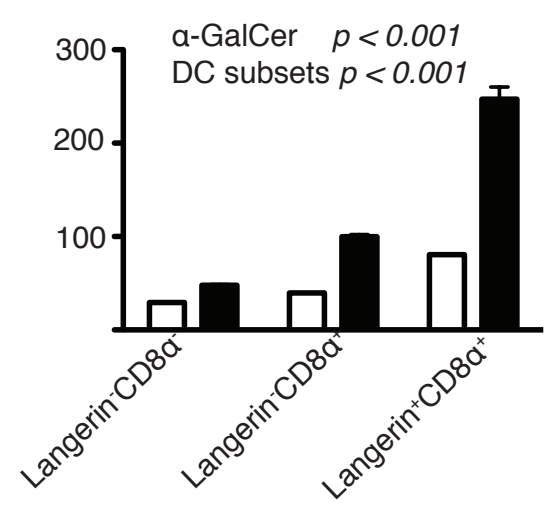

a-GalCer

Naive 
Figure 5.7 The characterization of splenic DC subsets after systemic $\alpha$-GalCer treatment for 5 and $\mathbf{2 4}$ h. (A) Histograms show relative geometric MFI CD86 and CD40 expression across all major splenic DC subsets in naive, $\alpha$-GalCer treated langerin-EGFP subjects after $5 \mathrm{~h}\left(1^{\text {st }}\right.$ and $2^{\text {nd }}$ column $)$ and (B) $24 \mathrm{~h}\left(3^{\text {rd }}\right.$ and $4^{\text {th }}$ columns $)$. (C) Bar graphs show geometric MFI values for CD86 (top) and CD40 (bottom) for major splenic DC populations in naïve and $\alpha$-GalCer treated langerin-EGFP recipients 5 and (D) $24 \mathrm{~h}$ after systemic administration. Results from one experiment are shown. Statistical significance was determined using 2-way ANOVA test where $* p=0.05-0.01, * * p=0.01-0.001$, $* * * \mathrm{p}<0.001, \mathrm{NS}=$ not significant.

and directly interacting with NKT cells to induce their release of pro-inflammatory cytokines and subsequent maturation of $\alpha$-GalCer presenting DCs. It was also possible that DC subsets were responding to pro-inflammatory cytokine release being induced by other $\alpha$-GalCer presenting cell types interacting with NKT cells or that even a combination of both processes was taking place. Further studies were conducted to determine the action of $\alpha$-GalCer on splenic DCs.

\subsubsection{The loading of $\alpha$-GalCer in-vitro onto vaccine BM-DCs compared with systemic administration}

The use of BM-DCs loaded with $\alpha$-GalCer in-vitro had been shown consistently to improve OVA specific $\mathrm{CD}^{+} \mathrm{T}$ cell responses measured in the blood one week after vaccination. The next issue to be addressed was whether $\alpha$-GalCer administered systemically was capable of mediating the generation of an OVA specific $\mathrm{CD}^{+} \mathrm{T}$ cell response and to determine if this response was superior to that seen if using $\alpha$-GalCer loaded onto BM-DCs. To address this, the OVA specific $\mathrm{CD} 8^{+} \mathrm{T}$ cell response was measured in vaccine recipients after giving $\alpha$-GalCer systemically and compared with that generated after the loading of $\alpha$-GalCer onto BM-DCs.

The experimental timeline used can be seen in Figure 5.8 A, while representative dot plots are presented in Figure 5.8 B and show the expansion of OVA specific T cell populations for the different treatment groups as seen in the blood one week after vaccination. Soluble $\alpha$-GalCer versus BM-DC loaded $\alpha$-GalCer groups were tested and compared with each 
A

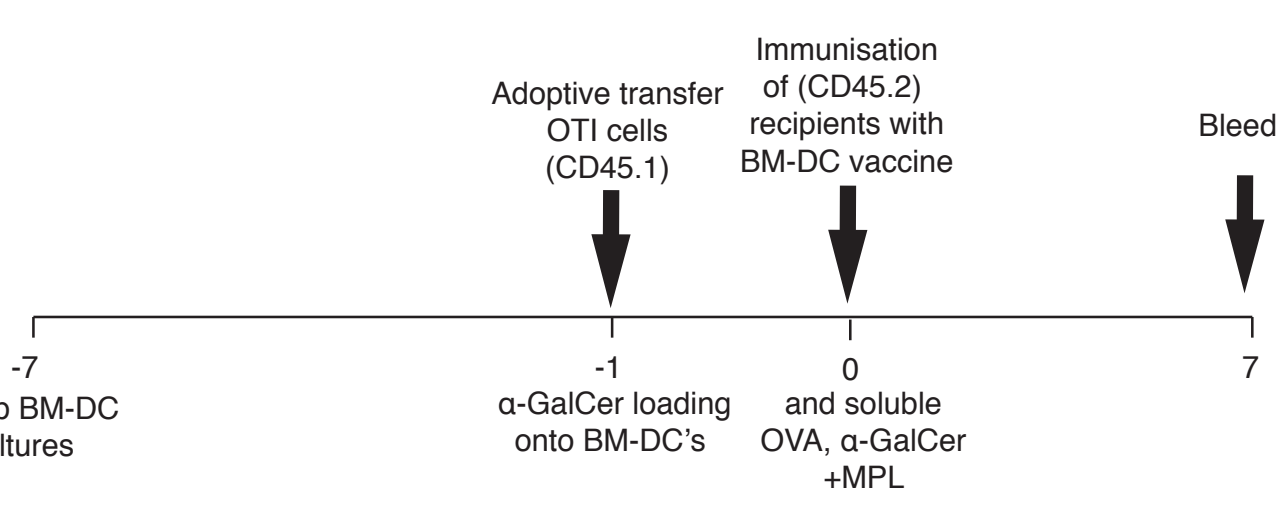

B

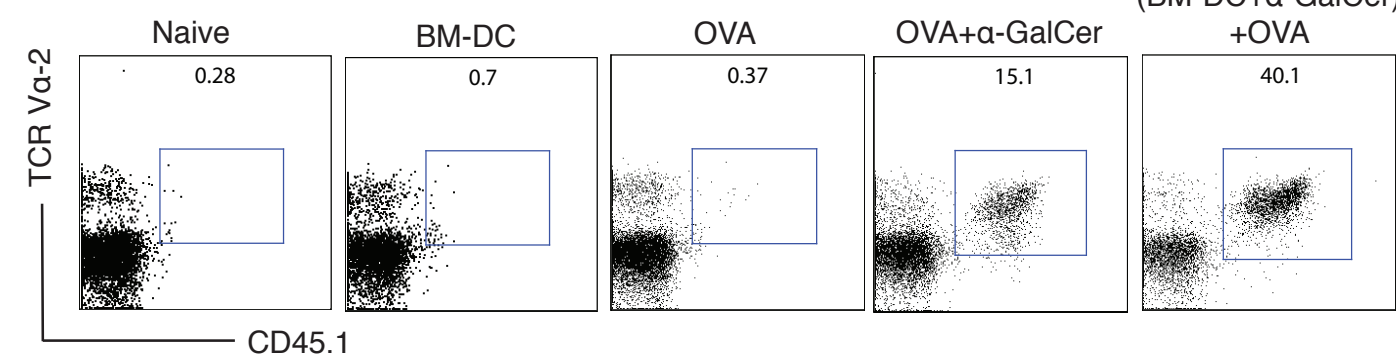

C

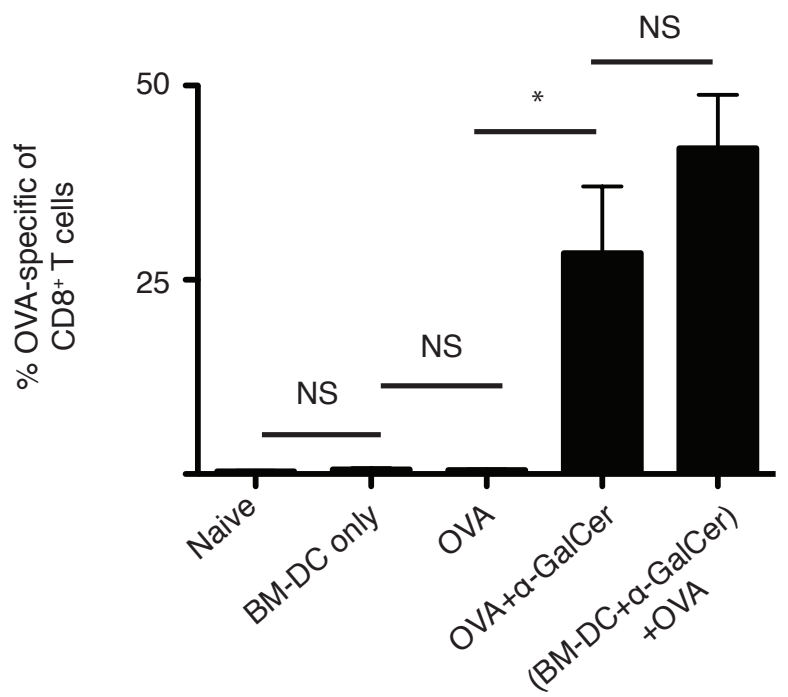

Figure 5.8 The loading of BM-DCs with $\alpha$ GC in-vitro increases OVA specific $T$ cell responses. (A) Timeline depicting the $\mathrm{BM}-\mathrm{DC}$ and soluble vaccination components protocol strategy. (B) Representative flow cytometry dot plots illustrate the OVA-specific $\mathrm{T}$ cell response generated with the adoptively transferred OVA specific $\mathrm{T}$ cells represented as TCRV $\alpha 2^{+} \mathrm{CD} 45.1^{+}$cells $(n=4-5)$. (C) Bar graph shows the mean percentages of the OVA specific $\mathrm{T}$ cell response expressed as a percentage of $\mathrm{CD} 8^{+} \mathrm{T}$ cells $( \pm \mathrm{SEM})$ as seen in the blood 7 days post BM-DC vaccination. Results from one experiment are shown. Statistical significance was determined using a 1-way ANOVA test and Tukey's post-test where $* \mathrm{p}=0.05-0.01, * * \mathrm{p}=0.01-0.001, * * * \mathrm{p}<0.001, \mathrm{NS}=$ not significant. 
other. The bar graph in Figure 5.8 C suggests that the loading of $\alpha$-GalCer onto BM-DCs generates superior OVA specific T cell response after vaccination when compared with the administration of soluble $\alpha$-GalCer and antigen. Although this difference is not statistically significant it is important to note that the amount of $\alpha$-GalCer being made available to the in-vivo environment by systemic administration is much greater than that being delivered after the in-vitro loading of $\alpha$-GalCer onto BM-DCs. Given that similar responses are generated, this indicates that the loading of $\alpha$-GalCer onto BM-DCs improves the presentation capacity for DCs carrying $\alpha-$ GalCer than when administered systemically.

What these results show is that soluble $\alpha$-GalCer is capable of mediating the induction of an antigen specific $\mathrm{CD}^{+} \mathrm{T}$ cell response when given systemically. However it appears that there are presentation requirements that exist for $\alpha$-GalCer which are carried out by vaccine BM-DCs that allow for proper $\mathrm{T}$ cell presentation activities and the generation of a superior OVA specific $\mathrm{CD}^{+} \mathrm{T}$ cell response in the blood after vaccination.

\subsubsection{Langerin ${ }^{+} \mathrm{CD8} \alpha^{+}$DCs mediate the increased $\mathrm{CD8}^{+} \mathrm{T}$ cell response to $\alpha$-GalCer containing vaccines (via antigen and glycolipid transfer)}

Previous work shown in chapter 4 had indicated that protein and peptide antigen was being transferred from injected vaccine DCs to endogenous DC populations. To further examine the action of NKT stimulation when used in combination with BM-DC based vaccines, langerin-DTREGFP mice were used as recipients so that the impact of selective depletion of the langerin ${ }^{+} \mathrm{CD} 8 \alpha^{+} \mathrm{DCs}$ could be examined on the induction of $\mathrm{CD} 8^{+} \mathrm{T}$ cell responses. Studies were carried out to further test protein antigen transfer, and determine if the glycolipid $\alpha$-GalCer was also being transferred from injected DCs to endogenous host populations.

At this stage, two important experiments were carried out by colleague Dr Troels Petersen that added considerably to the concept of glycolipid and antigen transfer. First, OVAspecific $\mathrm{CD}^{+} \mathrm{T}$ cell responses were markedly reduced in recipients depleted of 
A

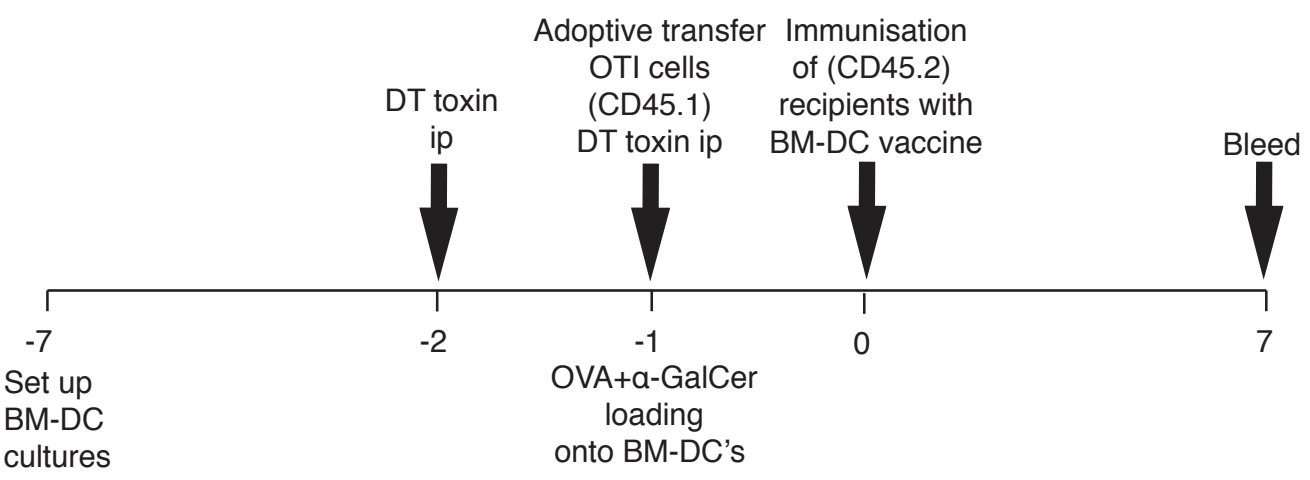

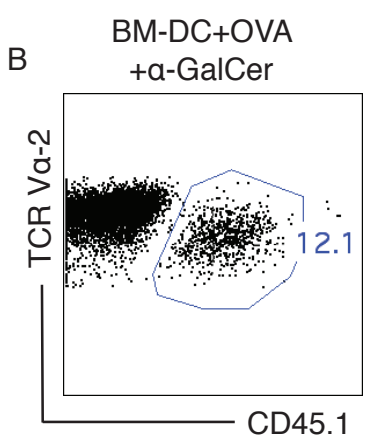
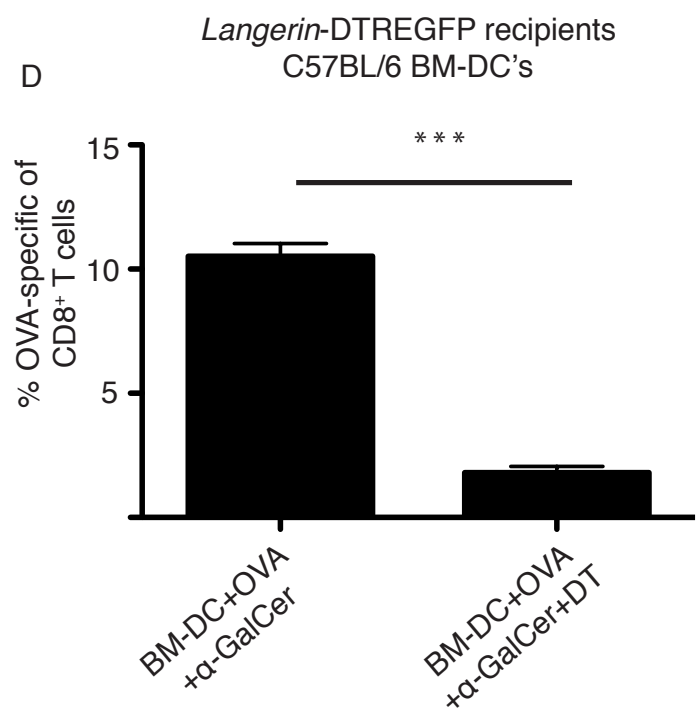

C

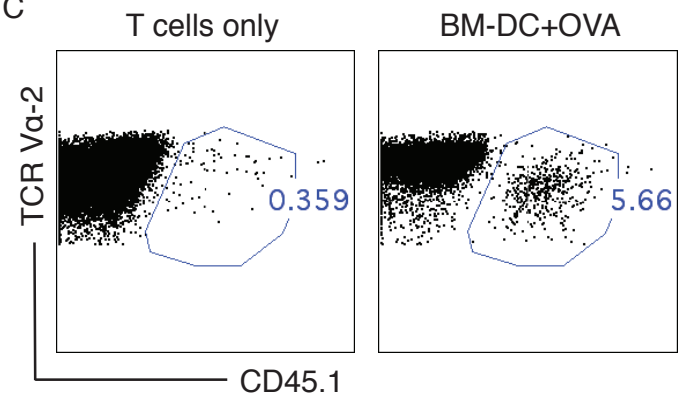

$\mathrm{E}$

C57BL/6 BM-DC's recipients C57BL/6 BM-DC's

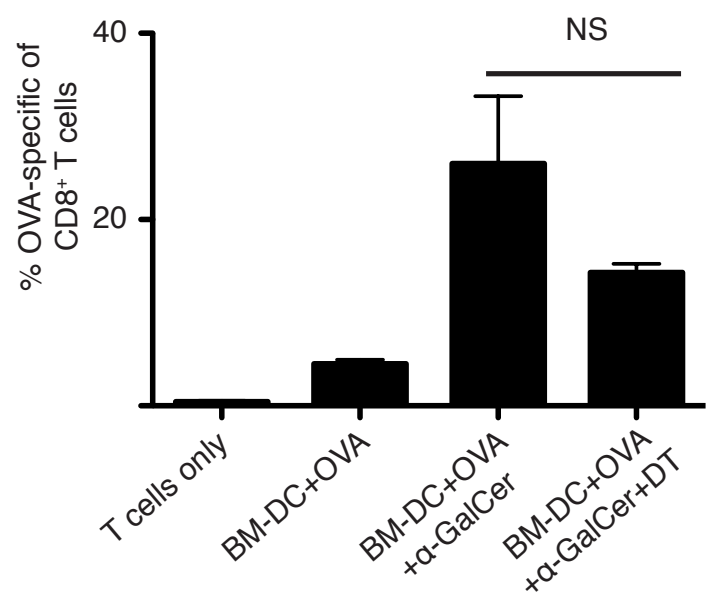

Figure 5.9 The depletion of langerin ${ }^{+} \mathrm{CD} \alpha^{+}$DCs reduces the antigen specific $\mathrm{T}$ cell response to $\boldsymbol{\alpha}$-GalCer loaded BM-DC vaccines. (A) Timeline depicting the BM-DC, OVA and $\alpha$-GC loading vaccination protocol strategy. (B) Representative flow cytometry dot plots illustrate the OVA-specific $\mathrm{T}$ cell responses generated by adoptively transferred OVA specific T cells after treatment with DT into langerin-DTREGFP and (C) C57BL/6 recipients $(n=4-5)$. (D) \& (E) Bar graphs shows the mean percentages of the OVA specific $\mathrm{T}$ cell response expressed as a percentage of $\mathrm{CD}^{+} \mathrm{T}$ cells $( \pm \mathrm{SEM})$ as seen in the blood 7 days post BM-DC vaccination. Results shown are from one experiment. Statistical significance was determined using a 1-way ANOVA test and Tukey's post-test where * $\mathrm{p}=$ $0.05-0.01, * * p=0.01-0.001, * * * p=<0.001, \mathrm{NS}=$ not significant. Experiment conducted by Dr Troels Petersen. 
langerin ${ }^{+} \mathrm{CD} 8 \alpha^{+}$DCs (Figure 5.9) and secondly OVA-specific responses induced by allogeneic BM-DCs were also reduced by the depletion of langerin ${ }^{+} \mathrm{CD} 8 \alpha^{+}$DCs (Figure $5.10)$.

For these experiments BM-DC-based vaccines were generated from C57BL/6 mice and injected into langerin-DTREGFP recipients. BM-DCs were not prepared from langerinDTREGFP mice to avoid the possibility that DT targets the injected cells. In-vitro BM-DC cultures were generated from C57BL/6 mice and loaded with OVA protein and $\alpha$-GalCer on day 6 as described in section 3.2.1.

The administration of $\alpha$-GalCer containing BM-DC vaccines clearly improved the OVAspecific $\mathrm{T}$ cell response generated, however this enhanced response is eliminated in the group where DT is given to deplete the langerin ${ }^{+} \mathrm{CD} 8 \alpha^{+} \mathrm{DC}$ population. Indeed, depletion of the langerin ${ }^{+} \mathrm{CD} 8 \alpha^{+}$population provides an OVA-specific T cell response level which is no different to that generated when BM-DCs loaded with OVA protein alone. Control groups were used to reveal if any detrimental effects of DT toxin administration could be shown on the induced $\mathrm{T}$ cell response using $\mathrm{C} 57 \mathrm{BL} / 6$ groups that received vaccination treatments in combination with DT toxin injections. No significant differences in the OVA-specific T cell responses were evident between the vaccine and MPL treated group, and the vaccine and MPL treated groups that received DT in these C57BL6 recipients which suggests, DT does not in itself impede T cell responses.

After noting the possible major involvement of the resident DC populations in $\mathrm{T}$ cell priming of the OVA-specific $\mathrm{T}$ cell responses after BM-DC vaccination, the fate of protein antigen initially loaded onto vaccine BM-DCs was investigated further. Data from within our laboratory suggested that allogeneic DCs could also stimulate OTI-T cell responses (Petersen personal communication). As these allogeneic DCs do not express the $\mathrm{H}-2 \mathrm{~K}^{\mathrm{b}}$ restriction element required to stimulate OT-1 cell, this result suggests that antigen has 


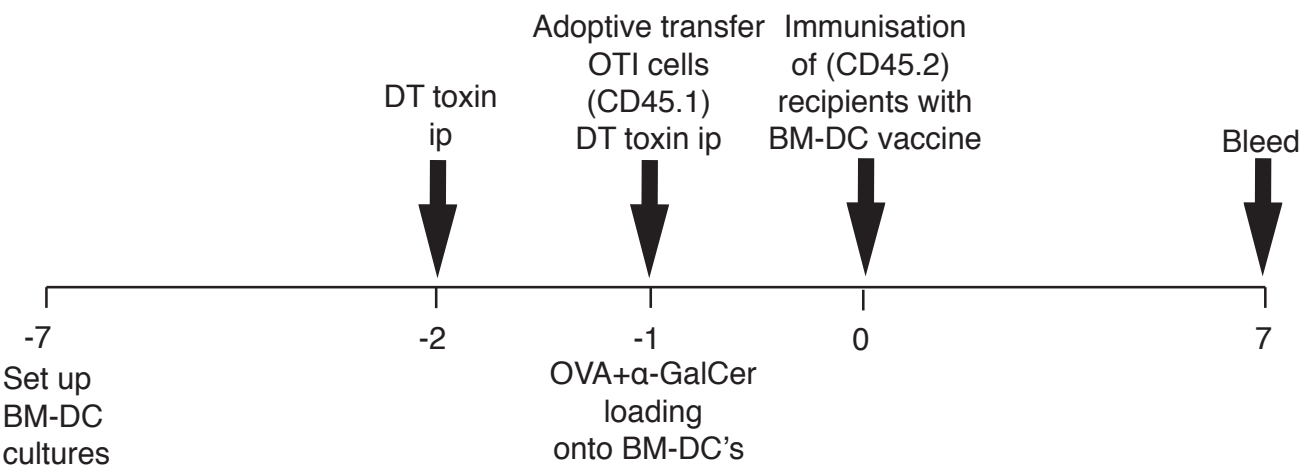

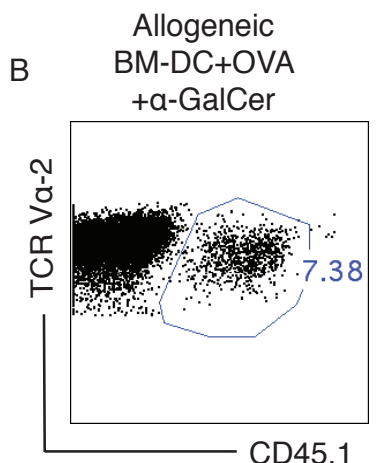
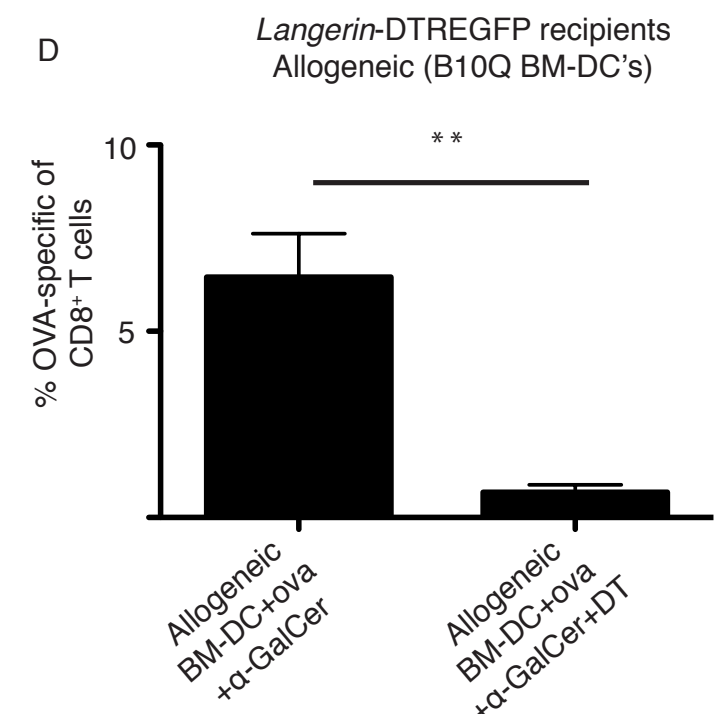

Allogeneic BM-DC+OVA $+\mathrm{a}-$ GalCer+DT

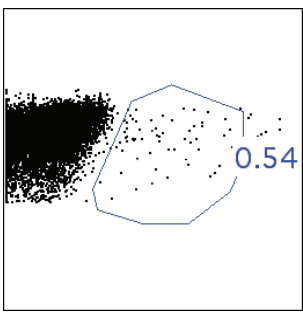
angerin-DTREGFP recipients Allogeneic (B10Q BM-DC's)

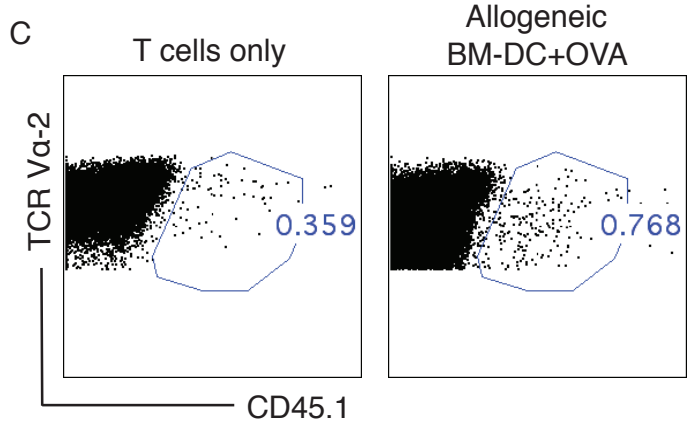

C57BL/6 recipients E Allogeneic (B10Q BM-DC's)

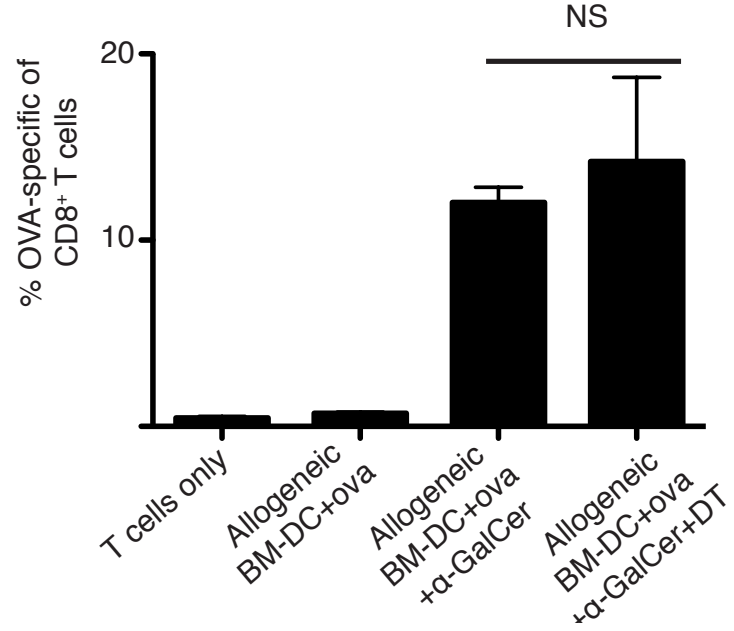

Figure 5.10 Protein antigen transfer from vaccine BM-DCs to resident langerin ${ }^{+} \mathrm{CD} 8 \alpha^{+} \mathrm{DC}$ populations is involved in cross presentation. (A) Timeline depicting the BM-DC, OVA and $\alpha$-GalCer loading vaccination protocol strategy. (B) Representative flow cytometry dot plots illustrate the OVA-specific $\mathrm{T}$ cell responses generated by adoptively transferred OVA specific $\mathrm{T}$ cells after treatment with DT into langerin-DTREGFP and (C) C57BL/6 recipients $(n=4-5)$. (D) \& (E) Bar graphs shows the mean percentages of the OVA specific $\mathrm{T}$ cell response expressed as a percentage of $\mathrm{CD}^{+} \mathrm{T}$ cells $( \pm \mathrm{SEM})$ as seen in the blood 7 days post BM-DC vaccination. Results shown are from one experiment. Statistical significance was determined using a 1-way ANOVA test and Tukey's post-test where ${ }^{*} p=0.05-0.01,{ }^{* *} p=0.01-0.001,{ }^{* *} p=<0.001$, NS $=$ not significant. Experiment conducted by Dr Troels Petersen. 
transferred to resident $\mathrm{H}-2 \mathrm{~K}^{\mathrm{b}+}$ cells. The possibility that antigen was transferred from allogeneic DCs to resident langerin ${ }^{+}$DCs was therefore investigated.

B10Q mice were used to generate allogeneic BM-DCs that were loaded with OVA protein and used to vaccinate C57BL/6 and langerin-DTREGFP recipients. Syngeneic BM-DCs were also prepared using C57BL/6 mice, and the OVA-specific immune responses compared with those seen after using the B10Q BM-DCs. B10Q mice (B10.Q; H-2 ${ }^{\mathrm{q}}$ ) differ in $\mathrm{MHC}$ haplotype from $\mathrm{C} 57 \mathrm{BL} / 6$ mice $\left(\mathrm{C} 57 \mathrm{BL} / 6 ; \mathrm{H}-2^{\mathrm{b}}\right)$, and therefore BM-DCs generated from B10Q mice cannot directly present processed antigen to OTI cells and prime the OVA-specific T cell response.

As shown in Figure 5.10 D, a significant OVA-specific $\mathrm{T}$ cell response was observed following injection of the allogeneic vaccine BM-DCs in the presence of systemic $\alpha$ GalCer stimulation, suggesting antigen had been transferred to resident cells. This response was eliminated in langerin mice where DT was also administered to deplete the langerin ${ }^{+}$ $\mathrm{CD} 8 \alpha^{+}$DC population suggesting transfer of antigen to this resident DC population is critical to inducing the cross-presented OVA-specific T cell response.

Collectively these experiments have provided strong evidence to support a previously described but less mainstream notion that vaccine DCs can act as antigen carriers, and that under appropriate conditions the antigen-specific $\mathrm{T}$ cell response can be primed by resident DC populations. 


\subsubsection{Antigen and glycolipid transfer from injected vaccine BM-DCs to resident DC populations}

To investigate how antigen transfer was being conducted between injected vaccine BMDCs and host DC populations, a study using langerin-DTREGFP recipients and peptide antigen was carried out. Peptide was substituted for OVA protein antigen and used to load BM-DCs in-vitro as this could indicate whether peptide antigen was actually being transferred. Langerin-DTREGFP recipients with groups treated with DT to deplete langerin ${ }^{+} \mathrm{CD} 8 \alpha^{+} \mathrm{DC}$ subsets were used for this study in order that host cells receiving antigen could be identified.

The timeline used for this study is shown in Figure 5.11 A with representative dot plots presented in Figure 5.11 B showing the expansion of OVA-specific T cell populations adoptively transferred into langerin-DTREGFP recipients after vaccination with $\alpha$-GalCer and peptide loaded BM-DCs. The bar graph seen in Figure 5.11 C shows that an OVA specific $\mathrm{CD}^{+} \mathrm{T}$ cell response is generated after administration of peptide loaded BM-DCs alone, however a superior response is achieved when using $\alpha$-GalCer also to load vaccine BM-DCs. When DT is used to treat recipients in order to deplete langerin ${ }^{+} \mathrm{CD} 8 \alpha^{+} \mathrm{DC}$ populations, the increased OVA specific $\mathrm{T}$ cell response is lost and blood levels of OVA specific $\mathrm{CD}^{+} \mathrm{T}$ cells return to that achieved when using peptide alone to load BM-DCs.

These results indicate that not only is peptide antigen being transferred, but also strongly suggests that $\alpha$-GalCer may also be transferred to resident cellular populations. The enhanced OVA specific $\mathrm{T}$ cell response seen after the use of $\alpha$-GalCer loaded BM-DCs and the subsequent loss after DT administration, indicates that the host DCs acquiring antigen from injected BM-DCs and priming the $\mathrm{T}$ cell response are those of the langerin ${ }^{+} \mathrm{CD} 8 \alpha^{+} \mathrm{DC}$ subset. It is evident that antigen and possibly $\alpha$-GalCer is being transferred but how this is being done is not known at this stage. 
A

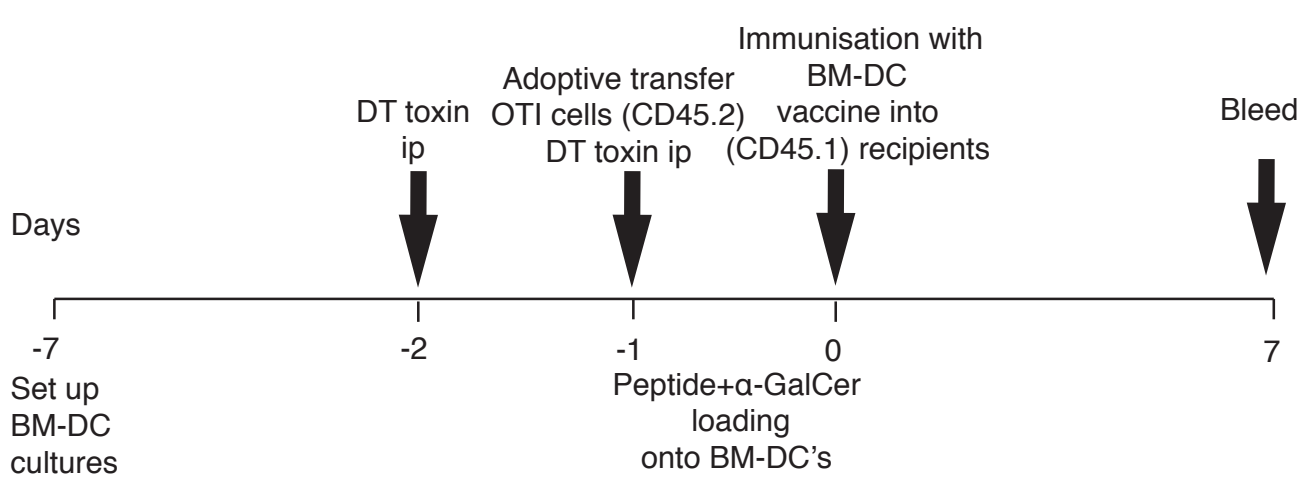

B

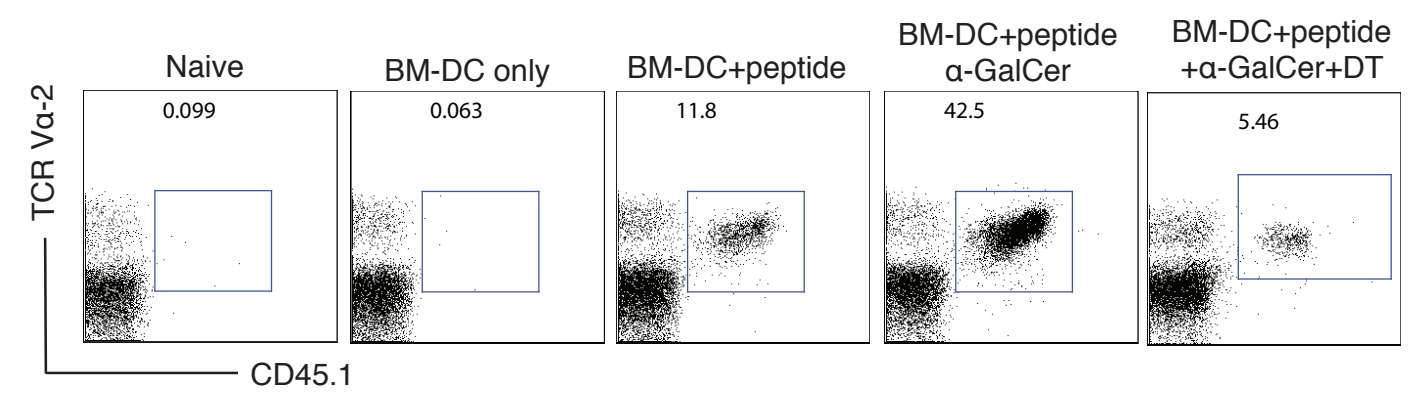

C

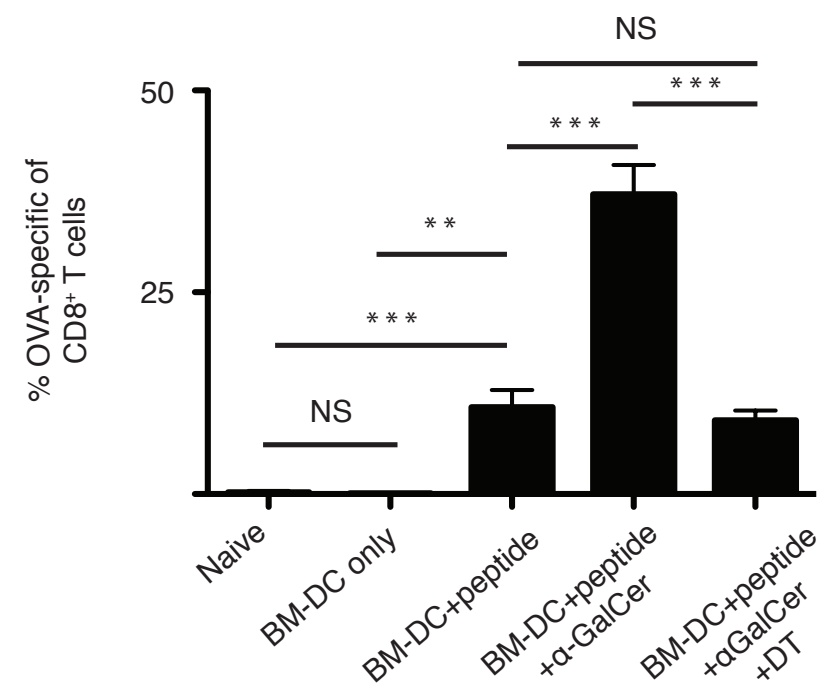

Figure 5.11 Peptide loaded BM-DCs treated with $\alpha-$ GalCer in-vitro transfer antigen in langerin-DTREGFP recipients. (A) Timeline depicts BM-DC and DT vaccination protocol. (B) Representative flow cytometry dot plots illustrate the OVA-specific T cell response generated with the adoptively transferred OVA specific $\mathrm{T}$ cells represented as TCRV $\alpha 2^{+} \mathrm{CD} 45.1^{+}$cells (C) Bar graph shows the mean percentages of the OVA specific $\mathrm{T}$ cell response expressed as a percentage of $\mathrm{CD}^{+} \mathrm{T}$ cells $( \pm$ SEM) as seen in the blood 7 days post vaccination. Results shown are from one experiment with ( $n=4$ - 5 recipients). Statistical significance was determined using a 1-way ANOVA test and Tukey's post-test where ${ }^{*} \mathrm{p}=0.05-0.01,{ }^{* *} \mathrm{p}=0.01-0.001,{ }^{* * *} \mathrm{p}<0.001, \mathrm{NS}=$ not significant. 
To address the possibility of glycolipid transfer, vaccine BM-DCs were prepared from CD1d-/- DCs, loaded with peptide antigen and $\alpha$-GalCer then administered to langerinDTREGFP recipients. Since $\alpha$-GalCer is presented to iNKT cells on CD1d surface molecules, any increased OVA specific $\mathrm{T}$ cell response generated after using CD1d -/vaccine BM-DCs would indicate that $\alpha$-GalCer has indeed been transferred to a host antigen presenting cell.

The OVA specific $\mathrm{T}$ cell response was measured on day 7 after vaccination and compared between treatment groups. The bar graph in Figure 5.12 $\mathrm{C}$ shows that even when using CD1d-/- BM-DCs loaded with $\alpha$-GalCer and peptide antigen, an increased OVA specific T cell response is still being generated indicating that $\alpha$-GalCer has not only been transferred, but is still somehow being presented. When DT is given to the relevant treatment group for langerin ${ }^{+} \mathrm{CD} 8 \alpha^{+} \mathrm{DC}$ depletion, the increased OVA specific $\mathrm{T}$ cell response is again lost indicating the langerin ${ }^{+} \mathrm{CD} 8 \alpha^{+} \mathrm{DC}$ subset is still acquiring peptide antigen and is also mediating the iNKT stimulated response by either directly presenting $\alpha$-GalCer or even possibly responding to proinflammatory cytokine release induced by other host DC subtype such as the plasmacytoid DCs that might be presenting $\alpha$-GalCer.

These results show that not only antigen but also the glycolipid $\alpha$-GalCer is being transferred between injected vaccine BM-DCs and host DC populations. In particular the langerin ${ }^{+} \mathrm{CD} 8 \alpha^{+}$DC subset is strongly implicated as the recipient DC population in this model system. 
A

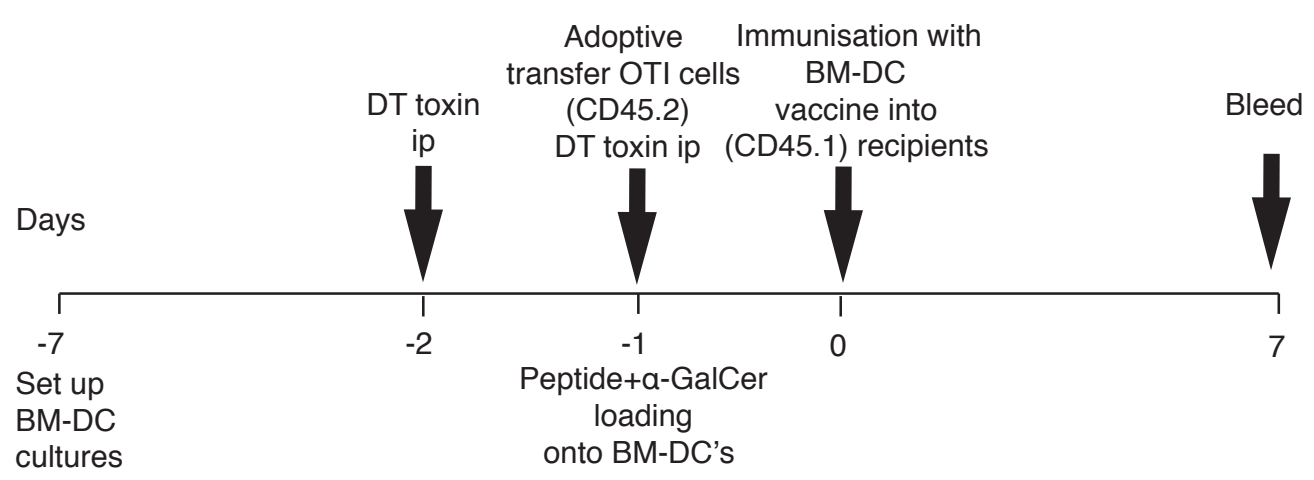

B

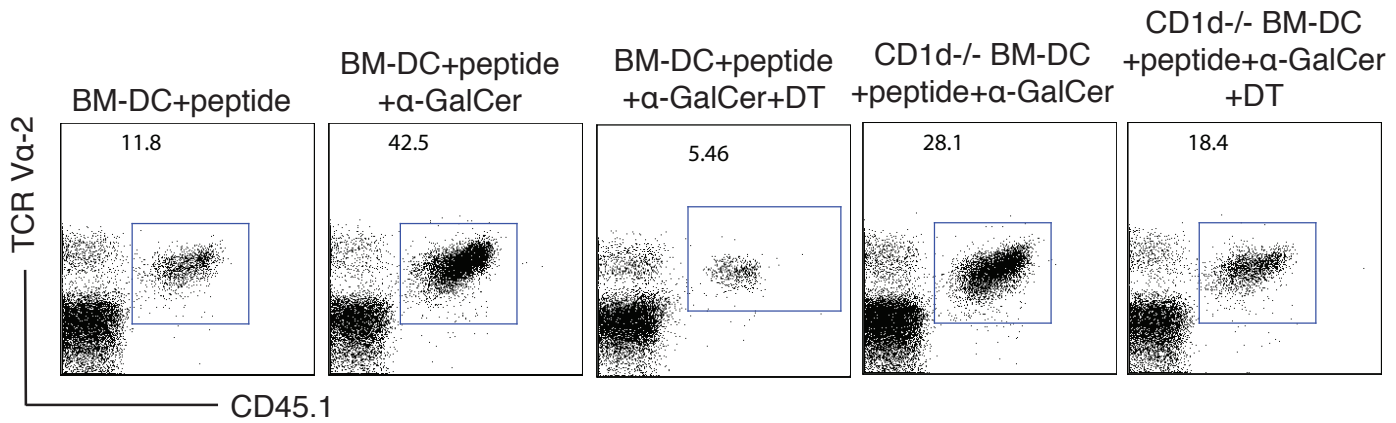

C

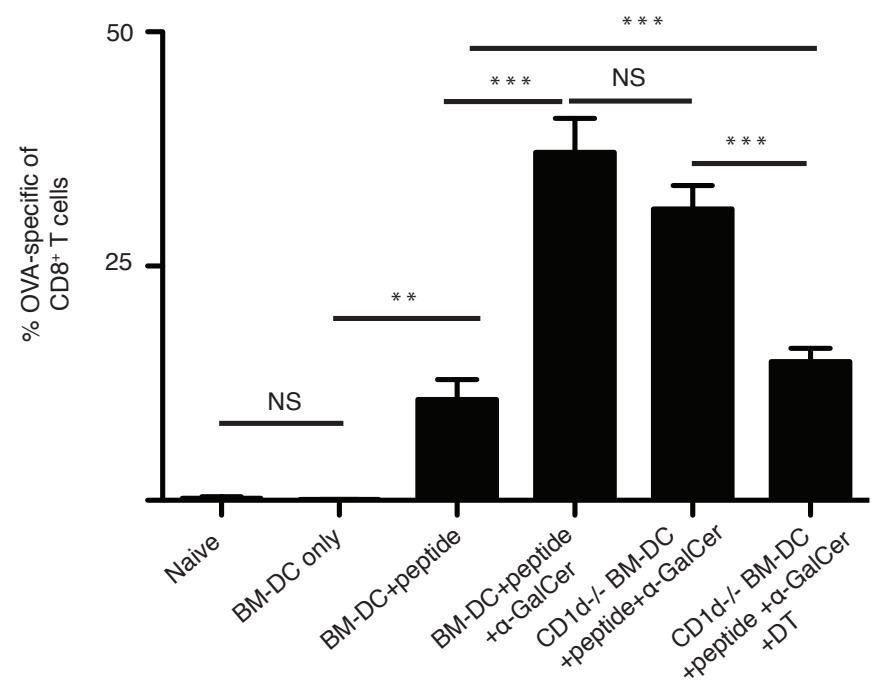

Figure 5.12 Peptide loaded BM-DCs and CD1d-/- BM-DCs treated with $\alpha-$ GalCer invitro transfer antigen in langerin-DTREGFP recipients. (A) Timeline depicts BM-DC and DT vaccination protocol. (B) Representative flow cytometry dot plots illustrate the OVA-specific $\mathrm{T}$ cell response generated with the adoptively transferred OVA specific $\mathrm{T}$ cells represented as TCRV $\alpha 2^{+} \mathrm{CD} 45.1^{+}$cells into recipients $(n=4-5)$. (C) Bar graph shows the mean percentages of the OVA specific $\mathrm{T}$ cell response expressed as a percentage of $\mathrm{CD} 8^{+} \mathrm{T}$ cells $( \pm$ SEM $)$ as seen in the blood 7 days post BM-DC vaccination. Results shown are from one experiment. Statistical significance was determined using a 1-way ANOVA test and Tukey's post-test where ${ }^{*} p=0.05-0.01,{ }^{* *} p=0.01-0.001,{ }^{* * *} p<0.001$, NS $=$ not significant. 


\subsubsection{TLR stimulation did not improve the action of $\alpha$-GalCer loaded BM-DCs in-vivo}

Having established the success of using $\alpha$-GalCer loaded BM-DCs as a vaccine preparation for generating superior OVA specific T cell responses, an effort was made to use TLR in combination with this established NKT stimulation to see if further enhancement could be achieved.

Samples of the different BM-DC cultures used for vaccine preparation were taken prior to $i v$ administration and treated with specific fluorescent antibody markers to indicate expression levels of the maturation markers CD40 and CD86. Histograms in Figure 5.13 show the geometric mean MFI expression levels for CD86 (Figure 5.13 A) and CD40 (Figure $5.13 \mathrm{~B}$ ) on untreated BM-DC samples (grey outline), OVA loaded BM-DCs (blue outline), $\alpha$-GalCer loaded BM-DCs (green outline), and OVA loaded and $\alpha$-GalCer treated BM-DCs (black outline). The CD86 and CD40 expression on these separate BM-DC population groups does not differ markedly indicating that treatment with OVA and $\alpha$ GalCer alone and combined together does not mature BM-DC cultures in-vitro prior to $i v$ administration.

Histograms in Figure 5.13 also show geometric mean MFI surface expression levels of CD86 (Figure $5.13 \mathrm{~A}$ ) and CD40 (Figure $5.13 \mathrm{~B}$ ) on BM-DCs after treatment with OVA and $\alpha$-GalCer in addition to MPL (top row), Resiquimod (second row), MPL and Resiquimod (bottom row) combined together. Contrary to the lack of maturation marker upregulation seen when OVA and $\alpha$-GalCer loading of BM-DCs is carried out, the effect of in-vitro TLR stimulation using MPL and Resiquimod alone and in combination with OVA and $\alpha$-GalCer loading markedly increased CD86 and CD40 expression levels on the BM-DC cultures used in vaccine experiments. The combined usage of MPL and Resiquimod together on OVA and $\alpha$-GalCer treated BM-DCs was able to increase the CD86 and CD40 expression on BM- DCs more so than if using MPL or Resiquimod alone.

This is consistent with the original 

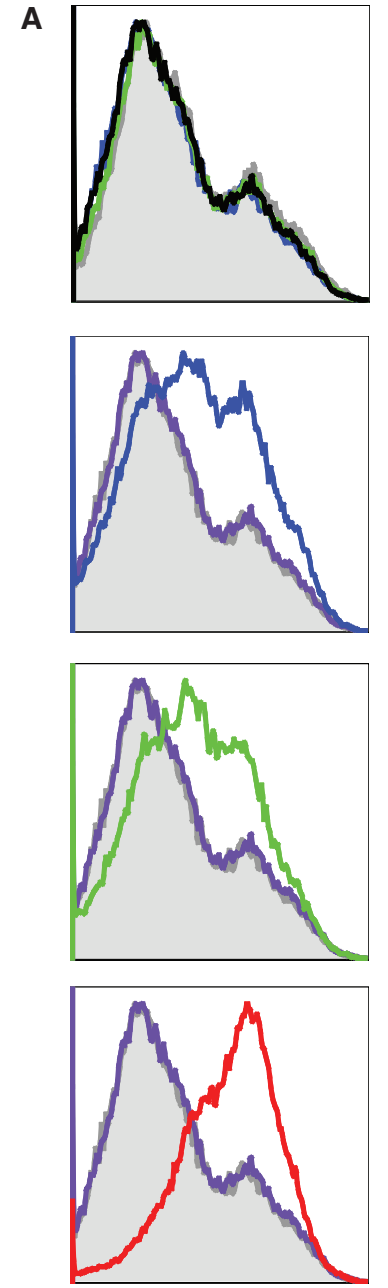

CD86
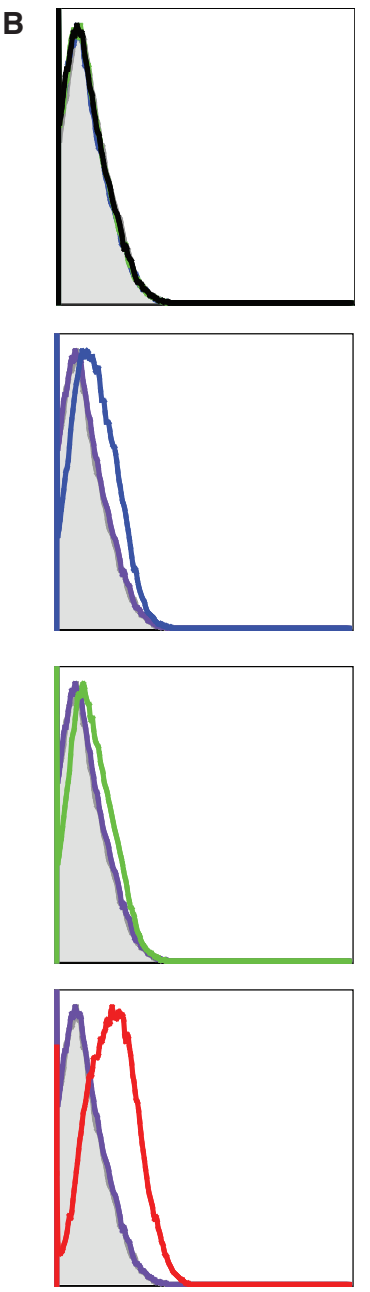

CD40
BM-DC+OVA+a-GalCer

BM-DC+a-GalCer

$B M-D C+O V A$

BM-DC

BM-DC+OVA+a-GalCer+MPL $\mathrm{BM}-\mathrm{DC}+\mathrm{OVA}+\mathrm{a}-\mathrm{GalCer}$ BM-DC

$\mathrm{BM}-\mathrm{DC}+\mathrm{OVA}+\mathrm{a}-\mathrm{GalCer}+$ Resiquimod $\mathrm{BM}-\mathrm{DC}+\mathrm{OVA}+\mathrm{a}-\mathrm{GalCer}$ BM-DC

BM-DC+OVA+a-GalCer+MPL+Resiquimod BM-DC+OVA+a-GalCer

BM-DC

Figure 5.13 The maturation status of BM-DCs treated in-vitro with $\alpha$-GalCer and TLR ligands. Histograms show (A) CD86 and (B) CD40 geometric mean fluorescence levels of BM-DCs after in-vitro culture with OVA, $\alpha$-GalCer and MPL.

in-vitro TLR studies conducted and presented earlier in this thesis (Chapter 3) which showed that selected TLR agonists could be used alone and in combination to induce maturation of BM-DCs in-vitro characterised by increased surface marker expression of CD40 and CD86.

Selected TLR agonists alone and in combination were used to treat OVA and $\alpha$-GalCer loaded BM-DC cultures before vaccinating B6 congenic recipient groups (Figure 5.14 A). 
Dot plots presented in Figure 5.14 B show the expansion of adoptively transferred OVA specific $\mathrm{T}$ cell populations achieved by different treatment groups one week post vaccination. The bar graph in Figure 5.14 C shows that treatment groups given OVA and $\alpha$-GalCer loaded BM-DC vaccines treated in-vitro with either one or two TLR agonists were unable to improve the OVA specific $\mathrm{CD}^{+} \mathrm{T}$ cell response above that seen when using the non TLR treated OVA and $\alpha$-GalCer loaded BM-DCs.

These results show that vaccine BM-DCs loaded with OVA protein and $\alpha$-GalCer and treated with TLR agonists alone and in combination are more mature than non-TLR treated vaccine BM-DCs. To explain why the OVA specific T cell response is not improved by the additional maturation status of the vaccine BM-DCs, previous chapters in this thesis have presented work that indicates all injected vaccine BM-DCs have matured by the time they reach the spleen and that resident DC populations such as the langerin expressing DCs participate in the generation of $\mathrm{CD}^{+} \mathrm{T}$ cell populations. It was also possible that the BMDCs might be overly stimulated and be unable to therefore exert any added increase in OVA specific T cell expansion. The possibility of over-stimulated BM-DCs was tested using a shorter incubation time of $16 \mathrm{~h}$ for the BM-DCs, which again showed that using invitro TLR stimulation to mature vaccine BM-DCs did not equate with an increased OVAspecific $\mathrm{T}$ cell response (results not shown).

The effect of in-vitro TLR stimulation was also tested with peptide loaded BM-DC vaccines as described for Figure 5.3 where MPL and Resiquimod was used alone and together to stimulate OVA and $\alpha$-GalCer loaded BM-DC cultures in-vitro prior to $i v$ 
A

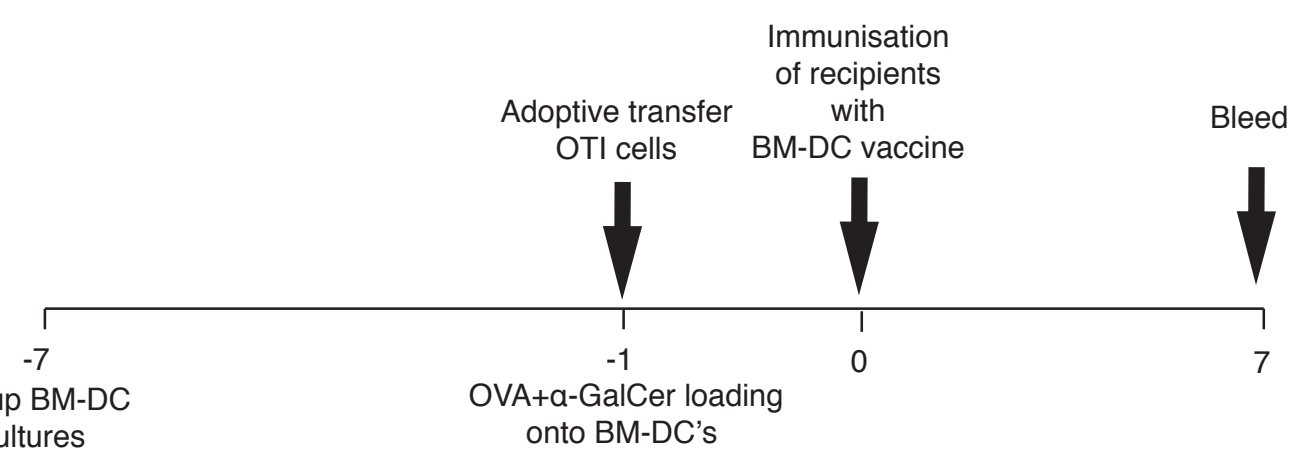

B

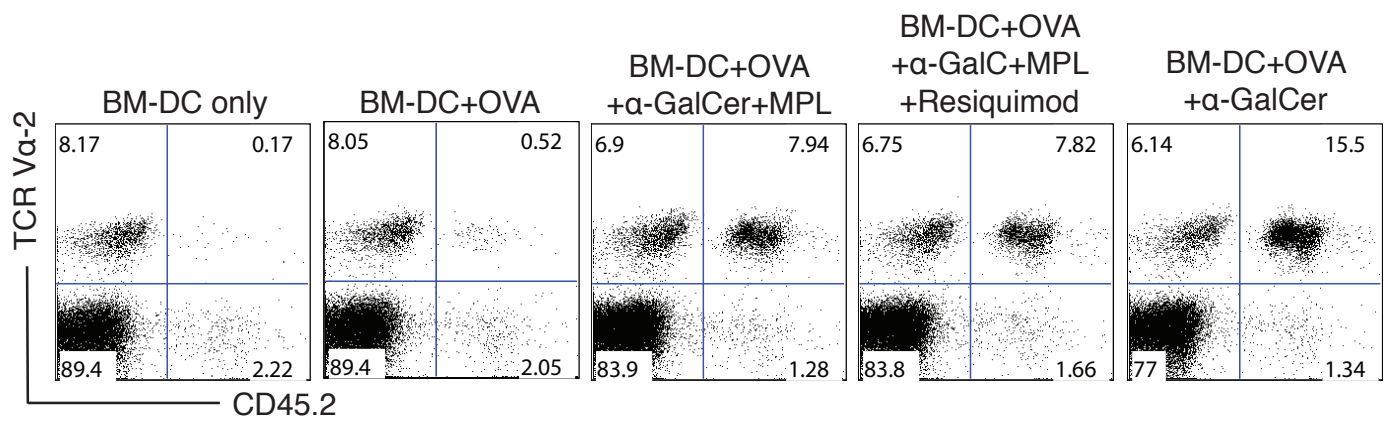

C

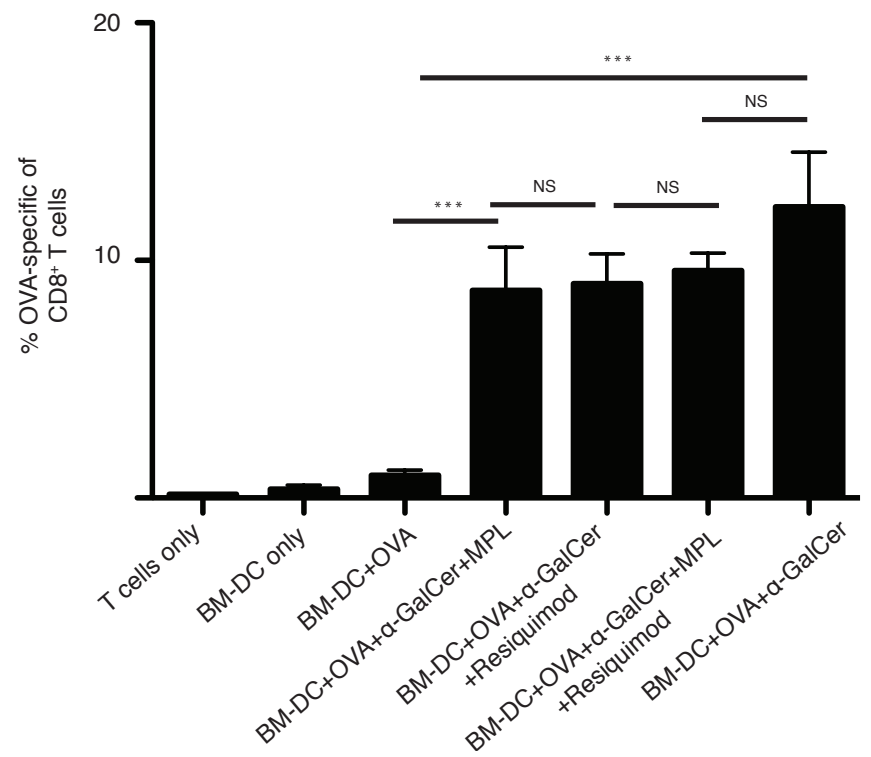

Figure 5.14 The in-vitro TLR treatment of $\alpha$-GalCer loaded BM-DC vaccines does not enhance OVA-specific $T$ cell responses generated in-vivo. (A) Timeline depicts BM-DC vaccination protocol. (B) Representative flow cytometry dot plots illustrate the OVA-specific $\mathrm{T}$ cell response generated with the adoptively transferred OVA specific $\mathrm{T}$ cells represented as TCRV $\alpha 2^{+} \mathrm{CD} 45.2^{+}$cells. (C) Bar graph shows the mean percentages of the OVA specific $\mathrm{T}$ cell response expressed as a percentage of $\mathrm{CD} 8^{+} \mathrm{T}$ cells $(+\mathrm{SEM})$ as seen in the blood 7 days post BM-DC vaccination. One representative experiment of 2 is shown ( $n=4$ - 5 recipients per group). Statistical significance was determined using a 1- 
Chapter 5: NKT stimulation enhances antigen specific responses to DC-based vaccines 226

way ANOVA test and Tukey's post-test where $* \mathrm{p}=0.05-0.01,{ }^{* *} \mathrm{p}=0.01-0.001,{ }^{* * *} \mathrm{p}$ $<0.001, \mathrm{NS}=$ not significant.

administration of vaccine recipients. Again no improvement over $\alpha$-GalCer treatment was observed (Figure 5.15).

Overall these results indicate that the processing of protein antigen does not interfere with or reduce the OVA specific $\mathrm{T}$ cell response being generated after vaccination of recipients with BM-DC vaccines as outlined in this model system. Similar levels of OVA specific $\mathrm{CD}^{+} \mathrm{T}$ cells were generated in vaccine recipients when using protein and peptide antigen loaded BM-DCs. 
A

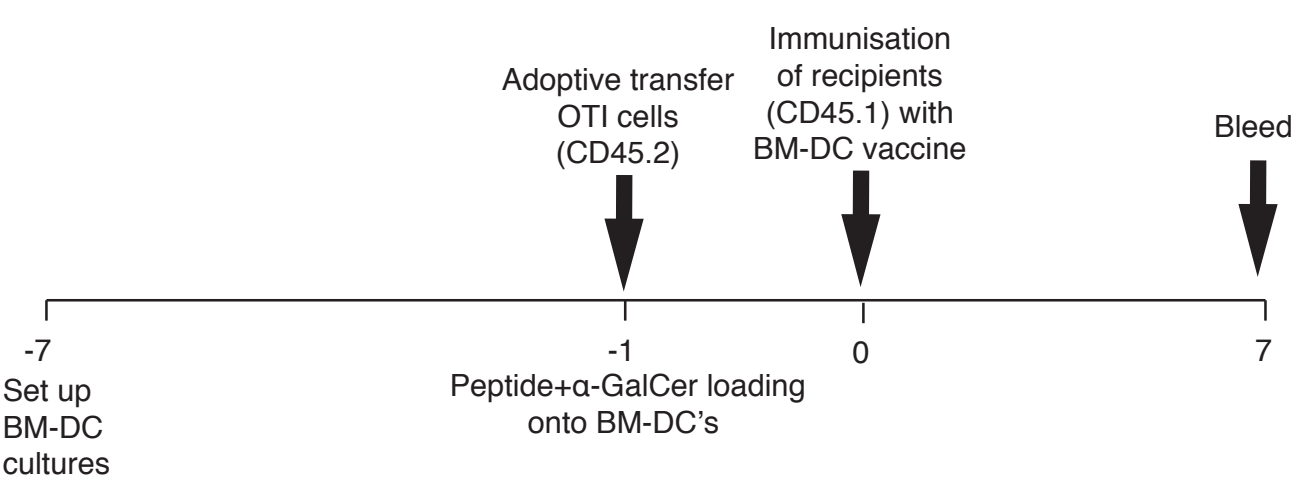

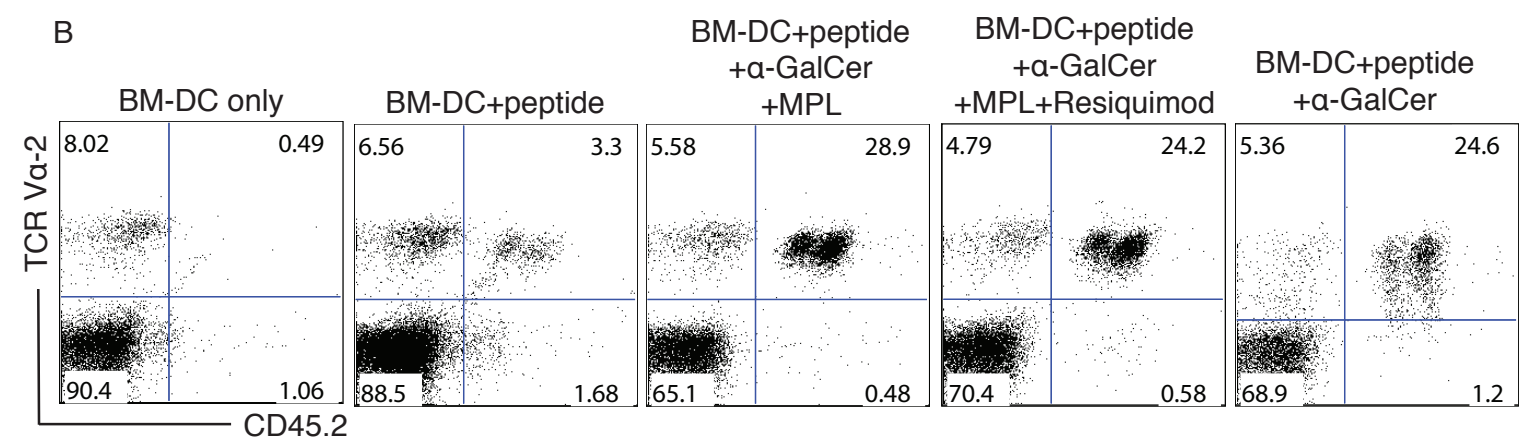

C

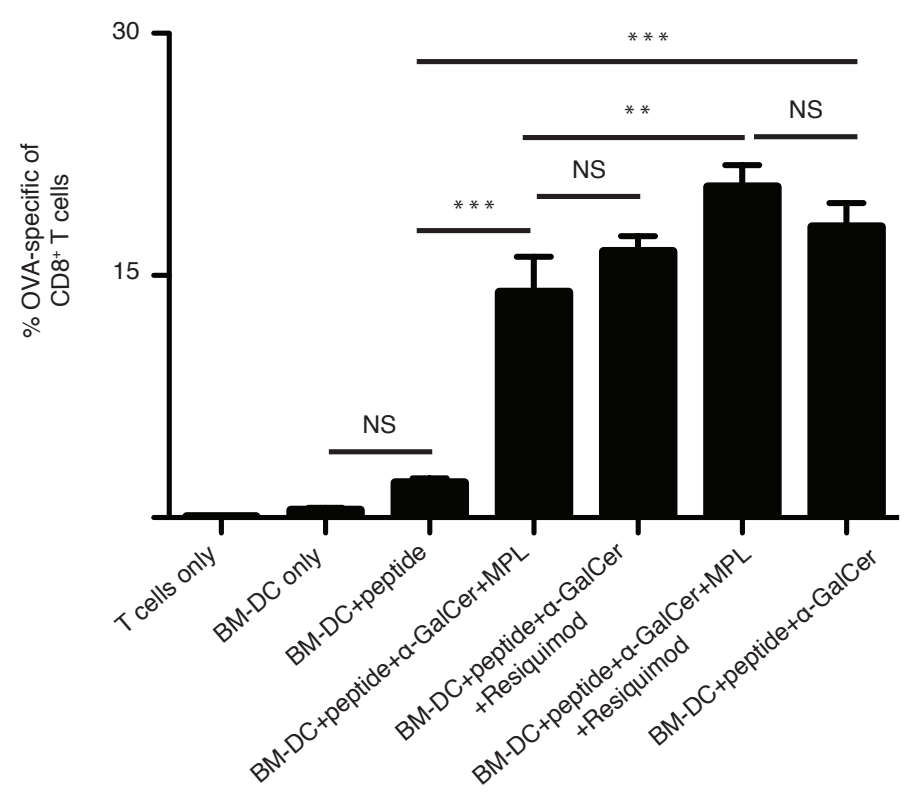

Figure 5.15 TLR treatment of $\alpha$-GalCer loaded peptide vaccine BM-DCs in-vitro does not enhance OVA-specific $T$ cell responses generated in-vivo. (A) Timeline shows experimental protocol. (B) Representative flow cytometry dot plots illustrate the OVAspecific $\mathrm{T}$ cell response generated with the adoptively transferred OVA specific $\mathrm{T}$ cells represented as TCRV $\alpha 2^{+} \mathrm{CD} 45.2^{+}$cells. (C) Bar graph shows the mean percentages of the OVA specific $\mathrm{T}$ cell response expressed as a percentage of $\mathrm{CD} 8^{+} \mathrm{T}$ cells $( \pm \mathrm{SEM})$ as seen 
in the blood 7 days post BM-DC vaccination. One representative experiment of 2 is shown $(n=4$ - 5 recipients per group). Statistical significance was determined using a 1-way ANOVA test and Tukey's post-test where $* \mathrm{p}=0.05-0.01,{ }^{*} \mathrm{p}=0.01-0.001,{ }^{* * *} \mathrm{p}<$ $0.001, \mathrm{NS}=$ not significant.

\subsubsection{The combined systemic effects of NKT and TLR stimulation on splenic DCs and the major splenic DC subsets}

The successful use of $\alpha$-GalCer to induce enhanced maturation of endogenous splenic DC populations after systemic administration was further developed by attempting to incorporate the use of TLR stimulation. This was done by evaluating systemic NKT and TLR stimulation with their combined effects on host endogenous DC populations after using $\alpha$-GalCer and selected TLR agonists. The timeline used for these experiments is shown in Figure 5.16 A.

Bar graphs presented in Figure 5.16 B, Figure 5.16 C and Figure 5.16 D show the geometric MFI measures for CD86 surface expression on splenic DC subsets $16 \mathrm{~h}$ after injection with $\alpha$-GalCer and MPL alone and together, $\alpha$-GalCer and Pam3Cys alone and together, $\alpha$-GalCer and Resiquimod alone and together. Systemic NKT and TLR stimulation given separately then together was able to induce increased CD86 expression on host splenic DC populations. The combined use of $\alpha$-GalCer and TLR stimulation was able to further enhance the maturation again with the exception of Resiquimod treated recipients (Figure 5.16 D). The CD86 expression of recipients treated with Resiquimod alone was not improved beyond that seen in naïve and PBS treated recipients, however the combined effect of using $\alpha$-GalCer and Resiquimod together was able to somehow induce a CD86 response that was significantly greater than that achieved with $\alpha$-GalCer treatment alone.

Collectively these results show that $\alpha$-GalCer and selected TLR agonists are able to work in co-operation with each other to increase the maturation and activation status of host DC 
A

B

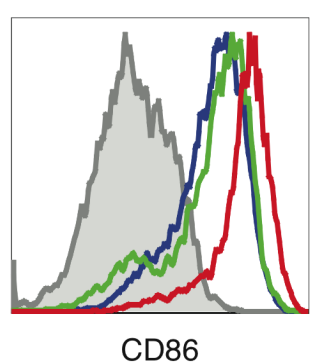

C

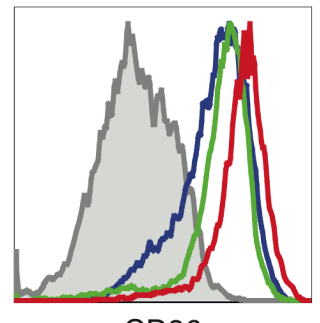

CD86

D

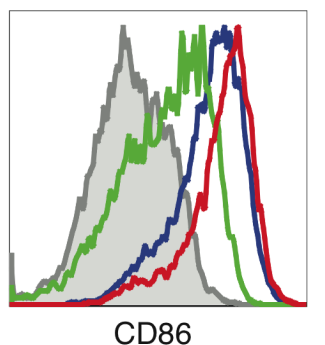

Inject TLR

agonist and/or

a-GalCer iv

Days

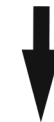

Harvest

after $16 \mathrm{~h}$

isolate splenic DC's
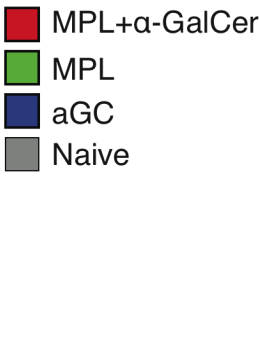
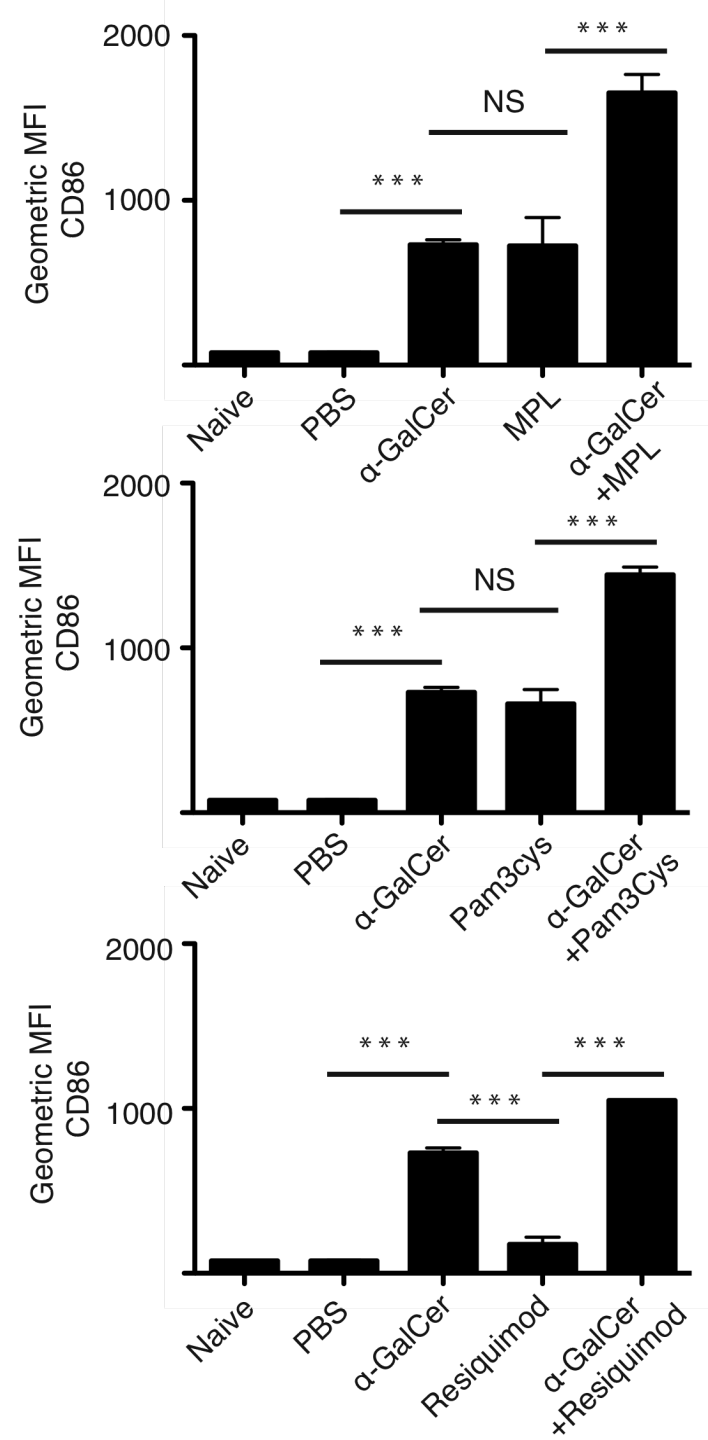

Figure 5.16 The characterization of splenic DC subsets after systemic $\alpha$-GalCer treatment for 16 h. (A) Timeline showing experimental plan. (B) Bar graph showing geometric MFI measures for CD86 expression on CD11 ${ }^{+}$DCs identified from splenocyte preparations isolated from C57BL/ 6 recipients treated with $\alpha$-GalCer, TLR agonists alone and in combination with $\alpha$-GalCer. Histograms show CD86 MFI expression comparing sample groups of $\alpha$-GalCer and MPL (B), $\alpha$-GalCer and Pam3Cys (C), $\alpha$-GalCer and Resiquimod (D). One representative experiment of two is shown $(n=3$ recipients per group) with \pm SEM. Statistical significance was determined using a 1-way ANOVA test and Tukey's post-test where ${ }^{*} \mathrm{p}=0.05-0.01,{ }^{* *} \mathrm{p}=0.01-0.001,{ }^{* * *} \mathrm{p}<0.001, \mathrm{NS}=$ not significant. 
populations. To further extend this work and examine the response to $\alpha$-GalCer and selected TLR agonists between the individual splenic DC subsets, the same experiments were carried out in langerin-EGFP recipients. Histograms in Figure 5.17 C and Figure 5.17 D show the CD86 and CD40 surface marker expression after systemic administration of $\alpha$ GalCer and MPL alone and together. Results for CD86 and CD40 geometric mean expression measures are show after $5 \mathrm{~h}$ (Figure 5.17 C) and $24 \mathrm{~h}$ (Figure 5.17 D).

As shown in the bar graphs displayed in Figure 5.17 A and Figure 5.17 B, the expression of CD86 and CD40 is already high on the langerin ${ }^{+} \mathrm{CD} 8 \alpha^{+} \mathrm{DC}$ subset even in the absence of stimulation. Treatment with $\alpha$-GalCer and MPL alone was able to improve both the CD40 and CD86 expression to a lesser degree on the remaining splenic DC subsets both after 5 and $24 \mathrm{~h}$ time points. The combined effects of using $\alpha$-GalCer and MPL together also showed an enhanced CD40 and CD86 expression at $5 \mathrm{~h}$ and $24 \mathrm{~h}$, however the response to $\alpha$-GalCer alone on CD40 expression superseded this at $24 \mathrm{~h}$. The histograms presented in Figure 5.17 C show CD86 and CD40 expression for each of the treatment groups tested at $5 \mathrm{~h}$, while Figure $5.17 \mathrm{D}$ shows the CD86 and CD40 expression on each of the recipient treatment groups but after $24 \mathrm{~h}$.

Overall these results show that NKT and TLR stimulation alone using selected TLR agonists can improve the maturation status of the major host splenic DC populations. A higher baseline level of CD86 and CD40 expression is seen on the langerin ${ }^{+} \mathrm{CD} 8 \alpha^{+} \mathrm{DC}$ subset even in the absence of stimulation when compared alongside other splenic DC subsets. It would appear however that this DC subset is preferentially targeted by maturation enhancing stimuli as evidenced by a marked increase in CD86 expression after treatment with MPL and $\alpha$-GalCer alone. The combined use of NKT and TLR stimulation is able to further improve the maturation status of resident splenic DC populations. 


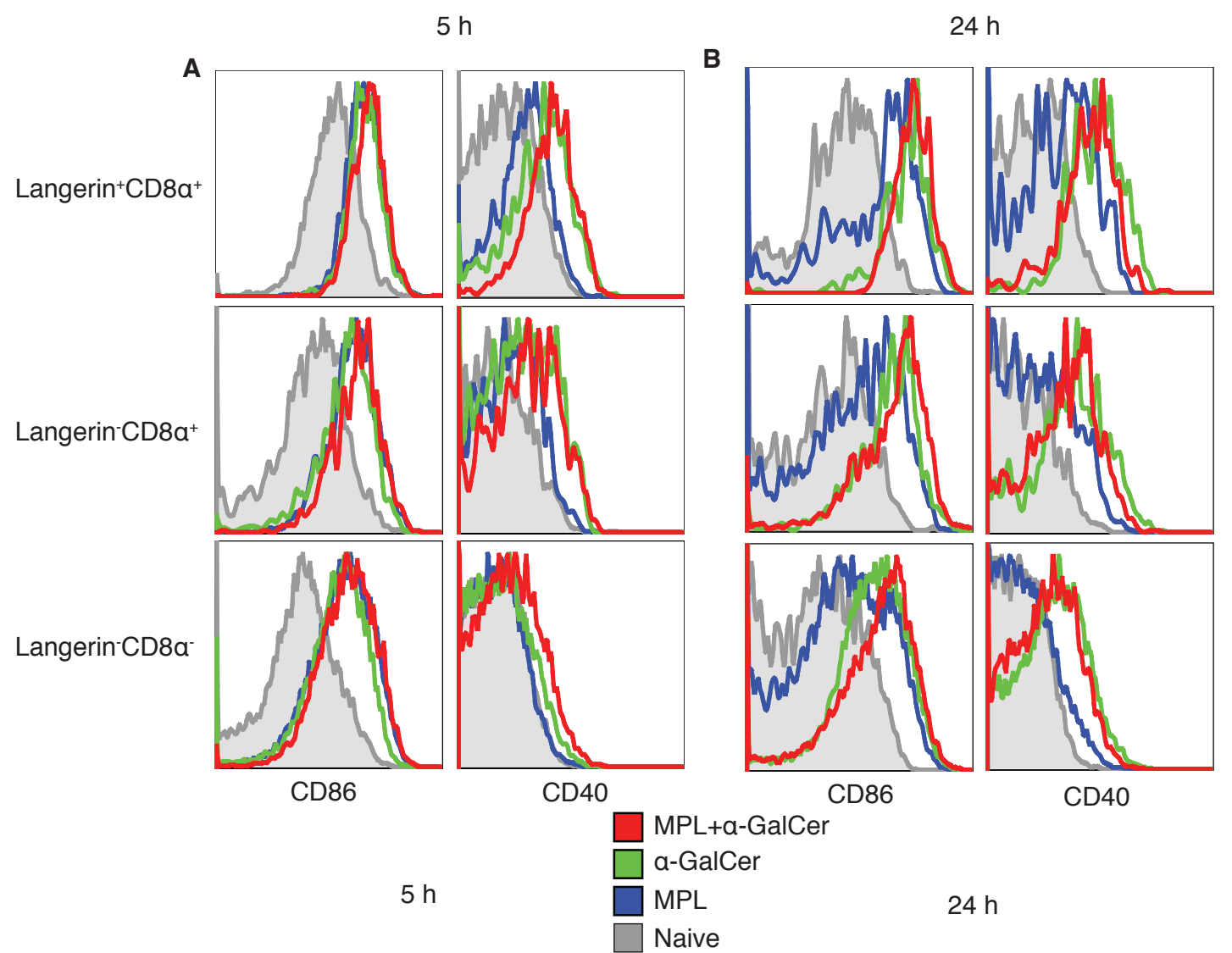

C
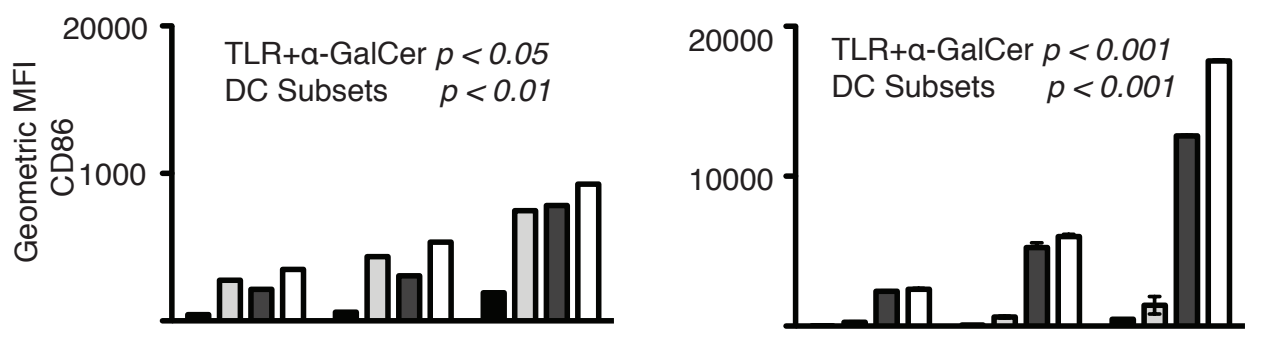

D

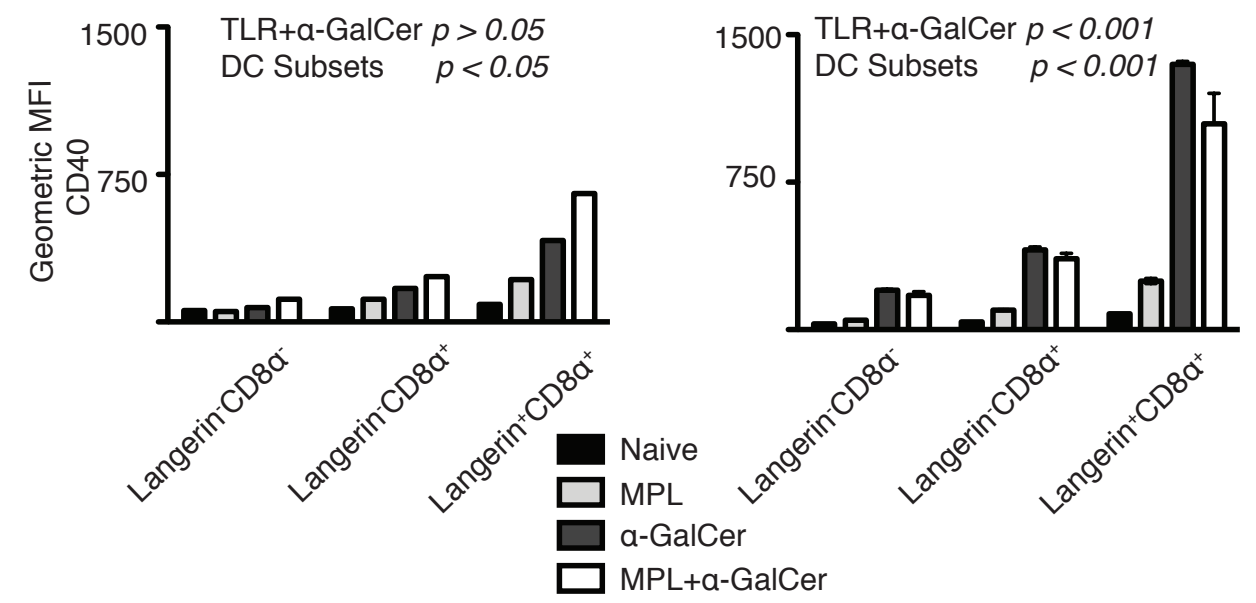


Figure 5.17 Characterization of the major splenic DC subsets after systemic $\alpha-$ GalCer and MPL treatment for 5 and $24 \mathrm{~h}$. (A) Histograms show relative geometric MFI CD86 $\left(1^{\text {st }}\right.$ column $)$ and CD40 ( $2^{\text {nd }}$ column $)$ expression on langerin ${ }^{+} C D 8 \alpha^{+}$, langerin ${ }^{-}$ $\mathrm{CD} 8 \alpha^{+}$, and langerin ${ }^{-} \mathrm{CD} 8 \alpha^{-}$DCs after no treatment, MPL, $\alpha-$ GalCer, MPL and $\alpha-$ GalCer treatment for $5 \mathrm{~h}$ and (B) $24 \mathrm{~h}$. (C) Bar graph shows geometric MFI values for CD86 expression as seen on the major splenic DC subsets after systemic treatment with $\alpha-G C$ and MPL for 5 and $24 \mathrm{~h}$. B. (D) As for (C) but showing CD40 expression. Results shown are from one experiment. Statistical significance was determined using a 2-way ANOVA test where ${ }^{*} p=0.05-0.01,{ }^{* *} p=0.01-0.001,{ }^{* * *} p<0.001, \mathrm{NS}=$ not significant.

\subsubsection{Systemic $\alpha$-GalCer and DC loaded $\alpha$-GalCer + TLR stimulation}

Further work was conducted to determine the effect of systemic TLR stimulation after the administration of either soluble $\alpha-$ GalCer or BM-DC loaded $\alpha-$ GalCer on antigen specific $\mathrm{T}$ cell responses.

The experimental protocol followed is outlined in Figure $5.18 \mathrm{~A}$, while bar graphs in Figures $5.18 \mathrm{~B}, \mathrm{C}$ and $\mathrm{D}$ represent the antigen specific $\mathrm{T}$ cell response as measured one week post vaccination. Representative dot plots seen in Figure 5.18 B show the expansion of adoptively transferred OVA-specific $\mathrm{T}$ cell populations one week after vaccination and correspond with the bar graphs seen directly below. The co-administration of systemic MPL in addition to the injection of soluble OVA with $\alpha-$ GalCer was able to significantly enhance the OVA specific $\mathrm{T}$ cell responses generated as seen in Figure $5.18 \mathrm{~B}$. The systemic co-administration of MPL was also able to significantly improve the antigen specific $\mathrm{T}$ cell response after vaccination with soluble antigen loaded and $\alpha$-GalCer loaded vaccine BM-DCs (Figure 5.18 C). Control groups consisting of Naïve, BM-DC and OVA only recipients can be seen in Figure 5.18 D.

In simple terms, NKT stimulation works in co-operation with systemic TLR stimulation to increase antigen specific $\mathrm{T}$ cell responses generated after vaccination. These results show that systemically administered $\alpha-G a 1 C e r$ and MPL can increase $C D 8^{+} \mathrm{T}$ cell responses 


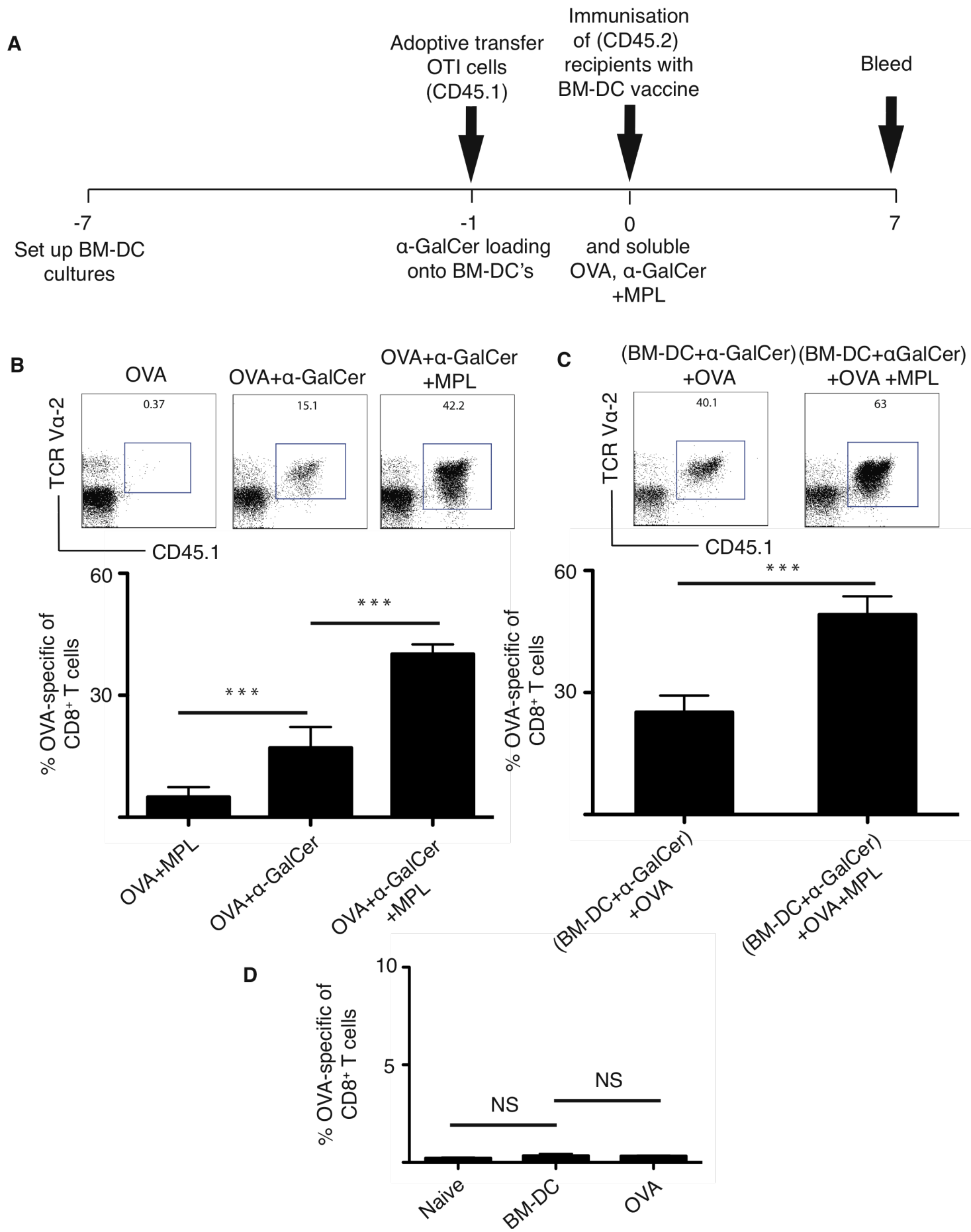

Figure 5.18 Systemic MPL improves antigen specific responses after vaccination with soluble $\boldsymbol{\alpha}$-GalCer and DC loaded $\boldsymbol{\alpha}$-GalCer. A. Timeline shows experimental protocol. Representative flow cytometry dot plots shown in (B) and (C) illustrate the OVA-specific $\mathrm{T}$ cell response generated with the adoptively transferred OVA specific $\mathrm{T}$ cells represented as TCRV $\alpha 2^{+} \mathrm{CD} 45.1^{+}$cells. Bar graphs in (B), (C) and (D) show the mean percentages of the OVA specific $T$ cell response expressed as a percentage of $C D 8^{+} \mathrm{T}$ cells $( \pm \mathrm{SEM})$ as seen in the blood 7 days post BM-DC vaccination for the different treatment groups. Results shown are from one experiment ( $n=3-5$ recipients per group). Statistical 
significance was determined using a 1-way ANOVA test and Tukey's post-test where * $\mathrm{p}=$ $0.05-0.01,{ }^{* *} \mathrm{p}=0.01-0.001,{ }^{* * *} \mathrm{p}<0.001, \mathrm{NS}=$ not significant.

when used with soluble protein antigen. Systemically administered MPL can also increase $\mathrm{CD}^{+} \mathrm{T}$ cell responses when administered with BM-DC loaded with $\alpha-$ GalCer in the presence of soluble protein antigen. It would appear that antigen specific responses can be increased by the combined the effects of TLR with NKT stimulation in the presence of soluble protein antigen, whether $\alpha-$ GalCer is administered systemically, or loaded onto BM-DCs.

What is important to note is that the systemic administration of $\alpha$-GalCer may provide greater availability of $\alpha$-GalCer to host populations mediating antigen specific $\mathrm{T}$ cell responses, than if loaded onto vaccine BM-DCs. The antigen specific $\mathrm{T}$ cell response generated after using TLR simulation combined with $\alpha$-GalCer loaded vaccine BM-DCs is still comparable to that seen when given systemically. This implies there is advantage to be gained by the loading of $\alpha-$ GalCer onto BM-DCs, which allows for more efficient antigen specific $\mathrm{T}$ cell generation after vaccination than if administered systemically. 


\subsubsection{TLR and NKT cell stimulation co-operate to improve OVA specific $T$ cell responses}

The information generated regarding TLR and NKT stimulation in previous studies was incorporated together and used to optimise the current BM-DC vaccine protocol.

BM-DCs were prepared as previously described in section 3.2.1 and loaded with the protein antigen OVA and $\alpha$-GalCer on day 6 before vaccination $24 \mathrm{~h}$ later. Systemic MPL stimulation was co-delivered with the $\alpha$-GalCer containing BM-DC vaccine using a separate injection into the contra-lateral tail vein at the time of vaccination. The experimental outline followed can be seen in Figure 5.19 A and the representative dot plots displayed in Figure 5.19 B show the expansion of OVA specific $\mathrm{T}$ cells adoptively transferred into $\mathrm{C} 57 \mathrm{BL} / 6$ recipients for the different treatment groups.

The bar graph in Figure 5.19 $\mathrm{C}$ shows the OVA specific $\mathrm{T}$ cell responses generated one week post vaccination and clearly indicates that systemic MPL co-administered with vaccine BM-DCs increases $\mathrm{CD}^{+} \mathrm{T}$ cell responses. The use of $\alpha$-GalCer loaded BM-DC vaccines also increases $\mathrm{CD}^{+} \mathrm{T}$ cell responses. The impact of incorporating and combining both the $\alpha$-GalCer loaded BM-DCs that were co-delivered with MPL at the time of vaccination, was that significantly increased $\mathrm{CD} 8^{+} \mathrm{T}$ cell responses were measured that surpassed that seen when using $\alpha$-GalCer or MPL treatment alone.

These results confirm that the careful usage of NKT and TLR stimulation together is capable of inducing a potent antigen specific $\mathrm{T}$ cell response that shows co-operation between these two adjuvant methods. To test the duration of vaccine action given the longlasting antigen specific $\mathrm{T}$ cell response after loading $\alpha$-GalCer onto BM-DCs shown in Figure 5.19 A, OVA specific $\mathrm{CD}^{+} \mathrm{T}$ cell levels generated were measured 2 weeks post vaccination and results between treatment groups compared (Figure 5.19 D). 
A

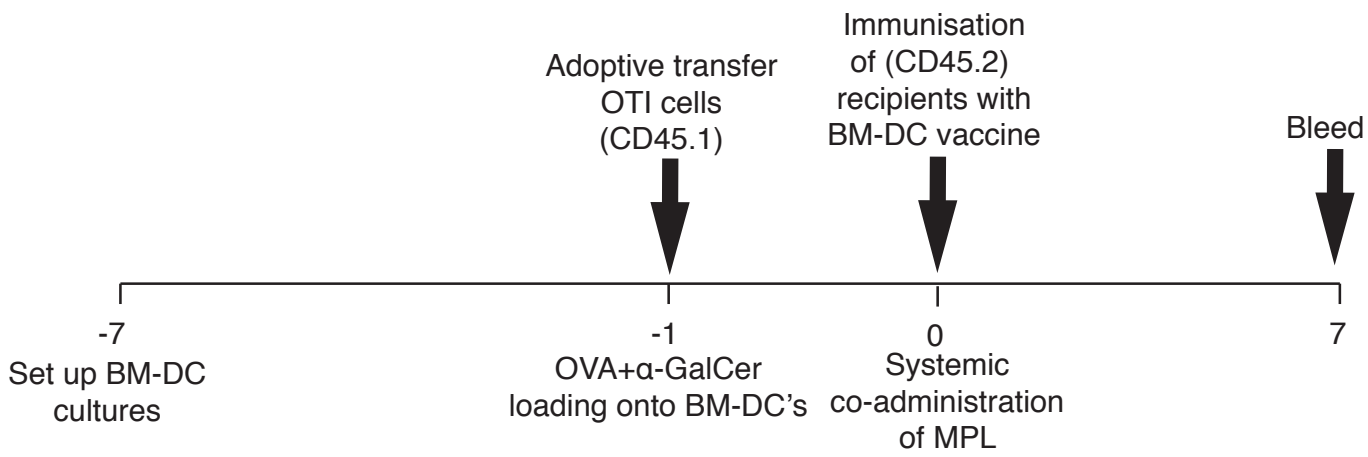

B

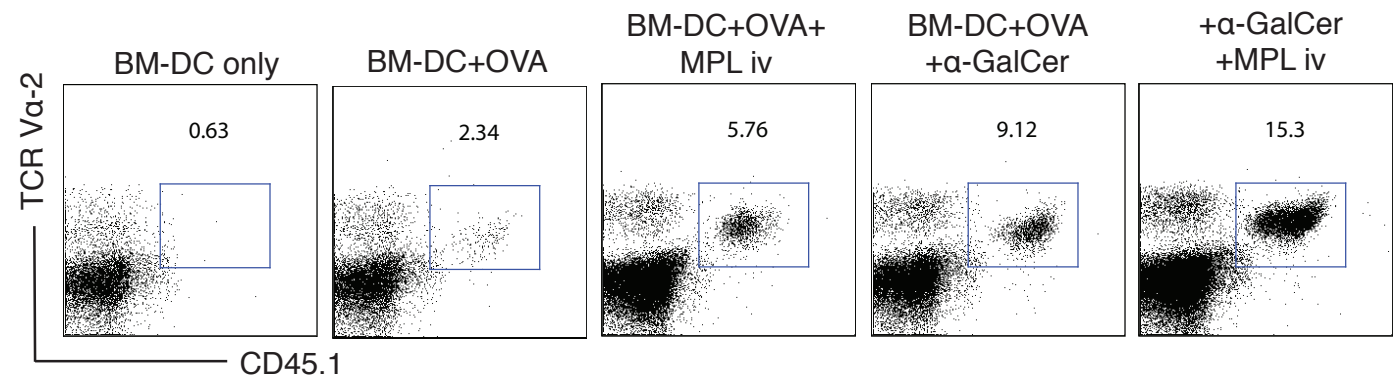

C

Day 7

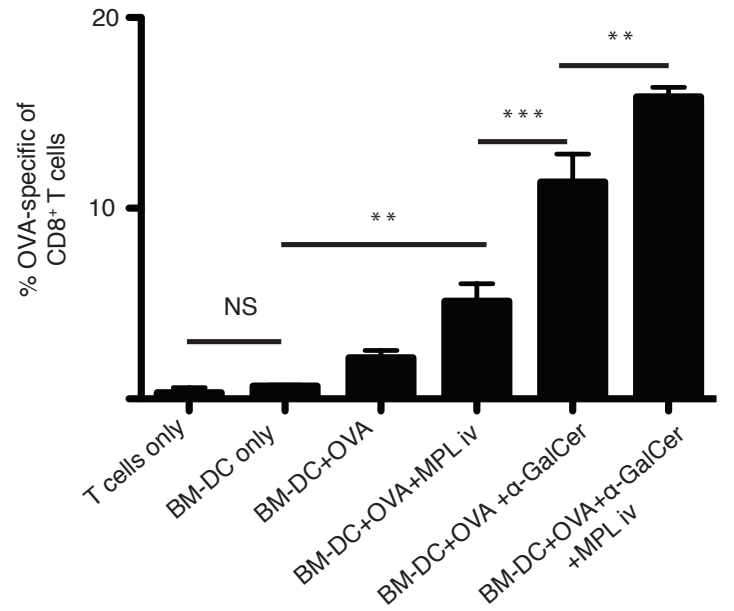

D

Day 14

Figure 5.19 Combined NKT and TLR stimulation increases the OVA-specific T cell responses in-vivo after vaccination. (A) Timeline depicts the BM-DC manufacture protocol used. (B) Representative flow cytometry dot plots illustrate the OVA-specific T cell response generated with the adoptively transferred OVA specific $\mathrm{T}$ cells represented as TCRV $22^{+}$CD $45.1^{+}$cells. (C) Bar graph shows the mean percentages of OVA specific $\mathrm{T}$ cell response expressed as a percentage of $\mathrm{CD} 8^{+} \mathrm{T}$ cells as seen in the blood 7 days post BM-DC vaccination. (D). Bar graph shows the mean percentages of OVA specific T cell response expressed as a percentage of $\mathrm{CD}^{+} \mathrm{T}$ cells as seen in the blood 14 days post vaccination. One representative experiment of 3 is shown with \pm SEM (4 - 5 recipients per group). Statistical significance was determined using 1-way ANOVA test and Tukey's post-test where ${ }^{*} p=0.05-0.01,{ }^{* *} p=0.01-0.001,{ }^{* *} p<0.001, \mathrm{NS}=$ not significant. 
Chapter 5: NKT stimulation enhances antigen specific responses to DC-based vaccines 237

The bar graph in Figure 5.19 D shows the OVA specific T cell response measure 2 weeks after vaccination. Although antigen specific $\mathrm{T}$ cell measures are lessened across all treatment groups at this time point, the response trends seen one week after vaccination are clearly evident with $\alpha$-GalCer loaded BM-DCs delivered with co-administration of MPL at the time of vaccination. 


\subsection{Discussion}

The work presented this chapter explored the utilization of $\alpha$-GalCer for the generation of increased antigen specific $\mathrm{CD} 8^{+} \mathrm{T}$ cell frequencies, the fate of $\alpha$-GalCer and protein and peptide antigen loaded onto BM-DCs after administration into recipients, the overall impact of employing both NKT and TLR stimulatory effects on antigen specific $\mathrm{CD}^{+} \mathrm{T}$ cell frequencies, and the application and usage of this work for the development of vaccination strategies against tumours. We showed that $\alpha$-GalCer was able to significantly and consistently increase antigen specific $\mathrm{T}$ cell responses generated after vaccination with protein and peptide antigen loaded vaccine BM-DCs. In addition to the movement of BMDC associated protein and peptide antigen from injected vaccine BM-DCs to resident langerin ${ }^{+} \mathrm{CD} 8 \alpha^{+}$DC populations, the movement of glycolipid components from injected vaccine BM-DCs was also demonstrated. The advantage of utilising $\alpha$-GalCer loaded onto BM-DCs compared with soluble $\alpha$-GalCer administration was also indicated. The combination of $\alpha$-GalCer loaded vaccine BM-DCs co-administered with systemic TLR stimulation was also shown consistently to significantly increase antigen specific $\mathrm{T}$ cell responses found in the blood one week post immunisation. This work is significant because $\alpha$-GalCer can be used in both mouse and humans and therefore presents enormous potential for the development of its anti-cancer properties for eliciting immediate tumour targeted killing in addition to longer lasting adaptive anti-tumour specific T cell responses capable of eliminating residual disease.

\subsubsection{NKT stimulation enhances antigen specific responses to BM-DC vaccines}

The efficacy of our vaccination model was based on the measuring antigen specific $\mathrm{T}$ cell frequencies generated after immunisation with varying treatment groups since previous work had shown that adoptive $\mathrm{T}$ cell therapy using antigen specific $\mathrm{T}$ cells could augment antitumour immunity (Yee et al., 2002). These aforementioned adoptively transferred $\mathrm{T}$ cells were also shown capable of localizing to tumour sites to cause ultimate regression of metastatic disease (Yee et al., 2002). The generation of increased antigen specific CD4 ${ }^{+}$ 
and $\mathrm{CD} 8^{+} \mathrm{T}$ cell responses after treatment with $\alpha$-GalCer has since been demonstrated by various groups (Fujii et al., 2003; Hong et al., 2006; Silk et al., 2004), although conflicting evidence has been presented by other sources (Moodycliffe et al., 2000; Palmer et al., 2006).

The antigen specific $\mathrm{CD}^{+} \mathrm{T}$ cell response being generated in-vivo using the current $\mathrm{BM}-$ DC vaccine model was manipulated by the use of NKT stimulation in the form of $\alpha$ GalCer-loaded vaccine BM-DCs. It was anticipated that molecular interactions such as CD40 signalling and pro-inflammatory cytokines released by iNKT cells engaged with antigen presenting cells bearing CD1d and bound with $\alpha$-GalCer would further mature antigen presenting DCs and hence increase the antigen specific $\mathrm{T}$ cell response being generated against the vaccine. Previous studies have shown that $\alpha$-GalCer loaded BM-DCs were indeed capable of enhancing T cell responses (Fujii et al., 2004; Fujii et al., 2003) (Hermans et al., 2007; Hermans et al., 2003; Silk et al., 2004; Stober et al., 2003).

The co-culture of $\alpha$-GalCer with BM-DCs in the presence of protein antigen generated $\alpha$ GalCer loaded vaccine BM-DCs that upon $i v$ administration, significantly and consistently increased the OVA specific $\mathrm{T}$ cell response measured one week post vaccination. The treatment of recipients with $\alpha$-GalCer-loaded BM-DCs in the absence of protein antigen was unable to elevate responses above that seen with control groups. The adjuvant effect of using $\alpha$-GalCer loaded vaccine BM-DCs to generate increased $\mathrm{CD} 8^{+} \mathrm{T}$ cell frequencies was still evident two weeks post vaccination, however by week three, this adjuvant effect had been lost.

Similar increased OVA-specific antigen $\mathrm{T}$ cell responses were also seen after vaccination when peptide antigen was substituted for OVA protein antigen in $\alpha$-GalCer loaded vaccine BM-DCs. The use of peptide antigen was expected to reduce the antigen processing workload of the BM-DCs by increasing the availability of peptide components ready for MHC-class presentation. The $\mathrm{CD}^{+} \mathrm{T}$ cell response seen after immunization experiments with $\alpha$-GalCer loaded protein and peptide antigen vaccine BM-DCs do not differ markedly from each other (Figure 5.1 and Figure 5.3). 
Adjuvants such as iNKT cell agonists can be utilised for the treatment of tumours with vaccination techniques (Exley and Nakayama, 2011; Vivier et al., 2012). It has also been reported that NKT ligands demonstrated superior in-vivo adjuvanticity when compared with individual TLR ligands (Cerundolo et al., 2009; Parekh et al., 2005b). CD40 signalling activities that occur via CD40/CD40L interactions between DCs and iNKT cells also work to enhance antigen specific cell responses (Kitamura et al., 1999). Previous work however has shown that the need for T cell help for CTL activation could be abolished by the triggering of CD40 on DCs to enhance CTL activation (Bennett, 1998; Lefrancois et al., 2000; Ridge 1998; Schoenberger et al., 1998).

The successful use of $\alpha$-GalCer for prophylactic antitumour effects shown in melanomas, carcinomas, hematopoietic malignancies, metastatic spread has been shown due to IFN- $\gamma$ production by NKT cells and bystander activation and function NK, CTLs and CD ${ }^{+} \mathrm{T}$ cells (Hayakawa et al., 2001a; Hayakawa et al., 2001b; Takeda et al., 2000). Type 1 IFNs are inflammatory cytokines that mediate important roles in $\mathrm{DC}$ activation and $\mathrm{CD} 8^{+} \mathrm{T}$ cell effector function and promoting cross-priming activities via stimulation of DCs (Lapenta et al., 2006; Le Bon et al., 2003; Le Bon and Tough, 2008). Recent work has shown that IFN1 facilitates cross priming activities of $\mathrm{CD} 8 \alpha^{+}$DCs against tumour derived cell associated antigens by improving cross presentation by enhancement of antigen persistence, by also increasing survival of antigen loaded DCs and finally by the activation of DCs (Lorenzi et al., 2011).

The engagement of a DC expressing an $\alpha$-GalCer bearing CD1d molecule with the TCR of an iNKT cell, results in the production of iNKT derived cytokines such as IL-4 and IFN- $\gamma$ in addition to the upregulation of CD40L, which causes the $\alpha$-GalCer-carrying DC to undergo maturation and activation processes including increased IL-12 production (Kitamura et al., 1999). The IL-12 produced in turn acts upon NK cells to induce a second burst of IFN- $\gamma$ production superior to that initially made by NKT cells (Eberl et al., 2000; Eberl and MacDonald, 2000). The cytokine activity induced after $i v$ administration of $\alpha$ GalCer can be easily detected in the murine serum with IL-4 production peaking at $2 \mathrm{~h}$, IL12 at $6 \mathrm{~h}$ post injection and IFN- $\gamma$ peaking between 12-24 h (Fujii et al., 2007). 
Chapter 5: NKT stimulation enhances antigen specific responses to DC-based vaccines 241

The upregulation of the CD80 and CD86 maturation markers on DCs carrying $\alpha$-GalCer loaded CD1d molecules, follows the proinflammatory cytokine production by iNKT cells after initial engagement. These cytokines include IL-12 and IFN- $\gamma$ which can be produced by both NKT and NK cells. These impacts collectively ensure that DCs become licensed to generate effective antigen specific $\mathrm{T}$ cell responses that are sufficient in magnitude and quality for the required response action and that also supersede the response generated when $\alpha$-GalCer is not present to provide the powerful amplification signals necessary for effective immunity.

\subsubsection{Systemic $\alpha$-GalCer administration versus in-vitro loading onto BM-DCs}

It had been reported that repeated free administration of soluble $\alpha$-GalCer causes decreases in NKT cell numbers (Fujii et al., 2002). Systemic $\alpha$-GalCer also causes decreases in the actual numbers of iNKT cells and eventual down regulation of TCR (Parekh et al., 2005a; Parekh et al., 2005b; Wilson et al., 2003; Wilson and Van Kaer, 2003). Recent work had also shown that soluble administration of $\alpha$-GalCer causes $\mathrm{CD}^{+} \mathrm{DC}$ numbers to be reduced in the spleen (Simkins et al., 2011). In humans, systemic $\alpha$-GalCer also leads to a decrease in iNKT frequencies (Chang et al., 2005; Giaccone et al., 2002). The systemic administration of $\alpha$-GalCer was shown to cause TCR downregulation within $24 \mathrm{~h}$, however this effect was rescued after $48 \mathrm{~h}$, but was followed by anergy in mice (Burdin et al., 1999; Burdin and Kronenberg, 1999; Fujii et al., 2002; Parekh et al., 2005a; Uldrich et al., 2005). A human study showed that $\alpha$-GalCer loaded onto BM-DCs could enhance $\mathrm{CD}^{+} \mathrm{T}$ cell responses (Chang et al., 2005), which was also seen in our murine work.

Studies highlighted in the previous chapter had suggested that antigenic components could be transferred from injected vaccine BM-DCs and be taken up by host splenic DC populations highlighting a role of the langerin ${ }^{+} \mathrm{CD} 8 \alpha^{+}$DCs. Experiments were carried out to examine the effect of direct NKT stimulation on host splenic DC populations in-vivo, after intravenous administration. These studies were also conducted in C57BL/6 subjects 
and then later in langerin-EGFP recipients so as to assess the impact of NKT cell stimulation on langerin ${ }^{+} \mathrm{CD} 8 \alpha^{+} \mathrm{DCs}$.

Systemic $\alpha$-GalCer treatment was shown to mature splenic CD11 $\mathrm{c}^{+} \mathrm{DCs}$, with responses in langerin-EGFP recipients also showing increased CD40 and CD86 expression 5 and $24 \mathrm{~h}$ after treatment but again highlighting the inherent enhanced basal expression of CD40 and CD86 on the langerin ${ }^{+} \mathrm{CD} 8 \alpha^{+}$DC subset in the absence of external stimulation. As seen with systemic TLR stimulation, the intravenous injection of $\alpha$-GalCer caused the percentage of langerin ${ }^{+} \mathrm{CD} 8 \alpha^{+}$splenic DCs to become significantly reduced when measured at $24 \mathrm{~h}$. What was unclear was whether this was due to down-regulation of the langerin marker in response to the $\alpha$-GalCer, or that whether cell death was occurring (results not shown) The langerin ${ }^{+} \mathrm{CD} 8 \alpha^{+}$DCs that remained were assessed for their CD86 and CD40 expression and incorporated into these results and showed enhanced CD86 expression at $24 \mathrm{~h}$. The increased CD86 expression seen on the splenic CD11c ${ }^{+}$DCs is likely to be caused by the direct presentation of $\alpha$-GalCer to NKT cell causing the release of pro-inflammatory cytokines acting to mature the host DCs presenting $\alpha$-GalCer on CD1d molecules.

These results indicate that systemic administration of $\alpha$-GalCer is able to increase the maturation status of all splenic DC subsets-but in particular the langerin ${ }^{+} \mathrm{CD} 8 \alpha^{+} \mathrm{DC}$ subset. It was likely that $\alpha$-GalCer was binding to CD1d molecules on splenic DC subsets and directly interacting with NKT cells to induce their release of pro-inflammatory cytokines and subsequent maturation of $\alpha$-GalCer presenting DCs. It was also possible that DC subsets were responding to pro-inflammatory cytokine release being induced by other $\alpha$-GalCer presenting cell types interacting with NKT cells or that even a combination of both processes was taking place. Further studies were conducted to determine the action of $\alpha$-GalCer on splenic DCs.

We also investigated $\mathrm{CD} 8^{+} \mathrm{T}$ cell responses after vaccination with systemic $\alpha$-GalCer and $\alpha$-GalCer loading onto DCs. Although we saw no statistical differences between groups that received $\alpha$-GalCer loaded onto DCs and those receiving systemic $\alpha$-GalCer, a general 
trend was evident which showed that $\alpha$-GalCer loading onto DCs induced increased and long lasting $\mathrm{CD}^{+} \mathrm{T}$ cell responses. The problem with this experiment was that the exact amount of $\alpha$-GalCer taken up by in-vitro cultured DCs could not be determined exactly and theoretically could not be compared directly with that amount delivered systemically. It is important to note however that essentially more $\alpha$-GalCer is being delivered to systemically treated groups however this administration is less controlled than when being delivered after loading onto BM-DCs. Nevertheless, far superior responses would have been expected from the systemically treated group, but what is actually shown by our results is that systemically treated $\alpha$-GalCer generates an antigen specific $T$ cell response that is comparable to that seen when loaded onto BM-DCs. These results would appear to highlight that loading $\alpha$-GalCer onto vaccine DCs as being desirable for "targeted" processing activities that allow delivery to T cell priming DCs.

A study was conducted to compare the antigen specific $\mathrm{T}$ cell responses achieved by systemic $\alpha$-GalCer and BM-DC vaccine administration versus that seen after loading of $\alpha$ GalCer onto the vaccine BM-DCs. The systemic administration of $\alpha$-GalCer was shown capable of generating an OVA specific T cell response, however this response was inferior in magnitude to that seen when using $\alpha$-GalCer loaded onto BM-DCs. It would appear that there are processing requirements that exist for $\alpha$-GalCer which are carried out by vaccine BM-DCs that allow for better $\mathrm{T}$ cell presentation activities and the generation of a superior OVA specific $\mathrm{T}$ cell response in the blood after vaccination. This is consistent with published reports that showed $\alpha$-GalCer pulsed onto DCs gives stronger and longer lasting immune response and that multiple doses of $\alpha$-GalCer could be given if loaded onto DCs and would not result in anergy of the immune response as repeated administration of free $\alpha$-GalCer causes decrease in NKT cell numbers (Fujii et al., 2002). The clinical relevance of this work is that loading $\alpha$-GalCer onto DCs in humans induced iNKT cell expansion (Chang et al., 2005; Giaccone et al., 2002).

Although $\alpha$-GalCer has been shown to be a powerful in-vivo adjuvant, recent work has also shown that its soluble administration causes $\operatorname{CD} 8 \alpha^{+}$DC numbers to be reduced in the spleen (Simkins et al., 2011). This effect on DC survival is undesirable as cross- 
presentation activities are needed to occur to ensure the generation of potent $\mathrm{CD}^{+} \mathrm{T}$ cell responses to exogenous antigens. The direct administration of $\alpha$-GalCer $i v$ is not conducive to the discriminative environment important for vaccination studies. The loading of $\alpha$-GalCer onto vaccine BM-DCs would provide a more controlled environment in comparison and also permit multiple administrations of $\alpha$-GalCer containing vaccines without attenuation of the immune response.

\subsubsection{The different routes of $\alpha$-GalCer administration}

Initial studies carried out using radioactive tracers to determine the fate of DC containing preparations administered by different route showed that i.v administered DCs collected initially in the lung \& liver, while i.d DC administration favoured migration to draining lymph nodes with varying efficiency (Morse et al., 1999). Mouse studies investigating the immunization capacity of DCs reported that the sc route of DC immunization was superior at generating CTL responses when compared with the $i v$ route (Eggert et al., 1999; Serody et al., 2000). Although a study in humans showed that i.d and i.l (intra-lymphatic) administration of DCs increased levels of Th1 immunity than iv DC administration, antibody responses of a greater magnitude and frequencies were demonstrated utilizing iv administration (Fong et al., 2001).

Although repeated vaccination through the skin has been the preferred method of immunizing and boosting immune responses, in humans, iNKT cells are found mainly in spleen, liver and bone marrow (MacDonald, 1995). In mice, large collections of iNKT cells are found in the spleen thereby favouring the $i v$ route of administration when considering $\alpha$-GalCer containing BM-DC vaccines. We investigated the subcutaneous route of administration for $\alpha$-GalCer containing vaccines and compared this with the $i v$ route.

Although it has been shown that $\alpha$-GalCer has worked after being administered intradermally, the action of $\alpha$-GalCer containing BM-DC loaded protein treated vaccines 
administered subcutaneously was confirmed by our work (Bontkes et al., 2010). In addition to demonstrating that $\alpha$-GalCer containing vaccines were capable of generating a measureable antigen specific $\mathrm{T}$ cell response one week post vaccination, results seen after $i v$ administration appeared more consistent while results seen from sc administration typically demonstrated heightened variation within treatment groups.

\subsubsection{The transfer of antigen and peptide after BM-DC vaccination}

Work shown in the previous chapter indicated that protein and peptide antigen loaded onto vaccine BM-DCs were being transferred to resident DC populations. It was also shown that resident splenic langerin ${ }^{+} \mathrm{CD} 8 \alpha^{+}$DCs were responsible for priming the antigen specific $\mathrm{T}$ cell response that was observed one-week post vaccination. It was also shown that the systemic delivery of TLR stimulation provided a more abundant cytokine source/pool than when used in-vitro to treat the vaccine BM-DCs. Using langerinDTREGFP recipients, BM-DC vaccines co-cultured with protein antigen and loaded with $\alpha$-GalCer were shown capable of generating increased antigen specific $\mathrm{T}$ cell responses that were also seen in $\mathrm{C} 57 \mathrm{BL} / 6$ recipients. After DT administration to deplete the langerin ${ }^{+} \mathrm{CD} 8 \alpha^{+} \mathrm{DC}$ subset however, this enhanced antigen specific $\mathrm{T}$ cell response was lost implicating their involvement in cross presentation processes required to generate the $\alpha$-GalCer induce antigen specific $\mathrm{T}$ cell response amplification.

An important question that needed to be addressed was whether $\alpha$-GalCer was being presented to iNKT cells simply by the injected vaccine BM-DCs, or if like the protein antigen, $\alpha$-GalCer was also being transferred to the langerin ${ }^{+} \mathrm{CD} 8 \alpha^{+} \mathrm{DC}$ subset (or other resident populations) and being directly presented by this host DC population. This leads to the possibility that the cytokine milieu induced by $\alpha$-GalCer could act on either the injected $\alpha$-GalCer presenting vaccine BM-DCs, or on the $\alpha$-GalCer bearing host langerin ${ }^{+} \mathrm{CD} 8 \alpha^{+} \mathrm{DC}$ subset. To further explore antigen and glycolipid transfer, peptide was substituted for OVA protein antigen and used to load BM-DCs in-vitro. The generation of 
antigen specific $\mathrm{T}$ cell response would indicate peptide antigen transfer had indeed taken place.

Increased OVA specific $\mathrm{CD} 8^{+} \mathrm{T}$ cell responses were seen generated after administration of peptide loaded BM-DCs carrying $\alpha$-GalCer, however when DT was used to deplete langerin ${ }^{+} \mathrm{CD} 8 \alpha^{+} \mathrm{DC}$ populations, this increased $\mathrm{CD}^{+} \mathrm{T}$ cell response was lost indicating that not only was peptide antigen being transferred, but that maybe $\alpha$-GalCer was also being transferred to resident cellular populations-namely the langerin ${ }^{+} \mathrm{CD} 8 \alpha^{+} \mathrm{DCs}$. It was evident that antigen and possibly $\alpha-G a l C e r$ was being transferred but the mechanism by which this was occurring was unknown. It is possible that the peptide bearing $\mathrm{MHC}$ complex is being lost from the vaccine BM-DCs to the host langerin ${ }^{+} \mathrm{CD} 8 \alpha^{+} \mathrm{DC}$ population somehow or that peptide and possibly MHC components are being recycled after phagocytosis of injected vaccine BM-DCs by langerin ${ }^{+} \mathrm{CD} 8 \alpha^{+}$host populations.

To explore the possibility that glycolipid $\alpha$-GalCer was also being transferred to host splenic DC populations, CD1d-/- DCs, vaccine BM-DCs were used to generate peptide loaded and $\alpha$-GalCer treated vaccines. The generation of antigen specific $\mathrm{T}$ cell responses after vaccination with these vaccines indicated that $\alpha$-GalCer had indeed been transferred onto host $\mathrm{T}$ cell priming $\mathrm{DC}$ populations. The ablation of the antigen specific $\mathrm{T}$ cell response after the administration of DT to deplete the langerin ${ }^{+} \mathrm{CD} 8 \mathrm{a}^{+} \mathrm{DC}$ subset again implicated these DCs in the T cell priming activities and in mediating the iNKT stimulated response by either directly presenting $\alpha$-GalCer or even possibly responding to proinflammatory cytokine release induced by another host DC subtype that might be presenting $\alpha$-GalCer.

These results show that not only antigen but also the glycolipid $\alpha$-GalCer is being transferred between injected vaccine BM-DCs and host DC populations. In particular the langerin ${ }^{+} \mathrm{CD} 8 \alpha^{+}$DC subset is strongly implicated as the recipient DC population in this model system. It does not rule out however the possibility that other CD1d expressing bystander cells such as other DC subsets, B cells or macrophages may have taken up the $\alpha$ GalCer, and have become the potent source of amplification signals that are impacting on 
the langerin ${ }^{+} \mathrm{CD} 8 \alpha^{+}$DCs driving the $\mathrm{CD} 8^{+} \mathrm{T}$ cell response being observed. It has been reported that $\alpha$-GalCer presentation is limited primarily to DCs, but also to macrophages (Bai et al., 2012; Bezbradica et al., 2005).

The langerin ${ }^{+} \mathrm{CD} 8 \alpha^{+} \mathrm{DC}$ subset could be described as providing an important method for targeting specialist cross-presenting cells which take advantage of the selective uptake ability of langerin ${ }^{+} \mathrm{CD} 8 \alpha^{+} \mathrm{DC}$ subset for the co-delivery of antigen and $\alpha$-GalCer for presentation and improved antigen specific $\mathrm{T}$ cell responses.

\subsubsection{Combined TLR \& NKT stimulation}

An effort was made to combine the systemic effects of NKT with TLR stimulation on the maturation and function of splenic DC subsets. Additive maturation phenotypes for especially CD86 were achieved after the combinations of $\alpha$-GalCer and MPL alone and together, $\alpha$-GalCer and Pam3Cys alone and together, $\alpha$-GalCer and Resiquimod alone and together were tested. This confirmed the ability of the selected TLR agonists to work in cooperation with each other to increase the maturation and activation status of host DC populations. The co-operation between NKT and TLR stimulation was also examined in further detail using langerin-EGFP recipients to reveal the separate effects on each of the major splenic DC subsets. Results showed that the maturation status of each of the major DC subsets was enhanced, although a preferential enhancement of the langerin ${ }^{+} \mathrm{CD} 8 \alpha^{+} \mathrm{DC}$ subset maturation status for CD86 and CD40 was also noted.

The ability of TLR and NKT stimulation to co-operatively improve antigen specific T cell responses to BM-DC vaccines was also tested by the co-administration of systemic MPL at the time of BM-DC vaccine delivery with our work clearly demonstrating that antigen specific $\mathrm{T}$ cell responses were significantly improved using this strategy. Careful usage of NKT and TLR stimulation together is capable of inducing a potent antigen specific T cell response that shows co-operation between these two adjuvant methods. 
Chapter 5: NKT stimulation enhances antigen specific responses to DC-based vaccines 248

\subsubsection{Conclusions}

- NKT stimulation is capable of increasing antigen specific T cell responses after invitro loading of BM-DCs with $\alpha$-GalCer and peptide and protein antigen

- BM-DCs loaded with $\alpha$-GalCer and protein antigen is capable of inducing antigen specific $\mathrm{T}$ cell responses after subcutaneous administration

- $\alpha$-GalCer is incorporated into the BM-DC membrane after in-vitro culture and is made available for transfer to host DC populations

- Host DCs receiving $\alpha$-GalCer can participate in CD8 T cell priming activities

- Direct TLR stimulation of antigen and $\alpha$-GalCer loaded BM-DC did not improve their capacity to induce $\mathrm{CD} 8^{+} \mathrm{T}$ cell response

- TLR stimulation of resident DCs did improve the capacity of antigen and $\alpha$-GalCer loaded BM-DCs to induce $\mathrm{CD} 8^{+} \mathrm{T}$ cell responses

- NKT co-operates with systemic TLR stimulation co-delivered at the time of vaccination to increase antigen specific $\mathrm{T}$ cell responses 
Chapter 5: NKT stimulation enhances antigen specific responses to DC-based vaccines 249 
Chapter 6:

\section{General Discussion}




\subsection{Introduction}

The incorporation of $\alpha$-GalCer as part of the DC-based vaccination strategy presented in this thesis consistently improved adaptive immune responses. This was characterized by an increase in the generation of antigen specific $\mathrm{T}$ cells found in the blood post vaccination. Further increases in antigen specific immune responses were achieved by the systemic coadministration of MPL at the time of vaccination alongside $\alpha$-GalCer containing DC-based vaccines. The transfer of peptide and protein antigenic components from injected vaccine DCs to resident DC populations was also reported. The langerin ${ }^{+} \mathrm{CD} 8 \alpha^{+}$splenic DC subset was found to mediate the cross-presentation and $\mathrm{T}$ cell priming activities required to drive the increased antigen specific $\mathrm{T}$ cell response after vaccination with antigen loaded $\mathrm{DC}$ based vaccines. The langerin ${ }^{+} \mathrm{CD} 8 \alpha^{+} \mathrm{DC}$ subset was also implicated in the crosspresentation and $\mathrm{T}$ cell activities induced after administration of $\alpha$-GalCer loaded DCbased vaccines delivered in combination with systemic MPL. This work describes a simple strategy for increasing the potency of DC-based vaccines applicable for clinical use in the treatment of many different cancers. Furthermore the recent identification of the human equivalent of the murine langerin ${ }^{+} \mathrm{CD} 8 \alpha^{+} \mathrm{DC}$ subset yields further value to the vaccine work concerning the langerin ${ }^{+} \mathrm{CD} 8 \alpha^{+} \mathrm{DC}$ subset and its potential relevance in defining a mechanism of DC-vaccine action in the human subject.

\subsection{DCs \& TLR stimulation}

\subsubsection{TLR stimulation increases antigen specific $\mathrm{CD8}^{+} \mathrm{T}$ cell responses to $\mathrm{DC}$ based vaccines}

The use of systemic TLR agonists has been described in this thesis as a strategy for improving the action of DC-based vaccines. Our initial work demonstrated that potent DC maturation and activation could be effectively achieved by the use of single TLR agonists to stimulate in-vitro BM-DC cultures. This maturation and activation response was further 
increased by the use of specific TLR combinations which typically utilized MPL in combination with another TLR agonist: Pam3Cys and Resiquimod.

Although used successfully to stimulated in-vitro BM-DC cultures, initial attempts to increase antigen specific immune responses in-vivo by the use of TLR treated BM-DCs in the vaccine preparation failed to achieve this aim. However subsequent experiments showed that the systemic co-administration of MPL alongside antigen loaded BM-DC based vaccines was in fact able to significantly increase antigen specific immune responses consistently by targeting resident DC populations. This increase in antigen specific immune responses was also observed when MPL was co-administered intravenously alongside antigen loaded and $\alpha$-GalCer carrying DC vaccines. This highlighted the importance of the resident DC populations and their interplay with injected DC vaccine components.

It was during the $18^{\text {th }}$ Century that Deidier made his observation that a positive correlation existed between infection and tumour regression, followed by William B Coley's cancer cure made from a mixture of gram-positive Streptococcus pneumoniae and gram-negative Serratia marcescens bacteria in the $19^{\text {th }}$ Century (Coley, 1893; Garay et al., 2007). The "haemorrhagic producing fraction" was identified by Shear and Turner as LPS, thought to be responsible for the anti-tumour effects of Coley's toxins (Garay et al., 2007). Once LPS was identified as a TLR4 stimulator, this implicated TLR stimulation as being connected to anti-tumour activity.

The use of TLR agonists to stimulate vaccine DCs stimulation using the previously described methods focussed upon MPL primarily as the TLR agonist of choice for this work. Given that MPL is the detoxified version of LPS and is typically used to generate activated and mature DC populations for positive controls, its selection was a deliberate attempt to generate DC populations that were superiorly stimulated. That MPL is currently being utilized within the clinical setting also provided support for its selection as TLR agonist of choice for this work. Another reason for the selection of MPL was due to its ability to function via the MyD88 adaptor signalling pathway and the MyD88 independent pathway - an ideal arrangement designed to ensure maximal stimulation of the DC is 
achieved given that all other TLRs typically exert their stimulatory effects via a single TLR signalling pathway.

Although the ability to improve DC activation and maturation using MPL combined with a second TLR agonist was clearly demonstrated in-vitro, this benefit unfortunately did not translate to improved antigen specific responses within the in-vivo setting. What was shown however was that the direct TLR stimulation of resident populations via systemic co-administration of MPL was able to ensure improved antigen specific T cell responses were generated. In fact the utilization of dual TLR agonists co-administered with DCbased vaccines highlighted the complexity of the vaccination response whereby antigen processing activities were affected by the presence of the more immediately potent TLR agonist. This strongly suggested that consideration should be given to the timing of administration when using multiple TLR agonists to allow for additive or synergistic antigen specific immune responses after vaccination using TLR stimulated adjuvanticity and was not explored further within these studies.

The utilization of TLR stimulation to achieve adjuvanticity involves both the innate and adaptive immune responses, the latter of which has been explored throughout this thesis. The induction of innate responses and processes within the tumour environment provides an immediate means to cause tumour destruction and regression under suitable conditions. The combination of innate immune responses with the achievement of potent longer lasting adaptive anti-tumour immunity could potentially serve as an effective treatment designed to target tumours and prevent the metastatic spread of tumour cells.

The in-vitro stimulatory action of CD40L was clearly shown to improve DC function measured by the upregulation of surface maturation markers and by the increase in cytokine production by such cells. This provided support for testing TLR treated BM-DC vaccines in-vitro using antigen loaded BM-DCs, which had also been treated with $\alpha$ GalCer. The use of $\alpha$-GalCer was a means of causing DCs to undergo CD40 mediated stimulation in-vivo by the recruitment of iNKT cells bearing generating CD40L and other cytokines capable of further enhancing DC stimulation. The incorporation of $\alpha$-GalCer 
within the vaccine protocol consistently yielded increased antigen specific immune responses after vaccination. $\alpha$-GalCer was shown to work safely and effectively to improve $\mathrm{CD}^{+} \mathrm{T}$ cell responses after using antigen loaded BM-DC vaccines alone, and in combination with the co-administration of systemic TLR stimulation using MPL coadministration at the time of vaccination.

The protein antigen processing and peptide antigen presentation capabilities of BM-DCs were markedly improved after treatment with MPL alone and in combination with either Pam3Cys or Resiquimod. Although this combinatorial impact worked favourably in-vitro, further work will be required to refine the in-vivo use of dual TLR agonists to generate antigen specific $\mathrm{T}$ cell responses. There is value in pursuing this line of investigation as the powerful immune response generated against infection with microorganisms typically involve exposure to more than a lone TLR agonist, but rather a combination of multiple TLR receptors acting in concert to induce potent immune responses against invading pathogens. The timing of TLR delivery appears to be an important factor that will require consideration and for optimal DC stimulation. The use of this as a refining tool should also be considered for future work.

Further examples of microbial based anti-tumour therapies include the intravesical BCG treatment of bladder cancer BCG stimulates TLR2 and TLR 4 receptors (Tsuji et al., 2000; Uehori et al., 2005) and has been used for more than 30 years as an effective treatment for this malignancy, OK-432 a concoction made from Streptococcus A (Okamoto et al., 1967) which stimulates TLR 4 (Hironaka et al., 2006; Okamoto et al., 2006) is used to treat gastric, cervical and oral squamous cell carcinoma (Kikkawa et al., 1993; Maehara et al., 1994; Sato et al., 1997).

The importance of the resident DC subset(s) and the impact of direct TLR stimulation was explored within this thesis with the langerin ${ }^{+} \mathrm{CD} 8 \alpha^{+} \mathrm{DC}$ subset identified as an important intermediary for vaccine responses. What was not determined was whether the langerin ${ }^{+}$ $\mathrm{CD} 8 \alpha^{+} \mathrm{DC}$ subset was responding to bystander cytokine effects derived from MPL acting on other cell types or whether in fact the MPL was acting directly and engaging with the 
langerin $\mathrm{CD} 8 \alpha^{+} \mathrm{DC}$ subset priming the antigen specific T cell response being measured. Preliminary efforts were made to determine the direct impact of TLR stimulation on the electronically purified and sorted splenic DC subsets with results suggesting splenic DCs can be further matured utilizing in-vitro TLR stimulation (data not shown).

Consideration should also be afforded to examine the impact of TLR stimulation on antigen processing activities and how this interplay supports or impedes antigen processing in the DC interior and the subsequent vaccination responses.

\subsection{Langerin ${ }^{+} \mathrm{CD8} \alpha^{+} \mathrm{DCs}$}

\subsubsection{Langerin ${ }^{+} \mathrm{CD8} \alpha^{+} \mathrm{DC}$ ' are involved in priming antigen specific $T$ cell responses to $\mathrm{DC}$ based vaccines}

The langerin-EGFP and langerin-DTREGFP transgenic murine models were utilized to investigate the action in-vivo action of the BM-DC vaccine tested in this study. The intravenous administration of antigen loaded BM-DCs ensured the exposure of splenic DC subsets to vaccine components. The langerin ${ }^{+} \mathrm{CD} 8 \alpha^{+} \mathrm{DC}$ subset characterized by the expression of langerin had been reported as being capable of superior cross-presentation activity when compared with other splenic DC subsets. The langerin-DTREGFP transgenic strain permitted the selective depletion of the langerin ${ }^{+} \mathrm{CD} 8 \alpha^{+}$subset using DT administration which revealed that this DC subset was responsible the cross-presentation and priming of the antigen specific $\mathrm{T}$ cell response being generated and measured in the blood. Antigenic components were also shown as being transferred from the injected vaccine preparation to the resident langerin ${ }^{+} \mathrm{CD} 8 \alpha^{+}$splenic DC subset which allowed the priming of the antigen specific $\mathrm{T}$ cell response after immunization.

The ability of systemic TLR stimulation to increase antigen specific immune responses was examined using the langerin-DTREGFP mice immunized with TLR 4-/- vaccine DCs. This investigation showed that the injected vaccine BM-DCs were not responsive to the 
systemic TLR administration, but rather the host langerin ${ }^{+} \mathrm{CD} 8 \alpha^{+} \mathrm{DC}$ subset was either receiving direct TLR stimuli or being influenced by cytokines released from bystander cells responding to TLR stimulation. Although it was unclear at this stage as to which process was taking place or if in fact both processes were being carried out together, it was clear the resident langerin ${ }^{+} \mathrm{CD} 8 \alpha^{+} \mathrm{DCs}$ were responsible for driving the antigen specific $\mathrm{T}$ cell response which could be increased using systemically administered TLR stimulation. The focal point regarding TLR adjuvanticity therefore became targeting resident DC populations, in particular the splenic langerin ${ }^{+} \mathrm{CD} 8 \alpha^{+} \mathrm{DC}$ subset.

Studies using the langerin-EGFP murine strain permitted the characterization of the langerin ${ }^{+} \mathrm{CD} 8 \alpha^{+}$DCs and showed elevated baseline expression of CD40 and CD86 on these DCs when compared with the remainder of the splenic DC compartment. The langerin ${ }^{+} \mathrm{CD} 8 \alpha^{+}$DCs were shown to be responsive to systemic TLR stimulation by further upregulating maturation markers in response. Based on the studies outlined in this chapter it would appear that benefit could be achieved by the preferential targeting of TLR stimulation to the langerin ${ }^{+} \mathrm{CD} 8 \alpha^{+} \mathrm{DC}$ subset since maturation marker expression was elevated and could be further increased using a TLR stimulus as was shown in-vivo and exvivo splenic DCs. This "semi-mature" phenotypic state of the langerin ${ }^{+} \mathrm{CD} 8 \alpha^{+} \mathrm{DCs}$ devoid of stimulation may support the superior cross-presentation abilities seen for $\mathrm{T}$ cell priming. This elevated baseline maturation status may induce preferential responsiveness to TLR stimulation and even confer a state of readiness for antigen acquisition and $\mathrm{T}$ cell priming by the langerin ${ }^{+} \mathrm{CD} 8 \alpha^{+} \mathrm{DCs}$.

The subsequent formal identification of the human langerin ${ }^{+} \mathrm{CD} 8 \alpha^{+} \mathrm{DC}$ equivalent as being the CD141 ${ }^{+}$BDCA-3 $^{+}$CLEC9A $^{+}\left(\right.$DNGR-1 ${ }^{+}$) DCs (Bachem et al., 2010; Crozat et al., 2010; Jongbloed et al., 2010; Poulin et al., 2010) was met with much enthusiasm given the large body of work being carried out using langerin ${ }^{+} \mathrm{CD} 8 \alpha^{+} \mathrm{DCs}$ and now a potential opening for their clinical relevance. Supporting evidence for their murine equivalence included the presence of both the langerin ${ }^{+} \mathrm{CD} 8 \alpha^{+} \mathrm{DCs}$ and $\mathrm{CD} 141^{+} \mathrm{BDCA}^{-}{ }^{+} \mathrm{CLEC} \mathrm{A}^{+}$ $\left(\mathrm{DNGR}-1^{+}\right)$DCs in the secondary lymphoid organs, the expression of the C-type lectin CLEC9A and the NECL2 adhesion molecule (Bachem et al., 2010; Caminschi et al., 2008; 
Crozat et al., 2010; Jongbloed et al., 2010; Poulin et al., 2010; Sancho et al., 2008). Other similarities between the human and murine DC counterparts include the expression of the chemokine receptor XCR1, the transcription factors Batf3 and IRF-8, their efficiency as superior cross-presenters of exogenous antigens, and their specific TLR expression profile (Bachem et al., 2010; Crozat et al., 2010; Jongbloed et al., 2010; Poulin et al., 2010).

When attempting to undertake electronic sorting activities to test and manipulate langerin ${ }^{+} \mathrm{CD} 8 \alpha^{+}$DCs processes ex-vivo, problems were experienced due to the labour intensive nature of the experimental techniques utilized and the spontaneous maturation induced after electronic sorting processing and associated activities. An attempt was made to address this by eliminating the electronic sorting process itself after initial purification steps leading to the production/isolation of splenic DC populations, which were stimulated in-vitro and separated by flow cytometric analytical and gating strategies.

Further work warranting investigation includes methods for targeting systemic TLR stimulation to the langerin ${ }^{+} \mathrm{CD} 8 \alpha^{+} \mathrm{DC}$ subset exclusively for the purpose of efficient increasing of antigen specific T cell responses, the use of langerin ${ }^{+} \mathrm{CD} 8 \alpha^{+} \mathrm{DCs}$ as vaccine DCs carrying antigen and the use of alternative synthetic TLR agonists alone and in combination together to generate superiorly stimulated langerin ${ }^{+} \mathrm{CD} 8 \alpha^{+}$DCs. Identification of the specific resident DC populations or other APCs that are responding directly to systemic TLR stimulation in the context of vaccination using antigen loaded BM-DC-based vaccines and the exploration of memory responses after immunization using these strategies would be valuable.

Although the work presented in this thesis would strongly suggest this vaccination strategy capable of indirectly targeting antigen and TLR stimulation to the langerin ${ }^{+} \mathrm{CD} 8 \alpha^{+} \mathrm{DC}$ subset, studies of immediate interest would involve the selective in-vivo targeting of antigen (and glycolipid) to langerin ${ }^{+} \mathrm{CD} 8 \alpha^{+} \mathrm{DCs}$. 


\subsection{DCs and NKT stimulation}

\subsubsection{NKT stimulation increases antigen specific $\mathrm{CD8}^{+} \mathrm{T}$ cell responses to $\mathrm{DC}$ based vaccines}

The use of NKT stimulation to improve the potency of DC based vaccines was shown to be a safe and effective method for increasing antigen specific $\mathrm{T}$ cell responses, the clinical relevance being the favourable induction of the adaptive immune response in an antigen specific manner by the incorporation of $\alpha$-GalCer within the vaccination protocol. Although primarily incorporated into a vaccination strategy that was administered intravenously to target the large NKT cell population found within the murine spleen, $\alpha$ GalCer was also found capable of influencing antigen specific immune responses after subcutaneous administration. Time constraints unfortunately prevented work to explore and develop this area further.

Importantly, the impact of $\alpha$-GalCer carrying BM-DC vaccines was highlighted after examination of the resident DC responses to vaccination. Work presented in this thesis showed that by the time vaccine BM-DCs had reached the spleen, groups all displayed a typical matured phenotype, regardless of the in-vitro manipulation protocol. This indicated that spontaneous maturation was taking place en-route to the spleen and it was also noted that $\alpha$-GalCer carrying vaccine BM-DCs were capable of maturing resident splenic DC populations.

The langerin-DTREGFP transgenic murine strain was utilized for studies, which showed that in addition to protein antigen, glycolipid antigen was also being transferred from the injected vaccine BM-DCs to resident DC populations. An extension of this work also showed this to be the case for peptide antigen as well. Although glycolipid transfer was occurring, it was unclear as to how exactly this was taking place. In addition, when CD1d/- BM-DCs were employed as vaccine DCs and treated with $\alpha$-GalCer, antigen specific immune responses remained significantly increased. Since CD1d-/- BM-DCs were deemed incapable of presenting $\alpha$-GalCer to NKT cell populations, it was surprising to see that $\alpha$ - 
GalCer had somehow been taken up and transferred to resident DC populations and subsequently presented to NKT cells.

An investigation was also carried out to determine whether benefit could be achieved from delivering systemic NKT stimulation. This was tested by comparing the antigen specific T cell response generated by using $\alpha$-GalCer loaded BM-DCs vs systemic $\alpha$-GalCer administration. These studies showed that superior responses were achieved when using $\alpha-$ GalCer loaded BM-DCs implying a processing requirement exists for $\alpha$-GalCer, which generates superior $\mathrm{T}$ cell responses compared with systemic administration. It was proposed that selective targeting of NKT stimulation to the resident $\mathrm{T}$ cell priming DC populations was prevented when $\alpha$-GalCer was administered systemically given the range of cell types expressing CD1d and capable of presenting $\alpha$-GalCer to NKT cells. Although glycolipid transfer to resident cells was demonstrated, the increased antigen specific immune effects were mediated by the langerin ${ }^{+} \mathrm{CD} 8 \alpha^{+} \mathrm{DC}$ subset. Again what was unknown was whether $\alpha$-GalCer was being presented to NKT cells by the langerin ${ }^{+} \mathrm{CD} 8 \alpha^{+}$ DCs driving the antigen specific immune response, or whether indirect cytokine effects were being induced in response to proinflammatory cytokines being produced by CD1d bystander APCs that had acquired $\alpha$-GalCer and were presenting to NKT cell.

\subsection{Implications for DC-based vaccines}

The antigen specific immune responses to DC based vaccines explored within this thesis focussed on varied methods to generate increased frequencies of $\mathrm{CD}^{+}$antigen specific $\mathrm{T}$ cells. The direction of TLR stimulation to T cell priming DCs would ensure the provision of sufficient and appropriate signals by carefully matured DCs to allow effective production of antigen specific $\mathrm{T}$ cells required for anti-tumour immunity. Balance is sought between the induction of appropriate anti-tumour immunity by these DC-based vaccines and the potential for autoimmunity to occur.

The direct targeting of TLR stimulation to resident DC populations was investigated. The physiological relevance of targeting TLR stimulation to resident murine splenic 
langerin ${ }^{+} \mathrm{CD} 8 \alpha^{+}$DCs studied in this thesis was connected to the knowledge that a human DC counterpart existed. Targeting these human cells for DC vaccination with TLR stimulation remains a possibility for future investigation and development.

The utilisation of $\alpha$-GalCer to induce NKT stimulation to enhance antigen specific T cell responses via DC based vaccination strategies was also shown to be an efficient method for consistently generating increased numbers of $\mathrm{CD}^{+} \mathrm{T}$ cells. The combined activities of TLR and NKT stimulation within the DC-based vaccine protocol generated increased $\mathrm{CD}^{+} \mathrm{T}$ cell frequencies.

\subsection{Final Remarks}

\subsubsection{Limitations and future work}

These DC based vaccination studies were not carried out in human subjects but in a murine model of B16.OVA. The B16.OVA cell line and OVA specific transgenic mice were utilised to observe $\mathrm{CD}^{+} \mathrm{T}$ cell responses to $\mathrm{DC}$ vaccination strategies tested within these studies. Systemic antigen specific responses were quantified as measure of anti-tumour activity potential, with therapeutic and prophylactic investigations possible for future experiments. The availability of clinical grade MPL and $\alpha$-GalCer would be required for future human translational studies.

Although studies showed that protein and peptide antigen was transferred after vaccination with antigen loaded DCs, the actual mechanism of antigen transfer detected after the use of allogeneic DC vaccine's in Langerin-DTREGFP recipients remains unknown. What was also unclear was the manner in which $\alpha$-GalCer was transferred and processed by CD1d-/DCs that permitted the induction and priming of antigen specific $\mathrm{T}$ cell responses after administration. 
The identity of the resident cells responsible for receiving antigen and glycolipid components and those cells responsible for the direct priming of the $\mathrm{T}$ cell response should be sought. In addition, the identity and full impact of cytokines and amplification signals released by bystander cells that impact upon the priming process should be sought. For example, the langerin ${ }^{+} \mathrm{CD} 8 \alpha^{+} \mathrm{DCs}$ were shown to prime antigen specific $\mathrm{T}$ cell responses to DC vaccines but what remained unknown was whether this splenic DC subset was presenting $\alpha$-GalCer or simply responding to cytokines released from DC- $\alpha$-GalCer interaction elsewhere between other DCs and NKT cells.

The selective targeting of the langerin ${ }^{+} \mathrm{CD} 8 \alpha^{+}$DCs subset in-vivo could potentially eliminate the need for ex-vivo DCs and their in-vitro manipulation. This could also possibly reduce the quantities of antigen and glycolipid required for direct delivery to resident DCs.

Similarly the exact nature and origin of TLR stimulation was unclear as it was unknown whether MPL was acting directly on langerin ${ }^{+} \mathrm{CD} 8 \mathrm{a}^{+} \mathrm{DCs}$ or other cell types that released cytokines in response capable of influencing the priming of the antigen specific $\mathrm{T}$ cell responses of resident DC populations that had received antigen. The systemic nature of the TLR co-delivery has its disadvantages in mice and humans. The functional plasticity and heterogeneity of the resident DC subsets hold the potential to generate non-protective or inappropriate $\mathrm{T}$ cell responses.

It should also be noted that although $\alpha$-GalCer has demonstrated powerful antitumour activity in murine models, it has had limited success in human patients in comparison. Possible explanations include the lower frequencies of NKT cells in humans, the even lower frequencies in patients with cancer and the presence of more complex and advanced disease when compared with animal models. Adoptive transfer of $\alpha$-GalCer pulsed DCs showed better success when compared to the utilization of soluble $\alpha$-GalCer (Chang et al., 2005; Ishikawa et al., 2005; Nieda et al., 2004). It has been suggested that IFN- $\gamma$ production post vaccination with $\alpha$-GalCer could be considered a predictive marker of success (Motohashi et al., 2006, 2009) given that some patients with advanced cancers 
have lower numbers of NKT cells or are defective in normal NKT cell function.

Another important area that require addressing include the quality and effector function of the antigen specific $\mathrm{T}$ cell response and memory response after vaccination using the $\mathrm{DC}$ based vaccination strategies outlined within this thesis.

Further work should also include DC vaccination as presented within this thesis being used in combination with other immune modulating agents such as PDL-1, CTLA-4 or 4-1BB blockade, T regulatory inactivation, or by administration with cytokines such as IL-15, IL17 or IL-12. The combination of DC vaccination immunotherapy with either adoptive $\mathrm{T}$ cell or NKT cell therapy is also highly feasible. 
Chapter 7:

\section{References}


Abdel Motal, U. M., Zhou, X., Joki, A., Siddiqi, A. R., Srinivasa, B. R., Stenvall, K., . . Jondal, M. (1993). Major histocompatibility complex class I-binding peptides are recycled to the cell surface after internalization. [Research Support, Non-U.S. Gov't]. European journal of immunology, 23(12), 3224-3229. doi: 10.1002/eji.1830231227

Abdi, K., Singh, N. J., \& Matzinger, P. (2012). Lipopolysaccharide-activated dendritic cells: "exhausted" or alert and waiting? [Research Support, N.I.H., Intramural]. Journal of immunology, 188(12), 5981-5989. doi: 10.4049/jimmunol.1102868

Abe, T., Hemmi, H., Miyamoto, H., Moriishi, K., Tamura, S., Takaku, H., . . Matsuura, Y. (2005). Involvement of the Toll-like receptor 9 signaling pathway in the induction of innate immunity by baculovirus. [Research Support, Non-U.S. Gov't]. Journal of virology, 79(5), 2847-2858. doi: 10.1128/JVI.79.5.2847-2858.2005

Abreu, M. T., Fukata, M., \& Arditi, M. (2005). TLR signaling in the gut in health and disease. [Research Support, U.S. Gov't, P.H.S. Review]. Journal of immunology, 174(8), 4453-4460.

Ackerman, A. L., Giodini, A., \& Cresswell, P. (2006). A role for the endoplasmic reticulum protein retrotranslocation machinery during crosspresentation by dendritic cells. [Research Support, Non-U.S. Gov't]. Immunity, 25(4), 607-617. doi: 10.1016/j.immuni.2006.08.017

Ackerman, A. L., Kyritsis, C., Tampe, R., \& Cresswell, P. (2003). Early phagosomes in dendritic cells form a cellular compartment sufficient for cross presentation of exogenous antigens. [Research Support, Non-U.S. Gov't Research Support, U.S. Gov't, P.H.S.]. Proceedings of the National Academy of Sciences of the United States of America, 100(22), 12889-12894. doi: 10.1073/pnas.1735556100

Ackerman, A. L., Kyritsis, C., Tampe, R., \& Cresswell, P. (2005). Access of soluble antigens to the endoplasmic reticulum can explain cross-presentation by dendritic cells. [Research Support, Non-U.S. Gov't Research Support, U.S. Gov't, P.H.S.]. Nature immunology, 6(1), 107-113. doi: 10.1038/ni1147

Adachi, O., Kawai, T., Takeda, K., Matsumoto, M., Tsutsui, H., Sakagami, M., . . Akira, S. (1998). Targeted disruption of the MyD88 gene results in loss of IL-1- and IL18-mediated function. [Research Support, Non-U.S. Gov't]. Immunity, 9(1), 143150.

Ahmed, R., \& Gray, D. (1996). Immunological memory and protective immunity: understanding their relation. [Research Support, Non-U.S. Gov't Research Support, U.S. Gov't, P.H.S. Review]. Science, 272(5258), 54-60.

Akira, S. (2001). Toll-like receptors and innate immunity. [Research Support, Non-U.S. Gov't Review]. Advances in immunology, 78, 1-56. 
Akira, S. (2006). TLR signaling. [Research Support, Non-U.S. Gov't Review]. Current topics in microbiology and immunology, 311, 1-16.

Akira, S., \& Takeda, K. (2004). Toll-like receptor signalling. [Research Support, Non-U.S. Gov't Review]. Nature reviews. Immunology, 4(7), 499-511. doi: 10.1038/nri1391

Albert, M. L., Jegathesan, M., \& Darnell, R. B. (2001). Dendritic cell maturation is required for the cross-tolerization of CD8+ T cells. [Research Support, Non-U.S. Gov't]. Nature immunology, 2(11), 1010-1017. doi: 10.1038/ni722

Albert, M. L., Pearce, S. F., Francisco, L. M., Sauter, B., Roy, P., Silverstein, R. L., \& Bhardwaj, N. (1998a). Immature dendritic cells phagocytose apoptotic cells via alphavbeta5 and CD36, and cross-present antigens to cytotoxic T lymphocytes. [Research Support, Non-U.S. Gov't Research Support, U.S. Gov't, P.H.S.]. The Journal of experimental medicine, 188(7), 1359-1368.

Albert, M. L., Sauter, B., \& Bhardwaj, N. (1998b). Dendritic cells acquire antigen from apoptotic cells and induce class I-restricted CTLs. Nature, 392(6671), 86-89. doi: $10.1038 / 32183$

Alexopoulou, L., Holt, A. C., Medzhitov, R., \& Flavell, R. A. (2001). Recognition of double-stranded RNA and activation of NF-kappaB by Toll-like receptor 3. [Research Support, Non-U.S. Gov't Research Support, U.S. Gov't, P.H.S.]. Nature, 413(6857), 732-738. doi: 10.1038/35099560

Aliquie, A. (1851). Inoculation de la syphilis au cancer. Gaz Hop(24), 546.

Allan, R. S., C. M. Smith, G. T. Belz, A. L. van Lint, L. M. Wakim, W. R. Heath, and F. R. Carbone. (2003). Epidermal viral immunity induced by CD8alpha + dendritic cells but not by Langerhans cells. Science, 301, 1925-1928.

Allan, R. S., J. Waithman, S. Bedoui, C. M. Jones, J. A. Villadangos, Y. Zhan, A. M. Lew, K. Shortman, W. R. Heath, and F. R. Carbone. (2006). Migratory dendritic cells transfer antigen to a lymph node-resident dendritic cell population for efficient CTL priming Immunity, 25, 153-162.

Allavena, P., Sica, A., Vecchi, A., Locati, M., Sozzani, S., \& Mantovani, A. (2000). The chemokine receptor switch paradigm and dendritic cell migration: its significance in tumor tissues. [Research Support, Non-U.S. Gov't Review]. Immunological reviews, 177, 141-149.

Ambrosino, E., Terabe, M., Halder, R. C., Peng, J., Takaku, S., Miyake, S., . . Berzofsky, J. A. (2007). Cross-regulation between type I and type II NKT cells in regulating tumor immunity: a new immunoregulatory axis. [Research Support, N.I.H., Intramural]. J Immunol, 179(8), 5126-5136. 
Amoureaux, A. (1760). Sur l'usage interieur de la belladonna. J Med Chir Pharm(13), 4765.

Andre, F., Chaput, N., Schartz, N. E., Flament, C., Aubert, N., Bernard, J., . . Zitvogel, L. (2004a). Exosomes as potent cell-free peptide-based vaccine. I. Dendritic cellderived exosomes transfer functional MHC class I/peptide complexes to dendritic cells. [Research Support, Non-U.S. Gov't]. Journal of immunology, 172(4), 21262136.

Andre, F., Escudier, B., Angevin, E., Tursz, T., \& Zitvogel, L. (2004b). Exosomes for cancer immunotherapy. [Review]. Annals of oncology : official journal of the European Society for Medical Oncology / ESMO, 15 Suppl 4, iv141-144. doi: 10.1093/annonc/mdh918

Andre, F. E. (2003). Vaccinology: past achievements, present roadblocks and future promises. [Historical Article]. Vaccine, 21(7-8), 593-595.

Anjuere, F., P. Martin, I. Ferrero, M. L. Fraga, G. M. del Hoyo, N. Wright, and C. Ardavin. . (1999). Definition of dendritic cell subpopulations present in the spleen, Peyer's patches, lymph nodes, and skin of the mouse. Blood, 93, 590-598.

Asea, A., Rehli, M., Kabingu, E., Boch, J. A., Bare, O., Auron, P. E., . . . Calderwood, S. K. (2002). Novel signal transduction pathway utilized by extracellular HSP70: role of toll-like receptor (TLR) 2 and TLR4. [Research Support, U.S. Gov't, P.H.S.]. The Journal of biological chemistry, 277(17), 15028-15034. doi: 10.1074/jbc.M200497200

Bachem, A., Guttler, S., Hartung, E., Ebstein, F., Schaefer, M., Tannert, A., . . Kroczek, R. A. (2010). Superior antigen cross-presentation and XCR1 expression define human CD11c+CD141+ cells as homologues of mouse CD8+ dendritic cells. [Research Support, Non-U.S. Gov't]. The Journal of experimental medicine, 207(6), 1273-1281. doi: 10.1084/jem.20100348

Bachmann, M. F., Oxenius, A., Pircher, H., Hengartner, H., Ashton-Richardt, P. A., Tonegawa, S., \& Zinkernagel, R. M. (1995). TAP1-independent loading of class I molecules by exogenous viral proteins. [Research Support, Non-U.S. Gov't Research Support, U.S. Gov't, P.H.S.]. European journal of immunology, 25(6), 1739-1743. doi: 10.1002/eji.1830250637

Backer, K. C., Hill, K. T., Shahin, A. J., \& Miller, L. M. (2010). Neural time course of echo suppression in humans. [Research Support, N.I.H., Extramural]. The Journal of neuroscience : the official journal of the Society for Neuroscience, 30(5), 19051913. doi: 10.1523/JNEUROSCI.4391-09.2010

Badovinac, V. P., Porter, B. B., \& Harty, J. T. (2002). Programmed contraction of CD8(+) T cells after infection. [Research Support, Non-U.S. Gov't Research Support, U.S. Gov't, P.H.S.]. Nature immunology, 3(7), 619-626. doi: 10.1038/ni804 
Bafica, A., Santiago, H. C., Goldszmid, R., Ropert, C., Gazzinelli, R. T., \& Sher, A. (2006). Cutting edge: TLR9 and TLR2 signaling together account for MyD88dependent control of parasitemia in Trypanosoma cruzi infection. [Research Support, Non-U.S. Gov't]. Journal of immunology, 177(6), 3515-3519.

Bafica, A., Scanga, C. A., Feng, C. G., Leifer, C., Cheever, A., \& Sher, A. (2005). TLR9 regulates Th1 responses and cooperates with TLR2 in mediating optimal resistance to Mycobacterium tuberculosis. [Comparative Study]. The Journal of experimental medicine, 202(12), 1715-1724. doi: 10.1084/jem.20051782

Bagchi, A., Herrup, E. A., Warren, H. S., Trigilio, J., Shin, H. S., Valentine, C., \& Hellman, J. (2007). MyD88-dependent and MyD88-independent pathways in synergy, priming, and tolerance between TLR agonists. [Research Support, N.I.H., Extramural]. Journal of immunology, 178(2), 1164-1171.

Bai, L., Constantinides, M. G., Thomas, S. Y., Reboulet, R., Meng, F., Koentgen, F., . . Bendelac, A. (2012). Distinct APCs explain the cytokine bias of alphagalactosylceramide variants in vivo. [Comparative Study Research Support, N.I.H., Extramural Research Support, Non-U.S. Gov't]. Journal of immunology, 188(7), 3053-3061. doi: 10.4049/jimmunol.1102414

Banchereau, J., Briere, F., Caux, C., Davoust, J., Lebecque, S., Liu, Y. J., . . Palucka, K. (2000). Immunobiology of dendritic cells. [Research Support, Non-U.S. Gov't Research Support, U.S. Gov't, P.H.S. Review]. Annual review of immunology, 18, 767-811. doi: 10.1146/annurev.immunol.18.1.767

Banchereau, J., Palucka, A. K., Dhodapkar, M., Burkeholder, S., Taquet, N., Rolland, A., . . . Fay, J. (2001). Immune and clinical responses in patients with metastatic melanoma to CD34(+) progenitor-derived dendritic cell vaccine. [Clinical Trial Research Support, Non-U.S. Gov't Research Support, U.S. Gov't, P.H.S.]. Cancer research, 61(17), 6451-6458.

Banchereau, J., \& Steinman, R. M. (1998). Dendritic cells and the control of immunity. [Research Support, U.S. Gov't, P.H.S. Review]. Nature, 392(6673), 245-252. doi: $10.1038 / 32588$

Bar-On, L., Birnberg, T., Lewis, K. L., Edelson, B. T., Bruder, D., Hildner, K., . . . Jung, S. (2010). CX3CR1+ CD8alpha+ dendritic cells are a steady-state population related to plasmacytoid dendritic cells. [Research Support, N.I.H., Extramural Research Support, Non-U.S. Gov't]. Proceedings of the National Academy of Sciences of the United States of America, 107(33), 14745-14750. doi: 10.1073/pnas.1001562107

Bardell, D. (1976). Smallpox during the American War of Independance. America Society for Microbiology(42), 526-530. 
Barnden, M. J., Allison, J., Heath, W. R., \& Carbone, F. R. (1998). Defective TCR expression in transgenic mice constructed using cDNA-based alpha- and beta-chain genes under the control of heterologous regulatory elements. [Research Support, Non-U.S. Gov't Research Support, U.S. Gov't, P.H.S.]. Immunology and cell biology, 76(1), 34-40. doi: 10.1046/j.1440-1711.1998.00709.x

Barquet, N., \& Domingo, P. (1997). Smallpox: the triumph over the most terrible of the ministers of death. [Historical Article]. Annals of internal medicine, 127(8 Pt 1), 635-642.

Barrat, F. J., Meeker, T., Gregorio, J., Chan, J. H., Uematsu, S., Akira, S., . . Coffman, R. L. (2005). Nucleic acids of mammalian origin can act as endogenous ligands for Toll-like receptors and may promote systemic lupus erythematosus. [Comparative Study Research Support, Non-U.S. Gov't]. The Journal of experimental medicine, 202(8), 1131-1139. doi: 10.1084/jem.20050914

Basner-Tschakarjan, E., Gaffal, E., O'Keeffe, M., Tormo, D., Limmer, A., Wagner, H., .. . Tuting, T. (2006). Adenovirus efficiently transduces plasmacytoid dendritic cells resulting in TLR9-dependent maturation and IFN-alpha production. [In Vitro Research Support, Non-U.S. Gov't]. The journal of gene medicine, 8(11), 13001306. doi: 10.1002/jgm.964

Bedoui, S., Prato, S., Mintern, J., Gebhardt, T., Zhan, Y., Lew, A. M., . . . Segura, E. (2009). Characterization of an immediate splenic precursor of CD8+ dendritic cells capable of inducing antiviral T cell responses. [Research Support, Non-U.S. Gov't]. Journal of immunology, 182(7), 4200-4207. doi: 10.4049/jimmunol.0802286

Bedoui, S., Whitney, P. G., Waithman, J., Eidsmo, L., Wakim, L., Caminschi, I., . . . Heath, W. R. (2009). Cross-presentation of viral and self antigens by skin-derived CD103+ dendritic cells. [Research Support, Non-U.S. Gov't]. Nature immunology, 10(5), 488-495. doi: 10.1038/ni.1724

Behbehani, A. M. (1983). The smallpox story: life and death of an old disease. [Historical Article Review]. Microbiological reviews, 47(4), 455-509.

Bekeredjian-Ding, I., Roth, S. I., Gilles, S., Giese, T., Ablasser, A., Hornung, V., . . . Hartmann, G. (2006). T cell-independent, TLR-induced IL-12p70 production in primary human monocytes. [Research Support, Non-U.S. Gov't]. Journal of immunology, 176(12), 7438-7446.

Belz, G. T., C. M. Smith, L. Kleinert, P. Reading, A. Brooks, K. Shortman, F. R. Carbone, and W. R. Heath. (2004). Distinct migrating and nonmigrating dendritic cell populations are involved in MHC class I-restricted antigen presentation after lung infection with virus. Proc Natl Acad Sci 101, 8670-8675.

Belz, G. T., Smith, C. M., Eichner, D., Shortman, K., Karupiah, G., Carbone, F. R., \& Heath, W. R. (2004). Cutting edge: conventional CD8 alpha+ dendritic cells are 
generally involved in priming CTL immunity to viruses. [Research Support, NonU.S. Gov't]. Journal of immunology, 172(4), 1996-2000.

Bendelac, A. (1995a). CD1: presenting unusual antigens to unusual T lymphocytes. [Comment Research Support, Non-U.S. Gov't Review]. Science, 269(5221), 185186.

Bendelac, A. (1995b). Mouse NK1+ T cells. [Review]. Current opinion in immunology, 7(3), 367-374.

Bendelac, A., Savage, P. B., \& Teyton, L. (2007). The biology of NKT cells. [Research Support, N.I.H., Extramural Research Support, Non-U.S. Gov't Review]. Annu Rev Immunol, 25, 297-336. doi: 10.1146/annurev.immunol.25.022106.141711

Benlagha, K., \& Bendelac, A. (2000). CD1d-restricted mouse V alpha 14 and human V alpha 24 T cells: lymphocytes of innate immunity. [Research Support, Non-U.S. Gov't Research Support, U.S. Gov't, P.H.S. Review]. Seminars in immunology, 12(6), 537-542. doi: 10.1006/smim.2000.0276

Benlagha, K., Weiss, A., Beavis, A., Teyton, L., \& Bendelac, A. (2000). In vivo identification of glycolipid antigen-specific $\mathrm{T}$ cells using fluorescent CD1d tetramers. [Research Support, Non-U.S. Gov't Research Support, U.S. Gov't, P.H.S.]. The Journal of experimental medicine, 191(11), 1895-1903.

Bennett, S. R., et al. (1998). Help for cytotoxic-T-cell responses is mediated by CD40 signalling. Nature, 393, 478-480.

Berland, R., Fernandez, L., Kari, E., Han, J. H., Lomakin, I., Akira, S., . . I Imanishi-Kari, T. (2006). Toll-like receptor 7-dependent loss of B cell tolerance in pathogenic autoantibody knockin mice. [Research Support, N.I.H., Extramural Research Support, Non-U.S. Gov't]. Immunity, 25(3), 429-440. doi: 10.1016/j.immuni.2006.07.014

Beutler, B. (2000). Endotoxin, toll-like receptor 4, and the afferent limb of innate immunity. [Review]. Current opinion in microbiology, 3(1), 23-28.

Bevan, M. J. (1976). Minor H antigens introduced on H-2 different stimulating cells crossreact at the cytotoxic T cell level during in vivo priming. [Research Support, U.S. Gov't, P.H.S.]. Journal of immunology, 117(6), 2233-2238.

Bezbradica, J. S., Stanic, A. K., Matsuki, N., Bour-Jordan, H., Bluestone, J. A., Thomas, J. W., . . Joyce, S. (2005). Distinct roles of dendritic cells and B cells in Va14Ja18 natural $\mathrm{T}$ cell activation in vivo. [In Vitro Research Support, Non-U.S. Gov't Research Support, U.S. Gov't, P.H.S.]. Journal of immunology, 174(8), 4696-4705.

Biragyn, A., Ruffini, P. A., Leifer, C. A., Klyushnenkova, E., Shakhov, A., Chertov, O., . . . Kwak, L. W. (2002). Toll-like receptor 4-dependent activation of dendritic cells 
by beta-defensin 2. [Research Support, U.S. Gov't, P.H.S.]. Science, 298(5595), 1025-1029. doi: 10.1126/science.1075565

Blander, J. M., \& Medzhitov, R. (2006). Toll-dependent selection of microbial antigens for presentation by dendritic cells. [Research Support, N.I.H., Extramural Research Support, Non-U.S. Gov't]. Nature, 440(7085), 808-812. doi: 10.1038/nature04596

Blander, J. M., \& Sander, L. E. (2012). Beyond pattern recognition: five immune checkpoints for scaling the microbial threat. [Research Support, N.I.H., Extramural Research Support, Non-U.S. Gov't]. Nature reviews. Immunology, 12(3), 215-225. doi: $10.1038 /$ nri3167

Blasius, A. L., \& Beutler, B. (2010). Intracellular toll-like receptors. [Research Support, N.I.H., Extramural Research Support, Non-U.S. Gov't Review]. Immunity, 32(3), 305-315. doi: 10.1016/j.immuni.2010.03.012

Bohnenkamp, H. R., Papazisis, K. T., Burchell, J. M., \& Taylor-Papadimitriou, J. (2007). Synergism of Toll-like receptor-induced interleukin-12p70 secretion by monocytederived dendritic cells is mediated through p38 MAPK and lowers the threshold of T-helper cell type 1 responses. [Research Support, Non-U.S. Gov't]. Cellular immunology, 247(2), 72-84. doi: 10.1016/j.cellimm.2007.07.008

Bonanni, P. (1999). Demographic impact of vaccination: a review. [Review]. Vaccine, 17 Suppl 3, S120-125.

Bontkes, H. J., Moreno, M., Hangalapura, B., Lindenberg, J. J., de Groot, J., Lougheed, S., Scheper, R. J. (2010). Attenuation of invariant natural killer T-cell anergy induction through intradermal delivery of alpha-galactosylceramide. [Comparative Study Research Support, Non-U.S. Gov't]. Clinical immunology, 136(3), 364-374. doi: 10.1016/j.clim.2010.04.019

Boule, M. W., Broughton, C., Mackay, F., Akira, S., Marshak-Rothstein, A., \& Rifkin, I. R. (2004). Toll-like receptor 9-dependent and -independent dendritic cell activation by chromatin-immunoglobulin G complexes. [Research Support, Non-U.S. Gov't Research Support, U.S. Gov't, P.H.S.]. The Journal of experimental medicine, 199(12), 1631-1640. doi: 10.1084/jem.20031942

Bowles, A. P., Jr., \& Perkins, E. (1999). Long-term remission of malignant brain tumors after intracranial infection: a report of four cases. [Case Reports Review]. Neurosurgery, 44(3), 636-642; discussion 642-633.

Breman, J. G., \& Arita, I. (1980). The confirmation and maintenance of smallpox eradication. The New England journal of medicine, 303(22), 1263-1273. doi: 10.1056/NEJM198011273032204

Brinkmann, M. M., Spooner, E., Hoebe, K., Beutler, B., Ploegh, H. L., \& Kim, Y. M. (2007). The interaction between the ER membrane protein UNC93B and TLR3, 7, 
and 9 is crucial for TLR signaling. [Research Support, N.I.H., Extramural Research Support, Non-U.S. Gov't]. The Journal of cell biology, 177(2), 265-275. doi: $10.1083 /$ jcb.200612056

Brossart, P., \& Bevan, M. J. (1997). Presentation of exogenous protein antigens on major histocompatibility complex class I molecules by dendritic cells: pathway of presentation and regulation by cytokines. [Research Support, Non-U.S. Gov't]. Blood, 90(4), 1594-1599.

Burdin, N., Brossay, L., \& Kronenberg, M. (1999). Immunization with alphagalactosylceramide polarizes CD1-reactive NK T cells towards Th2 cytokine synthesis. [Comparative Study Research Support, Non-U.S. Gov't Research Support, U.S. Gov't, P.H.S.]. European journal of immunology, 29(6), 2014-2025.

Burdin, N., \& Kronenberg, M. (1999). CD1-mediated immune responses to glycolipids. [Research Support, U.S. Gov't, P.H.S. Review]. Current opinion in immunology, 11(3), 326-331.

Burgdorf, S., Kautz, A., Bohnert, V., Knolle, P. A., \& Kurts, C. (2007). Distinct pathways of antigen uptake and intracellular routing in CD4 and CD8 T cell activation. [Research Support, Non-U.S. Gov't]. Science, 316(5824), 612-616. doi: 10.1126/science. 1137971

Burgdorf, S., \& Kurts, C. (2008). Endocytosis mechanisms and the cell biology of antigen presentation. [Research Support, Non-U.S. Gov't Review]. Current opinion in immunology, 20(1), 89-95. doi: 10.1016/j.coi.2007.12.002

Bursch, L. S., Wang, L., Igyarto, B., Kissenpfennig, A., Malissen, B., Kaplan, D.H. \& , \& Hogquist, K. A. (2007). Identification of a novel population of Langerin+ dendritic cells. J Exp Med(204), 3147-3156.

Busch, W. (1868). Niederrheinische Gesellshaft fur Natur und Heilkunder in Bonn. Berlin Klin Wochenschur(5), 137-138.

Byrd-Leifer, C. A., Block, E. F., Takeda, K., Akira, S., \& Ding, A. (2001). The role of MyD88 and TLR4 in the LPS-mimetic activity of Taxol. [Research Support, NonU.S. Gov't Research Support, U.S. Gov't, P.H.S.]. European journal of immunology, 31(8), 2448-2457. doi: 10.1002/15214141(200108)31:8\&\#60;2448::AID-IMMU2448\&\#62;3.0.CO;2-N

Cambi, A., Koopman, M., \& Figdor, C. G. (2005). How C-type lectins detect pathogens. [Research Support, Non-U.S. Gov't Review]. Cellular microbiology, 7(4), 481-488. doi: $10.1111 / \mathrm{j} .1462-5822.2005 .00506 . \mathrm{x}$

Caminschi, I., Proietto, A. I., Ahmet, F., Kitsoulis, S., Shin Teh, J., Lo, J. C., . . . Lahoud, M. H. (2008). The dendritic cell subtype-restricted C-type lectin Clec9A is a target 
for vaccine enhancement. [Research Support, Non-U.S. Gov't]. Blood, 112(8), 3264-3273. doi: 10.1182/blood-2008-05-155176

Casella, C. R., \& Mitchell, T. C. (2008). Putting endotoxin to work for us: monophosphoryl lipid A as a safe and effective vaccine adjuvant. [Research Support, Non-U.S. Gov't Research Support, U.S. Gov't, P.H.S. Review]. Cellular and molecular life sciences : CMLS, 65(20), 3231-3240. doi: 10.1007/s00018-0088228-6

Cella, M., Engering, A., Pinet, V., Pieters, J., \& Lanzavecchia, A. (1997). Inflammatory stimuli induce accumulation of MHC class II complexes on dendritic cells. [Research Support, Non-U.S. Gov't]. Nature, 388(6644), 782-787. doi: $10.1038 / 42030$

Cella, M., Scheidegger, D., Palmer-Lehmann, K., Lane, P., Lanzavecchia, A., \& Alber, G. (1996). Ligation of CD40 on dendritic cells triggers production of high levels of interleukin-12 and enhances $\mathrm{T}$ cell stimulatory capacity: T-T help via APC activation. [Research Support, Non-U.S. Gov't]. The Journal of experimental medicine, 184(2), 747-752.

Celluzzi, C. M., Mayordomo, J.I., Storkus, M.T., Lotze, M.T., Falo, Jr, L.D. (1996). Peptide-pulsed dendritic cells induce antigen-specific CTL-mediated protective tumour immunity. J Exp Med(183), 283-287.

Cerundolo, V., Silk, J. D., Masri, S. H., \& Salio, M. (2009). Harnessing invariant NKT cells in vaccination strategies. [Review]. Nature reviews. Immunology, 9(1), 28-38. doi: $10.1038 /$ nri2451

Cervantes, J. L., Dunham-Ems, S. M., La Vake, C. J., Petzke, M. M., Sahay, B., Sellati, T. J., . . . Salazar, J. C. (2011). Phagosomal signaling by Borrelia burgdorferi in human monocytes involves Toll-like receptor (TLR) 2 and TLR8 cooperativity and TLR8-mediated induction of IFN-beta. [Research Support, N.I.H., Extramural Research Support, Non-U.S. Gov't]. Proceedings of the National Academy of Sciences of the United States of America, 108(9), 3683-3688. doi: 10.1073/pnas. 1013776108

Chang, D. H., Osman, K., Connolly, J., Kukreja, A., Krasovsky, J., Pack, M., . . . Dhodapkar, M. V. (2005). Sustained expansion of NKT cells and antigen-specific T cells after injection of alpha-galactosyl-ceramide loaded mature dendritic cells in cancer patients. [Clinical Trial Comparative Study Research Support, N.I.H., Extramural Research Support, Non-U.S. Gov't Research Support, U.S. Gov't, P.H.S.]. The Journal of experimental medicine, 201(9), 1503-1517. doi: $10.1084 /$ jem.20042592

Chaudhuri, N., Dower, S. K., Whyte, M. K., \& Sabroe, I. (2005). Toll-like receptors and chronic lung disease. [Research Support, Non-U.S. Gov't Review]. Clinical science, 109(2), 125-133. doi: 10.1042/CS20050044 
Chen, K., Huang, J., Gong, W., Iribarren, P., Dunlop, N. M., \& Wang, J. M. (2007). Tolllike receptors in inflammation, infection and cancer. [Research Support, N.I.H., Extramural Review]. International immunopharmacology, 7(10), 1271-1285. doi: 10.1016/j.intimp.2007.05.016

Chen, L., Wang, T., Zhou, P., Ma, L., Yin, D., Shen, J., . . . Chong, A. (2006). TLR engagement prevents transplantation tolerance. [Research Support, N.I.H., Extramural Research Support, Non-U.S. Gov't]. American journal of transplantation : official journal of the American Society of Transplantation and the American Society of Transplant Surgeons, 6(10), 2282-2291. doi: 10.1111/j.1600-6143.2006.01489.x

Chen, Y. H., Chiu, N. M., Mandal, M., Wang, N., \& Wang, C. R. (1997). Impaired NK1+ $\mathrm{T}$ cell development and early IL-4 production in CD1-deficient mice. [Research Support, Non-U.S. Gov't]. Immunity, 6(4), 459-467.

Chinnery, H. R., Pearlman, E., \& McMenamin, P. G. (2008). Cutting edge: Membrane nanotubes in vivo: a feature of MHC class II+ cells in the mouse cornea. [Research Support, N.I.H., Extramural Research Support, Non-U.S. Gov't]. Journal of immunology, 180(9), 5779-5783.

Chiodoni, C., Stoppacciaro, A., Sangaletti, S., Gri, G., Cappetti, B., Koezuka, Y., \& Colombo, M. P. (2001). Different requirements for alpha-galactosylceramide and recombinant IL-12 antitumor activity in the treatment of C-26 colon carcinoma hepatic metastases. [Research Support, Non-U.S. Gov't]. Eur J Immunol, 31(10), 3101-3110.

Choi, Y. J., Im, E., Chung, H. K., Pothoulakis, C., \& Rhee, S. H. (2010). TRIF mediates Toll-like receptor 5-induced signaling in intestinal epithelial cells. [Research Support, N.I.H., Extramural Research Support, Non-U.S. Gov't]. The Journal of biological chemistry, 285(48), 37570-37578. doi: 10.1074/jbc.M110.158394

Christensen, S. R., Shupe, J., Nickerson, K., Kashgarian, M., Flavell, R. A., \& Shlomchik, M. J. (2006). Toll-like receptor 7 and TLR9 dictate autoantibody specificity and have opposing inflammatory and regulatory roles in a murine model of lupus. [Comparative Study Research Support, N.I.H., Extramural]. Immunity, 25(3), 417428. doi: 10.1016/j.immuni.2006.07.013

Cluff, C. W. (2009). Monophosphoryl lipid A (MPL) as an adjuvant for anti-cancer vaccines: clinical results. [Review]. Advances in experimental medicine and biology, 667, 111-123.

Coley, W. B. (1893). The treatment of malignant tumours by repeated inoculations of erysipelas: with a report of ten original cases. Am J Med Sci(105), 487-511. 
Coley, W. B. (1906). Late results of the treatment of inoperable sarcoma by the mised toxins of erysipelas and bacillus prodifiosus. Am J Med Sci(131), 375-430.

Coley, W. B. (1914). The treatment of maliganant inoperable tumours with the mixed toxins of erysipelas and bacillus prodigiosus with a brief report of 80 cases successfully treated with the toxins from 1893-1914. Brussels: M, Weinsbruch.

Colombo, M. P., \& Trinchieri, G. (2002). Interleukin-12 in anti-tumor immunity and immunotherapy. [Review]. Cytokine \& growth factor reviews, 13(2), 155-168.

Colonna, M., Trinchieri, G., \& Liu, Y. J. (2004). Plasmacytoid dendritic cells in immunity. [Review]. Nature immunology, 5(12), 1219-1226. doi: 10.1038/ni1141

Cook, E. B., Stahl, J. L., Esnault, S., Barney, N. P., \& Graziano, F. M. (2005). Toll-like receptor 2 expression on human conjunctival epithelial cells: a pathway for Staphylococcus aureus involvement in chronic ocular proinflammatory responses. [Research Support, Non-U.S. Gov't Research Support, U.S. Gov't, P.H.S.]. Annals of allergy, asthma \& immunology : official publication of the American College of Allergy, Asthma, \& Immunology, 94(4), 486-497. doi: 10.1016/S10811206(10)61120-9

Coombes, J. L., Siddiqui, K. R., Arancibia-Carcamo, C. V., Hall, J., Sun, C. M., Belkaid, Y., \& Powrie, F. (2007). A functionally specialized population of mucosal CD103+ DCs induces Foxp3+ regulatory $\mathrm{T}$ cells via a TGF-beta and retinoic acid-dependent mechanism. [Research Support, Non-U.S. Gov't]. The Journal of experimental medicine, 204(8), 1757-1764. doi: 10.1084/jem.20070590

Coquet, J. M., Chakravarti, S., Kyparissoudis, K., McNab, F. W., Pitt, L. A., McKenzie, B. S., . . . Godfrey, D. I. (2008). Diverse cytokine production by NKT cell subsets and identification of an IL-17-producing CD4-NK1.1- NKT cell population. [Research Support, Non-U.S. Gov't]. Proc Natl Acad Sci U S A, 105(32), 11287-11292. doi: 10.1073/pnas.0801631105

Creagh, E. M., \& O'Neill, L. A. (2006). TLRs, NLRs and RLRs: a trinity of pathogen sensors that co-operate in innate immunity. [Research Support, Non-U.S. Gov't]. Trends in immunology, 27(8), 352-357. doi: 10.1016/j.it.2006.06.003

Crowe, N. Y., Smyth, M. J., \& Godfrey, D. I. (2002). A critical role for natural killer T cells in immunosurveillance of methylcholanthrene-induced sarcomas. [Research Support, Non-U.S. Gov't]. J Exp Med, 196(1), 119-127.

Crozat, K., Guiton, R., Contreras, V., Feuillet, V., Dutertre, C. A., Ventre, E., . . . Dalod, M. (2010). The XC chemokine receptor 1 is a conserved selective marker of mammalian cells homologous to mouse CD8alpha+ dendritic cells. [Research Support, Non-U.S. Gov't]. The Journal of experimental medicine, 207(6), 12831292. doi: 10.1084/jem.20100223 
Cruveilhier, J. (1864). Traité d'anatomie pathologique générale. Paris: J.B.Bailliere.

Cui, Y., Le, Y., Yazawa, H., Gong, W., \& Wang, J. M. (2002). Potential role of the formyl peptide receptor-like 1 (FPRL1) in inflammatory aspects of Alzheimer's disease. [Research Support, U.S. Gov't, P.H.S. Review]. Journal of leukocyte biology, 72(4), 628-635.

Cui, J., Shin, T., Kawano, T., Sato, H., Kondo, E., Toura, I., . . Taniguchi, M. (1997). Requirement for Valpha14 NKT cells in IL-12-mediated rejection of tumors. [Research Support, Non-U.S. Gov't]. Science, 278(5343), 1623-1626.

Curtsinger, J. M., Johnson, C. M., \& Mescher, M. F. (2003a). CD8 T cell clonal expansion and development of effector function require prolonged exposure to antigen, costimulation, and signal 3 cytokine. [Research Support, U.S. Gov't, P.H.S.]. Journal of immunology, 171(10), 5165-5171.

Curtsinger, J. M., Lins, D. C., \& Mescher, M. F. (2003b). Signal 3 determines tolerance versus full activation of naive $\mathrm{CD} 8 \mathrm{~T}$ cells: dissociating proliferation and development of effector function. [Research Support, U.S. Gov't, P.H.S.]. The Journal of experimental medicine, 197(9), 1141-1151. doi: 10.1084/jem.20021910

Curtsinger, J. M., Schmidt, C. S., Mondino, A., Lins, D. C., Kedl, R. M., Jenkins, M. K., \& Mescher, M. F. (1999). Inflammatory cytokines provide a third signal for activation of naive CD4+ and CD8+ T cells. [Research Support, U.S. Gov't, P.H.S.]. Journal of immunology, 162(6), 3256-3262.

Cyster, J. G. (1999). Chemokines and the homing of dendritic cells to the T cell areas of lymphoid organs. [Comment]. The Journal of experimental medicine, 189(3), 447450 .

d'Ostiani, C. F., G. Del Sero, A. Bacci, C. Montagnoli, A. Spreca, A. Mencacci, P. Ricciardi-Castagnoli, and L. Romani. (2000). Dendritic cells discriminate between yeasts and hyphae of the fungus Candida albicans. Implications for initiation of $\mathrm{T}$ helper cell immunity in vitro and in vivo. . J Exp Med 191, 1661-1674.

da Silva Correia, J., Soldau, K., Christen, U., Tobias, P. S., \& Ulevitch, R. J. (2001). Lipopolysaccharide is in close proximity to each of the proteins in its membrane receptor complex. transfer from CD14 to TLR4 and MD-2. [Research Support, Non-U.S. Gov't Research Support, U.S. Gov't, P.H.S.]. The Journal of biological chemistry, 276(24), 21129-21135. doi: 10.1074/jbc.M009164200

Dahlberg, A., Auble, M. R., \& Petro, T. M. (2006). Reduced expression of IL-12 p35 by $\mathrm{SJL} / \mathrm{J}$ macrophages responding to Theiler's virus infection is associated with constitutive activation of IRF-3. [Research Support, N.I.H., Extramural Research Support, Non-U.S. Gov't]. Virology, 353(2), 422-432. doi: 10.1016/j.virol.2006.05.034 
Dai, P., Cao, H., Merghoub, T., Avogadri, F., Wang, W., Parikh, T., . . Deng, L. (2011). Myxoma virus induces type I interferon production in murine plasmacytoid dendritic cells via a TLR9/MyD88-, IRF5/IRF7-, and IFNAR-dependent pathway. [Research Support, N.I.H., Extramural Research Support, Non-U.S. Gov't]. Journal of virology, 85(20), 10814-10825. doi: 10.1128/JVI.00104-11

Dannull, J., Su, Z., Rizzieri, D., Yang, B. K., Coleman, D., Yancey, D., . . . Vieweg, J. (2005). Enhancement of vaccine-mediated antitumor immunity in cancer patients after depletion of regulatory $\mathrm{T}$ cells. [Clinical Trial Research Support, N.I.H., Extramural]. The Journal of clinical investigation, 115(12), 3623-3633. doi: 10.1172/JCI25947

Dar, W. A., \& Knechtle, S. J. (2007). CXCR3-mediated T-cell chemotaxis involves ZAP70 and is regulated by signalling through the T-cell receptor. [Research Support, N.I.H., Extramural]. Immunology, 120(4), 467-485. doi: 10.1111/j.13652567.2006.02534.x

Dasgupta, J. D., Watkins, S., Slayter, H., \& Yunis, E. J. (1988). Receptor-like nature of class I HLA: endocytosis via coated pits. [Research Support, U.S. Gov't, P.H.S.]. Journal of immunology, 141(8), 2577-2580.

Davila, S., Hibberd, M. L., Hari Dass, R., Wong, H. E., Sahiratmadja, E., Bonnard, C., . . . Seielstad, M. (2008). Genetic association and expression studies indicate a role of toll-like receptor 8 in pulmonary tuberculosis. [Research Support, Non-U.S. Gov't]. PLoS genetics, 4(10), e1000218. doi: 10.1371/journal.pgen.1000218

De Becker, G., Moulin, V., Pajak, B., Bruck, C., Francotte, M., Thiriart, C., . . Moser, M. (2000). The adjuvant monophosphoryl lipid A increases the function of antigenpresenting cells. [Research Support, Non-U.S. Gov't]. International immunology, 12(6), 807-815.

de Jong, J. M., Schuurhuis, D. H., Ioan-Facsinay, A., Welling, M. M., Camps, M. G., van der Voort, E. I., . . . Toes, R. E. (2006). Dendritic cells, but not macrophages or B cells, activate major histocompatibility complex class II-restricted CD4+ T cells upon immune-complex uptake in vivo. [Research Support, Non-U.S. Gov't]. Immunology, 119(4), 499-506. doi: 10.1111/j.1365-2567.2006.02464.x

De Smedt, T., Pajak, B., Muraille, E., Lespagnard, L., Heinen, E., De Baetselier, P., . . . Moser, M. (1996). Regulation of dendritic cell numbers and maturation by lipopolysaccharide in vivo. [Research Support, Non-U.S. Gov't]. The Journal of experimental medicine, 184(4), 1413-1424.

de Vries, I. J., Lesterhuis, W. J., Scharenborg, N. M., Engelen, L. P., Ruiter, D. J., Gerritsen, M. J., . . . Punt, C. J. (2003). Maturation of dendritic cells is a prerequisite for inducing immune responses in advanced melanoma patients. [Research Support, Non-U.S. Gov't]. Clinical cancer research : an official journal of the American Association for Cancer Research, 9(14), 5091-5100. 
del Rio, M. L., Rodriguez-Barbosa, J. I., Bolter, J., Ballmaier, M., Dittrich-Breiholz, O., Kracht, M., . . Forster, R. (2008). CX3CR1+ c-kit+ bone marrow cells give rise to $\mathrm{CD} 103+$ and CD103- dendritic cells with distinct functional properties. [Comparative Study Research Support, Non-U.S. Gov't]. Journal of immunology, 181(9), 6178-6188.

del Rio, M. L., Rodriguez-Barbosa, J. I., Kremmer, E., \& Forster, R. (2007). CD103- and CD103+ bronchial lymph node dendritic cells are specialized in presenting and cross-presenting innocuous antigen to CD4+ and CD8+ T cells. [Research Support, Non-U.S. Gov't]. Journal of immunology, 178(11), 6861-6866.

Delale, T., Paquin, A., Asselin-Paturel, C., Dalod, M., Brizard, G., Bates, E. E., . . Briere, F. (2005). MyD88-dependent and -independent murine cytomegalovirus sensing for IFN-alpha release and initiation of immune responses in vivo. [Research Support, Non-U.S. Gov't]. Journal of immunology, 175(10), 6723-6732.

Delbridge, L. M., \& O'Riordan, M. X. (2007). Innate recognition of intracellular bacteria. [Research Support, N.I.H., Extramural Research Support, Non-U.S. Gov't Review]. Current opinion in immunology, 19(1), 10-16. doi: 10.1016/j.coi.2006.11.005

Delmer, A., Heron, E., Marie, J. P., \& Zittoun, R. (1994). Spontaneous remission in acute myeloid leukaemia. [Case Reports Comment Letter]. British journal of haematology, 87(4), 880-882.

den Haan, J. M., \& Bevan, M. J. (2002). Constitutive versus activation-dependent crosspresentation of immune complexes by CD8(+) and CD8(-) dendritic cells in vivo. [Comparative Study Research Support, Non-U.S. Gov't Research Support, U.S. Gov't, P.H.S.]. The Journal of experimental medicine, 196(6), 817-827.

den Haan, J. M., Lehar, S. M., \& Bevan, M. J. (2000). CD8(+) but not CD8(-) dendritic cells cross-prime cytotoxic $\mathrm{T}$ cells in vivo. The Journal of experimental medicine, 192(12), 1685-1696.

Deng, H., Fosdick, L., \& Sercarz, E. (1993). The involvement of antigen processing in determinant selection by class II MHC and its relationship to immunodominance. [Review]. APMIS : acta pathologica, microbiologica, et immunologica Scandinavica, 101(9), 655-662.

Dhodapkar, M. V., Krasovsky, J., Steinman, R. M., \& Bhardwaj, N. (2000). Mature dendritic cells boost functionally superior CD8(+) T-cell in humans without foreign helper epitopes. [Research Support, Non-U.S. Gov't Research Support, U.S. Gov't, P.H.S.]. The Journal of clinical investigation, 105(6), R9-R14. doi: 10.1172/JCI9051

Dhodapkar, M. V., \& Steinman, R. M. (2002). Antigen-bearing immature dendritic cells induce peptide-specific $\mathrm{CD} 8(+)$ regulatory $\mathrm{T}$ cells in vivo in humans. [Research 
Support, Non-U.S. Gov't Research Support, U.S. Gov't, P.H.S.]. Blood, 100(1), 174-177.

Dhodapkar, M. V., Steinman, R. M., Krasovsky, J., Munz, C., \& Bhardwaj, N. (2001). Antigen-specific inhibition of effector $\mathrm{T}$ cell function in humans after injection of immature dendritic cells. [Clinical Trial In Vitro Randomized Controlled Trial Research Support, Non-U.S. Gov't Research Support, U.S. Gov't, P.H.S.]. The Journal of experimental medicine, 193(2), 233-238.

Dhodapkar, M. V., Steinman, R. M., Sapp, M., Desai, H., Fossella, C., Krasovsky, J., . . . Bhardwaj, N. (1999). Rapid generation of broad T-cell immunity in humans after a single injection of mature dendritic cells. [Research Support, Non-U.S. Gov't Research Support, U.S. Gov't, P.H.S.]. The Journal of clinical investigation, 104(2), 173-180. doi: 10.1172/JCI6909

Didot, A. (1852). Prophylaxie du cancer par la syphilization. Presse Med(4), 117-119, $143-$ 145.

Diebold, S. S., Kaisho, T., Hemmi, H., Akira, S., \& Reis e Sousa, C. (2004). Innate antiviral responses by means of TLR7-mediated recognition of single-stranded RNA. [Research Support, Non-U.S. Gov't]. Science, 303(5663), 1529-1531. doi: 10.1126/science. 1093616

Dieu-Nosjean, M. C., Vicari, A., Lebecque, S., \& Caux, C. (1999). Regulation of dendritic cell trafficking: a process that involves the participation of selective chemokines. [Research Support, Non-U.S. Gov't Review]. Journal of leukocyte biology, 66(2), 252-262.

Divito, S. J., Wang, Z., Shufesky, W. J., Liu, Q., Tkacheva, O. A., Montecalvo, A., . . . Morelli, A. E. (2010). Endogenous dendritic cells mediate the effects of intravenously injected therapeutic immunosuppressive dendritic cells in transplantation. [Research Support, N.I.H., Extramural]. Blood, 116(15), 26942705. doi: 10.1182/blood-2009-10-251058

Dolan, B. P., Gibbs, K. D., Jr., \& Ostrand-Rosenberg, S. (2006). Dendritic cells crossdressed with peptide MHC class I complexes prime CD8+ T cells. [Research Support, N.I.H., Extramural Research Support, U.S. Gov't, Non-P.H.S.]. Journal of immunology, 177(9), 6018-6024.

Donermeyer, D. L., \& Allen, P. M. (1989). Binding to Ia protects an immunogenic peptide from proteolytic degradation. [Research Support, U.S. Gov't, P.H.S.]. Journal of immunology, 142(4), 1063-1068.

Drummond, R. A., Saijo, S., Iwakura, Y., \& Brown, G. D. (2011). The role of Syk/CARD9 coupled C-type lectins in antifungal immunity. [Research Support, Non-U.S. Gov't Review]. Eur J Immunol, 41(2), 276-281. doi: 10.1002/eji.201041252 
Dudek, A. Z., Yunis, C., Harrison, L. I., Kumar, S., Hawkinson, R., Cooley, S., . . . Miller, J. S. (2007). First in human phase I trial of 852A, a novel systemic toll-like receptor 7 agonist, to activate innate immune responses in patients with advanced cancer. [Clinical Trial, Phase I Research Support, Non-U.S. Gov't]. Clinical cancer research : an official journal of the American Association for Cancer Research, 13(23), 7119-7125. doi: 10.1158/1078-0432.CCR-07-1443

Dudziak, D., Kamphorst, A. O., Heidkamp, G. F., Buchholz, V. R., Trumpfheller, C., Yamazaki, S., . . . Nussenzweig, M. C. (2007). Differential antigen processing by dendritic cell subsets in vivo. [Research Support, N.I.H., Extramural Research Support, Non-U.S. Gov't]. Science, 315(5808), 107-111. doi: $10.1126 /$ science. 1136080

Dummer, R., Hauschild, A., Becker, J. C., Grob, J. J., Schadendorf, D., Tebbs, V., . . . Urosevic, M. (2008). An exploratory study of systemic administration of the tolllike receptor-7 agonist $852 \mathrm{~A}$ in patients with refractory metastatic melanoma. [Clinical Trial, Phase II Multicenter Study Research Support, Non-U.S. Gov't]. Clinical cancer research : an official journal of the American Association for Cancer Research, 14(3), 856-864. doi: 10.1158/1078-0432.CCR-07-1938

Dussaussoy. (1787). Dissertations et observations sur la gangrene dans les hopitaux. Lyon.

Dzionek, A., Fuchs, A., Schmidt, P., Cremer, S., Zysk, M., Miltenyi, S., . . . Schmitz, J. (2000). BDCA-2, BDCA-3, and BDCA-4: three markers for distinct subsets of dendritic cells in human peripheral blood. [Comparative Study]. Journal of immunology, 165(11), 6037-6046.

Ebbell, B. (1937). The Papyrus ebers: the greatest Egyptian medical document. Oxford: Oxford University Press.

Eberl, G., Brawand, P., \& MacDonald, H. R. (2000). Selective bystander proliferation of memory CD4+ and CD8+ $\mathrm{T}$ cells upon NK $\mathrm{T}$ or $\mathrm{T}$ cell activation. Journal of immunology, 165(8), 4305-4311.

Eberl, G., \& MacDonald, H. R. (2000). Selective induction of NK cell proliferation and cytotoxicity by activated NKT cells. European journal of immunology, 30(4), 985992. $\quad$ doi: $10.1002 /($ SICI)1521-4141(200004)30:4\&\#60;985::AIDIMMU985\&\#62;3.0.CO;2-E

Eggert, A. A., Schreurs, M. W., Boerman, O. C., Oyen, W. J., de Boer, A. J., Punt, C. J., . . . Adema, G. J. (1999). Biodistribution and vaccine efficiency of murine dendritic cells are dependent on the route of administration. [Comparative Study Research Support, Non-U.S. Gov't]. Cancer research, 59(14), 3340-3345.

Enderlin, M., Kleinmann, E. V., Struyf, S., Buracchi, C., Vecchi, A., Kinscherf, R., . . Dinsart, C. (2009). TNF-alpha and the IFN-gamma-inducible protein 10 (IP- 
10/CXCL-10) delivered by parvoviral vectors act in synergy to induce antitumor effects in mouse glioblastoma. [Research Support, Non-U.S. Gov't]. Cancer gene therapy, 16(2), 149-160. doi: 10.1038/cgt.2008.62

Engelhardt, R., Mackensen, A., \& Galanos, C. (1991). Phase I trial of intravenously administered endotoxin (Salmonella abortus equi) in cancer patients. [Clinical Trial Research Support, Non-U.S. Gov't]. Cancer research, 51(10), 2524-2530.

Eriksson, U., Ricci, R., Hunziker, L., Kurrer, M. O., Oudit, G. Y., Watts, T. H., . . . Penninger, J. M. (2003). Dendritic cell-induced autoimmune heart failure requires cooperation between adaptive and innate immunity. [Research Support, Non-U.S. Gov't]. Nature medicine, 9(12), 1484-1490. doi: 10.1038/nm960

Escudier, B., Dorval, T., Chaput, N., Andre, F., Caby, M. P., Novault, S., . . Z Zitvogel, L. (2005). Vaccination of metastatic melanoma patients with autologous dendritic cell (DC) derived-exosomes: results of thefirst phase I clinical trial. Journal of translational medicine, 3(1), 10. doi: 10.1186/1479-5876-3-10

Ewald, S. E., Lee, B. L., Lau, L., Wickliffe, K. E., Shi, G. P., Chapman, H. A., \& Barton, G. M. (2008). The ectodomain of Toll-like receptor 9 is cleaved to generate a functional receptor. [Research Support, N.I.H., Extramural Research Support, NonU.S. Gov't]. Nature, 456(7222), 658-662. doi: 10.1038/nature07405

Exley, M. A., \& Nakayama, T. (2011). NKT-cell-based immunotherapies in clinical trials. [Editorial Introductory]. Clinical immunology, 140(2), 117-118. doi: 10.1016/j.clim.2011.04.015

Farrand, K. J., Dickgreber, N., Stoitzner, P., Ronchese, F., Petersen, T. R., \& Hermans, I. F. (2009). Langerin+ CD8alpha+ dendritic cells are critical for cross-priming and IL-12 production in response to systemic antigens. [Research Support, Non-U.S. Gov't]. Journal of immunology, 183(12), 7732-7742. doi: 10.4049/jimmunol.0902707

Fassas, A., Sakellari, I., Anagnostopoulos, A., \& Saloum, R. (1991). Spontaneous remission of acute myeloid leukemia in a patient with concurrent Pneumocystis carinii pneumonia. [Case Reports]. Nouvelle revue francaise d'hematologie, 33(5), 363-364.

Fehleisen, F. (1886). Recent essays on bacteria in relation to disease. London: New Sydenham Society.

Feng, Y., Wang, D., Yuan, R., Parker, C. M., Farber, D. L., \& Hadley, G. A. (2002). CD103 expression is required for destruction of pancreatic islet allografts by CD8(+) T cells. [Research Support, U.S. Gov't, P.H.S.]. The Journal of experimental medicine, 196(7), 877-886. 
Fenner, F., Henderson, D.A., Arita, I., Jezek, Z., Ladnyi, I.D. (1998). Smallpox and its Eradication (Vol. 6). Geneva: World Health Organization.

Finn, O. J. (2008). Cancer immunology. [Review]. The New England journal of medicine, 358(25), 2704-2715. doi: 10.1056/NEJMra072739

Fitzgerald, K. A., Palsson-McDermott, E. M., Bowie, A. G., Jefferies, C. A., Mansell, A. S., Brady, G., . . O'Neill, L. A. (2001). Mal (MyD88-adapter-like) is required for Toll-like receptor-4 signal transduction. [Research Support, Non-U.S. Gov't]. Nature, 413(6851), 78-83. doi: 10.1038/35092578

Fitzpatrick, F. A., \& Wheeler, R. (2003). The immunopharmacology of paclitaxel (Taxol), docetaxel (Taxotere), and related agents. [Research Support, Non-U.S. Gov't Research Support, U.S. Gov't, P.H.S. Review]. International immunopharmacology, 3(13-14), 1699-1714.

Fong, L., Brockstedt, D., Benike, C., Wu, L., \& Engleman, E. G. (2001). Dendritic cells injected via different routes induce immunity in cancer patients. [Clinical Trial Comparative Study Research Support, Non-U.S. Gov't Research Support, U.S. Gov't, P.H.S.]. Journal of immunology, 166(6), 4254-4259.

Forrester, J., Xu, H., Kuffova, L., Dick, A.D. \& McMenamin, P.G. (2010). Dendritic cell physiology and function in the eye. Immunol Rev, 234, 282-304.

Forsbach, A., Nemorin, J. G., Montino, C., Muller, C., Samulowitz, U., Vicari, A. P., . . Vollmer, J. (2008). Identification of RNA sequence motifs stimulating sequencespecific TLR8-dependent immune responses. [Comparative Study Research Support, N.I.H., Extramural]. Journal of immunology, 180(6), 3729-3738.

Frick, S., \& Frick, P. (1993). [Spontaneous remission in chronic lymphatic leukemia]. [Case Reports Review]. Schweizerische medizinische Wochenschrift, 123(8), 328334.

Fritz, J. H., Ferrero, R. L., Philpott, D. J., \& Girardin, S. E. (2006). Nod-like proteins in immunity, inflammation and disease. [Research Support, Non-U.S. Gov't Review]. Nature immunology, 7(12), 1250-1257. doi: 10.1038/ni1412

Fritz, J. H., \& Girardin, S. E. (2005). How Toll-like receptors and Nod-like receptors contribute to innate immunity in mammals. [Review]. Journal of endotoxin research, 11(6), 390-394. doi: 10.1179/096805105X76850

Fujii, S., Liu, K., Smith, C., Bonito, A. J., \& Steinman, R. M. (2004). The linkage of innate to adaptive immunity via maturing dendritic cells in vivo requires CD40 ligation in addition to antigen presentation and CD80/86 costimulation. [Research Support, Non-U.S. Gov't Research Support, U.S. Gov't, P.H.S.]. The Journal of experimental medicine, 199(12), 1607-1618. doi: 10.1084/jem.20040317 
Fujii, S., Shimizu, K., Hemmi, H., \& Steinman, R. M. (2007). Innate Valpha14(+) natural killer $\mathrm{T}$ cells mature dendritic cells, leading to strong adaptive immunity. [Research Support, N.I.H., Extramural Research Support, Non-U.S. Gov't Review]. Immunological reviews, 220, 183-198. doi: 10.1111/j.1600-065X.2007.00561.X

Fujii, S., Shimizu, K., Kronenberg, M., \& Steinman, R. M. (2002). Prolonged IFN-gammaproducing NKT response induced with alpha-galactosylceramide-loaded DCs. Nature immunology, 3(9), 867-874. doi: 10.1038/ni827

Fujii, S., Shimizu, K., Smith, C., Bonifaz, L., \& Steinman, R. M. (2003). Activation of natural killer $\mathrm{T}$ cells by alpha-galactosylceramide rapidly induces the full maturation of dendritic cells in vivo and thereby acts as an adjuvant for combined CD4 and CD8 T cell immunity to a coadministered protein. [Research Support, Non-U.S. Gov't Research Support, U.S. Gov't, P.H.S.]. The Journal of experimental medicine, 198(2), 267-279. doi: 10.1084/jem.20030324

Fuji, N., Ueda, Y., Fujiwara, H., Toh, T., Yoshimura, T., \& Yamagishi, H. (2000). Antitumor effect of alpha-galactosylceramide (KRN7000) on spontaneous hepatic metastases requires endogenous interleukin 12 in the liver. Clin Cancer Res, 6(8), 3380-3387.

Funami, K., Matsumoto, M., Oshiumi, H., Akazawa, T., Yamamoto, A., \& Seya, T. (2004). The cytoplasmic 'linker region' in Toll-like receptor 3 controls receptor localization and signaling. [Research Support, Non-U.S. Gov't]. International immunology, 16(8), 1143-1154. doi: 10.1093/intimm/dxh115

Galibert, L., Diemer, G. S., Liu, Z., Johnson, R. S., Smith, J. L., Walzer, T., . . . Yan, W. (2005). Nectin-like protein 2 defines a subset of T-cell zone dendritic cells and is a ligand for class-I-restricted T-cell-associated molecule. The Journal of biological chemistry, 280(23), 21955-21964. doi: 10.1074/jbc.M502095200

Gallucci, S., Lolkema, M., \& Matzinger, P. (1999). Natural adjuvants: endogenous activators of dendritic cells. [Research Support, Non-U.S. Gov't]. Nature medicine, 5(11), 1249-1255. doi: 10.1038/15200

Gantier, M. P., Irving, A. T., Kaparakis-Liaskos, M., Xu, D., Evans, V. A., Cameron, P. U., . . Williams, B. R. (2010). Genetic modulation of TLR8 response following bacterial phagocytosis. [Research Support, Non-U.S. Gov't]. Human mutation, 31(9), 1069-1079. doi: 10.1002/humu.21321

Garay, R. P., Viens, P., Bauer, J., Normier, G., Bardou, M., Jeannin, J. F., \& Chiavaroli, C. (2007). Cancer relapse under chemotherapy: why TLR2/4 receptor agonists can help. [Review]. European journal of pharmacology, 563(1-3), 1-17. doi: 10.1016/j.ejphar.2007.02.018 
Garcia-Rayo, S., Gurpide, A., Vega, F., \& Brugarolas, A. (1996). [Spontaneous tumor regression in a patient with multiple myeloma. Report of another case]. [Case Reports]. Revista de medicina de la Universidad de Navarra, 40(3), 41-42.

Gautier, G., Humbert, M., Deauvieau, F., Scuiller, M., Hiscott, J., Bates, E. E., . . . Garrone, P. (2005). A type I interferon autocrine-paracrine loop is involved in Tolllike receptor-induced interleukin-12p70 secretion by dendritic cells. [Comparative Study Research Support, Non-U.S. Gov't]. The Journal of experimental medicine, 201(9), 1435-1446. doi: 10.1084/jem.20041964

Giaccone, G., Punt, C. J., Ando, Y., Ruijter, R., Nishi, N., Peters, M., . . Pinedo, H. M. (2002). A phase I study of the natural killer T-cell ligand alpha-galactosylceramide (KRN7000) in patients with solid tumors. [Clinical Trial Clinical Trial, Phase I]. Clinical cancer research : an official journal of the American Association for Cancer Research, 8(12), 3702-3709.

Ginhoux, F. C., M.P., Bogunovic, M., Abel, M., Leboeuf, M., Helft, J., Ochando, J., Kissenpfennig, A., Malissen, B., Grisotto, M., Snoeck, H., Randolph, G. \& Merad, M. (2007). Blood-derived dermal langerin+ dendritic cells survey the skin in the steady state. $J$ Exp Med(204), 3133-3146.

Girardin, S. E., Travassos, L. H., Herve, M., Blanot, D., Boneca, I. G., Philpott, D. J., . . . Mengin-Lecreulx, D. (2003). Peptidoglycan molecular requirements allowing detection by Nod1 and Nod2. [Research Support, Non-U.S. Gov't]. The Journal of biological chemistry, 278(43), 41702-41708. doi: 10.1074/jbc.M307198200

Godfrey, D. I., MacDonald, H. R., Kronenberg, M., Smyth, M. J., \& Van Kaer, L. (2004). NKT cells: what's in a name? [Research Support, Non-U.S. Gov't Research Support, U.S. Gov't, P.H.S.]. Nat Rev Immunol, 4(3), 231-237. doi: $10.1038 /$ nri1309

Gondokaryono, S. P., Ushio, H., Niyonsaba, F., Hara, M., Takenaka, H., Jayawardana, S. T., . . . Ogawa, H. (2007). The extra domain A of fibronectin stimulates murine mast cells via toll-like receptor 4. [Research Support, Non-U.S. Gov't]. Journal of leukocyte biology, 82(3), 657-665. doi: 10.1189/jlb.1206730

Gonzalez-Aseguinolaza, G., Van Kaer, L., Bergmann, C. C., Wilson, J. M., Schmieg, J., Kronenberg, M., . . . Tsuji, M. (2002). Natural killer T cell ligand alphagalactosylceramide enhances protective immunity induced by malaria vaccines. [Research Support, U.S. Gov't, P.H.S.]. The Journal of experimental medicine, 195(5), 617-624.

Granucci, F., \& Ricciardi-Castagnoli, P. (2003). Interactions of bacterial pathogens with dendritic cells during invasion of mucosal surfaces. [Research Support, Non-U.S. Gov't Review]. Current opinion in microbiology, 6(1), 72-76. 
Grouard, G., Rissoan, M. C., Filgueira, L., Durand, I., Banchereau, J., \& Liu, Y. J. (1997). The enigmatic plasmacytoid $\mathrm{T}$ cells develop into dendritic cells with interleukin (IL)-3 and CD40-ligand. [Research Support, Non-U.S. Gov't]. The Journal of experimental medicine, 185(6), 1101-1111.

Guermonprez, P., Saveanu, L., Kleijmeer, M., Davoust, J., Van Endert, P., \& Amigorena, S. (2003). ER-phagosome fusion defines an MHC class I cross-presentation compartment in dendritic cells. [Research Support, Non-U.S. Gov't]. Nature, 425(6956), 397-402. doi: 10.1038/nature01911

Gumperz, J. E., Miyake, S., Yamamura, T., \& Brenner, M. B. (2002). Functionally distinct subsets of CD1d-restricted natural killer $\mathrm{T}$ cells revealed by CD1d tetramer staining. [Research Support, Non-U.S. Gov't Research Support, U.S. Gov't, P.H.S.]. The Journal of experimental medicine, 195(5), 625-636.

Guzylack-Piriou, L., Balmelli, C., McCullough, K. C., \& Summerfield, A. (2004). Type-A $\mathrm{CpG}$ oligonucleotides activate exclusively porcine natural interferon-producing cells to secrete interferon-alpha, tumour necrosis factor-alpha and interleukin-12. [Research Support, Non-U.S. Gov't]. Immunology, 112(1), 28-37. doi: $10.1111 / \mathrm{j} .1365-2567.2004 .01856 . x$

Hacker, H., Vabulas, R. M., Takeuchi, O., Hoshino, K., Akira, S., \& Wagner, H. (2000). Immune cell activation by bacterial CpG-DNA through myeloid differentiation marker 88 and tumor necrosis factor receptor-associated factor (TRAF)6. [Research Support, Non-U.S. Gov't]. The Journal of experimental medicine, 192(4), 595-600.

Hadeiba, H., Sato, T., Habtezion, A., Oderup, C., Pan, J., \& Butcher, E. C. (2008). CCR9 expression defines tolerogenic plasmacytoid dendritic cells able to suppress acute graft-versus-host disease. [Research Support, N.I.H., Extramural Research Support, Non-U.S. Gov't Research Support, U.S. Gov't, Non-P.H.S.]. Nature immunology, 9(11), 1253-1260. doi: 10.1038/ni.1658

Harding, C. V., \& Song, R. (1994). Phagocytic processing of exogenous particulate antigens by macrophages for presentation by class I MHC molecules. [Research Support, Non-U.S. Gov't Research Support, U.S. Gov't, P.H.S.]. Journal of immunology, 153(11), 4925-4933.

Haring, J. S., Badovinac, V. P., \& Harty, J. T. (2006). Inflaming the CD8+ T cell response. [Research Support, N.I.H., Extramural Research Support, Non-U.S. Gov't Review]. Immunity, 25(1), 19-29. doi: 10.1016/j.immuni.2006.07.001

Harshyne, L. A., Watkins, S. C., Gambotto, A., \& Barratt-Boyes, S. M. (2001). Dendritic cells acquire antigens from live cells for cross-presentation to CTL. [Research Support, U.S. Gov't, P.H.S.]. Journal of immunology, 166(6), 3717-3723.

Hart, A. L., Al-Hassi, H. O., Rigby, R. J., Bell, S. J., Emmanuel, A. V., Knight, S. C., . . Stagg, A. J. (2005). Characteristics of intestinal dendritic cells in inflammatory 
bowel diseases. [Research Support, Non-U.S. Gov't]. Gastroenterology, 129(1), 5065 .

Hart, D. N. (1997). Dendritic cells: unique leukocyte populations which control the primary immune response. [Review]. Blood, 90(9), 3245-3287.

Harty, J. T., Tvinnereim, A. R., \& White, D. W. (2000). CD8+ T cell effector mechanisms in resistance to infection. [Research Support, Non-U.S. Gov't Research Support, U.S. Gov't, P.H.S. Review]. Annual review of immunology, 18, 275-308. doi: 10.1146/annurev.immunol.18.1.275

Hatsuzawa, K., Tamura, T., Hashimoto, H., Yokoya, S., Miura, M., Nagaya, H., \& Wada, I. (2006). Involvement of syntaxin 18, an endoplasmic reticulum (ER)-localized SNARE protein, in ER-mediated phagocytosis. [Research Support, Non-U.S. Gov't]. Molecular biology of the cell, 17(9), 3964-3977. doi: 10.1091/mbc.E05-121174

Hawn, T. R., Verbon, A., Lettinga, K. D., Zhao, L. P., Li, S. S., Laws, R. J., . . Aderem, A. (2003). A common dominant TLR5 stop codon polymorphism abolishes flagellin signaling and is associated with susceptibility to legionnaires' disease. [Research Support, Non-U.S. Gov't Research Support, U.S. Gov't, P.H.S.]. The Journal of experimental medicine, 198(10), 1563-1572. doi: 10.1084/jem.20031220

Hayakawa, Y., Rovero, S., Forni, G., \& Smyth, M. J. (2003). Alpha-galactosylceramide (KRN7000) suppression of chemical- and oncogene-dependent carcinogenesis. [Research Support, Non-U.S. Gov't]. Proc Natl Acad Sci U S A, 100(16), 94649469. doi: 10.1073/pnas.1630663100

Hayakawa, Y., Takeda, K., Yagita, H., Kakuta, S., Iwakura, Y., Van Kaer, L., . . . Okumura, K. (2001a). Critical contribution of IFN-gamma and NK cells, but not perforin-mediated cytotoxicity, to anti-metastatic effect of alphagalactosylceramide. [Research Support, Non-U.S. Gov't]. Eur J Immunol, 31(6), $1720-1727$.

Hayakawa, Y., Takeda, K., Yagita, H., Smyth, M. J., Van Kaer, L., Okumura, K., \& Saiki, I. (2002). IFN-gamma-mediated inhibition of tumor angiogenesis by natural killer T-cell ligand, alpha-galactosylceramide. [Research Support, Non-U.S. Gov't]. Blood, 100(5), 1728-1733.

Hayakawa, Y., Takeda, K., Yagita, H., Van Kaer, L., Saiki, I., \& Okumura, K. (2001b). Differential regulation of Th1 and Th2 functions of NKT cells by CD28 and CD40 costimulatory pathways. [Comparative Study Research Support, Non-U.S. Gov't]. Journal of immunology, 166(10), 6012-6018.

Hayashi, F., Smith, K. D., Ozinsky, A., Hawn, T. R., Yi, E. C., Goodlett, D. R., . . . Aderem, A. (2001). The innate immune response to bacterial flagellin is mediated 
by Toll-like receptor 5. [Research Support, U.S. Gov't, P.H.S.]. Nature, 410(6832), 1099-1103. doi: 10.1038/35074106

Heemels, M. T., \& Ploegh, H. (1995). Generation, translocation, and presentation of MHC class I-restricted peptides. [Research Support, U.S. Gov't, P.H.S. Review]. Annual review of biochemistry, 64, 463-491. doi: 10.1146/annurev.bi.64.070195.002335

Heil, F., Hemmi, H., Hochrein, H., Ampenberger, F., Kirschning, C., Akira, S., . . Bauer, S. (2004). Species-specific recognition of single-stranded RNA via toll-like receptor 7 and 8. [Research Support, Non-U.S. Gov't]. Science, 303(5663), 15261529. doi: $10.1126 /$ science. 1093620

Heiser, A., Coleman, D., Dannull, J., Yancey, D., Maurice, M. A., Lallas, C. D., . . . Vieweg, J. (2002). Autologous dendritic cells transfected with prostate-specific antigen RNA stimulate CTL responses against metastatic prostate tumors. [Research Support, Non-U.S. Gov't Research Support, U.S. Gov't, P.H.S.]. The Journal of clinical investigation, 109(3), 409-417. doi: 10.1172/JCI14364

Helft, J., Ginhoux, F., Bogunovic, M. \& Merad, M. (2010). Origin and functional heterogeneity of non-lymphoid tissue dendritic cells in mice. Immunol Rev, 234, $55-75$.

Hemmi, H., Kaisho, T., Takeuchi, O., Sato, S., Sanjo, H., Hoshino, K., . . . Akira, S. (2002). Small anti-viral compounds activate immune cells via the TLR7 MyD88dependent signaling pathway. [Research Support, Non-U.S. Gov't]. Nature immunology, 3(2), 196-200. doi: 10.1038/ni758

Henri, S., D. Vremec, A. Kamath, J. Waithman, S. Williams, C. Benoist, K. Burnham, S. Saeland, E. Handman, and K. Shortman. (2001). The dendritic cell populations of mouse lymph nodes. . J Immunol 167, 741-748.

Hermans, I. F., Silk, J. D., Gileadi, U., Masri, S. H., Shepherd, D., Farrand, K. J., . . . Cerundolo, V. (2007). Dendritic cell function can be modulated through cooperative actions of TLR ligands and invariant NKT cells. [Research Support, Non-U.S. Gov't]. Journal of immunology, 178(5), 2721-2729.

Hermans, I. F., Silk, J. D., Gileadi, U., Salio, M., Mathew, B., Ritter, G., . . Cerundolo, V. (2003). NKT cells enhance CD4+ and CD8+ T cell responses to soluble antigen in vivo through direct interaction with dendritic cells. [Research Support, Non-U.S. Gov't]. Journal of immunology, 171(10), 5140-5147.

Hernandez, J., Aung, S., Redmond, W.L. \& Sherman, LA. (2001). Phenotypic and functional analysis of $\mathrm{CD} 8(+) \mathrm{T}$ cells undergoing peripheral deletion in response to coree-presentation of self-antigen. $J$ Exp Med, 194, 707-717.

Hertz, C. J., Kiertscher, S. M., Godowski, P. J., Bouis, D. A., Norgard, M. V., Roth, M. D., \& Modlin, R. L. (2001). Microbial lipopeptides stimulate dendritic cell maturation 
via Toll-like receptor 2. [Research Support, Non-U.S. Gov't Research Support, U.S. Gov't, P.H.S.]. Journal of immunology, 166(4), 2444-2450.

Hildenbrand, B., Sauer, B., Kalis, O., Stoll, C., Freudenberg, M. A., Niedermann, G., . . Azemar, M. (2007). Immunotherapy of patients with hormone-refractory prostate carcinoma pre-treated with interferon-gamma and vaccinated with autologous PSApeptide loaded dendritic cells--a pilot study. [Clinical Trial Research Support, NonU.S. Gov't]. The Prostate, 67(5), 500-508. doi: 10.1002/pros.20539

Hintzen, G., Ohl, L., del Rio, M. L., Rodriguez-Barbosa, J. I., Pabst, O., Kocks, J. R., . . . Forster, R. (2006). Induction of tolerance to innocuous inhaled antigen relies on a CCR7-dependent dendritic cell-mediated antigen transport to the bronchial lymph node. [Research Support, Non-U.S. Gov't]. Journal of immunology, 177(10), 73467354.

Hironaka, K., Yamaguchi, Y., Okita, R., Okawaki, M., \& Nagamine, I. (2006). Essential requirement of toll-like receptor 4 expression on $\mathrm{CD} 11 \mathrm{c}+$ cells for locoregional immunotherapy of malignant ascites using a streptococcal preparation OK-432. Anticancer research, 26(5B), 3701-3707.

Hochrein, H., Schlatter, B., O'Keeffe, M., Wagner, C., Schmitz, F., Schiemann, M., . . . Wagner, H. (2004). Herpes simplex virus type-1 induces IFN-alpha production via Toll-like receptor 9-dependent and -independent pathways. [In Vitro Research Support, Non-U.S. Gov't]. Proceedings of the National Academy of Sciences of the United States of America, 101(31), 11416-11421. doi: 10.1073/pnas.0403555101

Hoebe, K., Janssen, E. M., Kim, S. O., Alexopoulou, L., Flavell, R. A., Han, J., \& Beutler, B. (2003). Upregulation of costimulatory molecules induced by lipopolysaccharide and double-stranded RNA occurs by Trif-dependent and Trif-independent pathways. [Comparative Study Research Support, U.S. Gov't, Non-P.H.S. Research Support, U.S. Gov't, P.H.S.]. Nature immunology, 4(12), 1223-1229. doi: $10.1038 /$ ni1010

Hogquist, K. A., Jameson, S. C., Heath, W. R., Howard, J. L., Bevan, M. J., \& Carbone, F. R. (1994). T cell receptor antagonist peptides induce positive selection. [Research Support, Non-U.S. Gov't Research Support, U.S. Gov't, P.H.S.]. Cell, 76(1), 17-27.

Holtl, L., Ramoner, R., Zelle-Rieser, C., Gander, H., Putz, T., Papesh, C., . . Thurnher, M. (2005). Allogeneic dendritic cell vaccination against metastatic renal cell carcinoma with or without cyclophosphamide. [Clinical Trial Clinical Trial, Phase I Clinical Trial, Phase II Research Support, Non-U.S. Gov't]. Cancer immunology, immunotherapy : CII, 54(7), 663-670. doi: 10.1007/s00262-004-0629-2

Holtl, L., Rieser, C., Papesh, C., Ramoner, R., Herold, M., Klocker, H., . . Thurnher, M. (1999). Cellular and humoral immune responses in patients with metastatic renal cell carcinoma after vaccination with antigen pulsed dendritic cells. [Clinical Trial Research Support, Non-U.S. Gov't]. The Journal of urology, 161(3), 777-782. 
Holt1, L., Zelle-Rieser, C., Gander, H., Papesh, C., Ramoner, R., Bartsch, G., . . Thurnher, M. (2002). Immunotherapy of metastatic renal cell carcinoma with tumor lysatepulsed autologous dendritic cells. [Research Support, Non-U.S. Gov't]. Clinical cancer research : an official journal of the American Association for Cancer Research, 8(11), 3369-3376.

Honda, K., Yanai, H., Negishi, H., Asagiri, M., Sato, M., Mizutani, T., . . Taniguchi, T. (2005). IRF-7 is the master regulator of type-I interferon-dependent immune responses. [Research Support, Non-U.S. Gov't]. Nature, 434(7034), 772-777. doi: 10.1038 /nature 03464

Hong, C., Lee, H., Oh, M., Kang, C. Y., Hong, S., \& Park, S. H. (2006). CD4+ T cells in the absence of the CD8+ cytotoxic T cells are critical and sufficient for NKT celldependent tumor rejection. [Research Support, Non-U.S. Gov't]. Journal of immunology, 177(10), 6747-6757.

Hopkins, D. R. (1983). Princes and peasants : smallpox in history. Chicago: University of Chicago Press.

Hoption Cann, S. A., van Netten, J. P., \& van Netten, C. (2003). Dr William Coley and tumour regression: a place in history or in the future. [Biography Historical Article Research Support, Non-U.S. Gov't]. Postgraduate medical journal, 79(938), 672680.

Horng, T., Barton, G. M., \& Medzhitov, R. (2001). TIRAP: an adapter molecule in the Toll signaling pathway. [Research Support, Non-U.S. Gov't Research Support, U.S. Gov't, P.H.S.]. Nature immunology, 2(9), 835-841. doi: 10.1038/ni0901-835

Hoshino, K., Takeuchi, O., Kawai, T., Sanjo, H., Ogawa, T., Takeda, Y., . . . Akira, S. (1999). Cutting edge: Toll-like receptor 4 (TLR4)-deficient mice are hyporesponsive to lipopolysaccharide: evidence for TLR4 as the Lps gene product. [Research Support, Non-U.S. Gov't]. Journal of immunology, 162(7), 3749-3752.

Houde, M., Bertholet, S., Gagnon, E., Brunet, S., Goyette, G., Laplante, A., . . . Desjardins, M. (2003). Phagosomes are competent organelles for antigen cross-presentation. [Research Support, Non-U.S. Gov't Research Support, U.S. Gov't, P.H.S.]. Nature, 425(6956), 402-406. doi: 10.1038/nature01912

Hsu, F. J., Benike, C., Fagnoni, F., Liles, T. M., Czerwinski, D., Taidi, B., . . L Levy, R. (1996). Vaccination of patients with B-cell lymphoma using autologous antigenpulsed dendritic cells. [Case Reports Research Support, Non-U.S. Gov't Research Support, U.S. Gov't, Non-P.H.S. Research Support, U.S. Gov't, P.H.S.]. Nature medicine, 2(1), 52-58.

Huck, S. P., Tang, S. C., Andrew, K. A., Yang, J., Harper, J. L., \& Ronchese, F. (2008). Activation and route of administration both determine the ability of bone marrow- 
derived dendritic cells to accumulate in secondary lymphoid organs and prime CD8+ $\mathrm{T}$ cells against tumors. [Research Support, Non-U.S. Gov't]. Cancer immunology, immunotherapy : CII, 57(1), 63-71. doi: 10.1007/s00262-007-0350-z

Huysamen, C., Willment, J. A., Dennehy, K. M., \& Brown, G. D. (2008). CLEC9A is a novel activation C-type lectin-like receptor expressed on BDCA3+ dendritic cells and a subset of monocytes. [Research Support, Non-U.S. Gov't]. The Journal of biological chemistry, 283(24), 16693-16701. doi: 10.1074/jbc.M709923200

Iacobelli-Martinez, M., \& Nemerow, G. R. (2007). Preferential activation of Toll-like receptor nine by CD46-utilizing adenoviruses. [Research Support, N.I.H., Extramural]. Journal of virology, 81(3), 1305-1312. doi: 10.1128/JVI.01926-06

Ifrah, N., James, J. M., Viguie, F., Marie, J. P., \& Zittoun, R. (1985). Spontaneous remission in adult acute leukemia. [Case Reports]. Cancer, 56(5), 1187-1190.

Imai, J., Hasegawa, H., Maruya, M., Koyasu, S., \& Yahara, I. (2005). Exogenous antigens are processed through the endoplasmic reticulum-associated degradation (ERAD) in cross-presentation by dendritic cells. [Research Support, Non-U.S. Gov't]. International immunology, 17(1), 45-53. doi: 10.1093/intimm/dxh184

Imler, J. L., \& Hoffmann, J. A. (2003). Toll signaling: the TIReless quest for specificity. [Comment News]. Nature immunology, 4(2), 105-106. doi: 10.1038/ni0203-105

Inaba, K., Inaba, M., Naito, M., \& Steinman, R. M. (1993). Dendritic cell progenitors phagocytose particulates, including bacillus Calmette-Guerin organisms, and sensitize mice to mycobacterial antigens in vivo. [Research Support, Non-U.S. Gov't Research Support, U.S. Gov't, P.H.S.]. The Journal of experimental medicine, 178(2), 479-488.

Inaba, K., Metlay, J. P., Crowley, M. T., \& Steinman, R. M. (1990a). Dendritic cells pulsed with protein antigens in vitro can prime antigen-specific, MHC-restricted T cells in situ. [Research Support, U.S. Gov't, P.H.S.]. The Journal of experimental medicine, 172(2), 631-640.

Inaba, K., Metlay, J. P., Crowley, M. T., Witmer-Pack, M., \& Steinman, R. M. (1990b). Dendritic cells as antigen presenting cells in vivo. [Review]. International reviews of immunology, 6(2-3), 197-206. doi: 10.3109/08830189009056630

Inaba, K., Turley, S., Yamaide, F., Iyoda, T., Mahnke, K., Inaba, M., . . Steinman, R. M. (1998). Efficient presentation of phagocytosed cellular fragments on the major histocompatibility complex class II products of dendritic cells. [Research Support, U.S. Gov't, P.H.S.]. The Journal of experimental medicine, 188(11), 2163-2173.

Ismaili, J., Rennesson, J., Aksoy, E., Vekemans, J., Vincart, B., Amraoui, Z., . . . Dubois, P. M. (2002). Monophosphoryl lipid A activates both human dendritic cells and T 
cells. [Research Support, Non-U.S. Gov't]. Journal of immunology, 168(2), 926932.

Iwamura, T., Yoneyama, M., Yamaguchi, K., Suhara, W., Mori, W., Shiota, K., . . Fujita, T. (2001). Induction of IRF-3/-7 kinase and NF-kappaB in response to doublestranded RNA and virus infection: common and unique pathways. [Research Support, Non-U.S. Gov't]. Genes to cells : devoted to molecular \& cellular mechanisms, 6(4), 375-388.

Iwasaki, A., \& Medzhitov, R. (2004). Toll-like receptor control of the adaptive immune responses. [Research Support, Non-U.S. Gov't Research Support, U.S. Gov't, P.H.S. Review]. Nature immunology, 5(10), 987-995. doi: 10.1038/ni1112

Iyoda, T., Shimoyama, S., Liu, K., Omatsu, Y., Akiyama, Y., Maeda, Y., . . . Inaba, K. (2002). The CD8+ dendritic cell subset selectively endocytoses dying cells in culture and in vivo. [Research Support, Non-U.S. Gov't Research Support, U.S. Gov't, P.H.S.]. The Journal of experimental medicine, 195(10), 1289-1302.

Izhak, L., Berzofsky, J. A., \& Terabe, M. (2013). Balance is a key for happiness. Oncoimmunology, 2(5), e24211. doi: 10.4161/onci.24211

Jaensson, E., Uronen-Hansson, H., Pabst, O., Eksteen, B., Tian, J., Coombes, J. L., . . . Agace, W. W. (2008). Small intestinal CD103+ dendritic cells display unique functional properties that are conserved between mice and humans. [Research Support, Non-U.S. Gov't]. The Journal of experimental medicine, 205(9), 21392149. doi: $10.1084 /$ jem. 20080414

Jahng, A., Maricic, I., Aguilera, C., Cardell, S., Halder, R. C., \& Kumar, V. (2004). Prevention of autoimmunity by targeting a distinct, noninvariant CD1d-reactive $T$ cell population reactive to sulfatide. [Research Support, Non-U.S. Gov't Research Support, U.S. Gov't, P.H.S.]. $J$ Exp Med, 199(7), 947-957. doi: 10.1084/jem.20031389

Jakubzick, C., Tacke, F., Ginhoux, F., Wagers, A. J., van Rooijen, N., Mack, M., . . . Randolph, G. J. (2008). Blood monocyte subsets differentially give rise to CD103+ and CD103- pulmonary dendritic cell populations. [Comparative Study Research Support, N.I.H., Extramural Research Support, Non-U.S. Gov't]. Journal of immunology, 180(5), 3019-3027.

Janeway, C. (1989). Immunogenicity signals 1,2,3 ... and 0. [News]. Immunology today, 10(9), 283-286.

Jansen, J. H., Wientjens, G. J., Fibbe, W. E., Willemze, R., \& Kluin-Nelemans, H. C. (1989). Inhibition of human macrophage colony formation by interleukin 4. [In Vitro Research Support, Non-U.S. Gov't]. The Journal of experimental medicine, 170(2), 577-582. 
Janssen, E. M., et al. (2003). CD4+ T cells are required for secondary expansion and memory in CD8+ T lymphocytes. Nature, 421, 852-856.

Jenner, E. (1798). An inquiry into the causes and effects of the variolae vaccinae, a disease discovered in some of the western countries of England, particularly Gloucestershire, and known by the name of the cow pox. London.

Jensen, P. E. (2007). Recent advances in antigen processing and presentation. [Review]. Nature immunology, 8(10), 1041-1048. doi: 10.1038/ni1516

Jiang, Q., Akashi, S., Miyake, K., \& Petty, H. R. (2000). Lipopolysaccharide induces physical proximity between CD14 and toll-like receptor 4 (TLR4) prior to nuclear translocation of NF-kappa B. [Research Support, U.S. Gov't, P.H.S.]. Journal of immunology, 165(7), 3541-3544.

Jiang, W., Swiggard, W. J., Heufler, C., Peng, M., Mirza, A., Steinman, R. M., \& Nussenzweig, M. C. (1995). The receptor DEC-205 expressed by dendritic cells and thymic epithelial cells is involved in antigen processing. [Research Support, Non-U.S. Gov't Research Support, U.S. Gov't, P.H.S.]. Nature, 375(6527), 151155. doi: $10.1038 / 375151 \mathrm{a} 0$

Johansson-Lindbom, B., Svensson, M., Pabst, O., Palmqvist, C., Marquez, G., Forster, R., \& Agace, W. W. (2005). Functional specialization of gut CD103+ dendritic cells in the regulation of tissue-selective $\mathrm{T}$ cell homing. [Comparative Study Research Support, Non-U.S. Gov't]. The Journal of experimental medicine, 202(8), 10631073. doi: 10.1084/jem.20051100

Jongbloed, S. L., Kassianos, A. J., McDonald, K. J., Clark, G. J., Ju, X., Angel, C. E., . . Radford, K. J. (2010). Human CD141+ (BDCA-3)+ dendritic cells (DCs) represent a unique myeloid DC subset that cross-presents necrotic cell antigens. [Research Support, Non-U.S. Gov't]. The Journal of experimental medicine, 207(6), 12471260. doi: $10.1084 /$ jem.20092140

Jonuleit, H., Giesecke-Tuettenberg, A., Tuting, T., Thurner-Schuler, B., Stuge, T. B., Paragnik, L., . . Enk, A. H. (2001). A comparison of two types of dendritic cell as adjuvants for the induction of melanoma-specific T-cell responses in humans following intranodal injection. [Comparative Study Research Support, Non-U.S. Gov't]. International journal of cancer. Journal international du cancer, 93(2), 243-251. doi: 10.1002/ijc. 1323

Jude, B. A., Pobezinskaya, Y., Bishop, J., Parke, S., Medzhitov, R. M., Chervonsky, A. V., \& Golovkina, T. V. (2003). Subversion of the innate immune system by a retrovirus. [Research Support, Non-U.S. Gov't Research Support, U.S. Gov't, P.H.S.]. Nature immunology, 4(6), 573-578. doi: 10.1038/ni926 
June, C. H. (2007a). Adoptive T cell therapy for cancer in the clinic. [Research Support, N.I.H., Extramural Research Support, Non-U.S. Gov't Review]. The Journal of clinical investigation, 117(6), 1466-1476. doi: 10.1172/JCI32446

June, C. H. (2007b). Principles of adoptive T cell cancer therapy. [Research Support, N.I.H., Extramural Research Support, Non-U.S. Gov't Review]. The Journal of clinical investigation, 117(5), 1204-1212. doi: 10.1172/JCI31446

Jung, S., Unutmaz, D., Wong, P., Sano, G., De los Santos, K., Sparwasser, T., . . Lang, R. A. (2002). In vivo depletion of CD11c+ dendritic cells abrogates priming of CD8+ $\mathrm{T}$ cells by exogenous cell-associated antigens. [Research Support, Non-U.S. Gov't Research Support, U.S. Gov't, P.H.S.]. Immunity, 17(2), 211-220.

Jurk, M., Heil, F., Vollmer, J., Schetter, C., Krieg, A. M., Wagner, H., . . . Bauer, S. (2002). Human TLR7 or TLR8 independently confer responsiveness to the antiviral compound R-848. [Comment Letter]. Nature immunology, 3(6), 499. doi: $10.1038 /$ ni0602-499

Kabelitz, D., \& Medzhitov, R. (2007). Innate immunity--cross-talk with adaptive immunity through pattern recognition receptors and cytokines. [Review]. Current opinion in immunology, 19(1), 1-3. doi: 10.1016/j.coi.2006.11.018

Kaisho, T., \& Akira, S. (2001a). Dendritic-cell function in Toll-like receptor- and MyD88knockout mice. [Research Support, Non-U.S. Gov't Review]. Trends in immunology, 22(2), 78-83.

Kaisho, T., \& Akira, S. (2001b). Toll-like receptors and their signaling mechanism in innate immunity. [Research Support, Non-U.S. Gov't Review]. Acta odontologica Scandinavica, 59(3), 124-130.

Kaisho, T., Takeuchi, O., Kawai, T., Hoshino, K., \& Akira, S. (2001). Endotoxin-induced maturation of MyD88-deficient dendritic cells. [Research Support, Non-U.S. Gov't]. Journal of immunology, 166(9), 5688-5694.

Kanakaraj, P., Schafer, P. H., Cavender, D. E., Wu, Y., Ngo, K., Grealish, P. F., . . FungLeung, W. P. (1998). Interleukin (IL)-1 receptor-associated kinase (IRAK) requirement for optimal induction of multiple IL-1 signaling pathways and IL-6 production. The Journal of experimental medicine, 187(12), 2073-2079.

Karadimitris, A., Gadola, S., Altamirano, M., Brown, D., Woolfson, A., Klenerman, P., . . Cerundolo, V. (2001). Human CD1d-glycolipid tetramers generated by in vitro oxidative refolding chromatography. [Research Support, Non-U.S. Gov't]. Proceedings of the National Academy of Sciences of the United States of America, 98(6), 3294-3298. doi: 10.1073/pnas.051604498

Karlsson, H., Larsson, P., Wold, A. E., \& Rudin, A. (2004). Pattern of cytokine responses to gram-positive and gram-negative commensal bacteria is profoundly changed 
when monocytes differentiate into dendritic cells. [In Vitro Research Support, NonU.S. Gov't]. Infection and immunity, 72(5), 2671-2678.

Kasturi, S. P., Skountzou, I., Albrecht, R. A., Koutsonanos, D., Hua, T., Nakaya, H. I., . . Pulendran, B. (2011). Programming the magnitude and persistence of antibody responses with innate immunity. [Research Support, N.I.H., Extramural Research Support, Non-U.S. Gov't]. Nature, 470(7335), 543-547. doi: 10.1038/nature09737

Kawai, T., Adachi, O., Ogawa, T., Takeda, K., \& Akira, S. (1999). Unresponsiveness of MyD88-deficient mice to endotoxin. [Research Support, Non-U.S. Gov't]. Immunity, 11(1), 115-122.

Kawai, T., \& Akira, S. (2011). Toll-like receptors and their crosstalk with other innate receptors in infection and immunity. [Review]. Immunity, 34(5), 637-650. doi: 10.1016/j.immuni.2011.05.006

Kawai, T., Takeuchi, O., Fujita, T., Inoue, J., Muhlradt, P. F., Sato, S., . . . Akira, S. (2001). Lipopolysaccharide stimulates the MyD88-independent pathway and results in activation of IFN-regulatory factor 3 and the expression of a subset of lipopolysaccharide-inducible genes. [Research Support, Non-U.S. Gov't]. Journal of immunology, 167(10), 5887-5894.

Kawano, T., Cui, J., Koezuka, Y., Toura, I., Kaneko, Y., Motoki, K., . . . Taniguchi, M. (1997). CD1d-restricted and TCR-mediated activation of valpha14 NKT cells by glycosylceramides. [Research Support, Non-U.S. Gov't]. Science, 278(5343), 16261629.

Kawano, T., Cui, J., Koezuka, Y., Toura, I., Kaneko, Y., Sato, H., . . . Taniguchi, M. (1998). Natural killer-like nonspecific tumor cell lysis mediated by specific ligandactivated Valpha14 NKT cells. Proceedings of the National Academy of Sciences of the United States of America, 95(10), 5690-5693.

Kawano, T., Tanaka, Y., Shimizu, E., Kaneko, Y., Kamata, N., Sato, H., . . Taniguchi, M. (1999). A novel recognition motif of human NKT antigen receptor for a glycolipid ligand. [Research Support, Non-U.S. Gov't]. International immunology, 11(6), 881887.

Ke, Y., \& Kapp, J. A. (1996). Exogenous antigens gain access to the major histocompatibility complex class I processing pathway in B cells by receptormediated uptake. [Research Support, U.S. Gov't, P.H.S.]. The Journal of experimental medicine, 184(3), 1179-1184.

Keppler, S. J., Rosenits, K., Koegl, T., Vucikuja, S., \& Aichele, P. (2012). Signal 3 cytokines as modulators of primary immune responses during infections: the interplay of type I IFN and IL-12 in CD8 T cell responses. [Research Support, Non-U.S. Gov't]. PLoS One, 7(7), e40865. doi: 10.1371/journal.pone.0040865 
Kikkawa, F., Kawai, M., Oguchi, H., Kojima, M., Ishikawa, H., Iwata, M., . . . et al. (1993). Randomised study of immunotherapy with OK-432 in uterine cervical carcinoma. [Clinical Trial Randomized Controlled Trial]. European journal of cancer, 29A(11), 1542-1546.

Kim, Y. M., Brinkmann, M. M., Paquet, M. E., \& Ploegh, H. L. (2008). UNC93B1 delivers nucleotide-sensing toll-like receptors to endolysosomes. [Research Support, N.I.H., Extramural Research Support, Non-U.S. Gov't]. Nature, 452(7184), 234-238. doi: 10.1038/nature06726

Kissenpfennig, A., Henri, S., Dubois, B., Laplace-Builhe, C., Perrin, P., Romani, N., . . Malissen, B. (2005). Dynamics and function of Langerhans cells in vivo: dermal dendritic cells colonize lymph node areas distinct from slower migrating Langerhans cells. [In Vitro Research Support, Non-U.S. Gov't]. Immunity, 22(5), 643-654. doi: 10.1016/j.immuni.2005.04.004

Kissenpfennig, A., \& Malissen, B. (2006). Langerhans cells--revisiting the paradigm using genetically engineered mice. [Research Support, Non-U.S. Gov't Review]. Trends in immunology, 27(3), 132-139. doi: 10.1016/j.it.2006.01.003

Kitamura, H., Iwakabe, K., Yahata, T., Nishimura, S., Ohta, A., Ohmi, Y., . . Nishimura, T. (1999). The natural killer T (NKT) cell ligand alpha-galactosylceramide demonstrates its immunopotentiating effect by inducing interleukin (IL)-12 production by dendritic cells and IL-12 receptor expression on NKT cells. [Research Support, Non-U.S. Gov't]. The Journal of experimental medicine, 189(7), 1121-1128.

Kleijmeer, M. J., Escola, J. M., UytdeHaag, F. G., Jakobson, E., Griffith, J. M., Osterhaus, A. D., ... Geuze, H. J. (2001). Antigen loading of MHC class I molecules in the endocytic tract. [Research Support, Non-U.S. Gov't]. Traffic, 2(2), 124-137.

Kleijmeer, M. J., Morkowski, S., Griffith, J. M., Rudensky, A. Y., \& Geuze, H. J. (1997). Major histocompatibility complex class II compartments in human and mouse B lymphoblasts represent conventional endocytic compartments. [Research Support, Non-U.S. Gov't]. The Journal of cell biology, 139(3), 639-649.

Kleindienst, P., \& Brocker, T. (2003). Endogenous dendritic cells are required for amplification of $\mathrm{T}$ cell responses induced by dendritic cell vaccines in vivo. [Comparative Study Research Support, Non-U.S. Gov't]. Journal of immunology, $170(6), 2817-2823$.

Kobayashi, E., Motoki, K., Uchida, T., Fukushima, H., \& Koezuka, Y. (1995). KRN7000, a novel immunomodulator, and its antitumor activities. [Comparative Study]. Oncol Res, 7(10-11), 529-534.

Koch, F., Stanzl, U., Jennewein, P., Janke, K., Heufler, C., Kampgen, E., . . Schuler, G. (1996). High level IL-12 production by murine dendritic cells: upregulation via 
MHC class II and CD40 molecules and downregulation by IL-4 and IL-10. [Research Support, Non-U.S. Gov't]. The Journal of experimental medicine, 184(2), 741-746.

Kopp, E. B., \& Medzhitov, R. (1999). The Toll-receptor family and control of innate immunity. [Review]. Current opinion in immunology, 11(1), 13-18.

Kraal, G. (2002). [Inducing tolerance against antigens through initial contact via the mucous membrane]. [Review]. Nederlands tijdschrift voor geneeskunde, 146(50), 2406-2409.

Kronenberg, M. (2005). Toward an understanding of NKT cell biology: progress and paradoxes. [Research Support, U.S. Gov't, P.H.S. Review]. Annu Rev Immunol, 23, 877-900. doi: 10.1146/annurev.immunol.23.021704.115742

Krug, A., French, A. R., Barchet, W., Fischer, J. A., Dzionek, A., Pingel, J. T., . . . Colonna, M. (2004). TLR9-dependent recognition of MCMV by IPC and DC generates coordinated cytokine responses that activate antiviral NK cell function. [Research Support, Non-U.S. Gov't Research Support, U.S. Gov't, P.H.S.]. Immunity, 21(1), 107-119. doi: 10.1016/j.immuni.2004.06.007

Kuipers, H., Soullie, T., Hammad, H., Willart, M., Kool, M., Hijdra, D., . . Lambrecht, B. N. (2009). Sensitization by intratracheally injected dendritic cells is independent of antigen presentation by host antigen-presenting cells. [Research Support, Non-U.S. Gov't]. Journal of leukocyte biology, 85(1), 64-70. doi: 10.1189/jlb.0807519

Kurt-Jones, E. A., Popova, L., Kwinn, L., Haynes, L. M., Jones, L. P., Tripp, R. A., . . Finberg, R. W. (2000). Pattern recognition receptors TLR4 and CD14 mediate response to respiratory syncytial virus. [In Vitro Research Support, U.S. Gov't, P.H.S.]. Nature immunology, 1(5), 398-401. doi: 10.1038/80833

Labeur, M. S., Roters, B., Pers, B., Mehling, A., Luger, T. A., Schwarz, T., \& Grabbe, S. (1999). Generation of tumor immunity by bone marrow-derived dendritic cells correlates with dendritic cell maturation stage. [Research Support, Non-U.S. Gov't]. Journal of immunology, 162(1), 168-175.

Lamb, C. A., \& Cresswell, P. (1992). Assembly and transport properties of invariant chain trimers and HLA-DR-invariant chain complexes. [Research Support, U.S. Gov't, P.H.S.]. Journal of immunology, 148(11), 3478-3482.

Langenkamp, A., M. Messi, A. Lanzavecchia, and F. Sallusto. 2000. (2000). Kinetics of dendritic cell activation: impact on priming of TH1, TH2 and nonpolarized T cells. Nat Immunol, 1, 311-316.

Lantz, O., \& Bendelac, A. (1994). An invariant T cell receptor alpha chain is used by a unique subset of major histocompatibility complex class I-specific CD4+ and CD4- 
8- T cells in mice and humans. The Journal of experimental medicine, 180(3), 1097-1106.

Lanzavecchia, A., \& Sallusto, F. (2001a). The instructive role of dendritic cells on T cell responses: lineages, plasticity and kinetics. [Research Support, Non-U.S. Gov't Review]. Current opinion in immunology, 13(3), 291-298.

Lanzavecchia, A., \& Sallusto, F. (2001b). Regulation of T cell immunity by dendritic cells. [Review]. Cell, 106(3), 263-266.

Lapenta, C., Santini, S. M., Spada, M., Donati, S., Urbani, F., Accapezzato, D., . . . Belardelli, F. (2006). IFN-alpha-conditioned dendritic cells are highly efficient in inducing cross-priming CD8(+) T cells against exogenous viral antigens. European journal of immunology, 36(8), 2046-2060. doi: 10.1002/eji.200535579

Lau, C. M., Broughton, C., Tabor, A. S., Akira, S., Flavell, R. A., Mamula, M. J., . . Marshak-Rothstein, A. (2005). RNA-associated autoantigens activate B cells by combined B cell antigen receptor/Toll-like receptor 7 engagement. [Research Support, N.I.H., Extramural Research Support, Non-U.S. Gov't]. The Journal of experimental medicine, 202(9), 1171-1177. doi: 10.1084/jem.20050630

Lau, R., Wang, F., Jeffery, G., Marty, V., Kuniyoshi, J., Bade, E., . . Weber, J. (2001). Phase I trial of intravenous peptide-pulsed dendritic cells in patients with metastatic melanoma. [Clinical Trial Clinical Trial, Phase I Research Support, U.S. Gov't, P.H.S.]. Journal of immunotherapy, 24(1), 66-78.

Le Bon, A., Etchart, N., Rossmann, C., Ashton, M., Hou, S., Gewert, D., . . Tough, D. F. (2003). Cross-priming of CD8 $+\mathrm{T}$ cells stimulated by virus-induced type I interferon. [Research Support, Non-U.S. Gov't]. Nature immunology, 4(10), 10091015. doi: $10.1038 /$ ni978

Le Bon, A., \& Tough, D. F. (2008). Type I interferon as a stimulus for cross-priming. [Research Support, Non-U.S. Gov't Review]. Cytokine \& growth factor reviews, 19(1), 33-40. doi: 10.1016/j.cytogfr.2007.10.007

Leadbetter, E. A., Rifkin, I. R., Hohlbaum, A. M., Beaudette, B. C., Shlomchik, M. J., \& Marshak-Rothstein, A. (2002). Chromatin-IgG complexes activate B cells by dual engagement of IgM and Toll-like receptors. [Research Support, Non-U.S. Gov't Research Support, U.S. Gov't, P.H.S.]. Nature, 416(6881), 603-607. doi: $10.1038 / 416603 a$

Lee, A., Farrand, K. J., Dickgreber, N., Hayman, C. M., Jurs, S., Hermans, I. F., \& Painter, G. F. (2006). Novel synthesis of alpha-galactosyl-ceramides and confirmation of their powerful NKT cell agonist activity. [Research Support, Non-U.S. Gov't]. Carbohydrate research, 341(17), 2785-2798. doi: 10.1016/j.carres.2006.09.006 
Lee, B. O., Haynes, L., Eaton, S. M., Swain, S. L., \& Randall, T. D. (2002). The biological outcome of CD40 signaling is dependent on the duration of CD40 ligand expression: reciprocal regulation by interleukin (IL)-4 and IL-12. [In Vitro Research Support, Non-U.S. Gov't Research Support, U.S. Gov't, P.H.S.]. The Journal of experimental medicine, 196(5), 693-704.

Lefrancois, L., Altman, J. D., Williams, K., \& Olson, S. (2000). Soluble antigen and CD40 triggering are sufficient to induce primary and memory cytotoxic T cells. [Research Support, Non-U.S. Gov't Research Support, U.S. Gov't, P.H.S.]. Journal of immunology, 164(2), 725-732.

Lehmann, J., Huehn, J., de la Rosa, M., Maszyna, F., Kretschmer, U., Krenn, V., . . . Hamann, A. (2002). Expression of the integrin alpha Ebeta 7 identifies unique subsets of CD25+ as well as CD25- regulatory T cells. [Research Support, NonU.S. Gov't]. Proceedings of the National Academy of Sciences of the United States of America, 99(20), 13031-13036. doi: 10.1073/pnas.192162899

Leon, C. G., Tory, R., Jia, J., Sivak, O., \& Wasan, K. M. (2008). Discovery and development of toll-like receptor 4 (TLR4) antagonists: a new paradigm for treating sepsis and other diseases. [Review]. Pharmaceutical research, 25(8), 17511761. doi: 10.1007/s11095-008-9571-x

Liew, F. Y., Xu, D., Brint, E. K., \& O'Neill, L. A. (2005). Negative regulation of toll-like receptor-mediated immune responses. [Research Support, Non-U.S. Gov't Review]. Nature reviews. Immunology, 5(6), 446-458. doi: 10.1038/nri1630

Lin, M. L., Zhan, Y., Proietto, A. I., Prato, S., Wu, L., Heath, W. R., . . . Lew, A. M. (2008). Selective suicide of cross-presenting CD8+ dendritic cells by cytochrome c injection shows functional heterogeneity within this subset. [Research Support, Non-U.S. Gov't]. Proceedings of the National Academy of Sciences of the United States of America, 105(8), 3029-3034. doi: 10.1073/pnas.0712394105

Lin, M. L., Zhan, Y., Villadangos, J. A., \& Lew, A. M. (2008). The cell biology of crosspresentation and the role of dendritic cell subsets. [Research Support, Non-U.S. Gov't Review]. Immunology and cell biology, 86(4), 353-362. doi: 10.1038/icb.2008.3

Link, B. K., Ballas, Z. K., Weisdorf, D., Wooldridge, J. E., Bossler, A. D., Shannon, M., . . . Weiner, G. J. (2006). Oligodeoxynucleotide CpG 7909 delivered as intravenous infusion demonstrates immunologic modulation in patients with previously treated non-Hodgkin lymphoma. [Clinical Trial, Phase I Research Support, N.I.H., Extramural Research Support, Non-U.S. Gov't]. Journal of immunotherapy, 29(5), 558-568. doi: 10.1097/01.cji.0000211304.60126.8f

Lipscomb, M. F., \& Masten, B. J. (2002). Dendritic cells: immune regulators in health and disease. [Research Support, Non-U.S. Gov't Research Support, U.S. Gov't, P.H.S. Review]. Physiological reviews, 82(1), 97-130. doi: 10.1152/physrev.00023.2001 
Lister, J. (1906). The collected papers of Joseph, Baron Lister. Oxford: Oxford, Clarendon Press.

Liu, K., Iyoda,T., Saternus, M., Kimura, Y.,Inaba, K., Sreinman, R.M. (2002). Immune tolerance after delivery of dying cells to dendritic cells in-situ. J Exp Med, 196, 1091-1097.

Liu, K., Waskow, C., Liu, X., Yao, K., Hoh, J., Nussenzweig, M. (2007). Origin of dendritic cells in peripheral lymphoid organs of mice. Nat Immunol(8), 578-583

Liu, T., Matsuguchi, T., Tsuboi, N., Yajima, T., \& Yoshikai, Y. (2002). Differences in expression of toll-like receptors and their reactivities in dendritic cells in BALB/c and C57BL/6 mice. [Research Support, Non-U.S. Gov't]. Infection and immunity, 70(12), 6638-6645.

Liu, Y. J. (2005). IPC: professional type 1 interferon-producing cells and plasmacytoid dendritic cell precursors. [Research Support, Non-U.S. Gov't Research Support, U.S. Gov't, P.H.S. Review]. Annual review of immunology, 23, 275-306. doi: 10.1146/annurev.immunol.23.021704.115633

Lomaga, M. A., Yeh, W. C., Sarosi, I., Duncan, G. S., Furlonger, C., Ho, A., . . Mak, T. W. (1999). TRAF6 deficiency results in osteopetrosis and defective interleukin-1, CD40, and LPS signaling. [Research Support, Non-U.S. Gov't]. Genes \& development, 13(8), 1015-1024.

Lore, K., Betts, M. R., Brenchley, J. M., Kuruppu, J., Khojasteh, S., Perfetto, S., . . Koup, R. A. (2003). Toll-like receptor ligands modulate dendritic cells to augment cytomegalovirus- and HIV-1-specific $\mathrm{T}$ cell responses. [Comparative Study Research Support, Non-U.S. Gov't]. Journal of immunology, 171(8), 4320-4328.

Lorenzi, S., Mattei, F., Sistigu, A., Bracci, L., Spadaro, F., Sanchez, M., . . Schiavoni, G. (2011). Type I IFNs control antigen retention and survival of CD8alpha(+) dendritic cells after uptake of tumor apoptotic cells leading to cross-priming. [Research Support, Non-U.S. Gov't]. Journal of immunology, 186(9), 5142-5150. doi: $10.4049 /$ jimmunol.1004163

Lotze, M. T., \& Tracey, K. J. (2005). High-mobility group box 1 protein (HMGB1): nuclear weapon in the immune arsenal. [Review]. Nature reviews. Immunology, 5(4), 331-342. doi: 10.1038/nri1594

Lui, G., Manches, O., Angel, J., Molens, J. P., Chaperot, L., \& Plumas, J. (2009). Plasmacytoid dendritic cells capture and cross-present viral antigens from influenza-virus exposed cells. [Research Support, Non-U.S. Gov't]. PloS one, 4(9), e7111. doi: 10.1371/journal.pone.0007111 
Luketic, L., Delanghe, J., Sobol, P. T., Yang, P., Frotten, E., Mossman, K. L., . . Wan, Y. (2007). Antigen presentation by exosomes released from peptide-pulsed dendritic cells is not suppressed by the presence of active CTL. [Research Support, Non-U.S. Gov't]. Journal of immunology, 179(8), 5024-5032.

Lund, J., Sato, A., Akira, S., Medzhitov, R., \& Iwasaki, A. (2003). Toll-like receptor 9mediated recognition of Herpes simplex virus- 2 by plasmacytoid dendritic cells. [Research Support, Non-U.S. Gov't]. The Journal of experimental medicine, 198(3), 513-520. doi: 10.1084/jem.20030162

Lund, J. M., Alexopoulou, L., Sato, A., Karow, M., Adams, N. C., Gale, N. W., . . . Flavell, R. A. (2004). Recognition of single-stranded RNA viruses by Toll-like receptor 7. [Research Support, Non-U.S. Gov't Research Support, U.S. Gov't, P.H.S.]. Proceedings of the National Academy of Sciences of the United States of America, 101(15), 5598-5603. doi: 10.1073/pnas.0400937101

Luster, A. D. (2002). The role of chemokines in linking innate and adaptive immunity. [Research Support, U.S. Gov't, P.H.S. Review]. Current opinion in immunology, 14(1), 129-135.

Lyakh, L., Trinchieri, G., Provezza, L., Carra, G., \& Gerosa, F. (2008). Regulation of interleukin-12/interleukin-23 production and the T-helper 17 response in humans. [Review]. Immunological reviews, 226, 112-131. doi: 10.1111/j.1600065X.2008.00700.X

Lyons, A. S., Petrucelli, R.J. II. (1987). Medicine - An Illustrated History. New York: Harry N Abrams Inc, Abradale Press.

Ma, J. Z., Lim, S. N., Qin, J. S., Yang, J., Enomoto, N., Ruedl, C., \& Ronchese, F. (2012). Murine CD4+ T cell responses are inhibited by cytotoxic T cell-mediated killing of dendritic cells and are restored by antigen transfer. [Research Support, Non-U.S. Gov't]. PloS one, 7(5), e37481. doi: 10.1371/journal.pone.0037481

MacDonald, H. R. (1995). NK1.1+ T cell receptor-alpha/beta+ cells: new clues to their origin, specificity, and function. [Review]. The Journal of experimental medicine, 182(3), 633-638.

Mackensen, A., Herbst, B., Chen, J. L., Kohler, G., Noppen, C., Herr, W., . . Lindemann, A. (2000). Phase I study in melanoma patients of a vaccine with peptide-pulsed dendritic cells generated in vitro from CD34(+) hematopoietic progenitor cells. [Clinical Trial Clinical Trial, Phase I Research Support, Non-U.S. Gov't]. International journal of cancer. Journal international du cancer, 86(3), 385-392.

Maehara, Y., Okuyama, T., Kakeji, Y., Baba, H., Furusawa, M., \& Sugimachi, K. (1994). Postoperative immunochemotherapy including streptococcal lysate OK-432 is effective for patients with gastric cancer and serosal invasion. [Clinical Trial Randomized Controlled Trial]. American journal of surgery, 168(1), 36-40. 
Maekawa, T., Fujii, H., Horiike, S., Okuda, T., Yokota, S., Ueda, K., \& Urata, Y. (1989). [Spontaneous remission of four months' duration in hypoplastic leukemia with tetraploid chromosome after blood transfusions and infection]. [Case Reports]. Nihon Ketsueki Gakkai zasshi : journal of Japan Haematological Society, 52(5), 849-857.

Majumder, S., Bhattacharjee, S., Paul Chowdhury, B., \& Majumdar, S. (2012). CXCL10 is critical for the generation of protective CD8 $\mathrm{T}$ cell response induced by antigen pulsed CpG-ODN activated dendritic cells. [Research Support, Non-U.S. Gov't]. PloS one, 7(11), e48727. doi: 10.1371/journal.pone.0048727

Malley, R., Henneke, P., Morse, S. C., Cieslewicz, M. J., Lipsitch, M., Thompson, C. M., . . . Golenbock, D. T. (2003). Recognition of pneumolysin by Toll-like receptor 4 confers resistance to pneumococcal infection. [Comparative Study Research Support, Non-U.S. Gov't Research Support, U.S. Gov't, P.H.S.]. Proceedings of the National Academy of Sciences of the United States of America, 100(4), 1966-1971. doi: 10.1073/pnas.0435928100

Marcos Sanchez, F., Juarez Ucelay, F., Bru Espino, I. M., \& Duran-Perez Navarro, A. (1991). [A new case of spontaneous tumor regression]. [Case Reports Letter]. Anales de medicina interna, 8(9), 468.

Mariathasan, S., \& Monack, D. M. (2007). Inflammasome adaptors and sensors: intracellular regulators of infection and inflammation. [Review]. Nature reviews. Immunology, 7(1), 31-40. doi: 10.1038/nri1997

Marshak-Rothstein, A. (2006). Toll-like receptors in systemic autoimmune disease. [Research Support, N.I.H., Extramural Review]. Nature reviews. Immunology, 6(11), 823-835. doi: 10.1038/nri1957

Marten, A., Renoth, S., Heinicke, T., Albers, P., Pauli, A., Mey, U., . . Schmidt-Wolf, I. G. (2003). Allogeneic dendritic cells fused with tumor cells: preclinical results and outcome of a clinical phase I/II trial in patients with metastatic renal cell carcinoma. [Clinical Trial Clinical Trial, Phase I Clinical Trial, Phase II Research Support, Non-U.S. Gov't]. Human gene therapy, 14(5), 483-494. doi: 10.1089/104303403321467243

Martin-Fontecha, A., Sebastiani, S., Hopken, U. E., Uguccioni, M., Lipp, M., Lanzavecchia, A., \& Sallusto, F. (2003). Regulation of dendritic cell migration to the draining lymph node: impact on $\mathrm{T}$ lymphocyte traffic and priming. [Research Support, Non-U.S. Gov't]. The Journal of experimental medicine, 198(4), 615-621. doi: $10.1084 /$ jem. 20030448

Martinon, F., \& Tschopp, J. (2005). NLRs join TLRs as innate sensors of pathogens. [Review]. Trends in immunology, 26(8), 447-454. doi: 10.1016/j.it.2005.06.004 
Masuda, K., Makino, Y., Cui, J., Ito, T., Tokuhisa, T., Takahama, Y., . . . Taniguchi, M. (1997). Phenotypes and invariant alpha beta TCR expression of peripheral V alpha $14+$ NK T cells. [Research Support, Non-U.S. Gov't]. Journal of immunology, 158(5), 2076-2082.

Mata-Haro, V., Cekic, C., Martin, M., Chilton, P. M., Casella, C. R., \& Mitchell, T. C. (2007). The vaccine adjuvant monophosphoryl lipid A as a TRIF-biased agonist of TLR4. [Research Support, N.I.H., Extramural Research Support, Non-U.S. Gov't]. Science, 316(5831), 1628-1632. doi: 10.1126/science.1138963

Matsuda, J. L., Naidenko, O. V., Gapin, L., Nakayama, T., Taniguchi, M., Wang, C. R., . . . Kronenberg, M. (2000). Tracking the response of natural killer T cells to a glycolipid antigen using CD1d tetramers. [Research Support, Non-U.S. Gov't Research Support, U.S. Gov't, P.H.S.]. The Journal of experimental medicine, 192(5), 741-754.

Matsuda, J. L., Gapin, L., Baron, J. L., Sidobre, S., Stetson, D. B., Mohrs, M., . . . Kronenberg, M. (2003). Mouse V alpha 14i natural killer T cells are resistant to cytokine polarization in vivo. [Comparative Study Research Support, Non-U.S. Gov't Research Support, U.S. Gov't, P.H.S.]. Proc Natl Acad Sci U S A, 100(14), 8395-8400. doi: 10.1073/pnas.1332805100

Matsumoto, M., Funami, K., Tanabe, M., Oshiumi, H., Shingai, M., Seto, Y., . . Seya, T. (2003). Subcellular localization of Toll-like receptor 3 in human dendritic cells. [Research Support, Non-U.S. Gov't]. Journal of immunology, 171(6), 3154-3162.

Medzhitov, R., \& Janeway, C. A., Jr. (1998). Innate immune recognition and control of adaptive immune responses. [Research Support, Non-U.S. Gov't Research Support, U.S. Gov't, P.H.S. Review]. Seminars in immunology, 10(5), 351-353.

Medzhitov, R., Preston-Hurlburt, P., \& Janeway, C. A., Jr. (1997). A human homologue of the Drosophila Toll protein signals activation of adaptive immunity. [Research Support, Non-U.S. Gov't Research Support, U.S. Gov't, P.H.S.]. Nature, 388(6640), 394-397. doi: 10.1038/41131

Meier, O., Boucke, K., Hammer, S. V., Keller, S., Stidwill, R. P., Hemmi, S., \& Greber, U. F. (2002). Adenovirus triggers macropinocytosis and endosomal leakage together with its clathrin-mediated uptake. [Research Support, Non-U.S. Gov't]. The Journal of cell biology, 158(6), 1119-1131. doi: 10.1083/jcb.200112067

Mellman, I., \& Steinman, R. M. (2001). Dendritic cells: specialized and regulated antigen processing machines. [Comment Review]. Cell, 106(3), 255-258.

Menges, M., Rossner, S., Voigtlander, C., Schindler, H., Kukutsch, N. A., Bogdan, C., . . Lutz, M. B. (2002). Repetitive injections of dendritic cells matured with tumor necrosis factor alpha induce antigen-specific protection of mice from 
autoimmunity. [Research Support, Non-U.S. Gov't]. The Journal of experimental medicine, 195(1), 15-21.

Metcalf, D., Burgess, A. W., Johnson, G. R., Nicola, N. A., Nice, E. C., DeLamarter, J., . . . Mermod, J. J. (1986). In vitro actions on hemopoietic cells of recombinant murine GM-CSF purified after production in Escherichia coli: comparison with purified native GM-CSF. [Comparative Study Research Support, Non-U.S. Gov't Research Support, U.S. Gov't, P.H.S.]. Journal of cellular physiology, 128(3), 421-431. doi: $10.1002 /$ jcp. 1041280311

Michelsen, K. S., Aicher, A., Mohaupt, M., Hartung, T., Dimmeler, S., Kirschning, C. J., \& Schumann, R. R. (2001). The role of toll-like receptors (TLRs) in bacteriainduced maturation of murine dendritic cells (DCS). Peptidoglycan and lipoteichoic acid are inducers of DC maturation and require TLR2. [Research Support, NonU.S. Gov't]. The Journal of biological chemistry, 276(28), 25680-25686. doi: 10.1074/jbc.M011615200

Mitsui, H., Watanabe, T., Saeki, H., Mori, K., Fujita, H., Tada, Y., . . Tamaki, K. (2004). Differential expression and function of Toll-like receptors in Langerhans cells: comparison with splenic dendritic cells. [Comparative Study]. The Journal of investigative dermatology, 122(1), 95-102. doi: 10.1046/j.0022-202X.2003.22116.X

Mitterbauer, M., Fritzer-Szekeres, M., Mitterbauer, G., Simonitsch, I., Knobl, P., Rintelen, C., . . J Jaeger, U. (1996). Spontaneous remission of acute myeloid leukemia after infection and blood transfusion associated with hypergammaglobulinaemia. [Research Support, Non-U.S. Gov't]. Annals of hematology, 73(4), 189-193.

Miyagi, T., Takehara, T., Tatsumi, T., Kanto, T., Suzuki, T., Jinushi, M., . . Hayashi, N. (2003). CD1d-mediated stimulation of natural killer $\mathrm{T}$ cells selectively activates hepatic natural killer cells to eliminate experimentally disseminated hepatoma cells in murine liver. [Research Support, Non-U.S. Gov't]. Int J Cancer, 106(1), 81-89. doi: $10.1002 /$ ijc. 11163

Mocikat, R., Braumuller, H., Gumy, A., Egeter, O., Ziegler, H., Reusch, U., . . Rocken, M. (2003). Natural killer cells activated by MHC class I(low) targets prime dendritic cells to induce protective CD8 T cell responses. [Research Support, NonU.S. Gov't]. Immunity, 19(4), 561-569.

Montecalvo, A., Larregina, A. T., Shufesky, W. J., Stolz, D. B., Sullivan, M. L., Karlsson, J. M., . . Morelli, A. E. (2012). Mechanism of transfer of functional microRNAs between mouse dendritic cells via exosomes. [Research Support, N.I.H., Extramural Research Support, Non-U.S. Gov't]. Blood, 119(3), 756-766. doi: 10.1182/blood-2011-02-338004

Moodycliffe, A. M., Nghiem, D., Clydesdale, G., \& Ullrich, S. E. (2000). Immune suppression and skin cancer development: regulation by NKT cells. [Research 
Support, U.S. Gov't, P.H.S.]. Nature immunology, 1(6), 521-525. doi: $10.1038 / 82782$

Moore, A., McCarthy, L., \& Mills, K. H. (1999). The adjuvant combination monophosphoryl lipid A and QS21 switches $\mathrm{T}$ cell responses induced with a soluble recombinant HIV protein from Th2 to Th1. [Research Support, Non-U.S. Gov't]. Vaccine, 17(20-21), 2517-2527.

Moore, M. W., Carbone, F. R., \& Bevan, M. J. (1988). Introduction of soluble protein into the class I pathway of antigen processing and presentation. [Research Support, Non-U.S. Gov't Research Support, U.S. Gov't, P.H.S.]. Cell, 54(6), 777-785.

Morgan, A. J., \& Parker, S. (2007). Translational mini-review series on vaccines: The Edward Jenner Museum and the history of vaccination. [Biography Historical Article Portraits]. Clinical and experimental immunology, 147(3), 389-394. doi: 10.1111/j.1365-2249.2006.03304.x

Morgan, R. A., Dudley, M. E., Wunderlich, J. R., Hughes, M. S., Yang, J. C., Sherry, R. M., . . . Rosenberg, S. A. (2006). Cancer regression in patients after transfer of genetically engineered lymphocytes. [Clinical Trial Research Support, N.I.H., Intramural]. Science, 314(5796), 126-129. doi: 10.1126/science.1129003

Morita, M., Motoki, K., Akimoto, K., Natori, T., Sakai, T., Sawa, E., . . Fukushima, H. (1995). Structure-activity relationship of alpha-galactosylceramides against B16bearing mice. J Med Chem, 38(12), 2176-2187.

Morse, M. A., Coleman, R. E., Akabani, G., Niehaus, N., Coleman, D., \& Lyerly, H. K. (1999). Migration of human dendritic cells after injection in patients with metastatic malignancies. [Research Support, U.S. Gov't, P.H.S.]. Cancer research, 59(1), 56-58.

Moser, M., \& Murphy, K. M. (2000). Dendritic cell regulation of TH1-TH2 development. [Review]. Nature immunology, 1(3), 199-205. doi: 10.1038/79734

Mullins, D. W., Sheasley, S. L., Ream, R. M., Bullock, T. N., Fu, Y. X., \& Engelhard, V. H. (2003). Route of immunization with peptide-pulsed dendritic cells controls the distribution of memory and effector T cells in lymphoid tissues and determines the pattern of regional tumor control. [Research Support, Non-U.S. Gov't Research Support, U.S. Gov't, P.H.S.]. The Journal of experimental medicine, 198(7), 10231034. doi: $10.1084 /$ jem.20021348

Muzio, M., Bosisio, D., Polentarutti, N., D'Amico, G., Stoppacciaro, A., Mancinelli, R., . . . Mantovani, A. (2000). Differential expression and regulation of toll-like receptors (TLR) in human leukocytes: selective expression of TLR3 in dendritic cells. [Research Support, Non-U.S. Gov't]. Journal of immunology, 164(11), 5998-6004. 
Naik, S. H., Metcalf, D., van Nieuwenhuijze, A., Wicks, I., Wu, L., O'Keeffe, M., Shortman, K. (2006). Intrasplenic steady-state dendritic cell precursors that are distinct from monocytes. Nat Immunol(7), 663-671.

Nair, S. K., Hull, S., Coleman, D., Gilboa, E., Lyerly, H. K., \& Morse, M. A. (1999). Induction of carcinoembryonic antigen (CEA)-specific cytotoxic T-lymphocyte responses in vitro using autologous dendritic cells loaded with CEA peptide or CEA RNA in patients with metastatic malignancies expressing CEA. [Research Support, Non-U.S. Gov't Research Support, U.S. Gov't, P.H.S.]. International journal of cancer. Journal international du cancer, 82(1), 121-124.

Nair, S. K., Morse, M., Boczkowski, D., Cumming, R. I., Vasovic, L., Gilboa, E., \& Lyerly, H. K. (2002). Induction of tumor-specific cytotoxic T lymphocytes in cancer patients by autologous tumor RNA-transfected dendritic cells. [Clinical Trial In Vitro Research Support, Non-U.S. Gov't Research Support, U.S. Gov't, P.H.S.]. Annals of surgery, 235(4), 540-549.

Nakagawa, R., Inui, T., Nagafune, I., Tazunoki, Y., Motoki, K., Yamauchi, A., . . Seki, S. (2004). Essential role of bystander cytotoxic CD122+CD8+ T cells for the antitumor immunity induced in the liver of mice by alpha-galactosylceramide. $J$ Immunol, 172(11), 6550-6557.

Napolitani, G., Rinaldi, A., Bertoni, F., Sallusto, F., \& Lanzavecchia, A. (2005). Selected Toll-like receptor agonist combinations synergistically trigger a $\mathrm{T}$ helper type 1polarizing program in dendritic cells. [Research Support, N.I.H., Extramural Research Support, Non-U.S. Gov't Research Support, U.S. Gov't, P.H.S.]. Nature immunology, 6(8), 769-776. doi: 10.1038/ni1223

Nauts, H. C. (1980). The beneficial effects of bacterial infections on host resistance to cancer: end results in 449 cases. (Vol. 2nd Ed). New York: New York: Cancer Research Institue.

Nauts, H. C., Fowler, G. A., \& Bogatko, F. H. (1953). A review of the influence of bacterial infection and of bacterial products (Coley's toxins) on malignant tumors in man; a critical analysis of 30 inoperable cases treated by Coley's mixed toxins, in which diagnosis was confirmed by microscopic examination selected for special study. Acta medica Scandinavica. Supplementum, 276, 1-103.

Neijssen, J., Herberts, C., Drijfhout, J. W., Reits, E., Janssen, L., \& Neefjes, J. (2005). Cross-presentation by intercellular peptide transfer through gap junctions. [Research Support, Non-U.S. Gov't]. Nature, 434(7029), 83-88. doi: 10.1038 /nature 03290

Nestle, F. O., Alijagic, S., Gilliet, M., Sun, Y., Grabbe, S., Dummer, R., . . S Schadendorf, D. (1998). Vaccination of melanoma patients with peptide- or tumor lysate-pulsed dendritic cells. [Clinical Trial Research Support, Non-U.S. Gov't]. Nature medicine, 4(3), 328-332. 
Norbury, C. C., Basta, S., Donohue, K. B., Tscharke, D. C., Princiotta, M. F., Berglund, P., . . . Yewdell, J. W. (2004). CD8+ T cell cross-priming via transfer of proteasome substrates. [Research Support, Non-U.S. Gov't Research Support, U.S. Gov't, P.H.S.]. Science, 304(5675), 1318-1321. doi: 10.1126/science.1096378

Norbury, C. C., Hewlett, L. J., Prescott, A. R., Shastri, N., \& Watts, C. (1995). Class I MHC presentation of exogenous soluble antigen via macropinocytosis in bone marrow macrophages. [Research Support, Non-U.S. Gov't]. Immunity, 3(6), 783791.

Ohashi, K., Burkart, V., Flohe, S., \& Kolb, H. (2000). Cutting edge: heat shock protein 60 is a putative endogenous ligand of the toll-like receptor-4 complex. [Research Support, Non-U.S. Gov't]. Journal of immunology, 164(2), 558-561.

Ohnmacht, C., Pullner, A., King, S. B., Drexler, I., Meier, S., Brocker, T., \& Voehringer, D. (2009). Constitutive ablation of dendritic cells breaks self-tolerance of CD4 T cells and results in spontaneous fatal autoimmunity. [Research Support, Non-U.S. Gov't]. The Journal of experimental medicine, 206(3), 549-559. doi: $10.1084 /$ jem.20082394

Okamoto, H., Shoin, S., Koshimura, S., \& Shimizu, R. (1967). Studies on the anticancer and streptolysin S-forming abilities of hemolytic streptococci. [Review]. Japanese journal of microbiology, 11(4), 323-326.

Okamoto, M., Oshikawa, T., Tano, T., Ahmed, S. U., Kan, S., Sasai, A., . . Sato, M. (2006). Mechanism of anticancer host response induced by OK-432, a streptococcal preparation, mediated by phagocytosis and Toll-like receptor 4 signaling. [Research Support, Non-U.S. Gov't]. Journal of immunotherapy, 29(1), 78-86.

Okamura, Y., Watari, M., Jerud, E. S., Young, D. W., Ishizaka, S. T., Rose, J., . . Strauss, J. F., 3rd. (2001). The extra domain A of fibronectin activates Toll-like receptor 4. [Research Support, Non-U.S. Gov't Research Support, U.S. Gov't, P.H.S.]. The Journal of biological chemistry, 276(13), 10229-10233. doi: 10.1074/jbc.M100099200

Osada, T., Nagawa, H., \& Shibata, Y. (2004). Tumor-infiltrating effector cells of alphagalactosylceramide-induced antitumor immunity in metastatic liver tumor. $J$ Immune Based Ther Vaccines, 2(1), 7. doi: 10.1186/1476-8518-2-7

Otto, F., Schmid, P., Mackensen, A., Wehr, U., Seiz, A., Braun, M., . . . Engelhardt, R. (1996). Phase II trial of intravenous endotoxin in patients with colorectal and nonsmall cell lung cancer. [Clinical Trial Clinical Trial, Phase II Research Support, Non-U.S. Gov't]. European journal of cancer, 32A(10), 1712-1718. 
Palmer, C., Diehn, M., Alizadeh, A. A., \& Brown, P. O. (2006). Cell-type specific gene expression profiles of leukocytes in human peripheral blood. [Comparative Study Research Support, N.I.H., Extramural Research Support, Non-U.S. Gov't]. BMC genomics, 7, 115. doi: 10.1186/1471-2164-7-115

Palucka, K., Ueno, H., Fay, J., \& Banchereau, J. (2009). Harnessing dendritic cells to generate cancer vaccines. [Research Support, N.I.H., Extramural Research Support, Non-U.S. Gov't]. Annals of the New York Academy of Sciences, 1174, 88-98. doi: 10.1111/j.1749-6632.2009.05000.x

Pamer, E., \& Cresswell, P. (1998). Mechanisms of MHC class I--restricted antigen processing. [Research Support, Non-U.S. Gov't Research Support, U.S. Gov't, P.H.S. Review]. Annual review of immunology, 16, 323-358. doi: 10.1146/annurev.immunol.16.1.323

Parekh, V. V., Wilson, M. T., Olivares-Villagomez, D., Singh, A. K., Wu, L., Wang, C. R., ... Van Kaer, L. (2005a). Glycolipid antigen induces long-term natural killer T cell anergy in mice. [Research Support, N.I.H., Extramural Research Support, Non-U.S. Gov't Research Support, U.S. Gov't, P.H.S.]. The Journal of clinical investigation, 115(9), 2572-2583. doi: 10.1172/JCI24762

Parekh, V. V., Wilson, M. T., \& Van Kaer, L. (2005b). iNKT-cell responses to glycolipids. [Research Support, N.I.H., Extramural Research Support, U.S. Gov't, P.H.S. Review]. Critical reviews in immunology, 25(3), 183-213.

Parish, H. J. (1968). Victory with vaccines : the story of immunization. Edinburgh ; London: E. \& S. Livingstone.

Pasare, C., \& Medzhitov, R. (2004). Toll-dependent control mechanisms of CD4 T cell activation. [Research Support, Non-U.S. Gov't Research Support, U.S. Gov't, P.H.S.]. Immunity, 21(5), 733-741. doi: 10.1016/j.immuni.2004.10.006

Pasare, C., \& Medzhitov, R. (2005). Toll-like receptors: linking innate and adaptive immunity. [Review]. Advances in experimental medicine and biology, 560, 11-18. doi: 10.1007/0-387-24180-9_2

Pecher, G., Haring, A., Kaiser, L., \& Thiel, E. (2002). Mucin gene (MUC1) transfected dendritic cells as vaccine: results of a phase I/II clinical trial. [Clinical Trial Clinical Trial, Phase I Clinical Trial, Phase II Research Support, Non-U.S. Gov't]. Cancer immunology, immunotherapy : CII, 51(11-12), 669-673. doi: $10.1007 / \mathrm{s} 00262-002-0317-\mathrm{z}$

Pelayo, R., Hirose, J., Huang, J., Garrett, K. P., Delogu, A., Busslinger, M., \& Kincade, P. W. (2005). Derivation of 2 categories of plasmacytoid dendritic cells in murine bone marrow. [Research Support, N.I.H., Extramural Research Support, Non-U.S. Gov't Research Support, U.S. Gov't, P.H.S.]. Blood, 105(11), 4407-4415. doi: 10.1182/blood-2004-07-2529 
Pennant. (1777). British Zoology. 3.

Pertl, U., Luster, A. D., Varki, N. M., Homann, D., Gaedicke, G., Reisfeld, R. A., \& Lode, H. N. (2001). IFN-gamma-inducible protein-10 is essential for the generation of a protective tumor-specific CD8 T cell response induced by single-chain IL-12 gene therapy. [Research Support, Non-U.S. Gov't Research Support, U.S. Gov't, P.H.S.]. Journal of immunology, 166(11), 6944-6951.

Petersen, T. R., Sika-Paotonu, D., Knight, D. A., Simkins, H. M., \& Hermans, I. F. (2011). Exploiting the role of endogenous lymphoid-resident dendritic cells in the priming of NKT cells and CD8+ T cells to dendritic cell-based vaccines. [Research Support, Non-U.S. Gov't]. PloS one, 6(3), e17657. doi: 10.1371/journal.pone.0017657

Pfeifer, J. D., Wick, M. J., Roberts, R. L., Findlay, K., Normark, S. J., \& Harding, C. V. (1993). Phagocytic processing of bacterial antigens for class I MHC presentation to T cells. Nature, 361(6410), 359-362. doi: 10.1038/361359a0

Pham, N. L., Badovinac, V. P., \& Harty, J. T. (2011). Differential role of "Signal 3" inflammatory cytokines in regulating CD8 T cell expansion and differentiation in vivo. Front Immunol, 2, 4. doi: 10.3389/fimmu.2011.00004

Pierre, P., Denzin, L. K., Hammond, C., Drake, J. R., Amigorena, S., Cresswell, P., \& Mellman, I. (1996). HLA-DM is localized to conventional and unconventional MHC class II-containing endocytic compartments. [Research Support, Non-U.S. Gov't Research Support, U.S. Gov't, P.H.S.]. Immunity, 4(3), 229-239.

Pisitkun, P., Deane, J. A., Difilippantonio, M. J., Tarasenko, T., Satterthwaite, A. B., \& Bolland, S. (2006). Autoreactive B cell responses to RNA-related antigens due to TLR7 gene duplication. [Research Support, N.I.H., Intramural Research Support, Non-U.S. Gov't]. Science, 312(5780), 1669-1672. doi: 10.1126/science.1124978

Platt, C. D., Ma, J. K., Chalouni, C., Ebersold, M., Bou-Reslan, H., Carano, R. A., . . . Delamarre, L. (2010). Mature dendritic cells use endocytic receptors to capture and present antigens. [Research Support, N.I.H., Extramural Research Support, NonU.S. Gov't]. Proceedings of the National Academy of Sciences of the United States of America, 107(9), 4287-4292. doi: 10.1073/pnas.0910609107

Poltorak, A., He, X., Smirnova, I., Liu, M. Y., Van Huffel, C., Du, X., . . . Beutler, B. (1998). Defective LPS signaling in $\mathrm{C} 3 \mathrm{H} / \mathrm{HeJ}$ and $\mathrm{C} 57 \mathrm{BL} / 10 \mathrm{ScCr}$ mice: mutations in Tlr4 gene. [Research Support, Non-U.S. Gov't]. Science, 282(5396), 2085-2088.

Pooley, J. L., Heath, W. R., \& Shortman, K. (2001). Cutting edge: intravenous soluble antigen is presented to CD4 T cells by CD8- dendritic cells, but cross-presented to CD8 T cells by CD8+ dendritic cells. [Comparative Study Research Support, NonU.S. Gov't]. Journal of immunology, 166(9), 5327-5330. 
Poulin, L. F., Henri, S., de Bovis, B., Devilard, E., Kissenpfennig, A., Malissen, B. . (2007). The dermis contains langerin+ dendritic cells that develop and function independantly of Langerhans cells. J Exp Med(204), 3119-3131.

Poulin, L. F., Salio, M., Griessinger, E., Anjos-Afonso, F., Craciun, L., Chen, J. L., . . . Reis e Sousa, C. (2010). Characterization of human DNGR-1+ BDCA3+ leukocytes as putative equivalents of mouse CD8alpha+ dendritic cells. [Research Support, Non-U.S. Gov't]. The Journal of experimental medicine, 207(6), 12611271. doi: $10.1084 /$ jem. 20092618

Pribila, J. T., Itano, A. A., Mueller, K. L., \& Shimizu, Y. (2004). The alpha 1 beta 1 and alpha $\mathrm{E}$ beta 7 integrins define a subset of dendritic cells in peripheral lymph nodes with unique adhesive and antigen uptake properties. [Research Support, Non-U.S. Gov't Research Support, U.S. Gov't, P.H.S.]. Journal of immunology, 172(1), 282291.

Prussin, C., \& Foster, B. (1997). TCR V alpha 24 and V beta 11 coexpression defines a human NK1 T cell analog containing a unique Th0 subpopulation. [Research Support, U.S. Gov't, P.H.S.]. Journal of immunology, 159(12), 5862-5870.

Pufnock, J. S., Cigal, M., Rolczynski, L. S., Andersen-Nissen, E., Wolfl, M., McElrath, M. J., \& Greenberg, P. D. (2011). Priming CD8+ T cells with dendritic cells matured using TLR4 and TLR7/8 ligands together enhances generation of CD8+ T cells retaining CD28. [Evaluation Studies Research Support, N.I.H., Extramural Research Support, Non-U.S. Gov't]. Blood, 117(24), 6542-6551. doi: 10.1182/blood-2010-11-317966

Qiu, C. H., Miyake, Y., Kaise, H., Kitamura, H., Ohara, O., \& Tanaka, M. (2009). Novel subset of CD8 $\{$ alpha $\}+$ dendritic cells localized in the marginal zone is responsible for tolerance to cell-associated antigens. [Research Support, Non-U.S. Gov't]. Journal of immunology, 182(7), 4127-4136. doi: 10.4049/jimmunol.0803364

Qu, C., Nguyen, V. A., Merad, M., \& Randolph, G. J. (2009). MHC class I/peptide transfer between dendritic cells overcomes poor cross-presentation by monocyte-derived APCs that engulf dying cells. [Research Support, N.I.H., Extramural Research Support, Non-U.S. Gov't Research Support, U.S. Gov't, Non-P.H.S.]. Journal of immunology, 182(6), 3650-3659. doi: 10.4049/jimmunol.0801532

Rachitskaya, A. V., Hansen, A. M., Horai, R., Li, Z., Villasmil, R., Luger, D., . . Caspi, R. R. (2008). Cutting edge: NKT cells constitutively express IL-23 receptor and RORgammat and rapidly produce IL-17 upon receptor ligation in an IL-6independent fashion. [Research Support, N.I.H., Intramural Research Support, Non-U.S. Gov't]. J Immunol, 180(8), 5167-5171.

Ratzinger, G. e. a. (2002). Matrix metalloproteases 9 and 2 are necessary for the migration of Langerhans cells and dermal dendritic cells human and mice skin. J Immunol, 168, 4361-4371. 
Rebollo, J., Llorente, I., Yoldi, A. (1990). Spontaneous tumour remission in adult acute leukemia. Cancer(34), 141-142.

Reid, P. A., \& Watts, C. (1990). Cycling of cell-surface MHC glycoproteins through primaquine-sensitive intracellular compartments. [Research Support, Non-U.S. Gov't]. Nature, 346(6285), 655-657. doi: 10.1038/346655a0

Reis e Sousa, C. (2006). Dendritic cells in a mature age. Nat Rev Immunol, 6, 476-483.

Reis e Sousa, C., \& Germain, R. N. (1995). Major histocompatibility complex class I presentation of peptides derived from soluble exogenous antigen by a subset of cells engaged in phagocytosis. [Research Support, U.S. Gov't, P.H.S.]. The Journal of experimental medicine, 182(3), 841-851.

Reis e Sousa, C., \& Germain, R. N. (1999). Analysis of adjuvant function by direct visualization of antigen presentation in vivo: endotoxin promotes accumulation of antigen-bearing dendritic cells in the $\mathrm{T}$ cell areas of lymphoid tissue. [Research Support, U.S. Gov't, P.H.S.]. Journal of immunology, 162(11), 6552-6561.

Reis e Sousa, C., Sher, A., \& Kaye, P. (1999). The role of dendritic cells in the induction and regulation of immunity to microbial infection. [Review]. Current opinion in immunology, 11(4), 392-399. doi: 10.1016/S0952-7915(99)80066-1

Reits, E., Neijssen, J., Herberts, C., Benckhuijsen, W., Janssen, L., Drijfhout, J. W., \& Neefjes, J. (2004). A major role for TPPII in trimming proteasomal degradation products for MHC class I antigen presentation. [Research Support, Non-U.S. Gov't]. Immunity, 20(4), 495-506.

Ribi, E., Cantrell, J. L., Takayama, K., Qureshi, N., Peterson, J., \& Ribi, H. O. (1984). Lipid A and immunotherapy. Reviews of infectious diseases, 6(4), 567-572.

Richardson, M. A., Ramirez, T., Russell, N. C., \& Moye, L. A. (1999). Coley toxins immunotherapy: a retrospective review. [Comparative Study Research Support, Non-U.S. Gov't Research Support, U.S. Gov't, P.H.S.]. Alternative therapies in health and medicine, 5(3), 42-47.

Ridge, J. P., Di Rosa, F. \& Matzinger, P.A. (1998). A conditioned dendritic cell can be a temporal bridge between a CD4+ T helper and a T-killer cell. Nature, 393, 474478.

Riedel, S. (2005). Edward Jenner and the history of smallpox and vaccination. Proceedings, 18(1), 21-25.

Rietschel, E. T., Kirikae, T., Schade, F. U., Mamat, U., Schmidt, G., Loppnow, H., . . et al. (1994). Bacterial endotoxin: molecular relationships of structure to activity and function. [Research Support, Non-U.S. Gov't Review]. FASEB journal : official 
publication of the Federation of American Societies for Experimental Biology, 8(2), 217-225.

Robert, L. M. S. (1812). L'art de prevenir le cancer au sein chez les femmes qui taouchent a leur epoque critique. (155).

Rock, K. L., Hearn, A., Chen, C. J., \& Shi, Y. (2005). Natural endogenous adjuvants. [Historical Article Review]. Springer seminars in immunopathology, 26(3), 231246. doi: 10.1007/s00281-004-0173-3

Rodriguez, A., Regnault, A., Kleijmeer, M., Ricciardi-Castagnoli, P., \& Amigorena, S. (1999). Selective transport of internalized antigens to the cytosol for MHC class I presentation in dendritic cells. Nature cell biology, 1(6), 362-368. doi: $10.1038 / 14058$

Roelofs, M. F., Joosten, L. A., Abdollahi-Roodsaz, S., van Lieshout, A. W., Sprong, T., van den Hoogen, F. H., . . . Radstake, T. R. (2005). The expression of toll-like receptors 3 and 7 in rheumatoid arthritis synovium is increased and costimulation of toll-like receptors 3, 4, and 7/8 results in synergistic cytokine production by dendritic cells. [Research Support, Non-U.S. Gov't]. Arthritis and rheumatism, 52(8), 2313-2322. doi: 10.1002/art.21278

Romani, N., Gruner, S., Brang, D., Kampgen, E., Lenz, A., Trockenbacher, B., . . . Schuler, G. (1994). Proliferating dendritic cell progenitors in human blood. [Research Support, Non-U.S. Gov't Research Support, U.S. Gov't, P.H.S.]. The Journal of experimental medicine, 180(1), 83-93.

Ronchese, F., \& Hausmann, B. (1993). B lymphocytes in vivo fail to prime naive T cells but can stimulate antigen-experienced $\mathrm{T}$ lymphocytes. [Comparative Study Research Support, Non-U.S. Gov't]. The Journal of experimental medicine, 177(3), 679-690.

Ruffer, M. A., \& Moodie, R. L. (1921). Studies in the palaeopathology of Egypt. Chicago, Ill: The University of Chicago press.

Ruffer, M. A., \& Rietti, A. (1910). Remarks on the histology and pathological anatomy of Egyptian mummies. Alexandria: A. Moures.

Saito, M., Iwawaki, T., Taya, C., Yonekawa, H., Noda, M., Inui, Y., . . Kohno, K. (2001). Diphtheria toxin receptor-mediated conditional and targeted cell ablation in transgenic mice. [Research Support, Non-U.S. Gov't]. Nature biotechnology, 19(8), 746-750. doi: 10.1038/90795

Salim, S. Y., Silva, M. A., Keita, A. V., Larsson, M., Andersson, P., Magnusson, K. E., . . Soderholm, J. D. (2009). CD83+CCR7- dendritic cells accumulate in the subepithelial dome and internalize translocated Escherichia coli HB101 in the 
Peyer's patches of ileal Crohn's disease. [Research Support, Non-U.S. Gov't]. The American journal of pathology, 174(1), 82-90. doi: 10.2353/ajpath.2009.080273

Sallusto, F., Cella, M., Danieli, C., \& Lanzavecchia, A. (1995). Dendritic cells use macropinocytosis and the mannose receptor to concentrate macromolecules in the major histocompatibility complex class II compartment: downregulation by cytokines and bacterial products. [In Vitro Research Support, Non-U.S. Gov't]. The Journal of experimental medicine, 182(2), 389-400.

Sancho, D., Mourao-Sa, D., Joffre, O. P., Schulz, O., Rogers, N. C., Pennington, D. J., . . Reis e Sousa, C. (2008). Tumor therapy in mice via antigen targeting to a novel, DC-restricted C-type lectin. [Research Support, Non-U.S. Gov't]. The Journal of clinical investigation, 118(6), 2098-2110. doi: 10.1172/JCI34584

Saric, T., Chang, S. C., Hattori, A., York, I. A., Markant, S., Rock, K. L., . . Goldberg, A. L. (2002). An IFN-gamma-induced aminopeptidase in the ER, ERAP1, trims precursors to MHC class I-presented peptides. [Research Support, U.S. Gov't, P.H.S.]. Nature immunology, 3(12), 1169-1176. doi: 10.1038/ni859

Sato, M., Harada, K., Yoshida, H., Yura, Y., Azuma, M., Iga, H., . . Takegawa, Y. (1997). Therapy for oral squamous cell carcinoma by tegafur and streptococcal agent OK432 in combination with radiotherapy: association of the therapeutic effect with differentiation and apoptosis in the cancer cells. Apoptosis : an international journal on programmed cell death, 2(2), 227-238.

Sato, S., Nomura, F., Kawai, T., Takeuchi, O., Muhlradt, P. F., Takeda, K., \& Akira, S. (2000). Synergy and cross-tolerance between toll-like receptor (TLR) 2- and TLR4mediated signaling pathways. [Research Support, Non-U.S. Gov't]. Journal of immunology, 165(12), 7096-7101.

Sauter, B., Albert, M. L., Francisco, L., Larsson, M., Somersan, S., \& Bhardwaj, N. (2000). Consequences of cell death: exposure to necrotic tumor cells, but not primary tissue cells or apoptotic cells, induces the maturation of immunostimulatory dendritic cells. [Research Support, Non-U.S. Gov't Research Support, U.S. Gov't, P.H.S.]. The Journal of experimental medicine, 191(3), 423434.

Savina, A., \& Amigorena, S. (2007). Phagocytosis and antigen presentation in dendritic cells. [Review]. Immunological reviews, 219, 143-156. doi: 10.1111/j.1600065X.2007.00552.X

Schirmbeck, R., \& Reimann, J. (1996). 'Empty' Ld molecules capture peptides from endocytosed hepatitis B surface antigen particles for major histocompatibility complex class I-restricted presentation. [Research Support, Non-U.S. Gov't]. European journal of immunology, 26(12), 2812-2822. doi: 10.1002/eji.1830261204 
Schletter, J., Brade, H., Brade, L., Kruger, C., Loppnow, H., Kusumoto, S., . . . Ulmer, A. J. (1995). Binding of lipopolysaccharide (LPS) to an 80-kilodalton membrane protein of human cells is mediated by soluble CD14 and LPS-binding protein. [In Vitro Research Support, Non-U.S. Gov't]. Infection and immunity, 63(7), 25762580 .

Schletter, J., Heine, H., Ulmer, A. J., \& Rietschel, E. T. (1995). Molecular mechanisms of endotoxin activity. [Research Support, Non-U.S. Gov't Review]. Archives of microbiology, 164(6), 383-389.

Schmitz, M., Diestelkoetter, P., Weigle, B., Schmachtenberg, F., Stevanovic, S., Ockert, D., . . . Rieber, E. P. (2000). Generation of survivin-specific CD8+ T effector cells by dendritic cells pulsed with protein or selected peptides. [Research Support, NonU.S. Gov't]. Cancer research, 60(17), 4845-4849.

Schnare, M., Holt, A. C., Takeda, K., Akira, S., \& Medzhitov, R. (2000). Recognition of CpG DNA is mediated by signaling pathways dependent on the adaptor protein MyD88. [Research Support, Non-U.S. Gov't]. Current biology : CB, 10(18), 11391142.

Schnorrer, P., Behrens, G. M., Wilson, N. S., Pooley, J. L., Smith, C. M., El-Sukkari, D., . . . Villadangos, J. A. (2006). The dominant role of CD8+ dendritic cells in crosspresentation is not dictated by antigen capture. [Research Support, Non-U.S. Gov't]. Proceedings of the National Academy of Sciences of the United States of America, 103(28), 10729-10734. doi: 10.1073/pnas.0601956103

Schoenberger, S. P., Toes, R. E., van der Voort, E. I., Offringa, R., \& Melief, C. J. (1998). T-cell help for cytotoxic T lymphocytes is mediated by CD40-CD40L interactions. [Research Support, Non-U.S. Gov't]. Nature, 393(6684), 480-483. doi: $10.1038 / 31002$

Schuler-Thurner, B., Schultz, E. S., Berger, T. G., Weinlich, G., Ebner, S., Woerl, P., . . Schuler, G. (2002). Rapid induction of tumor-specific type $1 \mathrm{~T}$ helper cells in metastatic melanoma patients by vaccination with mature, cryopreserved, peptideloaded monocyte-derived dendritic cells. [Clinical Trial Research Support, NonU.S. Gov't]. The Journal of experimental medicine, 195(10), 1279-1288.

Schulz, O., Edwards, A. D., Schito, M., Aliberti, J., Manickasingham, S., Sher, A., \& Reis e Sousa, C. (2000). CD40 triggering of heterodimeric IL-12 p70 production by dendritic cells in vivo requires a microbial priming signal. [Research Support, NonU.S. Gov't Research Support, U.S. Gov't, P.H.S.]. Immunity, 13(4), 453-462.

Schulz, O., \& Reis e Sousa, C. (2002). Cross-presentation of cell-associated antigens by CD8alpha+ dendritic cells is attributable to their ability to internalize dead cells. [Research Support, Non-U.S. Gov't]. Immunology, 107(2), 183-189. 
Schuster, J. M., \& Nelson, P. S. (2000). Toll receptors: an expanding role in our understanding of human disease. [Review]. Journal of leukocyte biology, 67(6), 767-773.

Segura, E., Amigorena, S., \& Thery, C. (2005). Mature dendritic cells secrete exosomes with strong ability to induce antigen-specific effector immune responses. [Research Support, Non-U.S. Gov't]. Blood cells, molecules \& diseases, 35(2), 89-93. doi: 10.1016/j.bcmd.2005.05.003

Segura, E., and Villadangos, J.A. (2009). Antigen presentation by dendritic cells in-vivo. . Current Opinion in Immunology 21(105-110).

Semmling, V., Lukacs-Kornek, V., Thaiss, C. A., Quast, T., Hochheiser, K., Panzer, U., . . . Kurts, C. (2010). Alternative cross-priming through CCL17-CCR4-mediated attraction of CTLs toward NKT cell-licensed DCs. [Research Support, Non-U.S. Gov't]. Nature immunology, 11(4), 313-320. doi: 10.1038/ni.1848

Serody, J. S., Collins, E. J., Tisch, R. M., Kuhns, J. J., \& Frelinger, J. A. (2000). T cell activity after dendritic cell vaccination is dependent on both the type of antigen and the mode of delivery. [Research Support, U.S. Gov't, Non-P.H.S. Research Support, U.S. Gov't, P.H.S.]. Journal of immunology, 164(9), 4961-4967.

Serwold, T., Gonzalez, F., Kim, J., Jacob, R., \& Shastri, N. (2002). ERAAP customizes peptides for MHC class I molecules in the endoplasmic reticulum. [Research Support, U.S. Gov't, P.H.S.]. Nature, 419(6906), 480-483. doi: 10.1038/nature 01074

Sette, A., Adorini, L., Colon, S. M., Buus, S., \& Grey, H. M. (1989). Capacity of intact proteins to bind to MHC class II molecules. Journal of immunology, 143(4), 12651267.

Shastri, N., Schwab, S., \& Serwold, T. (2002). Producing nature's gene-chips: the generation of peptides for display by MHC class I molecules. [Research Support, U.S. Gov't, P.H.S.]. Annual review of immunology, 20, 463-493. doi: 10.1146/annurev.immunol.20.100301.064819

Shen, L., \& Rock, K. L. (2004). Cellular protein is the source of cross-priming antigen in vivo. [Research Support, Non-U.S. Gov't Research Support, U.S. Gov't, P.H.S.]. Proceedings of the National Academy of Sciences of the United States of America, 101(9), 3035-3040. doi: 10.1073/pnas.0308345101

Shortman, K., \& Heath, W. R. (2010). The CD8+ dendritic cell subset. [Research Support, Non-U.S. Gov't Review]. Immunological reviews, 234(1), 18-31. doi: 10.1111/j.0105-2896.2009.00870.x

Silk, J. D., Hermans, I. F., Gileadi, U., Chong, T. W., Shepherd, D., Salio, M., . . . Cerundolo, V. (2004). Utilizing the adjuvant properties of CD1d-dependent NK T 
cells in T cell-mediated immunotherapy. [Research Support, Non-U.S. Gov't]. The Journal of clinical investigation, 114(12), 1800-1811. doi: 10.1172/JCI22046

Simkins, H. M., Hyde, E., Farrand, K. J., Ong, M. L., Degli-Esposti, M. A., Hermans, I. F., \& Ronchese, F. (2011). Administration of alpha-galactosylceramide impairs the survival of dendritic cell subpopulations in vivo. [Research Support, Non-U.S. Gov't]. Journal of leukocyte biology, 89(5), 753-762. doi: 10.1189/jlb.0910480

Singh-Jasuja, H., Thiolat, A., Ribon, M., Boissier, M. C., Bessis, N., Rammensee, H. G., \& Decker, P. (2013). The mouse dendritic cell marker CD11c is down-regulated upon cell activation through Toll-like receptor triggering. [Research Support, Non-U.S. Gov't]. Immunobiology, 218(1), 28-39. doi: 10.1016/j.imbio.2012.01.021

Smith, C. M., et al. (2004). Cognate CD4(+) T cell licensing of dendritic cells in CD8(+) T cell immunity. Nature immunology, 5, 1143-1148.

Smith, G. E. (1912). The royal mummies. In C. g. d. A. é. d. M. d. Caire (Ed.). Cairo: Imprimerie de l'Institut français d'Archéologie orientale.

Smith, G. L., \& McFadden, G. (2002). Smallpox: anything to declare? [Historical Article Portraits]. Nature reviews. Immunology, 2(7), 521-527. doi: 10.1038/nri845

Smyth, M. J., Crowe, N. Y., \& Godfrey, D. I. (2001). NK cells and NKT cells collaborate in host protection from methylcholanthrene-induced fibrosarcoma. [Comparative Study Research Support, Non-U.S. Gov't]. Int Immunol, 13(4), 459-463.

Smyth, M. J., Crowe, N. Y., Pellicci, D. G., Kyparissoudis, K., Kelly, J. M., Takeda, K., . . . Godfrey, D. I. (2002). Sequential production of interferon-gamma by NK1.1(+) T cells and natural killer cells is essential for the antimetastatic effect of alphagalactosylceramide. [Research Support, Non-U.S. Gov't]. Blood, 99(4), 1259-1266.

Smyth, M. J., \& Godfrey, D. I. (2000). NKT cells and tumor immunity--a double-edged sword. [Comment News]. Nat Immunol, 1(6), 459-460. doi: 10.1038/82698

Snijders, A., Kalinski, P., Hilkens, C. M., \& Kapsenberg, M. L. (1998). High-level IL-12 production by human dendritic cells requires two signals. [Research Support, NonU.S. Gov't]. International immunology, 10(11), 1593-1598.

Song, R., \& Harding, C. V. (1996). Roles of proteasomes, transporter for antigen presentation (TAP), and beta 2-microglobulin in the processing of bacterial or particulate antigens via an alternate class I MHC processing pathway. [In Vitro Research Support, Non-U.S. Gov't Research Support, U.S. Gov't, P.H.S.]. Journal of immunology, 156(11), 4182-4190.

Spaner, D. E., Shi, Y., White, D., Shaha, S., He, L., Masellis, A., . . Gorczynski, R. (2010). A phase I/II trial of TLR-7 agonist immunotherapy in chronic lymphocytic 
leukemia. [Clinical Trial, Phase I Clinical Trial, Phase II Letter Research Support, Non-U.S. Gov't]. Leukemia, 24(1), 222-226. doi: 10.1038/leu.2009.195

Spisek, R., Charalambous, A., Mazumder, A., Vesole, D. H., Jagannath, S., \& Dhodapkar, M. V. (2007). Bortezomib enhances dendritic cell (DC)-mediated induction of immunity to human myeloma via exposure of cell surface heat shock protein 90 on dying tumor cells: therapeutic implications. [Research Support, N.I.H., Extramural Research Support, Non-U.S. Gov't]. Blood, 109(11), 4839-4845. doi: 10.1182/blood-2006-10-054221

Sporri, R., Joller, N., Albers, U., Hilbi, H., \& Oxenius, A. (2006). MyD88-dependent IFNgamma production by NK cells is key for control of Legionella pneumophila infection. [Research Support, U.S. Gov't, Non-P.H.S.]. Journal of immunology, 176(10), 6162-6171.

Srivastava, P. K., \& Heike, M. (1991). Tumor-specific immunogenicity of stress-induced proteins: convergence of two evolutionary pathways of antigen presentation? [Research Support, Non-U.S. Gov't Research Support, U.S. Gov't, P.H.S. Review]. Seminars in immunology, 3(1), 57-64.

Stark, R. B. (1977). Immunization saves Washington's army. [Biography Historical Article]. Surgery, gynecology \& obstetrics, 144(3), 425-431.

Steinman, R. M. (1991). The dendritic cell system and its role in immunogenicity. [Research Support, U.S. Gov't, P.H.S. Review]. Annual review of immunology, 9, 271-296. doi: 10.1146/annurev.iy.09.040191.001415

Steinman, R. M., Hawiger, D., \& Nussenzweig, M. C. (2003). Tolerogenic dendritic cells. [Review]. Annual review of immunology, 21, 685-711. doi: 10.1146/annurev.immunol.21.120601.141040

Steinman, R. M., \& Pope, M. (2002). Exploiting dendritic cells to improve vaccine efficacy. [Research Support, Non-U.S. Gov't Research Support, U.S. Gov't, P.H.S. Review]. The Journal of clinical investigation, 109(12), 1519-1526. doi: 10.1172/JCI15962

Stern, L. J., \& Wiley, D. C. (1994). Antigenic peptide binding by class I and class II histocompatibility proteins. [Comparative Study Research Support, Non-U.S. Gov't Review]. Structure, 2(4), 245-251.

Stetson, D. B., Mohrs, M., Reinhardt, R. L., Baron, J. L., Wang, Z. E., Gapin, L., . . . Locksley, R. M. (2003). Constitutive cytokine mRNAs mark natural killer (NK) and NK T cells poised for rapid effector function. [Research Support, Non-U.S. Gov't Research Support, U.S. Gov't, P.H.S.]. J Exp Med, 198(7), 1069-1076. doi: 10.1084/jem.20030630 
Stober, D., Jomantaite, I., Schirmbeck, R., \& Reimann, J. (2003). NKT cells provide help for dendritic cell-dependent priming of MHC class I-restricted CD8+ T cells in vivo. [Comparative Study Research Support, Non-U.S. Gov't]. Journal of immunology, 170(5), 2540-2548.

Strayer, D. R., Carter, W. A., Brodsky, I., Cheney, P., Peterson, D., Salvato, P., . . e et al. (1994). A controlled clinical trial with a specifically configured RNA drug, poly(I).poly(C12U), in chronic fatigue syndrome. [Clinical Trial Multicenter Study Randomized Controlled Trial Research Support, Non-U.S. Gov't]. Clinical infectious diseases : an official publication of the Infectious Diseases Society of America, 18 Suppl 1, S88-95.

Su, Y., Testaverde, J. R., Davis, C. N., Hayajneh, W. A., Adair, R., \& Colberg-Poley, A. M. (2003). Human cytomegalovirus UL37 immediate early target minigene RNAs are accurately spliced and polyadenylated. [Research Support, Non-U.S. Gov't Research Support, U.S. Gov't, P.H.S.]. The Journal of general virology, 84(Pt 1), 29-39.

Suleman, M., Galea, S., Gavard, F., Merillon, N., Klonjkowski, B., Tartour, E., \& Richardson, J. (2011). Antigen encoded by vaccine vectors derived from human adenovirus serotype 5 is preferentially presented to CD8+ T lymphocytes by the CD8alpha+ dendritic cell subset. [Research Support, Non-U.S. Gov't]. Vaccine, 29(35), 5892-5903. doi: 10.1016/j.vaccine.2011.06.071

Summers deLuca, L., \& Gommerman, J. L. (2012). Fine-tuning of dendritic cell biology by the TNF superfamily. [Research Support, Non-U.S. Gov't Review]. Nature reviews. Immunology, 12(5), 339-351. doi: 10.1038/nri3193

Sureda, M., Subira, M. L., Martin Algarra, S., Prieto Valtuena, J., \& Sangro, B. (1990). [Spontaneous tumor regression. Report of 2 cases]. [Case Reports]. Medicina clinica, 95(8), 306-308.

Suzuki, N., Suzuki, S., \& Yeh, W. C. (2002). IRAK-4 as the central TIR signaling mediator in innate immunity. [Research Support, Non-U.S. Gov't Review]. Trends in immunology, 23(10), 503-506.

Swantek, J. L., Tsen, M. F., Cobb, M. H., \& Thomas, J. A. (2000). IL-1 receptorassociated kinase modulates host responsiveness to endotoxin. [Research Support, Non-U.S. Gov't Research Support, U.S. Gov't, P.H.S.]. Journal of immunology, 164(8), 4301-4306.

Tabeta, K., Georgel, P., Janssen, E., Du, X., Hoebe, K., Crozat, K., . . B Beutler, B. (2004). Toll-like receptors 9 and 3 as essential components of innate immune defense against mouse cytomegalovirus infection. [Research Support, Non-U.S. Gov't Research Support, U.S. Gov't, P.H.S.]. Proceedings of the National Academy of Sciences of the United States of America, 101(10), 3516-3521. doi: 10.1073/pnas.0400525101 
Takeda, K., \& Akira, S. (2005). Toll-like receptors in innate immunity. [Research Support, Non-U.S. Gov't Review]. International immunology, 17(1), 1-14. doi: 10.1093/intimm/dxh186

Takeda, K., Hayakawa, Y., Atsuta, M., Hong, S., Van Kaer, L., Kobayashi, K., . . . Okumura, K. (2000). Relative contribution of NK and NKT cells to the antimetastatic activities of IL-12. [Research Support, Non-U.S. Gov't]. International immunology, 12(6), 909-914.

Takeda, K., Kaisho, T., \& Akira, S. (2003). Toll-like receptors. [Research Support, NonU.S. Gov't Review]. Annual review of immunology, 21, 335-376. doi: 10.1146/annurev.immunol.21.120601.141126

Takeuchi, O., Hoshino, K., \& Akira, S. (2000). Cutting edge: TLR2-deficient and MyD88deficient mice are highly susceptible to Staphylococcus aureus infection. [Research Support, Non-U.S. Gov't]. Journal of immunology, 165(10), 5392-5396.

Takeuchi, O., Sato, S., Horiuchi, T., Hoshino, K., Takeda, K., Dong, Z., . . . Akira, S. (2002). Cutting edge: role of Toll-like receptor 1 in mediating immune response to microbial lipoproteins. [Research Support, Non-U.S. Gov't]. Journal of immunology, 169(1), 10-14.

Takeuchi, O., Takeda, K., Hoshino, K., Adachi, O., Ogawa, T., \& Akira, S. (2000). Cellular responses to bacterial cell wall components are mediated through MyD88dependent signaling cascades. [Research Support, Non-U.S. Gov't]. International immunology, 12(1), 113-117.

Tan, J., Town, T., Mori, T., Obregon, D., Wu, Y., DelleDonne, A., . . Mullan, M. (2002). CD40 is expressed and functional on neuronal cells. The EMBO journal, 21(4), 643-652.

Tanchou, S. (1844). Recherches sur le traitement médical des tumeurs cancéreuses du sein. Ouvrage practique basé sur trois cents observations (extraits d'un grand nombre d'auteurs). Paris: Paris: G Bailliere.

Tang, H. L., \& Cyster, J. G. (1999). Chemokine Up-regulation and activated T cell attraction by maturing dendritic cells. [Research Support, Non-U.S. Gov't Research Support, U.S. Gov't, P.H.S.]. Science, 284(5415), 819-822.

Taniguchi, T., \& Takaoka, A. (2002). The interferon-alpha/beta system in antiviral responses: a multimodal machinery of gene regulation by the IRF family of transcription factors. [Research Support, Non-U.S. Gov't Review]. Current opinion in immunology, 14(1), 111-116. 
Taniguchi, M., Seino, K., \& Nakayama, T. (2003). The NKT cell system: bridging innate and acquired immunity. [Comment News]. Nat Immunol, 4(12), 1164-1165. doi: 10.1038/ni1203-1164

Tauszig-Delamasure, S., Bilak, H., Capovilla, M., Hoffmann, J. A., \& Imler, J. L. (2002). Drosophila MyD88 is required for the response to fungal and Gram-positive bacterial infections. [Comparative Study]. Nature immunology, 3(1), 91-97. doi: $10.1038 /$ ni747

Tel, J., Smits, E. L., Anguille, S., Joshi, R. N., Figdor, C. G., \& de Vries, I. J. (2012). Human plasmacytoid dendritic cells are equipped with antigen-presenting and tumoricidal capacities. [Research Support, Non-U.S. Gov't]. Blood, 120(19), 39363944. doi: 10.1182/blood-2012-06-435941

Terabe, M., Swann, J., Ambrosino, E., Sinha, P., Takaku, S., Hayakawa, Y., . . Berzofsky, J. A. (2005). A nonclassical non-Valpha14Jalpha18 CD1d-restricted (type II) NKT cell is sufficient for down-regulation of tumor immunosurveillance. [Comparative Study Research Support, N.I.H., Extramural Research Support, N.I.H., Intramural Research Support, Non-U.S. Gov't]. J Exp Med, 202(12), 1627-1633. doi: $10.1084 /$ jem.20051381

Thery, C., Duban, L., Segura, E., Veron, P., Lantz, O., \& Amigorena, S. (2002a). Indirect activation of naive CD4 $+\mathrm{T}$ cells by dendritic cell-derived exosomes. [Research Support, Non-U.S. Gov't]. Nature immunology, 3(12), 1156-1162. doi: $10.1038 /$ ni854

Thery, C., Zitvogel, L., \& Amigorena, S. (2002b). Exosomes: composition, biogenesis and function. [Research Support, Non-U.S. Gov't]. Nature reviews. Immunology, 2(8), 569-579. doi: 10.1038/nri855

Thoelen, S., Van Damme, P., Mathei, C., Leroux-Roels, G., Desombere, I., Safary, A., . . . Meheus, A. (1998). Safety and immunogenicity of a hepatitis B vaccine formulated with a novel adjuvant system. [Clinical Trial Randomized Controlled Trial]. Vaccine, 16(7), 708-714.

Thomas, J. A., Allen, J. L., Tsen, M., Dubnicoff, T., Danao, J., Liao, X. C., . . . Wasserman, S. A. (1999). Impaired cytokine signaling in mice lacking the IL-1 receptor-associated kinase. [Research Support, Non-U.S. Gov't Research Support, U.S. Gov't, P.H.S.]. Journal of immunology, 163(2), 978-984.

Thompson, K. A., Strayer, D. R., Salvato, P. D., Thompson, C. E., Klimas, N., Molavi, A., . . Carter, W. A. (1996). Results of a double-blind placebo-controlled study of the double-stranded RNA drug polyI:polyC12U in the treatment of HIV infection. [Clinical Trial Randomized Controlled Trial]. European journal of clinical microbiology \& infectious diseases : official publication of the European Society of Clinical Microbiology, 15(7), 580-587. 
Thurner, B., Haendle, I., Roder, C., Dieckmann, D., Keikavoussi, P., Jonuleit, H., . . . Schuler, G. (1999). Vaccination with mage-3A1 peptide-pulsed mature, monocytederived dendritic cells expands specific cytotoxic $\mathrm{T}$ cells and induces regression of some metastases in advanced stage IV melanoma. [Clinical Trial Multicenter Study Research Support, Non-U.S. Gov't]. The Journal of experimental medicine, 190(11), 1669-1678.

Thurner, B., Roder, C., Dieckmann, D., Heuer, M., Kruse, M., Glaser, A., . . Schuler, G. (1999). Generation of large numbers of fully mature and stable dendritic cells from leukapheresis products for clinical application. [Research Support, Non-U.S. Gov't Review]. Journal of immunological methods, 223(1), 1-15.

Thursfield, H. (1940). Smallpox in the American war of Independance. Annals of Medical History(2), 312-318.

Ting, J. P., \& Davis, B. K. (2005). CATERPILLER: a novel gene family important in immunity, cell death, and diseases. [Research Support, N.I.H., Extramural Research Support, Non-U.S. Gov't Research Support, U.S. Gov't, P.H.S. Review]. Annual review of immunology, 23, 387-414. doi: 10.1146/annurev.immunol.23.021704.115616

Toura, I., Kawano, T., Akutsu, Y., Nakayama, T., Ochiai, T., \& Taniguchi, M. (1999). Cutting edge: inhibition of experimental tumor metastasis by dendritic cells pulsed with alpha-galactosylceramide. [Research Support, Non-U.S. Gov't]. Journal of immunology, 163(5), 2387-2391.

Trinchieri, G. (2003). Interleukin-12 and the regulation of innate resistance and adaptive immunity. [Review]. Nature reviews. Immunology, 3(2), 133-146. doi: 10.1038/nri1001

Trinchieri, G., Rengaraju, M., D'Andrea, A., Valiante, N. M., Kubin, M., Aste, M., \& Chehimi, J. (1993). Producer cells of interleukin-12. [Comment Letter]. Immunology today, 14(5), 237-238. doi: 10.1016/0167-5699(93)90173-I

Trinchieri, G., \& Sher, A. (2007). Cooperation of Toll-like receptor signals in innate immune defence. [Research Support, N.I.H., Intramural Review]. Nature reviews. Immunology, 7(3), 179-190. doi: 10.1038/nri2038

Trombetta, E. S., \& Mellman, I. (2005). Cell biology of antigen processing in vitro and in vivo. [Research Support, Non-U.S. Gov't Research Support, U.S. Gov't, P.H.S. Review]. Annual review of immunology, 23, 975-1028. doi: 10.1146/annurev.immunol.22.012703.104538

Tsan, M. F., \& Gao, B. (2004). Endogenous ligands of Toll-like receptors. [Research Support, U.S. Gov't, Non-P.H.S. Review]. Journal of leukocyte biology, 76(3), 514519. doi: 10.1189/jlb.0304127 
Tsuji, S., Matsumoto, M., Takeuchi, O., Akira, S., Azuma, I., Hayashi, A., . . . Seya, T. (2000). Maturation of human dendritic cells by cell wall skeleton of Mycobacterium bovis bacillus Calmette-Guerin: involvement of toll-like receptors. Infection and immunity, 68(12), 6883-6890.

Tvinnereim, A. R., Hamilton, S. E., \& Harty, J. T. (2004). Neutrophil involvement in cross-priming CD8 $+\mathrm{T}$ cell responses to bacterial antigens. [Comparative Study Research Support, U.S. Gov't, P.H.S.]. Journal of immunology, 173(3), 1994-2002.

Tzankov, A., Ludescher, C., Duba, H. C., Steinlechner, M., Knapp, R., Schmid, T., . . . Stauder, R. (2001). Spontaneous remission in a secondary acute myelogenous leukaemia following invasive pulmonary aspergillosis. [Case Reports Research Support, Non-U.S. Gov't]. Annals of hematology, 80(7), 423-425.

Uebel, S., \& Tampe, R. (1999). Specificity of the proteasome and the TAP transporter. [Review]. Current opinion in immunology, 11(2), 203-208.

Uehori, J., Fukase, K., Akazawa, T., Uematsu, S., Akira, S., Funami, K., . . Seya, T. (2005). Dendritic cell maturation induced by muramyl dipeptide (MDP) derivatives: monoacylated MDP confers TLR2/TLR4 activation. [Comparative Study Research Support, Non-U.S. Gov't]. Journal of immunology, 174(11), 70967103.

Uicker, W. C., Doyle, H. A., McCracken, J. P., Langlois, M., \& Buchanan, K. L. (2005). Cytokine and chemokine expression in the central nervous system associated with protective cell-mediated immunity against Cryptococcus neoformans. [Research Support, Non-U.S. Gov't]. Medical mycology, 43(1), 27-38.

Uldrich, A. P., Crowe, N. Y., Kyparissoudis, K., Pellicci, D. G., Zhan, Y., Lew, A. M., . . Godfrey, D. I. (2005). NKT cell stimulation with glycolipid antigen in vivo: costimulation-dependent expansion, Bim-dependent contraction, and hyporesponsiveness to further antigenic challenge. [Research Support, Non-U.S. Gov't]. Journal of immunology, 175(5), 3092-3101.

Ulrich, J. T., \& Myers, K. R. (1995). Monophosphoryl lipid A as an adjuvant. Past experiences and new directions. [Review]. Pharmaceutical biotechnology, 6, 495524.

Underhill, D. M. (2007). Collaboration between the innate immune receptors dectin-1, TLRs, and Nods. [Review]. Immunological reviews, 219, 75-87. doi: 10.1111/j.1600-065X.2007.00548.X

Unterholzner, L., Keating, S. E., Baran, M., Horan, K. A., Jensen, S. B., Sharma, S., . . Bowie, A. G. (2010). IFI16 is an innate immune sensor for intracellular DNA. [Research Support, N.I.H., Extramural Research Support, N.I.H., Intramural Research Support, Non-U.S. Gov't]. Nature immunology, 11(11), 997-1004. doi: 10.1038/ni. 1932 
Uss, E., Rowshani, A. T., Hooibrink, B., Lardy, N. M., van Lier, R. A., \& ten Berge, I. J. (2006). CD103 is a marker for alloantigen-induced regulatory CD8+ T cells. Journal of immunology, 177(5), 2775-2783.

Vabulas, R. M., Ahmad-Nejad, P., da Costa, C., Miethke, T., Kirschning, C. J., Hacker, H., \& Wagner, H. (2001). Endocytosed HSP60s use toll-like receptor 2 (TLR2) and TLR4 to activate the toll/interleukin-1 receptor signaling pathway in innate immune cells. [Research Support, Non-U.S. Gov't]. The Journal of biological chemistry, 276(33), 31332-31339. doi: 10.1074/jbc.M103217200

Vacchelli, E., Galluzzi, L., Eggermont, A., Fridman, W. H., Galon, J., Sautes-Fridman, C., . . . Kroemer, G. (2012). Trial watch: FDA-approved Toll-like receptor agonists for cancer therapy. Oncoimmunology, 1(6), 894-907. doi: 10.4161/onci.20931

van Kooten, C., \& Banchereau, J. (2000). CD40-CD40 ligand. [Research Support, NonU.S. Gov't Review]. Journal of leukocyte biology, 67(1), 2-17.

van Mierlo, G. J., den Boer, A. T., Medema, J. P., van der Voort, E. I., Fransen, M. F., Offringa, R., ... Toes, R. E. (2002). CD40 stimulation leads to effective therapy of CD40(-) tumors through induction of strong systemic cytotoxic $\mathrm{T}$ lymphocyte immunity. [Research Support, Non-U.S. Gov't]. Proceedings of the National Academy of Sciences of the United States of America, 99(8), 5561-5566. doi: 10.1073/pnas.082107699

Villadangos, J. A., \& Schnorrer, P. (2007). Intrinsic and cooperative antigen-presenting functions of dendritic-cell subsets in vivo. [Research Support, Non-U.S. Gov't Review]. Nature reviews. Immunology, 7(7), 543-555. doi: 10.1038/nri2103

Vivier, E., Ugolini, S., Blaise, D., Chabannon, C., \& Brossay, L. (2012). Targeting natural killer cells and natural killer $\mathrm{T}$ cells in cancer. [Research Support, N.I.H., Extramural Research Support, Non-U.S. Gov't Review]. Nature reviews. Immunology, 12(4), 239-252. doi: 10.1038/nri3174

Vollmer, J., Tluk, S., Schmitz, C., Hamm, S., Jurk, M., Forsbach, A., . . Krieg, A. M. (2005). Immune stimulation mediated by autoantigen binding sites within small nuclear RNAs involves Toll-like receptors 7 and 8. The Journal of experimental medicine, 202(11), 1575-1585. doi: 10.1084/jem.20051696

Vosika, G., Giddings, C., \& Gray, G. R. (1984a). Phase I study of intravenous mycobacterial cell wall skeleton and trehalose dimycolate attached to oil droplets. [Research Support, Non-U.S. Gov't Research Support, U.S. Gov't, Non-P.H.S. Research Support, U.S. Gov't, P.H.S.]. Journal of biological response modifiers, $3(6), 620-626$.

Vosika, G. J., Barr, C., \& Gilbertson, D. (1984b). Phase-I study of intravenous modified lipid A. [Research Support, Non-U.S. Gov't Research Support, U.S. Gov't, Non- 
P.H.S. Research Support, U.S. Gov't, P.H.S.]. Cancer immunology, immunotherapy : CII, 18(2), 107-112.

Vremec, D., J. Pooley, H. Hochrein, L. Wu, and K. Shortman. (2000). CD4 and CD8 expression by dendritic cell subtypes in mouse thymus and spleen. J Immunol, 164, 2978-2986.

Walton, K. L., He, J., Kelsall, B.L., Sartor, R.B., Fisher, N.C. . (2006). Dendritic cells in germ-free and specific pathogen-free mice have similar phenotypes and in vitro antigen presenting function. . Immunol Lett (102), 16-24

Wang, H., Rayburn, E., \& Zhang, R. (2005). Synthetic oligodeoxynucleotides containing deoxycytidyl-deoxyguanosine dinucleotides (CpG ODNs) and modified analogs as novel anticancer therapeutics. [Review]. Current pharmaceutical design, 11(22), 2889-2907.

Wang, J., Shao, Y., Bennett, T. A., Shankar, R. A., Wightman, P. D., \& Reddy, L. G. (2006). The functional effects of physical interactions among Toll-like receptors 7, 8, and 9. [In Vitro]. The Journal of biological chemistry, 281(49), 37427-37434. doi: 10.1074/jbc.M605311200

Wang, T., Lafuse, W. P., \& Zwilling, B. S. (2001). NFkappaB and Sp1 elements are necessary for maximal transcription of toll-like receptor 2 induced by Mycobacterium avium. [Research Support, U.S. Gov't, P.H.S.]. Journal of immunology, 167(12), 6924-6932.

Warger, T., Osterloh, P., Rechtsteiner, G., Fassbender, M., Heib, V., Schmid, B., . . . Radsak, M. P. (2006). Synergistic activation of dendritic cells by combined Tolllike receptor ligation induces superior CTL responses in vivo. [Research Support, Non-U.S. Gov't]. Blood, 108(2), 544-550. doi: 10.1182/blood-2005-10-4015

Watts, T. H. (2005). TNF/TNFR family members in costimulation of T cell responses. [Research Support, Non-U.S. Gov't Review]. Annual review of immunology, 23, 23-68. doi: 10.1146/annurev.immunol.23.021704.115839

Weinzierl, A. O., Szalay, G., Wolburg, H., Sauter, M., Rammensee, H. G., Kandolf, R., . . . Klingel, K. (2008). Effective chemokine secretion by dendritic cells and expansion of cross-presenting CD4-/CD8+ dendritic cells define a protective phenotype in the mouse model of coxsackievirus myocarditis. [Research Support, Non-U.S. Gov't]. Journal of virology, 82(16), 8149-8160. doi: 10.1128/JVI.00047-08

White, G. (1890). Letter XVIII to Thomas Pennant, 27 July 1768 The Natural History of Selborne (pp. 56). London: G. Routledge and Sons.

Whitmore, M. M., DeVeer, M. J., Edling, A., Oates, R. K., Simons, B., Lindner, D., \& Williams, B. R. (2004). Synergistic activation of innate immunity by doublestranded RNA and CpG DNA promotes enhanced antitumor activity. [Research 
Support, U.S. Gov't, P.H.S.]. Cancer research, 64(16), 5850-5860. doi: 10.1158/0008-5472.CAN-04-0063

Wick, M. J., \& Pfeifer, J. D. (1996). Major histocompatibility complex class I presentation of ovalbumin peptide 257-264 from exogenous sources: protein context influences the degree of TAP-independent presentation. [Research Support, Non-U.S. Gov't]. European journal of immunology, 26(11), 2790-2799. doi: 10.1002/eji.1830261135

Wiertz, E. J., Tortorella, D., Bogyo, M., Yu, J., Mothes, W., Jones, T. R., . . Ploegh, H. L. (1996). Sec61-mediated transfer of a membrane protein from the endoplasmic reticulum to the proteasome for destruction. [Research Support, Non-U.S. Gov't Research Support, U.S. Gov't, P.H.S.]. Nature, 384(6608), 432-438. doi: $10.1038 / 384432 \mathrm{a} 0$

Williams, M. A., \& Bevan, M. J. (2007). Effector and memory CTL differentiation. [Review]. Annual review of immunology, 25, 171-192. doi: 10.1146/annurev.immunol.25.022106.141548

Willis, N. J. (1997). Edward Jenner and the eradication of smallpox. [Biography Historical Article Portraits]. Scottish medical journal, 42(4), 118-121.

Wilson, M. T., Johansson, C., Olivares-Villagomez, D., Singh, A. K., Stanic, A. K., Wang, C. R., . . . Van Kaer, L. (2003). The response of natural killer T cells to glycolipid antigens is characterized by surface receptor down-modulation and expansion. [Research Support, Non-U.S. Gov't Research Support, U.S. Gov't, P.H.S.]. Proceedings of the National Academy of Sciences of the United States of America, 100(19), 10913-10918. doi: 10.1073/pnas.1833166100

Wilson, M. T., \& Van Kaer, L. (2003). Natural killer T cells as targets for therapeutic intervention in autoimmune diseases. [Research Support, Non-U.S. Gov't Research Support, U.S. Gov't, P.H.S. Review]. Current pharmaceutical design, 9(3), 201220.

Wilson, N. S., Behrens, G. M., Lundie, R. J., Smith, C. M., Waithman, J., Young, L., . . Villadangos, J. A. (2006). Systemic activation of dendritic cells by Toll-like receptor ligands or malaria infection impairs cross-presentation and antiviral immunity. [In Vitro Research Support, Non-U.S. Gov't]. Nature immunology, 7(2), 165-172. doi: 10.1038/ni1300

Wilson, N. S., Young, L.J., Kupresanin, F., Naik, S.H., Vremec, D., Heath, W.R., Akira, S., Shortman, K., Boyle, J., Maraskovsky, E., et al. (2008). Normal proportion and expression of maturation markers in migratory dendritic cells in the absence of germs or Toll-like receptor signaling. Immunol Cell Biol(86), 200-205

Winberg, O. K. (1902). Inoperable round-celled sarcoma of the uppoer jaw with metastases successfully treated with the mixed toxins of erysipelas and bacillus prodigiosus. $\mathrm{Med} \operatorname{Rec}(61), 681-684$. 
World Health Organization (1967). Development of the smallpox eradication programme. (1967). WHO chronicle, 21(9), 389-393.

World Health Organization, G. C. f. C. o. S. E. (1980). The Global Eradication of Smallpox: Final Report of the Global Commission for the Certification of Smallpox Eradication. Geneva, Switzerland: World Health Organization.

Wyllie, D. H., Kiss-Toth, E., Visintin, A., Smith, S. C., Boussouf, S., Segal, D. M., . . . Dower, S. K. (2000). Evidence for an accessory protein function for Toll-like receptor 1 in anti-bacterial responses. [Research Support, Non-U.S. Gov't Research Support, U.S. Gov't, P.H.S.]. Journal of immunology, 165(12), 7125-7132.

Yamazaki, C., Miyamoto, R., Hoshino, K., Fukuda, Y., Sasaki, I., Saito, M., . . Kaisho, T. (2010). Conservation of a chemokine system, XCR1 and its ligand, XCL1, between human and mice. [Research Support, Non-U.S. Gov't]. Biochemical and biophysical research communications, 397(4), 756-761. doi: 10.1016/j.bbrc.2010.06.029

Yarovinsky, F., Zhang, D., Andersen, J. F., Bannenberg, G. L., Serhan, C. N., Hayden, M. S., . . . Sher, A. (2005). TLR11 activation of dendritic cells by a protozoan profilinlike protein. [Research Support, N.I.H., Extramural Research Support, N.I.H., Intramural Research Support, Non-U.S. Gov't Research Support, U.S. Gov't, P.H.S.]. Science, 308(5728), 1626-1629. doi: 10.1126/science.1109893

Yee, C., Thompson, J. A., Byrd, D., Riddell, S. R., Roche, P., Celis, E., \& Greenberg, P. D. (2002). Adoptive T cell therapy using antigen-specific CD8+ T cell clones for the treatment of patients with metastatic melanoma: in vivo persistence, migration, and antitumor effect of transferred T cells. [Clinical Trial Clinical Trial, Phase I Research Support, Non-U.S. Gov't Research Support, U.S. Gov't, P.H.S.]. Proceedings of the National Academy of Sciences of the United States of America, 99(25), 16168-16173. doi: 10.1073/pnas.242600099

Yewdall, A. W., Drutman, S. B., Jinwala, F., Bahjat, K. S., \& Bhardwaj, N. (2010). CD8+ $\mathrm{T}$ cell priming by dendritic cell vaccines requires antigen transfer to endogenous antigen presenting cells. [Research Support, N.I.H., Extramural Research Support, Non-U.S. Gov't]. PloS one, 5(6), e11144. doi: 10.1371/journal.pone.0011144

York, I. A., Brehm, M. A., Zendzian, S., Towne, C. F., \& Rock, K. L. (2006). Endoplasmic reticulum aminopeptidase 1 (ERAP1) trims MHC class I-presented peptides in vivo and plays an important role in immunodominance. [Research Support, Non-U.S. Gov't]. Proceedings of the National Academy of Sciences of the United States of America, 103(24), 9202-9207. doi: 10.1073/pnas.0603095103

York, I. A., Chang, S. C., Saric, T., Keys, J. A., Favreau, J. M., Goldberg, A. L., \& Rock, K. L. (2002). The ER aminopeptidase ERAP1 enhances or limits antigen 
presentation by trimming epitopes to 8-9 residues. [Research Support, U.S. Gov't, P.H.S.]. Nature immunology, 3(12), 1177-1184. doi: 10.1038/ni860

York, I. A., \& Rock, K. L. (1996). Antigen processing and presentation by the class I major histocompatibility complex. [Research Support, Non-U.S. Gov't Research Support, U.S. Gov't, P.H.S. Review]. Annual review of immunology, 14, 369-396. doi: 10.1146/annurev.immunol.14.1.369

Zamai, L., Ponti, C., Mirandola, P., Gobbi, G., Papa, S., Galeotti, L., . . . Vitale, M. (2007). NK cells and cancer. [Research Support, Non-U.S. Gov't Review]. Journal of immunology, 178(7), 4011-4016.

Zarember, K. A., \& Godowski, P. J. (2002). Tissue expression of human Toll-like receptors and differential regulation of Toll-like receptor mRNAs in leukocytes in response to microbes, their products, and cytokines. [Comparative Study]. Journal of immunology, 168(2), 554-561.

Zhang, D., Zhang, G., Hayden, M. S., Greenblatt, M. B., Bussey, C., Flavell, R. A., \& Ghosh, S. (2004). A toll-like receptor that prevents infection by uropathogenic bacteria. [Research Support, Non-U.S. Gov't Research Support, U.S. Gov't, P.H.S.]. Science, 303(5663), 1522-1526. doi: 10.1126/science.1094351

Zhang, Z., Kim, T., Bao, M., Facchinetti, V., Jung, S. Y., Ghaffari, A. A., . . L Liu, Y. J. (2011). DDX1, DDX21, and DHX36 helicases form a complex with the adaptor molecule TRIF to sense dsRNA in dendritic cells. Immunity, 34(6), 866-878. doi: 10.1016/j.immuni.2011.03.027

Zhao, D., Zhang, C., Yi, T., Lin, C. L., Todorov, I., Kandeel, F., . . Zeng, D. (2008). In vivo-activated $\mathrm{CD} 103+\mathrm{CD} 4+$ regulatory $\mathrm{T}$ cells ameliorate ongoing chronic graftversus-host disease. [Research Support, N.I.H., Extramural]. Blood, 112(5), 21292138. doi: 10.1182/blood-2008-02-140277

Zheng, R., Cohen, P. A., Paustian, C. A., Johnson, T. D., Lee, W. T., Shu, S., \& Koski, G. K. (2008). Paired Toll-like receptor agonists enhance vaccine therapy through induction of interleukin-12. [Research Support, N.I.H., Extramural]. Cancer research, 68(11), 4045-4049. doi: 10.1158/0008-5472.CAN-07-6669

Zhu, Q., Egelston, C., Vivekanandhan, A., Uematsu, S., Akira, S., Klinman, D. M., .. . Berzofsky, J. A. (2008). Toll-like receptor ligands synergize through distinct dendritic cell pathways to induce $\mathrm{T}$ cell responses: implications for vaccines. [Research Support, N.I.H., Intramural]. Proceedings of the National Academy of Sciences of the United States of America, 105(42), 16260-16265. doi: 10.1073/pnas.0805325105 


\section{Chapter 8:} Appendix 


\section{Appendix 1: Table 8.1 Fluorescent Antibodies}

\begin{tabular}{|l|l|l|l|}
\hline Marker Specificity & Fluorophore & Clone & Source \\
\hline B220 & PerCP & $6 \mathrm{~B} 2$ & BD Pharmingen, CA, USA \\
& Purified & $6 \mathrm{~B} 2$ & Purified in-house \\
& Biotin & $6 \mathrm{~B} 2$ & BD Pharmingen, CA, USA \\
\hline CD8 $\alpha$ (Ly-2) & APC & $53-6.7$ & eBioscience, San Diego, CA, USA \\
& Alexa 780 & $53-6.7$ & Purified in-house \\
\hline CD11c & APC & HL3 & BD Pharmingen, CA, USA \\
& PECy7 & N418 & Purified in-house \\
\hline CD40 & PE & $3 / 23$ & BD Pharmingen, CA, USA \\
\hline CD45.1 & PE & A20 & eBioscience, San Diego, CA, USA \\
\hline CD45.2 & PE & 104 & eBioscience, San Diego, CA, USA \\
\hline CD86 (B7-2) & PE & GL1 & eBioscience, San Diego, CA, USA \\
\hline MHC II & PE & AF6-120.1 & BD Pharmingen, CA, USA \\
\hline CD4 & PE & GK1.5 & BD Pharmingen, CA, USA \\
& APC & GK1.5 & eBioscience, San Diego, CA, USA \\
\hline V $\alpha 2 ~$ & B20.1 & BD Pharmingen, CA, USA \\
\hline V 35.1, V $\beta 5.2$ & FITC & MR9-4 & BD Pharmingen, CA, USA \\
\hline \multicolumn{2}{|l|}{ Strepdavidin conjugates } & \multicolumn{2}{|l|}{ Source } \\
\hline SA-FITC & eBioscience, San Diego, CA, USA \\
\hline SA-PE & BD Pharmingen, CA, USA \\
\hline SA-PerCP & BA-APC & BD Pharmingen, CA, USA \\
\hline SA Pharmingen, CA, USA
\end{tabular}




\section{Chapter 9:}

\section{Publications}




\title{
Potent anti-tumor responses to immunization with dendritic cells loaded with tumor tissue and an NKT cell ligand
}

\author{
Troels R Petersen, Dianne Sika-Paotonu, Deborah A Knight, Nina Dickgreber, Kathryn J Farrand, \\ Franca Ronchese and Ian F Hermans
}

Cancer immunotherapy is well tolerated and specific, but its efficacy remains variable. To enhance anti-tumor $\mathrm{CD}^{+} \mathrm{T}^{-}$-cell responses induced by immunization with antigen-loaded dendritic cells (DCs), we explored the impact of eliciting a potent source of T-cell help from activated invariant natural killer (NK)-like T cells (INKT cells) using the specific glycolipid ligand $\alpha$-galactosylceramide ( $\alpha$-GalCer). As cytokines released by $i N K T$ cells may drive proliferation of $C D 4+C D 25^{+}$regulatory T cells (Tregs), we assessed this immunization strategy in animals treated with anti-CD25 antibody to inactivate Treg function. Combining DC immunization with iNKT cell activation was found to significantly enhance anti-tumor activity, which was improved further by the prior inactivation of Tregs. The improved anti-tumor activity with Treg inactivation was associated with a prolonged proliferative burst of responding CD8 ${ }^{+} \mathrm{T}$ cells. We could find no evidence that inclusion of $\alpha$-GalCer in the vaccine enhanced Treg numbers, or that the 'helper' function of $i N K T$ cells was improved in the absence of Treg activity. Rather, the two activities appeared to act independently to improve the tumor-specific T-cell response. Inactivating regulatory $\mathrm{T}$ cells and eliciting NKT cell activation are therefore two useful strategies that can be used in combination to improve anti-tumor immunization with antigen-loaded DCs.

Immunology and Cell Biology advance online publication, 9 February 2010; doi:10.1038/icb.2010.9

Keywords: dendritic cells; T cells; invariant NKT cells; T regulatory cells; vaccination

Inducing T-cell-mediated immune responses to tumors by vaccination has the potential to be an effective cancer therapy, but obtaining sufficient potency is still a therapeutic challenge. Recent attention has been given to recruiting the adjuvant property of invariant natural killer (NK)-like T ( $i$ NKT) cells; a population of cells expressing a semiinvariant T-cell receptors (TCR) that respond to glycolipids of microbial or endogenous origin in the context of CD1d. ${ }^{1}$ Activation of $i$ NKT cells by administration of the synthetic glycolipid, $\alpha$-galactosylceramide ( $\alpha$-GalCer), results in a burst of iNKT cell-derived cytokines that, together with direct cellular interactions, result in global maturation of host APC. It is therefore possible to exploit the activity of $\alpha$-GalCer-stimulated $i$ NKT cells to enhance T-cell responses to co-administered antigens. ${ }^{2-5}$ The initial burst of $i$ NKT cell-derived cytokines is followed by a larger wave of NK cell-derived IFN- $\gamma$, in turn triggered by the IL- 12 released by resident APC. ${ }^{6,7}$ A number of anti-tumor activities can therefore be induced by administration of $\alpha$-GalCer; improved adaptive immunity, $i \mathrm{NKT}$ and NK cell-mediated cytolysis of tumor cells, and the anti-angiogenic function of IFN- $\gamma .{ }^{8,9}$ Although these observations suggest that $\alpha$-GalCer could be a useful adjuvant for anti-tumor vaccination strategies, it has been reported that IL- 2 secreted by $\alpha$-GalCer-activated $i$ NKT cells can expand populations of $\mathrm{CD}^{+} \mathrm{CD} 25^{+}$regulatory $\mathrm{T}$ cells (referred to as Tregs). ${ }^{10-12}$ Although Tregs protect the host against undesirable immune responses to self-antigen, ${ }^{13,14}$ they can also suppress antitumor T-cell responses. ${ }^{15,16}$ Accumulation of Tregs in tumor tissue has been associated with poor prognosis in humans, ${ }^{17}$ and depletion or inactivation of Tregs has been demonstrated to enhance T-cell priming and tumor rejection in tumor models. ${ }^{17-21}$ Significantly, Tregs have also been demonstrated to suppress proliferation and cytokine secretion of cultured human $i$ NKT cell lines ${ }^{22}$ and suppress NKT cell expansion in mice. ${ }^{23}$ Examples of Treg-mediated suppression of NK cell activation and NK cell-mediated tumor lysis have also been reported. ${ }^{24,25}$ It is therefore possible that the adjuvant properties of $i$ NKT cell activation may be negated to some extent by enhanced Treg activity. We therefore investigated whether $\alpha$-GalCer-mediated $i$ NKT cell activation can enhance anti-tumor immunity after immunization with bone marrow-derived dendritic cells (BM-DCs) loaded with stressed tumor cells, and specifically assessed the role of Tregs in this process.

We found that $i$ NKT cell activation by $\alpha$-GalCer-loaded BM-DC improves priming of tumor-reactive $\mathrm{CD}^{+} \mathrm{T}$ cells, and protection against tumor challenge, and that this activity can be further enhanced 
by inactivation of Treg before immunization. However, we could not find evidence that $i$ NKT cell activation drives proliferation of Tregs in vivo or that it enhances their suppressive function. Nor could we find evidence that Tregs suppressed $i$ NKT cell activation, suggesting that $i$ NKT cells and Tregs can independently modulate anti-tumor T-cell responses generated by immunization with antigen-loaded DC. Combining $i$ NKT cell activation with Treg inactivation is therefore a useful strategy to improve responses to vaccination.

\section{RESULTS}

Combining $i$ NKT cell activation and Treg inactivation enhances anti-tumor responses induced by vaccination with BM-DC loaded with stressed tumor cells

We, and others, have demonstrated earlier increased $\mathrm{CD} 4^{+}$and $\mathrm{CD} 8^{+}$ T-cell priming and anti-tumor activity after injection of the $i$ NKT cell ligand $\alpha$-GalCer with soluble tumor-associated antigen., ${ }^{2,3,5}$ To investigate whether $\alpha$-GalCer can enhance T-cell priming to non-defined tumor antigens loaded onto BM-DC as stressed tumor cells, we immunized mice with BM-DC loaded with irradiated and heatshocked B16.OVA melanoma cells alone or in combination with $\alpha$-GalCer. An additional group of animals received BM-DC loaded with $\alpha$-GalCer alone. Furthermore, to examine the role of Tregs in this process, mice were either pretreated with anti-CD25, or a control rat IgG antibody (rIgG). All animals were challenged with live B16.OVA cells 7 days later. Immunization with BM-DC loaded with stressed B16.OVA only had a small effect on tumor growth (Figure 1a) and did not delay the onset of tumors (Figure 1d). A similar trend toward a delay in tumor growth was observed in animals immunized with BMDC loaded with $\alpha$-GalCer alone (Figure 1b) although no statistical significant delay in the onset of tumor growth was detected over repeated experiments (Figure 1d). On the other hand, significant protection was seen in animals immunized with BM-DC loaded with a combination of stressed B16.OVA cells and $\alpha$-GalCer (Figure 1c). Tumor appearance was delayed by 10 days relative to control animals that received tumor challenge alone. Despite this significant activity, tumors eventually developed in approximately $70 \%$ of mice, indicating that the immune response is ultimately overwhelmed or disabled (Figure 1d). Anti-CD25 antibody had no significant effect on tumor growth after immunization with BM-DC loaded with stressed B16.OVA only (Figure 1a, open symbols). Nor did it significantly impact on the anti-tumor activity elicited by BM-DC loaded with $\alpha$-GalCer only (Figure $1 \mathrm{~b}$, open symbols), suggesting that $i \mathrm{NKT}$ cell activation is not responsible for driving broad Treg activity that masks de novo anti-tumor responses. This result also suggests that any direct $i$ NKT cell activity against the tumor is not influenced by Tregs. In contrast, Treg inactivation before immunization with BM-DC loaded with the combination of stressed B16.OVA cells and $\alpha$-GalCer significantly $(P<0.0001)$ enhanced protection against subsequent tumor challenge (Figure 1c, open symbols). In fact, all animals in this group were completely protected, with no tumors observed over 4 months of monitoring (Figure 1d). A similar pattern of protection was observed after DC immunization and tumor challenge with B16.F10 tumor cells, which do not express OVA, demonstrating that the induced T-cell response is not solely induced against the OVA antigen (Figure 1e). Inactivation of Tregs before immunization with BM-DC loaded with the combination of stressed B16.OVA cells and $\alpha$-GalCer also significantly $(P=0.02)$ delayed tumor growth when administered 3 days after tumor challenge demonstrating some therapeutic potential of this vaccination regime (Figure 1f). These experiments show that the powerful adjuvant activity of $i$ NKT cell activation improves anti-tumor responses in response to tumorloaded DC, and suggest that the responses induced are under a level of regulation by Tregs.

$i$ NKT cell activation by $\alpha$-GalCer- and tumor-loaded DC does not alter Treg function

To investigate whether cytokines released by $i$ NKT cell activation drive Treg proliferation, as has previously been suggested, ${ }^{10-12}$ we monitored the number and function of Tregs in response to immunization with BM-DC loaded with stressed B16.OVA and $\alpha$-GalCer. As Tregs express the transcription marker FoxP3, we could use the FoxP3 gfp mice to monitor Treg numbers by flow cytometry with very little manipulation. ${ }^{26}$ As has been reported earlier, ${ }^{7}$ incorporation of $\alpha$-GalCer into the vaccine leads to a twofold to threefold increase in both the total number of lymphocytes, as well as in the number of $\mathrm{CD}^{+}$cells in the spleen at day 6 post immunization (Figures $2 \mathrm{a}$ and $b$ ). However, no significant differences in the number of FoxP3 ${ }^{\text {gfp }}$ cells were observed between groups of mice immunized with tumor-loaded BM-DC in the presence or absence $\alpha$-GalCer, respectively (Figure 2c). Activation of $i$ NKT cells therefore did not lead to an expansion in Treg numbers. However, this experiment did not rule out the possibility of a change in function of these cells. We therefore purified FoxP3 $3^{\mathrm{gfp}+}$ cells from mice immunized with tumorloaded BM-DC plus or minus $\alpha$-GalCer, and compared their suppressive activities in vitro on purified $\mathrm{CD} 4^{+} \mathrm{CD} 25^{-} \mathrm{T}$ cells stimulated with splenic DC and anti-CD3. No differences in suppressive activity were observed between the two groups (Figure 3). These data suggest that $i$ NKT cell activation does not alter Treg function in this DC-based vaccination model.

$\alpha$-GalCer-mediated $i$ NKT cell activation is not suppressed by Tregs It has been reported earlier that Tregs can suppress cytokine secretion by human and mouse $\alpha$-GalCer-activated iNKT cells. ${ }^{22,27}$ Thus, although $i$ NKT cell activation did not affect Treg proliferation and activity in our model of DC-based vaccination, it was still possible that the $i$ NKT cells themselves were under a level of regulation by Tregs. We therefore examined whether Treg inactivation with anti-CD25 antibody before DC administration had any effect on DC-induced $i \mathrm{NKT}$ cell activity. Mice were administered anti-CD25 or control rIgG antibody, and were then immunized with BM-DC loaded with stressed B16.OVA cells and $\alpha$-GalCer 2 days later. The activation status of splenic $i$ NKT cells, as identified by surface labeling with $\alpha$-GalCerloaded CD1d tetramers and anti-TCR- $\beta$ antibody (Figure $4 \mathrm{a}$ ), was assessed $2 \mathrm{~h}$ after DC immunization by intracellular staining with antiIFN- $\gamma$, anti-IL-4, or control isotype antibodies (Figure $4 \mathrm{~b}$ ). Although the percentage of cytokine-positive $i$ NKT cells from immunized mice varied between experiments, anti-CD25 antibody did not increase the activation status of $i$ NKT cells as a similar percentage of $i$ NKT cells produced IFN- $\gamma$ and IL- 4 in mice treated with anti-CD25 or control rIgG antibody (Figure 4c). Hence, $i$ NKT cell activation was not suppressed by Tregs.

To investigate the impact of Tregs on $i$ NKT cell activation further, we assessed the impact of anti-CD25 treatment on additional in vivo readouts of $i$ NKT cell activity. The administration of $\alpha$-GalCer to animals, whether as a free agent, or loaded onto DC, results in global maturation of host APC, and release of cytokines into the serum, although the kinetics of cytokine release may vary depending on the administration method. ${ }^{8}$ We therefore assessed maturation of APC and cytokine release in animals that had been subjected to Treg inactivation before intravenous (i.v.) administration of DC loaded with stressed B16.OVA and $\alpha$-GalCer. Analysis of CD86 expression on 

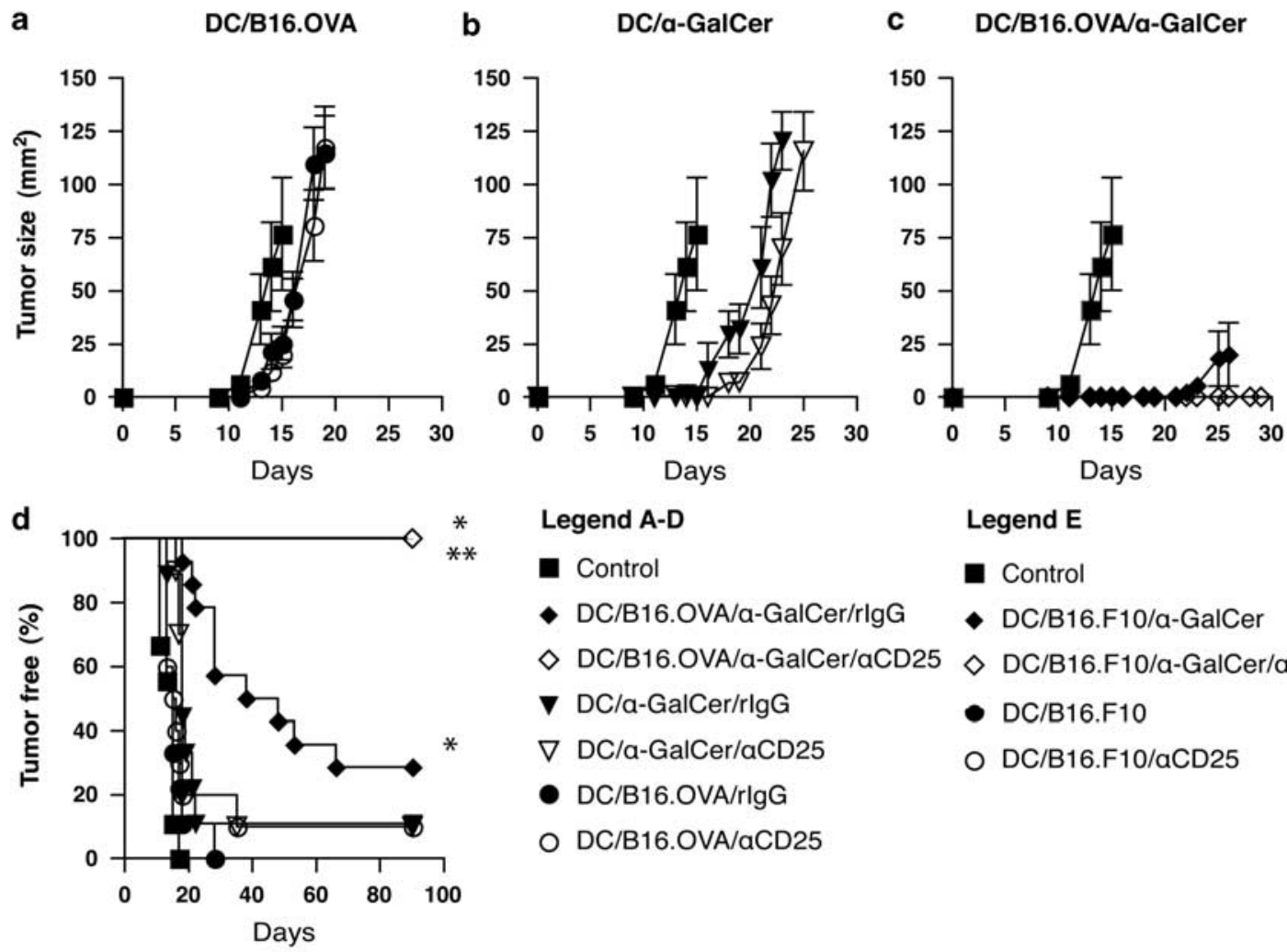

\author{
Legend $\mathrm{E}$ \\ - Control \\ - DC/B16.F10/a-GalCer \\ $\diamond$ DC/B16.F10/a-GalCer/aCD25 \\ - DC/B16.F10 \\ O DC/B16.F10/aCD25
}

O DC/B16.OVA/aCD25
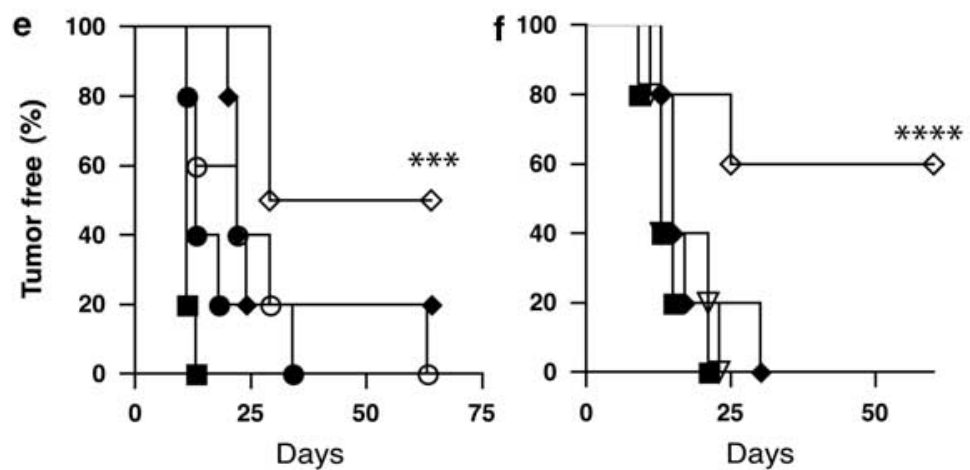

\author{
Legend $\mathrm{F}$ \\ - Control \\ - DC/B16.OVA/a-GalCer/rlgG \\ $\diamond$ DC/B16.OVA/a-GalCer/aCD25 \\ $\nabla$ DC/a-GalCer/aCD25
}

Figure 1 Anti-tumor responses elicited using DC loaded with stressed tumor cells are enhanced by concurrent iNKT cell activation, and most effective when also combined with Treg inactivation. Groups of mice $(n=5-10)$ received a single i.p. injection of either anti-CD25 (open symbols) or control rlgG (closed symbolds). Two days later, they were injected i.v. with BM-DC loaded with stressed B16.OVA cells (circles) (a), $\alpha$-GalCer (triangles) (b), or both stressed B16.OVA cells and $\alpha$-GalCer (diamonds) (c), and challenged 7 days later with live B16.OVA cells. Results are representative of two similar experiments with 5-10 mice per group. Percent tumor-free mice from both experiments (a total of 10-15 mice per group) are depicted in a Kaplan-Meier plot (d). ${ }^{*} P<0.0001$ (compared to unimmunized control mice). ${ }^{* *} P<0.0001$ (compared to control rlgG-treated animals receiving DC loaded with stressed B16.0VA cells and $\alpha$-GalCer). (e) Mice were injected with anti-CD25 (open symbols) or control rlgG (closed symbols) antibody as in (d) and 2 days later injected i.v. with BM-DC loaded with stressed B16.F10 cells (circles) or both stressed B16.F10 cells and $\alpha$-GalCer (diamonds). Results depicted show percent tumor-free mice of one representative experiment out of two $(n=5)$. ${ }^{* * *} P=0.0035$ (compared to control rlgG-treated animals receiving DC loaded with stressed B16.F10 cells and $\alpha$-GalCer). (f) Mice were challenged with live B16.OVA cells, injected with anti-CD25 2 days later (open symbols), and immunized i.v. with BM-DC loaded with stressed B16.OVA cells and $\alpha$-GalCer (diamonds) or only $\alpha$-GalCer (triangles) the following day (3 days after tumor challenge). Results depicted show percent tumor-free mice. ${ }^{* * *} P=0.02$.

CD11 $\mathrm{c}^{+}$APC in spleen showed typical $i$ NKT cell-mediated upregulation, regardless of whether animals had been pretreated with antiCD25 or control antibody. Analysis of cytokines in the serum showed a weak trend toward reduced cytokine levels in mice treated with antiCD25, which was most pronounced for IFN- $\gamma$ (Figure 5b). Tregs therefore do not suppress the immediate $i \mathrm{NKT}$ cell response to $\alpha$-GalCer. In fact, anti-CD25 administration paradoxically decreases some $i$ NKT cell-mediated activities to a limited extent.
Tregs suppress induction of vaccine-induced tumor-specific $\mathrm{CD8}^{+} \mathrm{T}$ cells

To establish the mechanism by which the combination of Treg inactivation and $i \mathrm{NKT}$ activation improves anti-tumor responses to DC vaccination, we investigated the impact of these treatments on induction of antigen-specific $\mathrm{CD}^{+} \mathrm{T}$-cell responses to the model antigen OVA expressed by B16.OVA tumor cells. To maximize the possibility of detecting a response to stressed tumor cells, initial 
a

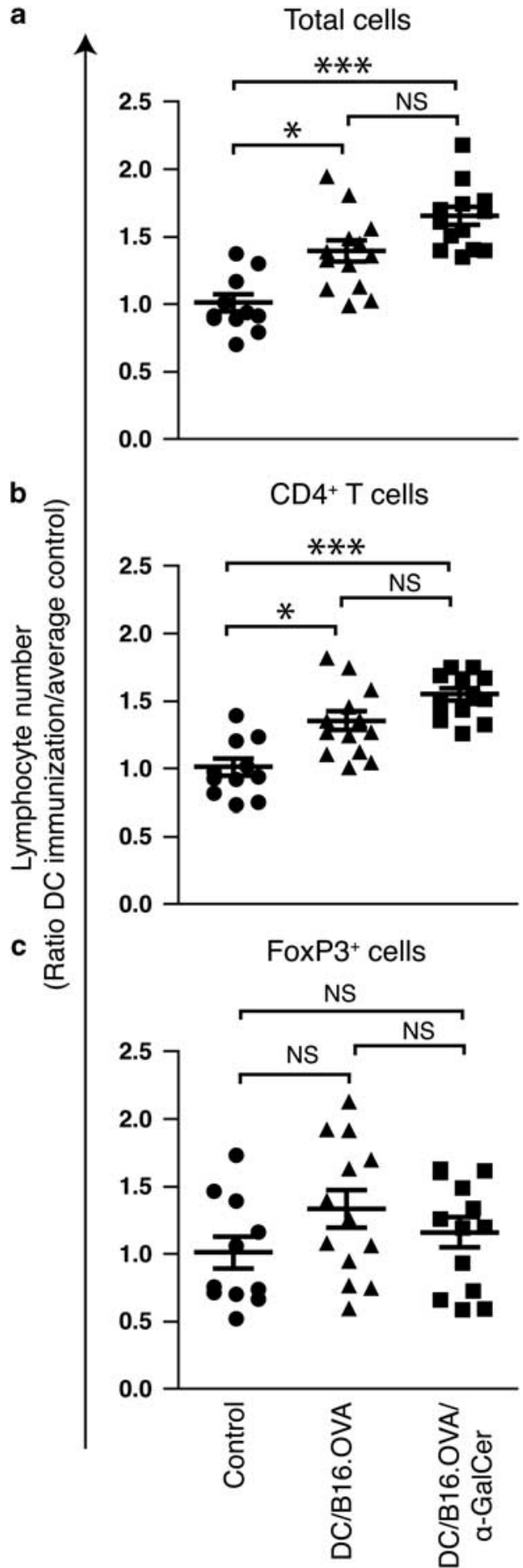

Figure 2 Vaccination with DC loaded with $\alpha$-GalCer and stressed B16.0VA cells does not induce proliferation of $\mathrm{FoxP}^{+}$Treg cells in vivo. Groups of FoxP3 $3^{\text {ffp }}$ mice $(n=3-5)$ were injected i.v. with BM-DC loaded with either stressed B16.0VA, or stressed B16.0VA and $\alpha$-GalCer. Six days later, the total number of lymphocytes (a), CD4 ${ }^{+} \mathrm{T}$ cells (b), and FoxP3-gfp ${ }^{+} \mathrm{T}$ cells (c) cells were determined in the spleen. Data from three separate experiments are depicted, with lymphocyte counts normalized to the average counts in the control non-immunized group. Statistical significance was determined by a Krusskall-Wallis test and Dunn's post test. NS, not significant, ${ }^{*} P=0.01-$ $0.05, * * * P<0.001$

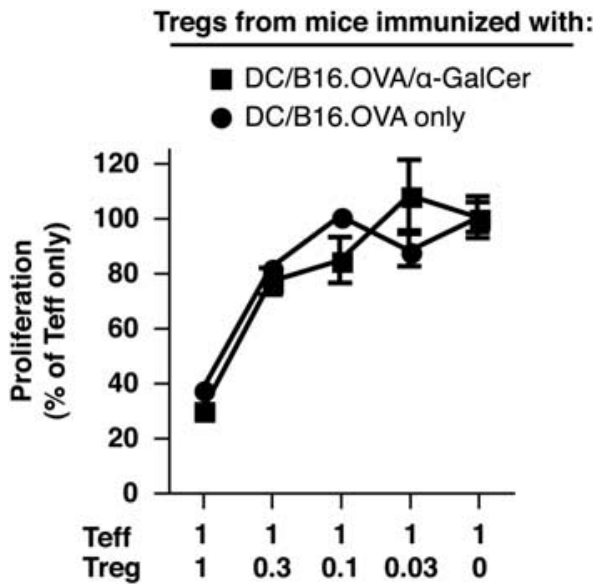

Figure 3 Vaccination with DC loaded with $\alpha$-GalCer and stressed B16.OVA cells does not alter the suppressive activity of $\mathrm{FoxP}^{+}$Treg cells. Groups of FoxP3 ${ }^{\text {ffp }}$ mice $(n=5-6)$ were injected i.v. with BM-DC loaded with either stressed B16.0VA, or stressed B16.0VA and $\alpha$-GalCer. Six days later CD4 ${ }^{+}$FoxP3 ${ }^{\text {ffp }}+$ Tregs were purified from the spleen and cultured (as described in Methods) with $\mathrm{CD}^{+}{ }^{+}$FoxP3 $3^{\text {gfp- }}$ cells from mice that had been vaccinated with BM-DC loaded with stressed B16.OVA 6 days previously. The graph is representative of two similar experiments.

experiments were performed using BM-DC loaded with stressed B16.OVA cells that had been electroporated with exogenous OVA to increase the specific antigen load. After electroporation, the tumor cells were irradiated and heat-shocked before being loaded onto BMDC together with $\alpha$-GalCer, as performed in previous experiments. In addition, the animals used for these initial studies received a small cohort $\left(25000\right.$ cells) of OVA-specific TCR transgenic CD8 ${ }^{+} \mathrm{T}$ cells (OT-I cells) before immunization to aid in monitoring the induced response. To assess the impact of Treg inactivation on immunization, recipients were treated with anti-CD25 or control rIgG antibodies before adoptive transfer of transgenic T cells and BM-DC administration. The percentage of $\mathrm{CD}^{+} \mathrm{T}$ cells specific for the $\mathrm{H}-2$ $\mathrm{K}^{\mathrm{b}}$-restricted peptide epitope of OVA $\left(\mathrm{OVA}_{257-264}\right)$ were identified in the blood 4,7 , and 11 days later by flow cytometry with $\mathrm{K}^{\mathrm{b}} / \mathrm{OVA}_{257-264}$-pentamers (Figures $6 \mathrm{a}-\mathrm{c}$ ). At 7 days after immunization, no significant accumulation of $\mathrm{K}^{\mathrm{b}} / \mathrm{OVA}_{257-264}$-pentamer-positive $\mathrm{CD}^{+} \mathrm{T}$ cells was detected in animals immunized with DC loaded with stressed B16.OVA cells only, irrespective of whether or not the mice had been pretreated with anti-CD25 antibody (Figure 6b). In contrast, a distinct population of antigen-specific $\mathrm{CD}^{+} \mathrm{T}$ cells was detected in all animals immunized with BM-DC loaded with electroporated B16.OVA and $\alpha$-GalCer (Figures $6 \mathrm{a}-\mathrm{c}$ ). Significantly, the percentage of antigen-specific $\mathrm{CD}^{+} \mathrm{T}$ cells were highest in animals pretreated with anti-CD25 antibody. Interestingly, this impact of Treg inactivation was only evident 7 days after immunization, despite there being a significant expansion of the antigen-specific T-cell population at earlier time points (Figure 6c). We repeated this experiment without the electroporation step, thereby reducing antigen dose to that delivered by the OVA transgene in the stressed tumor cells used to load the BM-DC. Again, prior Treg inactivation enhanced the $\mathrm{OVA}_{257-264}$-specific $\mathrm{CD}^{+}$T-cell response to immunization with BM-DC loaded with stressed tumor cells and $\alpha$-GalCer (Figure $6 \mathrm{~d}$ ). A similar trend, although not statistically significant, was observed when the same immunization protocol was used in recipients that did not receive OT-1 cells (Figure 6e). These data suggest that Tregs remain capable of suppressing tumor-specific $\mathrm{CD}^{+} \mathrm{T}$-cell responses in the presence of $i \mathrm{NKT}$ cell activation, and that the net impact of 


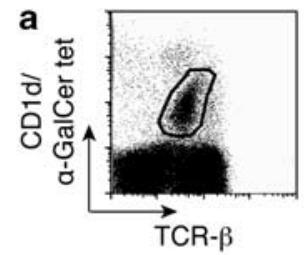

b
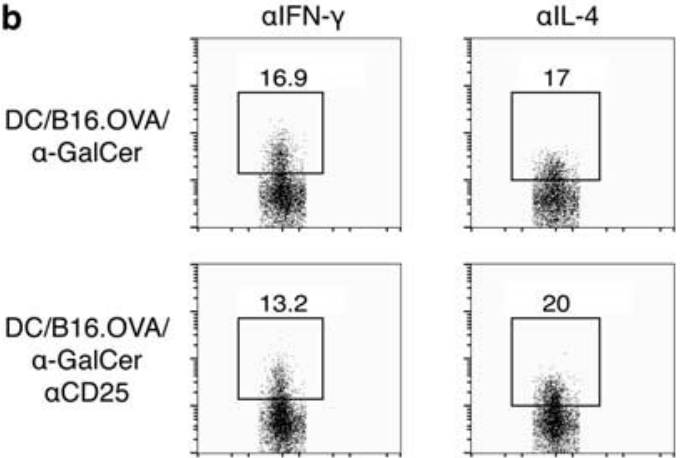

Naive
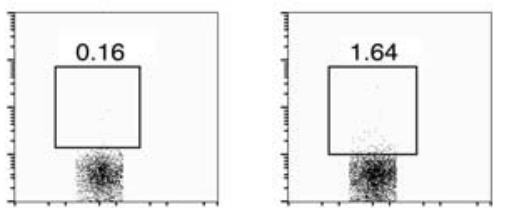

Isotype control

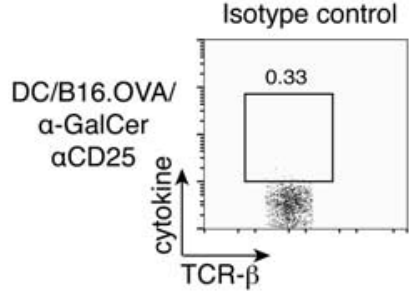

C

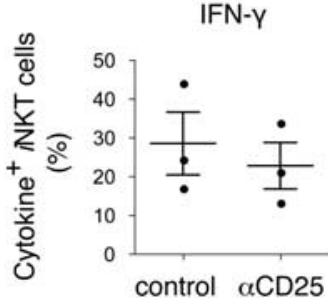

a

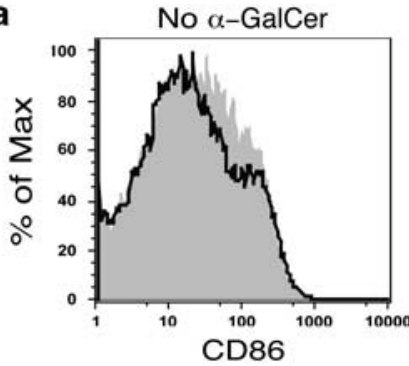

Naive

$\alpha \mathrm{CD} 25$

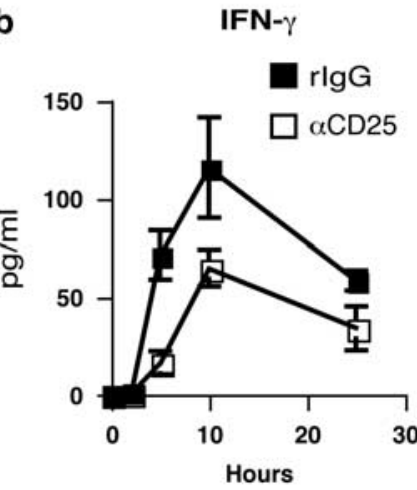

IL-12 p70

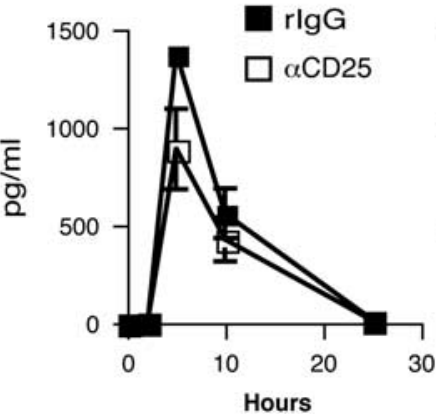

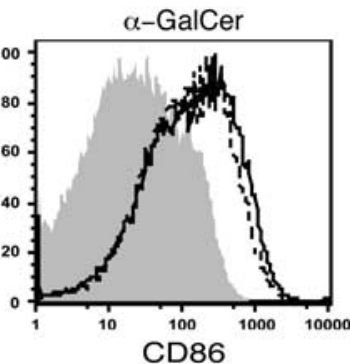

Naive

..... $\alpha$-GalCer

- $\alpha \mathrm{CD} 25+\alpha-$ GalCer

IL-4

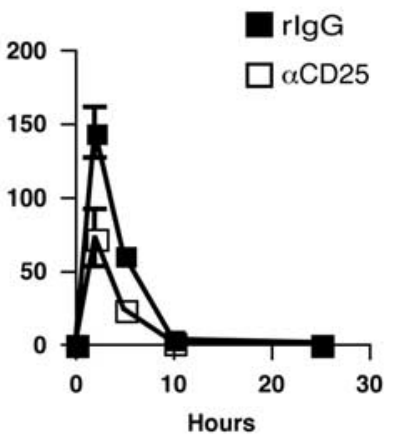

IL-6

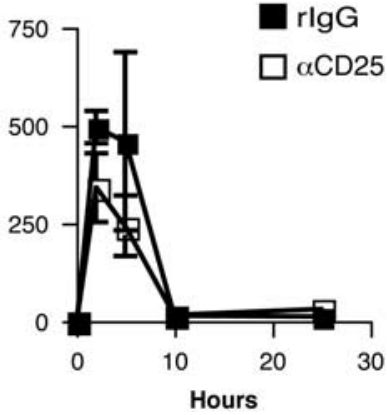

Figure 5 iNKT cell-mediated DC activation and release of cytokines into the serum is not suppressed by Tregs. (a) C57BL/6J mice received a single i.p. injection of either anti-CD25 or control rlgG antibody, and 2 days later all animals were injected i.v. with $200 \mathrm{ng} \alpha$-GalCer. Spleens were removed $18 \mathrm{~h}$ later and $\mathrm{CD} 86$ expression determined on $\mathrm{CD} 11 \mathrm{c}^{+}$cells by flow cytometry. A representative FACS profile of one experiment out of two is shown. (b) Groups of mice $(n=4-5)$ were injected with anti-CD25 or control rlgG antibody, and then vaccinated with BM-DC pulsed with stressed B16.OVA and $\alpha$-GalCer. Levels of cytokines in serum were evaluated at the indicated times. Mean values ( \pm s.e.m.) for one representative experiment out three are depicted. experiment out of three is depicted. (c) Percentage of cytokine-positive iNKT cells from mice immunized with DC loaded with B16.OVA and $\alpha$-GalCer in anti-CD25-treated and control rlgG-treated animals. The average and s.e.m. of three experiment with three mice per group are depicted.

combining iNKT cell activation and Treg depletion is to sustain induced anti-tumor responses to DC immunization.

\section{DISCUSSION}

In this study, we have investigated strategies to improve anti-tumor responses to vaccination with DC that have been loaded with whole,

Figure 4 Cytokine secretion by $\alpha$-GalCer-stimulated iNKT cells is v. with BM-DC loaded with stressed B16.OVA cells and $\alpha$-GalCer. After splenocytes were isolated and analyzed for cytokine production intracellular cytokine staining. (a) Typical gating of iNKT cells using antibody 
a

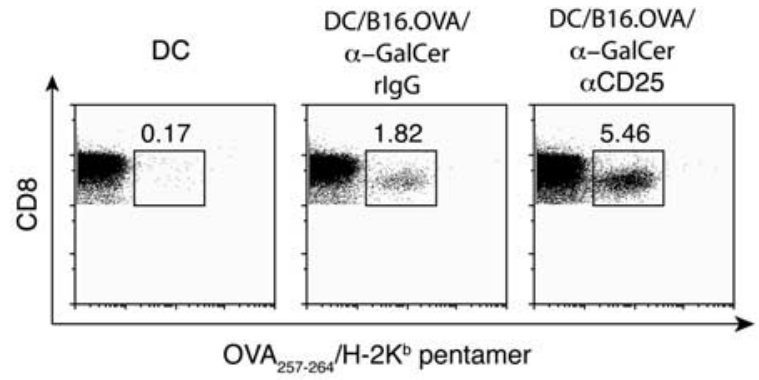

b

b

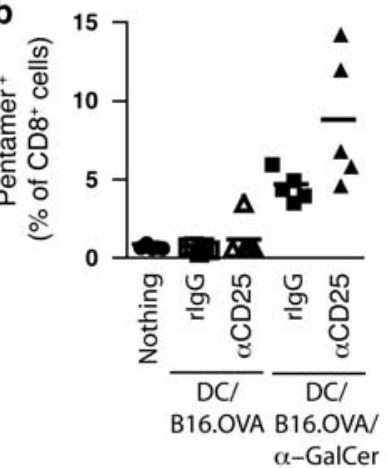

d

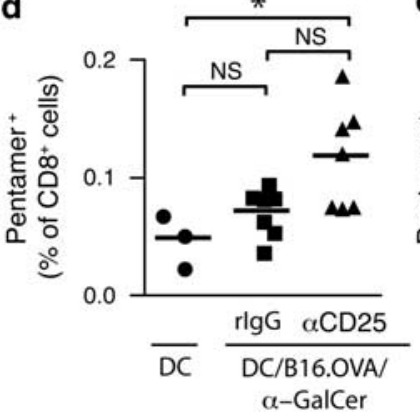

C

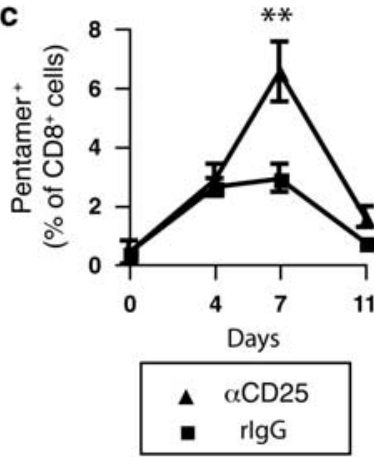

e

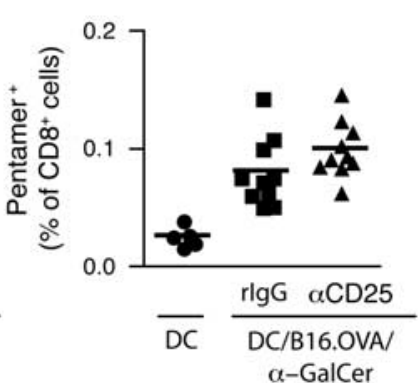

Figure 6 Inactivation of Tregs enhances antigen-specific $\mathrm{CD}^{+}{ }^{+} \mathrm{T}$-cell responses to vaccination with BM-DC loaded with stressed tumor cells and $\alpha$-GalCer. (a) Groups of mice received a single i.p. injection of anti-CD25 or control rlgG, or were left untreated. The next day all animals were donated a cohort of $\mathrm{OVA}_{257-264^{-}}$-specific OT-1 TCR transgenic CD8 ${ }^{+} \mathrm{T}$ cells, and then $24 \mathrm{~h}$ later all mice were vaccinated with BM-DC loaded with stressed B16.OVA cells (that had been electroporated with $2 \mathrm{mg} \mathrm{ml}^{-1}$ of extra OVA) alone or with $\alpha$-GalCer. The mice were bled at days 4,7 , and 11 , and the percentage of $\mathrm{CD}^{+} \mathrm{K}^{\mathrm{b}} / \mathrm{OVA}_{257-264}$-pentamer ${ }^{+} \mathrm{T}$ cells determined by flow cytometry. Representative day 7 flow cytometry profiles are shown. (b) The percentage of $\mathrm{K}^{\mathrm{b}} / \mathrm{OVA}_{257-264^{-}}$pentamer ${ }^{+}$cells of $\mathrm{CD} 8^{+}$cells in blood at day 7 after vaccination in one representative experiment out of two. Each symbol represents an individual mouse. (c) Kinetics of vaccine-induced $\mathrm{CD}^{+} \mathrm{K}^{\mathrm{b}}$ / $\mathrm{OVA}_{257-264}$-pentamer+-specific responses in blood. Mean values for each group ( \pm s.e.m.) combined from two separate experiments are presented. The statistical significance was determined by Mann-Whitney test. (d) Groups of mice were treated as above, except stressed B16.0VA cells were not electroporated with extra OVA before being loaded onto BM-DC. Percentages of $\mathrm{K}^{\mathrm{b}} / \mathrm{OVA}_{257-264}$-pentamer ${ }^{+}$cells of $\mathrm{CD}^{+}$cells in blood 7 after vaccination are depicted. One representative experiment out of two is shown. The statistical significance was determined by a Krusskall-Wallis test and Dunn's post test. (e) As in (d), except without adoptive transfer of OT-1 transgenic T cells. One representative experiment out of two is shown. Immunization with un-pulsed BM-DC served as control in all of the above experiments. NS, not significant, ${ }^{*} P=0.01-0.05,{ }^{*} P=0.001-0.01$.

improves protection against tumor challenge (Figure 1) by enhancing the priming of tumor-specific $\mathrm{CD}^{+} \mathrm{T}$ cells (Figure 6 ). Notably, the inclusion of $\alpha$-GalCer within the vaccine improves T-cell priming

without driving proliferation of Tregs (Figure 2), or enhancing their suppressive function (Figure 3).

We and others have previously demonstrated enhanced immunity to model antigens delivered as free proteins $s^{2,4,5}$ or antigen-loaded spleen cells ${ }^{3}$ when co-administrated with $\alpha$-GalCer. However, this study is the first to show enhanced anti-tumor activity of DC loaded with non-defined tumor antigens and $\alpha$-GalCer. Although the overall success rates of DC-based immunotherapy in clinical trials are still disappointing, trials using vaccines based on whole cells or cell extracts rather than individual proteins or peptides generally perform better. ${ }^{28}$ Including $\alpha$-GalCer in these vaccines could significantly improve the anti-tumor immune response to such non-defined antigens by eliciting a universal 'helper' function from activated $i$ NKT cells, thereby licensing APC to stimulate more effective CTL.

Recent studies showing that vaccination had the unexpected consequence of expanding or improving Treg function ${ }^{23,29,30}$ lead us to investigate whether the adjuvant effect of $i \mathrm{NKT}$ cell activation in the context of DC-based immunization impacted on Treg function, or vice versa. This was not merely a hypothetical question, as $\alpha$-GalCermediated activation of $i$ NKT cells has been shown to lead to the expansion of Treg populations in a model of experimental autoimmune myasthenia gravis. ${ }^{10}$ The authors of this study suggested that this expansion was supported by provision of IL-2 by the activated $i$ NKT cells. In another report, human $i$ NKT cells were shown to secrete IL-2 on stimulation with $\alpha$-GalCer, which was capable of driving proliferation of Tregs in vitro. ${ }^{11}$ To investigate the role of Tregs in our vaccination model, we injected mice with anti-CD25 antibody. Although significant protection had been observed when $\alpha$-GalCer was included in the vaccine, protection was only complete when Tregs had been inactivated. Under these conditions, tumor antigen-loaded DC without $\alpha$-GalCer provided only limited protection, and inactivation of Tregs made no difference. For these experiments the DC vaccines were administered by the i.v. route so that, where applicable, access to $i$ NKT cell-rich regions of the spleen and liver could be maximized. It is possible that the potent adaptive immunity induced in the presence of $i$ NKT cell activation involves transfer of antigen to spleen-resident DC, with these DC then actively participating in the response, as has previously been reported after injection of $\alpha$-GalCerloaded tumor cells. ${ }^{31}$ The recipient DC may be the CD8 $\alpha^{+}$subtype, which have been shown to cross-present cell-associated antigens derived from tumor cells, ${ }^{31}$ or migrating DC. ${ }^{32}$ The possibility that langerin ${ }^{+} \mathrm{CD} 8 \alpha^{+}$DC located within the marginal zone act as recipients is currently being investigated, as we have previously shown that these cells are particularly efficient at cross presentation of soluble antigens. ${ }^{33}$

The use of the i.v. route, as opposed to the subcutaneous route, is the only difference between this study, and our previous study in which administration of anti-CD25 was in itself capable of delaying tumor growth in response to immunization with DC and stressed tumor cells alone. ${ }^{34}$ The reason for this discrepancy is not clear. However, our data suggest that the negative impact of Tregs is only of significance when an immune response has been successfully invoked, which in this study was only relevant in animals receiving $\alpha$-GalCerloaded vaccines, and in the previous study was successfully achieved without $\alpha$-GalCer through the subcutaneous route, which is generally regarded as a more potent route of administration.

An alternative interpretation for the enhanced combined activity of $i$ NKT cell activation and Treg inactivation is that the suppressive activity of Tregs is driven concurrently with the anti-tumor response through the actions of activated $i$ NKT cells, and that Treg inactivation therefore unleashes a superior effector response. Yet another 
interpretation is that Tregs negatively regulate $\mathrm{iNKT}$ cells themselves, and removing this barrier improves the adjuvant activity elicited by activated $i$ NKT cells. One way of resolving these issues was to specifically investigate the activities of Treg and $i$ NKT cells independently in response to vaccination. Quantification of total Treg numbers in the spleen in response to DC-based vaccines with or without $\alpha$-GalCer did not reveal any significant differences in Treg population size. In addition, there was no difference in suppressive capacity of Tregs that had been purified from the different vaccinated groups, as assessed in vitro. It should be noted that there was no difference in Treg activity when the same experiments were repeated in mice carrying 7-day-old tumors (data not shown). Nor did the addition of $\alpha$-GalCer to the DC vaccine increases the percentage of intratumoral Tregs when compared with mice immunized without $\alpha$-GalCer (data not shown). Collectively, these data suggest that activation of $i$ NKT cells with $\alpha$-GalCer does not drive increased Treg proliferation or activity in our model of anti-tumor vaccination. The reason for this discrepancy with the experimental autoimmune myasthenia gravis model of Liu et al., ${ }^{10}$ where administration of $\alpha$-GalCer enhances Treg activity, is not clear but may reflect the different modes and frequency of $\alpha$-GalCer administration. Although our study involved a single i.v. injection of $\alpha$-GalCer-loaded DC, Liu and colleagues used three consecutive injections of free $\alpha$-GalCer. It has been reported that repeated injections of free $\alpha$-GalCer induce a skewed profile of cytokine release by $i$ NKT cells, which is biased toward the Th2 cytokines and ultimately results in an 'anergic' phenotype. ${ }^{35,36}$ Injection of $\alpha$-GalCer-loaded DC, on the other hand, is reported to elicit a greater Th1-biased response, and does not anergise the cells. ${ }^{37} \mathrm{~A}$ bias toward release of Th1 cytokines by $i$ NKT cells in our system may serve to override the capacity for these cells to drive Treg function. That Th1 cytokines can override Treg induction is supported by a report by Nishikawa et $a l .^{23}$ in which the unexpected induction of Tregs after DNA vaccination with a SEREX-defined self-antigen could be negated by incorporating in the vaccine an epitope that was capable of driving CTL-derived IFN- $\gamma$.

A number of reports have indicated that Tregs can suppress $i$ NKT cell responses ${ }^{22,27}$ and NK-mediated tumor lysis. ${ }^{24,38}$ It was possible, therefore, that inactivation of Tregs in our model of DC vaccination could unleash enhanced activity of $i \mathrm{NKT}$ and NK cells. However, we did not detect increased intracellular cytokine expression in $i$ NKT cells when Tregs were inactivated before administration of $\alpha$-GalCer-loaded DC vaccines. Nor could we find evidence that the absence of Treg activity enhanced the subsequent cascade of cytokine release into the serum and maturation of resident DC after administration of $\alpha$-GalCer. Instead, administration of anti-CD25 led to a slight trend toward a general reduction in serum cytokine levels. We cannot rule out the possibility that residual anti-CD25 antibody is depleting activated $i$ NKT cells as they rapidly upregulate CD25 on activation, thereby potentially reducing the interactions that lead to cytokine release. If true, this negative function of anti-CD25 treatment on NKT cells was certainly not sufficient to negate the enhancing effect of $\alpha$-GalCer on T-cell proliferation, as evident from data presented in Figures 1 and 6 . We conclude that Tregs do not significantly suppress $i \mathrm{NKT}$ cell-mediated activities in our model, and that the main impact of anti-CD25 treatment is to prevent Treg suppression of T-cell responses. It is unlikely that anti-CD25 is enhancing T-cell responses by blocking the IL-2 receptor, and thus preventing activation-induced cell death of responding $\mathrm{T}$ cells, as it has been shown that $\mathrm{CD} 25$ deficient $\mathrm{CD}^{+} \mathrm{T}$ cells display similar primary proliferative responses to CD25 sufficient cells. ${ }^{39}$ The impact of Treg depletion on antigen-specific $\mathrm{CD}^{+} \mathrm{T}$ cells was only evident between days 4 and 7 after DC immunization, in line with previous reports of a lag time of approximately 4 days from Treg stimulation to noticeable Treg suppression. ${ }^{40}$

The data presented here provide a strong incentive to use $\alpha$-GalCer in conjunction with DC-based vaccines loaded with whole, stressed, tumor cells, without concerns for driving Treg function. Importantly, because the CD1d structure is conserved, $\alpha$-GalCer can be used as a 'universal helper epitope' without the complication of MHC restriction associated with peptide antigens. Although not discussed here, $i$ NKT cell activation can also serve an adjuvant function on $\mathrm{CD}^{+}$ T-cell responses. ${ }^{2,3}$ Thus, by using DC loaded with $\alpha$-GalCer and whole stressed tumor cells, it is likely that T-cell responses can be induced toward a whole range of MHC classes I and II-restricted epitopes, including those derived from mutated neo-antigens individual to each tumor. Our results also suggest that this vaccination strategy can be combined successfully with inactivation of Tregs to improve antitumor responses. Strategies to deplete Tregs have now reached the clinic in the form of ONTAK, an IL-2-diphtheria toxin fusion protein that targets cells expressing the IL-2 receptor, ${ }^{41}$ and LMB-2, an antiCD25 antibody fused to Pseudomonas exotoxin A. Although the use of ONTAK to inactivate Tregs in cancer patients is controversial, ${ }^{42}$ it has been demonstrated to significantly improve the stimulation of tumor-specific T-cell responses in RCC patients vaccinated with RNA-transfected DCs. ${ }^{43}$ However, the real promise of ONTAK, or similar strategies to inactivate Tregs, may be in the context of combination therapies such as the inclusion of $\alpha$-GalCer to activate $i$ NKT cells, where the balance between regulation and immunity can be tipped in favor of anti-tumor function. This clinical strategy is therefore worthy of further investigation.

\section{METHODS}

\section{In vitro culture media and reagents}

B16.F10 and B16.OVA ${ }^{44}$ were maintained in complete RPMI medium (referred to as cRPMI) consisting of RPMI medium (Invitrogen, Auckland, New Zealand) supplemented with 5\% FBS (Sigma-Aldrich, Auckland, New Zealand), and $2 \mathrm{~mm}$ glutamax, $100 \mathrm{U} \mathrm{ml}^{-1}$ penicillin, $100 \mu \mathrm{g} \mathrm{ml}^{-1}$ streptomycin, and $50 \mu \mathrm{M} 2$-mercaptoethanol (all Invitrogen). Endotoxin-free OVA protein ${ }^{45}$ was kindly provided by Professor Thomas M Moran, Mount Sinai School of Medicine, New York, USA. The $i$ NKT cell ligand $\alpha$-GalCer was manufactured as described in Lee et al. ${ }^{46}$ and solubilized in $150 \mathrm{~mm} \mathrm{NaCl}, 0.5 \%$ Tween 20.

\section{Antibodies}

The antibodies anti-TCR- $\beta$ (clone H57-597), anti-V $\alpha 2$ (clone B20.1), antiV $35.1 / 5.2$ (clone MR9-4), anti-B220 (clone RA3-6B2), anti-IFN- $\gamma$ (XMG1.2), and $\mathrm{rIgG1}$ isotype control (clone R3-34) were all from BD-Pharmingen (BDPharmingen, Auckland, New Zealand). The antibodies anti-CD11c (clone N418), anti-CD86 (clone GL1), anti-CD4 (clone 11B11), anti-CD8 $\alpha$ (clone 53-6.7), anti-CD25 (clone PC61 and 7D4), and anti CD45.2 (clone 104) were from eBioscience (eBioscience, San Diego, CA, USA). The $\mathrm{H} 2-\mathrm{K}^{\mathrm{b}} / \mathrm{OVA}_{257-264^{-}}$ pentamer and CD1d tetramer were from Proimmune (Oxford, UK), the latter being loaded with $\alpha$-GalCer according to manufacturer's recommendations. PC61 (anti-CD25) and 24G2 (anti-Fc-receptor) were purified by low-pressure liquid chromatography from hybridoma supernatants. Control rIgG for in vivo studies was purchased from Caltag laboratories (Invitrogen).

\section{Mice}

C57BL/6J and the CD45 congenic mice B6-SJptprca (referred to as B6) mice were originally purchased from the Jackson Laboratory (Bar Harbour, ME, USA). OT-I mice expressing a transgenic V $\alpha 2, V \beta 5.1 / 5.2$ TCR specific for an $\mathrm{H}-2 \mathrm{~K}^{\mathrm{b}}$-binding peptide of chicken ovalbumin $\left(\mathrm{OVA}_{257-264}\right)$ were kindly provided by $\mathrm{F}$ Carbone. ${ }^{47}$ FoxP3 ${ }^{\text {gfp }}$ mice were kindly provided by $\mathrm{A}$ Rudensky. ${ }^{26}$ All mice were maintained by the Biomedical Research Unit, Malaghan Institute of Medical Research, Wellington, New Zealand. Experimental 
protocols were approved by Victoria University Animal Ethics Committee, and performed according to their guidelines. Mice used in this study were $7-10$ weeks of age and, when necessary, matched for age and gender.

\section{Culture of BM-DC}

BM-DCs were generated by culturing BM cells from the femur and tibia of 7-10-week-old female C57BL/6 mice in complete IMDM (IMDM media supplemented as cRPMI) with $10 \mathrm{ng} \mathrm{ml}^{-1}$ GM-CSF and $80 \mathrm{Uml}^{-1}$ IL-4 in $5 \mathrm{ml}$ of complete medium, with half of the medium replaced by new medium containing GM-CSF and IL-4 on days 2 and 5.

\section{Anti-CD25 administration}

Tregs were depleted/inactivated by a single intraperitoneal (i.p.) injection of $100 \mu \mathrm{g}$ of anti-CD25 antibody (clone PC61) 2 days before DC immunization. Depletion lasted for at least 12 days as determined in FoxP3gfp mice and by staining with anti-CD25 antibody clone 7D4.

\section{Generation of DC vaccine, vaccination, and tumor challenge}

For generation of the DC vaccine, B16.OVA or B16.F10 cells were first irradiated at $150 \mathrm{~Gy}$ and heat-shocked for $45 \mathrm{~min}$ at $43^{\circ} \mathrm{C}$ as described earlier. ${ }^{34}$ The 'stressed' B16.OVA cells were added to 6-days-old BM-DC cultures in the original six-well plates used to grow the DC at a 1:1 ratio either alone or in combination with $200 \mathrm{ng} \mathrm{ml}^{-1}$ of $\alpha$-GalCer. After incubation, non-adherent and loosely adherent cells were harvested and resuspended in $2 \mathrm{ml}$ of complete media. The cells were underlayed with $2 \mathrm{ml}$ of Ficoll (Pharmacia, Uppsala, Sweden), and centrifuged at 1700 r.p.m. at room temperature, after which live cells were collected from the interface. The cells were washed twice in IMDM and $5 \times 10^{5}$ cells per mouse were injected i.v. in $200 \mu \mathrm{l}$ of IMDM. In some experiments, B16.OVA cells were electroporated with endotoxin-free ovalbumin using the CellPorator electroporation system (Gibco-BRL). B16.OVA cells were washed once in 'cytomix buffer' ( $120 \mathrm{~mm} \mathrm{KCl}, 0.15 \mathrm{~mm} \mathrm{CaCl}_{2}, 25 \mathrm{~mm}$ Hepes, pH 7.6, 20 mM EGTA, $\left.5 \mathrm{~mm} \mathrm{MgCl}_{2}, 10 \mathrm{~mm} \mathrm{~K}_{2} \mathrm{HPO}_{4} / \mathrm{KH}_{2} \mathrm{PO}_{4}, \mathrm{pH} 7.6\right)^{48}$ and resuspended at $1 \times 10^{7}$ cells per $\mathrm{ml}$ in cytomix buffer supplemented with $2 \mathrm{~mm}$ ATP and $10 \mathrm{~mm}$ reduced glutathione. The CellPorator settings were $300 \mathrm{~V}$, $1080 \mu \mathrm{F}$, high resistance and fast pulse. The electroporation efficacy was $30-50 \%$ as determined by the uptake of FITC-labeled ovalbumin right after or the day after electroporation, or GFP-positive cells the day after electroporation with a DNA plasmid encoding GFP. For tumor challenge, mice were challenged 7 days after DC immunization by subcutaneous injection in the right flank of $1 \times 10^{5}$ live B16.OVA or B16.F10 cells in $100 \mu$ l of IMDM. Tumor size was thereafter calculated by caliper measurements as the product of bisecting tumor diameter. Mice were killed when the tumor size reached $150 \mathrm{~mm}^{2}$, or earlier if mice became visibly unwell or if the tumor ulcerated.

\section{Therapeutic DC vaccination}

Mice were challenged with $1 \times 10^{5}$ live B16.OVA, administered anti-CD25 antibody 2 days later, and immunized i.v. with BM-DC loaded as above with stressed B16.OVA cells and $\alpha$-GalCer or only $\alpha$-GalCer the following day (3 days after tumor challenge).

\section{Characterization of OVA-specific CD8 T-cell responses after DC vaccination}

CD8 $\mathrm{T}$ cells specific for the $\mathrm{H} 2-\mathrm{K}^{\mathrm{b}} / \mathrm{OVA}_{257-264} \mathrm{MHC} \mathrm{I} /$ peptide complex were detected in blood using PE-conjugated $\mathrm{H} 2-\mathrm{K}^{\mathrm{b}} / \mathrm{OVA}_{257-264}$-pentamer according to manufacturer's instructions. In some experiments, mice received a small cohort of $50 \times 10^{4}$ OVA-specific OT-I LN cells the day before DC immunization.

\section{Intracellular cytokine staining of $i$ NKT cells}

Mice either remained naive or were injected i.v. with either $200 \mathrm{ng} \alpha$-GalCer (diluted in PBS), or $0.5 \times 10^{6}$ BM-DC, which had been pulsed for $40 \mathrm{~h}$ with stressed B16.OVA cells and $200 \mathrm{ng} \mathrm{ml}^{-1} \alpha$-GalCer. The mice were killed $2 \mathrm{~h}$ later, and single cell suspensions from spleens were treated with RBC lysis buffer (Sigma-Aldrich), and cultured at $25 \times 10^{6}$ cells per $\mathrm{ml}$ in cIMDM supplemented with $0.2 \mu \mathrm{M}$ monensin (Merck, Palmerston North, New Zealand) for $2 \mathrm{~h}$ at $37^{\circ} \mathrm{C}$ and $5 \% \mathrm{CO}_{2}$. After incubation, the cells were washed twice with cold FACS buffer. Unspecific Fc-receptor-mediated antibody staining was blocked by incubation with anti-Fc-receptor antibody, after which the cells were stained with CD1d/ $\alpha$-GalCer tetramer (Proimmune, Oxford, England) for $20 \mathrm{~min}$ on ice. The cells were then spun down, and stained with anti-B220 and anti-TCR- $\beta$ before being fixed overnight at $4{ }^{\circ} \mathrm{C}$ in paraformaldehyde (Sigma-Aldrich). The following morning, the cells were washed twice in saponin buffer (PBS supplemented with $0.1 \%$ BSA, $0.1 \%$ saponin, and $0.05 \% \mathrm{NaN}_{3}$ (all Sigma-Aldrich)), before incubation with intracellular cytokine antibody or isotype control antibody in saponin buffer. The cells were finally washed twice in saponin buffer, resuspended in FACS buffer, and analyzed on a BD FacsCalibur. For analysis, B220-positive B cells were excluded, and $i$ NKT taken as TCR- $\beta^{+}$CD1d/ $\alpha$-GalCer doublepositive cells.

\section{Serum cytokine measurements}

C57BL/6J mice received an intra peritoneal injection of $100 \mu \mathrm{g}$ PC61 or control rIgG. Two days later, mice were injected i.v. with either $200 \mathrm{ng} \alpha$-GalCer or $0.5 \times 10^{6} \mathrm{BM}-\mathrm{DC} / \mathrm{B} 16$.OVA cells pulsed with $200 \mathrm{ng} \mathrm{ml}^{-1} \alpha$-GalCer. Serum was collected by tailbleeding after $2,5,10$, and $24 \mathrm{~h}$, and the concentration of IFN- $\gamma$, IL-4, IL-12 p70, and IL-6 was detected using bioplex cytokine bead arrays (Bio-Rad, Auckland, New Zealand).

\section{T-cell suppression assay}

FoxP3 ${ }^{\text {gfp }}$ mice were injected i.v. with $0.5 \times 10^{6} \mathrm{DC}$ pulsed with stressed B16.OVA cells with or without $\alpha$-GalCer. The mice were killed 6 days later and single cell suspensions from spleens of individual mice were generated. After red blood cell lysis, the total number of splenocytes was determined using a coulter counter and aliquots were stained with antibodies for CD4 and CD25. The remaining splenocytes were pooled, and CD4 cells purified by AutoMacs purification according to manufacturer's recommendations, followed by sorting of $\mathrm{CD} 4^{+} \mathrm{CD} 25^{+}$FoxP $3^{\text {gfp }}+$ (Tregs) and $\mathrm{CD} 4^{+}$FoxP3 ${ }^{\text {gfp }}-\mathrm{T}$ cells using a FACSVantage DIVA. The sorted cells were more than $95 \%$ pure. For suppression assays, $2 \times 10^{4} \mathrm{CD}^{+} \mathrm{FoxP}^{\text {gfp- }}{ }^{-}$cells per well in round 96-well plates were co-cultured with $4 \times 10^{4}$ spleen CD11c antigen presenting cells, $0.5 \mu \mathrm{g} \mathrm{ml}^{-1}$ of anti-CD3 antibody, and a titration of Treg cells. The cultures were incubated for $72 \mathrm{~h}$ with $0.5 \mu \mathrm{Ci} \mathrm{ml}^{-1}$ of ${ }^{3} \mathrm{H}$-thymidine added for the last $16 \mathrm{~h}$ of incubation.

\section{Statistical analyses}

A Mann-Whitney test was used to determine statistical significance in experiments with only two experimental groups and a Krusskall-Wallis test was used to determine statistical significance in experiments with three or more groups. Dunn's post test was used to determine statistical significance between two individual groups within a larger experiment. The time mice remained tumor free was plotted as Kaplan-Meier plots and statistical significance between individual curves determined using the logrank test. All statistical analyses were done with GraphPad Prism software (GraphPad Software Inc., La Jolla, CA, USA).

\section{ACKNOWLEDGEMENTS}

We thank the personnel of the Biomedical Research Unit of the Malaghan Institute of Medical Research for animal husbandry. We are very grateful to Thomas Moran and Bruno Moltedo for the supply of endotoxin-free OVA, Drs E Lord and JG Frelinger, Professor F Carbone, and A Rudensky for generously providing cell lines and mouse strains used in this study, and Dr GF Painter and A Lee for providing the $\alpha$-GalCer. This work was supported by the New Zealand Health Research Council and the Cancer Society of New Zealand. IFH was supported by a New Zealand Health Research Council Sir Charles Hercus Fellowship.

1 Bendelac A, Savage PB, Teyton L. The biology of NKT cells. Annu Rev Immunol 2007; 25: 297-336.

2 Hermans IF, Silk JD, Gileadi U, Salio M, Mathew B, Ritter G et al. NKT cells enhance $\mathrm{CD} 4+$ and $\mathrm{CD} 8+\mathrm{T}$ cell responses to soluble antigen in vivo through direct interaction with dendritic cells. J Immunol 2003; 171: 5140-5147.

3 Fujii S, Shimizu K, Smith C, Bonifaz L, Steinman RM. Activation of natural killer T cells by alpha-galactosylceramide rapidly induces the full maturation of dendritic cells 
in vivo and thereby acts as an adjuvant for combined CD4 and CD8 T cell immunity to a coadministered protein. J Exp Med 2003; 198: 267-279.

4 Hermans IF, Silk JD, Gileadi U, Masri SH, Shepherd D, Farrand KJ et al. Dendritic cell function can be modulated through cooperative actions of TLR ligands and invariant NKT cells. J Immunol 2007; 178: 2721-2729.

5 Silk JD, Hermans IF, Gileadi U, Chong TW, Shepherd D, Salio M et al. Utilizing the adjuvant properties of CD1d-dependent NK T cells in T cell-mediated immunotherapy. J Clin Invest 2004; 114: 1800-1811.

6 Kitamura H, Iwakabe K, Yahata T, Nishimura S, Ohta A, Ohmi Y et al. The natural killer $\mathrm{T}$ (NKT) cell ligand alpha-galactosylceramide demonstrates its immunopotentiating effect by inducing interleukin (IL)-12 production by dendritic cells and IL-12 receptor expression on NKT cells. J Exp Med 1999; 189: 1121-1128.

7 Eberl G. MacDonald HR. Selective induction of NK cell proliferation and cytotoxicity by activated NKT cells. Eur J Immunol 2000; 30: 985-992.

8 Fujii S, Shimizu K, Hemmi H, Steinman RM. Innate Valpha14(+) natural killer T cells mature dendritic cells, leading to strong adaptive immunity. Immunol Rev 2007; 220 183-198.

9 Colombo MP, Trinchieri G. Interleukin-12 in anti-tumor immunity and immunotherapy. Cytokine Growth Factor Rev 2002; 13: 155-168.

10 Liu R, La Cava A, Bai XF, Jee Y, Price M, Campagnolo Dl et al. Cooperation of invariant NKT cells and CD4+CD25+ T regulatory cells in the prevention of autoimmune myasthenia. J Immunol 2005; 175: 7898-7904.

11 Jiang S, Game DS, Davies D, Lombardi G, Lechler RI. Activated CD1d-restricted natural killer T cells secrete IL-2: innate help for CD4+CD25+ regulatory T cells? Eur J Immunol 2005; 35: 1193-1200.

12 La Cava A, Van Kaer L, Fu Dong S. CD4+CD25+ Tregs and NKT cells: regulators regulating regulators. Trends Immunol 2006; 27: 322-327.

13 Bluestone JA, Abbas AK. Natural versus adaptive regulatory T cells. Nat Rev Immunol 2003; 3: 253-257.

14 Sakaguchi S, Ono M, Setoguchi R, Yagi H, Hori S, Fehervari Z et al. Foxp3+ CD25+ CD4+ natural regulatory T cells in dominant self-tolerance and autoimmune disease. Immunol Rev 2006; 212: 8-27.

15 Baecher-Allan C, Anderson DE. Immune regulation in tumor-bearing hosts. Curr Opin Immunol 2006; 18: 214-219.

16 Wang RF. Immune suppression by tumor-specific CD4+ regulatory T-cells in cancer. Semin Cancer Biol 2006; 16: 73-79.

17 Beyer M, Schultze JL. Regulatory T cells in cancer. Blood 2006; 108: 804-811.

18 Shimizu J, Yamazaki S, Sakaguchi S. Induction of tumor immunity by removing CD25+CD4+ T cells: a common basis between tumor immunity and autoimmunity. J Immunol 1999; 163: 5211-5218.

19 Golgher D, Jones E, Powrie F, Elliott T, Gallimore A. Depletion of CD25+ regulatory cells uncovers immune responses to shared murine tumor rejection antigens. Eur J Immunol 2002; 32: 3267-3275

20 Quezada SA, Peggs KS, Simpson TR, Shen Y, Littman DR, Allison JP. Limited tumor infiltration by activated $T$ effector cells restricts the therapeutic activity of regulatory T cell depletion against established melanoma. J Exp Med 2008; 205: 2125-2138.

21 Turk MJ, Guevara-Patino JA, Rizzuto GA, Engelhorn ME, Sakaguchi S, Houghton AN. Concomitant tumor immunity to a poorly immunogenic melanoma is prevented by regulatory T cells. J Exp Med 2004; 200: 771-782

22 Azuma T, Takahashi T, Kunisato A, Kitamura T, Hirai H. Human CD4+ CD25+ regulatory T cells suppress NKT cell functions. Cancer Res 2003; 63: 4516-4520.

23 Nishikawa H, Kato T, Tanida K, Hiasa A, Tawara I, Ikeda H et al. CD4+ CD25+ T cells responding to serologically defined autoantigens suppress antitumor immune responses. Proc Natl Acad Sci USA 2003; 100: 10902-10906.

24 Smyth MJ, Teng MW, Swann J, Kyparissoudis K, Godfrey DI, Hayakawa Y. CD4+CD25+ T regulatory cells suppress NK cell-mediated immunotherapy of cancer. J Immunol 2006; 176: 1582-1587.

25 Ghiringhelli F, Menard C, Terme M, Flament C, Taieb J, Chaput N et al. CD4+CD25+ regulatory $T$ cells inhibit natural killer cell functions in a transforming growth factorbeta-dependent manner. J Exp Med 2005; 202: 1075-1085.

26 Fontenot JD, Rasmussen JP, Williams LM, Dooley JL, Farr AG, Rudensky AY. Regulatory T cell lineage specification by the forkhead transcription factor foxp3. Immunity 2005; 22: 329-341.
27 Ly D, Mi QS, Hussain S, Delovitch TL. Protection from type 1 diabetes by invariant NK T cells requires the activity of CD4+CD25+ regulatory T cells. J Immunol 2006; 177: 3695-3704.

28 Neller MA, Lopez JA, Schmidt CW. Antigens for cancer immunotherapy. Semin Immunol 2008; 20: 286-295.

29 Zhou G, Drake CG, Levitsky HI. Amplification of tumor-specific regulatory T cells following therapeutic cancer vaccines. Blood 2006; 107: 628-636.

30 Banerjee DK, Dhodapkar MV, Matayeva E, Steinman RM, Dhodapkar KM. Expansion of FOXP3high regulatory T cells by human dendritic cells (DCs) in vitro and after injection of cytokine-matured DCs in myeloma patients. Blood 2006; 108: 2655-2661.

31 Liu K, Idoyaga J, Charalambous A, Fujii S, Bonito A, Mordoh J et al. Innate NKT lymphocytes confer superior adaptive immunity via tumor-capturing dendritic cells. J Exp Med 2005; 202: 1507-1516.

32 Allan R, Waithman J, Bedoui S, Jones C, Villadangos J, Zhan Y et al. Migratory dendritic cells transfer antigen to a lymph node-resident dendritic cell population for efficient CTL priming. Immunity 2006; 25: 153-162.

33 Farrand KJ, Dickgreber N, Stoitzner P, Ronchese F, Petersen TR, Hermans IF. Langerin+CD8alpha+ dendritic cells are critical for cross-priming and IL-12 production in response to systemic antigens. J Immunol 2009; 183: 7732-7742.

34 Prasad SJ, Farrand KJ, Matthews SA, Chang JH, McHugh RS, Ronchese F. Dendritic cells loaded with stressed tumor cells elicit long-lasting protective tumor immunity in mice depleted of CD4+CD25+ regulatory T cells. J Immunol 2005; 174: 90-98.

35 Parekh VV, Wilson MT, Olivares-Villagomez D, Singh AK, Wu L, Wang CR et al. Glycolipid antigen induces long-term natural killer T cell anergy in mice. J Clin Invest 2005; 115: 2572-2583.

36 Sullivan BA, Kronenberg M. Activation or anergy: NKT cells are stunned by alphagalactosylceramide. J Clin Invest 2005; 115: 2328-2329.

37 Fujii S, Shimizu K, Kronenberg M, Steinman RM. Prolonged IFN-gamma-producing NKT response induced with alpha-galactosylceramide-loaded DCs. Nat Immunol 2002; 3: $867-874$

38 Ghiringhelli F, Menard C, Martin F, Zitvogel L. The role of regulatory T cells in the control of natural killer cells: relevance during tumor progression. Immunol Rev 2006; 214: 229-238.

39 Williams MA, Tyznik AJ, Bevan MJ. Interleukin-2 signals during priming are required for secondary expansion of CD8+ memory T cells. Nature 2006; 441: 890-893.

40 Deaglio S, Dwyer KM, Gao W, Friedman D, Usheva A, Erat A et al. Adenosine generation catalyzed by CD39 and CD73 expressed on regulatory $T$ cells mediates immune suppression. J Exp Med 2007; 204: 1257-1265.

41 Foss FM. DAB(389)IL-2 (ONTAK): a novel fusion toxin therapy for lymphoma. Clin Lymphoma 2000; 1: 110-116; discussion 117.

42 Attia P, Maker AV, Haworth LR, Rogers-Freezer L, Rosenberg SA. Inability of a fusion protein of IL-2 and diphtheria toxin (Denileukin Diftitox, DAB389IL-2, ONTAK) to eliminate regulatory $\mathrm{T}$ lymphocytes in patients with melanoma. J Immunother 2005; 28: 582-592.

43 Dannull J, Su Z, Rizzieri D, Yang BK, Coleman D, Yancey D et al. Enhancement of vaccine-mediated antitumor immunity in cancer patients after depletion of regulatory T cells. J Clin Invest 2005; 115: 3623-3633.

44 Lugade AA, Moran JP, Gerber SA, Rose RC, Frelinger JG, Lord EM. Local radiation therapy of B16 melanoma tumors increases the generation of tumor antigen-specific effector cells that traffic to the tumor. J Immunol 2005; 174: 7516-7523.

45 Brimnes MK, Bonifaz L, Steinman RM, Moran TM. Influenza virus-induced dendritic cell maturation is associated with the induction of strong $T$ cell immunity to a coadministered, normally nonimmunogenic protein. J Exp Med 2003; 198: 133-144.

46 Lee A, Farrand KJ, Dickgreber N, Hayman CM, Jurs S, Hermans IF et al. Novel synthesis of alpha-galactosyl-ceramides and confirmation of their powerful NKT cell agonist activity. Carbohydr Res 2006; 341: 2785-2798.

47 Kelly JM, Sterry SJ, Cose S, Turner SJ, Fecondo J, Rodda S et al. Identification of conserved T cell receptor CDR3 residues contacting known exposed peptide side chains from a major histocompatibility complex class I-bound determinant. Eur J Immunol 1993; 23: 3318-3326.

48 van den Hoff MJ, Moorman AF, Lamers WH. Electroporation in 'intracellular' buffer increases cell survival. Nucleic Acids Res 1992; 20: 2902 


\title{
Exploiting the Role of Endogenous Lymphoid-Resident Dendritic Cells in the Priming of NKT Cells and CD8+ T Cells to Dendritic Cell-Based Vaccines
}

\author{
Troels R. Petersen ${ }^{1 *}$, Dianne Sika-Paotonu ${ }^{1}$, Deborah A. Knight ${ }^{1}$, Helen M. A. Simkins ${ }^{1}$, lan F. Hermans ${ }^{1,2}$ \\ 1 Malaghan Institute of Medical Research, Wellington, New Zealand, 2 School of Biological Sciences, Victoria University of Wellington, Wellington, New Zealand
}

\begin{abstract}
Transfer of antigen between antigen-presenting cells (APCs) is potentially a physiologically relevant mechanism to spread antigen to cells with specialized stimulatory functions. Here we show that specific $\mathrm{CD} 8^{+} \mathrm{T}$ cell responses induced in response to intravenous administration of antigen-loaded bone marrow-derived dendritic cells (BM-DCs), were ablated in mice selectively depleted of endogenous lymphoid-resident langerin ${ }^{+} \mathrm{CD} 8 \alpha^{+}$dendritic cells (DCs), suggesting that the antigen is transferred from the injected cells to resident APCs. In contrast, antigen-specific CD4 ${ }^{+} \mathrm{T}_{\text {cells }}$ were primed predominantly by the injected BM-DCs, with only very weak contribution of resident APCs. Crucially, resident langerin ${ }^{+}$ $\mathrm{CD} 8 \alpha^{+} \mathrm{DCs}$ only contributed to the priming of $\mathrm{CD}^{+} \mathrm{T}$ cells in the presence of maturation stimuli such as intravenous injection of TLR ligands, or by loading the BM-DCs with the glycolipid $\alpha$-galactosylceramide $(\alpha$-GalCer) to recruit the adjuvant activity of activated invariant natural killer-like T (iNKT) cells. In fact, injection of $\alpha$-GalCer-loaded CD1d ${ }^{-1-}$ BM-DCs $^{-}$ resulted in potent $i$ NKT cell activation, suggesting that this glycolipid antigen can also be transferred to resident $C^{+} 1 \mathrm{~d}^{+}$ APCs. While iNKT cell activation per se was independent of langerin ${ }^{+} \mathrm{CD} 8 \alpha^{+} \mathrm{DC}$, some iNKT cell-mediated activities were reduced, notably release of IL-12p70 and transactivation of NK cells. We conclude that both protein and glycolipid antigens can be exchanged between distinct DC species. These data suggest that the efficacy of DC-based vaccination strategies may be improved by the incorporation of a systemic maturation signal aimed to engage resident APCs in CD8 ${ }^{+} \mathrm{T}$ cell priming, and $\alpha$-GalCer may be particularly well suited to this purpose.
\end{abstract}

Citation: Petersen TR, Sika-Paotonu D, Knight DA, Simkins HMA, Hermans IF (2011) Exploiting the Role of Endogenous Lymphoid-Resident Dendritic Cells in the Priming of NKT Cells and CD8+ T Cells to Dendritic Cell-Based Vaccines. PLoS ONE 6(3): e17657. doi:10.1371/journal.pone.0017657

Editor: William Halford, Southern Illinois University School of Medicine, United States of America

Received October 6, 2010; Accepted February 7, 2011; Published March 31, 2011

Copyright: (c) 2011 Petersen et al. This is an open-access article distributed under the terms of the Creative Commons Attribution License, which permits unrestricted use, distribution, and reproduction in any medium, provided the original author and source are credited.

Funding: This work was funded by Health Research Council of New Zealand (http://www.hrc.govt.nz) project grants 06/316 and 09/105D. I.F.H. was supported by a New Zealand Health Research Council Sir Charles Hercus Fellowship and DSP was supported by a Health Research Council of New Zealand Pacific Health Research Award. The funders had no role in study design, data collection and analysis, decision to publish, or preparation of the manuscript.

Competing Interests: Industrial Research Limited supplied $\alpha$-GalCer. This does not alter the authors' adherence to all the PLoS ONE policies on sharing data and materials.

*E-mail: tpetersen@malaghan.org.nz

\section{Introduction}

Migratory DCs perform a sentinel role as immature cells in the peripheral tissues. They respond to local danger signals, such as factors released by perturbed tissue in response to infection, by migrating to the draining lymphoid tissues with antigens they have acquired from the local environment. In the process migratory DCs undergo maturation, characterized by increased expression of costimulatory molecules, adhesion molecules and MHC molecules, to provide a stimulatory phenotype capable of driving activation and proliferation of antigen-specific $\mathrm{T}$ cells. There is also a level of constitutive migration of DCs from peripheral tissues to the lymphoid organs in the absence of danger, so that sizable populations of DCs with migratory phenotype can be detected in lymph nodes and spleen [1]. So-called 'lymphoid-resident' DCs (referred to as resident DCs), on the other hand, seed the lymphoid tissues directly as precursors released from the bone marrow. In the steady state, lymphoid-resident DGs represent approximately half of the DGs in the lymph nodes, and the majority of DCs in the spleen $[1,2]$. In mouse spleen three populations of lymphoidresident $\mathrm{DC}$ can be distinguished on the basis of expression of $\mathrm{CD} 4$ or $\mathrm{CD} 8 \alpha\left(\mathrm{CD} 4^{+} \mathrm{DC}, \mathrm{CD} 8 \alpha^{+} \mathrm{DC}\right.$, or double negative $\left.\mathrm{DC}\right)$
$[2,3,4]$, with some functional specialization between the subsets. In particular, $\mathrm{CD} 8 \alpha^{+}$DCs have a heightened capacity to crosspresent soluble and cell-associated antigens via MHC class I molecules to $\mathrm{CD}^{+} \mathrm{T}$ cells $[5,6,7,8]$, and are major producers of IL-12 in response to microbial activation stimuli $[9,10]$.

Lymphoid-resident DCs have been generally assumed to acquire antigens and danger signals directly from blood or lymph circulating through the lymphoid tissues. A less appreciated mechanism of acquiring antigen involves transfer from migratory DCs to lymphoid-resident DCs. It has been shown that migratory DCs that drain the skin following herpes simplex virus infection can transfer antigens to lymph node-resident $\mathrm{CD} 8 \alpha^{+} \mathrm{DCs}$, which are then responsible for stimulating $\mathrm{CD}^{+} \mathrm{T}$ cell responses [11]. A similar transfer process between migrating DCs and resident $\mathrm{CD} 8 \alpha^{+}$DCs has been reported in lymph-nodes draining the lung following influenza infection [12], suggesting antigen transfer is a physiologically relevant mechanism for spreading antigen to specialized antigen-presenting cells to enhance specific $\mathrm{T}$ cell responses. The phenotype and maturation status of the recipient lymphoid-resident DC populations may therefore be a critical factor in driving effective immune responses. In the case of an infection such as herpes simplex virus, it is possible that infection-driven 
maturation of recipient DCs ultimately drives the T cell response. In other situations, effective immunity may be reliant on circulation of factors capable of triggering maturation of lymphoid-resident DCs. In the absence of these stimuli, regardless of the maturation state of the migratory DC, the resident DCs may fail to stimulate immunity, or could potentially alter the response through, for example, the induction of $\mathrm{T}$ regulatory cells [13]. This possibility may be particularly relevant in the context of DC vaccination, as transfer of antigen from injected DCs to endogenous antigen presenting cells has been reported in animal models $[14,15,16]$, and has been suggested to contribute significantly to the vaccine induced $\mathrm{CD}^{+} \mathrm{T}$ cell responses [17]. Attempts to induce anti-tumor $\mathrm{T}$ cell responses in cancer patients typically involve culture of autologous DCs with tumor tissue or specific tumor antigens, followed by a DC maturation step prior to injection. While this maturation step may enhance migration of the injected DCs from the site of injection to the draining $\mathrm{LN}$, and enhance $\mathrm{T}$ cell priming by ensuring adequate expression of costimulatory markers, it may not support effective $\mathrm{T}$ cell stimulation by resident DGs that acquire antigen from injected cells.

We reasoned that $\mathrm{CD}^{+} \mathrm{T}$ cell activation in response to $\mathrm{DC}$ vaccination could be enhanced by strategies aimed at optimizing the function of lymphoid-resident DC capable of cross-presentation. In the mouse, a subset of lymphoid-resident CD8 $\alpha^{+}$DC expressing the c-type lectin langerin (CD207) has been shown to be primarily responsible for cross-presentation and IL-12 production following injection of soluble antigen and adjuvant [18]. We therefore investigated the role of langerin ${ }^{+} \mathrm{CD} 8 \alpha^{+} \mathrm{DCs}$ after injection of antigen-loaded DCs in a transgenic animal model in which langerin $^{+}$cells can be specifically depleted in vivo. We show that langerin ${ }^{+} \mathrm{CD} 8 \alpha^{+}$can indeed capture antigen from intravenously injected DGs, and can significantly contribute to induced CTL responses, but only if they receive appropriate maturation stimuli. In this context, loading the injected DCs with $\alpha$ galactosylceramide $(\alpha$-GalCer) to provoke 'licensing' by $i$ NKT cells $[19,20,21,22,23,24]$ proved to be particularly effective, as this glycolipid antigen was also effectively transferred to resident DCs, allowing the recipient cells to also become licensed for CTL induction. Thus, transfer of protein and glycolipid antigens to lymphoid resident DCs is a feature of DC vaccination that can be exploited to improve vaccine outcome.

\section{Materials and Methods}

\section{Mice}

Breeding pairs of the inbred strains C57BL/6j $\left(\mathrm{H} 2^{\mathrm{b}}\right.$, CD45.2 $\left.{ }^{+}\right)$ and B6.Sjptprca $\left(\mathrm{H} 2^{\mathrm{b}}, \mathrm{CD} 45.1^{+}\right)$were obtained from the Animal Resource Centre, Canning Vale, Western Australia. Also used were $\mathrm{B} 10 / \mathrm{Q}-\mathrm{H} 2^{\mathrm{q}} / \mathrm{SgAi}$ mice $\left(\mathrm{H}^{\mathrm{q}}\right)$ (referred to as B10.Q), (Taconic Repository, NIH, Bethesda MD, USA), OT-I mice expressing a transgenic $\mathrm{V} \alpha 2, \mathrm{~V} \beta 5.1 / 5.2 \mathrm{~T}$ cell receptor (TCR) specific for an $\mathrm{H}-2 \mathrm{~K}^{\mathrm{b}}$-binding peptide of chicken ovalbumin $\left(\mathrm{OVA}_{257-264}\right.$ ) [25], OT-II mice, expressing a transgenic V $\alpha 2, \mathrm{~V} \beta$ 5.1/5.2 TCR recognizing the $\mathrm{I}_{-} \mathrm{A}^{\mathrm{b}}$-restricted epitope $\mathrm{OVA}_{323-339}$ [26], Langerin-DTREGFP (referred to as lang-DTREGFP) mice expressing the human Diphtheria toxin (DT) receptor under control of the langerin promoter [27] (kindly provided by B. Malissen, Marseille, France), CDld ${ }^{-/-}$mice [28] (kindly provided by G-R Wang, University of Chicago, Illinois, USA), which are devoid of Val4 $i$ NKT cells, and TLR-4 $4^{-/-}$mice [29] (kindly provided by Dr S Akira, Hyogo College of Medicine, Japan). For some adoptive transfer experiments OT-I or OT-II animals were crossed with B6.Sjptprca animals (referred to as CD45.1/OT-I and CD45.1/OT-II), so that the congenic marker CD45.1 could be used to discriminate the transferred cells. All mice were maintained by the Biomedical Research Unit, Malaghan Institute of Medical Research, Wellington, New Zealand. Experimental protocols were approved by Victoria University Animal Ethics Committee (permit number 2007R17M), and performed according to their guidelines. Mice used in this study were 7-10 weeks of age and, when necessary, matched for age and gender.

\section{Culture media and reagents}

Bone marrow-derived DCs (BM-DCs) were cultured in complete IMDM (cIMDM) consisting of IMDM medium (Invitrogen, Auckland, New Zealand) supplemented with 5\% FBS (SigmaAldrich, Auckland, New Zealand), and $2 \mathrm{mM}$ glutamax, $100 \mathrm{U} / \mathrm{ml}$ penicillin, $100 \mu \mathrm{g} / \mathrm{ml}$ streptomycin, and $50 \mu \mathrm{M}$ 2-mercaptoethanol (all Invitrogen). The $i$ NKT cell ligand $\alpha$-GalCer was manufactured as described in [30] and solubilized in $150 \mathrm{mM} \mathrm{NaCl}, 0.5 \%$ Tween 20. Endotoxin free chicken OVA was purchased from Profos AG (Profos AG, Regensburg, Germany). The toll-like receptor (TLR) ligands used were polyinosinic polycytidylic acid $(\mathrm{p}(\mathrm{I}: \mathrm{G})$ ) (InvivoGen, San Diego, CA, USA) and monophosphoryl lipid A (MPL) (Sigma-Aldrich). The antibodies used were: anti-V $\alpha 2$ (clone B20.1), anti-IFN- $\gamma$ (clone XMG1.2), anti-B220 (clone RA3-6B2), antiTCR- $\beta$ (clone H57-597), and anti-V $\beta 5.1 / 5.2$ (clone MR9-4) from BD-Pharmingen (BD-Pharmingen, Auckland, New Zealand), and anti-IL-4 (clone 11B11), anti-CD4 (clone 11B11), anti-CD8 $\alpha$ (clone 53-6.7), anti-CD86 (clone GL1), and anti-CD45.1 (clone A20) from eBioscience (eBioscience, San Diego, CA, USA). Propidium iodide (PI) was from Sigma, CFSE from Molecular Probes (Molecular Probes, Invitrogen, New Zealand). Diphtheria toxin (DT) was from Sigma-Aldrich.

\section{Generation, antigen loading, and injection of bone marrow-derived DCs}

Bone marrow-derived dendritic cells (BM-DCs) were generated by culturing bone-marrow cells from the femur and tibia of 7-10 week old mice in cIMDM media with $10 \mathrm{ng} / \mathrm{ml}$ GM-CSF and $20 \mathrm{ng} / \mathrm{ml} \mathrm{IL-4}$ in $5 \mathrm{ml}$ of complete medium, with half of the medium replaced by new medium containing GM-CSF and IL-4 on day 2 and 5 . On day 6 , cultures were added OVA at $1 \mathrm{mg} / \mathrm{ml}$ and/or $\alpha$-GalCer at $200 \mathrm{ng} / \mathrm{ml}$. In some cultures OVA was added with MPL at $100 \mathrm{ng} / \mathrm{ml}$. After overnight incubation, loosely attached cells were spun down and washed at least three times in IMDM, and $5 \times 10^{5}$ DCs were injected intravenously in $200 \mu \mathrm{l}$ IMDM. In some experiments, $25 \mu \mathrm{g}$ MPL, $100 \mu \mathrm{g}$ p(I:C), or $200 \mathrm{ng} \alpha$-GalCer was injected $i . v$. in the contralateral tail vein after administration of OVA-loaded BM-DCs.

\section{Flow cytometry}

All antibody labeling was performed on ice in FACS buffer (PBS supplemented with $1 \%$ FCS, $0.05 \%$ sodium azide, and $2 \mathrm{mM}$ EDTA). Non-specific FcR-mediated antibody staining was blocked by incubation for $10 \mathrm{~min}$ with anti-CD16/32 Ab (24G2, prepared in-house from hybridoma supernatant). Flow cytometry was performed on a BD Biosciences FACSCalibur or BD LSRII SORP flow cytometer with data analysis using FlowJo software (Tree Star, Inc., OR, USA).

\section{Tracking of $\mathrm{T}$ cell responses}

To facilitate detection of OVA-specific $\mathrm{T}$ cells, mice received a cohort of $5 \times 10^{4} \mathrm{LN}$ cells from CD45.1/OT-I mice or $5 \times 10^{6} \mathrm{LN}$ cells from CD45.1/OT-II mice. Mice were tail-bled 7 days after vaccination and the percentage of OT-I or OT-II cells of all CD8 ${ }^{+}$ or $\mathrm{CD}^{+}$cells respectively, was determined by flow cytometry. In 
some experiments, OT-II cells were labeled with $1 \mu \mathrm{M}$ CFSE before injection, and the OT-II T cell proliferation was assessed in the spleen three days after DC vaccination.

\section{In vivo depletion of langerin ${ }^{+}$cells}

Lang-DTREGFP mice were injected i.p. with $350 \mathrm{ng}$ of DT $48 \mathrm{~h}$ and $24 \mathrm{~h}$ day prior to administration of DCs to deplete langerin ${ }^{+}$ cells [18]. In some experiments, control C57BL/6j mice were similarly injected with DT.

\section{Pentamer staining and in vivo killing assay}

Lang-DTREGFP mice were injected with DT or PBS and vaccinated with C57BL/6j BM-DCs loaded with OVA and $\alpha$ GalCer as described above. The percentage of $\mathrm{CD}^{+} \mathrm{T}$ cells specific for the $\mathrm{OVA}_{257-264}$-peptide was determined in the blood 6 days later by flow cytometry using anti-CD8 antibody and fluorescently labeled $\mathrm{K}^{\mathrm{b}} / \mathrm{OVA}_{257-264}$ pentamers (ProImmune, Oxford, UK) according to manufacturers instructions. The following day, in vivo cytotoxicity was assessed against syngeneic spleen cell populations loaded with $\mathrm{OVA}_{257-264}$ peptide and administered by i.v. injection. Mixtures of three populations were injected: a control population without peptide fluorescence-labeled with $10 \mu \mathrm{M}$ of the dye chloromethyl-benzoyl-aminotetramethylrhodamine (CTO) and two target populations loaded with 50 or $5 \mathrm{nM} \mathrm{OVA}_{257-264}$ peptide and fluorescence-labeled with CFSE at different dye concentrations (1.65 and $0.3 \mathrm{nM}$ respectively). Peptide-specific lysis was assessed by flow cytometry of blood $16 \mathrm{~h}$ later, with the mean percent survival of peptide-loaded targets cells calculated relative to that of peptide-negative controls. Cytotoxic activity was expressed as the percent specific lysis, calculated by the equation 100 - mean percent survival of peptideloaded targets.

\section{Intracellular cytokine staining of $i$ NKT cells}

Mice were injected i.v. with $5 \times 10^{5} \alpha$-GalCer-loaded BM-DCs. The mice were sacrificed $2 \mathrm{~h}$ later, and single cell suspensions prepared from spleens were depleted of red blood cells (RBC lysis buffer, Qiagen), washed twice with cold FACS buffer, and incubated with anti-Fc receptor antibody $2.4 \mathrm{G} 2$ to block nonspecific antibody binding. The cells were then incubated with CDld/ $\alpha$-GalCer tetramer (ProImmune) for $20 \mathrm{~min}$ on ice followed by incubation with anti-B220 and anti-TCR- $\beta$ antibodies for 15 min before being fixed overnight at $4^{\circ} \mathrm{C}$ in $4 \%$ formaldehyde (Sigma-Aldrich). The cells were then washed twice in saponin buffer (PBS supplemented with $0.1 \%$ BSA, $0.1 \%$ saponin, and $0.05 \%$ $\mathrm{NaN}_{3}$ (all Sigma-Aldrich), before incubation with anti-IL-4, antiIFN- $\gamma$, or isotype control antibody, in saponin buffer on ice for $15 \mathrm{~min}$. The cells were finally washed twice in saponin buffer, resuspended in FACS buffer, and analyzed on a BD FacsCalibur. For analysis, B220 ${ }^{+}$cells were excluded, and $i \mathrm{NKT}$ cells gated as TCR- $\beta^{+}$GDld $/ \alpha-$ GalCer $^{+}$cells.

\section{Analysis of cytokine release into serum}

Blood was collected from the lateral tail vein at different time intervals after BM-DC administration. Serum was collected after blood had clotted, and levels of the cytokines IL-12p70, IL-4, and IFN- $\gamma$ were assessed by bioplex cytokine bead arrays (Bio-Rad) according to the manufacturer's instructions.

\section{Uptake of BM-DCs by spleen DCs}

BM-DCs generated from B6.Sjptprca $\left(\mathrm{H} 2^{\mathrm{b}}, \mathrm{CD} 45.1^{+}\right)$mice were labeled with $1.5 \mu \mathrm{M}$ CFSE, and $10 \times 10^{6} \mathrm{BM}-\mathrm{DC}$ s were injected i.v. in C57BL/6j mice. Spleens were removed 9-16 h later, and digested with liberase and DNAse I to aid release of resident antigen-presenting cells (both Roche, Auckland, New Zealand). DCs were isolated from the splenocyte preparations with magnetic sorting (CD1lc-MACS MicroBeads, clone N418; Miltenyi Biotec, Bergisch Gladbach, Germany) according to manufacturers instructions, and analyzed by flow cytometry with antibodies against CD11c, CD8 $\alpha$ and CD45.2, and using DAPI (Invitrogen) to discriminate viable cells.

\section{Statistical analyses}

A Mann-Whitney test was used to determine statistical significance in experiments with two experimental groups. In experiments with three or more groups, statistical significance was determined using a Krusskal-Wallis test with Dunn's post test used to determine statistical significance between two individual groups. All statistical analyses were done with GraphPad Prism software (GraphPad Software, Inc., La Jolla, CA, USA).

\section{Results}

\section{Resident APCs cross-present antigen acquired from injected BM-DCs}

Vaccination with protein-loaded DCs has been demonstrated to prime antigen-specific $\mathrm{CD} 4^{+}$and $\mathrm{CD}^{+} \mathrm{T}$ cell responses, but the contribution of resident DCs in this process remains unclear. To establish whether resident DCs acquire and present antigen derived from injected DCs, we examined the in vivo priming of a cohort of OVA-specific $\mathrm{CD}^{+}{ }^{+}$transgenic T cells (OT-I T cells) in C57BL/6j recipients injected with OVA-loaded BM-DCs from syngeneic $\left(\mathrm{C} 57 \mathrm{BL} / 6 \mathrm{j} ; \mathrm{H}-2^{\mathrm{b}}\right)$ or allogeneic (B10.Q; H-2 ${ }^{\mathrm{q}}$ ) animals. As OT-I T cells recognize OVA as a processed peptide $\left(\mathrm{OVA}_{257-264}\right)$ presented by $\mathrm{H}-2 \mathrm{~K}^{\mathrm{b}}$ molecules, only syngeneic BM-DCs can provide a direct stimulus to these $\mathrm{T}$ cells; any OT-I T responses initiated after injection of allogeneic $\mathrm{H}-2^{\mathrm{q}}$ BM-DCs must therefore involve "indirect" cross-presentation via resident APCs. For these experiments, the BM-DCs were also loaded with the $i$ NKT-cell ligand $\alpha$ GalCer, which significantly enhances $\mathrm{T}$ cell responses by promoting $i$ NKT cell-mediated licensing of APCs [19,20,21,22,23,24]. To ensure interaction with $i \mathrm{NKT}$ cells, the BM-DCs were administered i.v. thereby gaining access to the $i \mathrm{NKT}$ cell-rich areas of the spleen. The accumulation of OT-I T cells was determined in the blood 7 days after BM-DC administration (Fig. 1A). As expected, injection of syngeneic BM-DCs loaded with OVA alone induced a moderate expansion of the OT-I T cell cohort, while BM-DCs loaded with both OVA and $\alpha$-GalCer provoked a much stronger response due to the adjuvant activity of stimulating $i$ NKT cells (Fig. $1 B$ ). Interestingly, injection of allogeneic BM-DCs loaded with OVA and $\alpha$-GalCer also resulted in accumulation of OT-I T cells, although to lower levels than syngeneic BM-DCs (Fig. 1G), despite the fact that the allogeneic BM-DCs were unable to directly stimulate the transgenic $\mathrm{T}$ cells. We conclude that antigen can be transferred from injected DCs to resident APCs for processing and indirect cross-presentation to $\mathrm{CD}^{+} \mathrm{T}$ cells.

\section{Depletion of langerin ${ }^{+}$DCs ablates indirect antigen- presentation by resident DCs}

We next determined which population of resident APCs was responsible for capture and indirect cross-presentation of antigen derived from injected BM-DCs. Lymphoid-resident DCs expressing a homodimer of CD8 $\alpha$ have previously been demonstrated to efficiently cross-present soluble and cell-associated antigens $[5,6,7,8]$. In addition, we have recently shown that the ability to cross-present soluble antigen resides almost exclusively within a subpopulation of $\mathrm{CD} 8 \alpha^{+}$DCs that express the c-type lectin 
A

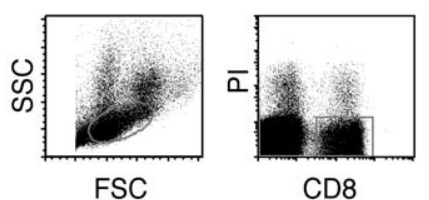

FSC

CD8

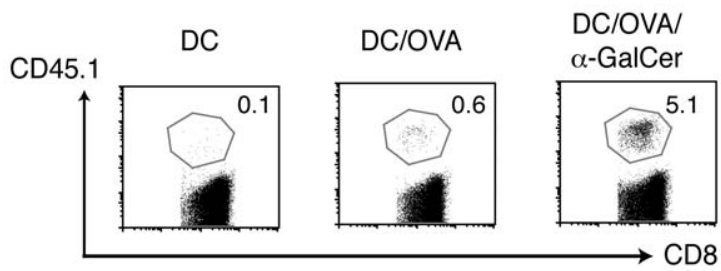

B

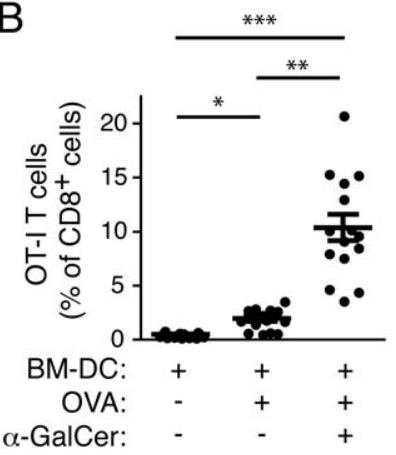

C

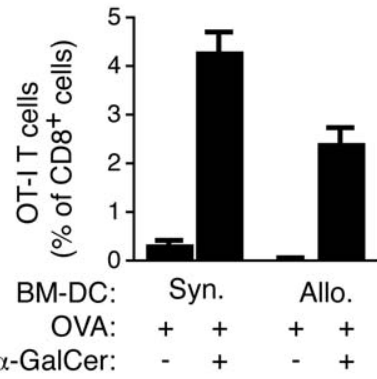

Figure 1. Antigen from injected allogeneic BM-DCs is crosspresented by resident APCs. C57BL/6j mice (CD45.2) were provided a cohort of OVA-specific CD8 ${ }^{+} \mathrm{T}$ cells from OT-I mice (CD45.1) by adoptive transfer and were vaccinated $1 \mathrm{~d}$ later with BM-DCs derived from syngeneic mice that had been loaded with OVA $\pm \alpha$-GalCer in vitro. Accumulation of OT-I cells was determined in blood $7 \mathrm{~d}$ later by flow cytometry with antibodies specific for CD8 and CD45.1. (A) Flow cytometry data outlining the gating strategy for the identification of OT-I T cells, and representative dot plots showing OT-I T cells as a percentage of all $\mathrm{CD}^{+}$cells in blood on day 7. (B) Pooled data \pm SEM from three experiments with 4-5 mice per group are shown with each symbol representing an individual mouse. Statistical significance was determined using a Kruskal-Wallis test and Dunn's post test. ${ }^{*} \mathrm{p}<0.05$, ${ }^{* *} p<0.01,{ }^{* * *} p<0.001$ (C) Mice were vaccinated with either syngeneic (C57BL/6j) or allogeneic (B10.Q) BM-DCs loaded with OVA $\pm \alpha$-GalCer and the percentage of OT-I T cells of all $\mathrm{CD} 8^{+}$cells determined as above. One representative experiment out of two with 4-5 mice per group is depicted with SEM.

doi:10.1371/journal.pone.0017657.g001

langerin (CD207) [18]. In order to determine if langerin ${ }^{+} \mathrm{CD} 8 \alpha^{+}$ DCs are responsible for cross-presentation of antigen derived from injected BM-DCs, we took advantage of the lang-DTREGFP mice [27], in which langerin-expressing DCs, including langerin ${ }^{+}$ $\mathrm{CD} 8 \alpha^{+}$DCs, can be selectively depleted by administration of Diphtheria toxin (DT). We treated lang-DTREGFP or control C57BL/6j mice with DT prior to vaccination with either syngeneic or allogeneic BM-DCs loaded with OVA and $\alpha$-GalCer, and assessed priming of OT-I T cells as described above. Depletion of langerin ${ }^{+} \mathrm{CD} 8 \alpha^{+}$DCs by DT injection was found to ablate OT-I T cell priming in response to allogeneic antigenloaded BM-DCs, indicating that langerin ${ }^{+} \mathrm{CD} 8 \alpha^{+}$DCs mediate indirect cross-presentation of antigen derived from the injected cells (Fig. 2A). Interestingly, depletion of langerin ${ }^{+} \mathrm{CD} 8 \alpha^{+}$DCs also reduced priming of OT-I cells in response to syngeneic antigen-loaded BM-DCs despite these cells being capable of directly stimulating OT-I T cells (Fig. 2A). In contrast, administration of DT had no effect on priming of OT-I T cells in C57BL/ 6 mice, demonstrating that this treatment specifically affects $\mathrm{T}$ cell priming by depleting langerin ${ }^{+}$cells (Fig. $2 B$ ). Priming of OVAspecific $\mathrm{CD}^{+} \mathrm{T}$ cells in the absence of transferred OT-I T cells was similarly ablated in lang-DTREGFP mice treated with DT and vaccinated with syngeneic BM-DCs loaded with OVA and $\alpha$ GalCer (Fig. 2C). This correlated with a significant impairment in the ability of DT-treated mice to kill target cells loaded with OVA $_{257-264}$-peptide (Fig. 2D). Transfer of antigen to resident langerin ${ }^{+} \mathrm{CD} 8 \alpha^{+} \mathrm{DCs}$ is therefore a major contributor to $\mathrm{CD}^{+} \mathrm{T}$ cell stimulation in this DC vaccination strategy.

Injected antigen-loaded DCs, and not resident DCs, prime $\mathrm{CD}^{+} \mathrm{T}$ cell responses

To determine whether transfer of antigen is required to stimulate $\mathrm{CD}^{+} \mathrm{T}$ cell responses to injected antigen-loaded BMDCs, and to also assess whether langerin ${ }^{+} \mathrm{CD} 8 \alpha^{+}$DCs are involved, we examined priming of a cohort of OVA-specific transgenic $\mathrm{CD}^{+}{ }^{+} \mathrm{T}$ cells $(\mathrm{OT}-\mathrm{II}$ cells) in $\mathrm{C} 57 \mathrm{BL} / 6 \mathrm{j}$ mice and langDTREGFP mice. Injection of syngeneic BM-DCs loaded with OVA and $\alpha$-GalCer resulted in priming of OT-II cells in C57BL/ $6 \mathrm{j}$ and untreated lang-DTREGFP recipients, with enhanced accumulation of OT-II cells in the blood at day 7 compared to non-vaccinated mice (Fig. 3A). However, this response was not dependent on resident langerin ${ }^{+} \mathrm{CD} \alpha^{+}$DCs because prior depletion of langerin ${ }^{+}$cells in lang-DTREGFP mice had no effect on the induced response (Fig. 3A). In addition, OT-II T cell priming was only induced when direct stimulation by the injected DCs was possible, as no expansion of the OT-II T cell population was observed in response to antigen-loaded allogeneic BM-DCs (Fig. 3A). Because the overall OT-II T cell response was low in these experiments, we performed similar experiments in mice receiving CFSE-labeled OT-II T cells, and used dilution of the CFSE dye as a measure of cell division to assess OT-II T cell activation. Using this more sensitive method, we confirmed the previous finding that maximal OT-II $\mathrm{T}$ cell priming occurred when the injected BM-DCs were capable of stimulating OT-II T cells directly (Fig. 3B), although there was some limited $\mathrm{T}$ cell proliferation in response to allogeneic BM-DC which may be attributed to antigen transfer (Fig. 3B). This latter response was partially ablated upon depletion of langerin ${ }^{+}$cells (Fig. $3 B$ and $C$ ), although the difference between DT-treated and not-treated mice did not reach significance (Fig. 3C). In contrast, the larger response induced to syngeneic BM-DCs was not obviously sensitive to langerin depletion (Fig. $3 B$ and $C$ ). Overall, injected BM-DCs are primarily responsible for directly stimulating $\mathrm{CD}^{+} \mathrm{T}$ cells, whereas a major proportion of the $\mathrm{CD}^{+} \mathrm{T}$ cell response is stimulated as a consequence of antigen transfer from injected cells to resident langerin ${ }^{+} \mathrm{CD} 8 \alpha^{+} \mathrm{DC}$.

\section{Resident DCs need a maturation signal to cross-present antigen acquired from injected BM-DCs}

DC maturation is a requirement for optimal stimulation of naive $\mathrm{T}$ cells. It is therefore likely that resident DCs are in similar need of a maturation signal in order to stimulate naïve $\mathrm{T}$ cells with antigen acquired from injected BM-DCs. To address whether this was the case, we assessed indirect cross-priming of OT-I T cells in C57BL/ $6 \mathrm{j}$ mice following injection of allogeneic OVA-loaded BM-DCs in the presence or absence of different adjuvant compounds known to mature DC. These compounds were injected $i . v$. at the same time as the BM-DCs in order to provide systemic access to the lymphoid 
A
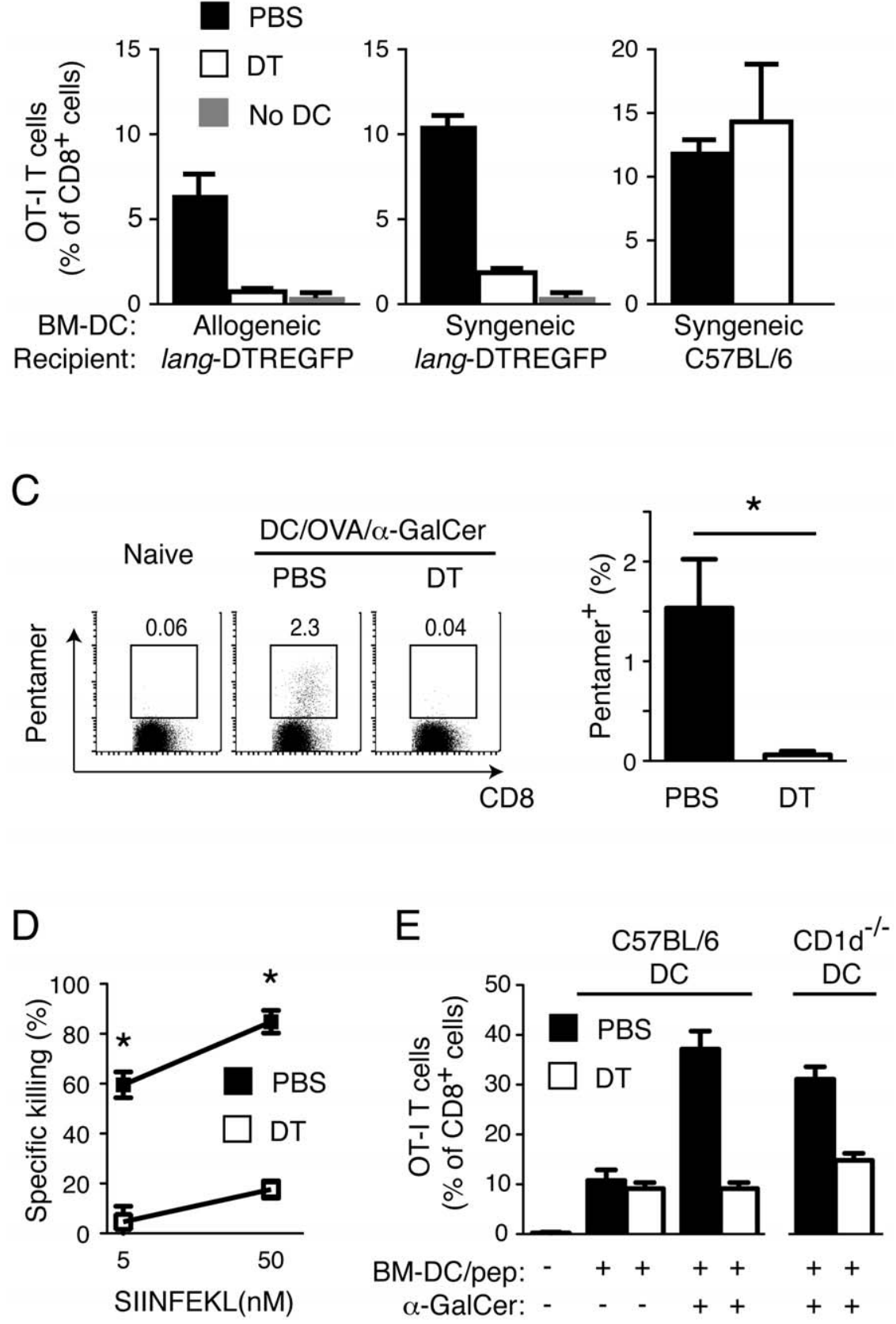

Figure 2. Resident langerin ${ }^{+}$CD8 $\alpha^{+}$DCs cross-present antigen derived from injected BM-DCs to CD8 ${ }^{+}$T cells. (A) Lang-DTREGFP mice were injected i.p. with DT to deplete resident langerin ${ }^{+}$cells, or with PBS vehicle, and then all mice were provided a cohort of transgenic OT-I T cells (CD45.1) by adoptive transfer. The following day, the mice were vaccinated i.v. with allogeneic (B10.Q) or syngeneic (C57BL/6j) BM-DCs loaded with OVA and $\alpha$-GalCer, and the percentage of OT-I T cells determined in the blood $7 \mathrm{~d}$ later by flow cytometry. One representative experiment of two with 4-5 mice per group is shown. The mean percentage \pm SEM is depicted. (B) As in $A$, except syngeneic antigen-loaded BM-DCs were injected into non-transgenic C57BL/6j mice. One representative experiment out of two with 4 mice per group is depicted with SEM. (C) Lang-DTREGFP mice were vaccinated with syngeneic BM-DCs loaded with OVA and $\alpha$-GalCer as in $A$ but without the transfer of transgenic OT-I T cells. The percentage of CD $8^{+} T$ cells specific for the $\mathrm{OVA}_{257-264}$ peptide (SIINFEKL) was determined in the blood six days later by flow cytometry using fluorescently labeled pentamers of $\mathrm{K}^{\mathrm{b}} / \mathrm{OVA}_{257-264}$. Representative dot plots from the indicated experimental groups are shown. The bar graph shows the mean percentage + SEM of pentamer positive cells. One experiment with 4 mice per group is depicted. (D) In vivo cytotoxic activity in mice from $C$ was determined by measuring the relative recovery of spleen cells loaded with 50 or $5 \mathrm{nM}$ of $\mathrm{OVA}_{257-264}$ peptide injected i.v. into vaccinated mice, and collected from blood $17 \mathrm{~h}$ later. Statistical significance was determined with a Mann-Whitney test. ${ }^{*} \mathrm{p}<0.05$.

doi:10.1371/journal.pone.0017657.g002

tissues, but injections were into the contralateral tail vein to limit direct interactions between the compounds and the injected cells. Injection of allogeneic OVA-loaded BM-DCs in the presence of the adjuvant $\alpha$-GalCer induced significant OT-I T cell priming. However, this response was almost undetectable in the absence of $\alpha$-GalCer (or other maturation signals) (Fig. 4A), suggesting that 
A

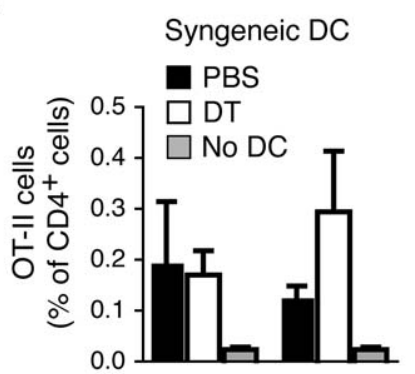

Mice: Lang-DTR C57BL/6

B

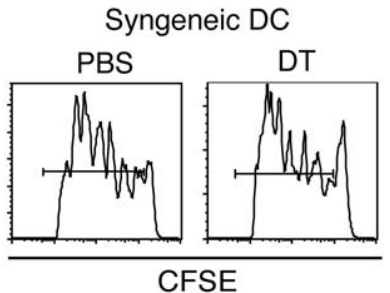

Allogeneic DC

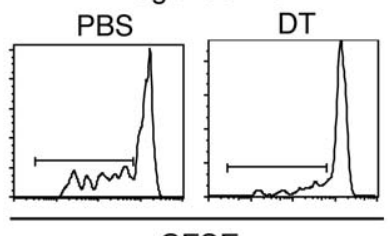

CFSE

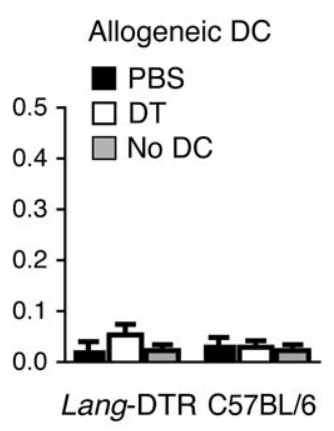

C Syngeneic DC

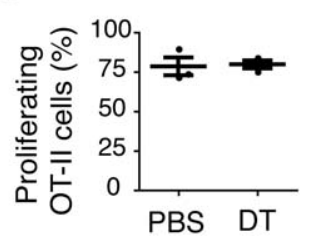

Allogeneic DC

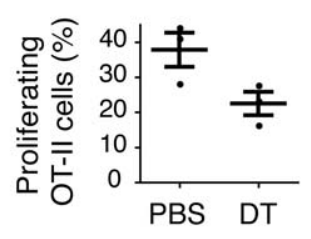

Figure 3. $\mathrm{CD4}^{+} \mathrm{T}$ cell responses are primed by injected antigenloaded BM-DCs with minimal involvement of resident langerin ${ }^{+}$CD8 $\alpha^{+}$DCs. (A) Lang-DTREGFP mice, or C57BL/6j controls, were injected i.p. with DT or PBS, and were then provided a cohort of OVAspecific $\mathrm{CD}^{+} \mathrm{T}$ cells from OT-II mice. The following day, the mice were vaccinated i.v. with OVA- and $\alpha$-GalCer-loaded BM-DCs from syngeneic (C57BL/6j), or allogeneic (B10.Q) mice, and the percentage of OT-II cells of all $\mathrm{CD}^{+}$cells determined in the blood 7 days later by flow cytometry. Mean percentages of one representative experiment of two with 5 mice per group are shown with SEM. $(B, C)$ Lang-DTREGFP mice were treated with PBS or DT and then provided CFSE-labeled OT-II cells before vaccination with OVA- and $\alpha$-GalCer-loaded BM-DCs from syngeneic or allogeneic mice. (B) Representative histogram plot showing CFSE dilution of OT-II T cells three days after administration of antigenloaded BM-DCs. (C) Mean percentages of divided OT-II T cells with SEM for one representative experiment of two, with three animals in each treatment group.

doi:10.1371/journal.pone.0017657.g003

resident DCs require an $i \mathrm{NKT}$ cell-mediated maturation signal in order to engage in $\mathrm{T}$ cell priming. It was possible that even a limited amount of binding of $\alpha$-GalCer to the injected cells in vivo could make the injected cells susceptible to $i \mathrm{NKT}$ cell-mediated lysis, thereby facilitating the release of antigen available to resident DCs. In order to rule out this potential contribution to the induced response, we repeated the above experiment using i.v. injection of the TLR ligands $\mathrm{p}(\mathrm{I}: \mathrm{C})$ or MPL as adjuvant compounds, thereby avoiding the involvement of $i \mathrm{NKT}$ cells altogether. Intravenous administration of each of these TLR ligands also enhanced the ability of the resident DCs to indirectly cross-present antigen derived from injected allogeneic BM-DCs (Fig. 4A). The enhancement mediated by MPL was completely negated by prior treatment with DT in lang-DTREGFP recipients, regardless of whether

syngeneic or allogeneic BM-DCs were injected (Fig. $4 B$ ), indicating that MPL enhance $\mathrm{CD}^{+} \mathrm{T}$ cell priming by maturing the langerin ${ }^{+}$ $\mathrm{CD}_{8} \alpha^{+}$DCs. It is unlikely that the i.v. administered MPL contributed to the $\mathrm{T}$ cell response by directly stimulating the injected BM-DCs, as treatment of the OVA-loaded BM-DCs with MPL in vitro before injection failed to significantly improve the OT-I response (Fig. 4C). Also, responses to OVA-loaded BM-DCs from TLR4-deficient mice, which cannot respond directly to MPL, could be enhanced by $i . v$. administration of MPL to levels similar to BMDCs from TLR4-sufficient mice (Fig. 4D). Overall, these data suggest that resident langerin ${ }^{+} \mathrm{CD} 8 \alpha^{+}$DCs require a maturation signal to participate in indirect cross-presentation.

\section{Resident antigen-presenting cells present $\alpha$-GalCer acquired from injected $\alpha$-GalCer-loaded BM-DCs}

We next examined how loading $\alpha$-GalCer onto the injected BM-DCs serves to enhance the stimulatory capacity of resident $\mathrm{CD}^{+}{ }^{+}$langerin ${ }^{+}$DC. The adjuvant effect of $\alpha$-GalCer-mediated $i$ NKT cell activation requires that $\alpha$-GalCer is presented on CDld molecules by APCs engaged in T cell stimulation [20,22,31]. This stimulates $i$ NKT cells to become activated and secrete IL-4 and IFN- $\gamma$, and the activated $i$ NKT cells in turn stimulate enhanced APC function, including secretion of proinflammatory cytokines such as IL-12 [32,33,34,35,36]. The release of IL-12 then instructs NK cells to produce more IFN- $\gamma$ [37]. While release of cytokines into the environment is sufficient to induce up-regulation of costimulatory molecules such as CD80 and CD86 on local APCs, the ability of these cells to promote $\mathrm{T}$ cell responses is critically dependent on direct cellular interactions between APG and $i$ NKT cells, with CD40/CD40L interactions particularly crucial $[20,22,38]$. We therefore hypothesized that resident DCs must acquire $\alpha$-GalCer from injected DCs in order to interact with $i$ NKT cells directly. To test this hypothesis, we examined whether injection of $\alpha$-GalCer-loaded CDld-deficient $\left(\mathrm{CD}^{-/-}\right)$BMDCs, which cannot directly present $\alpha-G a l C e r$ to $i$ NKT cells, can activate $i$ NKT cells in C57BL/6j recipients. Similar percentages of cytokine-positive $i$ NKT cells were induced by $\alpha$-GalCer-loaded $\mathrm{CD} \mathrm{d}^{-1-}$ and wild-type BM-DCs, when intracellular antibody staining for IFN- $\gamma$ and IL-4 was used as a measure for $i$ NKT cell activity (Fig. 5A). These data suggest that $\alpha$-GalCer is transferred from the injected BM-DG to resident $\mathrm{CDld}^{+}$APGs that can engage directly in stimulating $i$ NKT cells. We next examined the role of langerin ${ }^{+}$CD $8 \alpha^{+}$DCs in this process by injecting $\alpha$ GalCer-loaded CD1d ${ }^{-/-}$BM-DGs into DT-treated or untreated lang-DTREGFP mice. Similar percentages of activated $i$ NKT cells (as measured by intracellular cytokine staining) were seen regardless of DT treatment, indicating that langerin ${ }^{+} \mathrm{CD} 8 \alpha^{+}$ DCs were dispensable for initial $i$ NKT cell activation (Fig. $5 B$ ). Accordingly, serum levels of IL-4 two hours after administration of BM-DCs were similar in depleted and non-depleted mice (Fig. 5G). However, depletion of langerin ${ }^{+}$cells severely reduced the level of IL-12p70 released into the serum (Fig. 5C), demonstrating an important role of langerin ${ }^{+} \mathrm{CD} 8 \alpha^{+}$DCs in the production of this cytokine in response to $\alpha$-GalCer as we have recently reported [18]. Serum levels of IFN- $\gamma$ were also reduced in recipients depleted of langerin ${ }^{+}$cells, which is likely to reflect reduced transactivation of NK cells as a consequence of lower IL-12 levels (Fig. 5C). Overall, these data demonstrate that $\alpha$-GalCer is transferred from injected BM-DCs to APG populations within the lymphoid tissue that are capable of stimulating cytokine production by $i$ NKT cells. Crucially, the subsequent release of IL-12p70, which may help in driving cross-primed responses [39], is largely dependent on the presence of langerin ${ }^{+} \mathrm{CD} 8 \alpha^{+}$DCs among the recipient APGs. 
A
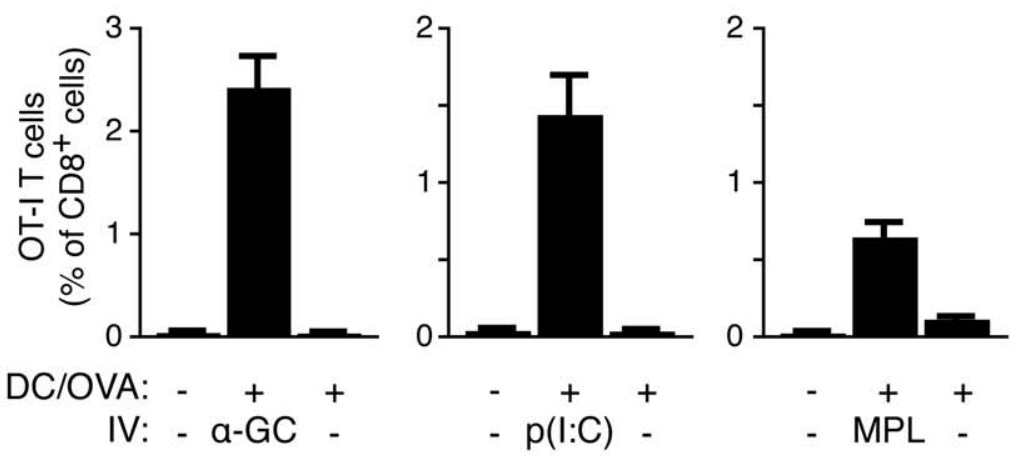

B
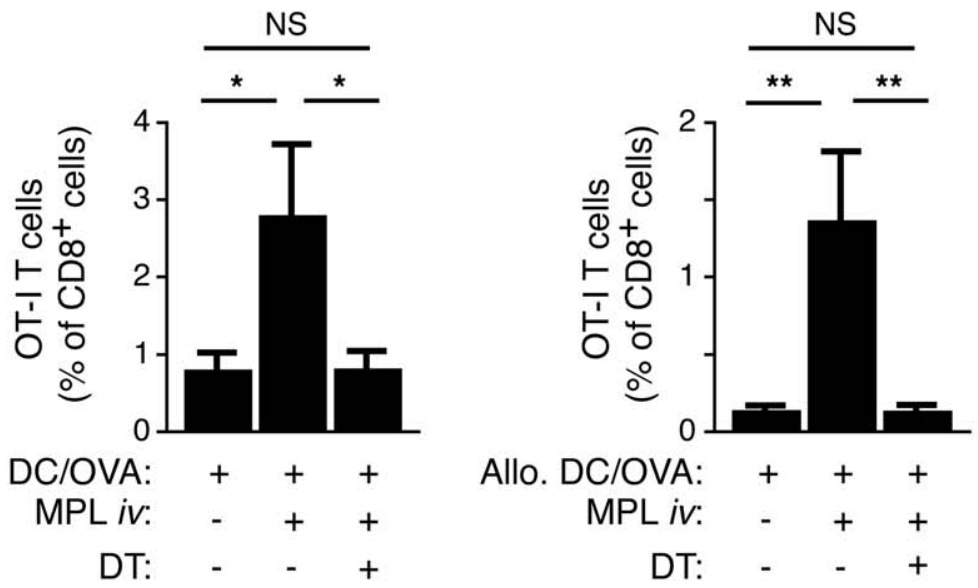

C
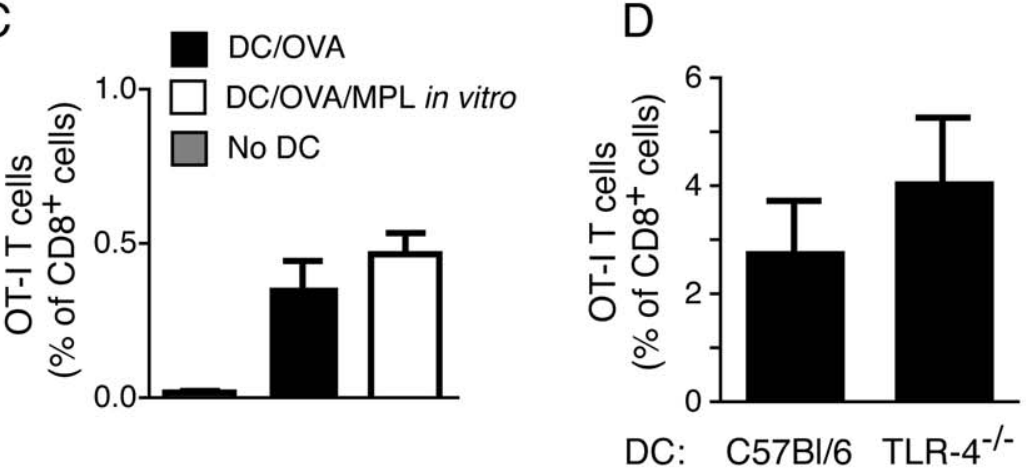

Figure 4. Resident langerin ${ }^{+}$DCs need a maturation signal to cross-present antigen derived from antigen-loaded BM-DCs. (A) C57BL/ 6j mice were provided a cohort of OT-I T cells and vaccinated i.v. with allogeneic (B10.Q) BM-DCs with or without OVA. The mice were injected in the contralateral tail vein with either $\alpha$-GalCer, $p(I: C)$, or MPL and the percentage of OT-I T cells of all CD8 ${ }^{+}$cells was determined in the blood $7 \mathrm{~d}$ later. One representative experiment out of two with 4-5 mice per group is shown with SEM. (B) Lang-DTREGFP mice were treated with DT, or PBS vehicle, provided a cohort of OT-I T cells, and then administered OVA-loaded BM-DCs from syngeneic or allogeneic mice with or without injection of MPL in the contralateral vein. The percentage of OT-I T cells of all CD8 ${ }^{+}$cells was determined in the blood $7 \mathrm{~d}$ later. Pooled data from two experiments with 4-5 mice per group is depicted with SEM. Statistical significance was determined with a Kruskal-Wallis test and Dunn's post test. ${ }^{*} p<0.05,{ }^{* *} p<0.01$, $\mathrm{NS}=$ not significant. (C) C57BL/6j mice received a cohort of OT-I T cells as above, and were vaccinated with syngeneic OVA-loaded BM-DCs that had been incubated with $100 \mathrm{ng} / \mathrm{ml}$ of MPL for $16 \mathrm{~h}$ prior to injection, or left untreated. The mean percentage of OT-I T cells of all CD8 ${ }^{+}$cells in the blood $7 \mathrm{~d}$ later is depicted using pooled data from three experiments with 4-5 mice per group, with SEM. (D) C57BL/6j mice received OT-I T cells as above and were vaccinated with OVA- and $\alpha$-GalCer-loaded BM-DCs derived from C57BL/6j or TLR-4 ${ }^{-1-}$ mice. The mean percentage of OT-I T cells of all $\mathrm{CD}^{+}$cells in the blood $7 \mathrm{~d}$ later is depicted with SEM from one representative experiment out of two.

doi:10.1371/journal.pone.0017657.g004 
A

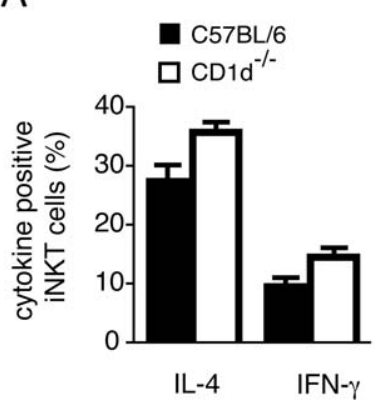

B

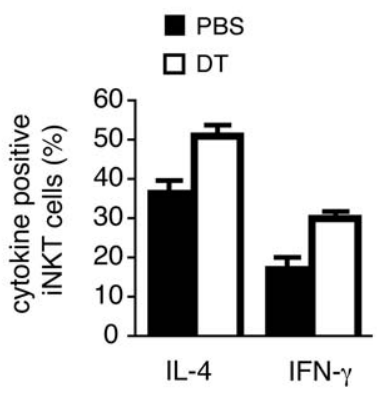

C

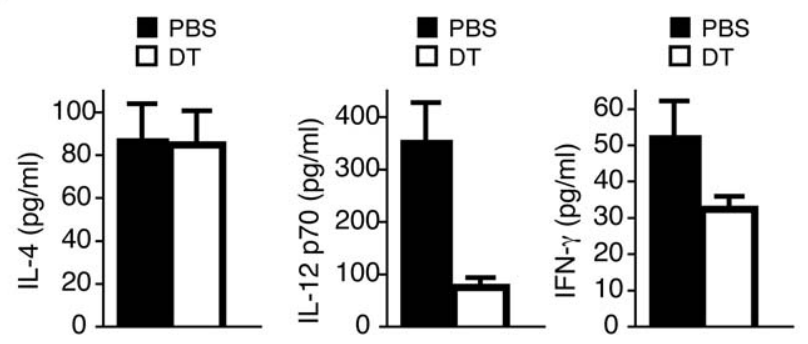

Figure 5. Resident APCs acquire $\alpha$-GalCer from $\alpha$-GalCer-loaded BM-DCs in vivo. (A) BM-DCs derived from C57BL/6j (black bars) or

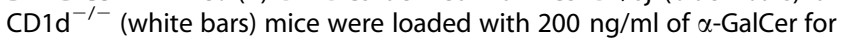
$16 \mathrm{~h}$ and injected i.v. into C57BL/6j mice. The percentage of splenic iNKT cells expressing intracellular IL-4 or IFN- $\gamma$ was determined $2 \mathrm{~h}$ later by flow cytometry. One representative experiment out of two with three mice per group is depicted, with SEM. (B) CD1d ${ }^{-1-}$ BM-DCs were incubated with $\alpha$-GalCer as above and injected into lang-DTREGFP mice, which had been injected with either PBS (black bars) or DT (white bars). The percentage of iNKT cells expressing intracellular IL-4 or IFN- $\gamma$ was determined $2 \mathrm{~h}$ later by flow cytometry. One representative experiment out of two with three mice per group is depicted with SEM. (C) LangDTREGFP mice were treated with PBS (black bars) or DT (white bars) and then injected i.v. with $\alpha$-GalCer-loaded BM-DCs from C57BL/6j mice. Serum concentrations of IL-4, IL-12 p70, and IFN- $\gamma$ were determined $2 \mathrm{~h}$, $5 \mathrm{~h}$, and $10 \mathrm{~h}$ later respectively. One representative experiment out of three with 3-5 mice per group is depicted with SEM. doi:10.1371/journal.pone.0017657.g005

\section{Resident langerin ${ }^{+} \mathrm{CD} 8 \alpha^{+} \mathrm{DCs}$ can acquire cellular material from injected BM-DCs}

The antigen-loaded BM-DCs used in all of the previous experiments were extensively washed before administration. Injection of the final washes, or supernatants from cells subsequently cultured in vitro, was not capable of inducing proliferation of OT-I cells in vivo, ruling out the possibility that OVA was 'leaching' as free antigen from the cells over time (data not shown). Some $\alpha$-GalCer did accumulate in culture supernatants, but at levels that were insufficient to account for the in vivo activities we have observed (data not shown). We therefore investigated the possibility that the antigens were transferred in an exchange of cellular material between injected and resident cells. To determine whether any cellular material could be exchanged in this manner, BM-DCs derived from CD45.1 congenic mice were CFSE-labeled and injected into CD45.2 ${ }^{+} \mathrm{C} 57 \mathrm{BL} / 6 \mathrm{j}$ mice, and the acquisition of CFSE fluorescence by resident (CD45.2 $2^{+}$DCs determined $16 \mathrm{~h}$ later. Analysis of CD $1 \mathrm{lc}^{+}$cells in the spleens of recipient animals showed that CFSE fluorescence was acquired by resident $\mathrm{CD} 8 \alpha^{+}$DCs as well as $\mathrm{CD} 8 \alpha^{-}$DCs (Fig. 6A), with fluorescence intensity highest in CD8 $\alpha^{+}$cells. To establish if CFSE fluorescence was acquired by the langerin ${ }^{+}$subset, we similarly injected CFSE-labeled CD45.1 BM-DCs into lang-EGFPDTR mice. In these mice, all langerin ${ }^{+} \mathrm{CD} 8 \alpha^{+}$resident DCs are weakly GFP positive (Fig. 6B, naïve). As above, a subset of resident CD8 $\alpha^{+}$ DCs acquire CFSE fluorescence upon injection of CFSE-labeled BM-DCs (Fig. 6B, CFSE ${ }^{+}$DC). However, the acquisition of CFSE fluorescence by resident $\mathrm{CD} 8 \alpha^{+}$DCs is ablated upon prior depletion of langerin ${ }^{+}$cells by DT (Fig. $6 \mathrm{~B}, C F S E^{+} D C+D T$, and Fig. 6C), suggesting cellular material is mainly acquired by $\mathrm{CD} 8 \alpha^{+}$ DCs expressing langerin.

\section{Discussion}

In this report we investigated the contribution of endogenous lymphoid-resident DCs to $\mathrm{T}$ cell priming after vaccination with antigen-loaded BM-DCs. We demonstrate that $\mathrm{CD}^{+} \mathrm{T}$ cells primed in response to i.v. injection of antigen-loaded BM-DCs can be primed by a subset of resident DCs expressing CD8 $\alpha$ and langerin. However, this subset of DCs only contributes to $\mathrm{T}$ cell priming if a potent maturation signal is received in combination with the administered BM-DGs. We also demonstrate that antigen transfer is not simply limited to protein antigens, as the glycolipid $\alpha$-GalCer is also acquired and presented by endogenous DC, which facilitates licensing of the resident DCs as a consequence of engaging $i$ NKT cells.

Two models were used to demonstrate that resident DCs acquire antigen from injected BM-DCs. The first model involved vaccination with allogeneic OVA-loaded BM-DCs, which were unable to directly stimulate OVA-specific OT-I T cells, and therefore required antigen to be transferred to host APCs to induce expansion of the transgenic $\mathrm{T}$ cell population. While this model showed that OT-I cells could indeed be primed if an adjuvant was provided, it is possible that this response was inflated because the allogeneic BM-DGs were lysed by NK cells, promoting release of antigen to the resident DCs [6]. In order to avoid this possibility, and characterize the resident DC population responsible for $\mathrm{CD}^{+} \mathrm{T}$ cell priming in more detail, we used a second model in which the recipient DCs were selectively depleted. Our previous studies have shown that langerin $^{+} \mathrm{CD} 8 \alpha^{+}$DCs in the spleen are critically involved in cross-presentation of systemic antigens and IL-12 induction [18]. We therefore examined DC vaccination in lang-DTREGFP mice in which all DCs expressing langerin could be depleted by injection of DT [27]. Typically, approximately $90 \%$ of the splenic langerin ${ }^{+} \mathrm{CD} 8 \alpha^{+} \mathrm{DC}$ subset is depleted for at least two days in response to DT (Fig. S1). In contrast, DT injection does not affect langerin ${ }^{-} \mathrm{CD} 8 \alpha^{+} \mathrm{DCs}, \mathrm{CD} 8 \alpha^{-} \mathrm{DCs}$, or the injected DCs themselves [18]. In addition to being expressed by a subset of $\mathrm{CD} 8 \alpha^{+} \mathrm{DCs}$ in the spleen, langerin is expressed by Langerhans cells in the epidermis, and a subset of dermal and lung DCs additionally expressing CD103 [27,40,41,42,43]. These DC subsets may therefore also be depleted by DT administration, and may be of relevance to the response to injected BM-DCs. BMDCs injected i.v. do initially accumulate in much higher numbers in the lungs compared to the spleen, but then disappear from the lungs within the first $24 \mathrm{~h}$. In contrast, the number of injected BMDCs in the spleen remains high for several days, and the spleen contains the largest number of injected BM-DCs a day after injection [44]. Although T cells are likely to be primed in the lungdraining lymph nodes as well as in the spleen following i.v. administration of antigen-loaded BM-DCs, the size of the spleen relative to lymph nodes combined with the continuous presence of the injected DCs, make the spleen the primary site of $\mathrm{T}$ cell priming [44]. Using the lang-DTREGFP model, depletion of 
A

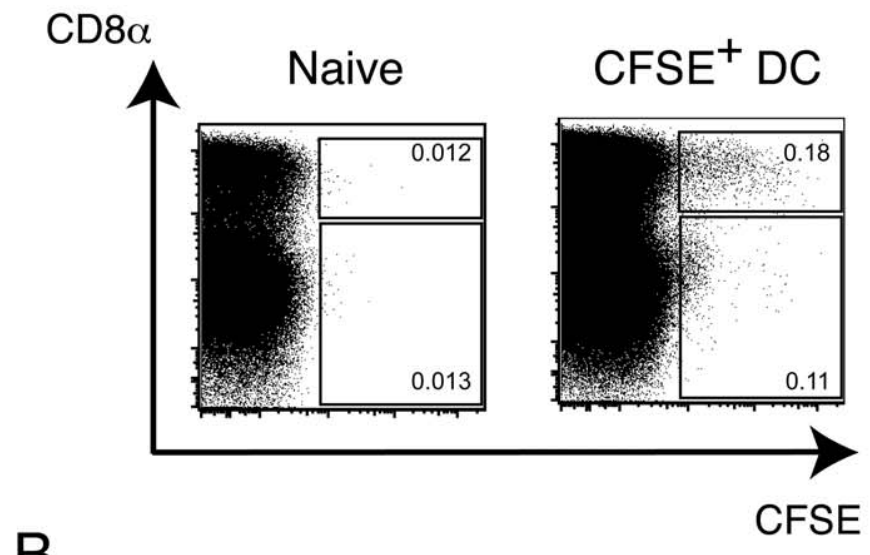

B
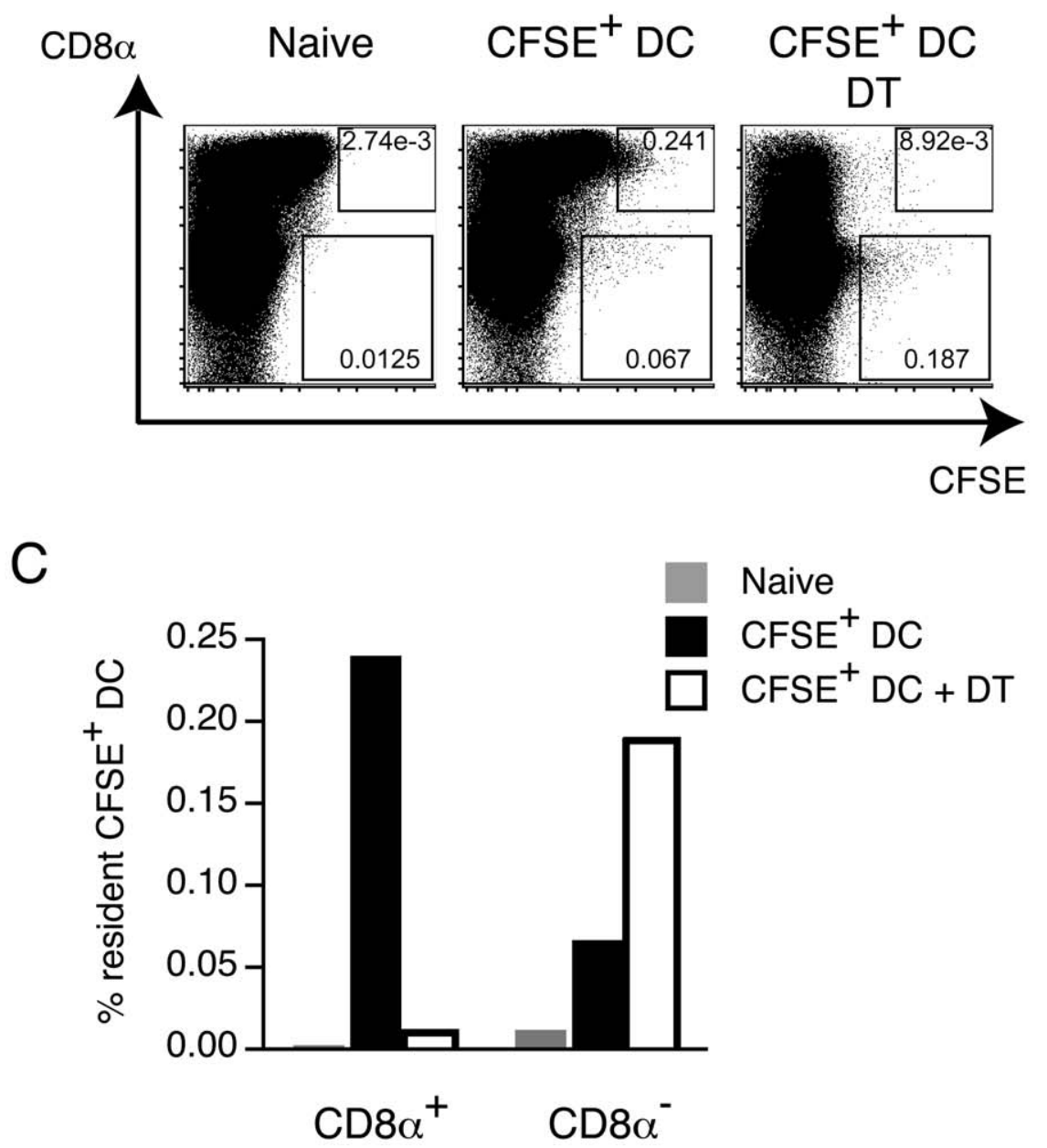

Figure 6. Injected BM-DCs are taken up by resident DCs. (A) Pools $(n=2)$ of C57BL/6j mice (CD45.2) were injected with CFSE-labeled BM-DCs from CD45.1 congenic mice, or left untreated. Spleens were removed $16 \mathrm{~h}$ later and CD11 $\mathrm{c}^{+}$cells were isolated using magnetic antibody-coated beads. Depicted dot plots show CFSE fluorescence in resident $\left(C D 45.2^{+}\right) C D 11 c^{+}$cells. One representative experiment of two is depicted. $(B)$ CFSElabeled BM-DCs from CD45.1 congenic mice were injected into lang-EGFPDTR mice with or without prior depletion of langerin ${ }^{+}$cells by DT, and the acquisition of CFSE by resident DCs determined as above. (C) Graphical representation of CFSE acquisition by resident CD8 $\alpha^{+}$and CD8 $\alpha^{-}$DCs in langDTREGFP mice with or without prior depletion of langerin ${ }^{+}$cells. doi:10.1371/journal.pone.0017657.g006 
langerin ${ }^{+}$DCs before i.v. injection of OVA and $\alpha$-GalCer-loaded allogeneic BM-DCs completely ablated OT-I T cell priming, and reduced OT-I T cell priming more than $75 \%$ when syngeneic BM-DCs were used (Fig. 2A). Resident langerin ${ }^{+}$DCs are therefore responsible for priming the majority of the OVA-specific $\mathrm{CD}^{+} \mathrm{T}$ cell response even when syngeneic BM-DCs were used, and NK-mediated killing of the injected BM-DCs is not required for antigen transfer.

Lymphoid resident DGs did not prime $\mathrm{CD}^{+} \mathrm{T}$ cells after injection of antigen-loaded BM-DCs unless the injected DCs are administered with a TLR agonist or $\alpha$-GalCer. As illustrated using MPL, the adjuvant effect of co-administered TLR ligand was negated by prior depletion of langerin ${ }^{+} \mathrm{DCs}$, suggesting that MPL enhances $\mathrm{CD}^{+} \mathrm{T}$ cell priming by inducing maturation of resident langerin ${ }^{+} \mathrm{DCs}$, and not by the stimulation of other cell types, or the generation of an inflammatory environment. Nor is the effect of MPL due to maturation of the injected BM-DCs because responses to TLR-4 deficient BM-DCs were similarly enhanced when MPL was co-administered. Surprisingly, maturation of BMDCs prior to injection had a very limited effect on OT-I T cell priming despite upregulation of costimulatory markers on the cells before injection, and induction of release of cytokines such as IL12 and IL-6 in vitro (not shown). In fact, our preliminary data suggest that injected BM-DCs that reach the spleen display a mature phenotype irrespective of whether they were matured in vitro prior to injection (data not shown). In contrast to OT-I T cells, only very few OT-II T cells were primed by allogeneic BMDCs loaded with OVA- and $\alpha$-GalCer, and this response was only mildly impacted by depletion of langerin ${ }^{+}$DCs. However, OT-II $\mathrm{T}$ cells were efficiently primed by syngeneic BM-DCs loaded with OVA and $\alpha$-GalCer, suggesting that it is the injected BM-DCs, and not lymphoid-resident DCs, that predominate in the stimulation of naïve $\mathrm{CD} 4^{+} \mathrm{T}$ cells.

The mechanism of antigen transfer in our experiments remains unclear. It has been shown that membrane fragments can be exchanged between live DCs following direct cell to cell contact [45], which may result in transfer of antigens presented on the surface. Similarly, antigens can be incorporated into exosomes released from a variety of hematopoietic and non-hematopoietic cell types (including DCs), and taken up by APCs [46]. Antigen can also be transferred by the uptake of DCs undergoing apoptosis, or as apoptotic bodies [14,47]. Our results showed that splenic APCs acquired fluorescence from injected CFSE-labeled BM-DCs, suggesting uptake of cellular material. Our experiments also showed that resident langerin ${ }^{+} \mathrm{CD} 8 \alpha^{+} \mathrm{DC}$ s acquired the most fluorescence from the CFSE-labeled BM-DCs; some CD8 $\alpha^{-}$DCs acquired fluorescence, but the intensity was lower, suggesting either less efficient uptake, a qualitatively different mechanism of uptake, or efficient uptake and subsequent degradation.

It is well-described that the in vivo priming of $\mathrm{CD}^{+} \mathrm{T}$ cells in response to intravenous injection of soluble or cell-associated antigens is critically dependent on CD8 $\alpha^{+}$DCs with capacity for cross-presentation $[5,6,7,8]$. On the other hand, priming of $\mathrm{CD} 4^{+}$ $\mathrm{T}$ cells in response to the same antigen sources is minimally affected by prior depletion of CD8 $\alpha^{+}$DCs, suggesting that other, CD8 $\alpha$-negative, APCs engage more efficiently in MHC class II presentation in vivo [18,48]. Inefficient targeting of antigen to APGs with high propensity for $\mathrm{CD}^{+} \mathrm{T}$ cell priming could therefore provide one explanation for the limited $\mathrm{CD} 4^{+} \mathrm{T}$ cell response primed as a consequences of antigen transfer in our model. It is possible that these differences in APC function can be explained not just by inherent differences in processing and presentation capacity, but also by differences in location in the lymphoid tissue, and capacity to respond to maturation stimuli.
Thus, while it is clear that CD8 $\alpha^{+}$DC possess superior crosspresenting function, the splenic langerin ${ }^{+}$fraction of this subset are also positioned within the marginal zone [49], and are therefore well-positioned to acquire antigens from the circulation to divert into the MHC class I presentation pathway. In fact, langerin ${ }^{+}$ $\mathrm{CD}_{103}{ }^{+} \mathrm{CD} 8 \alpha^{+}$DCs within the marginal zone have been shown to be particularly efficient at acquiring cellular material [50], which may be of particular relevance in our DG vaccination model. The $\mathrm{CD} 8 \alpha^{+} \mathrm{DC}$ population has also been shown to produce CCL17 in response to $i \mathrm{NKT}$ cell activation, which favors attraction of naïve $\mathrm{CD}^{+} \mathrm{T}$ cells via CCR4 [31], and the langerin ${ }^{+}$ subset responds more quickly to $i \mathrm{NKT}$ cell-mediated maturation stimuli in terms of costimulatory marker upregulation, and CD40 expression, than other DCs of the spleen [18]. Collectively these factors may favor induction of $\mathrm{CD} 8^{+} \mathrm{T}$ cell responses by langerin ${ }^{+}$ $\mathrm{CD} 8 \alpha^{+} \mathrm{DCs}$, rather than $\mathrm{CD} 4^{+} \mathrm{T}$ cell responses, although our data show that some minimal $\mathrm{CD} 4^{+} \mathrm{T}$ cell stimulation attributed to langerin ${ }^{+} \mathrm{CD} 8 \alpha^{+}$DCs may also occur.

Our findings do contrast with previous reports demonstrating MHC class II presentation as a consequence of antigen transfer from injected BM-DCs to resident DCs $[14,15]$. The reason for this discrepancy is not known, but may reflect the different forms of antigen and concentration used, particularly the use of high concentrations of an MHC class II-binding peptide ( $\left.\mathrm{MCC}_{88-103}\right)$ in one study where priming of $\mathrm{CD}^{+} \mathrm{T}$ cells by endogenous DCs was actually observed [15]. Alternatively, it has also been shown that OT-II T cells display low sensitivity to low doses of antigen when compared to OT-I T cells in vivo [51], which is supported by our studies (Fig. 3, and [18]), and may explain the negligible $\mathrm{CD} 4^{+} \mathrm{T}$ cell response induced as a consequence of antigen transfer between DCs in our experimental system. Further experimentation is required to resolve this issue.

Interestingly, vaccination with $\alpha$-GalCer-loaded CDld $\mathrm{d}^{-/-} \mathrm{BM}-$ DCs induced $i$ NKT cell activation to similar levels as $\alpha$-GalCerloaded wild-type BM-DCs. As presentation of $\alpha$-GalCer to $i$ NKT cells is dependent on CDld, this finding can only be explained by transfer of $\alpha$-GalCer from the injected CDld-negative BM-DCs to CDld-positive resident APGs. Resident DGs may acquire $\alpha$ GalCer following phagocytosis of whole antigen-loaded BM-DCs as previously suggested for the transfer of $\alpha$-GalCer from loaded tumor cells to resident DCs [52]. However, despite the proposed superior ability of $\mathrm{CD} 8 \alpha^{+}$DCs to phagocytose dying cells [6,53], which is supported by our observations (Fig. 6), the activation of $i$ NKT cells per se is not dependent on the langerin ${ }^{+}$fraction of this population, as the depletion of these cells in lang-DTREGFP mice did not influence initial $i$ NKT cell priming. We cannot rule out the possibility that other APC subsets such as CD11 $\mathrm{b}^{+} \mathrm{CD}^{+}$splenic DCs or marginal zone macrophages, can take up cellular material from antigen-loaded BM-DCs for presentation of $\alpha$-GalCer. In this context, subcapsular sinus $\mathrm{CD}_{16}{ }^{+}$macrophages were recently shown to mediate early activation of $i$ NKT cells in lymph nodes in response to particulate $\alpha$-GalCer [54].

Although not important for initial $i$ NKT cell activation, $\alpha$ GalCer-mediated IL-12 production was critically dependent on resident langerin ${ }^{+} \mathrm{CD} 8 \alpha^{+} \mathrm{DC}$, as IL-12 production was severely reduced in DT treated animals. This is consistent with our previous studies showing that the absence of langerin ${ }^{+}$cells has no impact on the ability of injected free $\alpha$-GalCer to elicit $i$ NKT cells to release IL-4 and IFN- $\gamma$, but that the subsequent release of large quantities of IL-12p70 into the serum, which is mediated through interaction of activated $i \mathrm{NKT}$ cells with APGs, is severely impaired [18]. We also noted in these earlier studies a heightened propensity of langerin ${ }^{+} \mathrm{CD} 8 \alpha^{+}$DC to upregulate CD40 in response to free $\alpha$ GalCer, which potentially explains the significant role these cells 
play in driving IL-12p70 release. The failure to produce IL-12p70 also prevented the release of large quantities of IFN- $\gamma$ into the serum, which has been previously described to be secreted by NK cells [37].

Using conditional ablation of langerin ${ }^{+}$cells in lang-DTREGFP mice, we find that a significant proportion of the $\mathrm{CD}^{+} \mathrm{T}$ cells responding to intravenous injection of antigen-loaded BM-DCs are primed by endogenous langerin ${ }^{+}$DCs. Yet, this subset of DCs only contributes to $\mathrm{T}$ cell priming if a potent maturation signal is received in combination with the administered BM-DCs. As targeting of antigen to immature DCs in vivo has been shown to induce $\mathrm{T}$ cell tolerance $[55,56,57]$, our results underscore the utility of providing a stimulus for endogenous resident DCs in DC vaccination protocols.

\section{Supporting Information}

Figure S1 Splenic $\mathrm{CD8}^{+}$langerin $^{+}$DGs are efficiently depleted by diphtheria toxin injections. F1 crosses of LangDTREGFP x Lang-EGFP were injected i.p. with $350 \mathrm{ng}$ of DT $48 \mathrm{~h}$ and $24 \mathrm{~h}$ prior to analysis. Single cell suspensions of spleen cells were generated by Liberase and DNAse treatment as described in Materials and Methods. Cells were stained with

\section{References}

1. Henri S, Vremec D, Kamath A, Waithman J, Williams S, et al. (2001) The dendritic cell populations of mouse lymph nodes. J Immunol 167: 741-748.

2. Shortman K, Naik SH (2007) Steady-state and inflammatory dendritic-cell development. Nat Rev Immunol 7: 19-30.

3. Vremec D, Pooley J, Hochrein H, Wu L, Shortman K (2000) CD4 and CD8 expression by dendritic cell subtypes in mouse thymus and spleen. J Immunol 164: 2978-2986.

4. McLellan AD, Kapp M, Eggert A, Linden C, Bommhardt U, et al. (2002) Anatomic location and T-cell stimulatory functions of mouse dendritic cell subsets defined by CD4 and CD8 expression. Blood 99: 2084-2093.

5. den Haan JM, Lehar SM, Bevan MJ (2000) CD8(+) but not CD8(-) dendritic cells cross-prime cytotoxic T cells in vivo. J Exp Med 192: 1685-1696.

6. Iyoda T, Shimoyama S, Liu K, Omatsu Y, Akiyama Y, et al. (2002) The CD8+ dendritic cell subset selectively endocytoses dying cells in culture and in vivo. J Exp Med 195: 1289-1302.

7. Pooley JL, Heath WR, Shortman K (2001) Cutting edge: intravenous soluble antigen is presented to CD4 T cells by CD8- dendritic cells, but cross-presented to CD8 T cells by CD8+ dendritic cells. J Immunol 166: 5327-5330.

8. Schnorrer P, Behrens GM, Wilson NS, Pooley JL, Smith CM, et al. (2006) The dominant role of CD8+ dendritic cells in cross-presentation is not dictated by antigen capture. Proc Natl Acad Sci U S A 103: 10729-10734.

9. Hochrein H, Shortman K, Vremec D, Scott B, Hertzog P, et al. (2001) Differential production of IL-12, IFN-alpha, and IFN-gamma by mouse dendritic cell subsets. J Immunol 166: 5448-5455.

10. Edwards AD, Manickasingham SP, Sporri R, Diebold SS, Schulz O, et al. (2002) Microbial recognition via Toll-like receptor-dependent and -independent pathways determines the cytokine response of murine dendritic cell subsets to CD40 triggering. J Immunol 169: 3652-3660.

11. Allan R, Waithman J, Bedoui S, Jones C, Villadangos J, et al. (2006) Migratory Dendritic Cells Transfer Antigen to a Lymph Node-Resident Dendritic Cell Population for Efficient CTL Priming. Immunity 25: 153-162.

12. Belz GT, Smith CM, Kleinert L, Reading P, Brooks A, et al. (2004) Distinct migrating and nonmigrating dendritic cell populations are involved in $\mathrm{MHC}$ class I-restricted antigen presentation after lung infection with virus. Proc Natl Acad Sci U S A 101: 8670-8675.

13. Kretschmer K, Apostolou I, Hawiger D, Khazaie K, Nussenzweig MC, et al. (2005) Inducing and expanding regulatory $\mathrm{T}$ cell populations by foreign antigen. Nat Immunol 6: 1219-1227.

14. Inaba K, Turley S, Yamaide F, Iyoda T, Mahnke K, et al. (1998) Efficient presentation of phagocytosed cellular fragments on the major histocompatibility complex class II products of dendritic cells. J Exp Med 188: 2163-2173.

15. Kleindienst P, Brocker T (2003) Endogenous dendritic cells are required for amplification of $\mathrm{T}$ cell responses induced by dendritic cell vaccines in vivo. J Immunol 170: 2817-2823.

16. Millrain M, Scott D, Addey C, Dewchand H, Ellis P, et al. (2005) Identification of the immunodominant HY H2-D(k) epitope and evaluation of the role of direct and indirect antigen presentation in HY responses. J Immunol 175: 7209-7217.

17. Yewdall AW, Drutman SB, Jinwala F, Bahjat KS O Bhardwaj N CD8+ T cell priming by dendritic cell vaccines requires antigen transfer to endogenous antigen presenting cell. PLoS One 5: e11144. antibodies against CD11c and CD8 as well as PI for live/dead cell exclusion. (A) Representative dot plots showing the percentage of CD11c high cells positive for CD8 and langerin-eGFP in DT treated or non-treated mice. $(B)$ Percentage of $\mathrm{CD}^{+}$langerin $^{+}$ cells of $\mathrm{CD} 1 \mathrm{lc}$ high cells in individual mice from a total of three experiments with three mice per group.

(TIF)

\section{Acknowledgments}

The authors wish to thank the personnel of the Biomedical Research Unit of the Malaghan Institute of Medical Research for animal husbandry. We are very grateful to F. Carbone, A. Kissenpfennig, B. Malissen, C-R Wang, and S. Akira for generously providing the mouse strains used in this study, and to R.J. Anderson and G.F. Painter at Industrial Research Limited, Lower Hutt, New Zealand for supplying $\alpha$-GalCer.

\section{Author Contributions}

Conceived and designed the experiments: TRP DSP HMS IFH. Performed the experiments: TRP DSP DAK HMS. Analyzed the data: TRP DSP. Wrote the paper: TRP IFH.

18. Farrand KJ, Dickgreber N, Stoitzner P, Ronchese F, Petersen TR, et al. (2009) Langerin+CD8alpha+ dendritic cells are critical for cross-priming and IL-12 production in response to systemic antigens. J Immunol 183: 7732 7742 .

19. Fujii S, Shimizu K, Smith C, Bonifaz L, Steinman RM (2003) Activation of natural killer $\mathrm{T}$ cells by alpha-galactosylceramide rapidly induces the full maturation of dendritic cells in vivo and thereby acts as an adjuvant for combined CD4 and CD8 T cell immunity to a coadministered protein. J Exp Med 198: 267-279.

20. Hermans IF, Silk JD, Gileadi U, Salio M, Mathew B, et al. (2003) NKT cells enhance CD4+ and CD8+ T cell responses to soluble antigen in vivo through direct interaction with dendritic cells. J Immunol 171: 5140-5147.

21. Stober D, Jomantaite I, Schirmbeck R, Reimann J (2003) NKT cells provide help for dendritic cell-dependent priming of MHC class I-restricted CD8+ T cells in vivo. J Immunol 170: 2540-2548.

22. Fujii S, Liu K, Smith C, Bonito AJ, Steinman RM (2004) The Linkage of Innate to Adaptive Immunity via Maturing Dendritic Cells In Vivo Requires CD40 Ligation in Addition to Antigen Presentation and CD80/86 Costimulation.J Exp Med 199: 1607-1618.

23. Hermans IF, Silk JD, Gileadi U, Masri SH, Shepherd D, et al. (2007) Dendritic cell function can be modulated through cooperative actions of TLR ligands and invariant NKT cells. J Immunol 178: 2721-2729.

24. Silk JD, Hermans IF, Gileadi U, Chong TW, Shepherd D, et al. (2004) Utilizing the adjuvant properties of CDld-dependent NK $\mathrm{T}$ cells in $\mathrm{T}$ cell-mediated immunotherapy. J Clin Invest 114: 1800-1811.

25. Kelly JM, Sterry SJ, Cose S, Turner SJ, Fecondo J, et al. (1993) Identification of conserved $\mathrm{T}$ cell receptor CDR3 residues contacting known exposed peptide side chains from a major histocompatibility complex class I-bound determinant. Eur J Immunol 23: 3318-3326.

26. Barnden MJ, Allison J, Heath WR, Carbone FR (1998) Defective TCR expression in transgenic mice constructed using cDNA-based alpha- and betachain genes under the control of heterologous regulatory elements. Immunol Cell Biol 76: $34-40$.

27. Kissenpfennig A, Henri S, Dubois B, Laplace-Builhe C, Perrin P, et al. (2005) Dynamics and function of Langerhans cells in vivo: dermal dendritic cells colonize lymph node areas distinct from slower migrating Langerhans cells. Immunity 22: 643-654.

28. Chen YH, Chiu NM, Mandal M, Wang N, Wang CR (1997) Impaired NK1+ T cell development and early IL-4 production in CD1-deficient mice. Immunity 6 : $459-467$.

29. Hoshino K, Takeuchi O, Kawai T, Sanjo H, Ogawa T, et al. (1999) Cutting edge: Toll-like receptor 4 (TLR4)-deficient mice are hyporesponsive to lipopolysaccharide: evidence for TLR4 as the Lps gene product. J Immunol 162: 3749-3752.

30. Lee A, Farrand KJ, Dickgreber N, Hayman CM, Jurs S, et al. (2006) Novel synthesis of alpha-galactosyl-ceramides and confirmation of their powerful NKT cell agonist activity. Carbohydr Res 341: 2785-2798.

31. Semmling V, Lukacs-Kornek V, Thaiss CA, Quast T, Hochheiser K, et al. (2010) Alternative cross-priming through CCL17-CCR4-mediated attraction of CTLs toward NKT cell-licensed DCs. Nat Immunol 11: 313-320. 
32. Kawano T, Cui J, Koezuka Y, Toura I, Kaneko Y, et al. (1997) CD1d-restricted and TCR-mediated activation of valpha14 NKT cells by glycosylceramides. Science 278: 1626-1629

33. Brossay L, Chioda M, Burdin N, Koezuka Y, Casorati G, et al. (1998) CD1dmediated recognition of an alpha-galactosylceramide by natural killer $\mathrm{T}$ cells is highly conserved through mammalian evolution. J Exp Med 188: 1521-1528.

34. Spada FM, Koezuka Y, Porcelli SA (1998) CDld-restricted recognition of synthetic glycolipid antigens by human natural killer T cells. J Exp Med 188: 1529-1534.

35. Tomura M, Yu WG, Ahn HJ, Yamashita M, Yang YF, et al. (1999) A novel function of Valphal4+CD4+NKT cells: stimulation of IL-12 production by antigen-presenting cells in the innate immune system. J Immunol 163: 93-101.

36. Kitamura H, Iwakabe K, Yahata T, Nishimura S, Ohta A, et al. (1999) The natural killer T (NKT) cell ligand alpha-galactosylceramide demonstrates its immunopotentiating effect by inducing interleukin (IL)-12 production by dendritic cells and IL-12 receptor expression on NKT cells. J Exp Med 189: $1121-1128$

37. Carnaud C, Lee D, Donnars O, Park S-H, Beavis A, et al. (1999) Cross-Talk Between Cells of the Innate Immune System: NKT Cells Rapidly Activate NK Cells. J Immunol 163: 4647-4650.

38. Taraban VY, Martin S, Attfield KE, Glennie MJ, Elliott T, et al. (2008) Invariant NKT cells promote CD8+ cytotoxic $\mathrm{T}$ cell responses by inducing CD70 expression on dendritic cells. J Immunol 180: 4615-4620.

39. Mescher MF, Curtsinger JM, Agarwal P, Casey KA, Gerner M, et al. (2006) Signals required for programming effector and memory development by CD8+ T cells. Immunol Rev 211: 81-92.

40. Bursch L, Wang L, Igyarto B, Kissenpfennig A, Malissen B, et al. (2007) Identification of a novel population of Langerin+ dendritic cells. J Exp Med 204: 3147-3156.

41. Takahara K, Omatsu Y, Yashima Y, Maeda Y, Tanaka S, et al. (2002) Identification and expression of mouse Langerin (CD207) in dendritic cells. Int Immunol 14: 433-444.

42. Sung SS, Fu SM, Rose CE, Jr., Gaskin F, Ju ST, et al. (2006) A major lung CD103 (alphaE)-beta7 integrin-positive epithelial dendritic cell population expressing Langerin and tight junction proteins. J Immunol 176: 2161-2172.

43. GeurtsvanKessel CH, Willart MA, van Rijt LS, Muskens F, Kool M, et al. (2008) Clearance of influenza virus from the lung depends on migratory langerin+CD11b- but not plasmacytoid dendritic cells. J Exp Med 205: 1621-1634.

44. Creusot RJ, Yaghoubi SS, Chang P, Chia J, Contag CH, et al. (2009) Lymphoid-tissue-specific homing of bone-marrow-derived dendritic cells. Blood 113: $6638-6647$
45. Harshyne LA, Watkins SC, Gambotto A, Barratt-Boyes SM (2001) Dendritic cells acquire antigens from live cells for cross-presentation to CTL. J Immunol 166: 3717-3723.

46. Thery C, Duban L, Segura E, Veron P, Lantz O, et al. (2002) Indirect activation of naive $\mathrm{CD} 4+\mathrm{T}$ cells by dendritic cell-derived exosomes. Nat Immunol 3: 1156-1162.

47. Fleeton MN (2004) Peyer's Patch Dendritic Cells Process Viral Antigen from Apoptotic Epithelial Cells in the Intestine of Reovirus-infected Mice. J Exp Med 200: $235-245$.

48. Lin ML, Zhan Y, Proietto AI, Prato S, Wu L, et al. (2008) Selective suicide of cross-presenting CD8+ dendritic cells by cytochrome c injection shows functional heterogeneity within this subset. Proc Natl Acad Sci U S A 105: 3029-3034.

49. Idoyaga J, Suda N, Suda K, Park CG, Steinman R (2009) Antibody to Langerin/CD207 localizes large numbers of CD8alpha+ dendritic cells to the marginal zone of mouse spleen. Proc Natl Acad Sci U S A 106: 1524-1529.

50. Qiu C, Miyake Y, Kaise H, Kitamura H, Ohara O, et al. (2009) Novel Subset of CD8 + Dendritic Cells Localized in the Marginal Zone Is Responsible for Tolerance to Cell-Associated Antigens. J Immunol 182: 4127-4136.

51. Li M, Davey GM, Sutherland RM, Kurts C, Lew AM, et al. (2001) Cellassociated ovalbumin is cross-presented much more efficiently than soluble ovalbumin in vivo. J Immunol 166: 6099-6103.

52. Shimizu K, Kurosawa Y, Taniguchi M, Steinman R, Fujii S (2007) Crosspresentation of glycolipid from tumor cells loaded with alpha-galactosylceramide leads to potent and long-lived T cell-mediated immunity via dendritic cells. J Exp Med 204: 2641-2653.

53. Schulz O, Reis e Sousa C (2002) Cross-presentation of cell-associated antigens by CD8alpha+ dendritic cells is attributable to their ability to internalize dead cells. Immunology 107: 183-189.

54. Barral P, Polzella P, Bruckbauer A, Rooijen Nv, Besra GS, et al. (2010) CD169+ macrophages present lipid antigens to mediate early activation of iNKT cells in lymph nodes. Nat Immunol. pp 1-12.

55. Finkelman FD, Lees A, Birnbaum R, Gause WC, Morris SC (1996) Dendritic cells can present antigen in vivo in a tolerogenic or immunogenic fashion. J Immunol 157: 1406-1414.

56. Bonifaz L, Bonnyay D, Mahnke K, Rivera M, Nussenzweig MC, et al. (2002) Efficient targeting of protein antigen to the dendritic cell receptor DEC-205 in the steady state leads to antigen presentation on major histocompatibility complex class I products and peripheral CD8+ T cell tolerance. J Exp Med 196: 1627-1638.

57. Bonifaz L (2004) In Vivo Targeting of Antigens to Maturing Dendritic Cells via the DEC-205 Receptor Improves T Cell Vaccination. J Exp Med 199: 815-824. 